\title{
Georg-August-University
}

Platz der Göttinger Sieben 5

37073 Göttingen

(Germany)

\section{A cross-cultural study of motivational factors and values influencing purchase of organic food in Germany and Mexico}

\author{
PhD Candidate: \\ Maria Isabel Juárez Hernández
}

Georg-August University

Prof. Dr. Achim Spiller
Georg-August University

Prof. Dr. Ludwig Theuvsen 


\section{Tables of Contents}

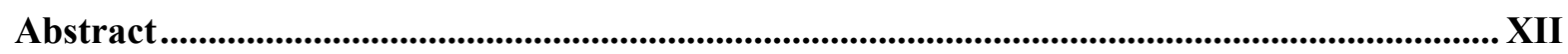

Kurzbeschreibung ....................................................................................................................

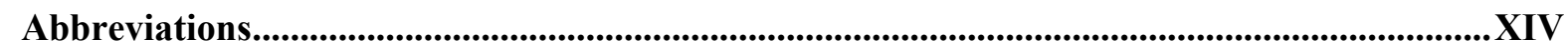

Acknowledgements............................................................................................................................................XVI

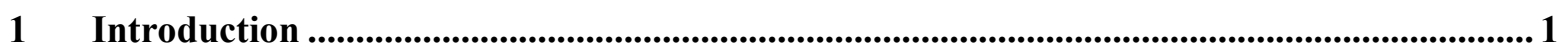

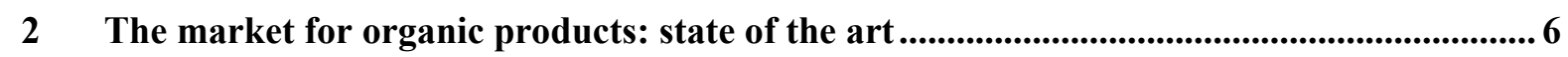

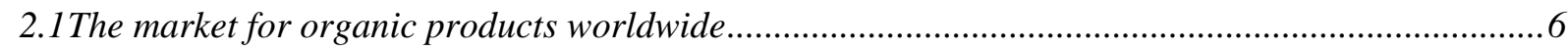

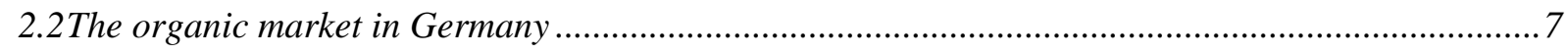

2.2.1 Historical factors that influenced the development of the organic market in

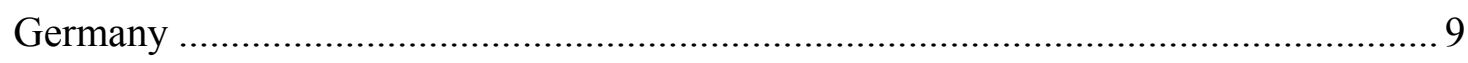

2.2.2 Political factors and national organic certification label ........................................ 10

2.2.3 Stage of the development of the market of organic food in Germany......................... 11

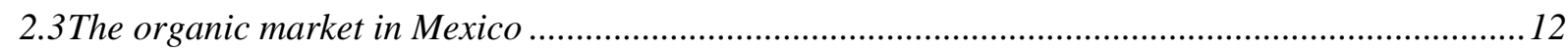

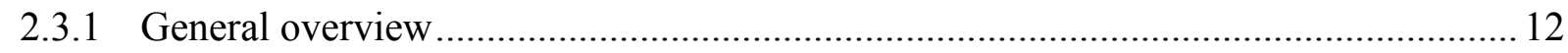

2.3.2 Social and economical implication of the export oriented scheme ........................... 13

2.3.3 Politics, programs and the consequences of the export orientation scheme................. 14

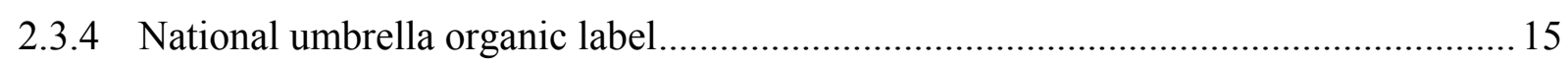

2.3.5 Stage of the market for organic food in Mexico ....................................................... 15

2.3.6 Distribution and mainstream retail channels of organic food.................................... 16

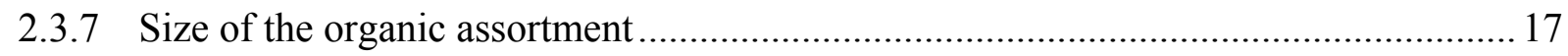

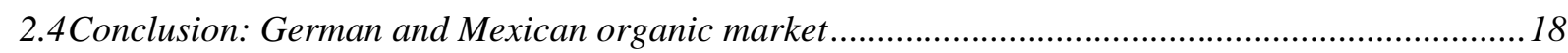

2.4.1 Future of organic market in Germany within conventionalization and

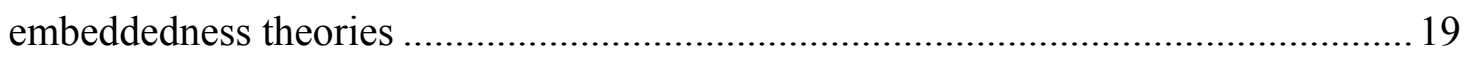

2.4.2 Future of organic market in Mexico within the export oriented and the PGS

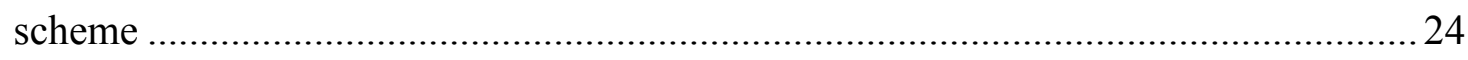

2.5Main features of the two organic markets to compare ...................................................................2 


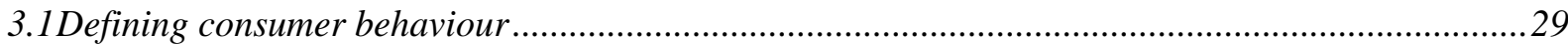

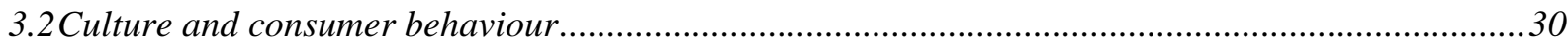

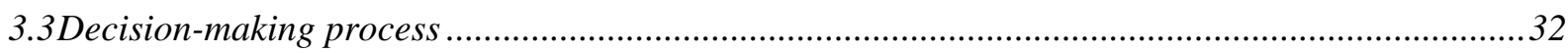

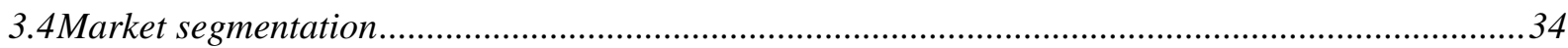

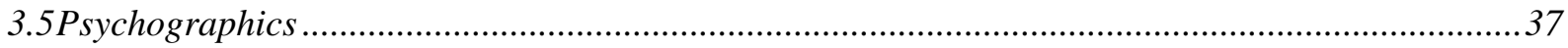

$4 \quad$ Theoretical framework of values and motivations .................................................................39

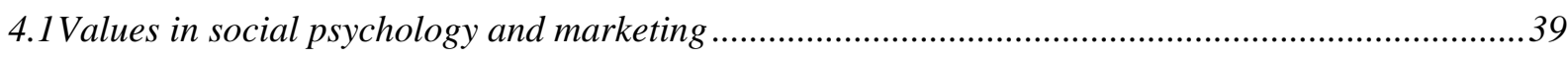

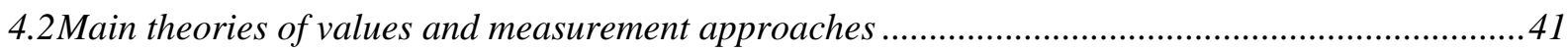

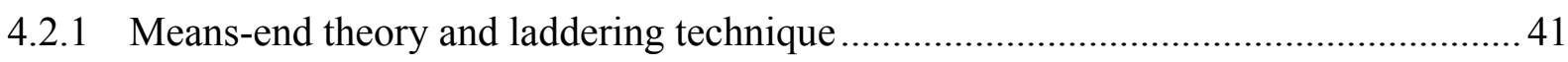

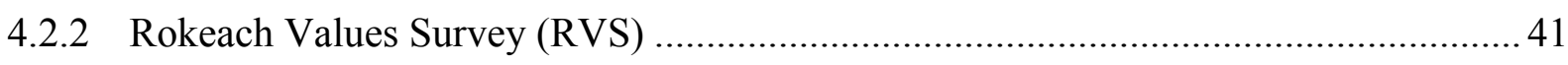

4.2.3 Schwartz's Value Inventory (SVI) or Schwartz's Value Survey (SVS) ..................... 43

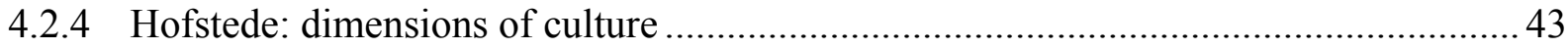

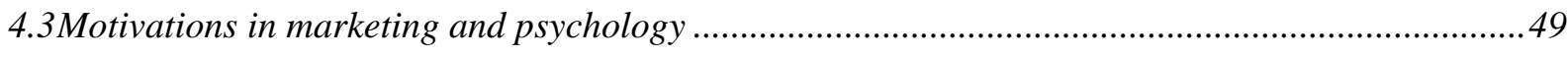

4.4Main theories of motivations and measurement approaches............................................................51

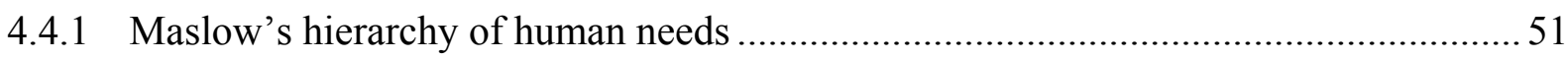

4.4.2 McClelland's theory: achievement, power, and affiliation ...................................... 52

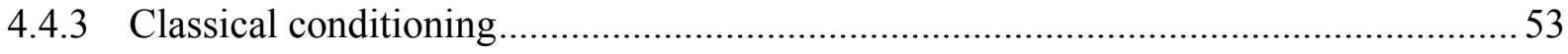

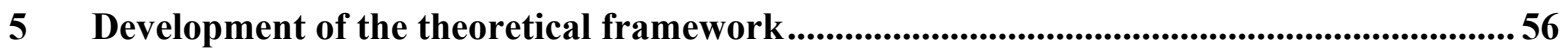

5.1International literature review on purchase of organic food.........................................................57

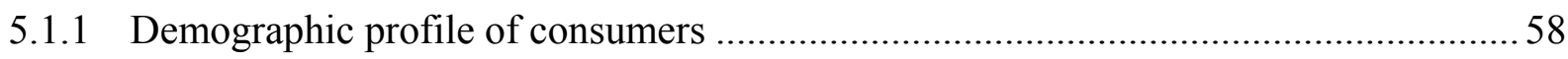

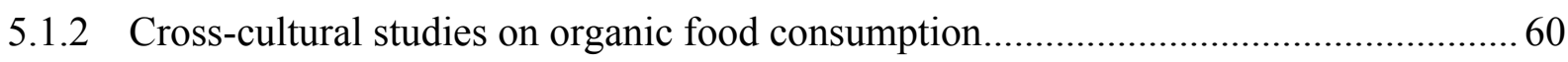

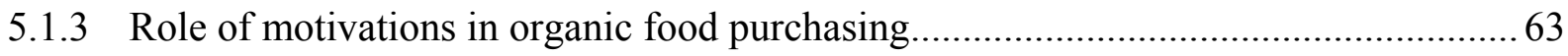

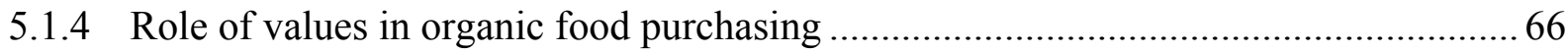

5.2Review of values and motivational measurement instruments in organic food studies .....................68

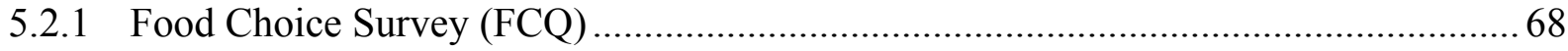

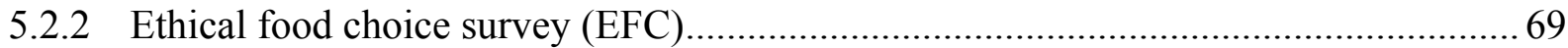

5.3Studies on organic food purchase which focus on Germany and Mexico.........................................70

5.4Situational assessment and development of research questions ....................................................72 
5.5.1 Theoretical constructs of motives to purchase organic food ..................................... 75

5.5.2 Theoretical constructs of values to purchase organic food....................................... 80

5.6Operationalization of the constructs: development and adaptation of previous measurement instruments

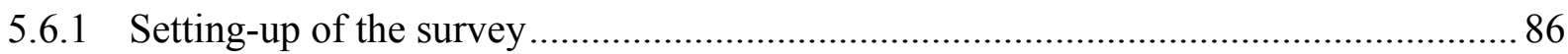

5.6.2 Conceptual considerations of cross-cultural studies............................................... 91

6 Pretesting the survey ............................................................................................................... 94

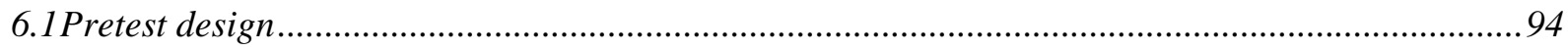

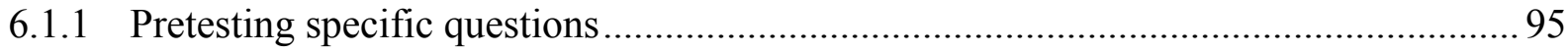

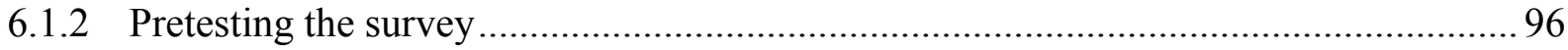

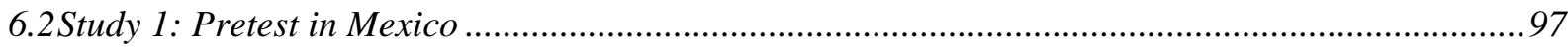

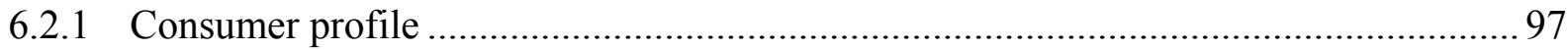

6.2.2 Supposed determinant attributes of the POS image: organic stores vs organic

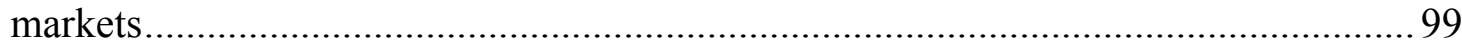

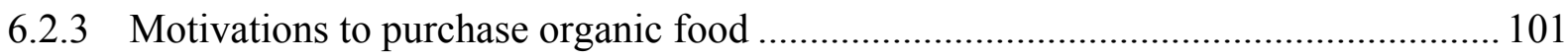

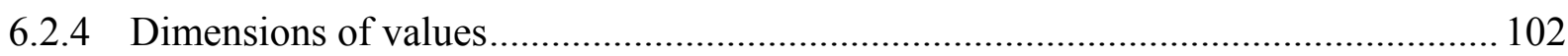

6.2.5 First conclusions: evaluation and refining of the survey ...................................... 103

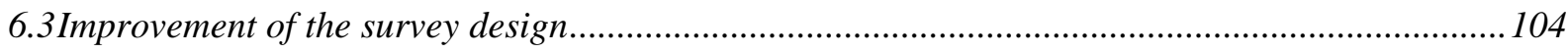

6.4Further literature review to improve the sections of motivations and values.................................105

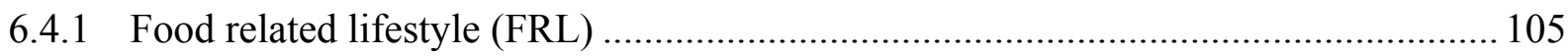

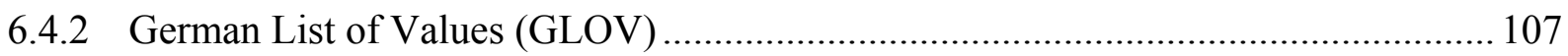

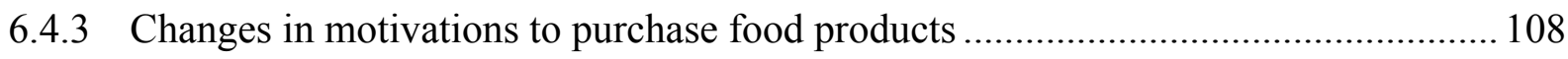

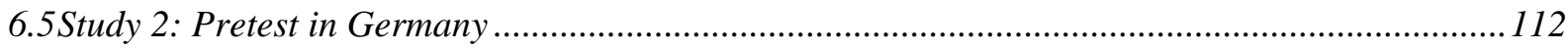

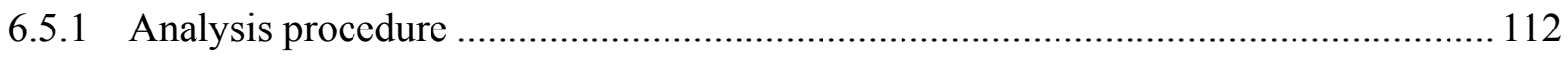

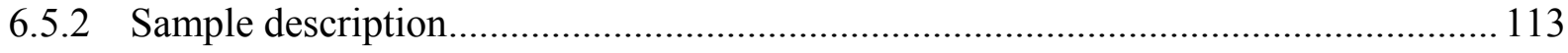

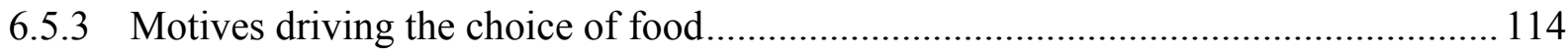

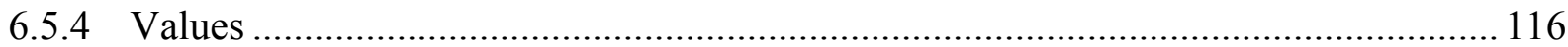

6.6Secondary conclusions: motivations are satisfactory and values are not all satisfactory..............119

6.7Final, optimised version of the cross-cultural survey .................................................................120 


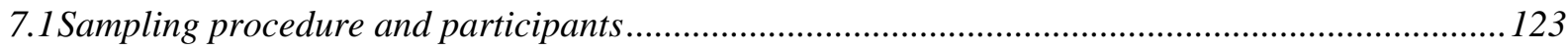

7.2Some remarks of measurement and methods ..............................................................................124

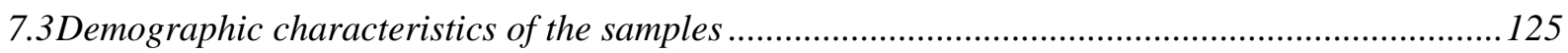

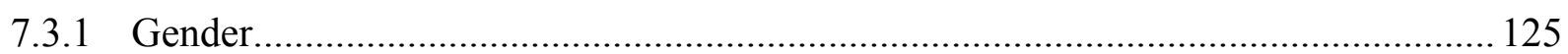

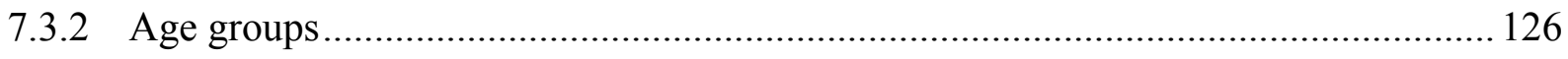

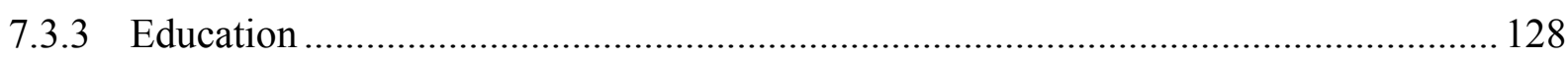

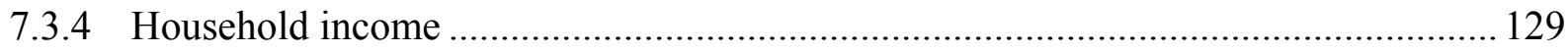

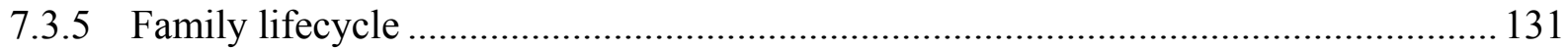

7.4Comparison of the frequency of motives in the purchase of food products between the German and

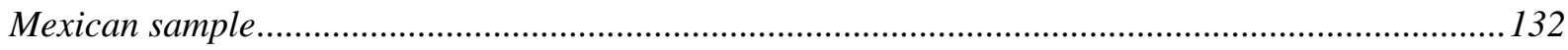

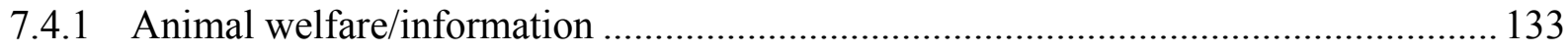

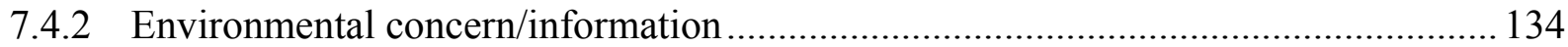

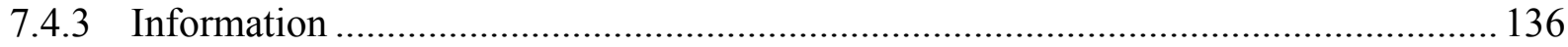

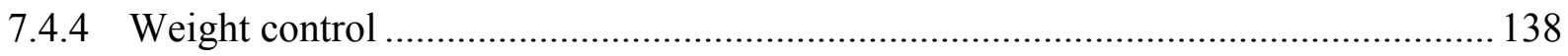

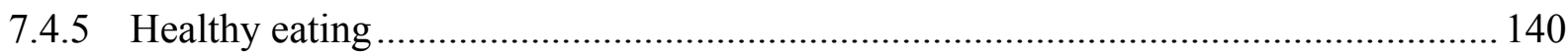

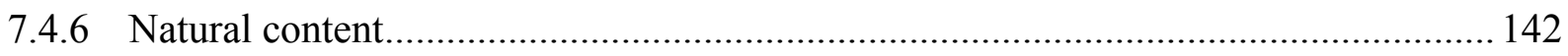

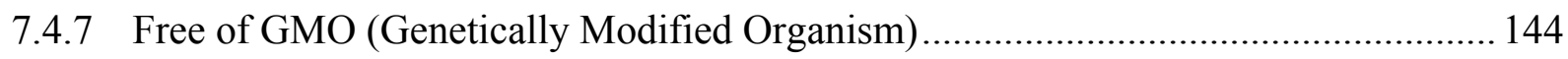

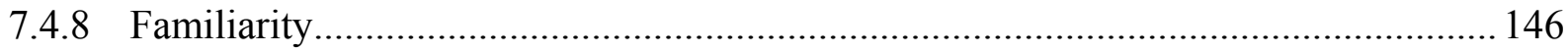

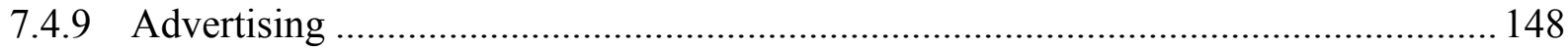

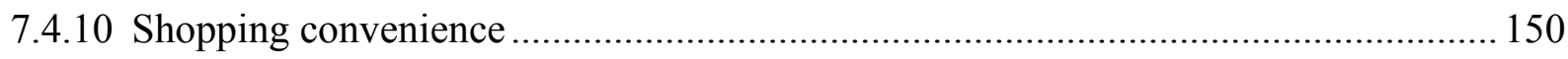

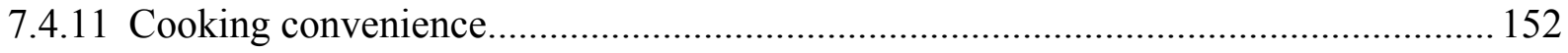

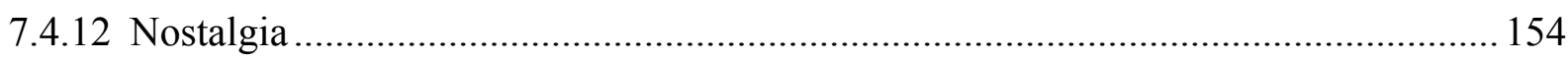

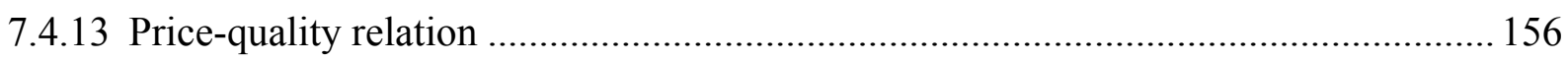

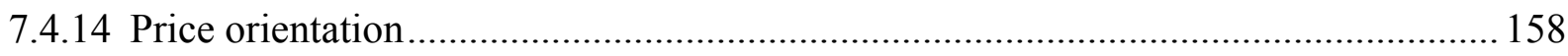

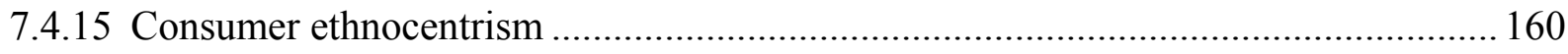

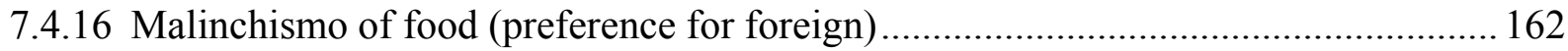

7.5Frequency of responses of values in the German and Mexican samples ......................................163

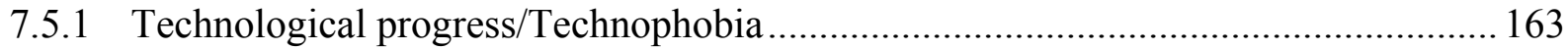

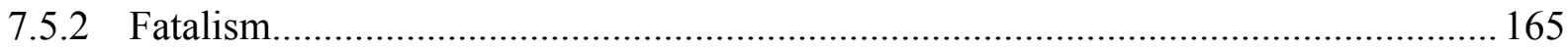

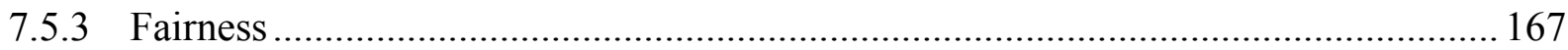

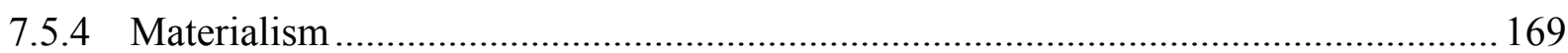


7.5.5 Variety in life/neophobia

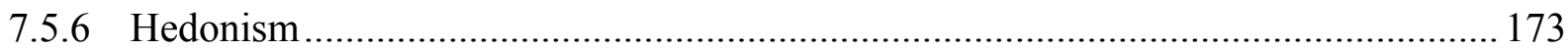

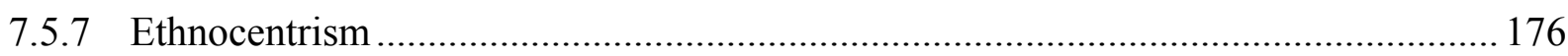

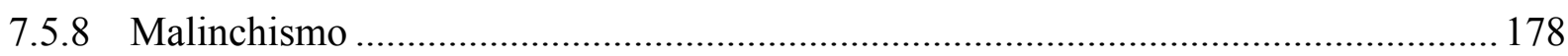

7.6Comparison of the average value priorities of groups of buyers and non-buyers of organic food in

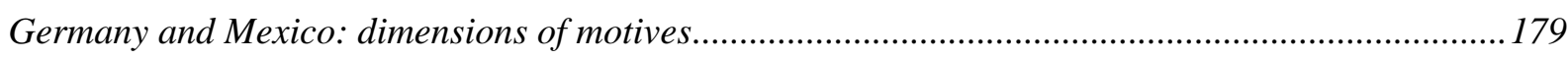

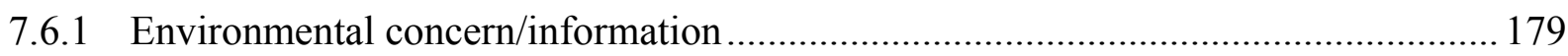

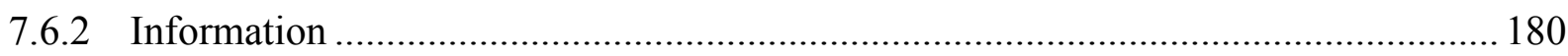

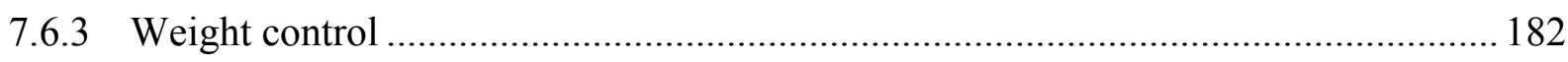

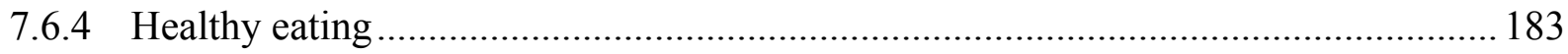

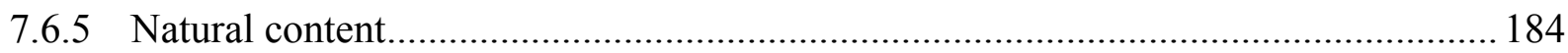

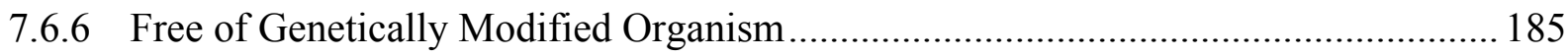

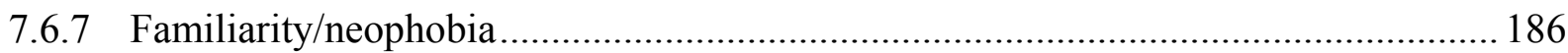

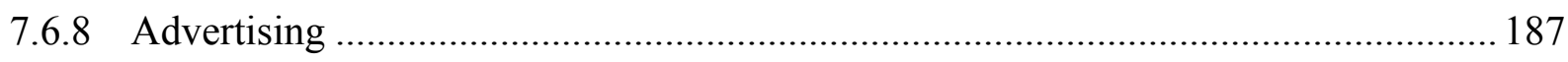

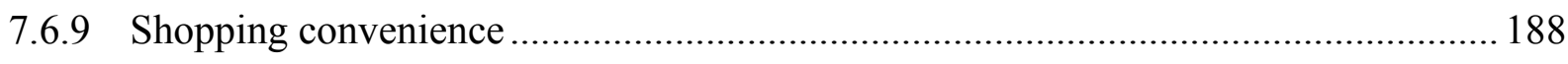

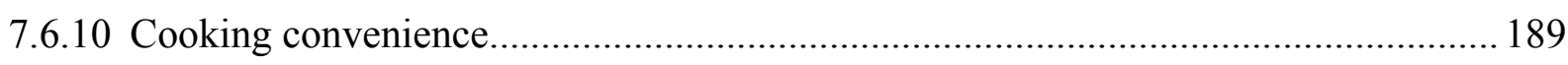

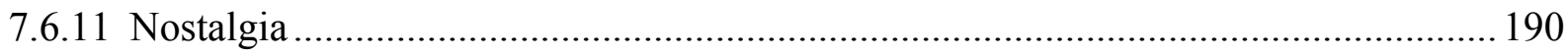

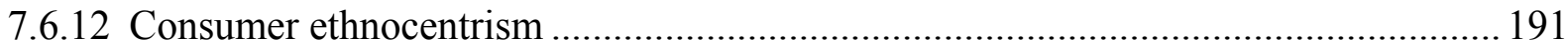

7.7Comparison of the average values priorities: dimensions of values...............................................193

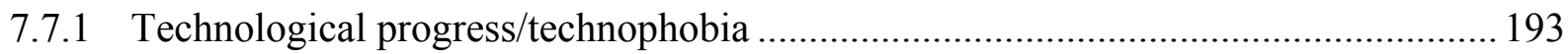

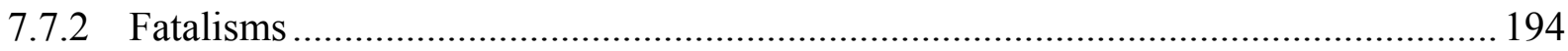

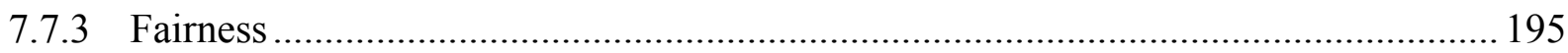

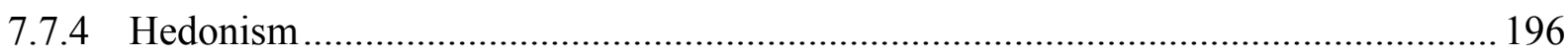

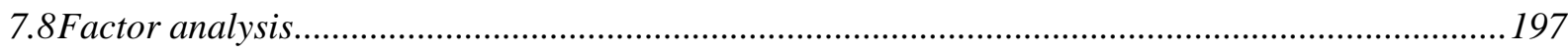

7.8.1 Exploratory factor analyses and construction of scales of motives related to

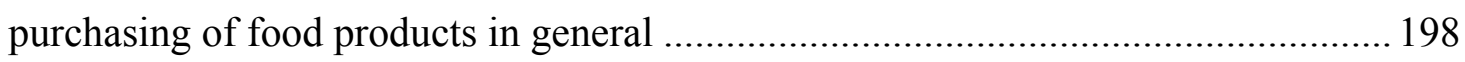

7.8.1.1 Exploratory factor analyses of weight control and healthy eating: German and

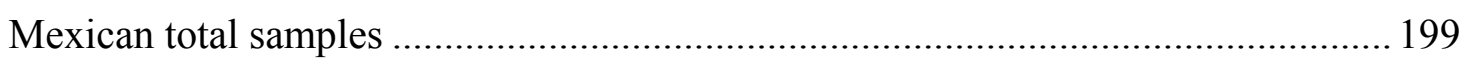

7.8.1.2 Exploratory factor analyses of the motives related to purchase of food products in general: German total sample

7.8.1.3 Exploratory factor analyses and construction of scales of motives related to purchase of food products in general: Mexican sample 
7.8.1.4 Exploratory factor analyses and construction of scales of values related to purchasing of food products in general: German sample

7.8.1.5 Exploratory factor analyses and construction of scales of values related to purchasing of food products in general: Mexican sample

7.8.2 Exploratory factor analysis of the motivations and values in the purchase of organic food

7.8.2.1 Exploratory factor analysis of the motivations in the purchase of organic food in Germany

7.8.2.2 Exploratory factor analysis of the motivations in the purchase of organic food in Mexico

7.8.2.3 Exploratory factor analyses and construction of index for the values to purchase organic food in the German sample

7.8.2.4 Exploratory factor analyses and construction of index for the values to purchase organic food in the Mexican sample

7.8.3 Multiple regression analysis

7.8.3.1 Common motives and values among the consumers of organic food in the German and Mexican samples: simple linear regression.

7.8.3.2 Motives and values in the purchase of organic food in Germany and Mexico:

MLR

7.8.4 Final discussion

8 Discussions and conclusions.

8.1Main findings on cross-cultural motives and values.

8.2Framework

8.3Limitations

8.4Guidelines for further research

References 


\section{List of figures}

Figure 1: Model of cross cultural comparison between Germany and Mexico.........................2

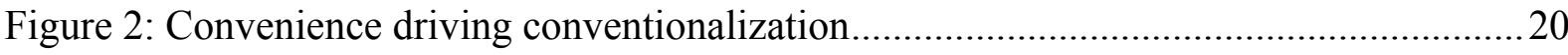

Figure 3: The "onion diagram": manifestations of culture at different levels of depth............44

Figure 4: Ranking of dimension of national culture in Germany and Mexico........................ 49

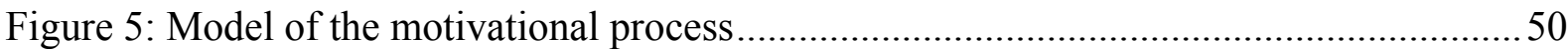

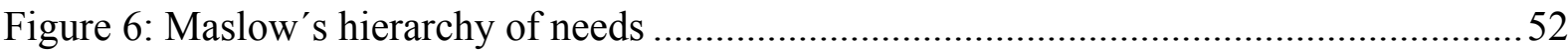

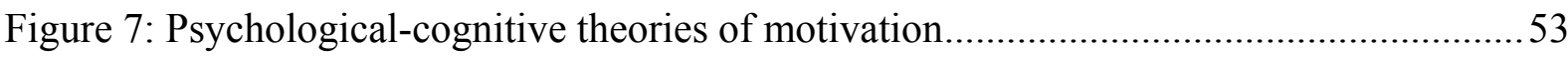

Figure 8: Conceptual model of motives of choice of organic food and domains of values in Mexico and Germany (explorative phase) ................................................... 75

Figure 9: Process of the development of multi-item scales measurement .............................. 84

Figure 10: Adaptation of the theoretical structure of relations among value domains.............86

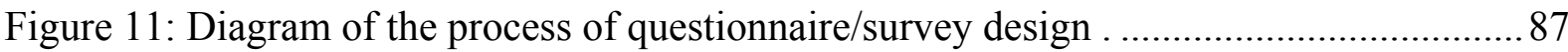

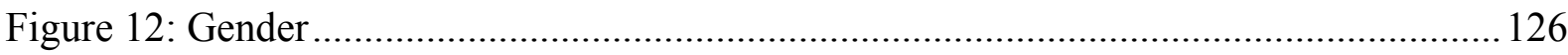

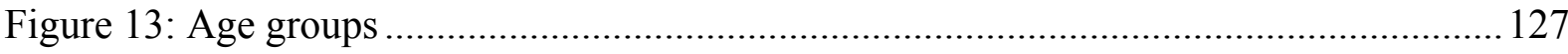

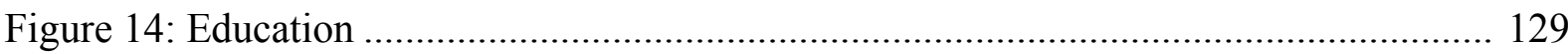

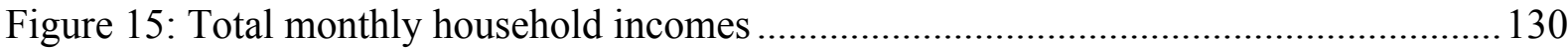

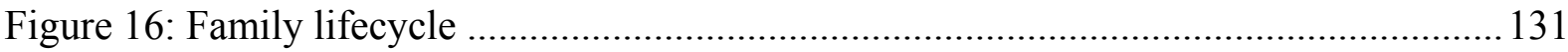

Figure 17: Dimension of animal welfare/information in the German sample....................... 133

Figure 18: Dimension of animal welfare/information in the Mexican sample...................... 134

Figure 19: Dimension of environmental concern/information in the German sample........... 135

Figure 20: Dimension of environmental concern/information in the Mexican sample.......... 136

Figure 21: Dimension of information in the German sample............................................ 137

Figure 22: Dimension of information in the Mexican sample............................................ 138

Figure 23: Dimension of weight control in the German sample ........................................ 139

Figure 24: Dimension of weight control in the Mexican sample ......................................... 140

Figure 25: Dimension of healthy eating in the German sample .......................................... 141

Figure 26: Dimension of healthy eating in the Mexican sample ......................................... 142

Figure 27: Dimension of natural content in the German sample....................................... 143

Figure 28: Dimension of natural content in the Mexican sample........................................ 144

Figure 29: Dimension of free of GMO in the German sample........................................... 145 


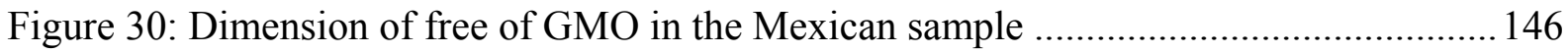

Figure 31: Dimension of familiarity in the German sample............................................. 147

Figure 32: Dimension of familiarity in the Mexican sample.............................................. 148

Figure 33: Dimension of advertising in the German sample ........................................... 149

Figure 34: Dimension of advertising in the Mexican sample............................................ 150

Figure 35: Dimension of shopping convenience in the German sample ............................. 151

Figure 36: Dimension of shopping convenience in the Mexican sample............................. 152

Figure 37: Dimension of cooking convenience in the German sample............................... 153

Figure 38: Dimension of cooking convenience in the Mexican sample................................ 154

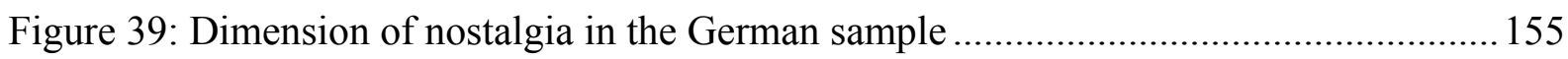

Figure 40: Dimension of nostalgia in the Mexican sample ............................................... 156

Figure 41: Dimension of price-quality relation in the German sample ............................... 157

Figure 42: Dimension of price-quality relation in the Mexican sample............................... 158

Figure 43: Dimension of price orientation in the German sample ...................................... 159

Figure 44: Dimension of price orientation in the Mexican sample ...................................... 160

Figure 45: Dimension of consumer ethnocentrism in the German sample ........................... 161

Figure 46: Dimension of consumer ethnocentrism in the Mexican sample ........................... 162

Figure 47: Dimension of malinchismo of food in the Mexican sample ................................ 163

Figure 48: Dimension of technological progress/technophobia in the German sample......... 164

Figure 49: Dimension of technological progress/technophobia in the Mexican sample........ 165

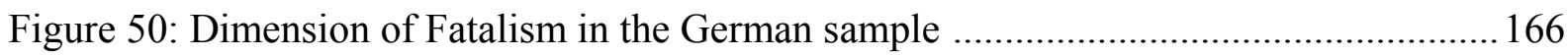

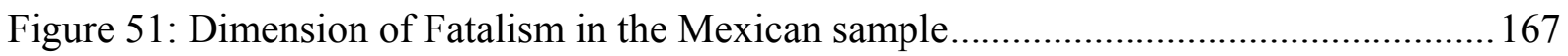

Figure 52: Dimension of fairness in the German sample .................................................. 168

Figure 53: Dimension of fairness in the Mexican sample ................................................. 169

Figure 54: Dimension of materialism in the German sample............................................ 170

Figure 55: Dimension of materialism in the Mexican sample............................................. 171

Figure 56: Dimension of variety in life/neophobia in the German sample ............................. 172

Figure 57: Dimension of variety in life/neophobia in the Mexican sample .......................... 173

Figure 58: Dimension of hedonism in the German sample ................................................. 174

Figure 59: Dimension of hedonism in the Mexican sample............................................. 175

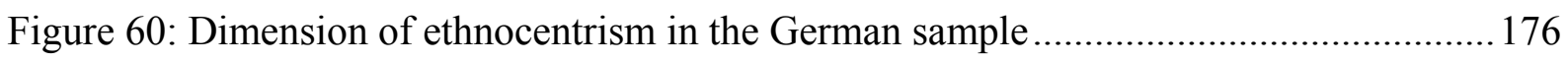

Figure 61: Dimension of ethnocentrism in the Mexican sample......................................... 178

Figure 62: Dimension of malinchismo in the Mexican sample.......................................... 179 
Figure 63: Environmental concern/information - average value priorities of group. 180

Figure 64: Information - average value priorities of group 181

Figure 65: Weight control - average value priorities of group .......................................... 182

Figure 66: Healthy eating - average value priorities of group ........................................... 183

Figure 67: Natural content - average value priorities of group .......................................... 184

Figure 68: Free of GMO - average value priorities of group ........................................... 186

Figure 69: Familiarity/neophobia - average value priorities of group ................................ 187

Figure 70: Advertising - average value priorities of group .............................................. 188

Figure 71: Shopping convenience - average value priorities of group............................... 189

Figure 72: Cooking convenience - average value priorities of group .................................. 190

Figure 73: Nostalgia - average value priorities of group................................................. 191

Figure 74: Consumer ethnocentrism - average value priorities of group ............................. 192

Figure 75: Technological progress - average value priorities of group............................... 194

Figure 76: Fatalism - average value priorities of group .................................................... 195

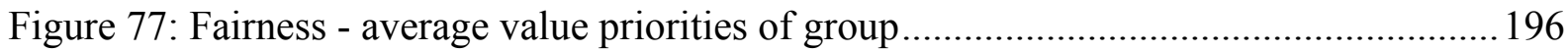

Figure 78: Hedonism - average value priorities of group..................................................... 197

Figure 79: Motivations and values related to the purchasing of food products and

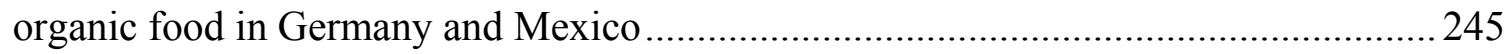

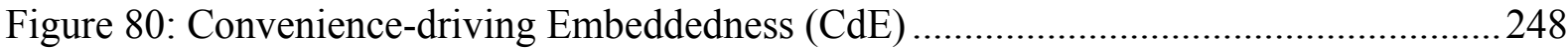




\section{List of Tables}

Table 1: Organic farming figures in Germany, Mexico and worldwide 2008 ........................ 7

Table 2: Development of the total turnover, total outlets and store formats in Germany 19952005

Table 3: Economic relevance of the organic produce in Germany, 1999-2008 ….................. 10

Table 4: Recent trends in Germany's organic marketing channels ....................................... 12

Table 5: Economic relevance of the organic produce in Mexico, 1996-2007/2008................ 13

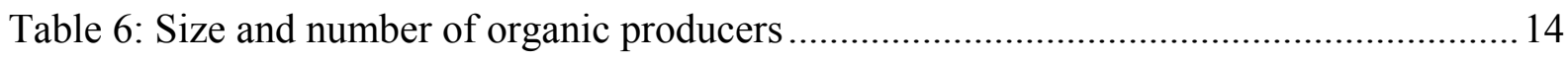

Table 7: Organic assortments in food retail channels in Mexico .......................................... 18

Table 8: Main features of the two organic markets to compare ........................................... 28

Table 9: Distinction between the desired and the desirable and associated distinctions..........46

Table 10: Hofstede's dimension of values across German and Mexican national culture....... 48

Table 11: Demographic features of organic food consumers................................................58

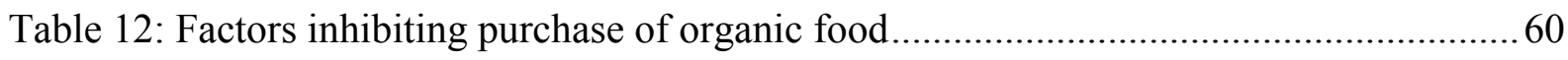

Table 13: Ranking of motivations for purchase of organic food.......................................... 65

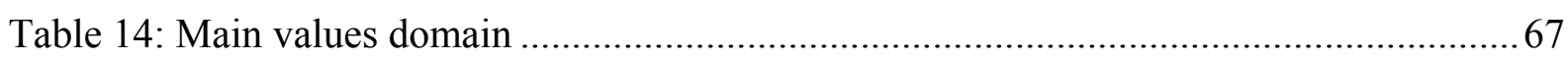

Table 15: Definition of variables included in the model ...................................................... 91

Table 16: Demographic profile of consumers of organic food in Mexico .............................. 99

Table 17: Supposed determinant attributes of the POS image (percent) ............................... 100

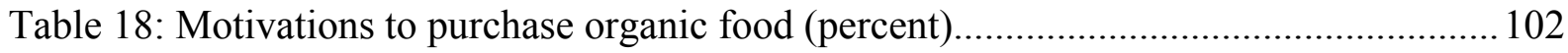

Table 19: Most frequent responses provided for the dimension of values............................ 103

Table 20: Conceptual framework of the dimensions included in the original FRL ............... 106

Table 21: Changes in the measurement of the theoretical constructs of motives and values in

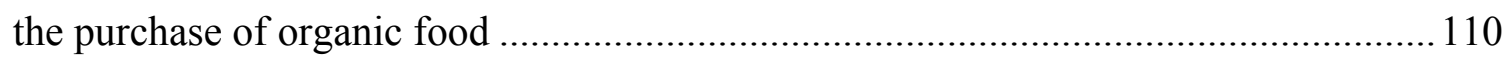

Table 22: Demographic profile of the consumers of organic food in Germany..................... 114

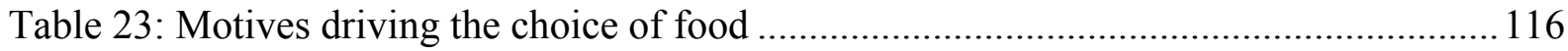

Table 24: Measuring of values with 7-point Likert-type scale (first measurement scale) ..... 118

Table 25: Mentioned importance of values (second measurement scale) .............................. 119

Table 26: Changes in the theoretical constructs of motivations and values driving the choice

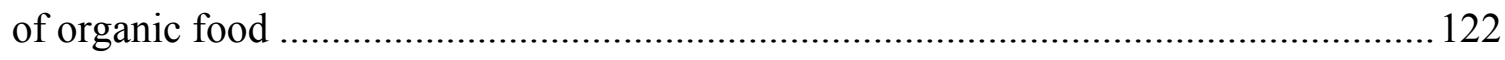


Table 27: Exploratory factor analyses of weight control and healthy motives related to purchase food products: German consumers and non-buyers of organic food.

Table 28: Exploratory factor analyses for the weight control and healthy motives related to purchase food products: Mexican consumers and non-buyers of organic food 200

Table 29: Exploratory factor analyses of the motives related to purchase food products: German non-buyers and consumers of organic food..... 201

Table 30: Exploratory factor analyses for the motives related to purchase food products: Mexican consumers and non-buyers of organic food. 204

Table 31: Exploratory factor analyses of values related to purchasing of food: German sample 206

Table 32: Exploratory factor analysis of values related to purchasing of food: Mexican sample 208

Table 33: Exploratory factor analysis for the motivations to purchase organic food in Germany

Table 34: Exploratory factor analysis for the motivations to purchase organic food in Mexico

Table 35: Exploratory factor analysis for values of German consumers of organic food......216

Table 36: Exploratory factor analysis of values of Mexican consumers of organic food .....218

Table 37: Dimension of motives related to the purchasing of food products and organic food in Germany and Mexico..... 219

Table 38: Dimension of values related to the purchasing of food products and organic food in Germany and Mexico 220

Table 39: Relationships between the independent variables and organic diet in Germany ...221 Table 40: Variance explained by the motivations to purchase organic food in Germany and Mexico 222

Table 41: Relationships between the independent variables and organic diet in Germany ...224

Table 42: Variance explained by factors in organic diet in Germany ..... 226

Table 43: Factors predicting organic diet in Germany 227

Table 44: Relationships between the independent variables and organic diet in Mexico..... 229

Table 45: Variance explained by factors in organic diet in Mexico. 230

Table 46: Factors of motives and values predicting an organic diet in Mexico 231 


\section{Abstract}

Keywords: consumer behavior, cross-cultural, motives, values, organic food.

The present dissertation compares the motives and values associated with the purchase of organic food in Germany and in Mexico. After an international literature review enough evidence suggests that in developed and someone developing countries motivations to purchase organic food are similar among the consumers. However, there is no evidence supporting this fact in Mexico. In most of the marketing literature culture appears as an external factor influencing consumer behavior. Moreover; in the present research culture seems useful to understand the motives and values in the purchase of organic food. Firstly, an overview of the stages of the market for organic food in Germany and Mexico will be given. Secondly, there will be a literature review of consumer behavior, psychographics, the main motivations, and values when it comes to the purchase of organic food. Thirdly, the methodological framework which contains the cross-cultural approach to identify the motives and values related to purchasing of organic food. After an exploratory factor analysis (EFA) two common factors of motives and one value were carried out. The factor conglomerating the motives of healthy, tasty, animal welfare, concern with environment, and fairness seem as stronger predictor associated with the purchase of organic food in both countries. Within the insights interestingly cultural differences on consumer behavior were uncover. 


\section{Kurzbeschreibung}

Stichwörter: Konsumentenverhalten, interkulturelle Unterschiede, Motivation, Werte, Biolebensmittel.

Die vorliegende Dissertation vergleicht die Motive und Werte für den Kauf von Biolebensmitteln in Deutschland und Mexiko. Die durchgeführte internationale Literaturrecherche gibt ausreichend Hinweise darauf, dass in entwickelten Staaten sowie Entwicklungsländern die Beweggründe und Motivationen zum Kauf von Biolebensmitteln bei den Konsumenten ähnlich sind. Ein vergleichbares Verhalten bei mexikanischen Verbrauchern konnte bis dato nicht nachgewiesen werden.

In der Marketingliteratur wird Kultur häufig als externer Faktor verstanden, der das Verbraucherverhalten beeinflusst. Auch in der vorliegenden Arbeit wird Kultur als sinnvoll und essentiell angesehen, um die Motive sowie Werte, die zum Kauf von Biolebensmitteln führen, zu verstehen.Zunächst wird ein Überblick über die Phasen des Biomarktes in Deutschland und Mexiko gegeben. Anschließend folgt ein Überblick über das generelle Konsumverhalten, psychographische Faktoren sowie die wichtigsten Motive und Werte, die den Kauf von Biolebensmitteln beeinflussen.

Des Weiteren wird näher auf den „,methodologischen Rahmen“ eingegangen, der durch seinen interkulturellen Ansatz die Motive und Werte, die den Kauf Biolebensmitteln forcieren, analysiert.

Anhand einer explorativen Faktoranalyse kristallisieren sich zwei gemeinsame Motiffaktoren sowie ein Wertefaktor heraus. Der Faktor, der die Motive: Gesundheit, Geschmack, Umweltbewusstsein, Tierschutz sowie Fairness umfasst, kann als starker Prädiktor für den Kauf von Biolebensmitteln in beiden Ländern gesehen werden.

Im Rahmen der Arbeit werden zudem auch kulturelle Unterschiede hinsichtlich des Verbraucherverhaltens in Deutschland und Mexiko aufgezeigt. 


\section{Abbreviations}

Community Supported Agriculture (CSA)

Consolidated Metropolitan Statistical area (CMSA)

Exploratory Factor Analysis (EFA)

Ethical Food Choice Survey (EFC)

European Social Survey (ESS)

Food Choice Survey (FCQ)

Food Related Lifestyle (FRL)

Food Supply Chain (FSC)

Genetically Modified Organisms (GMO)

German List of Values (GLOV)

List of Values (LOV)

Means-End Chain (MEC)

Metropolitan Statistical Area (MSA)

Multiple Linear Regression (MLR)

Multidimensional Health Locus Control (MHLOC)

Organic Trade Association (OTA)

Participatory Guarantee System (PGS)

Principal Component Analysis (PCA)

Primary Metropolitan Statistical Area (PMSA)

Point of Sale (POS)

Rokeach Values Survey (RVS)

Schwartz's Value Inventory (SVI)

Schwartz's Value Survey (SVS)

Short Schwartz Value Survey Short SVS (SSVS) 
Socially Desirable Responses (SDR)

Uncertainty Avoidance Index (UAI)

United State Department Agriculture (USDA)

Values and Life Style System (VALS) 


\section{Acknowledgements}

I would like to dedicate my words of thanks to all those people who have directly and indirectly contributed to this dissertation.

I am very grateful to Professor Dr. Achim Spiller for believing in my capabilities, allowing me a lot of freedom, and providing valuable input into developing and improving the quality of this dissertation as well as my quality as a researcher. I am also very grateful to him for providing me with such fascinating material to investigate. As a student in a foreign country, I also would like to thank to Petra Geile, Wendy Boyle and my colleagues for their support! For this, I am very grateful! I am very grateful to my whole family and my entire friend for all their words, encourage and support in all the moments.

It is worth mentioning that without the financial support by the German Academic Exchange Agency (DAAD) the conclusion of this would not be possible. 


\section{$1 \quad$ Introduction}

Although displaying a slower place than in the past the organic market is still showing a 'healthy growth' (Biovista, 2009). This dissertation is dedicated to the marketing of organic food. Organic farming is defined as "a production system that sustains the health of soils, ecosystems, and people. It relies on ecological processes, biodiversity, and cycles adapted to local conditions, rather than the use of inputs with adverse effects. Organic agriculture combines tradition, innovation, and science to benefit the shared environment and promote fair relationships and a good quality of life for all involved" (IFOAM, 2010).

The main aim of this dissertation is to discover cultural differences and similarities on the motivations and values with respect to purchase of organic food in Germany and Mexico. To this aim, culture is a key concept to understand the behavior of consumers in the two countries. Culture has been defined in different ways and by several disciplines for instances, Hofstede (2001) defines culture "as the collective programming of the mind that distinguishes the members of one group or category of people from another". Thus, when we compare cultures we compare individuals of a society, e.g., Mexican buyers and non-buyers of organic food with individuals belonging to other societies, e.g. German buyers and non-buyers of organic food.

As seen within this dissertation, motives and values were presupposed as proxies for measuring cultural dimensions affecting consumer behavior. Motives or motivations are defined as "an internal state of an organism that drives it to behave in a certain way" (Mooij, 2004). On the other hand, values, are defined as "an enduring belief that one mode of conduct or end-state of existence is preferable to an opposing mode of conduct or end-state of existence" (Rokeach, 1973 cited in Mooij, 2004). 


\section{Figure 1: Model of cross cultural comparison between Germany and Mexico}

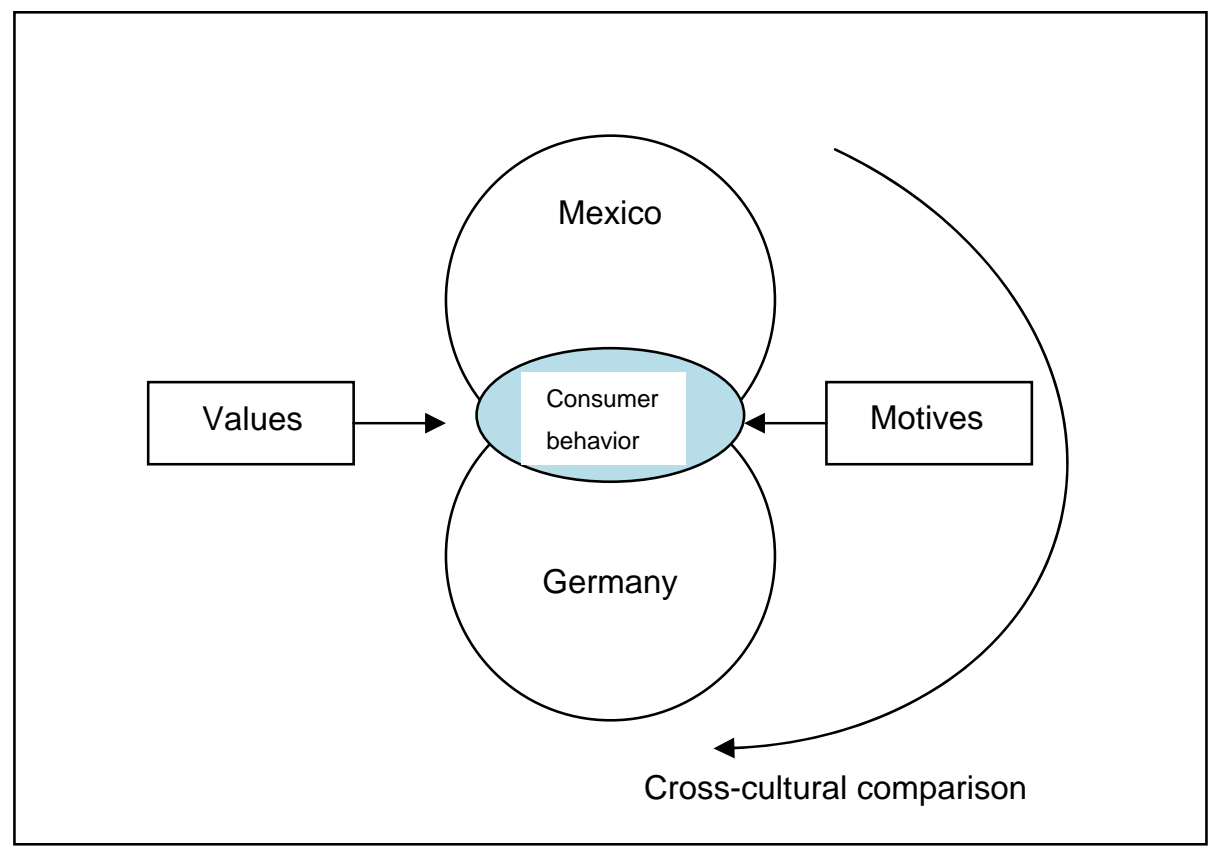

As the title of this dissertation suggests, the intention of this study is to trace a cross-cultural comparison between Germany and Mexico. The following will explain this approach in detail. Both the term "cross-cultural" and "comparison" presuppose the use of comparative research. In social sciences comparative research is used to understand complex realities. In fact, when we compare culture, nations, groups or countries we can distinguish between local and particular conditions and the commonalities shared by different groups, countries, or nations (Harkness et al., 2003). In comparative research not only differences or commonalities between social phenomena are uncovered, even unique aspects that are superficially impossible to detect are revealed (Mills et al., 2006).

Furthermore, even though the potentially invaluable usefulness of comparative research is well known, it has been underutilized in a few cross cultural or cross-national studies that have been conducted for several reasons; some of them are its financially costly and takes a considerable amount of time; it is difficult to do, and in methodological terms raise sometimes more interpretative problems and questions than they solve (Kohn 1989 cited by Harkness et al., 2003). Occasionally external factors such as political barriers to collecting data or publishing findings have to be considered also as limiting factors (Harkness et al., 2003). 
Comparative research cover quantitative (i.e. cross-cultural survey, average value priorities of groups, scores of individuals (person) and groups (national) levels) and qualitative methods (i.e. historical narratives, hermeneutic analysis are used). The number of disciplines using comparative methods is increasing, for instance, comparative sociology, comparative political sciences, intercultural communication, transcultural psychiatry, cross cultural psychology, and cross-cultural marketing (Harkness et al., 2003).

Moreover, as we stated before our comparative research is focused on cross-cultural dimensions. Kluckhohn (1951) defines culture as "patterned ways of thinking, feeling and reacting, acquired and transmitted mainly by symbols, constituting the distinctive achievement of human groups, including their embodiments in artifacts, the essential core of culture consists of traditional (i.e., historically derived and selected) ideas and especially their attached values".

As mentioned, when we compare culture we compare individuals of a society with individuals belonging to another society. As will be seen in more detail in the literature review and theoretical framework in Chapter 3, cross-cultural research can be established as withinsystem (sub-groups belonging to the same society) or between-system comparisons (different societies, groups nations or countries). In all these cases, is presupposes the existence of something to compare, this means something shared by the groups to compare.

More recently, in marketing the theoretical framework of cross-cultural psychology has been used to understand consumer behaviour, on the grounds of the roots of culture influence on the minds of consumers and habits of the past which often might predict practices and future behaviour. Moreover, it has been used in product branding to standardize operations and brands of multinational companies, advertising, and market segmentation (Mooij, 2004). As in other disciplines, qualitative and quantitative methods have been used in cross- cultural marketing (i.e. the Emic and Ethic approach, measuring of values, average values priorities of groups, Mean-End Theory, and searching for similarities and differences).

Finally, this dissertation focuses on two countries, Germany and Mexico, which could not differ more in terms of the development stage of the organic market. In fact, as seen in Chapter 2, Germany is a mature market with a long tradition of purchasing of organic food and one of the largest import countries of organic fresh food and raw materials. On the 
contrary, in Mexico organic farming is of paramount importance, though, mainly on the export level.

So why focus on two countries which are so different? As Stuart Mill (1843) prescribed with regard to the choice of cases and variables in cross-country studies there are two main principles which can be chosen: either concordance or difference. Thus, in this dissertation the "most different system design" by Przeworski and Teune (1970) was used as approach in our study with advantages of better understanding cultural dimension influencing the purchase of organic food in domestic markets within different stages of development. The main idea is that a comparative approach between the two countries could help both German and Mexican marketers and operators to develop marketing strategies according to the expectations of consumers to improve their businesses as well as to generate important insights into this sector.

As stated above, the general objectives of this dissertation are two-fold: firstly, we looked for differences of the motives to purchase organic food, and secondly, differences of the values of consumers of organic food in Germany and Mexico.

This dissertation is based upon a theoretical and an empirical study. The theoretical chapters support the basic knowledge of our cross-cultural comparison of the organic market of Germany and Mexico. The results and findings are described in the last part immediately previously to the conclusions. Below is a brief synopsis of the chapters.

Chapter 1 introduced the topic, explained the main objectives, and presented the structure of the dissertation.

In Chapter 2 the state of the art of the organic market in both countries, Germany and Mexico is discussed and the main issues related to the embededdness and conventionalization theories which form the current debate on the future of the organic market was examined.

In Chapters 3 and 4 a multidisciplinary overview and theoretical framework on motives and values are provided.

Chapter 5 presents both the relevant literature on motives and values related to the purchasing of organic food as well as the theoretical framework of our study. 
Chapter 6 explains the methodological considerations related to the results of the comprehensive pre-testing conducted in Mexico in 2008 and in Germany in 2009.

In Chapter 7 are presented the main results and insights of the findings of the online surveys in Germany and Mexico.

Finally, in Chapter 8 the main findings are discussed and positionated in a conceptual framework which can be taken as a point of departure for further research. 


\section{The market for organic products: state of the art}

In this chapter an overview of organic produce will be provide. Firstly, the relevance of organic farming for farmers, operators, and total turnover in the worldwide, Germany and Mexico is show by figures. Secondly, I focus the overview on the development of the German market which is one of the most developed and mature market for organic produce and the Mexican market which is an emergent market and focused on the export oriented scheme. Finally, in the part of conclusions I turn the discussion of the two markets and within conventionalization and embeddedness theories.

\subsection{The market for organic products worldwide}

Organic agriculture has developed rapidly in the recent past. Production, demand, and consumption of organic products have increased in most countries. Global sales of organic food and beverages have increased by 43\% from $\$ 23$ billion US (17.8 billion Euros) in 2002, to $\$ 46$ billion US in 2007 (Willer \& Yussefi, 2009). Until 2002, organic farming was practice in 130 countries dedicated to certified organic produce; approximately 90 are developing countries (Fotopoulos \& Krystallis, 2002). In 2009, Willer \& Yuseffi (2009) reported that organic agriculture is practiced in 141 countries.

Although in most countries around the world is practiced organic agriculture, demand for organic produce remains concentrated in Europe and North America. These two main areas are experiencing undersupply, because local production does not meet their demand (Willer \& Yussefi, 2007). Consequently, trade in organic food across and between continents is increasing, including exports from developing countries. Therefore, governments in developing countries have increased programs to support exports of organic produce rather than improving sales on local markets (Hall \& Mogyorody, 2001). However, an increase of the regional markets in developing countries is expected with economic development and more educated and affluent middle class consumers (Sinus Sociovision, 2006; Wier \& Calverley, 2002; Willer \& Yussefi, 2004). 
Table 1: Organic farming figures in Germany, Mexico and worldwide 2008

\begin{tabular}{|l|r|r|r|l|l|}
\hline & Germany & Europe & \multicolumn{1}{l|}{ Mexico } & \multicolumn{1}{l|}{$\begin{array}{l}\text { Latin } \\
\text { America }\end{array}$} & \multicolumn{1}{l|}{ World } \\
\hline $\begin{array}{l}\text { Total } \\
\text { operators }\end{array}$ & $18,703^{\mathrm{c}}$ & $213,297^{\mathrm{b}}$ & $128,819^{\mathrm{e}}$ & $220,000^{\mathrm{e}}$ & $1,219,526^{\mathrm{b}}$ \\
\hline $\begin{array}{l}\text { Total } \\
\text { hectares (ha) }\end{array}$ & $865,336^{\mathrm{c}}$ & $7,800,000^{\mathrm{b}}$ & $403,268^{\mathrm{d}}$ & $6,400,000^{\mathrm{e}}$ & $32,221,311^{\mathrm{b}}$ \\
\hline $\begin{array}{l}\text { Turnover } \\
\text { (billions } € \text { ) }\end{array}$ & $5.85^{\mathrm{a}}$ & $14.5^{\mathrm{d}}$ & n.d. & n.d. & $30^{\mathrm{b}}$ \\
\hline
\end{tabular}

Source: Hamm et al., 2009 ; Willer \& Yussefi, 2009 ; Oekolandbau, 2008 ${ }^{\mathrm{c}}$; Greenplanet.net, 2009 (www.greenplanet.net/biologico-a-biodinamico/19958-biologico-in-germania-oom.html [February, 14 2009]); Sinab, $2007^{\mathrm{e}}$ (www.sinab.it programmi/ biostatistiche .php ?tp=sit [February, 14 2009]), Gómez et al., 2008 .

The structure of the organic market in Europe has multiple facets. In terms of per capita consumption of organic food, Switzerland and Denmark are the leading European countries; however, in terms of total turnover, Germany has the largest European market of organic products followed by the United Kingdom (UK) and France (Willer \& Yussefi, 2006). In Switzerland, Norway, Finland, Denmark and the UK the market is characterized by the domination of a few large-scale distributors of organic food. In contrast, the Netherlands, Italy, and Germany developed a different market structure where small shops, i.e. health food shops and organic shops, have held a dominating position (Wier \& Calverley, 2002)

\subsection{The organic market in Germany}

Until 2008, Germany remains the largest market for organic food in whole Europe. The German market is supplied by domestic and import organic produce (Willer \& Yuseffi, 2009). In contrast to most European countries, different marketing channels for organic food exist in Germany ranging from direct selling to bakeries, and from health food stores to conventional supermarkets. Organic shops have held a dominate position for years (Wier \& Calverley, 2002; Jonas \& Roosen 2005; Hamm et al., 2002) that only recently has started to decline because of deep concentration in the retailing sector (Willer \& Yuseffi, 2009). Hence, to 
understand the special structure of the German organic market, a brief overview of the main changes in the structure of conventional supermarkets will be providing.

From a historical perspective, the retailing sector in Germany was developed in three major phases. Firstly, after 1945 with a strong influence from the U.S.A, retailing introduced selfservice into the food sector. Thereafter, the boom in supermarkets and neighborhood shops took place. Secondly, in the 1960s the design of various types of retailing establishments satisfied the different needs of consumers. These newly developed market segmentations and formats consolidated the sector. Between the 1970s and 1980s, new formats such as food hypermarkets and superstores and first discount outlets (discounters) appeared (Dawson, 2006). Thirdly, between 1990 and 2000 with the emergence of the common market in the European Union, internationalization of the food retailing sector began (Dawson, 2006).

Since 2000, food retailing has changed. Attempts have been made to develop a more centralized and integrated schematic approach to consumer needs in stores including nonfoodstuffs and leisure related items (KPMG \& EHI, 2006; Dawson, 2006). For instance, in 2000 the total turnover by discounters were around 33 million $€$, in contrast, in 2005, the total turnover concentrated by this retail channel increased between 52 million $€($ KPMG \& EHI, 2006) (see Table 2).

Table 2: Development of the total turnover, total outlets and store formats in Germany 1995-2005 (in Billion $€$ )

\begin{tabular}{|c|c|c|c|c|c|c|c|}
\hline \multirow{2}{*}{$\begin{array}{l}\text { Retail } \\
\text { Channels } \\
\text { (Vendors) }\end{array}$} & \multirow{2}{*}{$\begin{array}{c}\text { Total area } \\
\left(\text { in } \mathbf{m}^{2}\right)\end{array}$} & \multicolumn{2}{|c|}{$\begin{array}{c}\text { Total } \\
\text { Turnover } \\
\text { (in Mio } € \text { ) }\end{array}$} & \multicolumn{2}{|c|}{ Total outlets } & \multicolumn{2}{|c|}{ Sales areas } \\
\hline & & 1995 & 2005 & 1995 & 2005 & 1995 & 2005 \\
\hline Hypermarkets & $>4,000$ & 27.1 & 33.00 & 2,038 & 2,880 & 4.31 & 5.80 \\
\hline Discounter & $\begin{array}{r}800 \text { to } \\
2,000\end{array}$ & 32.6 & 51.75 & 10,630 & 14,610 & 4.92 & 9.48 \\
\hline Supermarkets & $400-800$ & 32.2 & 29.30 & 9,635 & 8,770 & 6.80 & 6.83 \\
\hline Small retailers & $<400$ & 19.6 & 13.40 & 54,100 & 35,200 & 7.70 & 6.46 \\
\hline
\end{tabular}

Source: KPMG \& EHI 2006. 
Moreover, the German market is characterized by discount and price orientation as main factors influencing the preference of consumption. In this context, change in preference of consumption lends itself to five scenarios of trends in food retailing: 1) strong price orientation, 2) experience in trading, 3) trends addressed to individualize offers, 4) changes in the traditional concept of retailing, and 5) value orientation (KPMG \& EHI, 2006). At this point, organic food and fair trade products offered as premium products by discounters are of special relevance to consumers with an ethical buying behavior oriented concerning environmental and fair trade practices. Due to the price strategies by discounters arises the question whether organic food offered by discounters is really ethically concerned? With this regard a discussion will be provided in the further sections.

\subsubsection{Historical factors that influenced the development of the organic market in Germany}

As mentioned, Germany has the largest European organic market in terms of total turnover. In contrast to other European countries, Germany has developed a different market structure for organic food. A historical overview of the German market is required to understand its development. Initially, organic food was sold directly by farmers, which means that originally organic food was not distributed by food retailers as other countries (i.e., in the United Kingdom or Switzerland). Moreover, the origins of organic farming were more ideological and political. The historical evolution of the organic market in Germany is provided below.

Modern organic farming was first recognized when Rudolf Steiner started biodynamic agriculture in the early 1920s, after which the marketing and distribution of organic food led to direct selling (Haccius \& Lünzer, 2000). In the 1970s, organic farming and organic food grew up as part of the environmental movement. During this time, the first organic shops and health food shops were opened, the last one was considered one of the main distribution channels until the early 2000s (Kreuzer 1996; Jonas \& Roosen, 2005). In 1985, some conventional supermarkets started to offer organic assortments (Richter \& Hempfling, 2003). In 1987, the first organic supermarket "Alnatura" was opened (Alnatura, 2007). In addition, more food retailers began to offer small assortments of organic food. In the early 2000 s, 
because of several food scandals (BSE, Nitrogen Scandal), consumers lost confidence in the food industry sector and organic food started to hold the interest of consumers as a reaction to the scandals. Thus, political efforts to increase organic farming started, as well as the participation of food retailers introducing massive quantities of organic food in conventional supermarkets.

Table 3: Economic relevance of the organic produce in Germany, 1999-2008

\begin{tabular}{|l|r|r|r|r|r|r|}
\hline & $\mathbf{1 9 9 9 / 2 0 0 0}$ & $\mathbf{2 0 0 2 / 2 0 0 3}$ & $\mathbf{2 0 0 4 / 2 0 0 5}$ & $\mathbf{2 0 0 5 / 2 0 0 6}$ & $\mathbf{2 0 0 6 / 2 0 0 7}$ & $\mathbf{2 0 0 8}$ \\
\hline Hectares & 452,279 & 546,023 & 734,027 & 767,891 & 807,403 & 865,336 \\
\hline Number of farmers & 10,400 & 12,732 & 16,603 & 16,476 & 17,020 & 18,703 \\
\hline Total of Turnover (in Billion $€$ ) & 1.8 & $2.2-2.4$ & $3.1-3.5$ & $3.9-4.6$ & $4.6-5.3$ & 5.8 \\
\hline
\end{tabular}

Source: Willer and Yuseffi, 2000; Willer and Yuseffi, 2001; Willer and Yuseffi, 2002; Willer and Yuseffi, 2006; Willer and Yuseffi, 2007; Willer and Yuseffi, 2009.

\subsubsection{Political factors and national organic certification label}

On the basis of its growing acceptance of organic agriculture the first private basic standard for organic produce were established in 1984 (Haccius \& Lünzer, 2000). In the 1990s, organic farming was included in the EU Common Agricultural Policies (CAP), and the common regulation for organic farming 2092/91 was developed (Kreuzer, 1996).

For several years different organic association and certification agencies were using their own organic label. However, in 2001 on the basis of the EU Regulation of organic farming, the national German umbrella label for organic products "Bio-Siegel” was introduced to regulate the organic produce from national produced and foreign origins with the aim to make easier for the consumers the recognition of the organic produce (Bio-Siegel, 2010). 


\subsubsection{Stage of the development of the market of organic food in Germany}

While the small organic shops were, since the earlier stage of the organic market, the main retail channels of organic food in Germany until the early 2000s, from this period onward the boom of the organic food ("Bio-boom"; in ZMP, 2007) expanded into the conventional supermarket and discount market. The latter succeeded within a few years to dominate the distribution channels (Willer \& Yuseffi, 2006) by means of different marketing strategies: some of them, by launching their own organic private labels, others by establishing their own organic supermarkets. Consequently, the structure of organic market in Germany has been changing extensively, indicative of the percentage of sales by the mainstream retail channels of rapid transfer from the small organic shops to the large multiple retailers (Hamm \& Rippin, 2007) (see Table 4).

As different consumer studies in Europe and Germany have shown, buyers in organic shops are demographically characterized as having high incomes and higher level of education (Aersten et al., 2009; Bolten et al., 2006; Bruhn, 2001; Hamm et al, 2002; Spiller, 2006; Wier $\&$ Calverley, 2002). Therefore, on the one hand, distribution of organic products by discounters can be interpreted as a democratization of organic food in relation to the price and extending into convenience and consumption. However, this democratization process detrimentally affected small health food shops, small organic shops, and direct selling. In reference to the experience of other organic markets in Europe, e.g. Switzerland, where the multiple retailers Coop and Migros initiated the organic segment or Tesco and Sainsbury in the UK, price orientation in these food retail channels is stronger than in Germany (Gerlach \& Spiller, 2006). Although small organic shops are not yet a discharged model, their success is due to non-tangible aspects such as personal service, face-to-face interaction, freshness, and high quality of products (Bolten et al., 2006; Kratochvil \& Leitner, 2005). 
Table 4: Recent trends in Germany's organic marketing channels

\begin{tabular}{|l|r|r|r|r|r|r|r|r|}
\hline Year & $\begin{array}{c}\text { Total } \\
\text { turnover } \\
\text { In billion } € \text { ) }\end{array}$ & $\begin{array}{c}\text { Increase in } \\
\text { turnover }\end{array}$ & $\begin{array}{c}\text { Small } \\
\text { retailers } \\
\text { (Inganic } \\
\text { shops) }\end{array}$ & $\begin{array}{c}\text { Food } \\
\text { retailing }\end{array}$ & Producers & $\begin{array}{c}\text { Health } \\
\text { food } \\
\text { shops }\end{array}$ & $\begin{array}{c}\text { Handi- } \\
\text { craft }\end{array}$ & Other \\
\hline $\mathbf{1 9 9 7}$ & 1.48 & & $31 \%$ & $28 \%$ & $19 \%$ & $10 \%$ & $5 \%$ & $7 \%$ \\
\hline $\mathbf{2 0 0 0}$ & 2.05 & & $28 \%$ & $33 \%$ & $17 \%$ & $10 \%$ & $7 \%$ & $5 \%$ \\
\hline $\mathbf{2 0 0 1}$ & 2.70 & $+32 \%$ & $27 \%$ & $35 \%$ & $17 \%$ & $9 \%$ & $7 \%$ & $4 \%$ \\
\hline $\mathbf{2 0 0 2}$ & 3.01 & $+11 \%$ & $26 \%$ & $35 \%$ & $17 \%$ & $9 \%$ & $7 \%$ & $6 \%$ \\
\hline $\mathbf{2 0 0 3}$ & 3.10 & $+3 \%$ & $26 \%$ & $35 \%$ & $17 \%$ & $8 \%$ & $7 \%$ & $7 \%$ \\
\hline $\mathbf{2 0 0 4}$ & 3.50 & $+13 \%$ & $26 \%$ & $37 \%$ & $16 \%$ & $8 \%$ & $7 \%$ & $6 \%$ \\
\hline $\mathbf{2 0 0 5}$ & 3.90 & $+11 \%$ & $25 \%$ & $41 \%$ & $14 \%$ & $6 \%$ & $6 \%$ & $8 \%$ \\
\hline $\mathbf{2 0 0 6}$ & 4.60 & $+18 \%$ & $23 \%$ & $49 \%$ & $11 \%$ & $5 \%$ & $5 \%$ & $7 \%$ \\
\hline $\mathbf{2 0 0 7}$ & 5.30 & $+15 \%$ & $22 \%$ & $53 \%$ & $10 \%$ & $4 \%$ & $5 \%$ & $6 \%$ \\
\hline $\mathbf{2 0 0 8}$ & 5.85 & $+15 \%$ & $22 \%$ & $57 \%$ & $8 \%$ & $3 \%$ & $4 \%$ & $6 \%$ \\
\hline
\end{tabular}

Source: Hamm \& Rippin, 2007; Hamm \& Rippin, 2009 in Haccius, 2009.

\subsection{The organic market in Mexico}

\subsubsection{General overview}

This section will provide an overview of the organic sector in Mexico. Agro-ecological practices' hold on modern organic farming have existed in Mexico since pre-Hispanic times; however, it was not until recently when practices and methods have been recognized by modern organic farming, in consequence certified organic farming has increased rapidly in the country. This growth is related to the increasing demand for organic produce in developed countries since the early 1990s. It has been stated that until 2007 there were 128,819 farmers involved in organic agriculture (Gómez et al., 2009 in Willer \& Yussefi, 2009) (see Table 5). The annual growth rate of the sector is $30 \%$ this make organic produce as the most dynamic farming sector. Because of the long tradition of using agro-ecological techniques by the majority of the indigenous and rural population, it was possible to increase the organic farming relatively rapidly.

The organic sector is characterized by the predominance of small-scale farmers. For instance, in 1996 , the total number of small-scale farmers was $97.5 \%$ and in 2008 , the total increased to 
$99.9 \%$. Despite the superior number of small-scale farmers, large farmers are gradually increasing their participation in terms of cultivated land. While in 1996 large producers cultivated only $11 \%$ of the total "organic" land, in 2005 this increased to $20 \%$. In general terms, the growth of the organic sector in Mexico has been encouraged by foreign technical and financial support, mostly by Non Governmental Organizations (NGOs), religious groups, private foundations, and international cooperative agencies (Gómez et al., 2005b).

Table 5: Economic relevance of the organic produce in Mexico, 1996-2007/2008

\begin{tabular}{|l|r|r|r|r|r|r|}
\hline & $\mathbf{1 9 9 6}$ & $\mathbf{1 9 9 8}$ & $\mathbf{2 0 0 0}$ & $\mathbf{2 0 0 2}$ & $\mathbf{2 0 0 4 / 0 5}$ & $\mathbf{2 0 0 7 / 2 0 0 8}$ \\
\hline Hectares & 23,265 & 54,457 & 102,802 & 215,843 & 307,692 & 403,268 \\
\hline $\begin{array}{l}\text { Number of } \\
\text { farmers }\end{array}$ & 13,176 & 27,914 & 33,587 & 53,577 & 83,174 & 128,819 \\
\hline $\begin{array}{l}\text { Workdays } \\
(1,000)\end{array}$ & 3,722 & 8,713 & 16,448 & 34,534 & 40,747 & 172,251 \\
\hline $\begin{array}{l}\text { Incomes (US\$ } \\
1,000)\end{array}$ & 34,293 & 72,000 & 139,404 & 215,000 & 270,503 & $394,149,000$ \\
\hline
\end{tabular}

Source: Gómez et al., 2009 in Willer \& Yuseffi, 2009.

\subsubsection{Social and economical implication of the export oriented scheme}

Organic farming in Mexico as in other developing countries attempts to satisfy the existing demand centralized in Europe. In fact, because of the number of domestic markets in a mature stage (Hall \& Mogyorody 2001) ggovernmental programs in Mexico are export-oriented. Consequently, medium and large-scale farmers were encouraged to follow the organic farming scheme in their overall production (Gómez et al., 2005b). Therefore, the country is considered as an organic producer and export oriented due to the fact that around 80 to $85 \%$ is exported mainly to the U.S:A., European countries and Japan (Lernoud \& Piovano 2006).

Organic farming relates to environmental, economical, and social implications to the country. Economical implications include satisfaction of an existing demand in external markets especially in the winter season. In fact, until 2005 organic farming covered 797 zones of production in 28 out of 32 federal states (Gómez et al., 2005a). Around 99.9\% of organic farmers were classified as small-scale farmers who cultivated $80 \%$ of the total organic land. Besides the small-scale farmers, there are 22 indigenous groups most of whom work together 
as cooperatives with plots of 3.02 hectares on average. Relating to social implications, organic farming reduces emigration because of the demand for local labour (Gómez et al., 2009). However, the high cost of certification negatively influences the development of the sector, as it represents a significant barrier for most of the small-scale farmers even for exporting larger farmers. Organic farmers in the country produce without financial support because of the absence of national regulation; governmental programs and support for the organic farming sector. These facts suppose the reduced number of larger farmers between the period of 2004 until 2008, who differently to small farmers altogether in cooperatives should self-realise the process of produce and export (see Table 6).

Table 6: Size and number of organic producers

\begin{tabular}{|c|c|c|c|c|c|c|c|c|}
\hline \multirow{2}{*}{$\begin{array}{c}\text { Size of } \\
\text { production }\end{array}$} & \multicolumn{2}{|c|}{1996} & \multicolumn{2}{|c|}{2000} & \multicolumn{2}{|c|}{ 2004-2005 } & \multicolumn{2}{|c|}{$2007 / 2008$} \\
\hline & Number & $\%$ & Number & $\%$ & Number & $\%$ & Number & $\%$ \\
\hline Small scale & 12,847 & 97.5 & 33,117 & 98.6 & 80,319 & 99.6 & 128,690 & 99.9 \\
\hline Large scale & 329 & 2.5 & 470 & 1.4 & 345 & 0.4 & $129^{\mathrm{a}}$ & $0.1^{\mathrm{a}}$ \\
\hline Total & 13,176 & 100 & 33,587 & 100 & 80,664 & 100 & 128,819 & 100 \\
\hline
\end{tabular}

Source: CIESTAAM, 2005; Gómez et al., 2009 in Willer \& Yuseffi, 2009; ${ }^{\mathrm{a}}$ own estimations based on Gómez et al., 2009.

\subsubsection{Politics, programs and the consequences of the export orientation scheme}

Although organic farming has the highest annual growth rate in the Mexican agricultural sector, several barriers, and limitations hinder this growth. One of the most important is the role of the government, which does not provide significant support in this area. In a study conducted by Gómez et al. (2005a) that encompassed 244 farmers/cooperatives of farmers, 153 farmers/cooperatives of farmers (63\%) reported receiving some financial support from the federal government, and 67 units of production (27\%) received financial support from regional governments. However, financial support covers only the cost of travelling, assisting at fairs to promote and merchandise organic produce and, in some cases, to cover part of the total cost of certification. 53 farmers/cooperatives of farmers $(21 \%)$ reported to have none financial support. There are other challenges with respect to the lack of specific programs supporting organic farming, such as the small size of the domestic and local markets and the high cost of certification. Other barriers pointed out in the study were the absence of political 
support, a regulatory framework, a national organic label, subsidies, technical and financial support, bureaucracy, and unclear governmental regulations with regards to its programs and support (Gómez et al., 2005a).

\subsubsection{National umbrella organic label}

In 1997 a national norm was created for organic produce based on the international norms established by the International Federation Organic Agriculture Movements (IFOAM) and the Codex Alimentarius by FAO, for instance, the Official Mexican Norm, NOM- 037- Fito1995; however, some aspects such as origin of animal products were incomplete. More recently in 2006, the Mexican government concluded a regulatory framework ${ }^{1}$, Iniciativa de Ley de Productos Orgánicos, to promote and regulate national organic produce; however, what kind of programs, strategies, and supports might be applied it is unclear including any existence of a national organic label.

The absence of a national umbrella label concerns the certification process, as there are twenty-one agencies certifying organic land in the country. Certimex is the only agency of national origin and most of them are foreign; for instance: the U.S.A., Germany, Italy, Switzerland, Sweden, Guatemala, and Holland.

\subsubsection{Stage of the market for organic food in Mexico}

The domestic market for organic food remains very small. As in other emerging markets, the organic market in Mexico is characterized by the lack of information regarding opportunities and challenges with its development, including information on the total turnover and sales,

\footnotetext{
${ }^{1}$ Iniciativa de ley de productos orgánicos retrieved July 12, 2009.http://vinculando.org/organicos/leyorganicos.html.
} 
demand, consumption, and consumer preferences. Some scholars estimate that around 80 or $85 \%$ of organic produce is exported and the remainder (15 or 20\%) is sold on the domestic market; $10 \%$ is sold as conventional produce and only $5 \%$ is sold with an organic label (Lernoud \& Paviano, 2006).

Regarding demand and consumption, some of the limitations argued on building an organic market in Mexico deal with inadequate knowledge about organic produce by the Mexican population and price sensitivity due to the national level of low household incomes, hence the unwillingness to pay premium prices. Concerning the Food Supply Chain (FSC), inconsistent supply in the retail channels has been mentioned as constraint consumption (Lernoud \& Paviano 2006). Regarding consumer behavior, there are few studies: one of them relates to the willingness to pay for organic food in three capital cities in northern Mexico (Padilla \& Perez 2006), and in Mexico City another study was undertaken on attitudes as well as target groups of consumers (OTA, 2004). However, there is not enough information on who, where, why, and how frequently consumers purchase organic products.

\subsubsection{Distribution and mainstream retail channels of organic food}

To understand the stage of the organic market in Mexico is provided and overview of the FSC. In 2006, Schwentesius and Gómez identified five mainstream retail channels where food is sold in Mexico: 1) traditional markets, 2) weekly markets (tianguis), 3) small traditional shops with limited assortment and quality dependent on incomes and preferences of neighbourhoods, 4) self-service stores including hypermarkets, supermarkets, members' clubs and convenience stores, and 5) organic shops are of little importance since consumers prefer to purchase at tianguis. Most of the self-service stores belong to chains of conventional supermarkets throughout the country. Some of the self-service stores are supported by national capital and others by foreign capital. There are also many local chains and small independent markets (Schwentesius \& Gómez, 2006).

Out of all these different outlets, organic food is mainly distributed by chains of conventional supermarkets; organic shops, and direct selling by tianguis orgánicos. This last one was 
inspired in the Community Supported Agriculture (CSA) scheme (DeMuth, 1993) and covers several cities and regions across the country because of the national networks of small-scale farmers, producers and consumers who organized themselves to encourage the organic movement (Nelson et al., 2009). The organic assortment offered by each one of the retail channels is described below.

\subsubsection{Size of the organic assortment}

Since the domestic market is still in the seedling stage, there is a relatively small organic assortment in the retail channels. The assortment consists of national and imported products. Regarding imported products, the assortment depends on the country of origin, for instance, some fresh food such as vegetables and salad ingredients come from the U.S.A. as well as cereals, muesli, soya milk, teas, bread, etc. Other products such as pastas, spices and wines come from Italy and Spain. Also, cosmetics and essences for aromatherapy or homeopathic medicine come from Germany and France. The national assortment consists of fresh and seasonal food; for instance, fruits, vegetables, and salads. As seen, the national assortment consists of products with low transformation levels (e.g., coffee, muesli, marmalades, animal and dairy products, honey, spices, cereals, bread and bakery). This is not only because of governmental policies and programs but also because of the limited knowledge about organic food in the domestic market. Therefore, the development of the organic farming sector even in the domestic organic market depends on the tendencies of the external markets.

The largest assortment is sold by: 1) Organic shops, including organic, green, environmentally friendly and fair-trade products; the size of the assortment depends on the size of the store; 2) Direct marketing, in most cases these are small-scale farmers or producers who organize themselves as cooperatives to sell one day per week; most of the assortment is seasonal including fresh vegetables, fruits, local crops, honey, and coffee with low inputs and low manufacturing processes or labor intensiveness. Regarding the chains of conventional supermarkets, the organic assortment depends on the format and location of the outlet according to the socio-demographic characteristics of the neighborhood or zone; 3) Supermarkets, for instance Superama, which is managed through foreign capital, offers an assortment of between 100 and 200 products including fresh vegetables and fruits, honey, 
coffee and milk from national origins and imported assortments of cereals, marmalades, juice and soya milk; 4) Hypermarkets-the largest assortment is offered by Wal-Mart Supercenter which is like superama because both belong to the same retailing group. The size of the assortment offered by hypermarkets of national origin (e.g. Chedraui, Comercial Mexicana and Soriana) is smaller, most of them from national origins and distributed under the national brand Aires de campo; and 5) the assortment offered by members' clubs (e.g. Costco and Sam's Club) is of minor importance (see Table 7). Other marketing channels are cafeterias and restaurants some of which are located within the organic stores.

Table 7: Organic assortments in food retail channels in Mexico

\begin{tabular}{|l|l|}
\hline \multicolumn{1}{|c|}{ Food retail channel } & \multicolumn{1}{c|}{ Number of organic products } \\
\hline Specialized stores & $1000-1200$ \\
\hline Direct marketing & $200-300$ \\
\hline Supermarkets & $100-200$ \\
\hline Hypermarkets (foreign origin) & $100-200$ \\
\hline Hypermarkets (national origin) & $50-70$ \\
\hline Member's clubs & $20-50$ \\
\hline
\end{tabular}

Source: Own estimations (2008).

\subsection{Conclusion: German and Mexican organic market}

The following provides a summarization of stage of the organic markets of the two countries to be compared. On the one side, Germany has developed one of the most mature markets of organic food in Europe as well as worldwide. The total turnover reflects the growth of the total market. With the "Bio-Boom" the phenomenon of conventionalization on the organic sector has emerged and demand of organic food has been concentrated in conventional supermarkets. While in Mexico, the export-oriented scheme of organic produce encouraged by the federal government comes in to question and the alternatives to develop a domestic market are discussed. 


\subsubsection{Future of organic market in Germany within conventionalization and embeddedness theories}

According to the literature, the consequences of changes in produce and consumption in Germany are analyzed twofold. Some scholars maintain that the organic sector is undergoing a "conventionalization" process, and its outcome will be a drastic alteration of the origins, ideologies and principles of the core of organic farming because of the objectives and achievements of the latter (Allen \& Kovach, 2000; Guthman, 2004; Hall \& Mogyorody, 2001; Kratochvil \& Leitner, 2005). This can be partly confirmed by observing the decreasing importance of traditional retail channels displayed above in Table 4. In contrast to this view, the scholars of the "embeddedness theory" - originally proposed by Granovetter (1985) interpret the recent trends of the organic market more optimistically (Kratochvil \& Leitner, 2005). For instance, the increasing interest in local and regional products should especially be seen as an opportunity for small organic farmers to strengthen production, distribution, and consumption in small organic shops and through direct selling. In the next section more details will be provided on the conventionalization and embeddedness theories.

In fact, convenience, larger assortments, and lower prices were basic marketing strategies that made organic food with private labels accessible in conventional supermarkets and discounters consequently; organic food with private labels has developed from a niche to a mainstream market and restored confidence in the food industry sector. Meanwhile, intrinsic profits for food retailers increases their branch positioning and consumer loyalty, in consequence because of the changes in preferences of purchasing in conventional supermarkets, discounter, and conveniences stores, traditional retail channels such as direct selling, small organic shops, health shops and organic shops have been shifting in the market structure. In fact, since the early 2000s, the process of conventionalization of the organic sector has threatened the original principles, commitments, and ideological objectives through modern organic farming and Germany is no exception to this process. Figure 2 depicts the process of conventionalization. 
Figure 2: Convenience driving conventionalization

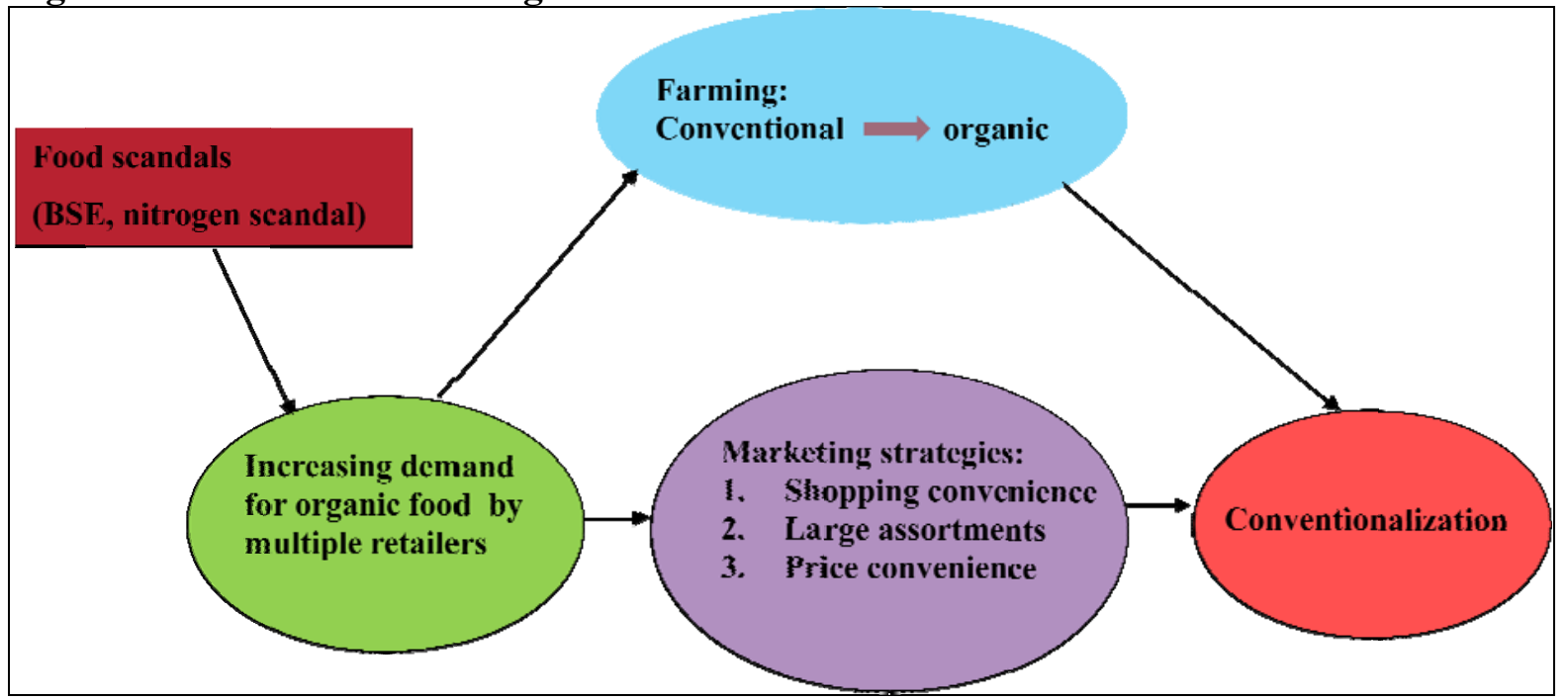

Source:Own elaboration (2007).

The conventionalization theory proposed by Buck et al. and recently reviewed by Guthman (2004), refers to the fact that "the most high-value crops and the most lucrative segmented organic commodity chains were being appropriated by agribusiness firms, many of which were abandoning the more sustainable agronomic and marketing practices associated with the principles and objectives of organic agriculture" (Buck et al., 1997 cited in Guthman 2004).

In 2004, Guthman argues that the participation of agribusiness firms in organic farming resulted in the commandeering of organic labeling, defining the rules of certification. Therefore, differences have developed in whom and what type of products can be included in organic production. There is a general tendency to invest capital in the most easily produced and profitable crops, marginalizing the profits of organic farmers and creating scale economies unattainable by producers who cannot adopt industrial production methods grounded in the conventionalization of the organic sector (Guthman, 2004).

In this way, conventionalization alters the organic sector twofold: firstly, altering methods of farming and production, increasing costs of merchandise and initial sale included in a direct marketing strategy; and secondly, ideological commitment in relation to the small amount of production and profits by farmers (Guthman, 2004). 
Even though the idea of conventionalization arose and was mainly discussed in the AngloAmerican countries, e.g. USA (Guthman, 2000; Guthman, 2004), Canada (Hall \& Mogyorody, 2001), New Zealand (Campbell, 2001), and Australia (Lockie et al., 2002), the Bio-boom in Germany averaged a two digit growth rate in total turnover; and in real terms, the structure of distribution channels was undergoing a process of change. Thus, the discussion of conventionalization of the organic sector in German speaking countries was opened creating special interest by some academics and scholars (Bahrdt et al., 2003; Brand, et al., 2004; Best, 2008; Gerlach \& Spiller, 2006).

The consequences of the marketing strategies by multiple retailers can be summarized as follow. When price premium it was an obstacle in purchasing organic food; food retailers could offer premium products with their own branches by means of some marketing strategies such as private label, price competitiveness, strong price policy, convenience, promotions, and advertising. Consequently, small shops that cannot adopt similar strategies but continue transmitting non-tangible goods such as the origins and principles of the earliest organic movement, which are still important for heavy consumers, were affected. However, occasionally new and non-committed consumers purchase organic food from multiple retailers and discounters where it is possible to purchase premium products at lower prices and in a more convenient way.

Food retailers distinguish themselves from competitors by using private labeling and at the same time improving brand positioning by selling premium products as organic food. Thus, private labeling represents changes in two aspects of the organic market.

On one hand, it alters the interactions and relationships among the actors in the supply chain because of the linkage of retailers with manufacturers and consumers. Food retailers are able to increase contractual obligations of manufacturers, encouraging them to become more dependent because of the foregoing reasons for concessions in price, quality, quantity, demands, and flexibility of payments (Jonas \& Roosen, 2005).

On the other, small manufacturers who cannot engage in this scheme are left out increasing the oligopoly structure of the market and changing the traditional market structure; conventionalization therefore emerged in the organic sector. For instance, in 2005 discounters, such as Aldi and Lidl, selling organic products with their own private label, 
entered into the market and imitation effects resulted. Lidl stated that their objective in increasing the organic market is approximately a $20 \%$ share of the total market in Germany (Spiller, 2006), and similar dynamics resulted in the U.S.A. market as a result of the incorporation of Wal Mart into the "organic segment".

In addition, this situation determines new conditions to the development of the organic market for small organic shops in a European country where nowadays the market structure is still considered as different. However, preferences on purchasing of organic food and habits on consumer behavior have changed. Whereas fifteen years ago, attributes of public concern, such as environmentally friendly, sustainable practices, animal welfare, social justice and fair trade were a motivation to purchasing organic food, nowadays there are hedonistic benefits such as wellness, lifestyle, fitness, health, and taste that are the main motivations in the purchasing of organic food by these new consumer segments (Aersten et al., 2009; Bruhn, 2001; Baker et al., 2004; Haccius, 2005).

Under conventionalization, different aspects are brought into question. Regarding production, aspects such as sustainable practices, quality, and guarantee are paramount; on the one hand, in the viewpoint of farmers, and manufacturers, aspects such as contractual obligations, elasticity of production, and flexibility in satisfying the demand and prices are of more concern. On the other hand, consumers with ethical issues such as ecological and environmental concerns, animal welfare, climatic change, global warming, and wildlife protection create a distinct precedence.

From an economical and social approach, aspects such as regionalism and localism, fairness, working conditions, and social justice are in question, since price competition in conventional channels has emerged. Nevertheless, academic scholars postulate the embeddedness theory with a local focus as an alternative orientation to strengthen the participation of small organic shops and direct sellers in the Food Supply Chain (FSC) (Granovetter, 1985; Krachtovil \& Leitner, 2005).

Moreover, the "social" within the economical exchanges which is the main propose of social embeddedness. On the premise that "quality" is a social construction in 2006, Kirwan proposed the Conventions Theory in his work about the interpersonal world of direct marketing in the farmers markets in UK. Conventions Theory is defined as "the 
understanding of how actors negotiate and coordinate exchange outcomes through their cognitive interaction, rather than relying solely on the price” (Kirwan, 2006). Consequently, the main proposal is the creation of new spaces of production and consumption by face-toface interaction between the participants on the FSC. All in all, both theories "social embeddedness" and "Conventions Theory," enhances social construct "trustworthiness" between the actors of the FSC to strengthen the sustainability of the distribution chains to overcome the current dominate policy by agribusiness.

Even so some of the theories were developed inspired by the direct selling their applicability is not restricted to organic farming if it is focused within a local or regional strategy. Nevertheless, the process of conventionalization in organic farming, the embeddedness and conventions theories seem to be alternatives for direct selling, and small organic shops by involving all the participants in the organic FSC. In fact, the relationship between the participants in production, distribution, and retailing as well as committed consumers is strengthened by face-to-face interaction, while the sustainability of the distribution chain is itself strengthened through the perceptions of trust and loyalty (Kirwan, 2006).

Furthermore, to recognize the process of conventionalization and its implications in the development of the organic sector, the embeddedness theory deals with the understanding of the organic food supply chain that is locally oriented, characterized by trust, transparency, connectivity, reciprocity and communication in order to encourage organic production, distribution and consumption in a local and regional context consolidating social interactions, face-to-face communication, confidence and loyalty between the FSC actors in a local context (Kratochvil \& Leitner 2005; Seyfang, 2005). Thus, the proposal of these initiatives represents the possibility of including smaller actors into the FSC such as farmers, producers, retailers, and concerned consumers, who do not have to make a commitment to participate in a scheme of private labeling and price competition.

The promotion by food retailers of "uninformed" consumption of organic products, as well as the excessive importation of seasonal or "exotic" food products in order to offer a large assortment resulted in part as entrapment by conventionalization. Moreover, to recognize the reasons for this process, the embeddedness theory also proposes conscious consumption emphasizing social interaction and incorporating small regional shops. Even if purchasing of 
organic food regionally produced was not initially intended, it is still a potential market segment (Stokebrand \& Spiller, 2007).

\subsubsection{Future of organic market in Mexico within the export oriented and the PGS scheme}

Regarding the emerging organic market in Mexico, as far as it is concerned, the overview of the organic sector shows that organic farming has been mainly an option for small-scale farmers to reduce emigration. It has enabled them to maintain agriculture as their main economic activity. In fact, it can be said that self-organized alternatives and agro-ecological agriculture have emerged in rural zones in Mexico as a consequence of social exclusion resulting from neoliberal policies and modern agriculture. Therefore, questions arise such as why might organic agriculture been an option for small-scale farmers in developing countries like Mexico? In which way do organic production systems favour small farming? How do they maintain themselves despite the weak institutional arrangement that supports them?

Given the remarkable external market orientation of the organic sector in Mexico, it is important to look at the tensions that this orientation produces. Firstly, the underlying discourses and trends of the organic movement in the developed countries have to be distinguished where Mexican organic crops are normally sold, in order to analyse how those tendencies affect available options for the organic sector of Mexico.

Noticeably, environmental protection and health care are at the core of organic farming discourses in developed countries (Allen \& Martin, 2000). However, the increasing intensive participation of agribusiness in the sector in developed countries and calls into question the achievement of ecological goals. The reason lies in that large agribusinesses find it difficult to meet strict ecological standards, since their land tends to be more contaminated by chemical residues. Therefore, they try to influence official standards by promoting the input 
substitution approach ${ }^{2}$ in organic agriculture legislation. This process is mentioned by several authors as the "conventionalization of organic agriculture".

Meanwhile, organic farming in Mexico has been identified as an alternative way of addressing rural poverty. Social exclusion is a more prominent issue than health care and environmental protection, although these are not ignored. In fact, organic food has become a niche market in Mexico that has mainly benefited from the holistic movement in the developed countries, because most of the produce of small-scale farmers is basically traded in Europe through alternative supply channels by Fair-Trade Labelling Organizations ${ }^{3}$. Thus, the difference between discourses in the developed countries regarding organic farming in Mexico reveals different priorities at work in each context; this also helps to identify the different nature of their threats and challenges. For example, the certification process poses an imminent threat for the small-scale farmers in Mexico, since they lack the financial capacity to cope with the certification costs. Also, if the input substitution approach becomes dominant in international organic standards, there will be less opportunity to develop alternative markets, thus the consolidation of the conventionalization process.

The certification process as it is defined in the developed countries attempts to simplify and homogenize the organic production system and make easy to consumers the recognition of organic products. The rules are focused on guaranteeing chemical-free crops but dismiss labour organization within the production process. Because the large scale systems are more homogeneous, centrally administered, and financially stronger, their certification process is easier. Thus, organic produce from large production units reaches the market faster. Therefore, the organic farming model based upon small-scale farmers is threatened by a green wash of large production systems.

\footnotetext{
2 Basically, the input substitution approach focuses on the replacement of chemicals such as pesticides and fertilizers with biological pesticides and fertilizers. Contrary to input substitution is the holism approach, which advocates a method of production committed to environmental protection compatible with the interests of consumers concerned by environmental and health-care related issues.

${ }^{3}$ www.flo.net
} 
Another potential setback to the external market orientation of Mexican small-scale farmers resides in the increasing promotion of local food consumption in the developed countries, which is gaining local consumers' support for (i.e. local embededdness theory by Winter, 2003). Also in the academic and governmental institutions, approaches such as food sheds, CSA and community food security are at the frontier stage of sustainable agriculture and food studies and policies (Stagl, 2002; Johston \& Baker, 2005; Kloppenburg, 1996). These approaches point out a direct connection between sustainable agriculture and local markets. Therefore, in continuation some of the potentialities to develop a domestic organic market in Mexico are explored.

Furthermore, in order to achieve more transparency of the rules of production and consumption of organic food in international organic regulations, direct marketing stands out as an alternative to developing local markets. This allows small-scale farmers to get better prices due to the reduction of the number of intermediaries in the FSC. By personal interaction in direct marketing, participants create social embeddedness throughout their communities -local embededdness- because of the flow of information, exchange of experiences, local knowledge and trustworthiness in the consolidation of locally sustainable production and consumption. Similar experiences regarding direct marketing schemes have been successful in developed countries; for instance, the CSA scheme in the United States and Farmer's Markets in the UK as well as organic markets in developing countries in Latin America including Brazil, Peru, and Uruguay. Regarding national experience, within the network of organic markets, the adoption and combination between the Participatory Guarantee System (PGS) and the CSA schemes seem promising.

Taking into consideration the traditional purchase of food products at farmer's markets called tianguis, there is an opportunity to build organic markets as an alternative retail channel. As mentioned, consumers who utilize organic markets are willing to pay premium prices not only for private benefits such as healthy and tasty food, but also because of a sense of community, interest in green worth, environmental friendliness and animal welfare. Moreover, the benefits of consuming organic food the scarce information and knowledge by the general population about organic produce and the existence of alternative retail channels is limited. 


\subsection{Main features of the two organic markets to compare}

Moreover, the trends in consumption, challenges encouraging and alternatives to overcome the process of conventionalization, the main features the organic market in Germany and Mexico are summarized in Table 8. On the one side in Germany, the first organic shops started in the decade of the 1970s. In the decade of the 1980s conventional supermarkets launched the first organic assortment, and in the earliest 2000 the Bio-boom. Could be observed nowadays the German market for organic food is one of the most mature markets in Europe and still growing. Since 2001, there is a national umbrella organic label (Biosiegel, 2010). The assortment has a domestic and imported origin, and is mainly distributed by five main retail channels. The market structure is changed due to the demand of organic food is concentrated by multiple retailers, whereas there is not growth in the sales by direct marketing (Willer \& Yuseffi, 2009). The stage of mature market is influencing the fact that consumers are committed with organic produce.

On the other side, since the earliest 2000 in Mexico a domestic market exists which is a relative new and small segment, but potential. There is not a national umbrella organic label; less than $5 \%$ is sold through natural shops, organic shops, and restaurants (Gómez et al., 2009). Furthermore, the low knowledge about environmental issues, and organic farming, the absence of food scandals, and the market characterised as price sensible. There is not enough information regarding the demand and purchasing preferences of organic food in the mainstream retail channels in the country. 
Table 8: Main features of the two organic markets to compare

\begin{tabular}{|c|c|c|}
\hline Feature & Germany & Mexico \\
\hline $\begin{array}{l}\text { Historical facts of organic } \\
\text { farming }\end{array}$ & $\begin{array}{l}\text { 1920s started as alternative } \\
\text { methods of farming, 1970s } \\
\text { organic farming was } \\
\text { encourage because of } \\
\text { environmental movement, } \\
\text { 1980s started the first organic } \\
\text { shops, and first organic } \\
\text { assortments by multiple } \\
\text { retailers, 1990s there were } \\
\text { different food scandals, } 2000 \\
\text { started the bio-boom. }\end{array}$ & $\begin{array}{l}\text { During the decade of } 1990 \mathrm{~s} \\
\text { modern organic farming } \\
\text { started to be produced under } \\
\text { the export oriented scheme. } \\
\text { Whereas the Network of } \\
\text { producers and consumers (as } \\
\text { social movement) encourage } \\
\text { the organic movement. }\end{array}$ \\
\hline $\begin{array}{l}\text { Destination of organic } \\
\text { produce }\end{array}$ & Domestic market & External markets \\
\hline $\begin{array}{l}\text { National umbrella organic } \\
\text { label }\end{array}$ & Yes & No \\
\hline Stage of the domestic market & Mature & Emerging \\
\hline Mainstream retail channels & $\begin{array}{l}\text { 1. Multiple retailers } \\
\text { 2. Organic shop } \\
\text { 3. Producers } \\
\text { 4. Health food shop } \\
\text { 5. Handicraft }\end{array}$ & $\begin{array}{l}\text { 1. Organic shops/restaurants } \\
\text { 2. Direct selling } \\
\text { 3. Multiple retailers }\end{array}$ \\
\hline Actual stage of the market & $\begin{array}{l}\text { 1. Coventionalization of } \\
\text { organic produce. } \\
\text { 2. Concentration of the } \\
\text { demand in multiple retailers. } \\
\text { 3. Stagnation of the demand } \\
\text { in direct marketing. }\end{array}$ & $\begin{array}{l}\text { 1. Remaining of the export } \\
\text { oriented scheme } \\
\text { 2. Slow development of the } \\
\text { domestic market } \\
\text { 3. Low knowledge among the } \\
\text { populations about organic } \\
\text { produce. }\end{array}$ \\
\hline
\end{tabular}

Source: Own elaboration. 


\section{$3 \quad$ Multidisciplinary perspective on motivations and values}

This chapter presents some definitions of consumer behavior from the American and European viewpoint in order to clearly explain our conceptual framework. In our viewpoint culture is a key concept in the core of our cross-cultural research, conceptualization of culture related to consumer behavior is provided. Consumer influences such as demographic, geographic, psychographic and other categories used to segment the market are also described, emphasising psychographics which is directly related to the guideline in our approach to this study of motives and values as the core meaning.

\subsection{Defining consumer behaviour}

In the following, a review of the most common definitions of consumer behavior is provided from both an American and European viewpoint. Concerning the former, Blackwell et al. (2006) defines consumer behavior as "the activities that people undertake when obtaining, consuming, and disposing of products and services". In addition, they propose a second definition of consumer behavior as "a field of study that focuses on consumer activities" (Blackwell et al., 2006). On the other hand, Hoyer and MacInnis (2007) define consumer behavior "as the reflect of the totality of consumers' decision with respect to the acquisition, consumption and disposition of goods, services, activities, experiences, people and ideas by human decision making over time". Since many factors might affect the behavior of consumers, they propose a model of consumer behavior consisting of four main domains, each one related to the others: 1) psychological core, 2) process of making decisions, 3 ) consumer's culture, and 4) outcome of consumer behavior (Hoyer \& MacInnis, 2007).

These authors emphasize the psychological core, because consumers need firstly to be informed. In addition, they highlight the importance of culture because of its affects not only on consumers' motives but also on how they process information and the kinds of decisions 
that they make. Other factors such as age, gender, social class, ethnicity, values and lifestyle influence the consumer decision-making process as well (Hoyer \& MacInnis, 2007). On the other hand, Mowen and Minor (2001) define consumer behavior "as the study of the purchase units and the exchange processes involved in acquiring, consuming, and disposing goods, services, experiences and ideas".

From a European viewpoint Solomon et al. (2000) defines consumer behavior "as the study of processes involved when individuals or groups select, purchase, use or dispose of products, services, ideas, or experiences to satisfy needs and desires". The author reminds us that only recently marketers have recognized consumer behavior as an ongoing process. In the words of Schiffman and Lazar (2004), consumer behavior is defined as "the behavior that consumers display in searching for, purchasing, using, evaluating and disposing of products and services that they expect will satisfy their needs"; thus, consumer behavior focuses on how individuals make decisions about spending their resources on consumption. Similarly, Arnould et al. (2004) define consumer behavior as "individuals or groups acquiring, using, and disposing of products, services, ideas, and experiences". According to Wright (2009) the study of consumer behavior should focus on the decision-making process which consequently analyzes how relationships are built before, during and after sales. In his criticism of marketing sciences, O'Shaughnessy (1992) reflected that marketers should study not only how and why people buy, but also individuals' activities and responses in different situations. Thus, he proposed the inclusion of interdisciplinary approaches in marketing to better understand consumer behavior.

\subsection{Culture and consumer behaviour}

Many consumer behavior models refer to culture as an external or environmental factor which influences manifest purchase behavior (Solomon et al., 2000; Mowen \& Minor, 2001; Schiffman et al., 2008; Hawkins et al., 2004; Hoyer \& MacInnis, 2007; Blackwell et al., 2006; Blythe, 2008). In 2004 Mooij, enlarges the sphere of influence of culture claiming that it influences the mind of consumers. Therefore, studying consumer behavior should also include 
cultural root factors. Whilst the latter is defined as "the physical actions of people that can be directly observed and measured by others," mental activities are not directly observed (Mooij, 2004). Values may explain consumer behavior, which has a stable component. Thus, values permit the explanation of individuals' habits from the past, which, at the same time, allows forecasting of future behavior. In fact, consumption is a matter of habit, and most of a consumer's behavior is based on long-time habits (Mooij, 2004). Being essentially anthropological, the scientific approach to analysing culture by Mooij contrasts with the current way to treat this construct in marketing science, as culture has mainly been viewed as an external, social, or environmental factor influencing consumer behavior. Hofstede (2001) backs Mooij's considerations on culture and refined its definition. According to him, culture is a system of mental programming (perceptual framework) which can be categorized into three main levels: universal, collective, and individual. Each person experiences a certain amount of mental programming which stabilizes over time, thus the same person within a certain level will behave similarly in comparable situations (Hofstede, 2001).

At the universal level, perceptual framework refers to common human behavior, whereby people react or behave similarly to satisfy similar needs, for instance, basic human needs. The collective level refers to behavior shared by people belonging to the same group or category. Therefore, their behavioral patterns will differ depending on the commonality of a group or category. The individual level refers to a unique part of mental programming such as personality (Hofstede, 2001). In marketing, the three categories of perceptual framework are useful in forecasting consumer behavior with respect to segmentation of the market and developing marketing strategies and advertisements.

In terms of agriculture and food marketing, another important consideration defining consumer behavior is the viewpoint of Grunert et al. (1997); food purchase is one of the most problematic areas in the understanding of consumer behavior because the purchase of food depends on a large number of characteristics. Some of these include taste, quality, the level of satisfaction and cooking and preparation as social activities that many times are not considered in a purchase situation.

Thus, in this study, we refer to the term "consumer behavior" in order to indicate the exchange process of individuals in acquiring, using, and disposing of products, services, 
ideas, or experiences by people. Because of the cross-cultural nature of analysis, we will draw upon the framework based on the main assumptions by Mooij (2004) and Hofstede (2001) regarding culture. Hence, we will not consider the latter as an external, environmental factor influencing the decision-making process of consumers but as an internal behavioral component which influences their everyday habits of purchase.

\subsection{Decision-making process}

In the following, we briefly describe the decision-making process, which is another key concept in understanding consumer behavior (Blackwell et al., 2006; Blythe, 2008; Hoyer \& MacInnis, 2007; Mowen \& Minor, 2001; Schiffman \& Lazar, 2004; Solomon et al., 2000). Mowen and Minor (2001) define consumer decision making as "the process involved in recognizing problems, searching for solutions, evaluating alternatives, choosing among opinions, and evaluating outcomes of choice". Thus, the underlying idea is that consumers make decisions in order to reach their goals: choosing the best alternative, reducing the effort in making decisions, minimizing negative emotions, and maximizing the ability to justify their decisions.

They also describe the generic decision-making model in five phases: 1) problem recognition, 2) search, 3) alternative evaluation, 4) choice, and 5) post-acquisition evaluation. They recognize that physical environment, experience and emotions might influence consumer behavior (ibid.).

Paraphrasing Mowen and Minor (2001), the decision-making process is a rational, cognitive process. This idea is shared by Arnould et al. (2004) who define consumers as adaptive decision makers; they adapt to aspects of the environment in order to make decisions that help them to achieve their goals. Also, consumers perceive and appraise situations of choice within a social and cognitive context and attempt to determine what aspects of choice are related to their relevant goals, potential losses, and gains according to the pursuit of their goals (Arnould et al., 2004). Similarly, Schiffman et al. (2008) define the decision-making process as an informational, rational process - cognitive process - that consists of three stages: need 
recognition, pre-purchase search, and evaluation of alternatives influenced by externals (input) and reflected in post-decision behavior (output). Culture is considered an external influence in correlation with the socio-cultural environment, affecting the decision-making process as well as family, informational sources, other non-commercial sources, and social class. Another external force mentioned by the authors is a firm's marketing efforts such as product, promotion, price, and channels of distribution (Schiffman et al., 2008).

In the consumer decision process model by Blackwell et al. (2006) two kinds of influences on behavior are mentioned: organizational and consumer (psychological aspects). The former includes all the efforts of market planning such as brand, advertising, promotions, price, services, conveniences, packaging, product features, word of mouth, retail displays, quality, store ambiance, loyalty programs, and product availability. Psychological influences are: culture, personality, life-stage, income, attitudes, motivations, feelings, knowledge, ethnicity, family, values, available resources, opinions, past experiences, peer groups which are considered as consumer influences (Blackwell et al., 2006).

Among scholars there is a tendency to consider food decision-making process of individuals, as a cognitive process. However, as we have already seen, in 2004 Mooij confutes this view, stressing that the cognitive approach to consumption is a limited model in explaining consumer behavior. Firstly, economic development explains human actions, i.e. manifest behavior but not people's minds. For instance, consumption is not necessarily similar in countries with economically similar circumstances. In contrast, this model fits well in postscarcity societies but is not useful in explaining consumption in societies with scarce resources where people have to make decisions or defer from making a decision (Mooij, 2004). According to the author, when people's stomachs are full and possess - more or less enough of everything and can afford proper housing and durable products, values become manifest and are reflected in the choice of brands and products This is because individuals will be eager to spend their exceeded income on what most fits their values. Thus, values form the main variable explaining consumer behavior and differences among consumers can be explained by cultural variables. Thus, values are an integral part of a consumer's actions rather than an environmental or external factors (Mooij, 2004) as have been classically considered in the study of consumer behavior. 


\subsection{Market segmentation}

Segmenting consumers is useful for marketers to better understand the needs, desires, and wants of consumers. In fact, because it is impossible to understand each individual, market segments are designed strategies to discover the needs, wants and desires of specific groups of consumers in order to position and plan the sale of a product according to each segment. Most common variables used to classify consumers include demographic, geographic, psychological and cultural (Solomon et al., 2000). For our purposes, nine major categories in market segmentation are provided by Schiffman and Lazar (2004) which are 1) geographic, 2) demographic, 3) psychological, 4) socio-cultural, 5) use-related, 6) use-situation, 7) benefit, 8) hybrid, and 9) psychographics. In the following these segmentation categories are described in more detail.

Geographic segmentation is used to divide the market by location. The theory behind this strategy is that people who live in the same area share similar needs and wants than those who live in other areas (Schiffman et al., 2008). Within geographic segmentation, cities are the most important unit of analysis to forecast consumption. According to Blackwell et al. (2006), cities are internally segmented in terms of suburbs that have grown rapidly. Today exurbs (areas beyond the suburbs) are experiencing the fastest growth. These areas are neither metropolitan nor rural but are adjacent to suburban or metropolitan areas. A metropolitan statistical area (MSA) is defined as a freestanding metropolitan area surrounded by nonmetropolitan areas and not closely related with other metropolitan areas. A primary metropolitan statistical area (PMSA) is a metropolitan area that is closely related to another city. A grouping of closely related PMSAs is a consolidated metropolitan statistical area (CMSA). Other geographic classifications are regions, states, and cities cited basically in advertising (Blackwell et al., 2006).

Demographic segmentation refers to the use of characteristics such as age, gender, marital status, income, occupation, and education as variables in locating the target market. Thanks to national census data, information on these characteristics is the most accessible. Furthermore, they are easy to measure (Schiffman \& Lazar, 2004). In addition, Blackwell et al. (2006) define demographic data as the size, structure, and distribution of population; however, they 
suggest demographics be used as additional variables in forecasting trends in consumer behavior.

Psychological segmentation refers to the inner or intrinsic qualities of people. Thus, psychological and sociological characteristics help to describe how the members of the target market think and feel. Motivation, personality, perceptions, learning, and attitudes are variables studied in this segmentation market (Schiffman et al., 2008). According to Blackwell et al. (2006) analyzing individual differences such as personality, values, and lifestyle provide a better understanding of consumer behavior (Blackwell et al., 2006), as psychoanalytic, sociological and psychological theories are useful in segmentation studies.

Sociocultural segmentation refers to the use of sociological and anthropological variables such as family lifecycle, social class, culture, subculture and religion to provide further bases for market segmentation (Schiffman et al., 2008). For instance, family lifestyle is based on the premise that many families pass through similar phases in their formation, growth, and final dissolution. Thus, among these phases there are specific needs and different usages of products and services. The concept of social class implies a hierarchy in which individuals in the same class generally have a common degree of status, while members of other classes have either higher or lower status. This is one of the reasons explaining why consumers of different social classes have different values, product preferences, and purchase habits. As a consequence, this method is commonly used in marketing and advertising. Within this type of market segmentation variables such as education, occupation, and income are used to establish a measurement index (Schiffman et al., 2008). Marketers use the concepts of culture, and sub-culture to segment domestic and international markets, due to the fact that members of a culture tend to share like values, beliefs, and customs. Thus, cultural and sub-cultural segmentations are used to segment groups of consumers on the basis of cultural heritage.

Use-related segmentation is another form of market segmentation. Within this type of segmentation, consumers can be categorized according to the use of products, brand usage characteristics and, sometimes, by brand loyalty (e.g. segmentation of heavy users, medium users, light users, and non-users). Use-related segmentation is commonly used to create marketing strategies and advertising focused on the differentiation of heavy users from light consumers (Schiffman et al., 2008). For example, Squires et al. (2002) use the concept of use- 
related segmentation to compare purchase frequency of organic food in New Zealand and Denmark.

Use-situation segmentation is a type of segmentation that consists of recognizing a situation and/or occasion that determines what consumers purchase or consume. Within this segmentation, occasional factors that can influence the purchase of products are analyzed (e.g. weekday, weekend, disposition of time, place, and occasion). Some examples include Saint Valentine's day, birthdays, Christmas, etc. In their study about food related lifestyles Grunert et al. (1993) searched for the factors influencing the usage and purchase of food products, that is, if consumption is determined by a social occasion, activity, etc.

Benefit segmentation is another category in segmenting the market, which consists of identifying the most important benefits of products and/or services as the most meaningful to consumers. For instance, features such as healthy, natural, tasty, etc., can be mentioned as benefits of organic products. This type of segmentation can be used in positioning various brands within the same product category (Schiffman et al., 2008).

As stated above, there are several categories in the segmentation of the market; however, in order to pursue precise target groups, hybrid segmentation is commonly used. "Hybrid segmentation refers to the combination of several variables [rather] than the use of only one basis of segmentation" (Schiffman et al., 2008). This segmentation has been exemplary in Germany by Sinus Sociovision (2006) in segmenting the different types of consumers of organic food according to their demographic and psychographic characteristics such as income, education, occupation, lifestyle, frequency of consumption and reasons for purchasing.

Finally, psychographic (lifestyle) segmentation is considered to be very powerful in examining individuals' preferences. Psychographic segmentation has been successfully implemented by marketers in many fields of research and will be used in the design of our study as well. Henceforth, we will devote the following section to providing more details on psychographic data. 


\subsection{Psychographics}

Psychographic segmentation, also commonly referred to as lifestyle analysis, is closely related to psychological segmentation because it implies measurement of personality and attitudes. Psychographic segmentation has proven its usefulness in identifying consumer segments that will likely be responsive to specific marketing strategies (Mowen \& Minor, 2001; Arnould et al., 2004; Blackwell et al., 2006; Solomon, 2006; Schiffman et al., 2008).

Several qualitative and quantitative measurement techniques and approaches have been developed in psychographic segmentation, i.e. to measure personality. The latter is defined as a consistent response to environmental stimuli (Blackwell et al., 2006). In the literature, scholars exploring psychographic characteristics of consumers often use the concept of lifestyle. The latter is a personal construct system continually adapting in response to changes in the environment consistent with people's own values and personality (ibid.).

Lifestyle AIO (Activities, Interest, and Opinions) is the most common measurement instrument (Mowen \& Minor, 2001; Blackwell et al., 2006; Schiffman et al., 2008). The theory of lifestyle has been widely used to explore purchase behavior and has therefore been employed in many areas of marketing: 1) market segmentation, 2) advertising, 3) development of post-material values, and 4) in the discussion of the possibility of standardization of marketing within global marketing strategies (Grunert et al., 1993). However, the main criticism towards lifestyle research has been addressed as: AA (activities and attitudes) and AIO (approaches). Grunert et al. (1993) for instance criticize the ways to derivate such lifestyle dimensions mainly by factor analysis and corresponding analysis, by stressing that these are unsatisfactory and not well documented, especially if they are employed in cross-cultural studies. According to the authors, the main reason is that lifestyle and values have not been distinguished, neither conceptually or at the operational level, in marketing.

More recently, lifestyles of health and sustainability (LOHAS) is also a market segmentation using psychographics to categorize consumers that purchase or use products and services on the basis of their personal, social or environmental values. Within this market segmentation, 
consumers who are aware of sustainable economy, healthy wellbeing, alternative health, personal growth, "green" products and ecological alternatives are targeted by this method of segmentation which was developed in the U.S.A. (Solomon, 2006). 


\section{Theoretical framework of values and motivations}

This chapter provides a theoretical framework about values and motivations from social psychology, anthropology, and marketing. All this with the aim of support our research and definition of the constructs inspiring our comparative research. Firstly, the theoretical framework of values is discussed. Secondly, the main theories of motivations and a discussion between motives and motivation are provided.

\subsection{Values in social psychology and marketing}

Understanding people's minds, values, and culture has been widely studied in the social sciences. The study of values has been an especially important issue in behavioral sciences since the second half of the 20th century. Therefore, in this section, we will consider the most useful theories and measurement approaches of values provided by social psychologists and anthropologists, as they have been widely used in marketing science as well.

Values are complex constructs that cannot be observed directly; they have henceforth been defined in different ways. The marketing scholars Hoyer and MacInnis (2007) define values as "beliefs that guide what people regard as important to themselves". According to Mowen and Minor (2001) "values are enduring beliefs about ideal end states and modes of conduct. In general, values tend to be few in number and more abstract than attitudes and serve as guidelines of actions, attitudes, judgments, and behavior". For Arnould et al. (2004) values are defined as "enduring beliefs about desirable outcomes that transcend specific situations and shape one's behavior". In line with Blackwell et al. (2006) values are another way of understanding consumer behavior: "Values are likely to attitudes and represent beliefs and acceptable behavior; however, values are unlikely to attitudes and transcend situations or events because they are guidelines in personality". According to Solomon (2006) values are defined as "a belief that some condition is preferable to its opposite". For Schiffman et al. (2008) "beliefs and values refer to the accumulated feelings and priorities that individuals have". Values are also beliefs; however, values differ from beliefs because 1) values are 
relatively few in number; 2) they serve as a guide for culturally appropriate behavior; 3) they are enduring or difficult to change; 4) they are not tied to specific objects or situations; and 5) they are widely accepted by members of a society (Schiffman et al.,2008).

Anthropologists and social psychologists have provided a wide body of literature on values. The work of the American anthropologist Kluckhohn (1951/1967) was one of the earliest studies on values and culture. The definition of the latter is especially well known, as it has reached anthropological consensus: "culture consists in patterned ways of thinking, feeling and reacting, acquired and transmitted mainly by symbols, constituting the distinctive achievements of human groups, including their embodiments in artifacts; the essential core of culture consists of traditional ideas and their attached values". He provides a definition of value as well: "value is a conception, explicit or implicit, distinctive of an individual or characteristic of a group, of the desirable which influences the selection from available modes, means and ends of actions" (Kluckhohn 1951/1967 cited in Hofstede, 2001). This definition underpinned the basis for subsequent value studies also widely used in marketing (e.g. Rokeach, 1973 and Hofstede, 1980 cited in Mooij, 2004; Schwartz \& Bilsky 1987 cited in Schwartz, 1992).

Rokeach (1973) defines values as "an enduring belief that one mode of conduct or end-state of existence is preferable to an opposing mode of conduct or end-state of existence. A value system is an enduring organization of beliefs concerning preferable modes of conducts or end states of existence along a continuum of relative importance" (cited in Mooij, 2004).

In addition, Schwartz and Bilsky (1987) define the following characteristics of values that: 1) are concepts or beliefs, 2) pertain to desirable end state or behavior, 3) transcended specific situations, 4) guide selection or evaluation of behavior and events, and 5) are ordered by relative importance. Thus, values are different from attitudes due to their generality or abstractness and hierarchical order of importance. In addition, values are a type of expressed goal or motivational concern. As consciousness goals, values represent three universal requirements of human existence: 1) the needs of individuals as a biological organism, 2) to coordinate social interaction, and 3) needs of survival and welfare groups (Schwartz \& Bilsky, 1987 cited in Schwartz, 1992). Consequently, in the research of food purchase behavior, Grunert and Juhl (1995) define values as "criteria used to select and justify actions and to 
evaluate people, including self, and events; also, values are self-centred and social-centred". In the study on behavior, values are important, as they interact with social cognitions and thus help to improve knowledge and understanding of the interpersonal world. Since people belonging to the same cultural group are expected to share similar values, similarities and differences in values can be used to characterize consumer purchase behavior across cultures. To this end, we will provide in the following section an overview of the main measurement approaches of values in literature.

\subsection{Main theories of values and measurement approaches}

\subsubsection{Means-end theory and laddering technique}

The Means-End Theory and laddering technique by Reynolds and Gutman (1988) is a useful qualitative approach in the research of values. The technique is based on the Means-End Theory, and it specifically focuses on the linkages between the attributes of the products (the "means"), the consequences (outcomes) for the consumer, and personal values (the "ends") (Reynolds \& Gutman, 1988).

The Means-End Theory is closely related to the Expectancy-Value Theory (Rosenberg,1956), whereby consumers learn to associate particular consequences with particular product attributes reinforcing, in this way, their purchase behavior (cited in Gutman and Reynolds, 1988). The Means-End Theory has been widely used in qualitative research of values.

\subsubsection{Rokeach Values Survey (RVS)}

The American social psychologist Rokeach (1972/73) tried to classify values into two main categories: terminal and instrumental. Terminal refers to desirable end states of existence. Instrumental refers to the means to get there (cited in Hofstede, 2001). Within the framework 
of the Rokeach Values Survey (RVS), the author individuated further categories of values (sub) belonging to the aforementioned ordered categories. Within the former he proposed true friendship, mature love, self-respect, happiness, inner harmony, equality, freedom, pleasure, social recognition, wisdom, salvation, family security, national security, sense of accomplishment, beauty, peace, comfortable life, and exciting life. The set of instrumental values consists of cheerfulness, ambitiousness, love, cleanliness, self-control, capabilities, courage, politeness, honesty, imagination, independency, intellect, broad-mindedness, logics, obedience, helpfulness, responsibility, and forgiveness. Based on these findings, Rokeach (1973) drafted eight motivational types of values listed as follows: 1) Self-direction, 2) Stimulation, 3) Hedonism, 4) Achievement, 5) Power, 6) Security, 7) Conformity, and 8) Tradition (cited in Hofstede, 2001).

The RVS is a controversial model in literature, as many marketing scholars are doubtful about the usefulness of this scale whereas others back its validity. For instance, Schiffman et al. (2008) mention the usefulness of RVS in categorizing the Brazilian market. Blackwell et al., (2006) mention that several studies have successfully linked personal values with choice of specific brands by means of the RVS. In contrast, academic scholars in the field of crosscultural research downsize the usefulness of the RVS claiming that this method of value measurement is only appropriate for U.S.A. society. Thus, the measurement of values in other countries may require other measurement scales (Grunert et al., 1993; Hofstede, 2001; Mooij, 2004; Solomon, 2006).

Mooij (2004) claims that in his definition Rokeach (1972/1973) deliberately excludes the conception of desirableness (i.e. those general norms of society are described in absolute terms of right or wrong,). She underlines that, especially when measuring values, people give different answers depending upon whether they are asked to judge values as moral guides for the whole society, or whether they are asked to evaluate them as preferred states of being which directly or indirectly affect their personal living sphere. This discussion will be further developed by Schwartz and Bilsky (1987) (cited in Schwartz, 1992). Alternative Value Measurements to RVS within the U.S.A. are the List of Values (LOV) developed by Kahle and Timmer, 1986 cited by Mooij, 2004, and the VALS (Values and Life Style System) developed by SRI (Stanford Research Institute) (cited in Blackwell et al., 2006). 


\subsubsection{Schwartz's Value Inventory (SVI) or Schwartz's Value Survey (SVS)}

Inspired by the eight motivational values proposed by Rokeach (1972/1973), the Israeli social psychologist Schwartz $(1987,1992)$ proposed a theory of universal values to explore human behavior and developed a measurement instrument of 56 values. This theory and instrument has been widely used in the social sciences and marketing alike. In addition to Rokeach's eight motivational types, Schwartz (1992) drafted three other motivational domains of values: Spirituality, Benevolence, and Universalism (Schwartz, 1992). Schwartz's model underlies three types of domains of values concerning human pursuit of personal interest, collective interest and both individual and collective interest. The pursuit of the specific goal of each value may be either compatible or in conflict with other values. For example, the pursuit of a personal interest may be in conflict with the pursuit of a collective interest (Schwartz, 1992).

\subsubsection{Hofstede: dimensions of culture}

Another important theory explaining values in human behavior is the work of the Dutch psychologist Hofstede. His model is one of the most useful for studying values in manifold research areas such as cross-cultural comparisons, management theories, marketing, and advertising. Hofstede developed four dimensions of culture: power distance, uncertainty avoidance, individualism, masculinity, and long-term orientations, all of which could be analyzed separately within the cultural dimension.

According to Hofstede (2001), within the construct of culture it is important to distinguish three main levels: 1) universal level, which is shared by all individuals as the expressive behavior (from a biological viewpoint); 2) collective level, a learned behavior that is shared among people belonging to the same group or category therefore different than people belonging to other groups or categories; finally, 3) the individual level which refers to the 
internal, unique part of individuals, for instance personality. However, the borders between individual personality and collective culture are not clear (Hofstede, 2001).

Hofstede defines value as a "broad tendency to prefer certain states of affairs over others". Thus, the same value can be activated in a variety of situations. Values are also feelings depicted as arrows on a plus or minus pole, as feelings also include intensity and direction. For instance, if we "hold" a value, this means that the issue involved has some relevance for us (intensity). Direction is established whenever we identify outcomes as "good" or "bad" (see Figure 3) (Hofstede, 2001). Values are mutually related and are integrated systems which are arranged internally by hierarchies. However, these value systems need to be in a state of harmony with each other, otherwise a change in behavior is produced. It is generally assumed that individuals are not fully aware of their value systems, because value systems or hierarchies are not clear at the conscious level.

Therefore, Hosftede (2001) classifies values into two main levels: desired values, i.e. what people actually desire, and desirable values, i.e. what they think they ought to desire. This implies that, in contrast to other studies, in Hofstede's study of values social desirability is not an undesirable effect which one has to control, for it is part of the whole studied phenomena.

Figure 3: The "onion diagram": manifestations of culture at different levels of depth

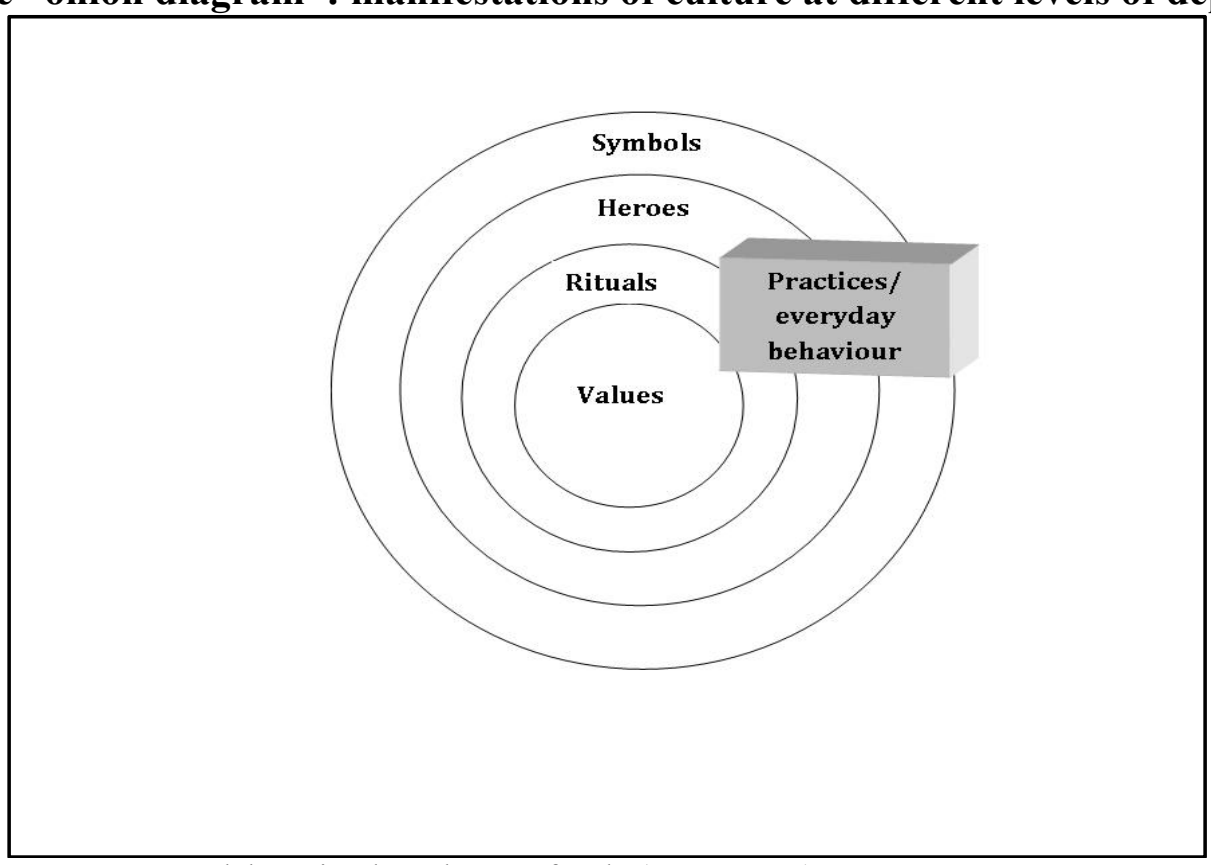

Source: Own elaboration based on Hofstede (2001, p. 11). 
This means that asking for the desirable is a perfectly legitimate research approach and serves to differentiate between desired and desirable values. It appears clear that desired values are more related to pragmatic issues. The exploration of such a dimension results in the values actually held by the majority of individuals within a society. On the other hand, the desirable dimension is more related to a subordinate ideology, an absolute norm, which is deontologically analyzed (see Table 9) (Hofstede, 2001).

To measure culture across nations, Hosftede developed a model of five dimensions to understand value differences: power distance, individualism/collectivism, masculinity/femininity, uncertainty avoidance, and long-term orientation. These dimensions are measured by means of interval scales with a score ranging from 0 to 100 . The samples were collected in 75 countries (Hofstede, 2001). In comparative cross-cultural studies Hosftede's dimensions have been widely used as independent variables to explain consumer behavior. Several comparative studies have confirmed and supported Hosftede's indices and measurement usefulness. An overview of Hofstede's dimensions regarding the case of our two- country comparison is summarized in both Table 10 and Figure 4. In the following, five dimensions underlying value differences are explained in detail. 
Table 9: Distinction between the desired and the desirable and associated distinctions

\begin{tabular}{|l|l|l|}
\hline \multicolumn{1}{|c|}{ Nature of a value } & \multicolumn{1}{|c|}{ Desirable } & \multicolumn{1}{c|}{ Desired } \\
\hline Dimension of value & Direction & Intensity \\
\hline $\begin{array}{l}\text { Nature of corresponding } \\
\text { norm of value }\end{array}$ & $\begin{array}{l}\text { Absolute, deontological, } \\
\text { ideological }\end{array}$ & $\begin{array}{l}\text { Statistical, } \\
\text { phenomenological, } \\
\text { pragmatic }\end{array}$ \\
\hline Corresponding behavior & Approval or disapproval & $\begin{array}{l}\text { Choice and differential } \\
\text { effort allocation }\end{array}$ \\
\hline Dominant outcome & Words & Deeds and/or words \\
\hline $\begin{array}{l}\text { Terms used in the } \\
\text { measurement instrument }\end{array}$ & Good, right, agree, should & $\begin{array}{l}\text { Important, } \\
\text { attractive, preferred }\end{array}$ \\
\hline Affective meaning of a term & Evaluation only & Activity plus evaluation \\
\hline $\begin{array}{l}\text { Person referred to in the } \\
\text { measurement instrument. }\end{array}$ & People in general. & Me, you. \\
\hline
\end{tabular}

Source: Hofstede (2001, p. 7).

Power distance is defined by Hofstede (2001) as "the extent to which less powerful members of a society accept and expect that power is distributed unequally". This dimension is reflected in the values of both less and more powerful members of a society. In societies with a large power distance culture (high scoring), everybody is in the right place in the social hierarchy; as a result, acceptance of power and legitimacy of authority come naturally. Within these societies older people are important, because respect for the aged plays an important role in demonstrating social position. In cultures with small power distance equality scores lower (Hofstede, 2001). According to Hosftede, the national culture in Mexico shows traits of large power distance (81 points); whereas, power distance in Germany is low (31 points).

Individualism/collectivism refers to people who look after themselves and their immediate family only (individualism) and to people who belong to groups (collectivism) that are taken care of in exchange for loyalty. Individualistic cultures are universalistic with more explicit verbal communication. Values are intrinsic on a personal level and include the desire for differentiation from others. Within these cultures, people attach priority to values such as 
variety and adventure (Hosftede, 2001). Conversely, collectivistic cultures are more particularistic and share a "we-consciousness". Furthermore, identity is based on social systems. Harmony is developed because the goals of a group(s) are preferred to individual aims (e.g. Latin American countries) (Hosftede, 2001). According to these parameters, the national culture in Mexico is collectivistic and score low (30 points) and the German national culture is individualistic and scored higher (63 points).

Masculinity/Femininity: within this dimension from Hofstede, "the dominant values in a masculine society are achievement and success, whereas dominant values in feminine societies are nurturing and quality of life". Within masculine societies, what is big and fast is beautiful. Conversely, feminine societies are more service-oriented, people oriented, and "small is see as beautiful" (Hosftede, 2001). Thus, regarding this dimension Mexico and Germany both demonstrate masculine like societies, but their scorings are different. The Mexican society scored (69 points) and the German society scored (62 points).

Uncertainty avoidance refers to "the extent to which people feel threatened by uncertainty and ambiguity and try to avoid these situations" (ibid.). Some people despise uncertainty or ambiguity, and therefore involve themselves in making rules and prescribing behavior; whereas, other people do not mind ambiguity (Hosftede, 2001). Within cultures of strong uncertainty avoidance (high scoring) there is a need for rules and formality in structuring life, and experts' beliefs are seen to be very trustworthy. People are more interested in how a product works than in results. Purity is an important value, and communication traits are formal. Within high uncertainty avoidance cultures, people tolerate a higher level of anxiety and aggressiveness. Furthermore, showing emotions is acceptable. Conflict and competition are considered threatening (Hosftede, 2001). On the contrary, in countries with low uncertainty avoidance cultures (low scoring), people feel that there should be as few rules as possible. Furthermore, individuals are more result-oriented and are more likely to believe in generalization and common sense. There is less ritual behavior. They do not consider conflict and competition as threatening (Hosftede, 2001). Regarding this dimension, Mexico scores high (82), whilst Germany scores high (61 points).

Long-term orientation was the latest dimension discovered by Hofstede and Bond (Mooij, 2004). This dimension focuses on long-term versus short-term orientation in life. Long-term 
orientation cultures consider that there is no one truth; thus, changes are acceptable. Within such cultures, individuals form values such as perseverance, thrift, and pursuit of peace of mind. In contrast, in short-term orientation cultures "spend now" is more important than saving for tomorrow (Anglo-Saxon societies). This dimension is particularly interesting in combination with the previous ones. For instance, countries with a combination of long-term orientation and the collectivistic dimension might be characterized by the following values: importance of family ties, filial piety, and paternalism (e.g. Chinese populations). A paradoxical value is generated by the combination of strong respect for tradition and shortterm orientation; this is the case in a large part of the Western world (Hosftede, 2001). For the interest of this study, Germany scored within this dimension (27 points); while no single score is available for Mexico.

Table 10: Hofstede's dimension of values across German and Mexican national culture

\begin{tabular}{|l|l|l|}
\hline \multicolumn{1}{|c|}{ Dimension of value } & \multicolumn{1}{|c|}{ Mexico } & \multicolumn{1}{|c|}{ Germany } \\
\hline Power distance & $\begin{array}{l}\text { Large distance } \\
\text { (high scoring: 81 points). }\end{array}$ & $\begin{array}{l}\text { Small distance (low } \\
\text { scoring: 31 points) }\end{array}$ \\
\hline Individualistic/ & $\begin{array}{l}\text { Collectivistic (low scoring: } \\
30 \text { points). }\end{array}$ & $\begin{array}{l}\text { Individualistic (scoring: } \\
63 \text { points). }\end{array}$ \\
\hline Masculine/Feminine & $\begin{array}{l}\text { Masculine (scoring: 69 } \\
\text { points). }\end{array}$ & $\begin{array}{l}\text { Masculine (scoring: 62 } \\
\text { points). }\end{array}$ \\
\hline Uncertainty avoidance & $\begin{array}{l}\text { High uncertainty (high } \\
\text { scoring: 82 points). }\end{array}$ & $\begin{array}{l}\text { Middle } \\
\text { (scoring: 61 points). }\end{array}$ \\
\hline Long-term orientation & $\begin{array}{l}\text { Information not available } \\
\text { (none scoring). }\end{array}$ & $\begin{array}{l}\text { Low term-orientation (low } \\
\text { scoring: 27 points). }\end{array}$ \\
\hline
\end{tabular}

Source: Own elaboration based on Hosftede (2009). 
Figure 4: Ranking of dimension of national culture in Germany and Mexico

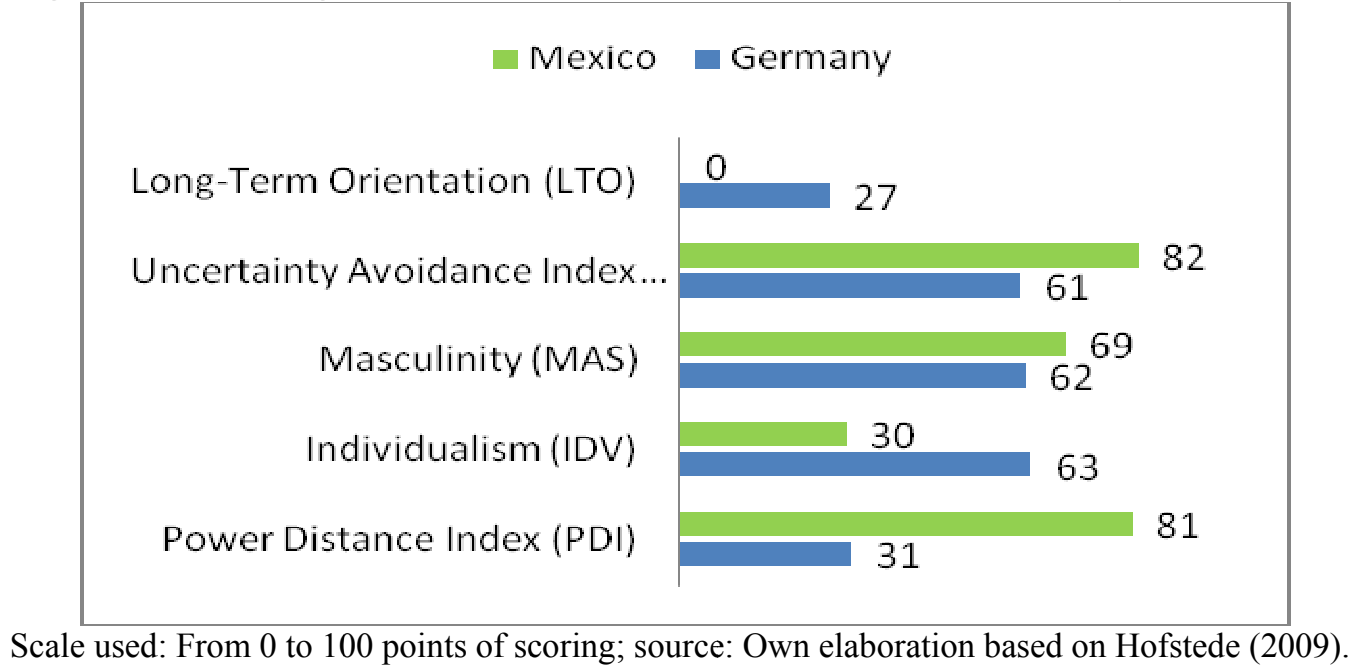

\subsection{Motivations in marketing and psychology}

Motivations are closely linked to a system of values and mainly to the satisfaction of needs. Similarly, motivations, a psychical process, have been widely studied in relation to consumer behavior in order to understand why people behave the way they do. Human motivations have been defined differently in social sciences. Mooij (2004) states that motivations should be distinguished as internal drivers, needs, primary drivers or motives, and it is assumed that their origin is to satisfy the organismic sphere of individuals (from a physiological/biological viewpoint). In psychology, motivations are also generally conceived as a process - or a series of processes - which somehow starts and guides one to the achievement of a goal. Behavioral explanations consider motivations as external drivers, since people have learned to adapt to external or environmental circumstances (O'Shaughnessy, 1992; Mooij, 2004).

Although in psychology, motives and motivations are defined in a different way, in marketing textbooks both terms are used interchangeably. In the following, we will show how marketing scholars generally refer to motivations. According to Mowen and Minor (2001), motivations are defined as "an active state within a person that leads to goal-directed behavior". Mooij (2004) defines motivation "as the internal state of an organism that drives it to behave in a certain way. Drivers are the motivational forces that cause individuals to be active and to strive for certain goals". Similarly, Blackwell et al. (2006) define motivation as "an internal 
state that activates goal-oriented behavior". Arnould et al. (2004) define motivation as "an inner drive-internal stimulus. Motivations drive behavior in the pursuit of a goal and create willingness to spend time and energy to achieve a goal". Human motivations are developed within a social context and culture. Although it is possible that all human cultures share some common basis of motivations (organismic, biological or physiological needs), a consistent theoretical framework has not been developed regarding universal motives of human behavior (Arnould et al., 2004).

In 2006, Solomon defined motivations as "the processes that lead people to behave as they do". When a need has been activated, a state of tension drives the individual to attempt to reduce or eliminate the tension caused by the need; this process may be described in terms of degrees of strength. The particular way(s) that consumers attempt to reduce their motivational tension describe(s) the direction. In addition, a drive refers to the degree of arousal. Accordingly, Hoyer and McInnis (2007) define motivation as "an inner state of arousal" with aroused energy directed toward achievement of a goal. Schiffman et al. (2008) claim that motivation "is the driving force that moves people to actions". Driving force is produced by a state of tension which exists as a result of an unfulfilled need, wants, and desires. The way people strive to reduce tension may be conscious or unconscious. Thus, motivation is a component of the motivational process (Figure 5).

\section{Figure 5: Model of the motivational process}

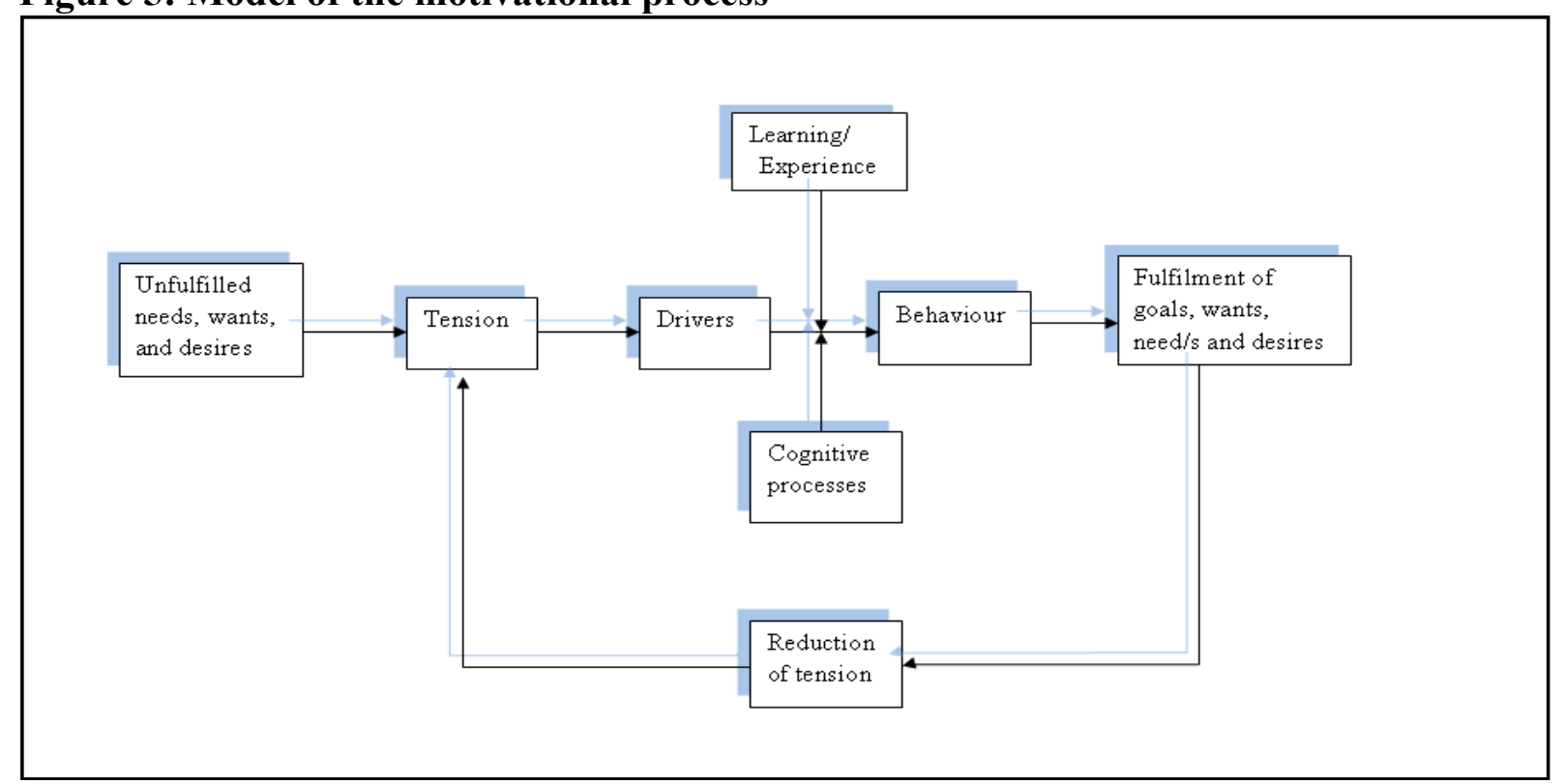

Source: Own elaboration based on Schiffman et al. (2008, p.105). 


\subsection{Main theories of motivations and measurement approaches}

According to Mooij (2004) and Blackwell et al. (2006), most motivations are classified in dichotomous needs categories (e.g. utilitarian/functional versus hedonic/experimental needs). The theory of unconscious motivation which relies on the concepts of Id, Ego and Superego by Freud is widely used in marketing as well (Blackwell et al., 2006; Mowen \& Minor 2001; Schiffman et al., 2008). However, Mooij (2004) highlights the Austrian-Hungarian cultural context of Freud in the early 1900s. Hence, the facts observed by Freud correspond to the cultural background of that period and therefore might not be universalized in all countries, cultures, and societies. The theory might still be valid for Hungarians and Austrians and those sharing a similar culture. This is confirmed by the findings of Hofstede (2001) who scores Austria and Hungary extremely low on power distance and high on uncertainty avoidance.

\subsubsection{Maslow's hierarchy of human needs}

Regarding human motivations, one of the most common and relevant theories is Maslow's hierarchy of needs. Mowen and Minor (2001) mention that the classification proposed by Maslow since the 1970s has been criticized and inspired as a broad theory of motivation. For Blackwell et al. (2006), in marketing the Maslow's hierarchy is a useful concept in order to understand consumer motivations, because it reminds us that people attach different priorities to their needs. All in all, the most relevant classification of needs is the Maslow's Hierarchy which "classified the human needs into five categories: 1) physiological, 2) safety, 3) social, 4) esteem, and 5) self-actualization". Maslow's hierarchy ranks "needs" from the most important to the least important (Figure 6). 


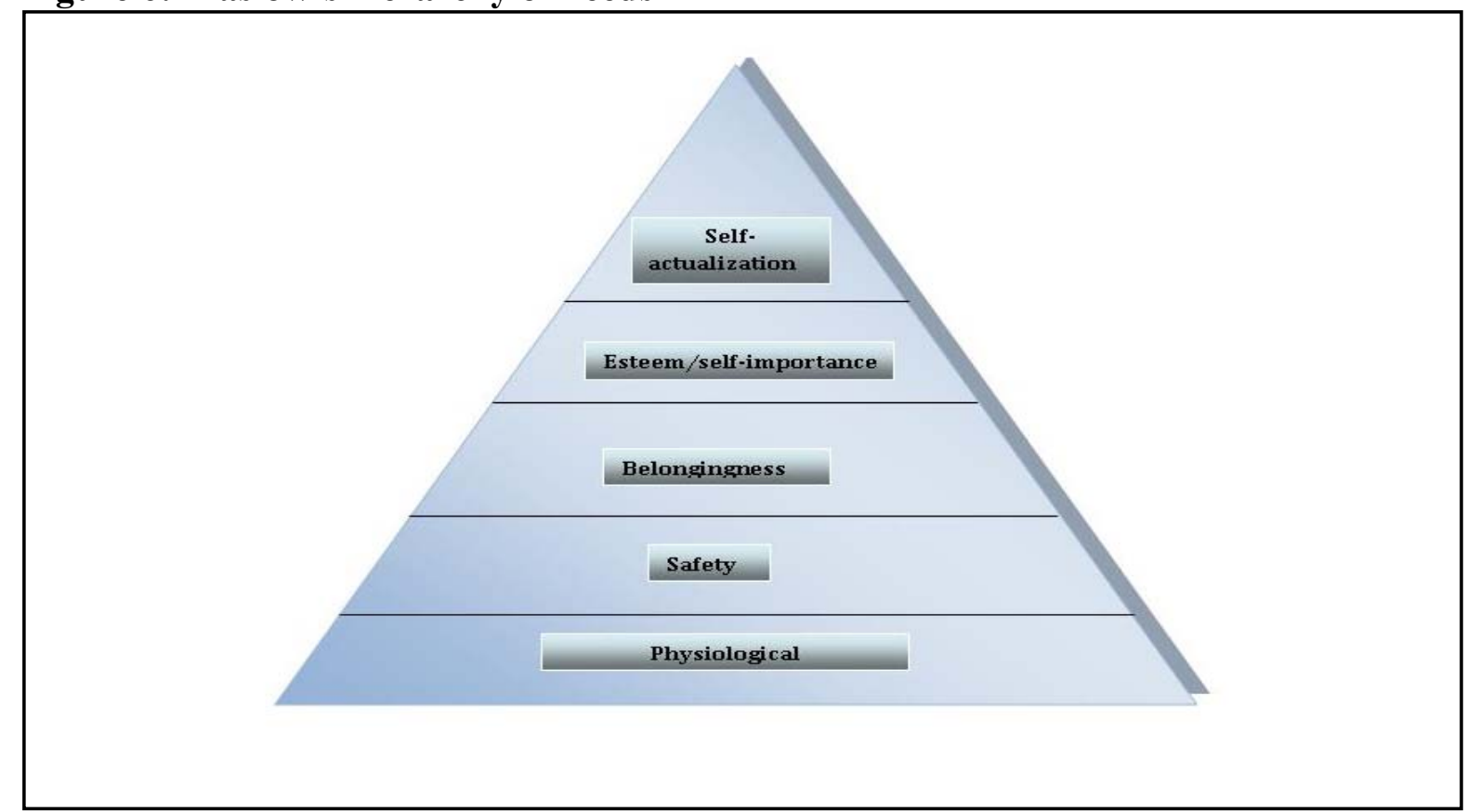

Source: Own Elaboration based on Solomon et al. (2000, p.126).

Although Maslow's hierarchy is useful in pointing out that people attach different priorities to their needs and refers to the priorities of many, it does not reflect the priorities of everyone in all situations, nor how social environment and culture influence the priorities of need to explain consumer behavior.

\subsubsection{McClelland's theory: achievement, power, and affiliation}

Another important theory on motivations was developed by McClelland. According to Mowen and Minor (2001) this theory proposes four basic learned needs-achievement, affiliation, power and uniqueness or novelty-to explain human motives. The need for achievement is linked to getting ahead, striving for success, and taking responsibility for solving problems. The need for affiliation relates to the need for people to become a member, to be associated with others, or belong to a group. The need for power refers to the desire to obtain and exercise control over others. This need might go in two directions: in a positive way, resulting in persuasive and inspirational power, and in a negative way, resulting in the 
desire to dominate and obtain submission from others. Finally, the need for uniqueness coincides with the desire to perceive oneself as different and original.

\subsubsection{Classical conditioning}

This label indicates a set of theories used to explain motivations as well. According to Mowen and Minor (2001), these theories are based on the idea that a motivational state can be created to lead people to engage in a variety of behaviors.

The so-called vicarious learning, or observational learning, is based on the premise that there is a phenomenon whereby people observe the actions of others and develop their own "patterns of behavior" accordingly. Thus, observational learning creates a motivational state that might influence a variety of behaviors.

\section{Figure 7: Psychological-cognitive theories of motivation}

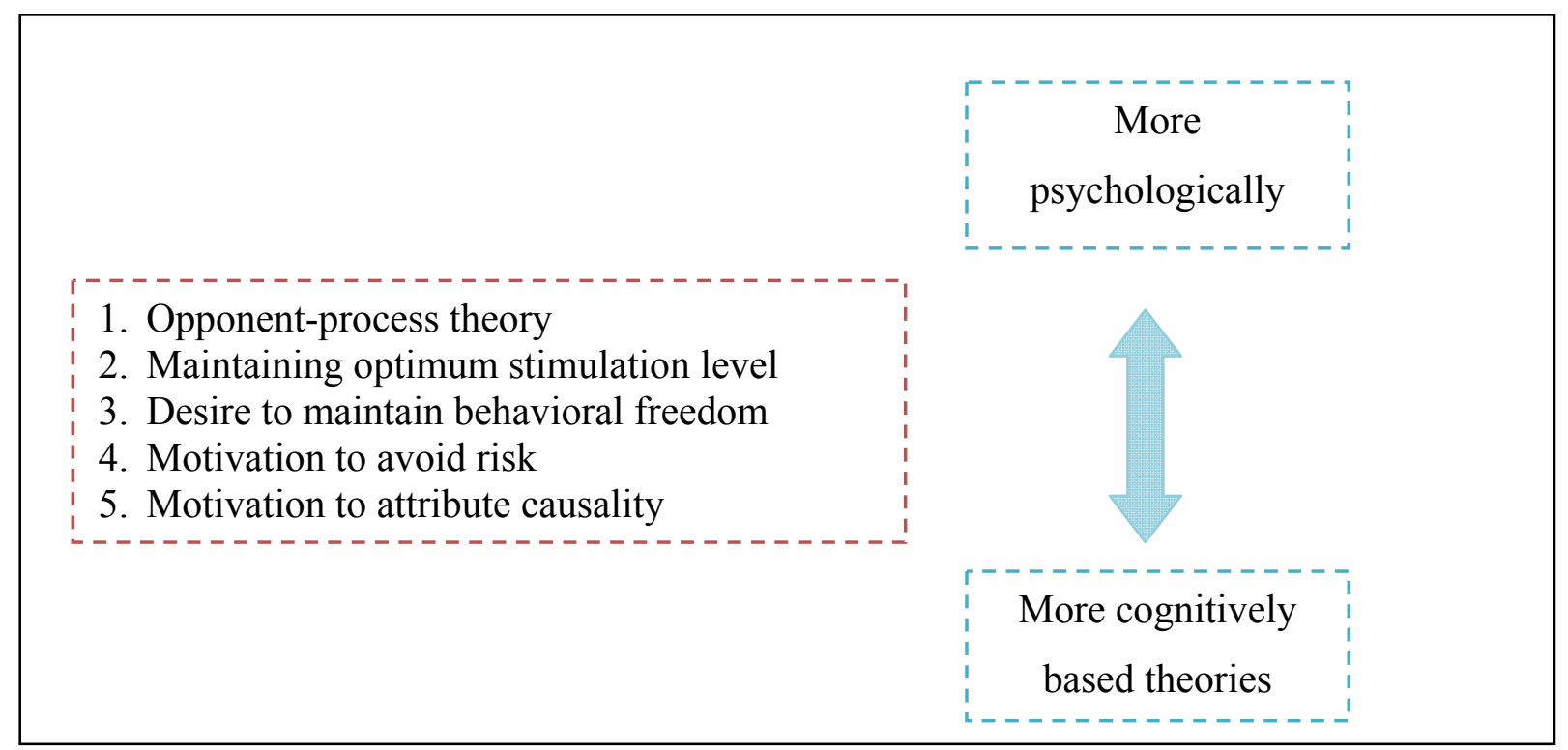

Source: Own elaboration based on Mowen and Minor (2001, p. 354)

The opponent-process theory refers to the situation where a person receives a stimulus that elicits an immediate positive or negative emotional reaction. In this case two new situations 
may be elicited: firstly, the immediate positive or negative reactions felt. Secondly, an emotional reaction occurs that acts in opposition to the initial experience. The output of the combination of both emotional reactions is the gradual decline of feelings (Mowen \& Minor, 2001). According to Mowen and Minor, individuals seek an optimum stimulation level and take action to correct it when it becomes too high or too low. Internal or external factors may influence a person's level of stimulation. Within internal factors are personal history and personality characteristics. Within the external factors are uncertainty and risk perceived in the environment.

The desire to maintain behavioral freedom refers to the reaction to preserve freedom if it has been threatened. Two kinds of threats can lead to reactance: social threats involve external pressure from others; impersonal threats are barriers restricting the ability to buy a particular product or service (Mowen \& Minor, 2001).

Motivation to avoid risk implies a consumer's perception of an overall course of action based on an assessment of possible negative outcomes and of the likelihood that those outcomes will occur (Mowen \& Minor, 2001).

Motivation to attribute causality: this theory is based on the premise that consumers look for explanations about events that happen in everyday life. Meaning, the explanation of the processes by which people make such determinations of causality of action has been labelled attribution theory, because people attempt to determine whether the cause of an action was either internal or external to the person or object in question (Mowen \& Minor, 2001). In the words of Arnould et al. (2004) "consumers adapt their motivations". For instance, in a new environment, consumers take action to adapt to change. As a consequence, motivations tend to vary among cultures; therefore it can be concluded that there is no direct relationship between culture and motivations. Specific motivations would be better supported in some cultures than in others".

In summary, in the literature there is evidence that although people's motivations are different, they are related to a values system. As a consequence, individuals strive first and foremost to satisfy their conscious or subconscious needs. Thus, studying motivation which is inclusive with other elements of human behavior is a challenging task. Understanding why people behave in the way they do is often a difficult endeavor. In line with Blackwell et al. 
(2006), difficulty arises because people may not be willing to disclose the actual reasons behind their actions. Another challenge stems from the fact that reality changes continuously. Consequently, what motivates consumers to buy today may not be what motivates them in the future. 


\section{Development of the theoretical framework}

Food satisfies one of the universal physiological needs of humans. However, in the study of human behavior what and how people choose food is a complex phenomenon. Cognitive and affective variables have an influence on the choice of food within and across cultures. In line with Magistris and Gracia (2008) "food satisfies one of the universal human requirements; however preparation of meals and surroundings of social activities might differ among cultural groups and individuals". Similarly, Steptoe et al. (1995) recognize that "cultural factors influence food selection as well as habitual consumption of certain foods and habits of preparation of meals, social environment or social interaction which plays a role in the consumption, cooking, preparation, and eating of food products".

Hence, the definition of cultural levels according to Hofstede (2001) also applies to food consumption. The latter is from the physiological viewpoint as universal activity and has a social dimension (i.e. search and consumption imply interaction). Finally, since each person has a personal attitude toward food, it is an individual activity. These considerations are shared by a vast number of scholars (Krondl \& Coleman, 1984 cited in Steptoe et al., 1995; Baker et al., 2004; Magistris \& Gracia, 2008). For instance, what Mexicans, Italians or Chinese prefer or expect from meals might be different from the expectations of Germans, Norwegians, or Algerians. As a consequence, cultural differences are important when examining habits of consumption and preparation of food.

In this chapter, the theoretical framework of the study is provided, beginning with international literature on consumer buying behavior with special emphasis on, above all, motives and values driving the choice to purchase organic food. After that, the theoretical constructs, as well as the first tentative model, are described and finally some considerations concerning cross-cultural studies are made. 


\subsection{International literature review on purchase of organic food}

Since the 1990s, the production and consumption of organic food has increased in the world, especially in European countries. Thus, several studies concerning consumption of organic food show that this is an interesting topic in the study of consumer behavior. To estimate demand, consumption, and the development of markets for organic products most of the earliest studies focus on the demographic profile of the consumers. However, an increasing number of studies with emphasis on psychographic variables have recently appeared to understand better the purchase of organic food. Psychographic variables have been suggested to contribute to a more complex understanding of organic food purchase.

Overall, studies seem to devote attention to the following topics: 1) perception of organic food (Cicia et al., 2002; Harper \& Makatouni, 2002; Roddy, 1994; Roitner-Schobesberger et al., 2008; Wier et al., 2008); 2) beliefs/attitudes toward organic food (Baker et al., 2004; Bruhn, 2001; Chinnici et al., 2002; Magnusson et al., 2001; Magistris \& Gracia, 2008; Radman, 2005; Roddy, 1994; Roininen, 2001; Tarkiainen \& Sundyvist, 2005; von Alvensleben, 1998;); 3) lifestyle (Gil \& Sanchez, 2000; Grunert et al., 1993); 4) values (Grunert et al., 1995; Honkanen et al., 2006; v. Alvensleben, 1998; Zanoli \& Naspetti, 2002;); 5) motivations (Baker et al., 2004; Fotopoulos \& Krystallis, 2002; Honkanen et al., 2006; Lockie et al., 2002; Makatouni, 2002; Sirieix et al., 2007; Zanoli \& Naspetti, 2002;); 6) willingness to pay (Chinnici et al., 2002; Davies et al., 1995; Gil \& Sanchez, 2000); and 7) intention to buy (Tarkiainen \& Sundyvist, 2005).

Consumers of organic food have often been analyzed by means of hybrid models whose main constructs are perception, attitudes and motivations. The samples used in the majority of studies include both consumers of organic food and non-buyers. The survey approach has been the most common research instrument in collecting information by self-reporting, faceto-face interviews, or postal surveys (Chinnici et al., 2002; Cicia et al., 2002; Davies et al., 1995; Fotopoulos \& Krystallis, 2002; Grunert \& Juhl, 1995; Gil \& Sanchez, 2000; Honkanen et al., 2006; Magistris \& Gracia, 2008; Schifferstein \& Oude Ophuis, 1998; Radman, 2005; Roitner-Schobesberger et al., 2008; Squires et al., 2001; Tarkiainen \& Sundyvist, 2005). 
Other methods used by academic scholars are in-depth interviews to explore attitudes/beliefs and values, and focus groups of consumers of organic food to explain the purchase thereof (Roddy, 1994; Makatouni, 2002; Padel \& Foster 2005). In these studies, research methods such as Means-End-Chain and laddering technique are widely used (Zanoli \& Naspetti, 2002; Baker et al., 2004; Padel \& Foster, 2005). Additionally, first studies use panel data (Niessen \& Hamm, 2008; Padel \& Foster, 2005; Wier et al., 2008; Garcia et al., 2010).

\subsubsection{Demographic profile of consumers}

Many studies have focused on the demographic features of individuals with a preference for organic food. According to the international literature review, consumers of organic food are considerably alike internationally. The "typical" consumer of organic food is female with children holding a higher level of education and high-income level; thus, she usually belongs to the upper-middle class (Table 11).

Table 11: Demographic features of organic food consumers

\begin{tabular}{|l|l|}
\hline \multicolumn{1}{|c|}{$\begin{array}{l}\text { Demographic } \\
\text { characteristic }\end{array}$} & \multicolumn{1}{c|}{ Sources } \\
\hline Female & $\begin{array}{l}\text { Chinnici et al., 2002; Davies et al., 1995; Fotopoulos \& Krystallis, } \\
\text { 2002; Magistris \& Gracia, 2008; Wier \& Calverley, 2002. }\end{array}$ \\
\hline $\begin{array}{l}\text { High education } \\
\text { level }\end{array}$ & $\begin{array}{l}\text { Chinnici et al., 2002; Davies et al., 1995; Fotopoulos \& Krystallis, } \\
\text { 2002; Gil \& Sanchez, 2000; Magistris \& Gracia, 2008; Roitner- } \\
\text { Schobesberger et al., 2008; Wier \&Calverley, 2002. }\end{array}$ \\
\hline $\begin{array}{l}\text { Higher disposible } \\
\text { income }\end{array}$ & $\begin{array}{l}\text { Chinnici et al., 2002; Davies et al., 1995; Fotopoulos \& Krystallis, } \\
\text { 2002; Magistris \& Gracia, 2008; Roitner-Schobesberger et al., 2008; } \\
\text { Wier \& Calverley, 2002. }\end{array}$ \\
\hline $\begin{array}{l}\text { Children in the } \\
\text { household }\end{array}$ & $\begin{array}{l}\text { Chinnici et al., 2002; Davies et al., 1995; Fotopoulos \& Krystallis, } \\
\text { 2002; Roitner-Schobesberger et al., 2008; Wier \& Calverley, 2002. }\end{array}$ \\
\hline Couples & Fotopoulos \& Krystallis, 2002; Magistris \& Gracia, 2008. \\
\hline
\end{tabular}

Source: Own elaboration.

It is worth mentioning that because most of the studies have been conducted in Europe, the demographic profile of consumers of organic food is similar because of the stage of economic 
development of these countries. In a study conducted in Australia by Lockie et al. (2004), the two demographic variables which mostly explained the purchase of organic food were age and education. In addition, because of the gap of information about the demand and consumption of organic food in developing countries, there is not enough evidence to suggest that the demographic profile of "typical" organic consumers described above also applies to these countries.

All in all, the main differences among consumers of organic food and non-buyers are of a demographic and social nature. For instance, in the majority of the studies, lower level of education and lower income levels are mentioned as characteristics of non-buyers (Cicia et al., 2002; Fotopoulos \& Krystallis, 2002; Magistris \& Gracia, 2008; Roitner-Schobesberger et al., 2008). Similarly, in a study conducted in Southern Italy, Magistris and Gracia (2008) mentioned that among the consumers of organic food who were interviewed, the subgroup with lower incomes are more likely to purchase organic food only occasionally. Thus, the overall belief that consumers with lower incomes are less likely to purchase organic food seems to be confirmed. The findings of Cicia et al. (2002) who conducted a study in the same Italian region with consumers of organic food and non-buyers/consumers of conventional food confirmed homogeneity of demographic and social characteristics among the consumers of organic food. However, heterogeneity at the individual level still exists which can be better explained by means of psychographic variables. The factors inhibiting the purchase of organic food are summarized in Table 12. 
Table 12: Factors inhibiting purchase of organic food

\begin{tabular}{|l|l|}
\hline \multicolumn{1}{|c|}{ Factor } & \multicolumn{1}{|c|}{ Source } \\
\hline Lower availability & $\begin{array}{l}\text { Chinnici et al., 2002; Davies et al., 1995; Gil \& Sanchez, 2000; } \\
\text { Fotopoulos \& Krystallis, 2002. }\end{array}$ \\
\hline Higher price & $\begin{array}{l}\text { Chinnici et al., 2002; Davies et al., 1995; Fotopoulos \& Krystallis, } \\
\text { 2002; Gil \& Sanchez, 2000; Harper \& Makatouni, 2002; Magistris \& } \\
\text { Gracia, 2008; Roitner-Schobesberger et al., 2008. }\end{array}$ \\
\hline Poor quality with & Davies et al., 1995; Fotopoulos \& Krystallis, 2002. \\
\hline $\begin{array}{l}\text { Satisfaction } \\
\text { conventional food }\end{array}$ & $\begin{array}{l}\text { Fotopoulos \& Krystallis, 2002. } \\
\text { Gracia, 2008 -visual attractiveness-. }\end{array}$ \\
\hline Appearance & Fotopoulos \& Krystallis, 2002. \\
\hline Nothing special to try & Gil \& Sanchez, 2000. \\
\hline Fashion & Roitner-Schobesberger et al., 2008. \\
\hline Lack of information &
\end{tabular}

Source: Own elaboration.

\subsubsection{Cross-cultural studies on organic food consumption}

Few cross-cultural studies on the purchasing of organic food have been conducted. One of them is the work of Baker et al. (2004), who provided an in-depth understanding of the purchase of organic food by means of a cross-cultural comparison of attitudes, motivations and values between German and England consumers.

Overall, the reasons mentioned by respondents for purchasing organic food were health, wellbeing and enjoyment of life. However, these values were different between the two groups of consumers. Because values are the core element of culture, the purpose of their study was to search for the differences between the value systems of consumers in the two countries in order to identify which values, desirable end states of existence, (Rokeach, 1973, cited in Schwartz, 1992) were influencing the choice of organic food.

From the interviews conducted within specialized stores and at weekend markets with consumers of organic food by Baker et al. (2004), three dominant perceptual orientations were derived: 1) health/enjoyment, 2) belief in nature, and 3) animal welfare. In the German group the construct of health/enjoyment reflected the products' attributes and was the dominant 
perceptual orientation. Enjoyment was the second perceptual orientation which indicates a major concern for nature through the hierarchical value chain. In addition, animal welfare was the least dominant perceptual orientation and showed no correlation with the other two. Overall, similarities between consumers' perceptual orientations in the two countries were found only at the superficial level. As already mentioned, the health/enjoyment perceptual orientation was widely different. That said, the unique attribute shared by the consumers of organic food was "Not Genetically Modified" which suggests a similarity with the concept of food security by Squires et al. (2002).

The "need for security" and the "need of safety" are concepts included in the cultural dimension of the Uncertainty Avoidance Index (UAI) developed by Hofstede (2001) who used a scale ranging from 0 to 100 to measure this dimension. According with this, the national culture in the UK was characterized by a low UAI score of 30 points; whereas the national culture in Germany scored 60 points. This means that although the need for safety and security is important in the two cultures, it is more so in Germany than in the $\mathrm{UK}^{4}$.

Also, the second domain of perceptual orientation "belief in nature" was quite different between the two countries with a higher score among German consumers. The value "absence of pesticides and chemicals" was weakly associated with other attributes among English consumers. Again, this domain of perceptual orientation was more important among the German group than the UK group. Among the German group, the value of quality was connected to taste, while among the UK group, the value of quality was not mentioned at all.

In the study of Baker et al. (2004), the conceptual maps obtained from the German group were more elaborate than the hierarchical maps obtained from the UK group. Thus, according to the authors (ibid.), this has an important role in the drawing of conceptual maps. Another important difference in the results is the availability of organic food on the market which is greater in Germany than in the UK. As a consequence, German consumers are more familiarized than England consumers with organic food supply. In a further stage, Baker et al.

\footnotetext{
${ }^{4}$ http://www.geert-hofstede.com/hofstede_united_kingdom.shtml, http://www.geert-hofstede.com/hofstede_germany.shtml
} 
(2004) simplified their measurement model. In the final stage the latter consisted, of only two main constructs: one related to health, enjoyment and achievement, and the other regarding respect for others/workers.

Another cross-cultural study regarding organic food consumption was conducted between Germany and France by Sirieix and Schaer (2000). According to the authors, the purpose of the study was the comparison of perception of image and attitudes toward organic food locally produced in two regions. Data were collected by means of telephone interviews and statistically analysed by means of univariate statistic frequencies and cross tables. As a result, five variables were established on the image of organic food. The highest response frequency related to environment, followed by health, and animal welfare. One difference mentioned was that Germans considered taste the most important attribute. Finally, trustworthiness was investigated; the French scored organic food as highly trustworthy; tasteful scored in second place.

Another comparative study was undertaken by Squires et al. (2002) between the Danish and New Zealand markets. The study used a quantitative approach, and data were collected in the two countries by means of a survey. A logic regression model was used to measure attitudes toward health, environment, and conventional food and included demographic information influencing the frequency or intensity of organic food purchase. The authors suggested that demographic variables are a limited predicator in organic food purchase.

Contrary to developed countries, only a few studies concerning purchase behavior of organic food have been conducted in developing countries. As far as we know, there is only one comparative study between Brazil and France, first case area from a total of six, by Sirieix et al. (2007). Personal interviews were conducted taking into consideration urban socioeconomic features, motives, and barriers to purchase organic food. The importance of organic food quality for personal and family health was also included in the survey. Preference for local organic produce was common in both countries; however, the motives for purchase were different regarding environmental concerns and the support of small or local farmers (Sirieix et al., 2007). Whereas environmental reasons were the most important motives for purchase in France, in Brazil the main motive was clearly health, followed by altruistic values and 
concerns for small organic farmers. Environmental concerns were of minor importance (Sirieix et al., 2007).

\subsubsection{Role of motivations in organic food purchasing}

Before summarizing the main motivations to purchase organic food reported in international literature, it is worth mentioning that most of the studies use the words "reason," "motive" or "motivation" interchangeably (Baker et al. 2004; Chinnici et al., 2002; Davies et al., 1995; Fotopoulos \& Krystallis, 2002; Hughner et al., 2007; Magistris \& Gracia, 2008; RoitnerSchobesberger et al., 2008; Schifferstein \& Oude Ophuis, 1998; Wier \& Calverley, 2002).

A different terminology was employed by Makatouni (2002) who prefers to speak of "the process of motives" or "motivating factors"; Honkanen et al. (2006) define ethical food choice motives "as more specific than values, but more abstract than attitudes". They identified two levels of value domains: "The first refers to the desired end states (Schwartz, 1992); these are very abstract and it can be difficult to find direct relations between these values and specific attitudes; and the second level corresponds to Vinson's specific domain values which are relevant at a more superficial level (e.g. economic and social activities)".

Several academic scholars consider that the motivation to purchase organic food could be attributed to some environmental/ethical beliefs, to quality/health concerns, as well as to exploratory food purchase behaviors (neo-philia). Others assign them to sensory features and price level (Baker et al., 2004; Chinnici et al., 2002; Davies et al., 1995; Grunert \& Juhl, 1995; Honkanen et al., 2006; Magnusson et al., 2003; Radman, 2005; Roddy et al., 1996; Tarkiainen \& Sundyvist, 2005; Zanoli \& Naspetti, 2002). All in all, it appears that "demand of organic food is mostly based on value concepts, living situation, and access to information instead of demographic and sociological factors" (Worner \& Meier-Ploeger, 1999 cited in Fotopoulus \& Krystallis, 2002).

Although there is no evidence that organic food is healthier or more nutritious than conventional food, health is the most frequently mentioned reason as the main motivation for 
purchasing organic food in international literature (see Table 13). The second most motivation is environmental protection/environmental benefits. Taste is also frequently mentioned as a motivation to purchase organic food. Animal welfare is frequently linked to food safety, and lifestyle is linked to environmental consciousness (Fotopoulos \& Krystallis, 2002). 
Table 13: Ranking of motivations for purchase of organic food

\begin{tabular}{|c|c|}
\hline Motive & Study \\
\hline Health & $\begin{array}{l}\text { Baker et al., 2004; Chinnici et al., 2002; Cicia, et al., 2002; Chyssohoidis \& } \\
\text { Krystallis, 2005; Davies et al, 1995; Fotopoulos \& Krystallis, 2002; Harper \& } \\
\text { Makatouni, 2002; Makatouni, 2002; Magnusson, et al., 2003; Magistris \& Gracia, } \\
\text { 2008; Michelsen et al., 1999; Padel \& Foster, 2005; Roitner-Schobesberger et al., } \\
\text { 2008; Sirieix et al., 2007; Schifferstein \& Oude Ophuis, 1998; Roitner-Schobesberger } \\
\text { et al., 2008; v. Alvensleben, 1998; Zanoli \& Naspetti 2002. }\end{array}$ \\
\hline Better taste & $\begin{array}{l}\text { Baker et al., 2004; Chinnici et al., 2002; Chyssohoidis \& Krystallis, 2005; Davies et } \\
\text { al., 1995; Fotopoulos \& Krystallis, 2002; Magnusson et al., 2001, Magistris \& Gracia, } \\
\text { 2008; Michelsen et al., 1999; Roddy et al., 1996; Roitner-Schobesberger, et al., 2008; } \\
\text { Sirieix et al., 2007; Schifferstein \& Oude Ophuis, 1998; v. Alvensleben, } 1998 .\end{array}$ \\
\hline $\begin{array}{l}\text { Environmental } \\
\text { concern }\end{array}$ & $\begin{array}{l}\text { Baker et al., 2004; Chinnici et al., 2002; Cicia et al., 2002; Davies, 1995; Fotopoulos } \\
\text { \& Krystallis, 2002; Honkanen et al., 2006; Lockie et al., 2004; Michelsen et al., } \\
\text { 1999; Makatouni, 2002; Magnusson, et al., 2003; Padel \& Foster, 2005; Roddy et } \\
\text { al., 1996; Sirieix et al., 2007; Schifferstein \& Oude Ophuis, 1998; Squires et al., } \\
\text { 2002; Magistris \& Gracia, 2008; v. Alvensleben, 1998; Zanoli \& Naspetti } 2002 .\end{array}$ \\
\hline Food safety & $\begin{array}{l}\text { Baker et al., 2004; Harper \& Makatouni, 2002; Schifferstein \& Oude Ophuis, 1998; } \\
\text { Squires et al., 2002. }\end{array}$ \\
\hline Food scandals & Sirieix et al., 2007 \\
\hline $\begin{array}{l}\text { Animal welfare } \\
\text { concern }\end{array}$ & $\begin{array}{l}\text { Baker et al., 2004; Davies et al., 1995; Harper \& Maktouni 2002; Honkanen et al., } \\
\text { 2006; Michelsen et al., 1999; }\end{array}$ \\
\hline $\begin{array}{l}\text { Support local } \\
\text { economy }\end{array}$ & $\begin{array}{l}\text { Baker et al., 2004; Magistris \& Gracia, 2008; Padel \& Foster, 2005; Wier \& } \\
\text { Calverley 2002, Worner \& Meier-Ploeger, } 1999 \text { cited in Fotopoulos \& Krystallis, } \\
2002 .\end{array}$ \\
\hline $\begin{array}{l}\text { Wholesome/ } \\
\text { nostalgic }\end{array}$ & $\begin{array}{l}\text { Chinnici et al., 2002; Davies et al., 1995; Squires et al., 2002; Zanoli and Naspetti, } \\
2002 .\end{array}$ \\
\hline $\begin{array}{l}\text { Curiosity/try } \\
\text { something new/ } \\
\text { fashionable }\end{array}$ & Chinnici et al., 2002; Roitner-Schobesberger et al., 2008; v. Alvensleben, 1998; \\
\hline $\begin{array}{l}\text { Nutrition } \\
\text { concern }\end{array}$ & Baker et al., 2004; Chinnici et al., 2002; Magnusson et al., 2003. \\
\hline Superior Quality & Fotopoulos \& Krystallis, 2002; Magistris \& Gracia, 2008. \\
\hline Naturalness & Lockie et al., 2004; Schifferstein \& Oude Ophuis, 1998; v. Alvensleben, 1998; \\
\hline Fair trade & Harper \& Makatouni, 2002; Padel \& Foster, 2005. \\
\hline Freshness & Chinnici et al., 2002; Fotopoulos \& Krystallis, 2002; Magistris \& Gracia, 2008. \\
\hline Political motives & Honkanen et al., 2006; Sirieix et al., 2007 \\
\hline Ethical act & Sirieix et al., 2007. \\
\hline
\end{tabular}

Source: Own international literature review based on Hughner et al. (2007). 


\subsubsection{Role of values in organic food purchasing}

Values have been widely used to predict human as well as consumer behavior, because values are a core element guiding cultural behavior. Thus, values might provide information about people/individuals and societies or cultural groups to understand how people purchase. As mentioned in earlier studies dealing with purchase of organic food, scholars suggest that ethical behavior, concern for environment, and support of local economies are main values affecting the purchase of organic food products. The majority of these studies have been conducted in Northern European countries (Baker et al., 2004; Davies et al., 1995; Grunert \& Juhl, 1995; Honkanen et al., 2006; v. Alvensleben, 1998).

Most studies focusing on the role of values in the purchase of organic food are undertaken using a theoretical framework on social psychology findings. For instance, Grunert and Juhl (1995) tested the Schwartz Value Survey (SVS) to assess self-reported environmental attitudes reflected in purchasing organic food in Denmark. The findings of the cluster analysis and the discriminate analysis confirmed the relationship between environmental attitudes and purchase of organic food. Thus, the SVS was suggested as a useful instrument of measurement for cross-cultural psychology and consumer behavior research.

Makatouni (2002) used in-depth consumer interviews to explain consumption of organic food. Further, by means of Means-End Chain and laddering interviews he was able to determine that the purchase of organic food is related to individualistic and social values. Another study providing an important theoretical framework of values is that conducted by Honkanen et al. (2006) in Norway (see Table 14). The purpose of this study was to examine the structural relationships between ethical behavior and the choice of organic food. As measurement instrument was used the Ethical Food Choice Survey by Lindeman and Väänänen (2000). The authors could extrapolate two ethical motives -environmental and political motives-. Furthermore, they could demonstrate the usefulness of the study of values in explaining attitudes toward organic food purchase. For instance, consumers who were ecologically oriented were more likely to purchase not only organic food but also demonstrated a penchant for fair trade products. They also declared a higher commitment to human rights. 
Table 14: Main values domain

\begin{tabular}{|c|c|c|}
\hline Values domain & Values & Source \\
\hline \multicolumn{3}{|l|}{ A) Human level: } \\
\hline \multirow{8}{*}{ 1. Individual } & Health & $\begin{array}{l}\text { Baker et al., 2004; } \\
\text { Makatouni, 2002. }\end{array}$ \\
\hline & Responsibility for family and self & Makatouni, 2002; \\
\hline & Wellbeing & $\begin{array}{l}\text { Baker et al., 2004; } \\
\text { Makatouni, 2002. }\end{array}$ \\
\hline & Enjoy life & Baker et al., 2004. \\
\hline & Relaxation and satisfaction & Makatouni, 2002. \\
\hline & Nostalgia & Makatouni, 2002. \\
\hline & Longer life & Makatouni, 2002. \\
\hline & Happiness & Makatouni, 2002. \\
\hline \multirow{4}{*}{$\begin{array}{l}\text { 2. Collective: } \\
\text { Political motives }\end{array}$} & Egalitarian/respect for others & $\begin{array}{l}\text { Baker et al., 2004; } \\
\text { Makatouni, 2002. }\end{array}$ \\
\hline & \begin{tabular}{|lll}
$\begin{array}{l}\text { From a } \\
\text { country }\end{array}$ & politically acceptable \\
\end{tabular} & Honkanen et al., 2006. \\
\hline & $\begin{array}{l}\text { From a country with no violations of } \\
\text { human rights }\end{array}$ & Honkanen et al., 2006. \\
\hline & \begin{tabular}{|l} 
Does not conflict with personal \\
political values.
\end{tabular} & Honkanen et al., 2006. \\
\hline \multicolumn{3}{|l|}{$\begin{array}{l}\text { B) Animal level } \\
\text { (Animal welfare): }\end{array}$} \\
\hline 1. Animal benefits: & $\begin{array}{l}\text { Health and human feelings/beliefs } \\
\text { about life in general }\end{array}$ & Makatouni, 2002 \\
\hline 2. Animal friendliness: & Produced without pain to animals & Honkanen et al., 2006. \\
\hline \multirow{5}{*}{$\begin{array}{c}\text { Environment level } \\
\text { (Concern about environment): }\end{array}$} & Pesticides & Makatouni, 2002 \\
\hline & $\begin{array}{l}\text { Consequences of importation of } \\
\text { organic food }\end{array}$ & Makatouni, 2002 \\
\hline & $\begin{array}{l}\text { Responsibility and protection of the } \\
\text { environment }\end{array}$ & $\begin{array}{l}\text { Honkanen et al., 2006; } \\
\text { Makatouni, 2002; }\end{array}$ \\
\hline & $\begin{array}{l}\text { "Healthy" planet for future } \\
\text { generations }\end{array}$ & Makatouni, 2002. \\
\hline & Production without disturbing nature & Honkanen et al., 2006. \\
\hline
\end{tabular}

Sources: Own elaboration based on Makatouni, 2002; Baker et al., 2004; Honkanen et al., 2006. 


\subsection{Review of values and motivational measurement instruments in organic food studies}

\subsubsection{Food Choice Survey (FCQ)}

Since the early 1990s, the subject of interest of consumer behavior studies in different European countries focused on the exploration of how people choose and purchase food. One of the most important works in this field is that of Steptoe et al. (1995). The study was conducted in London and employed the multidimensional measurement instrument named Food Choice Survey (FCQ). By means of factor analysis, Steptoe et al. (ibid.) succeeded to extrapolate a set of nine motives which explain the choice of food: health, mood, convenience, sensory appeal, natural content, price, weight control, familiarity, and ethical concern. In addition, significant demographic variables were denoted. The FCQ has been used in several developed countries (Eertmans et al., 2005; Fotopoulos et al., 2009; Pollard et al., 1998; Prescott et al., 2001; Roininen, 2001; Scheibehenne et al., 2007) as well as in one developing country, i.e. in Uruguay by Ares and Gambaro (2007). In the original FCQ Steptoe et al. (1995) postulated a general "ethical factor" as a "reason" to choose food. However, after the analysis, the authors refined the ethical concern factor, which consisted of three items, two regarding political reasons and one concerning environmentally friendly packaging. The ethical concern factor was considered as an independent factor in the choice of food, because it was not statistically correlated with the social desirability score, but instead with age and gender, showing that it increases with age and scored higher among women than men.

The original FCQ consisted of a total of 36 items measured with a Likert type scale of 4 points - not at all important to very important. Other variations are the Health Value Scale by Lau et al., (1986) and the Marlowe Crowne Social Desirability scale by Strahan and Gerbasi (1972) (cited by Steptoe et al., 1995); these scales also included dietary information, eating style and demographic variables. A study to assess the validity of the nine FCQ factors was carried out, including two personality traits from the NEO-Five factors inventory and from the 
Multidimensional Health Locus Control (MHLOC). Again, correlations between the nine factors were visible, and their influences on the choice of food and demographic information were found. However, from the cultural viewpoint, the outcome of the study is limited to the Londoner, since validation of the instrument for application to other cultural groups was not provided.

Nevertheless, further studies have confirmed the validity of the FCQ in other countries, both singly and cross-culturally, i.e. in Finland by Roininen (2001) and in Germany by Scheibehenne, et al. (2007). Prescott et al. (2001) conducted a confirmatory study across three countries in Taiwan, Malaysia, and Japan; similarly, Eertmans et al., 2005 also conducted a cross-cultural study in Belgium, Canada, and Italy. An adaptation of the original FCQ by Ares and Gambaro (2007) was also developed for Uruguay. Moreover, Fotopoulos et al., (2009) applied the FCQ in Greece.

\subsubsection{Ethical food choice survey (EFC)}

One of the main criticisms of the study by Steptoe et al. (1995) was provided by Lindeman and Väänänen (2000) who argued that the ethical dimension was underrepresented due to environmental and political reasons were included in the same factor of political value. Thus, Lindeman and Väänänen (2000) suggested the creation of a new measurement instrument considering environmental concern and political values as different dimensions. Furthermore, because of the growing number of vegetarians, the animal welfare dimension was included together with religion, as it was deemed that both dimensions influence the preference for some kinds of food. As a result, a new measurement instrument named as Ethical Food Choice Survey (Ethical FC) was proposed. The Ethical FC consists of the ethical dimension from the original FCQ and political values, ecological welfare/animal welfare and environmental protection, and religion.

The Ethical FC Survey consisted of sixteen items: three items of the ethical concern dimension from the original FCQ and thirteen new items divided into three factors. The 
measurement instrument was tested in Finland with a sample of students. Quite surprisingly, the ethical reason rated less important than health, sensory appeal, and price factors in the choice of food. Lindeman and Väänänen (2000) explained it, affirming that in some situations and in the presence of some subgroups of people, ethical reasons may override other motives in the choice of food (e.g. boycott of products of a particular country). Religion influenced the choice of food the least. Nonetheless, it is worth mentioning that the study was done in Finland where the majority of the respondents (79.2\%) are Lutheran and consisted of students. Concerning demographic variables, differences between genders were not found. However, vegetarian women scored higher in the ecological welfare dimension than non-vegetarian women.

More recently, this measurement scale was also tested in Norway by Honkanen et al. (2006) to explore the structure of attitudes and ethical motives in the choice of organic food. The study purports the usefulness of a measurement instrument explaining organic food, fair trade products, environmental and/or ecological product consumption to categorize consumers environmentally or politically who were interested but not active consumers. Notably, the national culture in the two countries is alike which may explain the usefulness of the measurement instrument in this instance. As a consequence, its validity in countries or societies with other cultural backgrounds has not yet been established.

\subsection{Studies on organic food purchase which focus on Germany and Mexico}

In Germany, several studies on organic food have been conducted (Baker et al., 2004; Gerlach et al., 2005; Siriex \& Schaer, 2000; Schultz et al., 2003; Sinus Sociovision, 2006; Worner and Meier-Ploeger, 1999 cited in Fotopoulus \& Krystallis, 2002). Thus, the market for organic food in Germany may be characterized as mature. As a consequence, information about who purchases organic food is more complete. In fact, within the wide body of existing literature, many studies have been devoted to analyzing the demographic profile of consumers, the 
frequency of purchasing (consistent buyers, infrequent users, non-buyers/occasional) as well as socio-demographic categories and lifestyle (Sinus sociovision, 2006).

Regarding motivations, the consumer survey conducted by the German umbrella association of participants in the organic food market (BNN) in 1999 has confirmed health as a central motivation in organic food purchasing across the country, followed by better taste and concern for the environment.

The purpose of this study is to detect whether there are new tendencies in the motivational patterns of organic consumers. Whereas earlier reports and studies mentioned environmental reasons and support for organic farming (Worner and Mier-Ploeger, 1999 cited in Fotopoulus $\&$ Krystallis, 2002), recent reports state that the reported frequency of these motivations has decreased, and wellness, quality and lifestyle are gaining importance (Sinus Sociovision, 2006; Haccius, 2005). Health still remains a strong driver in the purchase of organic products (ibid.).

Little is known about who purchases organic food in Mexico. Worth mentioning is the study conducted by the Organic Trade Association (OTA) (2004) in Mexico City. Two main target groups were included: people with alternative "ways of living" and those belonging to the "upper-middle and upper class". The former are generally labelled "hippy", "Buddhist" and "vegetarian consumers". They understand the term "organic" and seem to appreciate its attributes. However, they do not necessarily have the purchasing power required. Members of the second group are health conscious, understand the benefits of organic food, and have purchasing power. However, they are not willing to pay a premium price solely because of the status of a premium product. More insights about the willingness to pay for organic food (regional) are provided in the study by Padilla and Perez (2006). Attitudes and motivations toward organic food were examined in three cities in northern Mexico (Zacatecas, Aguascalientes and San Luis Potosi). This study reported that consumers are unknowledgeable and confused about the term of "organic food". This is reflected in respondents' statements who affirmed that if organic food were labelled or sold in a different place as "conventional" food, they would be willingness to pay more.

As a result, higher prices and trustworthiness of conventional food products explained the lower interest to purchase organic food. Nevertheless, some motives for purchasing organic 
food reported were health consciousness, environmentally friendly, fairness, and protection of biodiversity (Padilla \& Perez, 2006). Furthermore, the authors mention freshness as the most hedonistic value when making food purchase decisions and price as the main criterion when deciding on the appropriate store.

In the research for this study, it was found that studies on the level of values are scarce in both Germany and Mexico. As mentioned above, v. Alvensleben (1998) performed an analysis on values and attitudes in Germany, and Baker et al. (2004) performed an analysis on values that induces the purchase of organic products between Germans and Englanders. On the other hand, to the best of our knowledge there are no studies profiling consumers in terms of their values related to the purchase of organic products in Mexico.

Thus, this study has both an explorative and confirmative nature. Regarding the former, exploration of motivations and values that influence the purchasing of organic food in Mexico, as well as exploration of values that are inherent in the consumer of organic food in Germany, were undertaken. In addition, the study is directed within a confirmative approach toward to detect the motivations to purchase organic food in Germany.

\subsection{Situational assessment and development of research questions}

Although organic farming is present in most countries around the world, demand for organic food remains concentrated in Europe, the U.S.A., and Canada. Consequently, most of the studies regarding consumption and consumer behavior have been conducted in these countries; whereas little is known about the stage of the market, consumption, and consumer behavior in developing countries (Sirieix et al., 2007). This establishes, therefore, the necessity to assess whether motives to purchase organic food are similar in developed and developing countries.

As previously mentioned, only a few cross-cultural studies on the purchase of organic food have been conducted in Europe, i.e. by Germany and the UK (Baker et al., 2004); Germany 
and France (Sirieix \& Schaer, 2000); France and Brazil (Sirieix et al., 2007). The focus of this research is twofold: firstly, to identify the motives driving the purchasing of organic food in Mexico and in Germany; therefore, the first research question is:

\section{Are different motives driving the purchase of organic food in Germany and Mexico?}

Secondly, the determination of cultural dimensions influencing behavioral patterns of consumers in both countries was undertaken. A comparison of the domains of values in both Germany and Mexico led to the second research question as follows:

\section{Are values different between the consumers of organic food in Germany and Mexico?}

Among the expected results, the most important issues are the following: to find relevant differences on motivations and values in the purchasing of food products between the groups of study, as well as differences on motives and values between the consumers of organic food. For instance, food safety is expected to score lower in Mexico, since this country has not experienced food scandals and is considered a producer of fruits and vegetables. After food scandals in Europe, food safety, higher quality, and confidence in organic food were selfreported as main motivations to purchase organic food (Baker et al., 2004; Latacz-Lohmann \& Foster, 1997; Sirieix \& Schaer, 2000; Schultz, 2003; Sinus Sociovision, 2006; v. Alvensleben 1998; Wandel, 1994; Worner \& Meier-Ploeger, 1999 cited in Fotopoulus \& Krystallis, 2002).

Due to the longer tradition of organic production in Germany, this group of consumers has more knowledge and are more familiarized with organic food and have intense environmental attitudes than consumers in the Mexican group. Germans are expected to relate more to hedonistic benefits. Because of the lower household incomes within the Mexican population, and the Mexican market is characterized as very price sensitive; we expected to find a significant influence of price affecting the proposed motives to purchase organic food. 


\subsection{Development of the theoretical model}

Social science models are simplified designs for visualizing complex understanding that is explained herein. Models describe patterns into which events, items, or situations can be grouped (O'shaughnessy, 1992; Hofstede, 2001). To build any model, it is fundamental to identify appropriate theoretical concepts or constructs. Hosftede (2001) defines a construct as "a product of our imagination, supposed to help our understanding"; similarly O'Shaughnessy (1992) defines a concept as "abstracting common features of objects or situations" with an explanatory usefulness of the phenomena studied. In consumer behavior research, constructs are commonly used to understand mentality of people and are directly related conceptually to specific behavior (intentions, attitudes/beliefs, values, motivations, and perceptions) (Hofstede, 2001). To explain how constructs might be measured, the use of hypotheses and how these are inter-correlated, models are commonly used (O'Shaughnessy, 1992). A constant challenge in developing a model lies in determining the right degree of complexity while taking into consideration whether very simple models may be distant from reality or the phenomena under study and that complex models are difficult to understand.

As stated, the main goals of the current research are to detect the differences in motives for purchasing organic food; secondly, to analyze cultural differences regarding the purchase of organic products through identification of different domains of values. As displayed in Figure 8, a model has been developed which includes constructs regarding motives of purchasing organic food and values to be probed in both countries.

Accordingly, in the next sections an explanation is provided of the stages undergone in order to identify the motives driving the purchasing of organic food and values to be included in our theoretical model as well as the operationalization of constructs using a survey approach. 
Figure 8: Conceptual model of motives of choice of organic food and domains of values in Mexico and Germany (explorative phase)

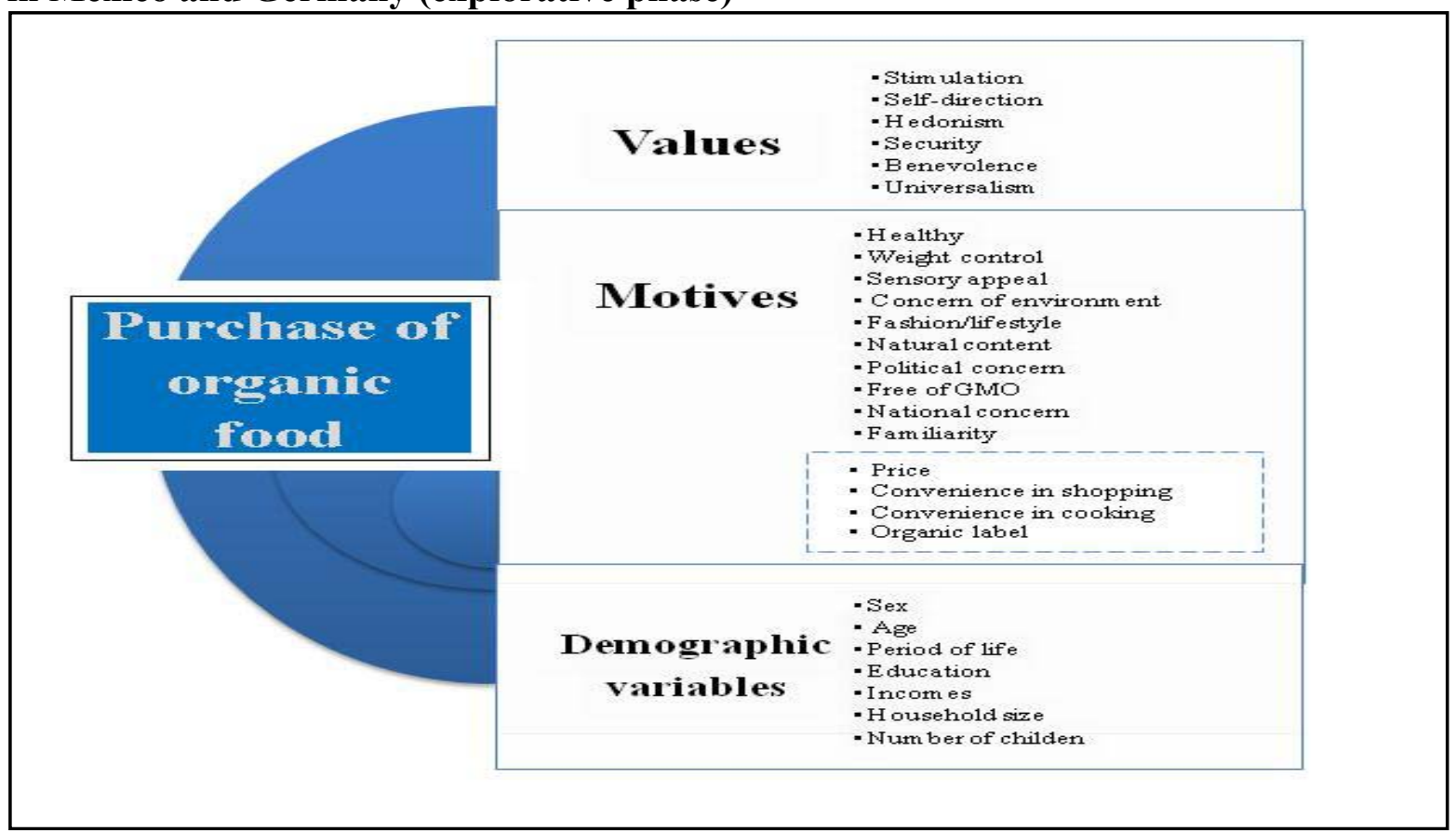

Source: Own elaboration (2008).

\subsubsection{Theoretical constructs of motives to purchase organic food}

Firstly, the term motives or motivations in the study are used to refer to "the drivers/reasons to satisfy physiological, psychological, or social needs, desires, or wants through the purchase of organic food". Following this line, the constructs of motives to purchase organic food are defined as follows:

Healthy is often mentioned as the main motivation in purchasing organic food among consumers in several countries. In the cross-cultural study between Germany and the UK hierarchical maps of value chains were developed using the Means-End Theory by Baker et al. (2004). Among the findings, the hierarchical value maps of the German consumers were more elaborate than those of Englanders. The strongest chains among the Germans were health-related perceptual orientations which were derived from quality and taste as product attributes via the consequences of health-related aspects. These were linked to the value chain of well-being and health and strongly supported by the chains absence of pesticides, chemical 
fertilizers post-harvest chemicals and wax and avoidance of unnatural things that can be interpreted as naturalness, chemical free and food safety.

The author regards the findings as a linkage of coherent and sensible aspects, except for the connection between taste and health inferring that taste depends on quality and is indicative of healthy food. Similarly, Hughner et al. (2007) found in their literature review that for many consumers the perception of healthier food is a parameter for quality. In addition, consumers purchase organic food because of their desire to avoid the use of chemicals in conventional food and because of their mistrust in the food industry due to food scandals, such as the cases of BSE across Europe during a decade in the 1990s. In this explorative study, the terminology of concern about health, healthy and health conscious was used as the core meaning to interpret the construct of Health.

Weight control: although in the literature review it is not referred to as a motivation to purchase organic food, there is enough empirical evidence to postulate that the purchasing of organic food in Germany is linked with the desire for a trim figure and fitness. Thus, in this study, the question of whether weight control is a motivation to purchase organic food was explored, because it is linked with health and the perception of nutritional attributes. Conversely, Steptoe et al. (1995) within the FCQ propose a relationship between taste and choice of fatty food, since "taste may be particularly important in the selection of high fat diets". The core meaning used to operationalize this construct is "weight control", "low calories" and "low fat".

Sensory appeal: international literature reveals that taste/better taste is often self-reported by consumers as a main motivation in purchasing of organic food. As mentioned in the construct of Health, consumers in some countries link "taste" (product attribute) to "quality" and “health” (Baker et al., 2004; Gil \& Sanchez; 2000; Makatouni, 2002). In other countries, taste is linked to "hedonistic values" such as "nostalgia" as in Chinnici et al. (2002). Unattractiveness is a reason to reject the purchase of organic food; however, consumers of organic food mention that it is not an important criterion influencing their purchase of organic food (Fotopoulos \& Krystallis, 2002).

There is enough evidence to postulate that the level of involvement with organic food is connected with the criteria of appearance in the choice of organic food. For instance, 
consumers in mature markets regard appearance as an unimportant factor in deciding to purchase organic food. Consumers in emerging markets, first-time buyers or occasional consumers regard sometimes organic food as unattractive. On this ground, "taste", "smell", and "appearance" have been used as core meaning to explore the construct of sensory appeal in the Mexican emerging market, as well as to confirm the previous findings within the mature market in Germany.

Concern of environment follows health as the second main motivation to purchase organic food. Some authors regard attitudes toward environmental concern as a reflection on consumption of organic food because of the "absence of chemicals and "pesticides" in organic farming methods, thus organic produce is perceived as "environmentally friendly," this fact is also linked with healthy food. On this basis, environmentally friendly is one of the core meanings of this construct. The second core meaning corresponds to animal welfare which is a multi-level construct linked to "health" because of "food safety", "quality and better treatment of livestock". It is also linked to social components because of "respect for other creatures" and "animal rights” (Baker et al., 2004; Hughner et al., 2007; Makatouni, 2002).

Lifestyle and fashion are both terms mentioned in some of the international literature. The proposal for this construct is to explore the involvement of consumers with organic food. On the one hand, organic food is perceived as "fashionable" due to intensive advertising and higher prices and thus is also perceived as not available or not purchasable by all people (Chinnici et al., 2002). On the other hand, occasionally, new or first-time consumers regard the purchase of organic food as a curiosity. Consequently, because the Mexican market of organic food is just emerging, to provide empirical evidence we explored the possibility of "fashion", "fashion/curiosity" or "lifestyle" as a motivation to purchase organic food in Mexico. Although lifestyle is a complex construct that implies at least one study to provide evidence, awareness of this involvement by consumers of organic food in Mexico was not apparent. We further explored whether frequent or intensive buyers would report the purchase of organic food as a lifestyle decision; or because of the stage of the domestic market, they would report it as fashion/curiosity. Thus, the term lifestyle has been used as a core meaning in this construct. 
Natural content-within the motivations to purchase organic food, naturalness is rarely mentioned. However, in studies using Means-End theory as a research approach, the attribute of "natural product" is often linked to "health" and "food safety" (Schifferstein \& Oude Ophuis, 1998; Zanoli \& Naspetti, 2002). In 2004, Baker et al. placed "avoiding unnatural” within the "health related" domain of perceptual orientation. Because of the product attribute of naturalness, it is postulated as a construct in explorative research. The core meanings within the construct are naturalness and additives.

Free of Genetically Modified Organisms (GMO) - the conception of this construct is closely related to the construct of "natural content". A few findings about the attitudes and perceptions of consumers of organic food concerning genetically modified food (GM Food) have been reported in quantitative research. However, Makatouni (2002) reports "GM Free" as a product attribute within the hierarchical value map of "health". Similar findings were reported by Baker et al. (2004) due to the product attribute Not Genetically Modified which lies within the "health related" domain of perceptual orientation. Because of the emerging stage of the market for organic food in Mexico, with the construct of Free of GMO, we explored attitudes of consumers with respect to the use of GM and organic food. The term Genetically Modified Organism has been used as core meaning.

Political concern: because of fair trade, working conditions of agricultural workers, and support of the local economy were mentioned as motivations to purchase organic food (Baker et al., 2004; Makatouni, 2002; Worner \& Mier-Ploeger 1999 cited in Fotopoulus \& Krystallis, 2002) we explore if this motives reported in earliest studies still been important for German consumers, whereas we explore their relevance for the Mexican consumers, the terms of "fair trade" and "working/human rights" will be used as core meaning.

National concern: the basis of support of the local economy and preference for regional products are often mentioned as reasons to purchase organic food among European consumers. Therefore, a search was undertaken in this explorative study regarding national concern. This construct was conceived as a variant of regional concern. On the basis of the domestic market of organic products in Mexico, is centralized in Mexico City, and including demographic reasons, asking for regional concern within the largest city in Mexico was 
considered as unreliable. Thus, national concern, profitable for Mexican and German farmers respectively, "national origin" will be use as core meaning for this construct.

Apart from the mentioned motives, the model developed for the study also included some motives from a psychological viewpoint that are often considered antecedents of motives/motivations. For the purpose of this study, they are considered as motives impelling consumers' buying behavior of organic products. These are as follows:

Price is obviously an influencing factor in the purchase of food, and the Mexican market is characterized as a sensibly priced market (USDA, 2002). Moreover, in considering price as a motivation to purchase, we explore the influence of price in the purchase of organic food, especially in Mexico because of the sensible market price as well as the emerging stage of the domestic market of organic food. The terms expensive and good value will be used as core meanings in the measurement of price.

Convenience, some scholars such as Steptoe et al. (1995) regard purchase convenience and convenience of preparation as convenience. Meanwhile, Grunert et al. (1993) consider convenience solely as cooking/preparation. However, we consider that convenience will be measured first as "shopping convenience", and secondly, as "cooking convenience", with the premise that convenience in cooking organic food does not imply that organic food is easily available in the different food supply channels and vice versa. Although organic food might be available in different retail channels and point of sales (POS), it does not necessarily imply convenience of cooking. Thus, for the purposes of this study, "shopping convenience" will be used as a core meaning, and "easy preparation" will be a core meaning of "cooking convenience".

Even familiarity has not been referenced as a motivation to purchase organic food in the literature review. There is enough information to suppose that "familiarity" is conceptually linked to "involvement", "trustworthiness" (Grunert et al., 1993) as above mentioned in the study conducted by Baker et al. (2004). Therefore, it is the intention of this study to explore if familiarity with organic food might be a motivation in its purchasing. The core meaning within this construct will be "familiar". 
Finally, Organic labelling will be explored in Mexico due to the emerging stage of the domestic market to determine if this might be also a motivation to purchase organic food; the core meaning for this construct will be "organic label", and "trustworthiness".

\subsubsection{Theoretical constructs of values to purchase organic food}

In our research the term "values" will refer to the definition of values by Grunert and Juhl (1995) as "the self-centred and social-centred criteria used to select and justify actions and to evaluate people, including the self and events". In line with the international literature review and marketing textbooks, the SVS is regarded as a useful measurement instrument of values also used in cross cultural studies. The original SVS scale provided by Schwartz (1992) consists of eleven value domains measured by 56 terminal values.

It is worth mentioning that in our study we postulate the search of six dimensions linked with the purchase of organic food on the basis of the hedonistic and altruistic motivations referred in the theoretical framework. The six dimensions to be explored are in following described. Hedonism, self-direction, and stimulation are dimensions regarding values in the pursuit of individual interests. Benevolence and universalism are dimensions of values in the pursuit of collective interests. Even security, is the last one dimension that will be search, "security" remains between individuals and collective goal domains, which implies the need for "selfsecurity" i.e. "healthy" or the "security of others" i.e. "family", "particularly members", and "national security" Schwartz (1992). In continuation, the theoretical framework of the sixvalue domains considered in these theoretical constructs is summarized below.

1. Stimulation, in line with Schwartz (1992): the stimulation value domain derived from "organismic needs for variety and stimulation to maintain an optimal level of activations. Although the need of stimulation is conditioned by social experience, there are differences among the individuals". This dimension was postulated to explore the basis for consumers of organic food self-reporting curiosity and trying something new as motivations to purchase organic food. The core meanings are variety, excitement, and life.

2. Self-direction, according to Schwartz (1992), biological needs for control and mastery as well as social interactional requirements of autonomy and independence lay within the self- 
direction dimension. In line with Schwartz (1992), stimulation and self-direction dimensions are closely related. Thus, curiosity and trying something new as motivations to purchase organic food justifies the exploration of this values domain. Freedom and creativity have been used as core meanings.

On the basis of the scores of the two countries within the individualism/collectivism dimension of national culture by Hosftede (2001), Mexican culture scored higher as a collectivistic culture, and the German culture scored higher as an individualistic culture. Therefore, it was expected that the average value priorities of the German group would score higher than the Mexican group in both stimulation and self-direction domains of values.

3. Hedonism: According to Schwartz (1992), the origin of this motivational domain refers to organismic needs and pleasure in the satisfaction of these needs. The core meanings used in this dimension are pleasure and enjoyment. This value domain was postulated because of sensory appeal motivations to purchase organic food such as better taste.

4. Security: referencing Schwartz (1992), the motivational domain of security refers to the satisfaction of needs in the pursuit of individual interest (e.g. health) as well as in the pursuit of collective interests (e.g. family security, social order, and national security). The core meanings used in this theoretical construct will be healthy and sense of belonging. Because health is the main reason for purchasing organic food across the two countries, security was proposed as a measurement within this explorative research.

Due to its psychographic nature, the value of security has been studied by Schwartz (1992) and, whether safety was studied by Maslow (1959) within the Maslow's hierarchy of needs (cited in Solomon et al., 2000). Regarding the motivations to purchase organic food, security could be inferred as a sensational meaning with respect to health, natural content, and food security. Regarding cultural differences, within the dimension of uncertainty avoidance by Hosftede (2001), German culture scored higher (61 points); therefore, it is understood that there is a higher need for security among the members of this cultural group. Conversely, because German national culture is characterized as individualistic and Mexican national culture as collectivistic, the importance of sense of belonging by the two groups inferring cultural differences was explored. 
5. Benevolence: within the theory of universal content of values by Schwartz (1992), the value domain of benevolence was inspired by previous findings (Kluckhohn, 1951; Maslow, 1959; Schwartz \& Bilsky, 1987 cited in Schwartz, 1992); that is, "the dimension focuses on the concern about the welfare of others in one's realm of everyday interaction". Consequently, the terminal values of helpful, loyal, forgiving, honest, responsible, true friendship and mature love lay within this value domain. This value domain is included in this explorative research on the grounds of altruistic traits that have been mentioned in the purchase of organic food. "Helpful" and "honest" have been used as a core meaning.

6. Universalism: like benevolence, the value domain of universalism is included in the explorative research due to altruistic reasons for the purchase of organic food reported in the international literature. According to Schwartz (1992), this is a "pro-social value dimension, due to survival needs of groups and individuals which becomes apparent when people are exposed to those outside the extended primary group and become aware of the scarcity of natural resources". "Awareness of other people" and "nature" are within the dimension of "universalism" in this study. It was concluded that a distinction between people and nature on the premise of respect and awareness does not imply sameness concerning nature and vice versa; consequently, "environment," "nature," "tolerance," and "respect for fellow humans" have been used as core meanings in the interpretation of this construct.

\subsection{Operationalization of the constructs: development and adaptation of previous measurement instruments}

To measure constructs in social sciences there are single-item and multi-item scales. As the name suggests single-item scales have only one item to measure a construct. Whereas, multiitem scales have several and are commonly used to measure complex constructs. In line with Aaker et al. (2007) to develop a multi-item scale is a complex procedure, thus they propose eight steps to develop multi-item scales summarized as follows: 1) determine what will be measured; this point regards the definition of the constructs and their theoretical foundations as well as the meanings and definitions that would be distinguishable from other constructs. 2) Generate as many items as possible; this phase deals with the need to reflect the primary 
interest of the constructs on the content of the items. 3) Ask experts in the field to evaluate the initial pool of items; in this phase, it is recommended because an expert's review of the items pool can confirm or invalidate the constructs. 4) Determine the type of attitudinal scale to be used; this step is linked with the fact that wording of items varied according to the format of the scale, thus the decision about the type of scale to be used in the measurement is one of the earliest tasks. 5) Including of some items that will help in the validations of the scale; the inclusion of socially desirable items is recommended to improve the scale validity on the basis of some of the respondents answering in a certain fashion because they want to be socially desirable. Thus, items of social desirability are added to the scale, in an aim to drop out responses that are highly correlated with social desirability. 6) Administer the items to an initial sample; to check the validity of the items, it is recommended to administer the scale to an initial sample. Better results are related with the large of the sample and the representativeness of the population. 7) Evaluate and refine the items; this step refers to the idea that high correlation in an item is sought as a characteristic of the quality of the items to be included in the scale. In this way, the following are criteria to determine the items to include in a scale: "items of a scale should posses a high intercorrelation, high items scale correlation, high items variance, and mean close to the centered of the range of possible scores, and a high coefficient alpha. Finally, 8) Optimize of the scale length. The last step regards the balance between the reliability and brevity in order to determine the optimal scale length. Due to the larger the scale, the greater the reliability, shorter scales are easier to answer to respondents according to Aaker et al. (2007). Figure 9 summarizes the steps of this process. 


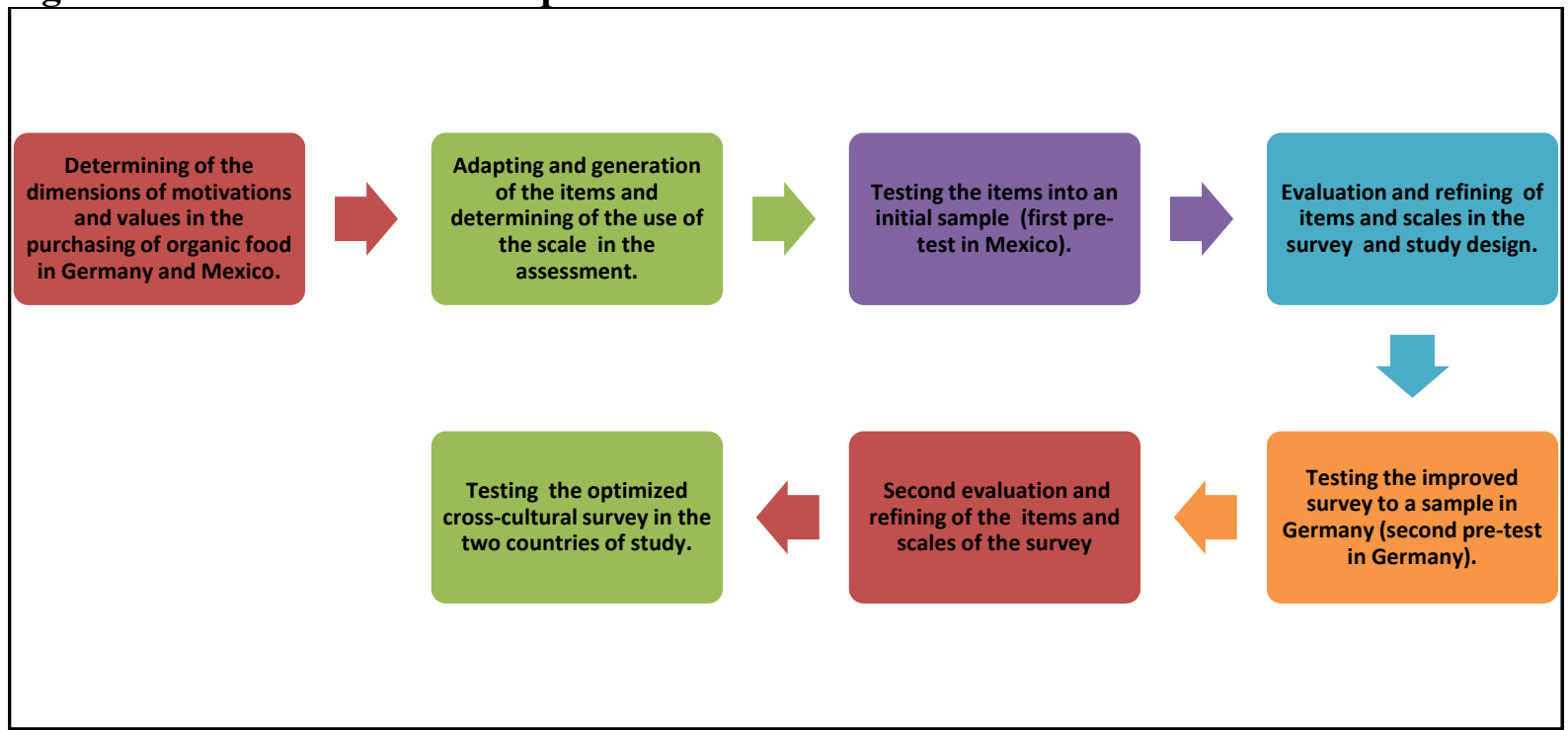

Source: Own elaboration based on Aaker et al. (2007, p. 302).

To operationalize the theoretical constructs of the first tentative model for the purpose of this research, some new constructs were developed based on the literature review, as well as adaptation of previous measurement models. Regarding motivations, the FCQ has been adapted, as these motives are intended to influence the purchase of organic food. In particular, the ethical food choice has been adapted to extrapolate the motivations regarding an ethical buying behavior. The main adaptation from the original measurement instruments are explained in the following:

The original FCQ by Steptoe et al. (1995) was designed to glean information regarding, above all, health, eating habits, and consumer psychology. As mentioned, the measurement instrument contained items related to health, attitudes, and beliefs connected to healthy eating. The influence of cultural factors on habits of consumption, preparation of food, social interaction, and hedonistic factors such as taste, weight control, and emotions were also included, as well as the growing environmental concern. However, since not all the dimensions correspond to motivations to purchase organic food, it was decided to retain only seven dimensions in this model: health, convenience, sensory variables, natural content, price, weight control, and familiarity. In the original FCQ a Likert type scale of four points (not at all important, a little important, moderately important and very important) was used, but because of the interest in the mean value, a Likert type scale of five points was adopted: $(-2)$ 
not at all important (-1) somewhat unimportant; (0) neither important not important; (1) somewhat important; (2) very important.

The usefulness of the Ethical FC represents a paradox that would be taken into consideration in the measurement. As mentioned, the Ethical FC measurement instrument was successfully employed in Scandinavian countries. Ethical motives supposedly influence choice of food, but, especially in cross-cultural comparisons, their usage can be misleading. In fact, as they relate to the domains of values, which, in turn, are a core element of culture (Kluckhohn, 1951/1967 cited in Hofstede, 2001; Mooij, 2004), they may be valid only for the specific country studied. Nevertheless, since concern about environment, environmentally friendly attitudes, and animal welfare are considered in international literature as significant motivations to purchase organic food, the following dimensions have been included in the model: environmental protection, animal welfare, and political values.

As far as values are concerned, an adaptation of the SVS measurement model was undertaken. In the following, some considerations toward the adaptation of this measurement instrument will be provided.

The usefulness of the SVS by Schwartz (1992) to categorize groups of consumers across cultures is often mentioned in marketing textbooks (Solomon et al., 2000; Mooij, 2004; Blackwell et al., 2006). Grunert and Juhl (1995) provide evidence of the usefulness of the SVS in environmental attitudes and purchase of organic food. As mentioned, the original SVS consisted of ten dimensions - domains of values - measured by 56 items referring to terminal values/end states. More recently, Schwartz (2006) proposed a new model of seven cultural dimensions (see Figure 10), which is useful in this research study to graphically show the cultural differences between the two countries where the comparative research was conducted. 
Figure 10: Adaptation of the theoretical structure of relations among value domains

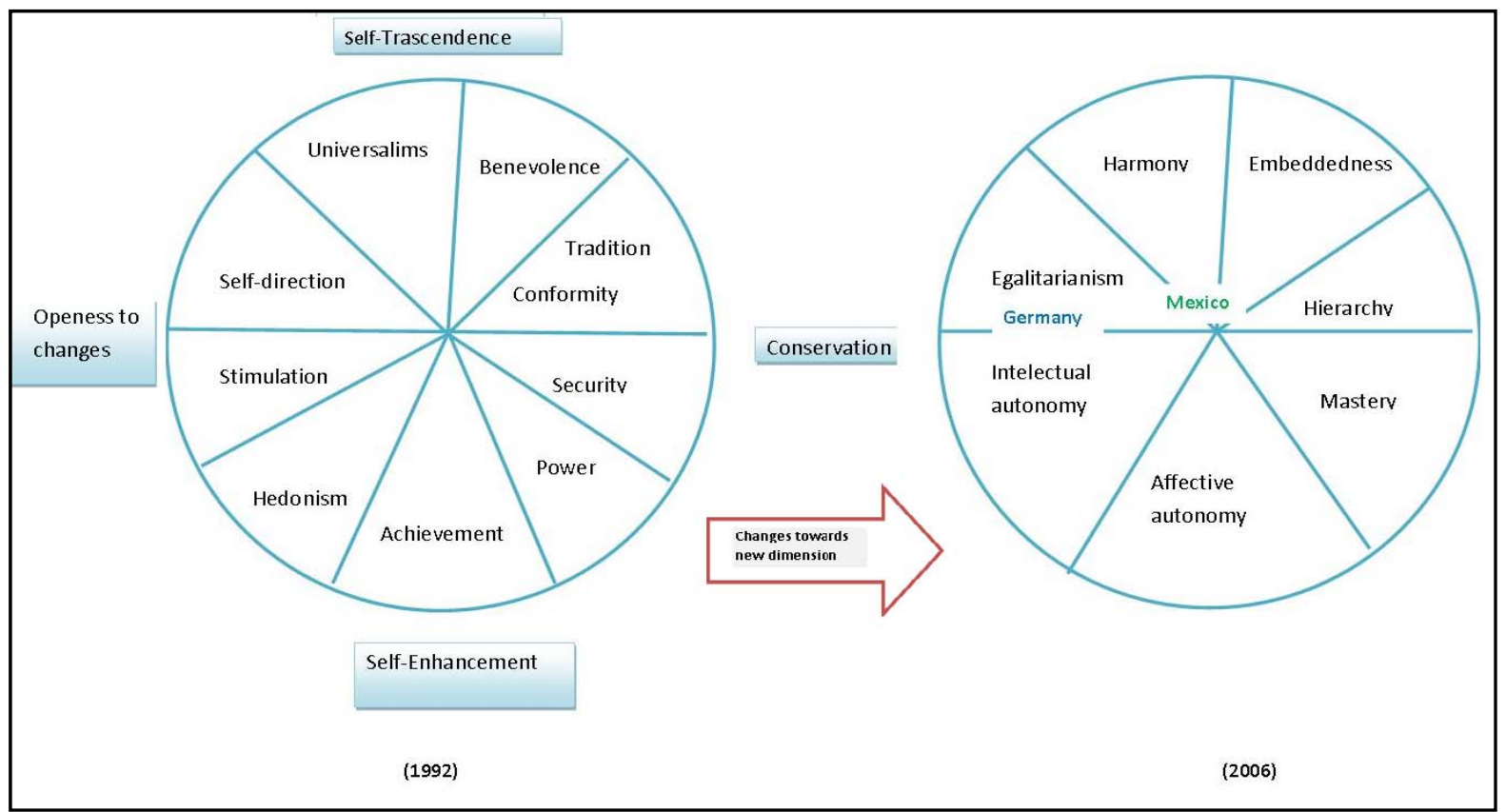

Source: Own elaboration based on Schwartz (1992, p.14; and 2006, p. 142).

\subsubsection{Setting-up of the survey}

To operationalize the measurement of the proposed theoretical constructs of motivations and values, a survey was designed; including some determinant attributes of image at the point of sale (POS), frequency, and motives of purchasing of organic food, and values that were adapted. The diagram proposed by Aaker et al. (2007) as shown in Figure 11 provides guidance in the design and pretesting of the cross-cultural survey. 


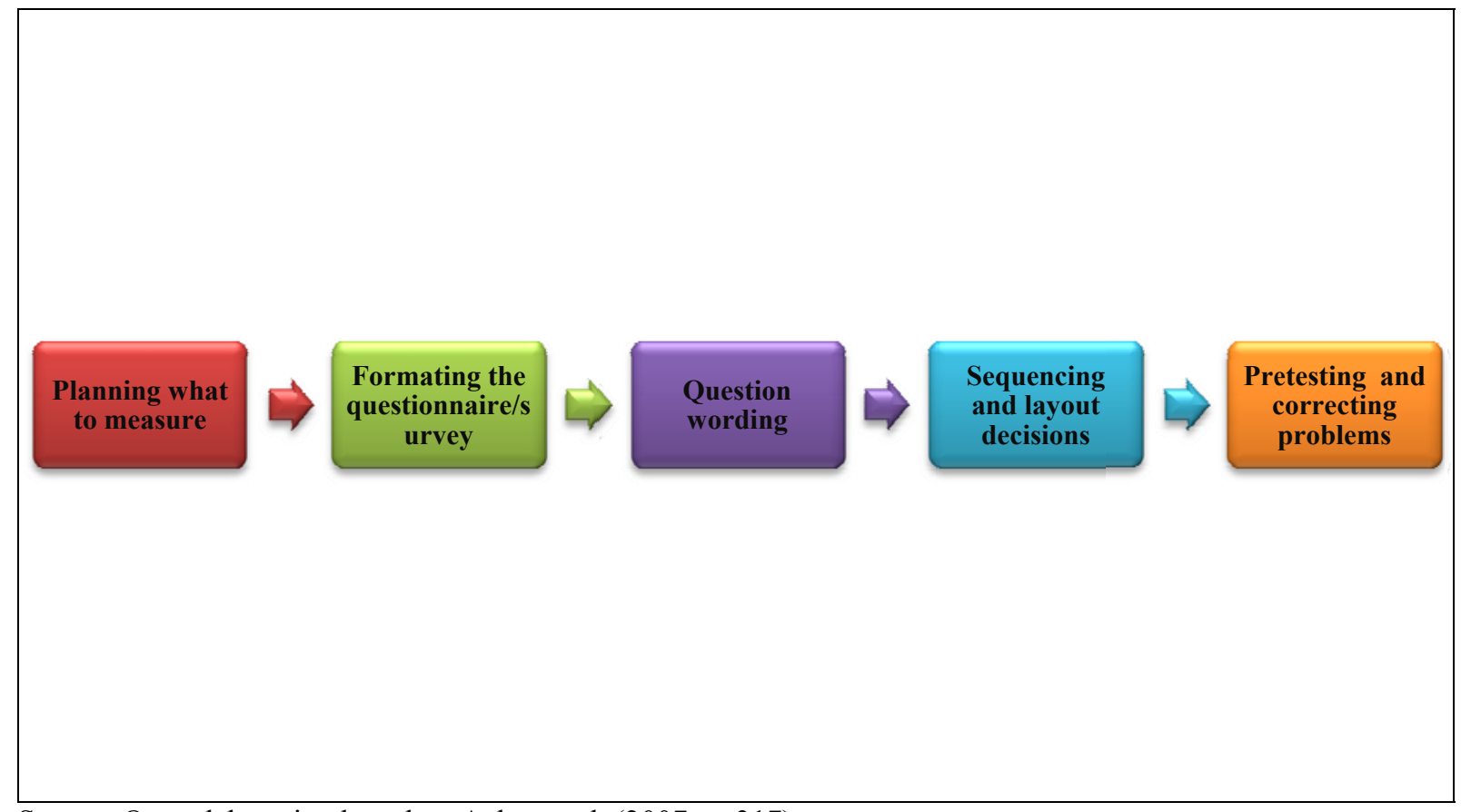

Source: Own elaboration based on Aaker et al. (2007, p. 317).

The survey conceived for pretesting in Mexico addresses consumers of organic food who were meant to be interviewed face to face in organic shops or organic markets (direct marketing). It consists of five sections described below. In the first section, by means of an open question, respondents were asked to list three attributes of organic food. Other questions concern the number of years that consumers have been purchasing organic food and the frequency of purchasing in mainstream retail channels.

A question asked in the second section referred to the perceived determinant attributes of image at the point of sale (POS). These supposed determinant attributes are linked with the fact of the emerging stage of the domestic market in Mexico. Thus, some product attributes and marketing strategies in the POS to attract or encourage new consumers to purchase organic food were considered in the pretesting. The factors are: 1) available information, 2) promotions, 3) quality and freshness, 4) trustworthiness, 5) assortment, 6) convenience, and 7) price. These factors are briefly described in the following:

Trustworthiness: because of the stage of the emerging market, trustworthiness plays an important role in the purchase of organic food. There is empirical evidence to predict that in 
the Mexican market trustworthiness is higher in organic market places, due to the interpersonal and face-to-face interaction with farmers and producers. Moreover, trustworthiness is linked to the fact that most of the consumers are first-time buyers. In this sense, there is enough evidence to contend that retail channels play an important role not only in distribution and supply but also in perceptions, image, and attitudes toward organic food.

Assortment: the size of organic assortment is another factor that can influence store preferences. As mentioned in chapter two, the largest assortment of organic food is offered by organic shops; thus, the question arose as to what extent consumers evaluate it.

Store convenience is considered an influencing factor in the purchase of organic food, as it is impossible to determine organic assortment overall in conventional supermarkets, and most of the time the size offered depends on the format of the store. Regarding the organic shops, many of them are located in neighbourhoods or exurbs where inhabitants have middle to higher incomes level. Whereas, organic markets are located in exurbs, most of them are groups of consumers who organize themselves and use the CSA scheme. Thus, organic markets are located in neighbourhoods of slightly lower-middle incomes or higher income levels. However, not all the consumers of organic food are residents, or are in close proximity to stores; many consumers reported travelling a considerable distance to purchase organic food. Therefore, consumers' perception of store convenience has been an important issue to explore.

Price orientation: as mentioned in the literature review and theoretical framework, the Mexican market is characterized as price sensitive (USDA, 2002). Furthermore, the premium price of organic food and how consumers perceive the prices within stores were also explored due to demographic segmentation which is a common marketing strategy in Mexico. Typically consumers in the country are able to purchase the same products at a different price, depending on the store or the neighbourhood where offered.

Quality/freshness: both are characteristic perceptions in both countries. Meanwhile, taste is a criterion for quality of food in Germany (Baker et al., 2004). For Italians familiarity, usability, naturalness, and healthy are perceived as important in food products (Bredahl et al., 2001). In search of quality and freshness, we explored the level of influence that they have on the purchase of organic food. As provided in the second chapter, country inhabitants are 
characterized as organic producers and exporters rather than consumers. This fact is linked to the FSC, as most organic food is either overproduced or produced on a small scale and destined for the domestic market. Consequently, farmers who sell through direct marketing in the organic markets are also within the FSC suppliers of conventional supermarkets and organic shops. Therefore, overproduction that is not sold by the other supply channels is destined for the organic markets, thus our interest to explore how consumers perceive the quality and freshness at the POS.

Available information: this factor deals with information concerning organic farming such as principles, and methods of produce (e.g. brochures, flyers, etc.) provided by stores, and advice provided by staff or producers in the organic markets. Due to the emerging stage of the Mexican organic market, information plays an important role in attracting new consumers, increasing consumers' knowledge, and involvement in purchasing organic food by occasional consumers. Another marketing strategy that was explored is promotion.

Since the third section is devoted to motivations driving consumption of organic food, an adaptation of the FCQ of Steptoe et al. (1995) was undertaken. As already mentioned, this measurement instrument contains items related to attitudes, and beliefs connected with healthy consumption. The influence of cultural factors on the habits of consumption, preparation of food, social interaction, and hedonistic factors such as taste, weight control, and emotions were also included, as well as environmental growing concerns. However, since not all the dimensions correspond to motivations to purchase organic food, it was decided to retain only seven dimensions in this model: 1) health, 2) convenience, 3) sensory appeal, 4) natural content, 5) price, 6) weight control, and 7) familiarity. In the original FCQ the Likerttype scale of 4-points (not at all important, a little important, moderately important and very important) was used, but because of our interest in the mean value -average values of groups-, we adopted a 5-points Likert-type scale: (-2) not at all important; (-1) somewhat unimportant; (0) neither important not important; (1) somewhat important; (2) very important.

To extrapolate the motivations regarding an ethical buying behavior, some items of the ethical food choice survey were adapted for this study, which had been successfully employed in Scandinavian countries. This decision was made after long reflection, since the employment of this instrument in a developing country could be seen as risky. In fact, ethical motives are supposed to have an influence on choice of organic food, but, especially in cross-cultural 
comparisons, their usage can be misleading. As they relate to the domains of values, which, in turn, are a core element of culture (Kluckhohn, 1951/1967 cited in Hofstede, 2001; Mooij, 2004) they may be valid only for the specific country under study. Nevertheless, since concern about environment, environmentally friendly attitudes, and animal welfare are considered in international literature as significant motivations to purchase organic food, the following dimensions have been included in this model: 1) environmental protection, 2) animal welfare, and 3) political values.

The fourth section concerns the measurement of values which are considered a proxy for detecting underlying cultural differences among countries. To this end, the SVS measurement model was adapted according to the six dimensions of values that are intended to be strictly related to purchasing organic food. To operationalize the measurement of values, the original SVS was adapted. The changes are summarized as follows: in the SVS Schwartz (1992) also used a 9-points Likert-type scale: (7) supremely important, (6) very important, (5) unlabeled, (4) unlabeled, (3) important, (2) unlabeled, (1) unlabeled, (0) not important, (-1) opposite to my values. However, to facilitate answers by respondents, it was considered more functional to use the Likert type scale of five points (-2) not at all important to (2) supreme important which is also used in the measurement of values. Regarding the section on presumed purchase factors at the store image level, similarly a 5-points Likert type-scale: (-2) strongly disagree; (-1) disagree; (0) neither agree nor disagree; (1) agree; (2) strongly agree was used.

Finally, a set of questions regarding socio-demographic information was included in the fifth section. Table 15 provides an overview of the motivation and value constructs included in the pretest of the multi-item scale to provide a cross-cultural survey. The survey was designed in English (appendix) and translated simultaneously into German and Spanish by native speakers using back translation procedures and sent to the authors. Discrepancies regarding the meaning of words were reviewed and modified (more details are provided in the next section). 
Table 15: Definition of variables included in the model

\begin{tabular}{|c|c|c|}
\hline Theoretical Construct & Items & Source \\
\hline \multicolumn{3}{|l|}{ Motivations to purchase organic food: } \\
\hline Environmental concern & 2 & Adapted from Lindeman and Väänänen, 2000. \\
\hline Animal Welfare & 2 & Adapted from Lindeman and Väänänen, 2000. \\
\hline Political concern & 2 & Adapted from Lindeman and Väänänen, 2000. \\
\hline National concern & 2 & Author \\
\hline Lifestyle & 2 & Author \\
\hline Weight control & 2 & Adapted from Steptoe et al., 1995. \\
\hline Sensory appeal & 2 & Adapted from Steptoe et al., 1995. \\
\hline Health & 2 & Adapted from Steptoe et al., 1995. \\
\hline Natural content & 2 & Adapted from Steptoe et al., 1995. \\
\hline Free of Genetically Modified Organisms (GMO) & 2 & Author \\
\hline Familiarity & 2 & Adapted from Steptoe et al., 1995. \\
\hline Price & 2 & Adapted from Steptoe et al., 1995. \\
\hline Cooking convenience & 2 & Adapted from Steptoe et al., 1995. \\
\hline Shopping Convenience & 2 & Adapted from Steptoe et al., 1995. \\
\hline Organic labeling & 2 & Author \\
\hline \multicolumn{3}{|l|}{ Dimensions of values: } \\
\hline Stimulation & 2 & Adapted from Schwartz, 1992. \\
\hline Self-direction & 2 & Adapted from Schwartz, 1992. \\
\hline Hedonism & 2 & Adapted from Schwartz, 1992. \\
\hline Security & 2 & Adapted from Schwartz, 1992. \\
\hline Benevolence & 2 & Adapted from Schwartz, 1992. \\
\hline Universalism & 2 & Adapted from Schwartz, 1992. \\
\hline
\end{tabular}

\subsubsection{Conceptual considerations of cross-cultural studies}

Some considerations have to be taken into account to avoid misinterpretations when cultures are compared. "Culture consists of individuals, however it should not been seen as a kingsized personality and try to understand it functioning by measuring only individuals," because patterns observed at the cultural level (ecological level) can be different from patterns at the individual level (personality) (Mooij, 2004).

Similarities or differences can be found when cultures are compared. Culture level-ecological level variables are used to find similarities by determining types of subsets within cultures that are similar among societies but differ from other subsets (i.e. young people, lifestyle 
groups). If the focus of research is on differences between cultures, dimensions of societies demographic variables such as age, gender, income, and education- can be used to create typologies or categories within cultures in order to individuate people who share a common set of values and beliefs (i.e. affinities) and who can be categorized into groups (Mooij, 2004).

Comparative studies might also be conducted as a "within-system" and "between-country comparison" (also called "between-system" comparison). The latter requires the use of variables at the national level of a country to find explanations for some phenomena (countrylevel variables). Within-system comparison involves the measurement of individual behavior within social systems, countries, or cultures (Mooij, 2004).

The exploration of motives driving the choice of organic food, which is the first aim of this study, and the identification of values influencing the purchase of organic food as second aim, categorized the research approach as a between-countries comparison, as it is the goal of this study to recognize differences between the consumers of organic food in a mature market in Germany and in an emerging market in Mexico.

Different approaches can be used in cross-cultural studies, such as interviews, focus groups, and surveys mentioned above. Some considerations should be provided concerning the so called back-translation techniques: translation equivalences, construct equivalence and measurement equivalence as well as perceptional meaning and functional meanings.

In line with Grunert et al. (1993), the term of translation equivalence refers to the "conceptual, functional, and experiential equivalence" of the concepts. The achievement of the translation equivalence depends on the conceptual functional and experiential equivalence of the concepts to be translated. "Measurement equivalence refers to the construct operationalisation, item, and scalar equivalence"; whereas the measurement of the constructs refers to the factor invariance, that is defined as good criteria for research of the degree of translation and measurement equivalence Grunert et al. (1993).

According to Grunert et al. (1993) conceptual equivalence presupposes that the term to be measured can be meaningfully expressed and understood in the cultures to be compared; for instance, the concepts of "animal welfare" or "well being". Experiential equivalence refers to the existence of equivalent referent symbols and meanings in different cultures; "good taste" 
or "fashion", the meaning or thought evoked by these words for Germans may not be the same for Mexicans. Within measurement equivalences, the psychological processes occurring in the respondent are presupposed, and it is presupposed that the process of answering has the same or at least comparable degree in the cultures under comparison. In addition, scales are referred to as having some equivalence if there are categories and metrics, due to scales being sometimes culture-bound because of language. According to O'Shaughnessy (1992) meaning in use requires recognizing the situation and occasion in which the word or expression occurs in a particular context or language: "a word would have meaning only if it has a use and its use could be taught" O'Shaughnessy (1992). Because of the differences between the two countries and the complicity of meanings within them, the meaning-in-use approach has been used in the interpretation of the analysis in this research. 


\section{$6 \quad$ Pretesting the survey}

In this chapter, the design and the results of the pretest of the survey in Mexico providing data concerning consumption of organic food in the domestic market are provided. This chapter consists of two parts. Firstly, an overview of factors describing consumption of organic food in Mexico such as total of years of purchase of organic food in general, frequency of purchasing in the mainstream retail channels, perception of price premium, and factors related to store image is provided. Secondly, details concerning the measurement of the model and the theoretical constructs of motivations and values of the cross-cultural comparison between Germany and Mexico.

In addition, the demographic profile of consumers of organic food is depicted by cross table analysis of demographic variables. After changes, a second version was tested by an online survey in Germany. The results of the first and second pretests as well as some considerations toward a final version of the multi-item scale measurement instrument as an online survey in the two countries are presented below.

\subsection{Pretest design}

In the design of a survey, is necessary to realize at least one pretest to recognize problems and limitations in the clearness and comprehension of meanings on the questions, items, as well as the measurement scale and method approach used. According to Aaker et al. (2007) the pretesting of a survey has two aims. Firstly, pretesting the specific questions, and secondly, pretesting the survey. In the pretest of a survey the representativeness of a sample of the target population is necessary to recognize limitation or problems in the survey design.

Although the authors, suggest the pretest with a sample of 15 respondents if the survey is short and straightforward and with 25 respondents if the survey is long and complex, which is our case due to the multi-item scale and cross-cultural survey. Other criterion regards the need 
of done personal or telephone interviews to get direct feedback on problems in order to improve survey. In the following paragraphs are sum up the factors regarding these factors within the pretest design.

\subsubsection{Pretesting specific questions}

In line with Aaker et al. (2007), some some very specific reasons for pretest questions are the variation, meaning, task difficulty and respondents' interest and attention. The theoretical background for each one of these reasons is summarized as follow:

1. Variation, is most of the time a common goal of a pretest. According to the authors, in a pre/test researchers look at an the acceptable level of variation of the items in a target population -variability- a greater variability is useful to detect sub-groups of people whereas, much skewed distributions can be considered as warning signal that question is not tapping the intended constructs.

2. Meaning, is also an important aim of a pretesting. In the ground of the intended meaning of the questions for the researchers might not be the same meaning interpret for respondents. Thus, in a pretest we look at possible distortions of the meaning of the questions to deal with the re-wording of the items and questions to be improved in the survey.

3. Task difficulty, is other factor to be look at in a pretest, due to even a meaningful and clear question can still be difficult to answer if it requires that respondents make connections or put together information in unfamiliar ways.

4. Respondents interest and attention. Finally, the interest and attentions that respondents give is other important factor to look at in a pretest. In fact, excessive repetitions of a question or the use of the same format asking for the same question can reduce the amount of attention paid by respondents. 


\subsubsection{Pretesting the survey}

To pretest the survey is also an aim of the pretesting design thus Aaker et al. (2007) suggest also four aspects to consider.

1. Flow of the survey, is the first aspect to be considered. In line with the authors, testing the flow of the survey is often a matter of intuitive judgment. Since respondents do not know exactly what will be the next question, questions must have a logical sequence and be part of a coherent flow, transitions from one topic to another must be also pretested to ensure their clear and logical order.

2. Skip patterns, is regarded by the authors, as the question that have been skipped depending on the questions. This is concerning mainly with filter questions. However, in on pretest design we will not use them.

3. Length, the third aspect is assessing the length of the each section of the survey; it is aimed by timing to ensure that none of them is too long to respondents. This regards, the idea that respondents with personal interest on the topic, are willing to spend more time on surveys thus, an upper limit to the durations might be considered. On the other side, other respondents might experience fatigue, interview break/off, and initial refusal if the length of the survey is larger as they expected. Thus, this information will be useful to improve the survey after an evaluation based on the result of a pretest.

4. Respondents interest and attention, the fourth aspect to be considered regards the attention and interest that respondents maintain throughout the entire survey. This is a major design challenge due to often the answering task varied throughout the survey.

The results and findings of the pretesting design conducted in Mexico deals with the improvement of the survey. These finding are summarize in the following chapter. 


\subsection{Study 1: Pretest in Mexico}

The survey described in the previous chapter was pretested between the months of July and September 2008 in Mexico City suburbs with a sample of 257 consumers (72\% female and $28 \%$ male) who were interviewed face to face in organic stores (217 respondents) and organic markets-tianguis ${ }^{5}$ (40 respondents). The collected data were then analysed using univariate statistics with the statistical program SPSS $17^{\text {th }}$ version.

\subsubsection{Consumer profile}

As in other countries, Mexican female consumers are responsible for nutrition within most households which was confirmed by the findings of the pretest. For ease of readability in the following, the consumer profiles of both the organic shops and organic markets have been grouped. All in all, consumers of organic food are mainly female (76.7\% versus $23.3 \%$ male) (see Table 16). Most consumers are between 30 and 50 years of age (58.8\%), followed by a group of mature consumers who are more than 50 years old (23\%). Finally, the youngest consumers who are less than 30 years old constitute the smallest group (17.9\%). Most of the consumers are characterized by a higher education level: $50.2 \%$ are graduates and $22.2 \%$ are postgraduates; it is worth mentioning that in the latter 1970s an historical change took place in the educational system due to the first massive entrance of women into universities. Most of the members belonging to the older group have an elementary education $(1.2 \%)$, high school degree $(5.4 \%)$, other degrees $(1.9 \%)$ or no formal education $(1.9 \%)$ respectively; whilst the youngest group consists of university students (data not shows). Regarding Household income, the Socio-Economical level classification by Lopez (2005) was assumed. According to their monthly income, most of the respondents (44.4\%) have mid-level incomes; $17.9 \%$ are

\footnotetext{
${ }^{5}$ A brief description of this market type was already provided in Chapter 1
} 
slightly higher-middle incomes, and $14.8 \%$ have slightly lower-middle household incomes. Interestingly, there are two less representative groups of consumers. The first group consists of consumers who have the lowest income $(10.5 \%)$; the second group belongs to consumers with the highest household income (4.7\%). Regarding the size of the household, most of them are younger families, as respondents self-reported having a partner and one/two children $(31.9 \%)$; within the second group are households with two or three members $(24.1 \%)$; additionally, there are households consisting of a single person (16.3\%); another group live with parents $(11.3 \%)$; and the last group belongs to households with a partner and no children (9.7\%). Finally, the smallest group belongs to people sharing an apartment (living with friends) (5.8\%).

In line with the findings of previous studies, consumers of organic food in Mexico seem to share a high degree of similarity with consumers of mature markets at least in demographic terms, such as female, younger adults, highly educated and high income as well as households with children (Davies et al., 1995; Gil \& Sanchez, 2000; Chinnici et al., 2002; Fotopoulos \& Krystallis, 2002; Wier \& Calverley, 2002). Furthermore, these findings are confirmed in studies carried out in other emerging markets; for instance, Croatia, Brazil and Thailand (Radman, 2005; Sirieix et al., 2007; Roitner-Schobesberger et al., 2008). 
Table 16: Demographic profile of consumers of organic food in Mexico $(n=257)$

\begin{tabular}{|c|c|c|}
\hline & Responses & $\%$ \\
\hline \multicolumn{3}{|l|}{ Gender: } \\
\hline Female & 197 & 76.7 \\
\hline Male & 60 & 23.3 \\
\hline \multicolumn{3}{|l|}{ Age groups: } \\
\hline Less than 30 years old & 46 & 17.9 \\
\hline Between $30-50$ years old & 151 & 58.8 \\
\hline More than 50 years old & 59 & 23.0 \\
\hline \multicolumn{3}{|l|}{ Education level: } \\
\hline Elementary School & 3 & 1.2 \\
\hline High School & 14 & 5.4 \\
\hline Intermediate School & 44 & 17.1 \\
\hline University & 129 & 50.2 \\
\hline Postgraduate & 57 & 22.2 \\
\hline Other & 5 & 1.9 \\
\hline No formal education & 5 & 1.9 \\
\hline \multicolumn{3}{|l|}{ Household incomes (monthly in US Dollars): } \\
\hline$\$ 670.00$ (Lower level) & 27 & 10.5 \\
\hline$\$ 671.00$ - \$1,142.00 (slightly lower-middle level) & 38 & 14.8 \\
\hline$\$ 1,143.00-\$ 3,451.00$ (middle level) & 114 & 44.4 \\
\hline$\$ 3,452.00$ - $\$ 8,382.00$ (slightly higher-middle level) & 46 & 17.9 \\
\hline More than $\$ 8,382.00$ (higher level) & 14 & 4.7 \\
\hline Missing values & 18 & 5.5 \\
\hline \multicolumn{3}{|l|}{ Family lifestyle: } \\
\hline Alone & 42 & 16.3 \\
\hline As part of a couple & 62 & 24.1 \\
\hline As part of a couple with children & 82 & 31.9 \\
\hline As part of a couple without children & 25 & 9.7 \\
\hline Living with parents & 29 & 11.3 \\
\hline Living with friends (shared apartment) & 15 & 5.8 \\
\hline Missing values & 2 & .8 \\
\hline
\end{tabular}

Source: -Own estimations (2008).

\subsubsection{Supposed determinant attributes of the POS image: organic stores vs organic markets}

In general terms, consumers who purchase in organic stores and organic markets claimed to be satisfied. This is confirmed by the findings of the pretest (see Table 17). On a range from strongly disagree to strongly agree: (-2) strongly disagree; (-1) disagree; (0) neither agree nor disagree; (1) agree; (2) strongly agree. Most of the responses are concentrated in the category of agree for most of the supposed determinant attributes measuring the image of the POS: information availability, trustworthiness, assortment, shopping convenience, price 
orientation, quality/freshness, and promotion. If on the one hand this shows a high satisfaction of customers within the market of organic products, then on the other hand, it raises methodological problems due to the low variation of responses and skewness of distribution (Aaker et al., 2007). Thus, these findings were considered as the first warning signal for further refinement of the proposed factors at the level of POS.

Table 17: Supposed determinant attributes of the POS image (percent)

\begin{tabular}{|c|c|c|c|c|c|c|c|c|c|c|c|c|}
\hline & $\mathbf{n}(217)^{\mathrm{a}}$ & $n(40)^{\mathrm{a} a}$ & n(217) & $n(40)$ & $\mathbf{n}(217)$ & $n(40)$ & $\mathbf{n}(217)$ & $n(40)$ & $\mathbf{n}(217)$ & $n(40)$ & $\mathbf{n}(217)$ & $n(40)$ \\
\hline \multicolumn{13}{|l|}{ Available information: } \\
\hline I find information and recomendations about the organic products her & 1.8 & 7.5 & 11.1 & 2.5 & 24.9 & 2.5 & 51.2 & 60.0 & 8.8 & 22.5 & 97.7 & 95.0 \\
\hline There is enough information about the organic products that are sold & 2.8 & 2.5 & 10.1 & 5.0 & 26.3 & 12.5 & 48.4 & 50.0 & 10.1 & 22.5 & 97.7 & 92.5 \\
\hline \multicolumn{13}{|l|}{ Trustworthiness: } \\
\hline I' $m$ sure that the organic products that I buy here are really organic & .5 & 2.5 & 5.5 & 5.0 & 10.6 & 5.0 & 61.8 & 57.5 & 19.4 & 25.0 & 97.7 & 95.0 \\
\hline The organic products that are sold here are authentic & .9 & 2.5 & 2.3 & 2.5 & 8.8 & $(---)$ & 66.4 & 62.5 & 18.4 & 27.5 & 96.8 & 95.0 \\
\hline \multicolumn{13}{|l|}{ Assortment: } \\
\hline I found an organic assortment that is not easy to find in other shops & .9 & 5.0 & 7.4 & 22.5 & 11.5 & 5.0 & 57.1 & 47.5 & 20.7 & 15.0 & 97.7 & 95.0 \\
\hline I like the assortment available & 1.4 & 5.0 & 6.9 & 7.5 & 6.5 & 7.5 & 67.7 & 52.5 & 15.2 & 20.0 & 97.7 & 92.5 \\
\hline \multicolumn{13}{|l|}{ Allocation (Shopping convenience): } \\
\hline The store is close to home & 6.9 & 5.0 & 20.7 & 27.5 & 20.7 & 15.0 & 36.9 & 30.0 & 12.4 & 17.5 & 97.7 & 95.0 \\
\hline \multicolumn{13}{|l|}{ Price orientation: } \\
\hline The prices are better than in other shops & 2.3 & 2.5 & 24.9 & 17.5 & 37.8 & 15.0 & 27.2 & 45.0 & 5.1 & 15.0 & 97.2 & 95.0 \\
\hline \multicolumn{13}{|l|}{ Q uality-f reshness: } \\
\hline There is a guarantee of quality & $(---)$ & 2.5 & 3.7 & $(--)$ & 13.8 & (----) & 62.7 & 70.0 & 17.1 & 22.5 & 97.2 & 95.0 \\
\hline The store is well known because of the fresh food & $(---)$ & 2.5 & $(----)$ & (---) & (----) & (----) & $(-)$ & 62.5 & (----) & 30.0 & $(---)$ & 95.0 \\
\hline \multicolumn{13}{|l|}{ Promotions: } \\
\hline There are promotions for organic food & 16.6 & n.d. ${ }^{\text {aaa }}$ & 53.0 & n.d. & 19.4 & n.d. & 5.5 & n.d. & .9 & n.d. & 95.4 & n.d. \\
\hline
\end{tabular}

Source: own estimations (2008).

This reduces the leeway of possible methods to be employed in order to attain this study's purposes. Henceforth, a new research design was conceived for the follow-up to the interviews of both consumers and non-buyers of organic food. 


\subsubsection{Motivations to purchase organic food}

The results of the motivational responses follow. For ease of discussion, the findings concerning both the organic stores and organic markets are grouped. Table 18 shows the results of health and environmental concerns which are exemplarily presented in order to again show a problem with lower variation of responses and skewed distribution of data regarding the dimensions of health and environmental concern.

The dimension of healthy eating, "keeps me healthy", is firstly analysed: most of the responses are within the category of "very important" $(86.8 \%) ; 10.1 \%$ were within the category of "important" and around 1.2\% within "moderately important" and "a little important" and $0.8 \%$ of the respondents fall into the category of "not at all important". The scoring for the second item "are good for me" is the following: $87.2 \%$ of the respondents scored "very important", $8.9 \%$ "important", and 1.9\% "moderately important", and 1.2\% chose of "little importance". Finally, $0.8 \%$ of the respondents scored "not at all important".

The scoring of the two items of dimensions of environmental concern are as follows. The item "has been transported with a low environmental cost" scored at the level of "very important" by $77 \%$ of the respondents; $11.7 \%$ scored it as "important" and $6.2 \%$ scored it as "moderately important". Least mentioned were the categories of "little importance" shared by $1.6 \%$ of the respondents and "not at all important" by $1.9 \%$. The scoring of the item "has been produced in a way which has not changed the balance of nature" by respondents is summarized as follows: most (72.8\%) agree with "very important", 14\% with "important", $8.9 \%$ with "moderately important", and the category of "a little important" was again chosen by $1.9 \%$ of the respondents including "not at all important". An overview of all motivations is available in Appendix. The above findings were interpreted as a second signal resulting in the necessity to consider refining the theoretical constructs, the measurement scale and rewording questions to improve the operationalization of multi-item motivations to purchase organic food. 
Table 18: Motivations to purchase organic food (percent)

\begin{tabular}{|c|c|c|c|c|c|c|c|c|}
\hline Statements & $\begin{array}{l}\text { Not at all } \\
\text { important }\end{array}$ & $\begin{array}{c}\text { A little } \\
\text { important }\end{array}$ & Neutral & Important & $\begin{array}{c}\text { Very } \\
\text { important }\end{array}$ & $\begin{array}{c}\text { Total } \\
\%\end{array}$ & $\mu$ & $\sigma$ \\
\hline \multicolumn{9}{|l|}{ Healthy eating: } \\
\hline Keeps me healthy. & .8 & 1.2 & 1.2 & 10.1 & 86.8 & 100 & 1.81 & .59 \\
\hline Are good for me. & .8 & 1.2 & 1.9 & 8.9 & 87.2 & 100 & 1.81 & .60 \\
\hline \multicolumn{9}{|l|}{ Environmental concern: } \\
\hline Has been transported with a low environmental cost. & 1.9 & 1.6 & 6.2 & 11.7 & 77.0 & 98.4 & 1.63 & .83 \\
\hline Has been produced in a way which has not disturbed the balance of nature. & 1.6 & 3.1 & 3.9 & 10.5 & 80.9 & 100 & 1.66 & .82 \\
\hline
\end{tabular}

Source: Own estimation (2008).

\subsubsection{Dimensions of values}

The results regarding values as motivations to purchase provided similar answers by respondents. Again, the findings of both the organic stores and the organic markets are grouped. Thus, the frequency of responses is described in the following. Table 19 shows the results of the dimensions of self-direction and universalism which are exemplarily presented in order to again depict the problem of low variation and skewness of data distribution due to the concentration of data responses.

The scoring of the dimensions of "self-direction" are summed up as follows: most of the respondents $(87.9 \%)$ agree within the category of "very important" regarding the item "freedom of (action and thought)", and 9.7\% were within the category of "important". Less mentioned were the categories of "moderately important", $(0.4 \%) ; 1.2 \%$ scored within the category of "a little importance". Meanwhile, $0.8 \%$ of the respondents agreed at the "not at all important" level.

Concerning the dimension of value of universalism, most of the respondents (82.5\%) agreed to "protecting the environment (preserving nature)" within the category of "very important"; $14 \%$ within this category scored it as "important"; $2.3 \%$ agreed with the category of "moderatey important"; $0.8 \%$ with "a little important"; and least mentioned was the category of "not at all important" as only $0.4 \%$ of respondents chose it. Regarding the item "broadminded (tolerant of different ideas and beliefs)" again most of the respondents (82.1\%) agreed that it is "very important"; $13.2 \%$ scored "important"; $2.7 \%$ "moderately important" and 1.2\% agreed with "a little important"; $0.4 \%$ of the respondents scored within the category of "not at 
all important". All in all, the lower variation among the responses regarding the dimensions of values is a third warning signal for the reconsideration of the design of the cross-cultural survey. An overview of all dimensions of values is available in Appendix.

Table 19: Most frequent responses provided for the dimension of values (percent)

\begin{tabular}{|l|r|r|r|r|r|r|r|r|}
\hline \multicolumn{1}{|c|}{ Statements } & $\begin{array}{c}\text { Not at all } \\
\text { important }\end{array}$ & $\begin{array}{c}\text { A little } \\
\text { important }\end{array}$ & Neutral & Important & $\begin{array}{c}\text { Very } \\
\text { important }\end{array}$ & $\begin{array}{c}\text { Total } \\
\mathbf{\%}\end{array}$ & $\boldsymbol{\mu}$ & $\boldsymbol{\sigma}$ \\
\hline Self-direction: & & & & & & & \\
\hline Freedom (of action and thought). & .8 & 1.2 & .4 & 9.7 & $\mathbf{8 7 . 9}$ & 100 & 1.8 & .6 \\
\hline Creativity (uniqueness and imagination). & .8 & 1.2 & 9.7 & 20.2 & $\mathbf{6 8 . 1}$ & 100 & 1.5 & .8 \\
\hline Universalism: & & & & & & & & \\
\hline Protecting the environment (preserving nature). & .4 & .8 & 2.3 & 14.0 & $\mathbf{8 2 . 5}$ & 100 & 1.8 & .6 \\
\hline Broad minded (tolerant of different ideas and beliefs). & .8 & 1.2 & 2.7 & 13.2 & $\mathbf{8 2 . 1}$ & 100 & 1.7 & .6 \\
\hline
\end{tabular}

Source: Own estimation (2008).

\subsubsection{First conclusions: evaluation and refining of the survey}

After the pretesting, several discrepancies were detected of both the survey design and wording. Regarding the survey design, the overall structure was not considered problematic. However, some difficulties were detected regarding the length of the survey and respondents' general interest and attention. It appeared that after the section on "motivations to purchase", interest and attention by the respondents were reduced. Consumers with more time, interest in the topic, interest in participation, or without a companion were more willing to continue with the interviews, and they also took the time to provide some reasons to support their responses. On the contrary, respondents who had limited time to shop or were accompanied with someone else, or had little involvement with organic food showed more fatigue, intention to walk away, and an initial attitude of refusal to continue the interview.

Regarding the pretest questions, as mentioned above problems with the levels of variation of responses were detected as well as meanings, task difficulty and measurement scales in the sections of motivations and values. Accordingly, an evaluation of the theoretical constructs and the need for refining the meanings and the wording of items were necessary. The specific problem areas will be described in the section referring to changes of motivations and values. 
All in all, the major problems which arose from the face-to-face interviews were recognised as the following: influence of the social class, characteristics of the Mexican culture, and Socially Desirable Responses (SDR) (Ross et al., 1983; Ross \& Mirowsky, 1984). The usefulness of the pretest highlighted the need to improve the questionnaire design, to refine the measurement of the items, to modify some theoretical constructs, and to include other groups of respondents. The improved version of the survey was pre-tested in a second sample; the changes are summarized in the following section.

\subsection{Improvement of the survey design}

After evaluate the results of the findings of the pretest conducted in Mexico and with respect to the homogeneity of the sample, it was decided to include "non-buyer" in the sample to improve the cross-cultural survey. Accordingly, the second pretest did not take place in the organic stores or organic markets. Thus, the second section regarding the supposed determinant attributes at the level of POS were deleted. However, further literature review was done to improve the multi-item batteries of motivations and values. Thus, important changes in the theoretical constructs of motivations and values were undertaken on both meaning and wording of the items as well as on the scales to be used in the approach. Further literature review included Food Related Lifestyle (FRL) by Grunert et al. (1993) and Hofstede (2001) supporting changes to motivation and value sections to improve the survey to be pretested in Germany. 


\subsection{Further literature review to improve the sections of}

\section{motivations and values}

\subsubsection{Food related lifestyle (FRL)}

Food related lifestyle (FRL) is a multidimensional measurement instrument designed by Grunert et al. (1993) to understand which product characteristics are perceived by consumers as superior. His measurement approach is based on principles derived by cognitive psychology. Considering the use of the cognitive-inductive approach traditionally used in marketing in lifestyle research, Grunert et al. (1993) proposed the concept of a consumption related lifestyle that according to the authors "identifies a system of cognitive categories, scripts, and associations according to which a set of products can be linked to a set of values".

According to Grunert et al. (1993), to understand how people purchase food products it is important to know their values. In fact, because of culture, purchasing of food products and values can be indirectly linked. However, expectations regarding the consumption of food products depend not only on values but also on other factors such as how food is purchased, used and disposed and social interaction. Thus, the degree of freedom that a consumer has in preparing/cooking, eating, and using food products deals with the existence of differently related lifestyles. The FRL was designed to provide information on the determinants of value perception of consumers and, subsequently, their lifestyles. In its design the FRL includes a theoretical framework consisting of the theory of lifestyle, cognitive structure research, and Mean-End-Chain (MEC). This measurement instrument detects long-term trends among consumer's dealings. Finally, the measurement instrument was cross-culturally empirically tested in Denmark, the UK, and France. Some important constructs of food related lifestyle by Grunert et al. (1993) is summarized below. 
Table 20: Conceptual framework of the dimensions included in the original FRL

\begin{tabular}{|c|c|c|}
\hline Dimension & Conceptual framework & Constructs \\
\hline $\begin{array}{l}\text { Meal preparation } \\
\text { scripts }\end{array}$ & $\begin{array}{l}\text { This dimension refers to the food } \\
\text { preparation process. For instance, whether it } \\
\text { is a spontaneous/planned activity, a social } \\
\text { activity/division of labor is characterized by } \\
\text { efficiency/indulgence or requirement for } \\
\text { technical/human assistance. }\end{array}$ & $\begin{array}{l}\text { Involvement with } \\
\text { cooking, looking for new } \\
\text { ways, convenience, } \\
\text { whole family, spontaneity } \\
\text { and as a woman's task. }\end{array}$ \\
\hline Shopping scripts & $\begin{array}{l}\text { This question refers to the decision-making } \\
\text { process. For instance, whether the purchase } \\
\text { of food is an impulsive/extensive } \\
\text { deliberation, if consumers read the } \\
\text { information displayed on the labels, and if } \\
\text { advice by experts, friends, or others is } \\
\text { considered, and whether they shop } \\
\text { singularly or with others. }\end{array}$ & $\begin{array}{l}\text { Importance of product } \\
\text { information, attitude } \\
\text { towards advertising, joy } \\
\text { of shopping, organic } \\
\text { shops, price orientation, } \\
\text { and shopping list. }\end{array}$ \\
\hline $\begin{array}{ll}\text { Desire } & \text { higher- } \\
\text { order } & \text { product } \\
\text { attributes } & \end{array}$ & $\begin{array}{l}\text { Refers to indeterminate attributes such as } \\
\text { healthier, nutritious, natural, exclusive, } \\
\text { convenient, and better taste. }\end{array}$ & $\begin{array}{l}\text { Health, price-quality } \\
\text { relationship, novelty, and } \\
\text { organic products. }\end{array}$ \\
\hline Usage situations & $\begin{array}{l}\text { Refers to social context of consumption. For } \\
\text { instance, the perception of meals eaten } \\
\text { alone, with family, friend, or guests. }\end{array}$ & $\begin{array}{l}\text { Snacks versus meals, as } \\
\text { well as social events. }\end{array}$ \\
\hline $\begin{array}{l}\text { Desired } \\
\text { consequences }\end{array}$ & $\begin{array}{l}\text { Refers to consumers' expectations of } \\
\text { consuming food within a nutritional sphere } \\
\text { or a social event in addition to the } \\
\text { emotional/feelings and/or hedonism } \\
\text { consequences. }\end{array}$ & $\begin{array}{l}\text { Self-fulfillment by food, } \\
\text { security, and social } \\
\text { relationships. }\end{array}$ \\
\hline
\end{tabular}

Source: Grunert et al. (1993).

Grunert et al. (1993) also proposed that these dimensions were connected to cognitive categories, which, in turn, relates to products on the one side and values on the other.

Because the FRL was designed to glean an in-depth understanding of consumer behavior and because the measurement instrument was empirically tested in three countries, it seemed useful for this study's cross-cultural comparison. It should be noted that the FRL was tested in Australia by Reid et al. (2000). However, not all five dimensions carried out by Grunert et al. (1993) are related to this study's proposal regarding motivations to purchase organic food. 
Thus, some items of the eight factors including attitude towards advertising, price criterion, joy of shopping, health, price-quality relation, convenience, and involvement with cooking were adapted in our measurement to reinforce the motives driving choice of food carried out by Steptoe et al. (1995) in the FCQ and frequently mentioned in the review of international literature on motivations to purchase organic food.

\subsubsection{German List of Values (GLOV)}

To improve the section on values, some theoretical approaches were reviewed including the European Social Survey 2006 (ESS), Sinus Milieu (Sinus Sociovision, 2006); Short Schwartz Value Survey Short SVS (SSVS) (Lindeman \& Verkasalo, 2005); and the German List of Values (GLOV) by Grunert and Scherhorn (1990). Frequently, the List of Values by Kahle and Timmer (1986) is a polemical measurement instrument of values: some marketing scholars regard it as a useful scale (Blackwell et al., 2006; Solomon, 2006) while some others claim that its usefulness is limited to the measurement of values of American societies (Mooij, 2004; Hofstede, 2001). Furthermore, Grunert and Scherhorn (1990) provide the validity of the German version of the List of Values in comparing the values of four countries: (West) Germany, the United States, Canada (Montreal, Toronto, and Vancouver) and Norway. Among the results, cultural differences were provided as well as the wording of the values that differ among the countries and the language-related specificities. Regarding this study's comparative research, it was concluded that the GLOV would be useful in the measurement scale of values for both Mexican and German societies. Regarding the former, it was also concluded that, because of the closeness of the two countries, the values of the US society influencing the Mexican society as well. As far as Germany is concerned, a pretest was undertaken of the measurement scale to determine its usefulness. The changes provided in the sections of motivations and values are summarized below. 


\subsubsection{Changes in motivations to purchase food products}

A description is provided in this section of the changes in the survey to improve the multiitems scale of motivations for pretesting in Germany using the multiple-item scale developing process proposed by Aaker et al. (2007) which was used as a guideline in the design of the cross-cultural survey. Next, the findings of the pretest in Mexico will be evaluated and items refined.

It is worth mentioning that the constructs and items administered to the initial sample in Mexico were inspired by the international literature review on food choice and motivations to purchase organic food mostly provided by Europe and Germany. Therefore, most of the statements were adapted and translated into Spanish. After the pretesting in Mexico some problems were detected on the set of questions related to motivations and values as well (see appendix). As a result of the evaluation, some constructs and items were deleted. The changes are described in the following:

The two items of political concern were difficult to answer by most of the respondents. Also the meaning was commonly confused. The aim of the measurement of the construct in the cross-cultural comparison was to find out significant cultural differences among the respondents in Germany and Mexico. However, after the Mexican pretest, it was discovered that most respondents shared some difficulty in linking the construct's items of human rights and political values (adapted from the Ethical FC) with the production and consumption of food products. As a consequence, this theoretical construct was dismissed. Also, for most of the respondents, the item related to political values was not clear. It was assumed that the limited reliability of the political concern construct in Mexico is related to several factors such as the stage of development of the domestic market for organic products, the development of the food industry sector, and in a certain way, the socio-economic structure of the society.

To summarize, because most respondents scored the two items of national concern at the "very important" level, this construct was withdrawn and two new constructs, consumer ethnocentrism and malinchismo, were added. Consumer ethnocentrism is defined as 
consumers' beliefs in moral appropriateness in favour of domestic products (Shim \& Sharma 1987 cited in Nguyen et al., 2008); whereas malinchismo refers to the opposed attitude of preferences for people or products of foreign origin. Thus, this construct was only applied to the Mexican sample.

Due to the complexity of its measurement, lifestyle was removed and its sub-dimensions were replaced by the following new constructs: fair price, nostalgia, product information, advertising, cooking involvement and price quality. Finally, other items were added to the constructs of animal welfare, environmental concern, natural content, healthy eating, sensory variables, and weight control. Familiarity was refined as familiarity/advertising and the 5 point Likert measurement scale was also improved to a 7-point Likert-type scale: (-3) Strongly disagree; (-2) Disagree; (-1) Somewhat disagree; (0) Neither agree or disagree; (1) Somewhat agree; (2) Agree; and (3) Strongly agree to improve the variation of the categories of answers (See Table 21). 
Table 21: Changes in the measurement of the theoretical constructs of motives and values in the purchase of organic food

\begin{tabular}{|c|c|c|c|c|}
\hline \multicolumn{3}{|c|}{ First study: pretest in Mexico } & \multicolumn{2}{|c|}{ Second study: pretest in Germany } \\
\hline \multicolumn{3}{|c|}{ Dimension of motivations to ftem Source: } & \multicolumn{2}{|c|}{ Dimension of motivations to purd Item Source: } \\
\hline Animal welfare & 2 & $\begin{array}{l}\text { Adapted from Lindeman and } \\
\text { Väänänen, } 2000 .\end{array}$ & Animal welfare & 2 |Author \\
\hline National concern & 2 & Author & $(----)$ & $(---)$ \\
\hline Environmental concern & 2 & $\begin{array}{l}\text { Adapted from Lindeman and } \\
\text { Väänänen, } 2000 .\end{array}$ & Environmental concern & $3 \begin{array}{l}\text { Adapted from Steptoe et al., 1995; Lindeman and } \\
\text { Väänänen, 2000; Author. }\end{array}$ \\
\hline Political concern & 2 & $\begin{array}{l}\text { Adapted from Lindeman and } \\
\text { Väänänen, } 2000 .\end{array}$ & $\mid(----)$ & $(---) \mid(---)$ \\
\hline Lifestyle & 2 & Author & $(----)$ & $(---)$ \\
\hline Weight control & 2 & Adapted from Steptoe et al., 1995. & Weight control & 3 Adapted from Steptoe et al., 1995. \\
\hline Sensory variables & 2 & Adapted from Steptoe et al., 1995. & Sensory variables & 3 Adapted from Steptoe et al., 1995. \\
\hline Healthy eating & 2 & Adapted from Steptoe et al., 1995. & Healthy eating & $\begin{array}{l}\text { Adapted from Grunert et al., 1993; Steptoe et al, 1995; } \\
\text { Author. }\end{array}$ \\
\hline Natural content & 2 & Adapted from Steptoe et al., 1995. & Natural content & $\begin{array}{l}\text { Adapted from Grunert et al., 1993; Steptoe et al, 1995; } \\
\text { Author. }\end{array}$ \\
\hline Free of GM0 & 1 & Author & Free of GM0 & 1 Author \\
\hline Familiarity & 2 & Adapted from Steptoe et al., 1995. & Familiarity/Advertising & 3 Adapted from Steptoe et al., 1995; Author. \\
\hline Shopping convenience & 2 & Adapted from Steptoe et al., 1995. & Shopping convenience & $\begin{array}{l}\text { Adapted from Grunert et al., 1993; Steptoe et al, 1995; } \\
\text { Author. }\end{array}$ \\
\hline Cooking convenience & 2 & Adapted from Steptoe et al., 1995. & Cooking convenience & 2 Adapted from Steptoe et al., 1995. \\
\hline Price & 2 & Adapted from Steptoe et al., 1995. & $(----)$ & $(---) \mid(---)$ \\
\hline \multirow[t]{7}{*}{ Organic label } & 2 & Author & $(----)$ & (----) \\
\hline & & & Cooking involvement & 4 Author \\
\hline & & & Fair price & 3 Author \\
\hline & & & Nostalgia & 3 Author \\
\hline & & & Price Orientation/Price Quality Rel| & 3 Adapted from Grunert et al., 1993; Author. \\
\hline & & & \begin{tabular}{|l|l} 
Information & \\
\end{tabular} & 2 Adapted from Grunert et al., 1993; Author. \\
\hline & & & Consumer ethnocentrism & \begin{tabular}{l|l|l|}
2 & Author \\
\end{tabular} \\
\hline \multicolumn{3}{|l|}{ Dimensions of Values: } & \multicolumn{2}{|l|}{ Dimensions of Values: } \\
\hline Hedonism & 2 & SVS by Schwartz 1992. & Hedonism & 1 Adapted from Schwartz, 1992. \\
\hline Stimulation & 2 & SVS by Schwartz 1992. & Stimulation & $\begin{array}{l}\text { Adapted from Grunert and Scherhorn, 1990; Schwartz, } \\
1992\end{array}$ \\
\hline Security & 2 & SVS by Schwartz 1992. & Security & $\begin{array}{l}\text { Adapted from Grunert and Scherhorn, 1990; Schwartz, } \\
1992 .\end{array}$ \\
\hline Self-direction & 2 & SVS by Schwartz 1992. & Self-direction & $\begin{array}{l}\text { Adapted from Grunert and Scherhorn, 1990; Schwartz, } \\
1992 .\end{array}$ \\
\hline Universalism & 2 & SVS by Schwartz 1992. & Universalism & 3 Adapted from Schwartz, 1992. \\
\hline \multirow[t]{3}{*}{ Benevolence } & 2 & SVS by Schwartz 1992. & Unity with nature & 2 Adapted from Schwartz, 1992. \\
\hline & & & Tradition & \begin{tabular}{l|l}
1 & Adapted from Schwartz, 1992. \\
\end{tabular} \\
\hline & & & Power/achivement & $\begin{array}{l}\text { Adapted from Grunert and Scherhorn, 1990; Schwartz, } \\
1992 .\end{array}$ \\
\hline
\end{tabular}

Source: Own estimations (2008). 
Due to the lack of studies applying Schwartz's SVS (1992) in Mexico, it was decided within an explorative approach to adapt some dimensions of values and labels. This decision was endorsed through the recommendations of some expert researchers who pointed out the need for abbreviating the original measurement scale of 57 items and modifying the sample including a wider range of respondents beyond the educated population. Eventually, it was combined with other measurement instruments such as those referred to by Grunert and Juhl (1995). This adaptation process to the measurement instrument involved phases of the items' conception, measurement, meaning (wording) and method of analysis as follows: firstly, the sole usage of six dimensions of values that we considered exemplified the purchasing of organic food which was problematic due to the strong interdependency of the ten dimensions measured originally by Schwartz (1992). In fact, in further review of literature on measuring values provided by Lindeman and Verkasalo (2005), it pointed out "that the reason for selecting the 57 items in their Short version of the SVS was that the mean of the 45 items of the seven cross-cultural dimensions (in a second SVS provided by Schwartz in 1994 cited in Lindeman \& Verkasalo, 2005) had caused the problem of linear dependency in some analyses".

Secondly, not all the participants of the sample were educated as in the samples of the studies provided by Schwartz (1992) and Grunert and Juhl (1995). Thirdly, in the original SVS instrument, Schwartz (1992) used a 9 point Likert type scale. However in the aim of reducing the survey duration and improving its fluency, the measurement scale used was a 5 point Likert type scale on the basis suggested by researchers that a scale between 5 to 7 responses of choice is optimal (Betz, 1996 cited in Lindeman \& Verkasalo, 2005). Thus, the items to measure values were replaced by others conceived by Grunert and Scherhorn (1990) in order to adapt the GLOV instrument.

Therefore, in the second pretest in Germany two measurement methods: (a) 7-point Likerttype scale ranging from (3) extremely important to (-3) contrary to my viewpoint was used ${ }^{6}$.

\footnotetext{
6 the whole scale was conceived as follows: (3) extremely important (-2) very important, (-1) moderately important, (0) moderate, (1) low importance, (2) not at all important (-3) contrary to my viewpoint
} 
Thirdly, respondents were required to state the importance if any of listed values (See Table 19). Fourthly, confusion regarding meaning was detected that was postulated as being a result of the Spanish language; that is: the specific meaning of words in the Mexican national culture (culture bias) and the overestimation of meanings by respondents in the case of value items. Finally, the dimension of universalism by Schwartz's (1992) SVS should be mentioned. Whereas Schwartz's intention of affinity with both humanity and nature were mixed, in this study they separated. Specifically, universalism in the questionnaire refers to affinity with fellow men, whereas unity with nature refers to affinity with nature. As a consequence, the dimension of benevolence was removed. In addition, two dimensions tradition and power/achievement- were included in the survey design because of the usefulness of GLOV.

\subsection{Study 2: Pretest in Germany}

\subsubsection{Analysis procedure}

The online data were collected by the Unipark Company, and analysed using univariate statistics (frequencies and cross table) with the statistical program SPSS $17^{\text {th }}$ version. As a filtering question, a question concerning the participation in purchasing food products in the household was presented. Accordingly, respondents who do not participate were eliminated. To classify the groups of respondents as buyers and non-buyers, an inquiry on the purchasing of organic food was included. Consumers of organic food were also required to answer a set of general questions regarding frequency of purchasing of organic food, knowledge about organic labels, and purchasing of the nutritional groups of organic quality. 


\subsubsection{Sample description}

After improving the multi-item scale, the new version of the survey was tested in Germany by means of an online survey of 63 respondents (see Table 22). The sample consists of 47 male (73\%) and 17 female (27\%) respondents. Regarding the purchasing of organic food, 46 respondents (73\%) are consumers of organic food and 13 are non-buyers (23.8\%). Regarding the federal states, 16 respondents (25.4\%) are from Nord Rhine-Westphalia, 8 from BadenWürttemberg (12.7\%), 7 from Lower Saxony (11.1\%) and 7 from Bavaria (11.1\%). The other federal states (Bremen, Hamburg, Schleswig Holstein, Hessen, Rhineland-Palatinate, Mecklenburg-Vorpommern, Saxony, and Saxony-Anhalt) had less than 5 respondents respectively (data not shows).

Most of the respondents are between 30 and 50 years old (55.6\%) followed by respondents who are less than $30(36.5 \%)$; within the smallest group are those of more than 50 years of age $(7.9 \%)$. Regarding education, most of the respondents are university graduates $(54 \%)$, others are intermediate graduates $(33 \%)$, and among the smallest group are those with an elementary education (9.5\%); information regarding other levels of education was not included. Regarding household income, most of the respondents belong to the three middle income level bands, i.e. most (30.2\%) in the middle level (second band) with incomes between 2,000 and 3,199 $€$; the second group are in the middle income level (first band) with incomes between 1,500 - 1,999 €, and in third place were those in the middle level (third band) (20.6\%) with higher incomes than the two previous groups of between 3,200 and 4,499 $€$. As in the pretest in Mexico, respondents belonging to the incomes groups located in the extreme are underrepresented. In fact, $4.8 \%$ of the respondents belong to the slightly lowermiddle income level between 900 and $1,499 €$ and $4.8 \%$ of respondents belong to the lower income level with less than $900 €$. However, $6.3 \%$ of the respondents belong to the slightly higher-middle level with incomes between 4.500 and $5.499 €$. Among the smallest groups were respondents with the highest level of incomes - more than 5,500€.

Regarding lifestyle, the first group corresponds to family households where $44.4 \%$ of respondents live as a couple with children. The second household group are couples (19\%); among the third group there are households with one person (18.6\%), and others $(6.3 \%)$ share 
accommodation; $5.8 \%$ of the respondents live with their parents and the smallest group belongs to respondents who live as a couple without children (see Table 22).

Table 22: Demographic profile of the consumers of organic food in Germany

\begin{tabular}{|c|c|c|}
\hline & $\mathbf{n}$ & $\%$ \\
\hline \multicolumn{3}{|l|}{ Gender: } \\
\hline Female & 17 & 27.0 \\
\hline Male & 46 & 73.0 \\
\hline \multicolumn{3}{|l|}{ Age groups: } \\
\hline Less than 30 years old & 23 & 36.5 \\
\hline Between $30-50$ years old & 35 & 55.6 \\
\hline More than 50 years old & 5 & 7.9 \\
\hline \multicolumn{3}{|l|}{ Education level: } \\
\hline Elementary School & 6 & 9.5 \\
\hline High School/Bachelor & $(----)$ & $(----)$ \\
\hline Intermediate School & 21 & 33.3 \\
\hline University & 34 & 54.0 \\
\hline Post-graduate & $(----)$ & $(----)$ \\
\hline Other & $(----)$ & $(----)$ \\
\hline No formal education & $(---)$ & $(----)$ \\
\hline \multicolumn{3}{|l|}{ Household incomes (monthly in $€$ ): } \\
\hline More than 5.500 (higher level) & 2 & 3.2 \\
\hline 4.5005 .499 (slightly higher-middle level) & 4 & 6.3 \\
\hline 3.2004 .499 (middle level third band) & 13 & 20.6 \\
\hline 2.0003 .199 (middle level second band) & 19 & 30.2 \\
\hline $1.500-1.999$ (middle level first band) & 14 & 22.2 \\
\hline $900-1.499$ (slightly lower-middle income level) & 3 & 4.8 \\
\hline Less than $900.00 €$ (lower level) & 3 & 4.8 \\
\hline Missing values & 5 & 7.9 \\
\hline \multicolumn{3}{|l|}{ Family lifestyle: } \\
\hline Alone & 11 & 18.6 \\
\hline As a couple & 12 & 19.0 \\
\hline Family with children & 28 & 44.4 \\
\hline In a shared apartment (with friends) & 4 & 6.3 \\
\hline As a couple without children & 1 & 1.6 \\
\hline With parents & 3 & 4.8 \\
\hline Missing values & 4 & 6.3 \\
\hline
\end{tabular}

Source: Own estimation (2009).

\subsubsection{Motives driving the choice of food}

The inclusion of "non-buyer" in the new design of the survey led to the rewording of items related to the purchase of food products. In the pretest in Mexico respondents were asked by the purchase organic food (e.g. "It is important to me that organic food... contains no additives"), and respondents were required to express their agreement using a scale ranging 
from "very important" to "not important at all"; in the second version of the survey, the term "organic food" was eliminated, and the question regarding the importance of the above mentioned factors were reworded (e.g. "It Now it comes to issues that may play a role in purchasing of food. Please tick each case whether the following question apply to you or not").

The results of the frequencies of responses regarding motivations to purchase food products are described in the appendix. Table 23 shows the results of health and environmental concerns which are an exemplary presentation in order to portray that lower variations of data experienced previously in the Mexican pretest had been overcome.

Concerning the scoring of the construct "Healthy eating", $33.3 \%$ of respondents strongly agree with the item: "I try to eat food containing lots of vitamins and minerals," followed by $22.7 \%$ who minimally agree and $7.6 \%$ who neither agree nor disagree and $21.2 \%$ of the respondents somewhat disagree. No respondents disagree or totally disagree. Regarding the item: "I eat as much protein as possible in my diet," $22.7 \%$ of the respondets neither agree nor disagree; $19.7 \%$ strongly agree and $18.2 \%$ agree and somewhat disagree; finally, $12.1 \%$ somewhat agree. Similarly to the previous item, there were no responses in the categories of disagree or totally disagree. The frequencies of answers provided by the item "I eat healthily" are provided as follows: $33.3 \%$ of the respondents strongly agree; $24.2 \%$ agree; $1.5 \%$ somewhat agree, $18.2 \%$ neither agree nor disagree; $13.6 \%$ somewhat disagree; and again there are no responses in the categories of disagree and totally disagree. Regarding the item "I eat as much fibre as possible in my diet," $31.8 \%$ of the respondents strongly agree; $10.6 \%$ agree; 9.1\% somewhat agree; $18.2 \%$ neither agree nor disagree; $21.2 \%$ somewhat disagree; and again, none disagree or totally disagree.

Concerning environmental concern, the scoring of the item "I am careful to buy food products with environmentally friendly packaging" is the following: $15.2 \%$ of respondents strongly agree, $30.3 \%$ agree, $16.7 \%$ somehwat agree, $18.2 \%$ neither agree nor disagree, $6.1 \%$ somewhat disagree, $1.5 \%$ disagree and 3\% strongly disagree. Regarding the item "It is difficult to know if the production of my food has a negative impact on the environment", $18.2 \%$ of the respondents strongly agree; $18.2 \%$ neither agree nor disagree; $19.7 \%$ somehwat agree and 1.5\% somewhat disagree. Finally, there are 1.5\% who strongly disagree. Regarding the item "I am careful to buy food produced in an enviromentally friendly way", $16.7 \%$ of the 
respondetns strongly agree; however, most of the respondents agree (33.3\%); 12.1\% somehwat agree; $19.7 \%$ neither agree nor disagree; $4.5 \%$ somehwat disagree; $1.5 \%$ disagree; and $3 \%$ strongly disagree. An overview of all motivations is available in Appendix. The scores for the items included in values is displayed below.

Table 23: Motives driving the choice of food

\begin{tabular}{|c|c|c|c|c|c|c|c|c|c|c|}
\hline & $\begin{array}{l}\text { Strongly } \\
\text { disagree }\end{array}$ & Disagree & $\begin{array}{l}\text { Somewhat } \\
\text { disagree }\end{array}$ & $\begin{array}{l}\text { Neither } \\
\text { agree or } \\
\text { disagree }\end{array}$ & $\begin{array}{c}\text { Somewhat } \\
\text { agree }\end{array}$ & Agree & $\begin{array}{c}\text { Strongly } \\
\text { agree }\end{array}$ & Total \% & $\mu$ & 0 \\
\hline \multicolumn{11}{|l|}{ Request: Please give your assessment as to the following statements on nutrition: } \\
\hline \multicolumn{11}{|l|}{ Healthy: } \\
\hline I try to eat food containing lots of vitamins and minerals. & & & 21.2 & 7.6 & 6.1 & 22.7 & 33.3 & 90.9 & 1.4 & 1.6 \\
\hline I eat as much protein as possible in my diet & & & 18.2 & 22.7 & 12.1 & 18.2 & 19.7 & 90.9 & 1.0 & 1.5 \\
\hline I eat healthily & & & 13.6 & 18.2 & 1.5 & 24.2 & 33.3 & 90.9 & 1.5 & 1.5 \\
\hline I eat as much fibre as possible in my diet & & & 21.2 & 18.2 & 9.1 & 10.6 & 31.8 & 90.9 & 1.2 & 1.6 \\
\hline \multicolumn{11}{|l|}{ Now it comes to issues that may play a role in purchasing of food. } \\
\hline \multicolumn{11}{|l|}{ Request: Please tick each case whether the following questions apply to you, or not } \\
\hline \multicolumn{11}{|l|}{ Enviromental concern: } \\
\hline I am careful to buy food products with environmentally friendly packaging. & 3.0 & 1.5 & 6.1 & 18.2 & 16.7 & 30.3 & 15.2 & 90.9 & 1.2 & 1.5 \\
\hline It is difficult to know if the production of my food has a negative impact on the environment & 1.5 & & 1.5 & 18.2 & 19.7 & 31.8 & 18.2 & 90.9 & 1.5 & 1.2 \\
\hline I am careful to buy food produced in an enviromentally friendly way. & 3.0 & 1.5 & 4.5 & 19.7 & 12.1 & 33.3 & 16.7 & 90.9 & 1.2 & 1.5 \\
\hline
\end{tabular}

Source: Own estimation (2009).

\subsubsection{Values}

Among the results of the findings on values, the most frequent answers provided by the respondents according to the two different measurement scales for the constructs of selfdirection and the two new dimensions related to the construct of "Universalism" are presented in Table 24 and Table 25 and below described. 
Regarding self-direction, the scoring of the item "self-fulfillment and self-development" is as follows: most respondents agree with "very important" (37.9\%), and others $(24.2 \%)$ as being "extremely important"; $1.5 \%$ judge this item as contrary to their own viewpoint; there were no responses in the "not at all important" and "low important" categories; $12.1 \%$ were in the "moderate" category, $13.6 \%$ agree with the item as being "moderately important". Regarding the item "a job which fulfils me", similar responses were provided with the categories of contrary to my viewpoint where $1.5 \%$ of the respondents agree, and none of them placed their responses in the category of "not at all important" or "low importance". Again, 12.1\% chose the category "moderate" and $16.7 \%$ chose "moderately important"; $31.8 \%$ of respondents indicated that it is "very important" and $27.3 \%$ as "extremely important".

The scoring for the dimension of "fellow humans oriented" contains the item "social justice". Hereby, $1.5 \%$ of the respondents judge it as "contrary to my viewpoint" and "low importance"; there was no response in the category of "not at all important"; $9.1 \%$ judge it as "moderate"; $18.2 \%$ as "moderately important" and most of the respondents (39.4\%) placed their response in the category of "very important" with $19.7 \%$ in the category of "extremely important". Regarding the item "respect for fellow human beings", $1.5 \%$ of the respondents repute it is "contrary to my viewpoint"; none placed their answer in the categories of "not at all important" and "low importance"; 9.1\% chose the category "moderate"; $21.2 \%$ chose "moderately important"; $25.8 \%$ chose "very important" and most of the respondents $(31.8 \%)$ placed their answer in the category of "extremely important". The scoring of the item "readiness to help people in need" is as follows: $1.5 \%$ of the respondents scored the category "contrary to my viewpoint", and as many as the previous ones chose the category "low importance"; none of them think that it is "not at all important"; $18.2 \%$ were in both categories of "moderate" and "moderately important"; most of the respondents $(25.8 \%)$ agree with the category of "very important"; slightly similar are the responses in the category of "extremely important" $(24.2 \%$ of respondents). Regarding the item "environmental protection" the scoring of the responses in this category was: $1.5 \%$ chose "contrary to my viewpoint"; again as in the previous item none chose "not at all important"; $3 \%$ agree with "low importance"; $9.1 \%$ with "moderate" and $24.2 \%$ with "moderately important"; $28.8 \%$ with "very important". Others (22.7\%) agree that it is "extremely important". 
Table 24: Measuring of values with 7-point Likert-type scale (first measurement scale)

\begin{tabular}{|c|c|c|c|c|c|c|c|c|c|c|}
\hline & $\begin{array}{c}\text { Extremely } \\
\text { important }\end{array}$ & $\begin{array}{c}\text { Very } \\
\text { important }\end{array}$ & $\begin{array}{c}\text { Moderately } \\
\text { important }\end{array}$ & Neutral & $\begin{array}{c}\text { Low } \\
\text { important }\end{array}$ & $\begin{array}{l}\text { Not at all } \\
\text { important }\end{array}$ & $\begin{array}{c}\text { Contrary to } \\
\text { my } \\
\text { viewpoint }\end{array}$ & $\begin{array}{c}\text { Total } \\
\%\end{array}$ & $\mu$ & $\sigma$ \\
\hline \multicolumn{11}{|l|}{ Self-direction: } \\
\hline Self-fulfillment and self-development. & 24.2 & 37.9 & 13.6 & 12.1 & & & 1.5 & 89.4 & -1.8 & 1.2 \\
\hline A job which fulfils me. & 27.3 & 31.8 & 16.7 & 12.1 & & & 1.5 & 89.4 & -1.8 & 1.2 \\
\hline \multicolumn{11}{|l|}{ Universalism: } \\
\hline \multicolumn{11}{|l|}{ 1) Benevolence: } \\
\hline Social justice. & 19.7 & 39.4 & 18.2 & 9.1 & 1.5 & & 1.5 & 89.4 & -1.7 & 1.2 \\
\hline Respect for fellow human beings. & 31.8 & 25.8 & 21.2 & 9.1 & & & 1.5 & 89.4 & -1.8 & 1.2 \\
\hline Readiness to help people in need. & 24.2 & 25.8 & 18.2 & 18.2 & 1.5 & & 1.5 & 89.4 & -1.5 & 1.3 \\
\hline \multicolumn{11}{|l|}{ 2) Unity with nature: } \\
\hline Enjoy the world and life. & 24.2 & 31.8 & 22.7 & 7.6 & 1.5 & & 1.5 & 89.4 & -1.7 & 1.2 \\
\hline Environmental protection. & 22.7 & 28.8 & 24.2 & 9.1 & 3.0 & & 1.5 & 89.4 & -1.6 & 1.2 \\
\hline
\end{tabular}

Source: Own estimation (2009).

Next, we describe the responses referring to the second kind of scale instrument conceived for measuring values. Regarding the dimension of "Self-direction", "self-fulfillment and selfdevelopment" was scored by most respondents (82.5\%) as "important"; while 11.1\% mentioned it as "non important"; the item "a job which fulfils me" was scored by most respondents (79.4\%) as "important" whereas 14.3\% stated it as "not important". Regarding the dimension of humanistic orientation, "social justice" was recognized by $88.9 \%$ of respondents as important and $4.8 \%$ as not important (not mentioned). With respect to the construct fellow human beings, the scoring is as follows: "respect for fellow human beings" $82.5 \%$ recognized this as "important" and $11.1 \%$ as not. The item of "readiness to help people in need" was recognized as "important" by most of the respondents (90.5\% versus 3.2\%). Regarding the dimension of unity with nature, the item of "enjoying the world and life" was recognized as "important" by most respondents $(88.9 \%)$ versus $4.8 \%$ who do not. Finally, "environmental protection" was recognized as "important" by most of the respondents $(82.5 \%$ versus $11.1 \%)$. 
Table 25: Mentioned importance of values (second measurement scale)

\begin{tabular}{|l|r|r|r|}
\hline \multicolumn{2}{|c|}{ Important } & Not important & \multicolumn{1}{c|}{$\%$} \\
\hline Self-direction: & & & \\
\hline Self-fulfillment and self-development & 82.5 & 11.1 & 93.7 \\
\hline A job which fulfils me & 79.4 & 14.3 & 93.7 \\
\hline Universalis m: & & & \\
\hline Benevolence: & & & \\
\hline Social justice & 88.9 & 4.8 & 93.7 \\
\hline Respect for fellow human beings & 82.5 & 11.1 & 93.7 \\
\hline Readiness to help people in need & 90.5 & 3.2 & 93.7 \\
\hline Unity with nature: & & & \\
\hline Enjoying the world and life & 88.9 & 4.8 & 93.7 \\
\hline Environmental protection & 82.5 & 11.1 & 93.7 \\
\hline
\end{tabular}

Source: Own estimation (2009).

\subsection{Secondary conclusions: motivations are satisfactory and values are not all satisfactory}

To summarize, the findings of the second pretest in Germany indicate more variation among the responses regarding the multi-item scale of motives to purchase food products. Regarding values, these had been tested with a twofold measurement: on the one hand a 7-point Likerttype scale was used; and on the other hand, a dichotomous variable-important/not importantwas employed to detect the level of importance of values of respondents. However, since most responses were distributed on the left side thus showing a skewed distribution (see Table 24 and Table 25). The author is obliged to regard the findings related to values as not yet satisfactory. This can be partially explained by the following:

Due to semantics, respondents may interpret the meaning of items differently to the meaning originally assigned. Thus a second rewording is necessary.

Probably a cultural bias occurred in the process of adaptation of the Anglo-Saxon language and cultural context into the Spanish language and adaptation to the Mexican national culture. Again, a second rewording is necessary to determine cultural differences between German and Mexican consumers. 
This is confirmed by the literature and is in line with Hofstede (2001): "we can use questionnaires measuring constructs such as beliefs, attitudes, and personality to infer values". Similarly to Hofstede's approach, the measurement of attitudes is not the primary purpose of our study; on the contrary, values will be inferred by asking about attitudes and beliefs. Thus, in order to reword the items related to values, consideration was given to the difference between desired and desirable values stated by Hofstede (2001) as well as including the previous findings of the two pretests conducted in Mexico and Germany.

Thus, after a second evaluation, the constructs of the dimensions of values were refined and the items were reworded. The changes in the dimension of values are provided in Table 26. In the following section, the second improvement toward developing the final version of the cross-cultural survey is shows.

\subsection{Final, optimised version of the cross-cultural survey}

A second evaluation addressed the level of theoretical constructs, survey design, and wording. Regarding the survey design, adjustments at the level of conceptual constructs, measurement scale and length were made. In order to improve variation at the question level, rewording at the semantic level of items were performed in order to reduce respondents' difficulty in interpreting them. Regarding survey design, items respecting information about organic food purchase and nutritional behavior were included. As already shows, items referring to motivation to purchase food products were used to infer differences between buyers and nonbuyers of organic food. Items related to attitudes/beliefs were added. In this way we could infer values to provide cultural differences. As mentioned, because of the usefulness of the online survey in reducing the problem of social desirability of responses, data were simultaneously collected in the two countries. For all items, the measurement scale based on a 7-point Likert-type scale was introduced as follows: (-3) strongly disagree; (-2) disagree; (-1) somewhat disagree; (0) neither agree nor disagree; (1) somewhat agree; (2) agree; and (3) strongly agree. All adjustments in the theoretical constructs on motivations and values are shows in Table 26 and are described in the following: 
The theoretical constructs of the motives related to "Animal welfare" on the one hand and "Environmental concern" on the other hand was adjusted to 1) animal welfare and information, and 2) environmental concern and information. The justification for this procedure is the following: some consumers may be concerned about animal welfare and/or environmental issues. However, as long as this information is not available or shows on a label of the product packaging it's difficult for the researchers to discern any environmental or animal welfare motives behind the purchase of organic food. "Weight control", "Natural content" and "Nostalgia" remained the same, whereas sensory variables were replaced by an item designated as "Appearance", "Healthy eating" and "Free of GMO". "Shopping convenience", "Cooking convenience”, and "Fair price” were kept with slight adjustments; familiarity and advertising were separated as well as "Price orientation" and "Price-quality relation"; "Cooking involvement” was eliminated, because its measurement in Mexico was considered uncertain. Finally, "Consumer ethnocentrism" was retained, and one item regarding "Malinchismo of food" was included in the measurement of the Mexican sample.

Regarding values, the items included in the dimension of "Hedonism" were adjusted slightly, stimulation was replaced by "Variety in life/neo-phobia" and became a dimension; materialism/status consumption were added instead of security. "Self-direction" was removed. The two dimensions of universalism previously mentioned as humanistic orientation were refined as "Fairness", and unity with nature was refined as "Fatalism". Tradition and power/achievement were deleted, and the construct "Ethnocentrism/malinchismo" was included for measuring values, as they are considered as guidelines of behavior in everyday life. 
Table 26: Changes in the theoretical constructs of motivations and values driving the choice of organic food

\begin{tabular}{|c|c|c|c|c|c|}
\hline \multicolumn{3}{|c|}{ Second study: pre-test in Germany } & \multicolumn{3}{|c|}{ Cross-cultural survey } \\
\hline Motivations to purchase food products: It & Item & Source: & Motivations to purchase organic food: & Item & Source: \\
\hline Animal welfare & & Author & Animal welfare/information & & Author \\
\hline Environmental concern & 3 & Adapted from Steptoe et al, 1995; Lindeman and Väänänen, 2000; Author & Environmental concern/information & 3 & Lindeman and Väänänen, 2000; author. \\
\hline Information & & Adapted from Grunert et al., 1993; author. & Information & 3 & Adapted from Grunert et al., 1993; author \\
\hline Weight control & $3 \mid$ & Adapted from Steptoe et al., 1995. & Weight control & 3 & Adapted from Steptoe et al, 1995; author. \\
\hline Sensory variables & $3 \mid$ & Adapted from Steptoe et al., 1995. & Sensory variables & $(-\cdots)$ & $(--)$ \\
\hline Healthy eating & & Adapted from Grunert et al., 1993; Steptoe et al., 1995; author. & Healthy eating & 2 & Adapted from Steptoe et al, 1995; author. \\
\hline Natural content & & Adapted from Grunert et al., 1993; Steptoe et al., 1995; author. & Natural content & & Adapted from Grunert et al., 1993; Steptoe et al, 1995; author. \\
\hline Free of GM0 & & Author & Free of GMO & 2. & Author \\
\hline \multirow[t]{2}{*}{ Familiarity/Advertising } & & Steptoe et al, 1995; author. & Familiarity/Neo-phobia & 3. & Adapted from Steptoe et al, 1995 ; author. \\
\hline & & & Advertising & 2 & Adapted from Grunert et al., 1993; author. \\
\hline Shopping convenience & & Adapted from Grunert et al., 1993; Steptoe et al., 1995; author. & Shopping convenience & 3. & Adapted from Grunert et al., 1993; Steptoe et al, 1995; author. \\
\hline Cooking convenience & 2 & Adapted from Steptoe et al., 1995. & Cooking convenience & 2. & Adapted from Steptoe et al, 1995. \\
\hline Cooking involvement & & Author & $(--\cdot)$ & $(-\cdots)$ & $(--)$ \\
\hline Fair price & $3 \mid$ & Author & Fair price & 2 & Author \\
\hline Nostalgia & $3 \mid$ & Author & Nostalgia & 3 & Author \\
\hline \multirow[t]{2}{*}{ Price Orientation/Price Quality Relationship } & 3 & Adapted from Grunert et al., 1993; author. & Price-quality relationship & 2 & Adapted from Grunert et al., 1993; author \\
\hline & & & Price orientation & 3 & Adapted from Grunert et al., 1993; author \\
\hline \multirow[t]{3}{*}{ Consumer ethnocentrism } & & Author & Consumer ethnocentrism & 3 & Author \\
\hline & & & Malinchismo of food & & Author \\
\hline & & & Appereance & & Author \\
\hline \multicolumn{3}{|l|}{ Dimensions of Values: } & \multicolumn{3}{|l|}{ Dimensions of Values: } \\
\hline Hedonism & $1 \mid A$ & Adapted from Schwartz, 1992. & Hedonism & & Author \\
\hline Stimulation & 2 & Adapted from Grunert and Scherhorn, 1990; Schwartz, 1992 & $(---)$ & & \\
\hline Security & $3 \mid$ & Adapted from Grunert and Scherhorn, 1990; Schwartz, 1992. & $(--)$ & & \\
\hline Self-direction & & Adapted from Grunert and Scherhorn, 1990; Schwartz, 1992. & $(---)$ & & \\
\hline \multicolumn{6}{|l|}{ Universalism: } \\
\hline 1. Fellow humans oriented & $3 \mid$ & Adapted from Schwartz, 1992. & $(---)$ & & \\
\hline 2. Unity with nature & 2 & Adapted from Schwartz, 1992. & $(-\cdots)$ & & \\
\hline Tradition & $1 \mid$ & Adapted from Schwartz, 1992. & $(---)$ & & \\
\hline \multirow[t]{8}{*}{ Power/achivement } & 3 & Adapted from Grunert and Scherhorn, 1990; Schwartz, 1992. & $(--\cdot)$ & & \\
\hline & & & Technological progress/Techno-phobia & 3. & Author \\
\hline & & & Fatalism & & Author \\
\hline & & & Fairness & & Author \\
\hline & & & Materialism & 5 & Author \\
\hline & & & Variety in life/neo phobia & 2 & Author \\
\hline & & & Ethnocentrism & 3. & Author \\
\hline & & & Malinchismo & 1 & Author \\
\hline
\end{tabular}




\section{$7 \quad$ Main study: Results of the online surveys in Germany and Mexico}

After the final version of the cross cultural survey was designed. The data was online collected in both countries. In this chapter the main results are discussed. Firstly, the frequency of the answers and the mean average was compared. After that, exploratory factor analyses were loaded to reduce the number of items in order to build a scale to measure the motivations and values. Finally, to explain the motives and values in the purchasing of organic food a multiple lineal regression analysis was done.

\subsection{Sampling procedure and participants}

In order to develop a cross cultural comparison, consumers of organic food and non-buyers in Germany and Mexico were surveyed to determine the differences in their purchasing motives. An online data collection (poll) of a total of 5,852 respondents in Germany and Mexico $(100 \%)$ was undertaken by Unipark Company using a panel sampling approach. Surveys that were not completed were excluded from the data analysis. In Germany 501 samples of the survey were completed. The same procedure was employed in Mexico where a poll of 2,422 people was undertaken; 507 surveys were completed and analyzed. Therefore, the total sample of this survey consists of 1,008 respondents. To match the samples, the functional equivalence approach proposed by Hofstede (2001) was used, thus the German sample consists of 501 respondents; to match the sample, 269 are consumers of organic food $(26.8 \%)$ and $232(23 \%)$ are non-buyers. The Mexican sample consists of 507 respondents, 263 $(25.6 \%)$ of which are consumers of organic food and 250 are non-buyers $(24.4 \%)$.

All in all, the response range in Germany was $12 \%$ and $20 \%$ in Mexico, respectively. Furthermore, a filter question was asked regarding the respondent's responsibility or participation in the purchase of food products within the household, thus those who did not match these criteria were excluded. It was found that women were responsible for the purchase of food and nutrition in the household in both countries: 350 women and 150 men were required to quote the sample in each country, respectively. Among the respondents, vegetarians who self reported abstaining from eating meat or meat products consisted of $2.8 \%$ 
of the German sample of which $2.2 \%$ are consumers of organic food. This mean not all vegetarians purchase organic food. Within the Mexican sample, all the vegetarians (3.4\%) are also consumers of organic food.

In demographic terms, the German sample of respondents who matched the survey criteria were located in 16 federal states: $22.7 \%$ from Nord Rhine-Westphalia, 14.7\% from Bavaria, $11.2 \%$ from Baden Württemberg and the remaining from the other 13 federal states; the percentage match to the sample represents less than $10 \%$ of each federal state, respectively.

However, because of the seed stage of the domestic market described in the previous chapter, most of the retail channels of organic food are in Mexico City, thus 282 respondents (55.6\%) are from that city and 225 respondents $(44.4 \%)$ are from other cities. This is a result of the fact that in several other cities organic food is not sold, especially in the North and South of the country. The data were collected during the last week of August and the first week of September in 2009.

\subsection{Some remarks of measurement and methods}

For all items, attitudes regarding organic food by consumers and non-buyers were required previously to their motivations to purchase; after that, the set of items about values was requested. The question about attitudes toward organic food was: "In relation to organic produce, which of the following do you agree with?" To find out the differences in motives to purchase food products, respondents were requested to answer the following: "Considering the criteria that you use normally to purchase food products, how do you agree with the following statements?" Finally, regarding the section on values, the question was: "In the following there are several attitudes about some current issues, which do you agree with?" In all cases the measurement scale used was based on a 7-point Likert-type scale which was introduced as follows: (-3) strongly disagree; (-2) disagree; (-1) somewhat disagree; (0) neither agree nor disagree; (1) somewhat agree; (2) agree; and (3) strongly agree.

The total sample was analyzed with the statistical program SPSS version 17.00. First, the univariate methods were used, i.e. one-way tabulation, to observe the variability of the statement responses and the elimination of the missing items, i.e. data cleaning, to observe 
cumulative frequency, mean value, standard deviation, and variance between the German group and the Mexican group.

To determine the differences between countries, the average value priorities of individual members of a society are compared with the average value priorities of the other society. Thus, the average value priorities (mean value) of the group of non-buyers in Germany and Mexico are compared as well as the average value priorities of consumers of organic food. Factor analysis is of one of the most common methods of determining the dimension of crosscultural comparison, and at the same time, it is also a method of data reduction. Thus, after the comparison of the average value priorities, a factor analysis was done to reduce the dimension of motivations and values of the German sample and the Mexican sample. The factors were then compared.

The results are described in the following. Firstly, the sample is described in demographic terms from the most frequent responses and for each country as well. After that, the average value priorities (mean average) are compared; finally, the factors arrived at for each country was also compared.

\subsection{Demographic characteristics of the samples}

In continuation, the demographic characteristics such as gender, age group, education, household income, household size, and family lifecycle of the German and Mexican subsamples are described below:

\subsubsection{Gender}

The total sample consists of 681 female (68.6\%) and 317 male (31.4\%). Within the German sample: 350 female (69.7\%) and 152 male (30.3\%) from the sixteen federal states participates 
in the study. The Mexican sample consist of 342 female (67.4\%) and 165 male (32.5\%) (see Figure 12 below).

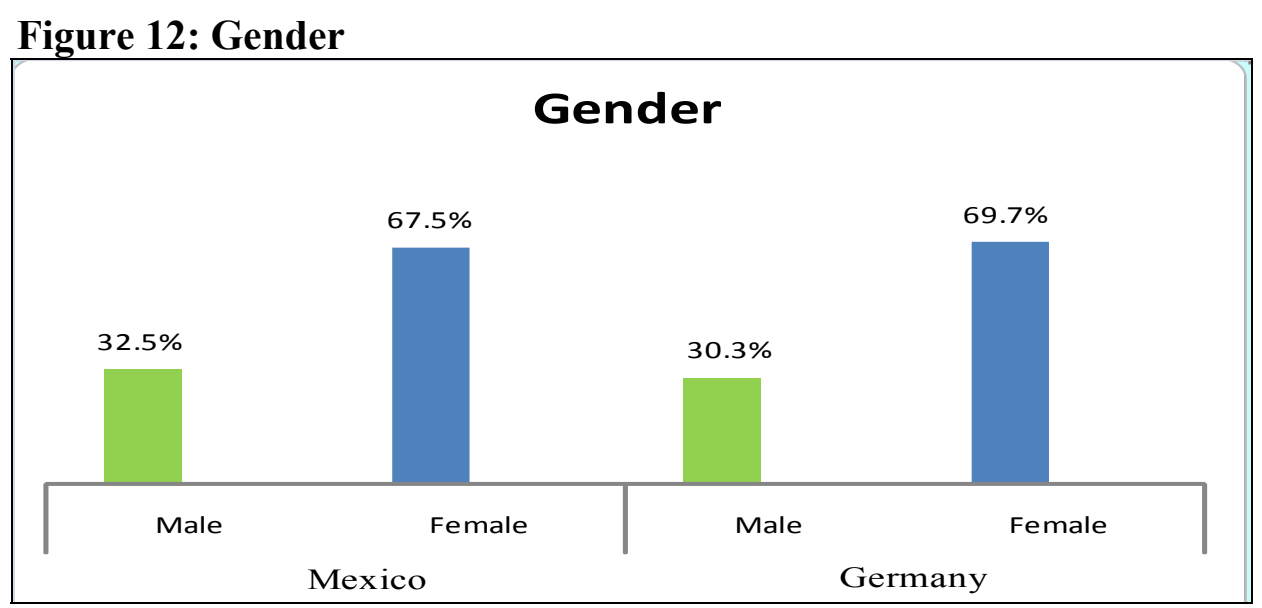

Source: Own estimation (2009).

Most of the time, the female gender is mentioned as the characteristic demographic profile of consumers of organic food (Davies et al., 1995; Chinnici et al., 2002; Fotopoulos \& Krystallis, 2002). Some scholars regard the consumption of organic food as an ethical buying behavior, thus with this viewpoint in mind, females tend to buy more organic food than men. However, an explanation of this is that in the majority of Western countries females are responsible for household expenditures including food consumables and preparation. Thus, females are more concerned with nutritional issues, product attributes, brands, prices, and convenience of food products.

\subsubsection{Age groups}

Age groups of the total sample shown in Figure 13 are described as follows: most of the respondents are younger adults as 30\% of the respondents are between 20 and 30 years of age. The second main group consists of respondents between 30 and 40 years of age $(25 \%)$; the third group consists of respondents between 40 and 50 years of age (22\%); the fourth group consists of $16 \%$ of the respondents, and the smallest group consists of the youngest respondents (between 18 and 21 years old) (6\%). 
Hence, each one of these descriptions corresponds with the demographic characteristics of each country; thus, the age groups of the German sample is the following: the largest group corresponds to respondents between 40 and 50 years of age (27\%); the second group consists of respondents of more than 50 years of age $(26 \%)$; the third group consists of respondents between 21 and 30 years of age; the group of respondents between 31 and 40 years of age is slightly smaller (20\%); and the smallest group is the youngest consumers who are between 18 and 21 years of age. In contrast, among the Mexican sample the largest age group consists of respondents between 21 and 30 years old (40\%) followed by the group of respondents between 31 and 40 years (31\%); 17\% between 41 and 50 years old; youngest respondents (between 18 and 21 years of age) represent $17 \%$ and slightly smaller is the group of respondents of more than 50 years of age $(6 \%)$. These differences correspond to the population pyramid for each country.

\section{Figure 13: Age groups}

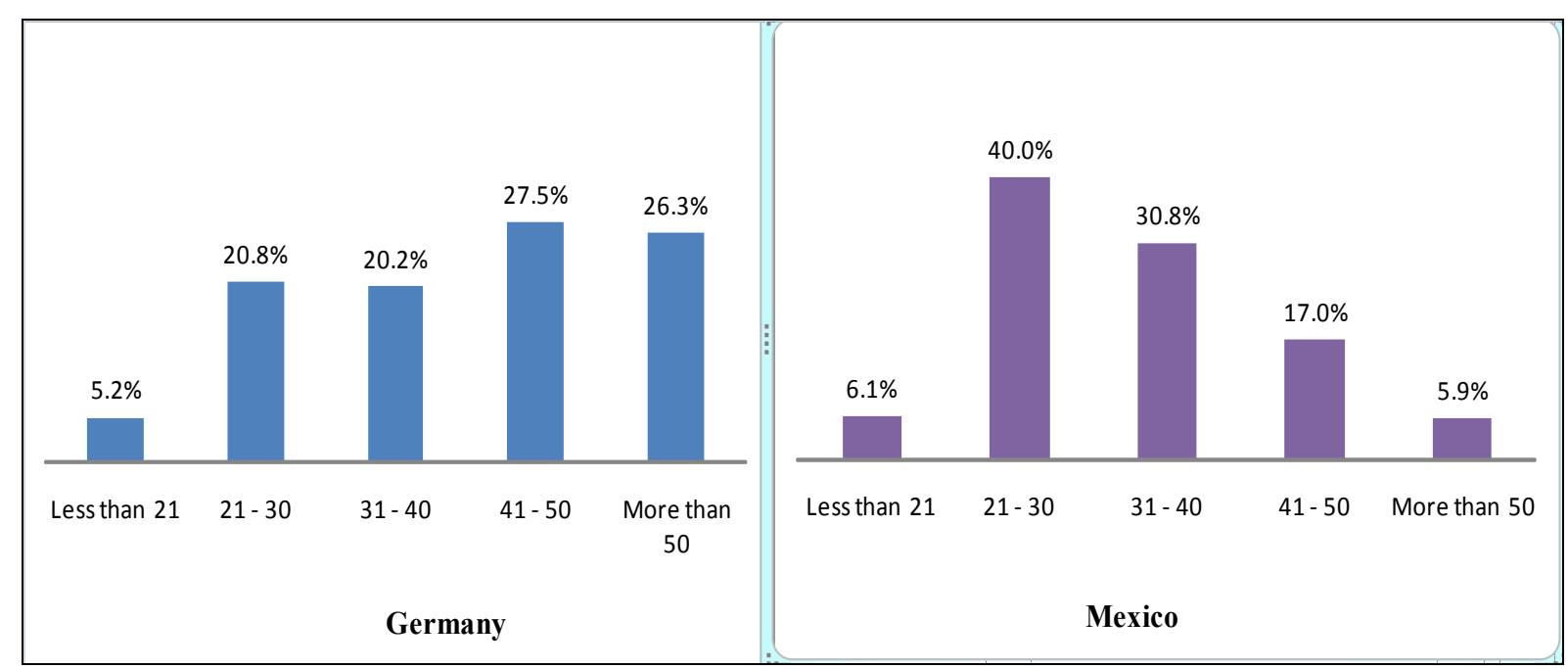

Source: Own estimation (2009).

Several studies regard age groups also as a demographic feature in the profiling of organic food consumers in other countries. According to Lindeman and Väänänen (2000), age plays a role in the consumption of products in relation to ethical values. However, most studies on organic food buying behavior do not regard differences in age among consumers of organic food as important. For instance, no apparent differences among the age groups were found in 
England in 1995 by Davies et al. whereas in Italy in 2002, Chinnici et al. observed slight differences.

Age is considered playing threefold role: firstly, age is linked to the family lifecycle and gender; secondly, age is also linked with the system of values at two levels of the individual, i.e. personality and cultural level. Thirdly, age is also linked with the national demographic population factor. For instances in 2008, the median age in Germany is 43 , whereas in Mexico it is 26 (Kinsella \& He, 2009) this is represented among or the participants in the study.

\subsubsection{Education}

Because of the German and Mexican national education systems, there are also differences among the samples regarding the variable of education. Thus, the most important sub-groups of the total sample are described as follows: $43 \%$ are university graduates, $23 \%$ are intermediate school graduates, and $21 \%$ are elementary school graduates. Hence, as shown in Figure 14, most of the respondents in the German sample have a basic education (43\%); $5 \%$ have advanced vocational certification; $21 \%$ are A-level7, $18 \%$ are university graduates, $10 \%$ are $\mathrm{PhDs}, 2 \%$ are still studying and $1 \%$ are no graduated.

\footnotetext{
${ }^{7}$ A-level is an intermediate school level provided by the German Education System.
} 


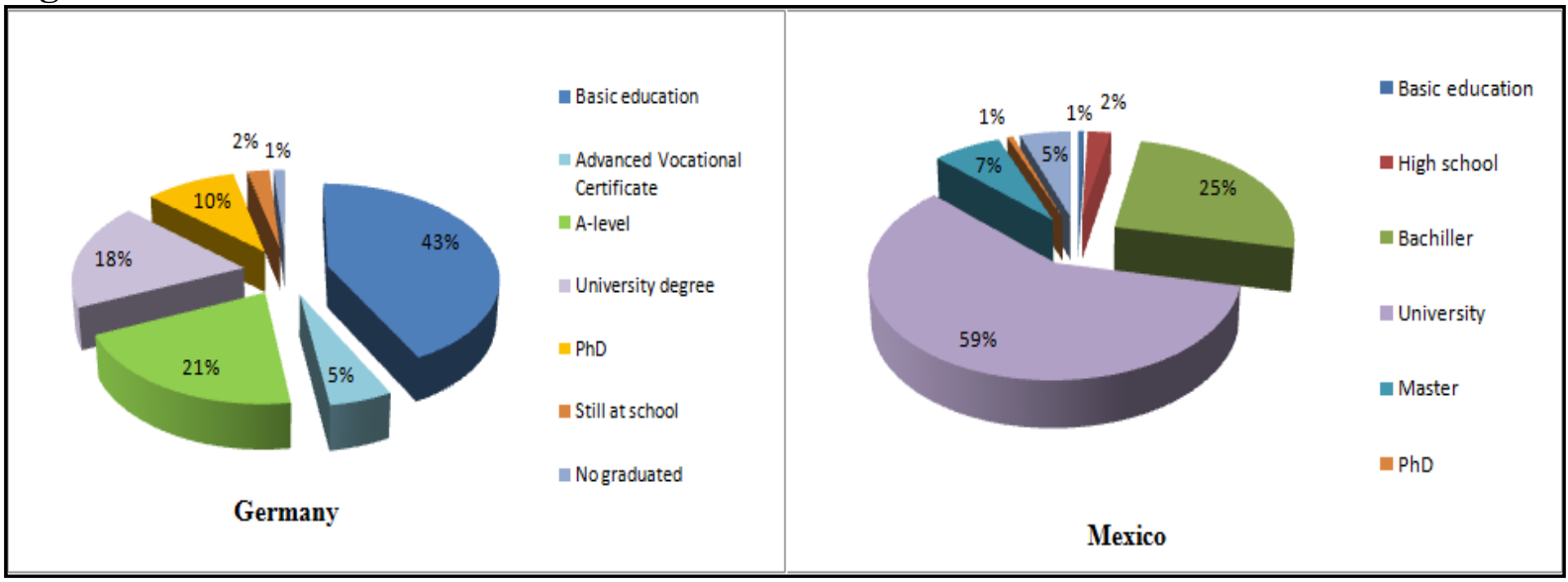

Source: Own estimation (2009).

Among the Mexican sample most of the respondents are university graduates (59\%); 1\% are basic school and $\mathrm{PhD}$ graduates, respectively; 2\% are high school graduates; $25 \%$ are intermediate school graduates; $7 \%$ are master graduates; $5 \%$ are technical/vocational graduates and $0.2 \%$ correspond to the missing values.

\subsubsection{Household income}

Concerning the total monthly household income, there are also differences because of the social structure between the two countries as shown in Figure 15. There are six household income bands for the German sample: $14.8 \%$ of the respondents belong to the lower income band (less than $900.00 €$ ); there is also a similar percentage of respondents who did not provide an answer; $20.6 \%$ of the respondents belong to the slightly lower income band (between 900.00 and $1499.00 €$ ); the other $15.6 \%$ of the respondents belong to the middle income band (between 1500.00 and $1999.00 €$ ); the higher percentage of $22.6 \%$ consists of respondents belonging to the higher middle income band (between 2,000.00 to 3,199.00 €); $9 \%$ of the respondents belong to the first higher income band (between 3,200.00 and 4,499.00 $€)$; finally, the lower percentage of $2.8 \%$ consists of respondents belonging to the highest income band (with more than 4,500.00€). 
Figure 15: Total monthly household incomes

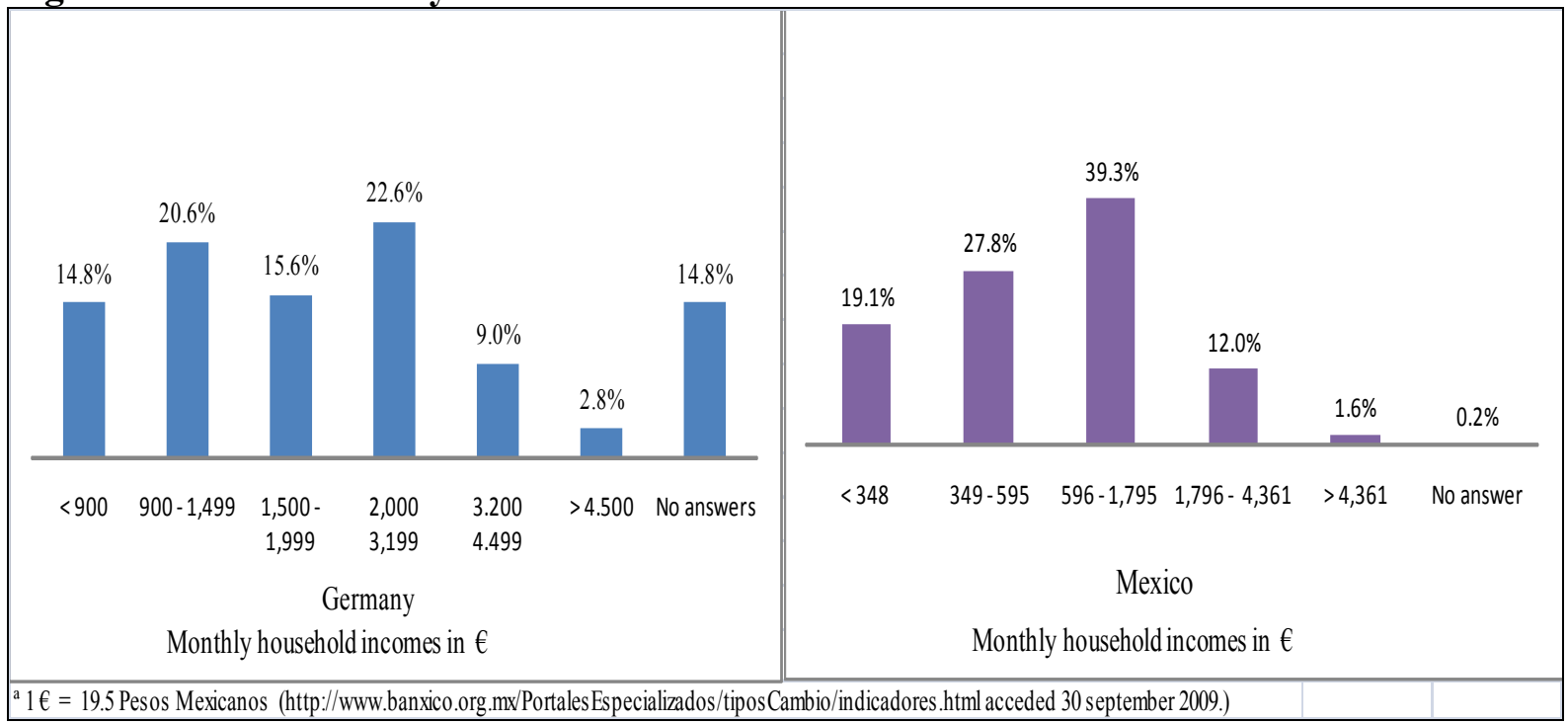

Source: Own estimation (2009).

Regarding the Mexican sample, there are five household income bands; however, similar, to the German sample is the fact that the lowest percentage of respondents belong to the lower income band and the higher income bands. Hence, differences in variability of the respondents pertains to the middle income bands; that is: $19.1 \%$ of the respondents belong to the lower income band (less than $348.00 €$ monthly); $27.8 \%$ belong to the second lower income band (between 349 and $595.00 €$ ); and the highest percentage (39.3\%) consists of respondents belonging to the lower middle income band (between 596.00 and 1,795.00 €); the lowest percentage $(12 \%)$ of respondents belong to the higher middle income band (between $1,796.00$ to $4,361 €$ ); and $1.6 \%$ belong to the highest income band (more than $4,361.00 €$ ); finally, respondents who did not provide an answer represent $0.2 \%$.

In previous studies when a direct relationship between household income and consumption of organic food was tested at various income bands, no one significant factor was found (Davies et al., 1995), thus, a "non" relationship between household income and consumption of organic food was suggested as well as the fact that consumption remains stable over time. 


\subsubsection{Family lifecycle}

The family lifecycle variable is related to age, and sometimes is linked with national culture as well. In general terms, the sample consists of families, as most of the respondents' lifestyles were as a couple with children (35.8\%). This high percentage corresponds to both samples; whereas $17.5 \%$ of the respondents live with parents; $14.5 \%$ live as a couple without children (children have moved).

The German sample is described as follows: $18 \%$ consist of individuals who live alone; $1 \%$ consists of individuals who live alone because the children have moved as well as individuals who live with children, respectively. $30 \%$ are families of couples with children; $22 \%$ are couples without children; $6 \%$ consists of couples without children (the children have moved); $9 \%$ are single parents, $4 \%$ share apartment; $9 \%$ live with parents (see Figure 16 ).

Figure 16: Family lifecycle

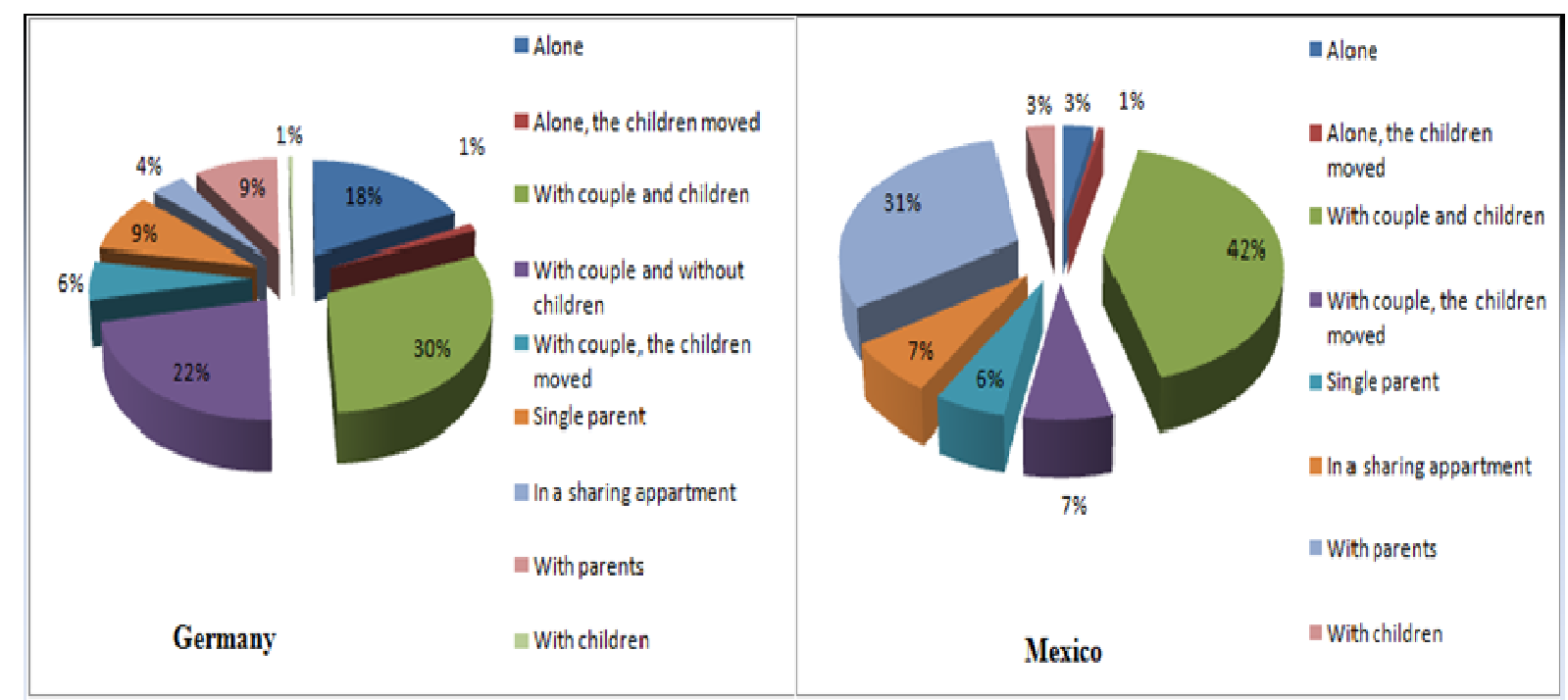

Source: Own estimation (2009).

The Mexican sample is described in the following. The percentages of individuals who live alone consist of $3 \%$ as well as the individuals who live with children, respectively; $1 \%$ consists of individuals who live alone because the children have moved; $42 \%$ are familiesindividuals living as a couple with children. The Mexican sample does not deal with the category of individuals who live as a couple without children; $7 \%$ consists of individuals who 
live as a couple because the children have moved and individuals who share apartments, respectively; $6 \%$ consists of single parents, $31 \%$ are individuals who live with parents (Figure 16).

Among the findings comparing the family life cycle, it is possible to determine differences regarding the social structure of the two countries as well as cultural differences regarding the concept of collectivist and individualist societies widely studied by Hofstede (2001) who describes the German national cultural society as individualistic, whereas the Mexican national cultural society is collectivistic. The concept of family in the two countries being compared is also different, as the percentages in the Mexican sample of people living alone and single parents are lower than in the German sample. In contrast, the Mexican sample indicates that the percentage of individuals who live with parents, with children and sharing apartment is higher than in the German sample.

All in all, demographic characteristics of the sample reflect differences in the social systems between the two countries. However, these differences might function at different levels including the cultural level.

\subsection{Comparison of the frequency of motives in the purchase of food products between the German and Mexican sample}

After the description of the demographic characteristics of the German and Mexican samples, the dimensions were found to be statistically distinct in the two countries. The dimension of "Appearance" dropped from the analysis because it was a single-item scale and is was negative skewed in both countries. The dimension of "Fair price" consisting of two items was also negative skewed among the Mexican sample. The social desirability among the responses provided is a reason explaining this fact. Consequently, in order to avoid cultural bias in further analysis to establish the comparison, both dimensions were also dropped.

In the following are describes the more interesting relative frequency of responses to the statements of motivations and values. Firstly, the responses by the German group are provided followed by the Mexican group. 


\subsubsection{Animal welfare/information}

\section{German sample:}

The statement: "Regarding dairy and meat products, it is difficult to know whether the animals have been treated well" was used on a single item scale to measure the dimension of animal welfare. Figure 17 displays responses to the above statement in the German sample. They are: 1.2 "totally disagree"; $0.8 \%$ "disagree"; $1.6 \%$ "somewhat disagree"; $21.4 \%$ "neither agree nor disagree"; $18.2 \%$ "somewhat agree"; 26.5\% "agree"; and 30.3\% "totally agree". The concentration of responses in the "zero-to-three range" indicates a clear concern of Germans for the welfare of animals.

\section{Figure 17: Dimension of animal welfare/information in the German sample}

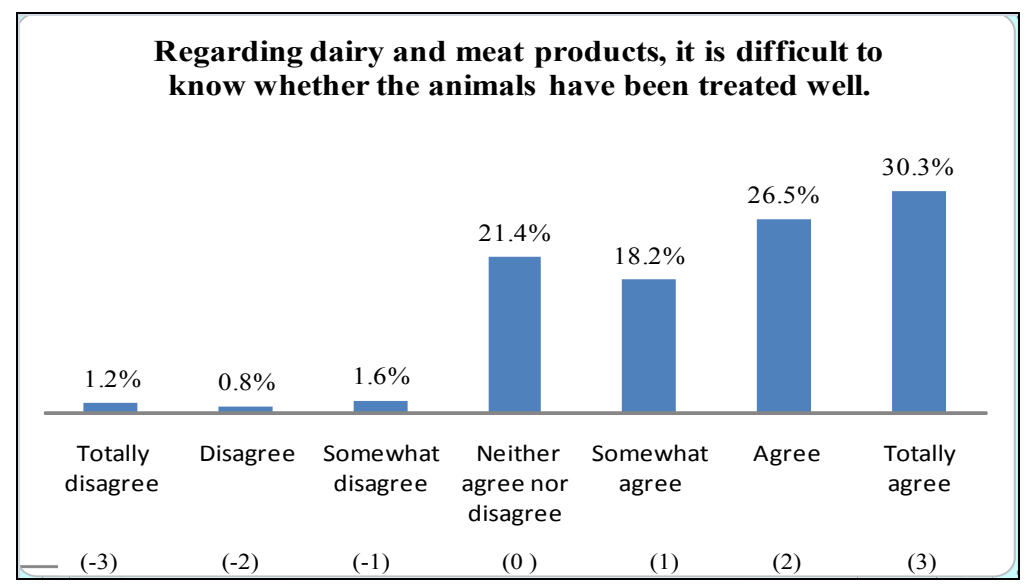

Source: Own estimation (2009).

\section{Mexican sample:}

Figure 18 shows the responses in the Mexican sample. The percentage of responses provided for each category are: $1.8 \%$ "totally disagree"; $1.4 \%$ "disagree"; $2.2 \%$ "somewhat disagree"; $10.3 \%$ "neither agree nor disagree"; $15.6 \%$ "somewhat agree"; $28.2 \%$ "agree"; and 40.6\% "totally agree". Also in the Mexican sample responses are concentrated in the "zero-to-three range" what demonstrates a similar concern as Germans for the welfare of animals. 


\section{Figure 18: Dimension of animal welfare/information in the Mexican sample}

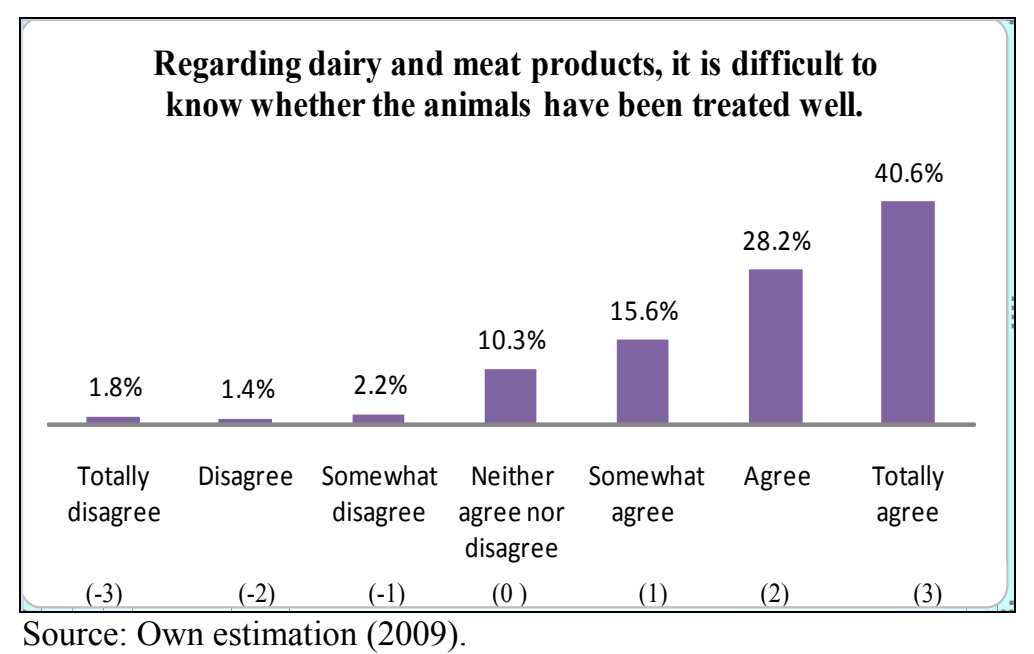

\subsubsection{Environmental concern/information}

\section{German sample:}

The frequency of responses to the statement: "It is difficult to know if the production of food has a negative impact on the environment" used in the measurement of the dimension of environmental concern is displayed in Figure 19 described in the following: $0.8 \%$ "totally disagree" and "disagree" respectively; 1.8\% "somewhat disagree"; 25.7\% "neither agree nor disagree"; $21.2 \%$ "somewhat agree"; $27.1 \%$ "agree"; and 22.6\% "totally agree". Hence, as for the former statement the majority of responses are concentrated in the "zero-to-three range" what demonstrates certain skepticism among the German population toward the production of food in terms of environmental pollution.

The frequency of responses to the statement: "I am careful to buy food products that have been produced in an environmentally friendly way". In Figure 19 are described as follows: 6.8\% "totally disagree"; 5.6\% "disagree"; 15.6\% "somewhat disagree"; 35.9\% "neither agree nor disagree"; $16.6 \%$ "somewhat agree"; $12.4 \%$ "agree"; and 7.2\% "totally agree". It is interesting to notice that "only" $36 \%$ of Germans somewhat-to-totally agree to this statement. This shows that the nurturing of environmental friendly behavioral patterns by an accurate selection of food products is pursuit by only a minority of Germans. 
Figure 19: Dimension of environmental concern/information in the German sample

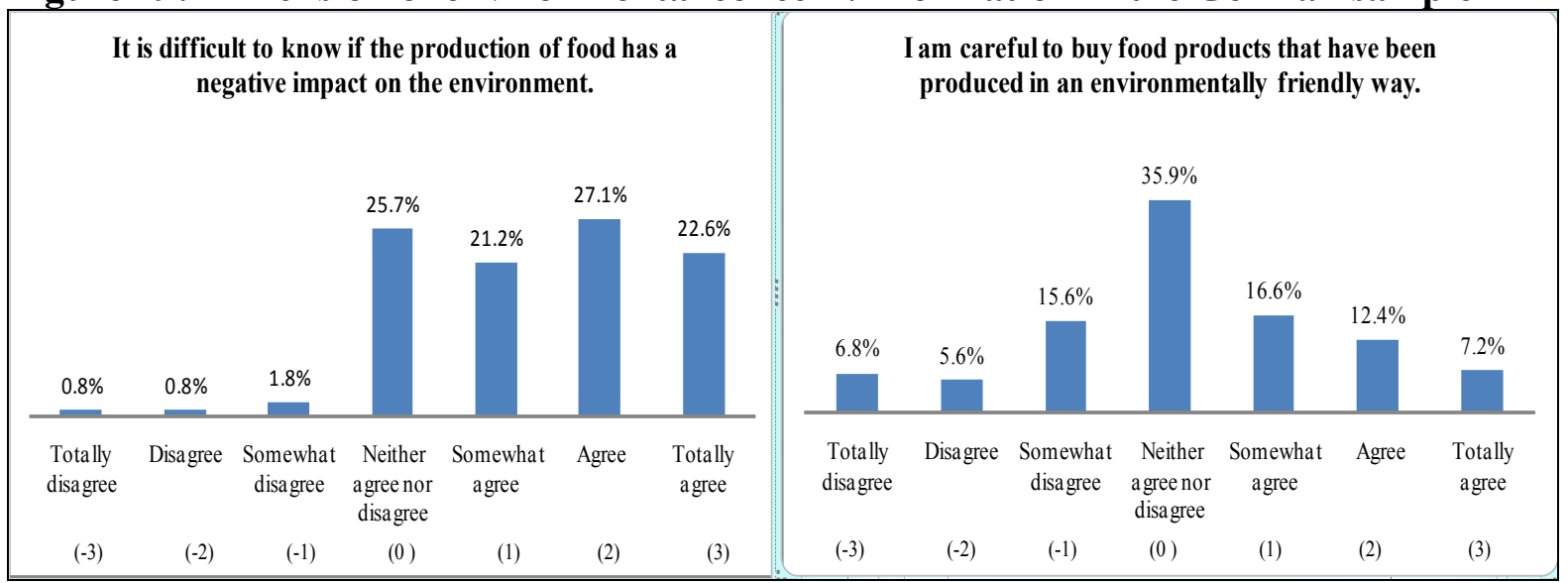

Source: Own estimation (2009).

\section{Mexican sample:}

Figure 20 displays the frequency of responses provided to the statement: "It is difficult to know if the production of food has a negative impact on the environment". Which is described in the following: $4.3 \%$ "totally disagree"; $5.5 \%$ "disagree"; $9.1 \%$ "somewhat disagree"; 14.4\% "neither agree nor disagree"; 20.3\% "somewhat agree"; 25.8\% "agree"; and $20.5 \%$ "totally agree," respectively. Here, the majority of responses are still concentrated in the "zero-to-three range" what demonstrates a similar skepticism among the Mexican population toward the production of food in terms of environmental pollution. However, in comparison with the German sample there is a higher number of responses also in the area "totally disagree to somewhat disagree" what could indicate a lower concern among Mexicans for environmental issues.

The frequency of responses provided to the statement: "I am careful to buy food products that have been produced in an environmentally friendly way". Are the following: $1.4 \%$ "totally disagree"; $2.6 \%$ "disagree"; $5.9 \%$ "somewhat disagree"; $23.1 \%$ "neither agree nor disagree"; $25.2 \%$ "somewhat agree"; similarly, 25\% "agree"; and 16.8\% "totally agree". In comparison to the German sample the majority of Mexicans somewhat-to-totally agree to this statement (66\%). This can be interpreted in twofold ways: either Mexicans show a higher concern for the environmentally friendly and they translate it into purchase of food products, or, we are facing a problem of "social desirability". The latter means that it is plausible to retain those Mexican respondents felt "forced" to answer in a socially desired manner. 
Figure 20: Dimension of environmental concern/information in the Mexican sample

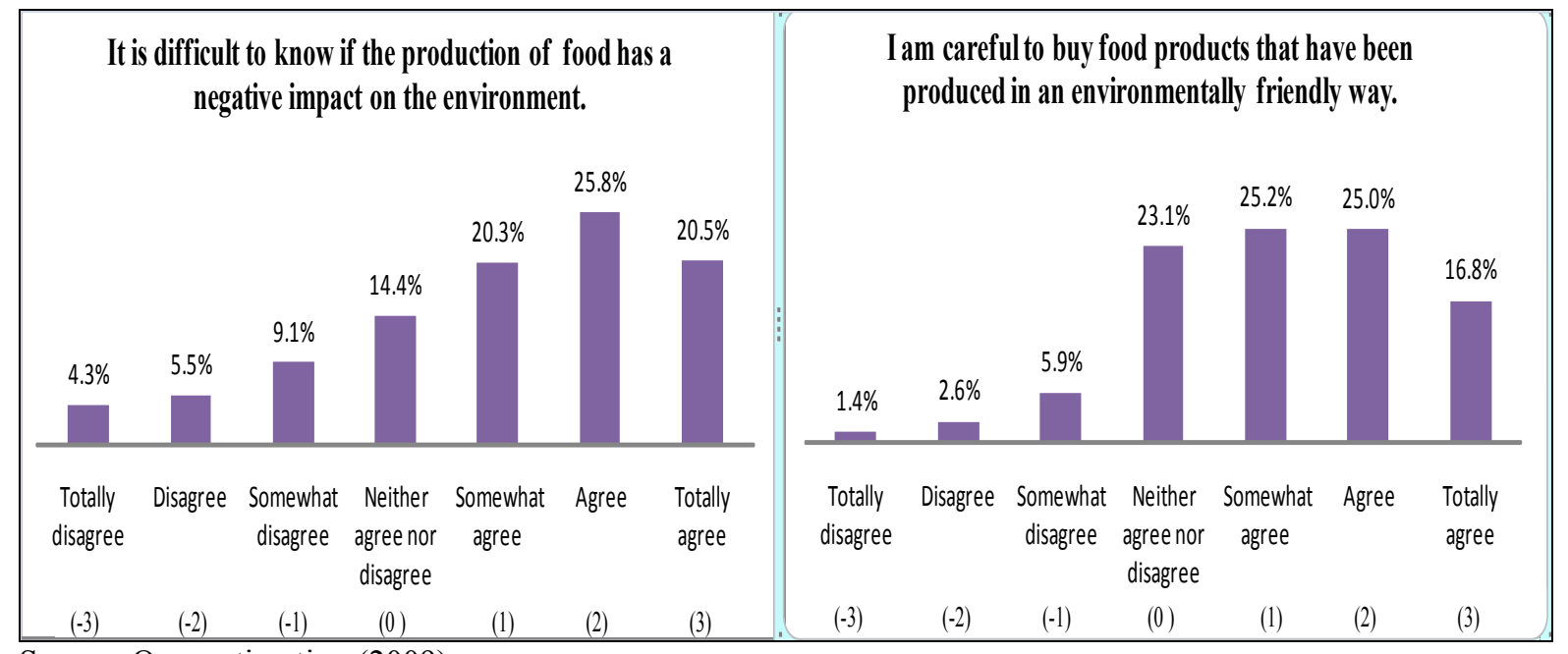

Source: Own estimation (2009).

\subsubsection{Information}

\section{German sample:}

In measuring the dimension of information, the statement: "I would like more information to be provided on the packaging" was used. Figure 21 displays the following: $2.6 \%$ "totally disagree"; similarly, 3\% "disagree"; 6.6\% "somewhat disagree"; 28.9\% "neither agree nor disagree"; 19\% "somewhat agree"; 19.4\%"agree"; and 20.6\% "totally agree". The majority of responses are concentrated in the "one-to-three range" what voices a certain desire among Germans for being provided with information concerning food products.

The frequency of responses to the statement: "Before buying food, I carefully read the information on the package" displayed in Figure 21 are the following: $7.6 \%$ "totally disagree"; similarly, 7.4\% "disagree"; 16.4\% "somewhat disagree"; the highest percentage (29.9\%) "neither agree nor disagree"; 18.8\% "somewhat agree"; 11.6\% "agree", and 8.4\% "totally agree". Hence, this statement divides the German population in (about) three thirds, one third shows no interest in the product information provided in the packaging, one third show low commitment regarding this issue. Finally, one third of the German population believes that product information on the package is important. 


\section{Figure 21: Dimension of information in the German sample}

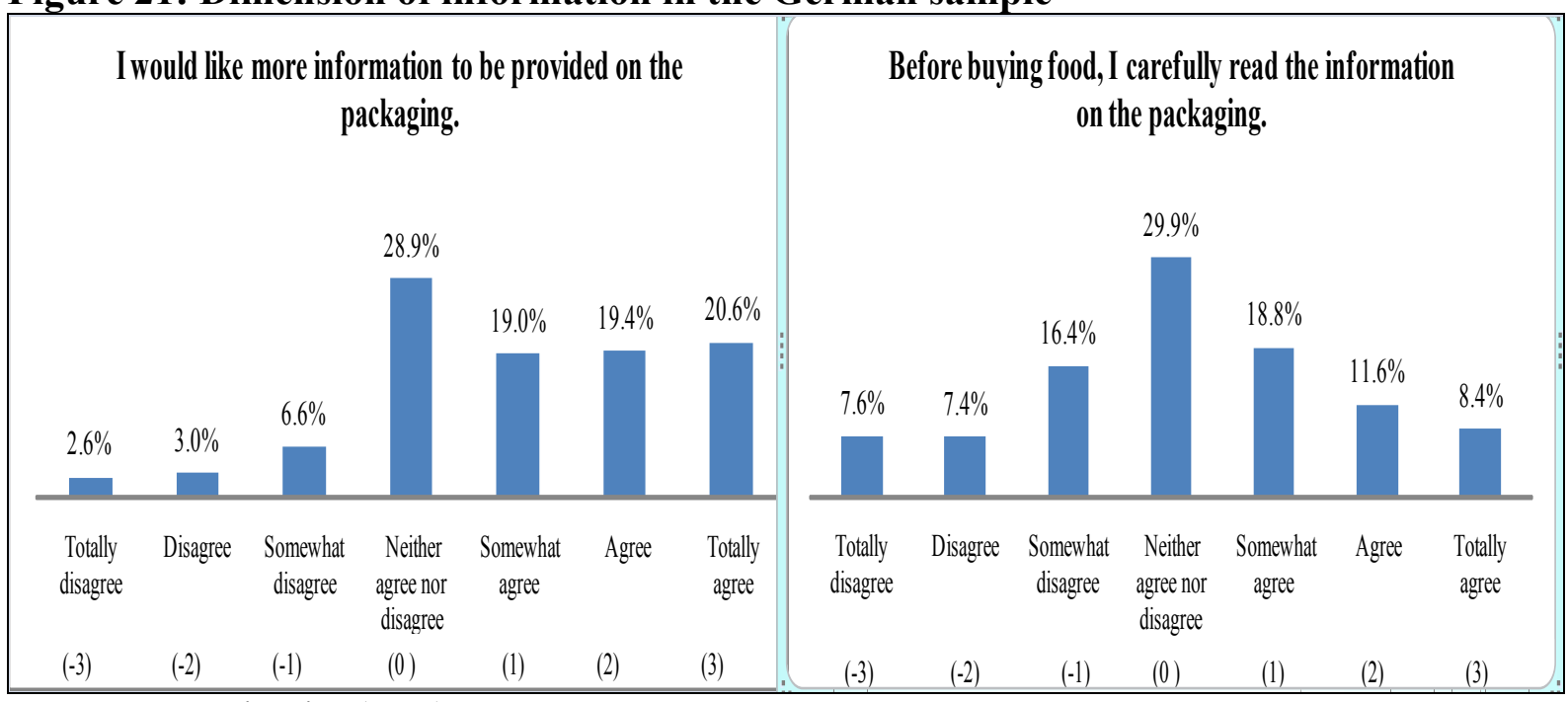

Source: Own estimation (2009).

\section{Mexican sample:}

Regarding the frequency of responses to the statement: "I would like more information to be provided on the packaging". Figure 22 describes the following: $0.6 \%$ "totally disagree" and "disagree," respectively; 2.4\% "somewhat disagree"; $8.9 \%$ "neither agree nor disagree"; $11.2 \%$ "somewhat agree"; $26.4 \%$ "agree"; and 49.9\% "totally agree". Hence, in the Mexican sample the vast majority of responses are concentrated in the range "agree-to-totally agree" as solely these two categories contain more than $70 \%$ of responses! As was the case of previous responses it is plausible to think that we have to cope with a problem of "social desirability". On the other side, it is possible that among Mexicans there is a profound feeling of insecurity concerning food production which, in turns, originates a strong desire for more information concerning food products.

The responses provided for the statement: "Before buying food, I carefully read the information on the package" displayed in Figure 22 are described as: 1.6\% "totally disagree"; similarly, 1.8\% “disagree"; $4.7 \%$ "somewhat disagree"; $16.6 \%$ "neither agree nor disagree"; the highest percentage (29.4\%) "somewhat agree"; 26.2\% "agree"; and 19.7\% "totally agree". More than two thirds of responses are concentrated in the area somewhat-to-totally agree. Taking into account the former statement, we can state that Mexicans not only strive for more information on food but also they appreciate (and use it) whenever it is provided on the packaging of food products. 
Figure 22: Dimension of information in the Mexican sample

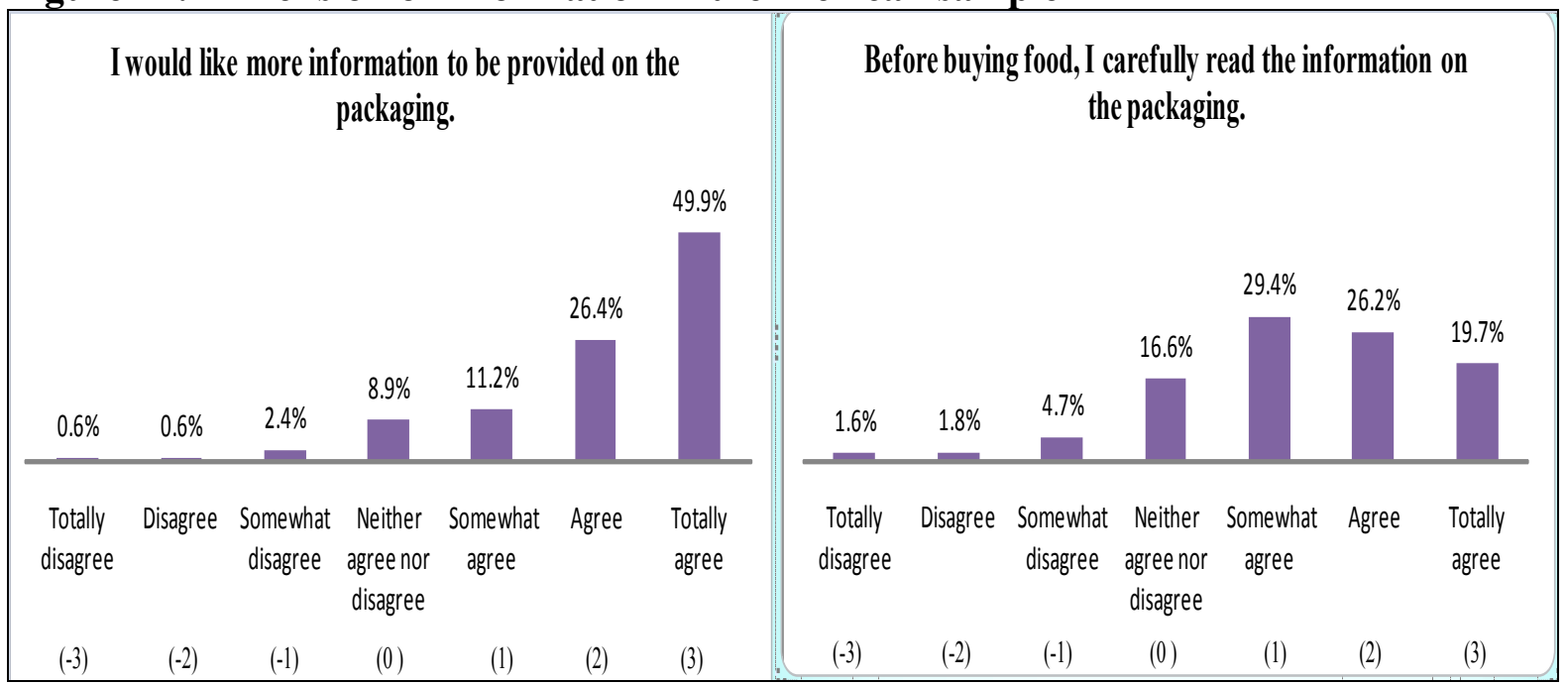

Source: Own estimation (2009).

\subsubsection{Weight control}

\section{German sample:}

Regarding the dimension of "Weight control", the statement: "As much as possible, I eat lowfat foods" was used. The frequency of responses displayed in Figure 23 are described as follows: $6.2 \%$ "totally disagree"; similarly, 5.8\% "disagree"; $15.6 \%$ "somewhat disagree"; 29.1\% "neither agree nor disagree"; $18.6 \%$ "somewhat agree"; 14.4\% "agree"; and 10.4\% "totally agree".

The frequency of responses to the statement: "As far as possible, I eat a low-calorie diet". are described as follows: $7.8 \%$ "totally disagree"; similarly, 6.6\% "disagree"; 19\% "somewhat disagree"; $32.9 \%$ "neither agree nor disagree"; 15.2\% "somewhat agree"; 10.6\% "agree"; and $8 \%$ "totally agree" (see Figure 23 ). All in all, both statements divide the German population in (about) three thirds, one third show no interest in a low-fat/low-calorie diet, one third show low commitment regarding these issues. Finally, one third of the German population believes that such food habits are important. 


\section{Figure 23: Dimension of weight control in the German sample}

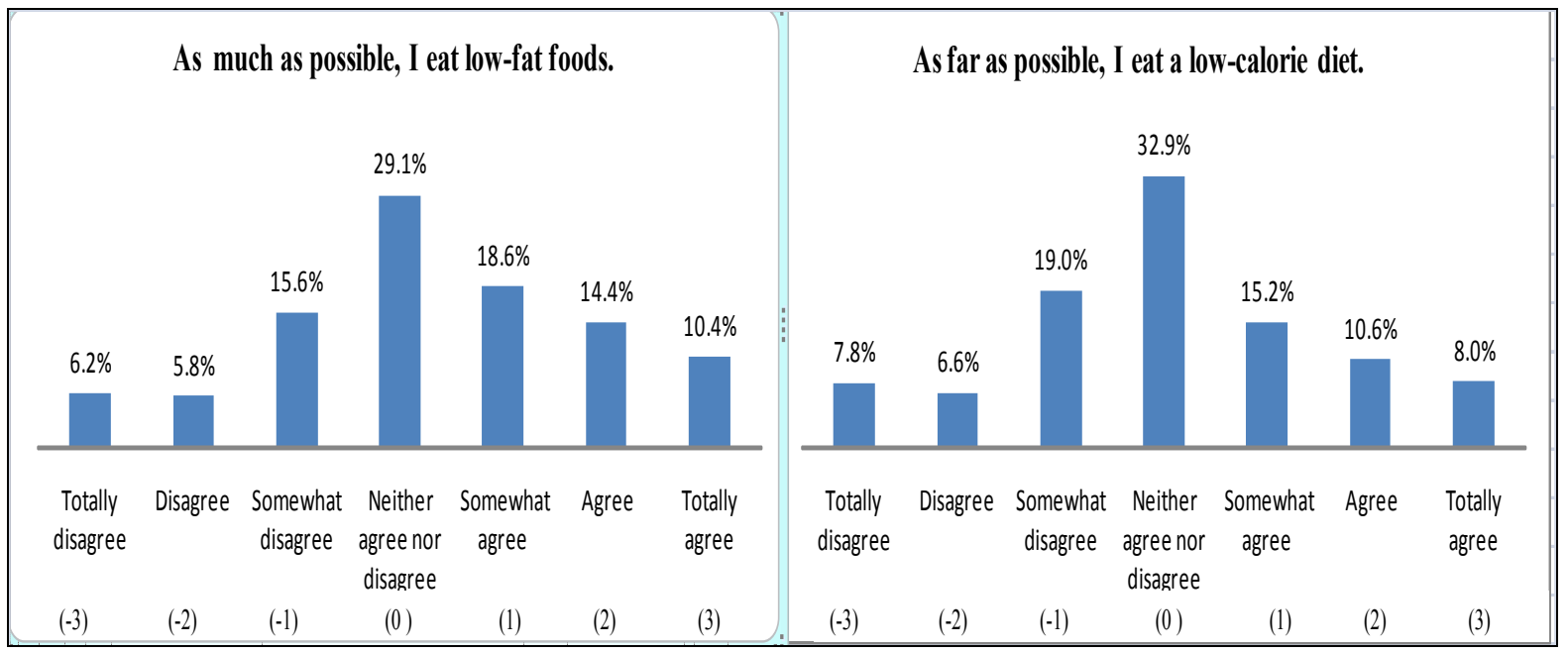

Source: Own estimation (2009).

\section{Mexican sample:}

The responses provided in the Mexican sample regarding the statement: "As much as possible, I eat low-fat foods" are shown in Figure 24 described as follows: 1.6\% "totally disagree"; $2.8 \%$ disagree"; $4.5 \%$ "somewhat disagree"; 11.8\% "neither agree nor disagree"; $22.1 \%$ "somewhat agree"; $34.7 \%$, "agree"; and 22.5\% "totally agree".

The frequency of responses to the statement: "As far as possible, I eat a low-calorie diet" displayed in Figure 24 are described as follows: 2.8\% "totally disagree"; $3.2 \%$ "disagree"; and $6.3 \%$ "somewhat disagree"; $17 \%$ "neither agree nor disagree"; $26 \%$ "somewhat agree"; 27.4\% "agree"; and 17.4\% "totally agree" respectively. Both statements show a similar distribution of responses with a high concentration in the "one-to-two" range. Hence, in comparison to Germans, Mexicans show a higher commitment for a low-fat/calorie diet. 
Figure 24: Dimension of weight control in the Mexican sample

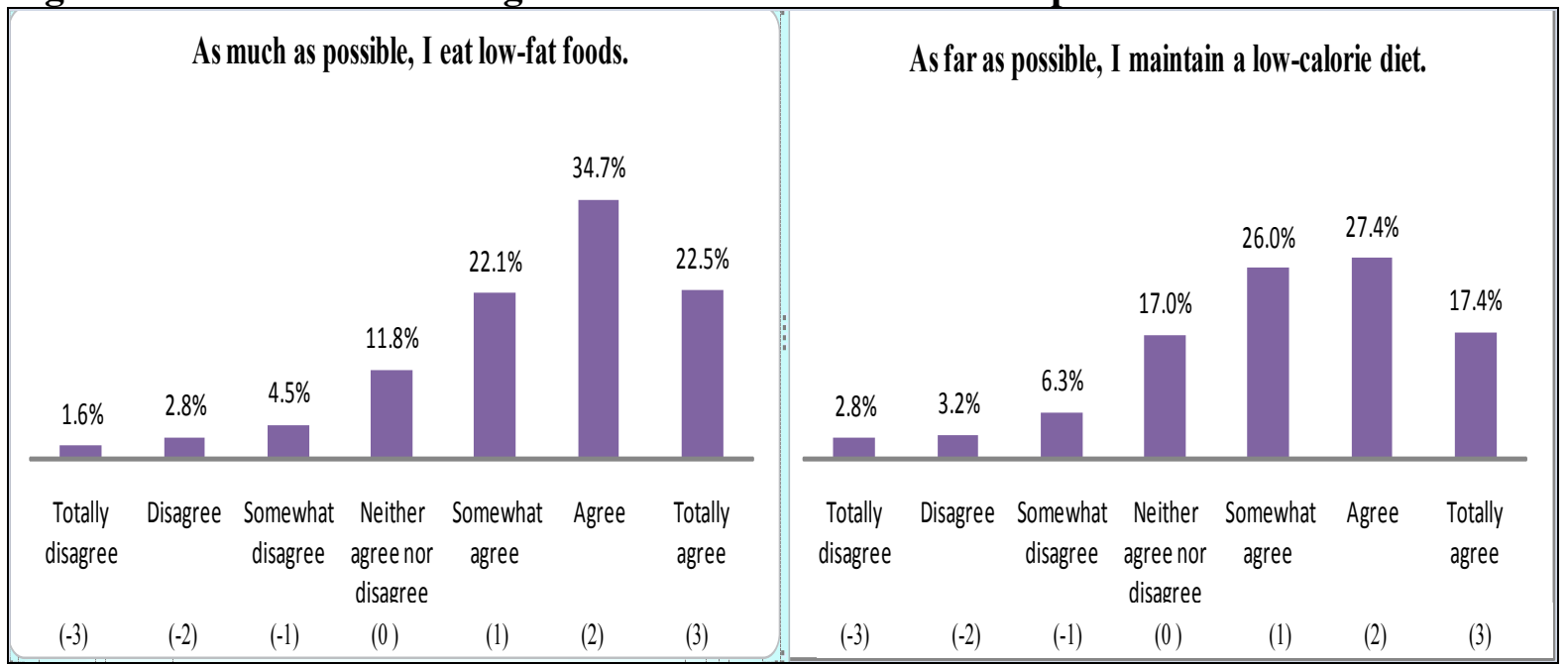

Source: Own estimation (2009).

\subsubsection{Healthy eating}

\section{German sample:}

Regarding the dimension of healthy eating, the statement: "I force myself to eat food that doesn't taste good but is healthy". was used. The frequency of responses in Figure 25 are described as follows: 15\% "totally disagree"; 13.8\% "disagree"; $24.2 \%$ "somewhat disagree"; 27.5\% "neither agree nor disagree"; 9.2\% "somewhat agree"; 5.8\% "agree"; and 4.6\% "totally agree". As depicted, the distribution of responses is skewed on the left-side of the chart. In fact, more than $70 \%$ of responses are concentrated in the "minus three-to-zero" area. Hence, we can assume that Germans are not very willing to undergo sacrifices, not even in the name of health.

The responses provided to the statement: "I know I should follow a healthy eating, but I don't manage it". are described as: $4.8 \%$ "totally disagree"; $4.4 \%$ "disagree"; $12.6 \%$ "somewhat disagree"; 29.9\% "neither agree nor disagree"; 19.4\% "somewhat agree"; 17.2\% "agree"; and $11.8 \%$ "totally agree", respectively (see Figure 25). Here the responses of the German sample are more normally distributed. However, since about $50 \%$ of responses are placed on the range "somewhat to totally agree" Germans try to follow a healthy diet however, they are not successful. 
Figure 25: Dimension of healthy eating in the German sample

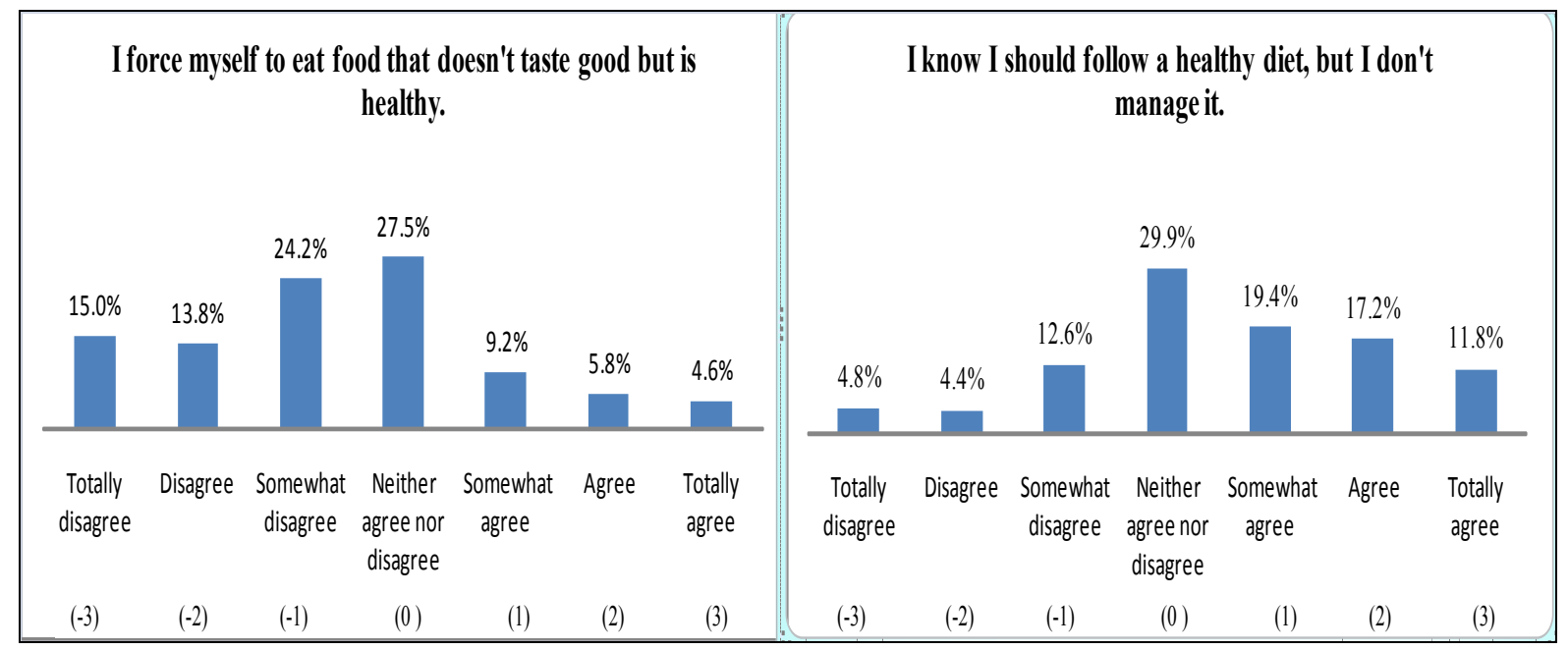

Source: Own estimation (2009).

\section{Mexican sample:}

The responses to the statement: "I force myself to eat food that doesn't taste good but is healthy" depicted in Figure 26, are described as: 7.5\% "totally disagree"; 11.6\% "disagree"; 14.4\% "somewhat disagree"; $17.8 \%$ "neither agree nor disagree"; $22.7 \%$ "somewhat agree"; $16.8 \%$ "agree"; and 9.3\% "totally agree". In comparison to the German sample, the distribution of responses is more normally distributed. Moreover, more responses are concentrated in the "one to three" range thus showing that Mexicans are more likely to undergo sacrifices when their health is at stake.

The responses to the statement: "I know I should follow a Healthy eating, but I don't manage it". Are the following: $4.9 \%$ "totally disagree"; $10.3 \%$ "disagree"; similarly, $10.7 \%$ "somewhat disagree"; 15\% "neither agree nor disagree"; 19.5\% "somewhat agree"; similarly, 20.9\% "agree"; and 18.7\% "totally agree" (see Figure 26). Here the responses of the Mexican sample are more normally distributed. Similarly as for the Germans, responses are concentrated on the range "somewhat-to-totally agree" (59 \%). This shows that even Mexicans try to follow a healthy diet it is difficult for them to achieve it. 
Figure 26: Dimension of healthy eating in the Mexican sample

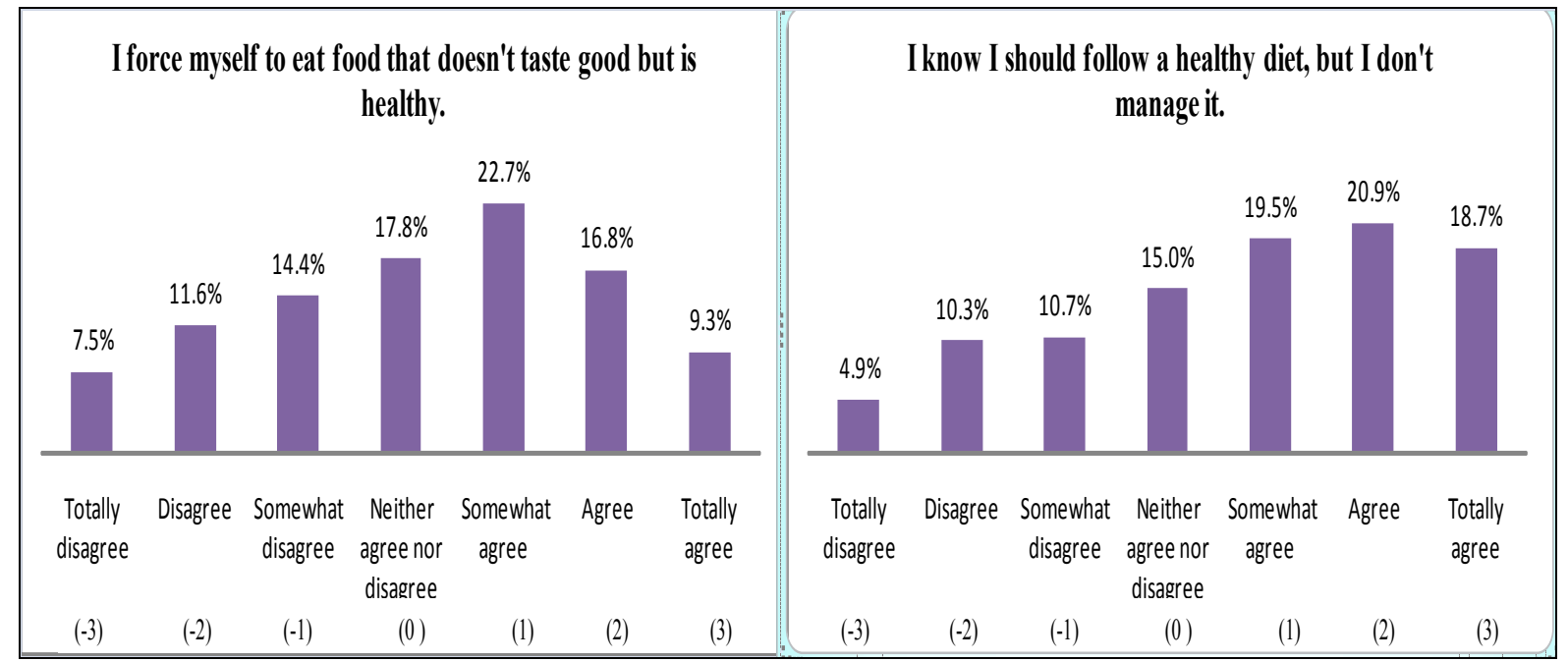

Source: Own estimation (2009).

\subsubsection{Natural content}

\section{German sample:}

In the measuring of the dimension of natural content, the statement: "I make sure that no artificial flavors have been added to my food" was used. The frequency of responses are shown in Figure 27 and are described as follows: 6.8\% "totally disagree"; 4.8\% "disagree"; 13\% "somewhat disagree"; $32.9 \%$ "neither agree nor disagree"; 16.6\% "somewhat agree"; $15.4 \%$ "agree"; and $10.6 \%$ "totally agree", respectively.

Regarding the statement: "I avoid all products containing flavor enhancers" the frequencies of responses are as follows: 7.6\% "totally disagree"; 10.2\% "disagree"; $18.8 \%$ "somewhat disagree"; $34.3 \%$ "neither agree nor disagree"; 12.4\% "somewhat agree"; 10.4\% "agree"; and $6.4 \%$ "totally agree", respectively (see Figure 27). All in all, both statements show a similar distribution of responses: Both for artificial and flavor enhancers about one third of the German population show a low commitment regarding these artificial ingredients. A higher willingness to avoid artificial flavor is shown in comparison to flavor enhancers: in fact for the former more than $40 \%$ of respondents somewhat to totally agree to avoid them versus almost $28 \%$ of respondents place their agreement in the same range of responses. 
Figure 27: Dimension of natural content in the German sample

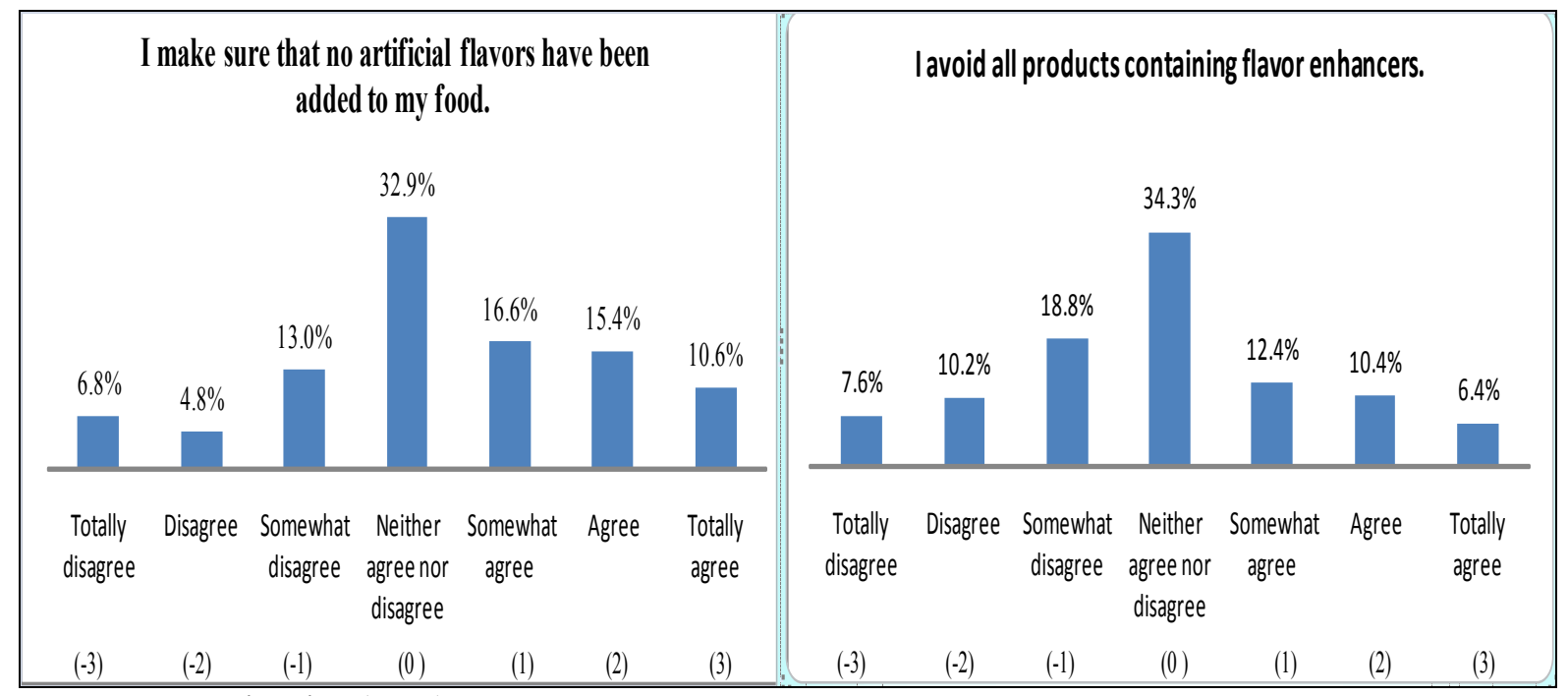

Source: Own estimation (2009).

\section{Mexican sample:}

Regarding the responses to the statement: "I make sure that no artificial flavors have been added to my food". Figure 28 displays the frequency of responses as follows: $2 \%$ "totally disagree"; similarly, 2.6\% "disagree"; 5.3\% "somewhat disagree"; 23.3\% "neither agree nor disagree"; $18.3 \%$ "somewhat agree"; $24.1 \%$ "agree"; similarly, $24.5 \%$ "totally agree".

In regard to the statement: "I avoid all products containing flavor enhancers" the frequency of responses shown in Figure 28 are as follows: 2.4\% "totally disagree"; similarly, 3.4\% "disagree"; 7.7\% "somewhat disagree"; 21.7\% "neither agree nor disagree"; similarly, 21.3\% "somewhat agree"; $25.4 \%$ "agree"; and 18.1\% "totally agree". Both statements show a similar distribution of responses with a high concentration in the "one-to-two" range. Hence, in comparison to Germans, Mexicans show a higher commitment to avoid artificial flavors/ flavor enhancers. 
Figure 28: Dimension of natural content in the Mexican sample

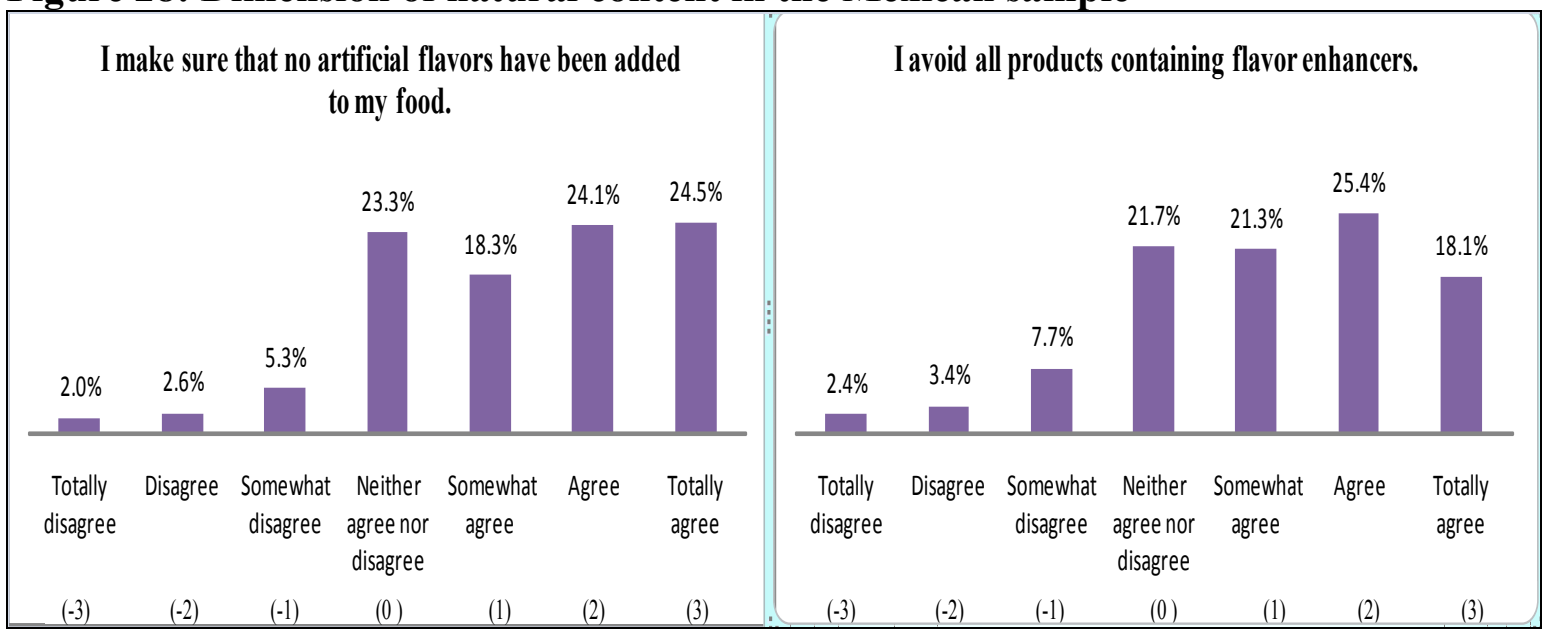

Source: Own estimation (2009).

\subsubsection{Free of GMO (Genetically Modified Organism)}

\section{German sample:}

To measure the dimension of free of GMO, the statement: "Sometimes I buy products that may contain genetically modified material" was used. Figure 29 shows the frequency of responses as described in the following: 6.6\% "totally disagree"; $7.2 \%$ "disagree"; $16 \%$ "somewhat disagree"; 33.5\% "neither agree nor disagree"; 15.4\% "somewhat agree"; 14.4\% "agree"; and 7\% "totally agree". The high concentration of responses in the "neither-nor" category let presume that this is still a "tabu" issue, where still a high number of respondents do not have a clear opinion.

The frequency of responses to the statement: "I buy only non-genetically modified food products". are as follows: 12.6\% "totally disagree"; $11.2 \%$ "disagree"; $14 \%$ "somewhat disagree"; $30.7 \%$ "neither agree nor disagree"; $12.2 \%$ "somewhat agree"; similarly, 11.2\% "agree"; and 8.2\% "totally agree" (see Figure 29). Both statements show a similar distribution of responses within the German sample thus showing approximately one third of responses in the "totally disagree-to-somewhat-disagree" range, about one third in the "neither-nor" category and one third in the "totally agree-to-somewhat agree" range. The high concentration of responses in the "neither-nor" category let presume that this is still a "tabu" issue, where still a high number of respondents do not have a clear opinion again. 
Figure 29: Dimension of free of GMO in the German sample

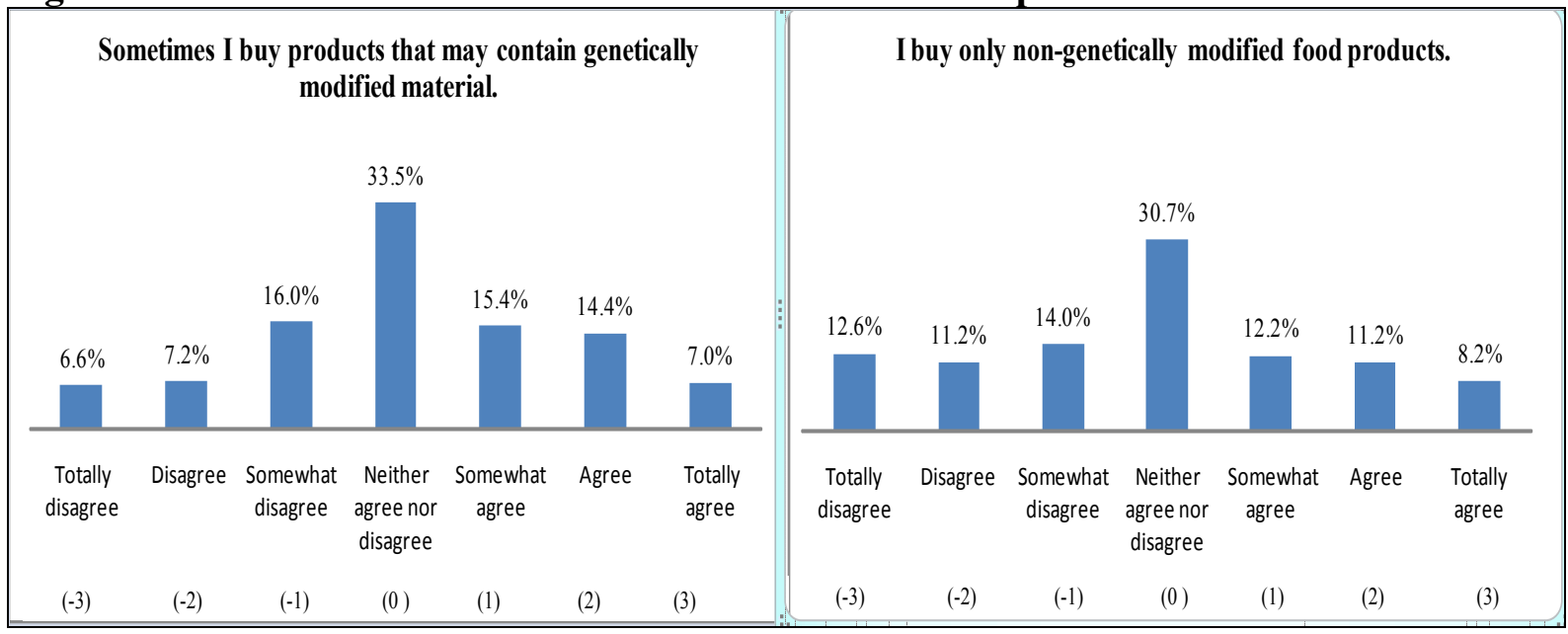

Source: Own estimation (2009).

\section{Mexican sample:}

The responses to the statement: "Sometimes I buy products that may contain genetically modified material" displayed in Figure 30 are described as follows: 7.1\% "totally disagree"; similarly, 6.9\% "disagree"; 9.1\% "somewhat disagree"; 16.2\% "neither agree nor disagree"; 22.9\% "somewhat agree"; similarly, 22.1\% "agree"; and 15.8\% "totally agree," respectively.

The responses to the statement: "I buy only non-genetically modified food products" are also described in Figure 30 as follows: 4.7\% "totally disagree"; 5.5\% "disagree"; 8.5\% "somewhat disagree," 34.3\% "neither agree nor disagree"; 18.7\% "somewhat agree"; 16.8\% "agree"; and $11.4 \%$ "totally agree," respectively. Both statements show a similar distribution of responses within the Mexican sample which partially differ to the German, more normally distributed, responses. In fact, whereas for the first statement only about $23 \%$ of Mexicans "totally-tosomewhat-disagree", in the second statement -which premises a more radical opposition against the GMO technology- only approximately $18 \%$ of responses are placed in the "totally disagree-to-somewhat-disagree" range and a higher number of respondents "neither agree nor disagree". 
Figure 30: Dimension of free of GMO in the Mexican sample

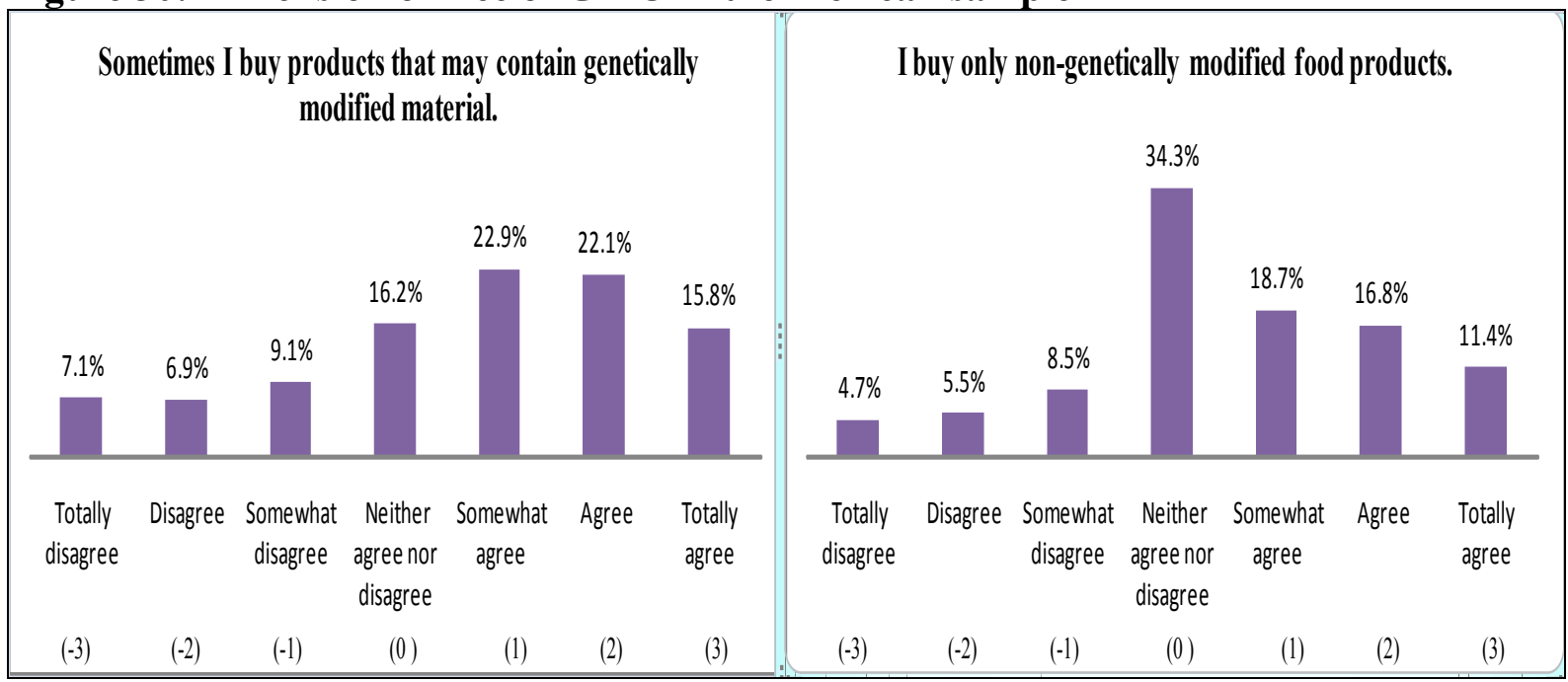

Source: Own estimation (2009).

\subsubsection{Familiarity}

\section{German sample:}

To measure the dimension of familiarity, was used the statement: "I tend to buy well-known brands of food products" was used. The percentage description of the number of responses in each category in Figure 31 are; 5.4\% "totally disagree"; 4.4\% "disagree"; 13.2\% "somewhat disagree"; 35.1\% "neither agree nor disagree"; 18.4 "somewhat agree"; 16.2\% "agree"; and $7.4 \%$ "totally agree".

The frequency of responses to the statement: "Concerning food, I like to try new things" are as follows: $1.8 \%$ "totally disagree"; $2.4 \%$ "disagree"; $7.8 \%$ "somewhat disagree"; $33.5 \%$, "neither agree nor disagree"; $23.6 \%$ "somewhat agree"; $18.4 \%$ "agree"; and 12.6\% "totally agree", respectively (see Figure 31). Both statements disclose two (above all in Europe, cfr. Schwartz, 2006) opposite values: the former is a proxy for measuring "neophobia" tendencies whereas the second measure "neophilia". Concerning the former we see that the distribution of responses approaches "normal distribution" with a peak of responses in the "neither/nor category". Concerning the second item, although the percentage of responses placed in the "neither/nor category" is similar, the majority of responses concern the rage "somewhat to totally agree". Hence, among Germans the tendency to try new types of food seems to prevail. 
Figure 31: Dimension of familiarity in the German sample

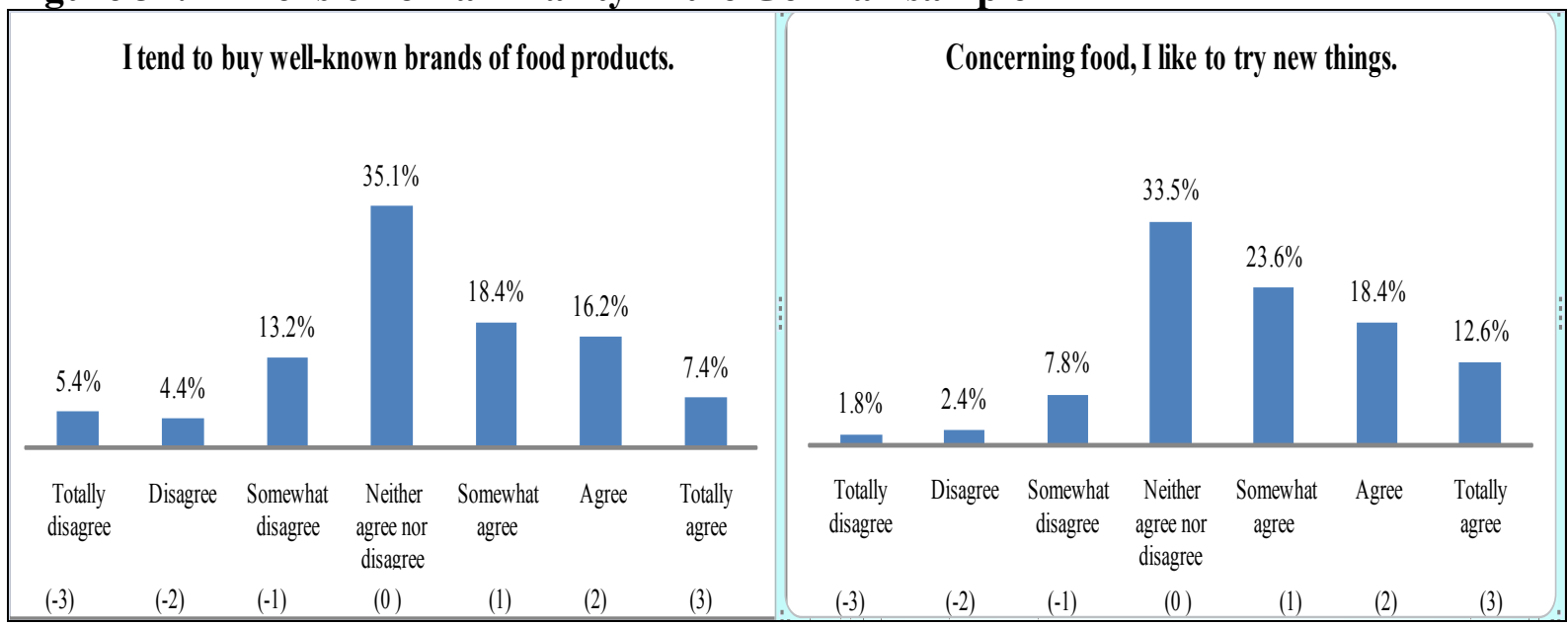

Source: Own estimation (2009).

\section{Mexican sample:}

The variability of responses to the statement: "I tend to buy well-known brands of food products" in Figure 32 is described as: 1.2\% "totally disagree"; $0.8 \%$ "disagree"; $2.8 \%$ "somewhat disagree"; 10.1\% "neither agree nor disagree"; 19.5\% "somewhat agree"; 35.7\% "agree"; and 30\% "totally agree".

Similarly, the frequency of responses to the statement: "Concerning food, I like to try new things" are: $0.6 \%$ "totally disagree"; $1.8 \%$ "disagree"; $4.1 \%$ "somewhat disagree"; $11.6 \%$ "neither agree nor disagree"; $27.6 \%$ "somewhat agree"; $31.8 \%$ "agree"; and 22.5\% "totally agree", respectively (see Figure 32).

Both statements show similar responses patterns. In fact, the majority of responses are placed on the range "somewhat-to-totally agree". This "apparent contradiction" can be explained by Schwartz's theory (2006). Whereas the German culture tends to emphasize opposite categories, the Mexican culture tend to reject such chiasm: these two inclinations are summarized in Schwartz's definitions of mastery and harmony, respectively. 
Figure 32: Dimension of familiarity in the Mexican sample

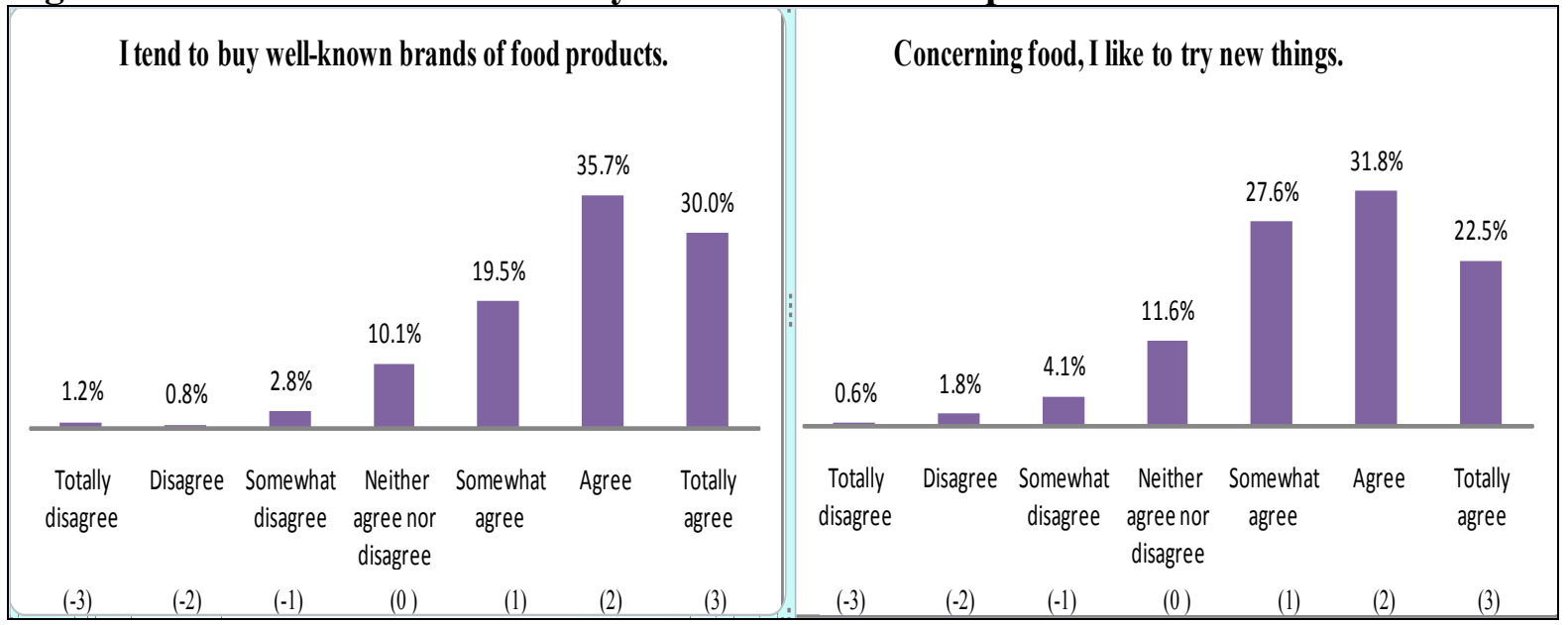

Source: Own estimation (2009).

\subsubsection{Advertising}

\section{German sample:}

To measure the dimension of advertising, the statement: "I frequently buy food because of the packaging" was used. The frequency of responses in Figure 33 are as follows: $14.4 \%$ "totally disagree"; 12.4\% "disagree"; 25.3\% "somewhat disagree"; similarly, 25.5\% "neither agree nor disagree"; $12 \%$ "somewhat agree"; 6.4\% "agree"; and 4\% "totally agree". All in all, Germans seem to undervalue the role of packaging on their buying behavior.

The frequency of responses to the statement: "I frequently buy food that I have previously seen advertised" in Figure 33 are described as follows: 9\% "totally disagree"; similarly, 10\% "disagree"; 20.4\% "somewhat disagree"; 39.5\% "neither agree nor disagree"; 9.8\% "somewhat agree"; 6.6\% "agree"; and 4.8\% "totally agree", respectively. Hence, as for the previous statement, Germans disagree on the role of advertising on their buying behavior with a high response of "insecure" respondents, who place their answer on the "neither-nor" range. 
Figure 33: Dimension of advertising in the German sample

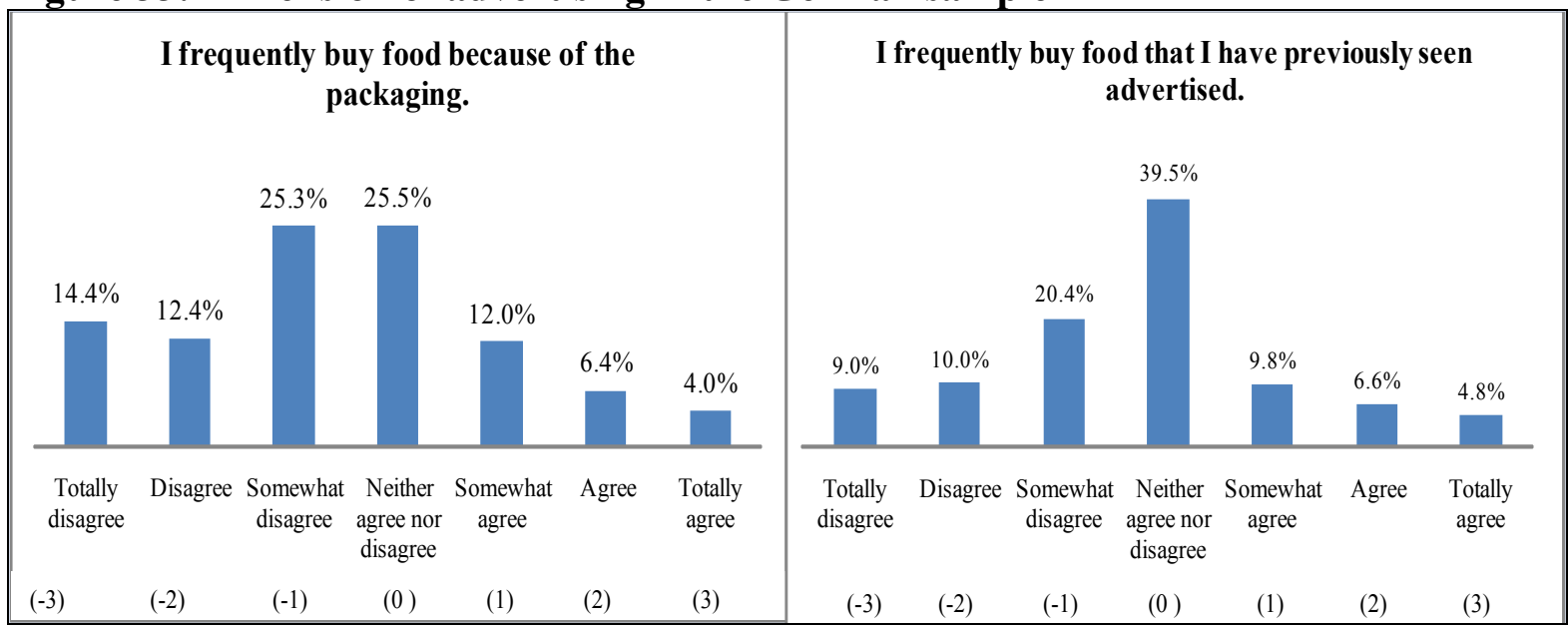

Source: Own estimation (2009).

\section{Mexican sample:}

The frequency of responses to the statement: "I frequently buy food because of the packaging" are described Figure 34 as follows: 13.4\% "totally disagree"; 14\% "disagree"; $17.9 \%$ "somewhat disagree"; similarly, $17.8 \%$ "neither agree nor disagree"; $19.5 \%$ "somewhat agree"; $12 \%$ "agree"; and 5.3\% "totally agree".

The frequency of responses to the statement: "I frequently buy food that I have previously seen advertised" are as follows: 2.2\% "totally disagree"; similarly, 4.1\% "disagree"; $7.3 \%$ "somewhat disagree"; 19.1\% "neither agree nor disagree"; 33.9\% "somewhat agree"; $22.7 \%$ "agree"; and 10.7\% "totally agree," respectively (see Figure 34). In contrast to Germans, Mexicans provide more normally distributed responses. Moreover, since 19.5\% of Mexicans "somewhat agree," it seems that the perceived role of packaging has a stronger influence on the buying behavior of Mexicans than on Germans. 
Figure 34: Dimension of advertising in the Mexican sample

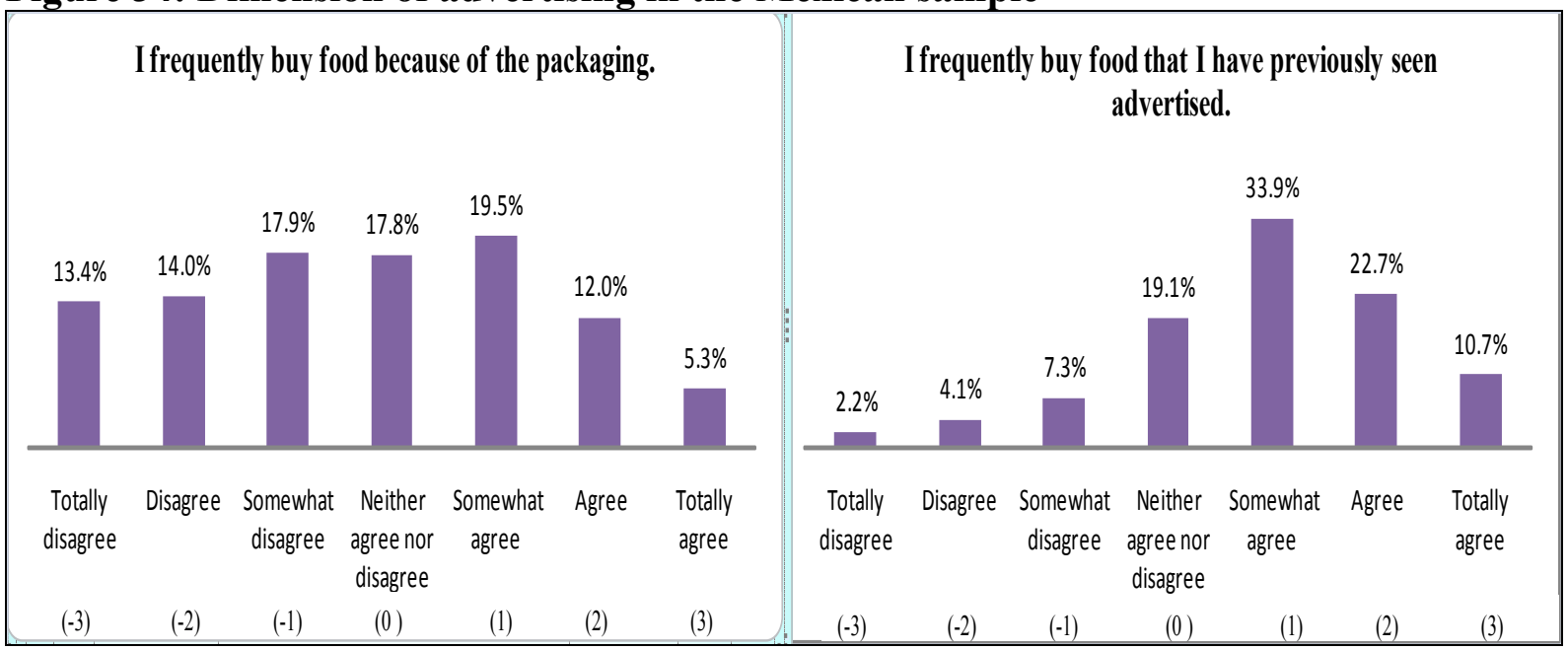

Source: Own estimation (2009).

\subsubsection{Shopping convenience}

\section{German sample:}

Regarding the dimension of shopping convenience, the frequency of responses to the statement: "For me, food shopping is an unpleasant task" displayed in Figure 35 are described as follows: $10.6 \%$ "totally disagree"; $11.8 \%$ "disagree"; $23 \%$ "somewhat disagree"; $31.5 \%$ "neither agree nor disagree"; 10\% "somewhat agree"; 6.2\% "agree"; and 7\% "totally agree".

The frequency of responses to the statement: "Food shopping should be quick" are described in the following: $2.8 \%$ "totally disagree"; $3.6 \%$ "disagree"; $11.4 \%$ "somewhat disagree"; 30.1\% "neither agree nor disagree"; 19.8\% "somewhat agree"; 17.6\% "agree"; and 14.8 "totally agree". Both statements mirror an interesting feature of the German 'food-related culture': although food shopping is not seen as an unpleasant task (about 44\% somewhat-tototally disagrees the first assertion of food shopping being "unpleasant"), this should notwithstanding follow a rather quick pace within the daily schedule. 
Figure 35: Dimension of shopping convenience in the German sample

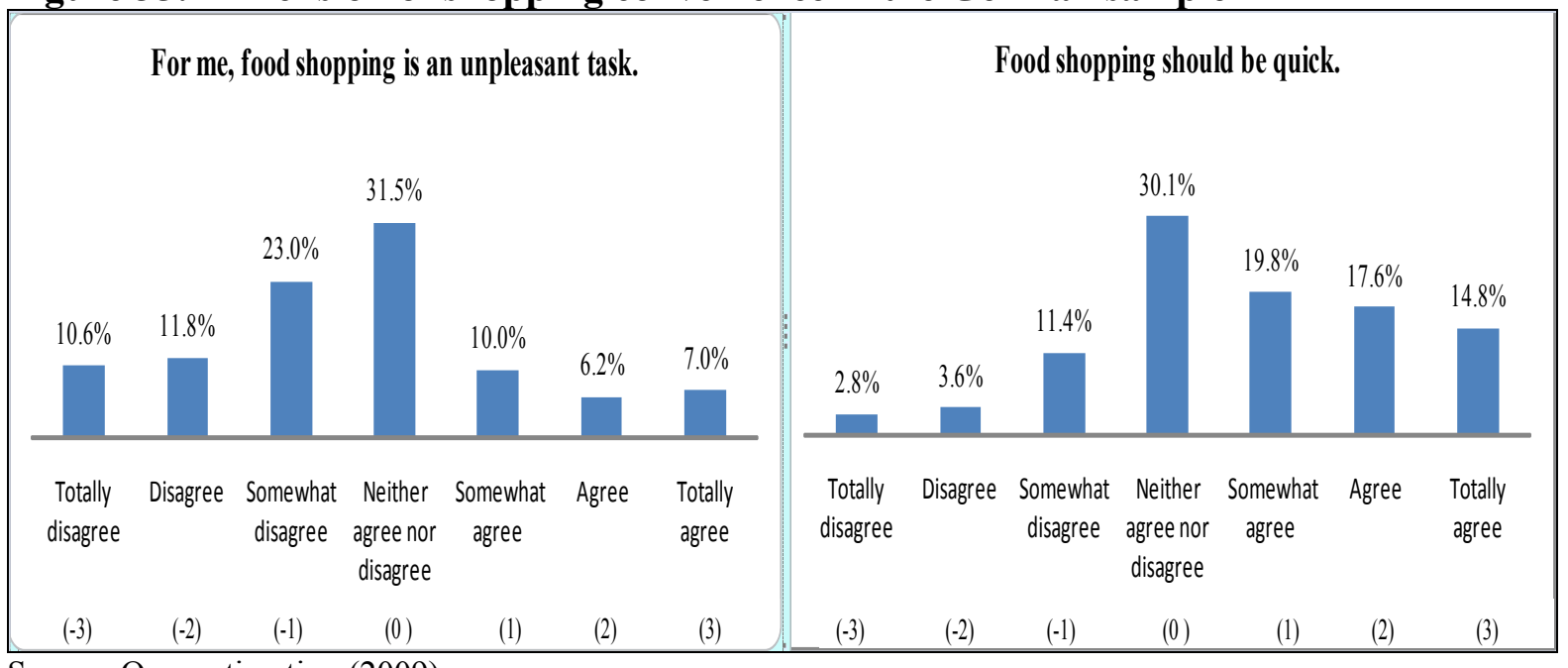

Source: Own estimation (2009).

\section{Mexican sample}

Regarding the frequency of responses to the statement: "For me, food shopping is an unpleasant task" show in Figure 36 the following described: 21.3\% "totally disagree"; similarly, 20.5\% "disagree"; 17.4\% "somewhat disagree"; 19.9\% "neither agree nor disagree"; $11 \%$ “somewhat agree"; 5.3\% "agree"; and 4.5\% "totally agree".

Regarding the statement "Food shopping should be quick". The following frequencies of responses are described as: 7.7\% "totally disagree"; 10.8\% "disagree"; and 13.8\% "somewhat disagree"; 21.1\% "neither agree nor disagree"; 18.7\% "somewhat agree"; 15\% "agree"; and $12.8 \%$ "totally agree" (see Figure 36 ), respectively. Both statements show sharp contrasts to the German sample: the majority of Mexicans strongly disagree to the statement that food shopping is an unpleasant task (about 10\% more responses than Germans in the "somewhatto-totally disagree" range). Concerning the second statement responses are more equally distributed than within the German sample. Interestingly, already $45 \%$ of Mexicans "somewhat-to-totally agree" that shopping should be quick $(51 \%$ for the same range among the Germans). 
Figure 36: Dimension of shopping convenience in the Mexican sample

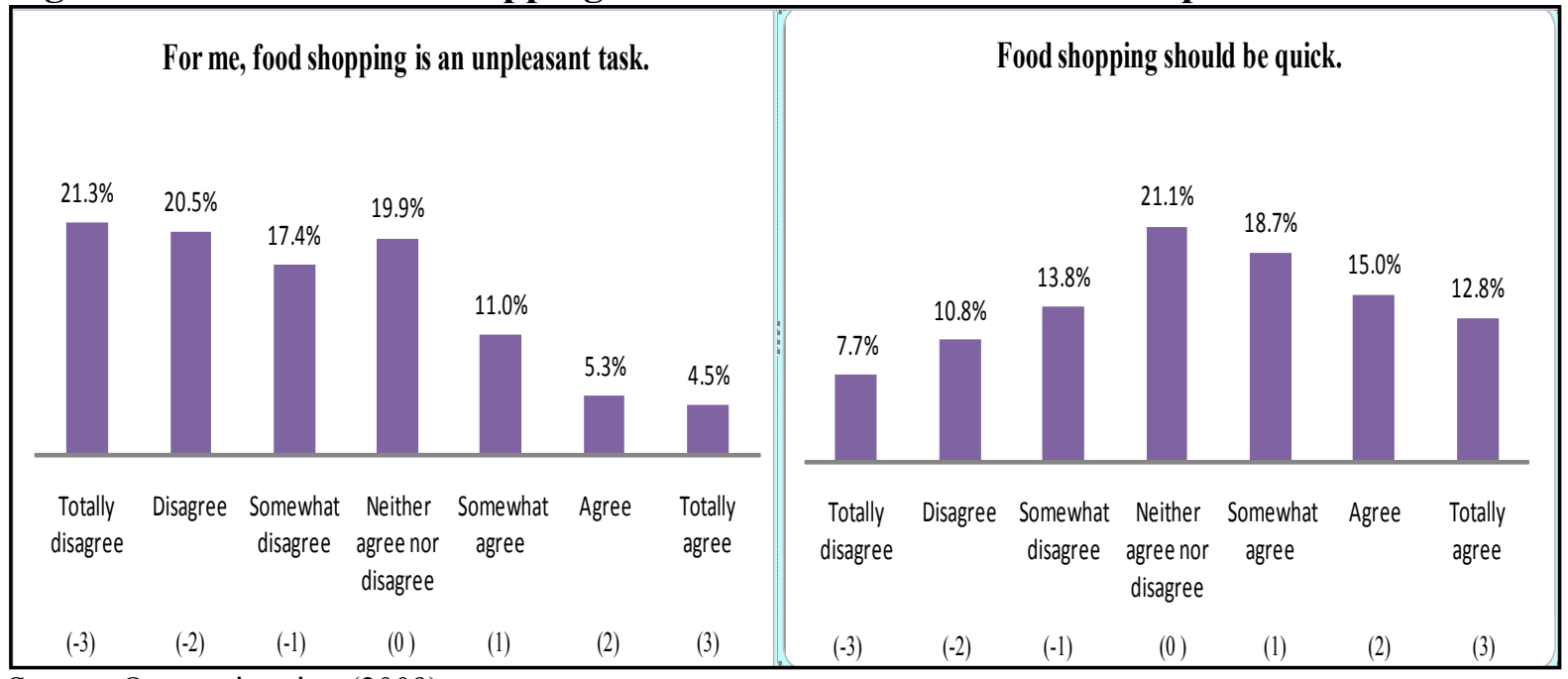

Source: Own estimation (2009).

\subsubsection{Cooking convenience}

\section{German sample:}

To measure the dimension of cooking convenience, the statement: "I think that meals should be easy to prepare" was used. The frequency of responses provided are as follow and displayed in Figure 37 2.6\% "totally disagree"; 4.6\% "disagree"; 9.4\% "somewhat disagree"; $28.1 \%$ "neither agree nor disagree"; $21.8 \%$ "somewhat agree"; $19.4 \%$ "agree"; and 14.2\% "totally agree".

The most frequent responses provided for the statement: "I think it is good that the assortment of convenience food is growing" are the following: 3.8\% "totally disagree"; $4 \%$ "disagree"; 9\% "somewhat disagree"; $32.1 \%$ "neither agree nor disagree"; $20 \%$ "somewhat agree"; similarly, 19\% "agree"; and 12.2\% "totally agree" (see Figure 37 ). Both statements show a high concentration of responses in the range "somewhat-to-totally agree" thus showing a high appreciation among Germans for convenience issues in food. 
Figure 37: Dimension of cooking convenience in the German sample

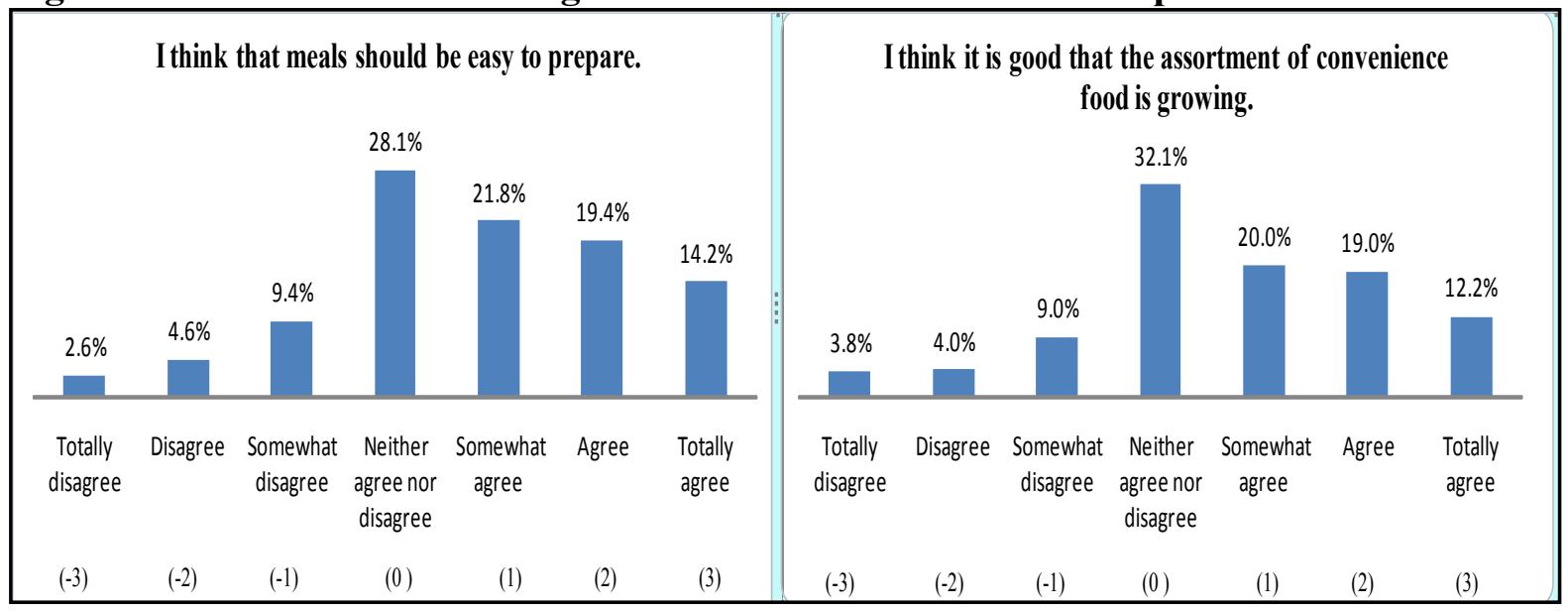

Source: Own estimation (2009).

\section{Mexican sample:}

The frequency of responses provided to the statement: "I think that meals should be easy to prepare". In Figure 38 is described as follows: 2.4\% "totally disagree"; 4.9\% "disagree"; 5.3\% "somewhat disagree"; $14.2 \%$ "neither agree nor disagree"; $22.3 \%$ "somewhat agree"; 29\% "agree"; and $21.9 \%$ "totally agree," respectively.

The responses to the statement: "I think it is good that the assortment of convenience food is growing" are described in the following: 7.5\% "totally disagree"; $10.3 \%$ "disagree"; $14.2 \%$ "somewhat disagree"; $24.9 \%$ "neither agree nor disagree"; $15.8 \%$ "somewhat agree"; $17.9 \%$ "agree"; and 9.5\% "totally agree," respectively (see Figure 38). The first statement shows a high concentration of responses in the range "somewhat-to-totally agree" whereas the distribution of responses for the second statement is more normally distributed. All in all, also for the Mexican sample it seems that there is a high appreciation for convenience in cooking and food assortment. 
Figure 38: Dimension of cooking convenience in the Mexican sample

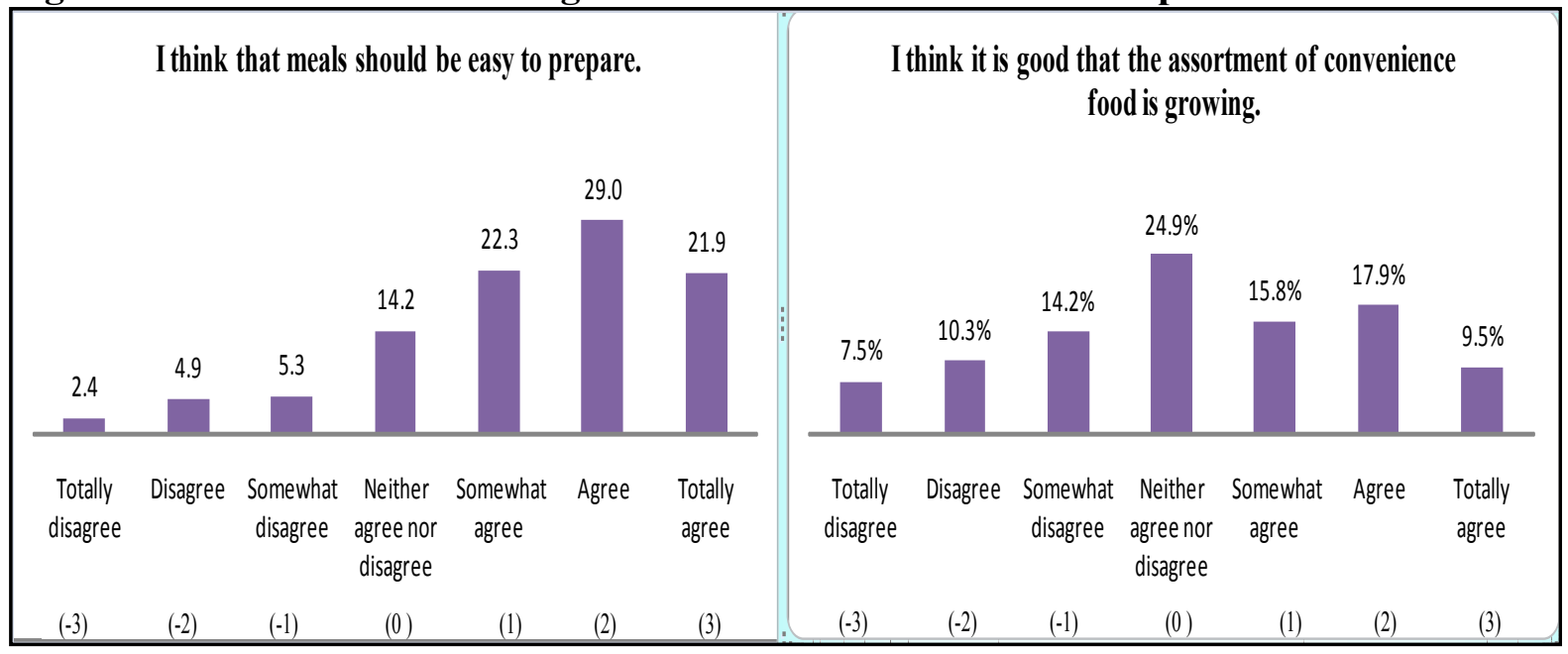

Source: Own estimation (2009).

\subsubsection{Nostalgia}

\section{German sample:}

To measure the dimension of nostalgia was used the statement: "When I come across foods from my childhood, I immediately buy them". Figure 39 displays the frequency of responses as follows: 3.8\% "totally disagree"; 5\% "disagree"; 7.4\% "somewhat disagree"; $28.7 \%$ "neither agree nor disagree"; 19.2\% "somewhat agree"; similarly, 20\% "agree"; and 16\% “totally agree," respectively.

The frequency of responses to the statement: "In my childhood, food tasted better" in Figure 39 are described as: $2.4 \%$ "totally disagree"; $3.2 \%$ "disagree"; $8.8 \%$ "somewhat disagree"; $32.9 \%$ "neither agree nor disagree"; $15.4 \%$ "somewhat agree"; $18.2 \%$ "agree" and 19.2\% within the category of "totally agree", respectively. Both statements show a high concentration of responses in the range "somewhat-to-totally agree". Moreover, almost one third of respondents concentrate their response on the "neither-nor" category. All in all, Germans seem to share nostalgic feelings concerning food. 
Figure 39: Dimension of nostalgia in the German sample

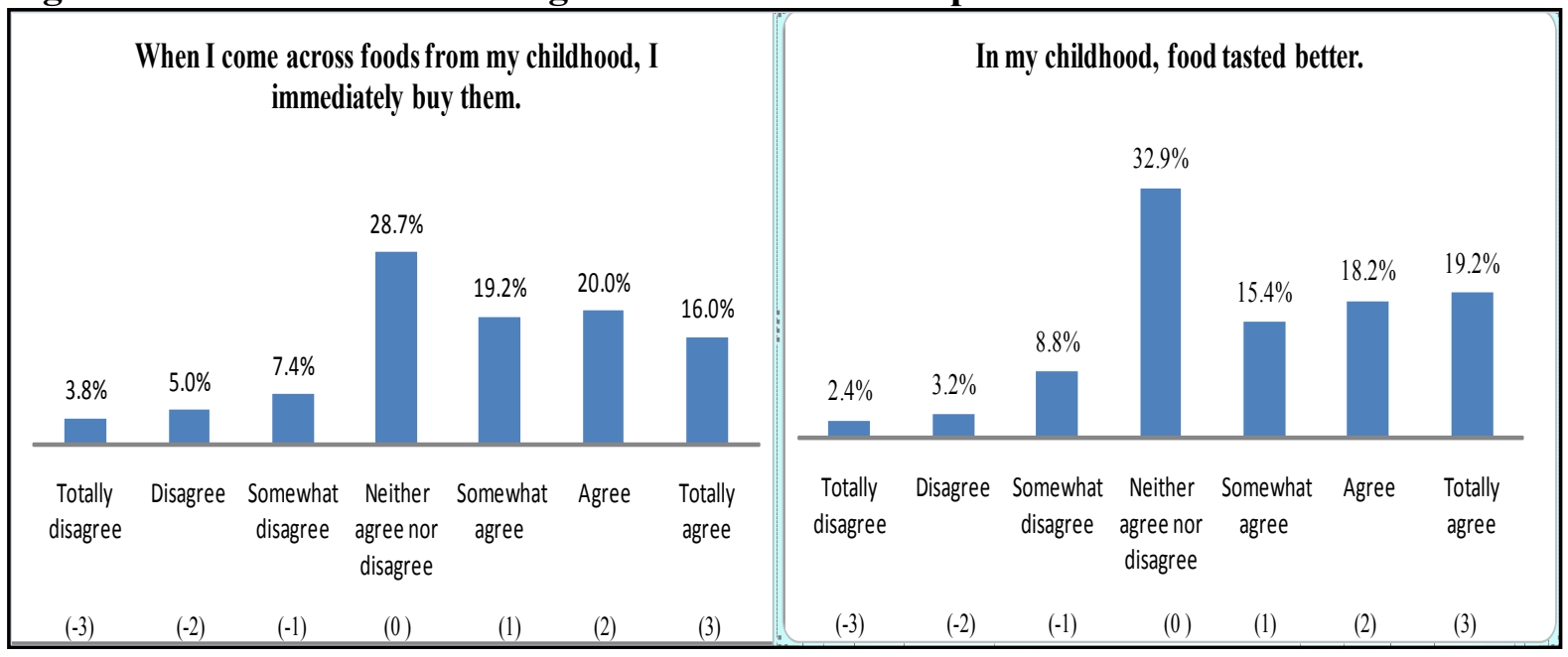

Source: Own estimation (2009).

\section{Mexican sample:}

Regarding the responses to the statement: "When I come across foods from my childhood, I immediately buy them" refer to Figure 40 described as follows: 1.8\% "totally disagree"; $4.1 \%$ “disagree"; similarly, 5.5\% "somewhat disagree"; 17.4\% "neither agree nor disagree"; 27.2\% "somewhat agree"; $22.9 \%$ "agree"; and 21.1\% "totally agree," respectively.

Regarding the statement, "In my childhood, food tasted better". The frequency of responses in Figure 40 are the following: 1\% "totally disagree"; similarly, $2.8 \%$ "disagree"; $3.9 \%$ "somewhat disagree"; 19.7\% "neither agree nor disagree"; 15.2\% "somewhat agree"; $24.9 \%$ "agree"; and 32.5\% "totally agree", respectively. In comparison to Germans, the two statements show even higher concentration of responses in the range "somewhat-to-totally agree" and a lesser concentration of on the "neither-nor" category. Hence, Mexicans seem even more nostalgic than Germans. 
Figure 40: Dimension of nostalgia in the Mexican sample

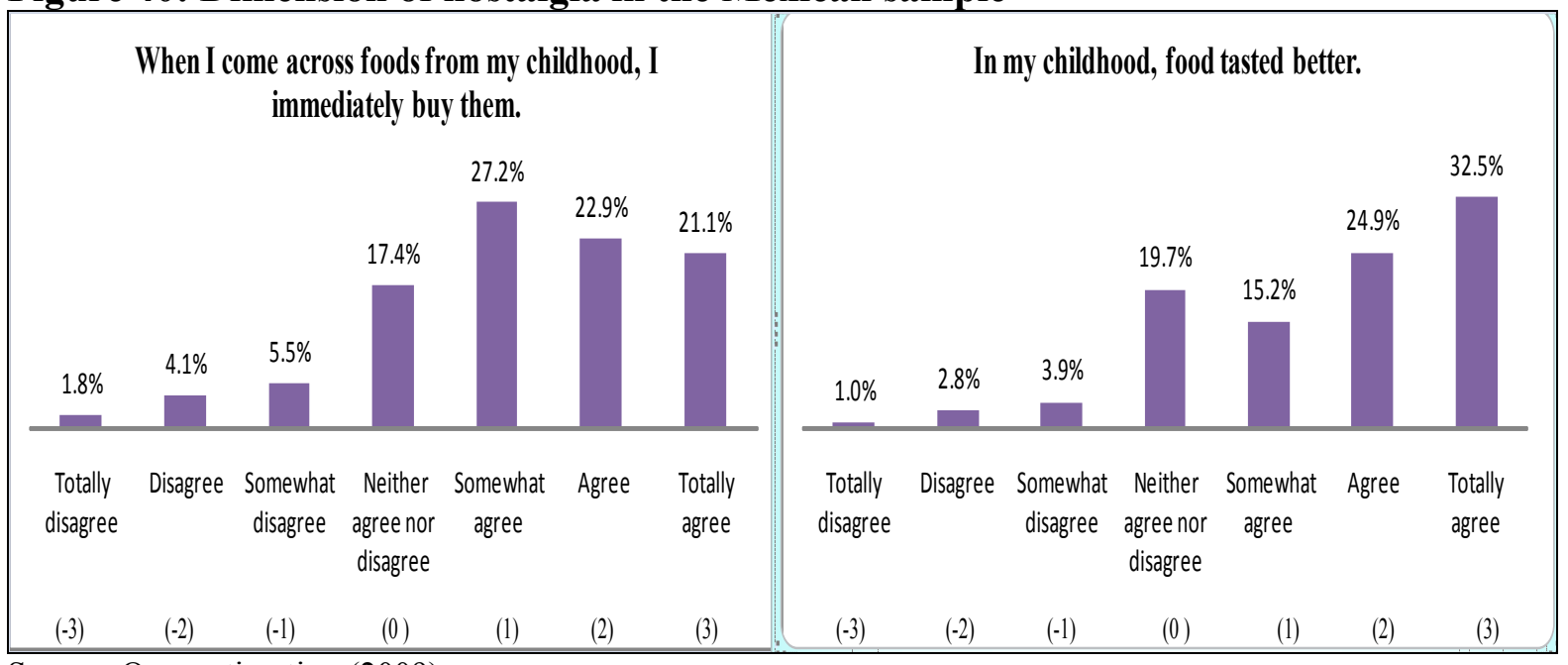

Source: Own estimation (2009).

\subsubsection{Price-quality relation}

\section{German sample:}

To measure the dimension of price-quality relation, the statement of "I prefer to buy higher priced foods, because these are better quality" was used. The frequency of responses are depicted in Figure 41; that is: $13.2 \%$ "totally disagree"; $9.4 \%$ "disagree"; $25.9 \%$ "somewhat disagree"; 31.9\% "neither agree nor disagree"; 10\% "somewhat agree"; 6.6\% "agree"; and $3.0 \%$ "totally agree", respectively.

With respect to the statement: "Low price is usually a sign of poor quality food". $15.4 \%$ "totally disagree"; $16.0 \%$ "disagree"; $25.7 \%$ "somewhat disagree"; $31.5 \%$ "neither agree nor disagree"; 6.2\% "somewhat agree"; 3.4\% "agree"; and 1.8\% "totally agree," respectively (see Figure 41). Because of the high concentration of responses in the range "totally-to-somewhat disagree" we think that Germans do not steer their buying behavior upon the height of the price. Thus, in the country of "Lidl" and "Aldi" it is not surprising that price do not signal quality in the eyes of consumers. Price-quality relation is more common in markets with higher food safety problems, whereas in Germany the discounters guarantee good prices for quality. 
Figure 41: Dimension of price-quality relation in the German sample

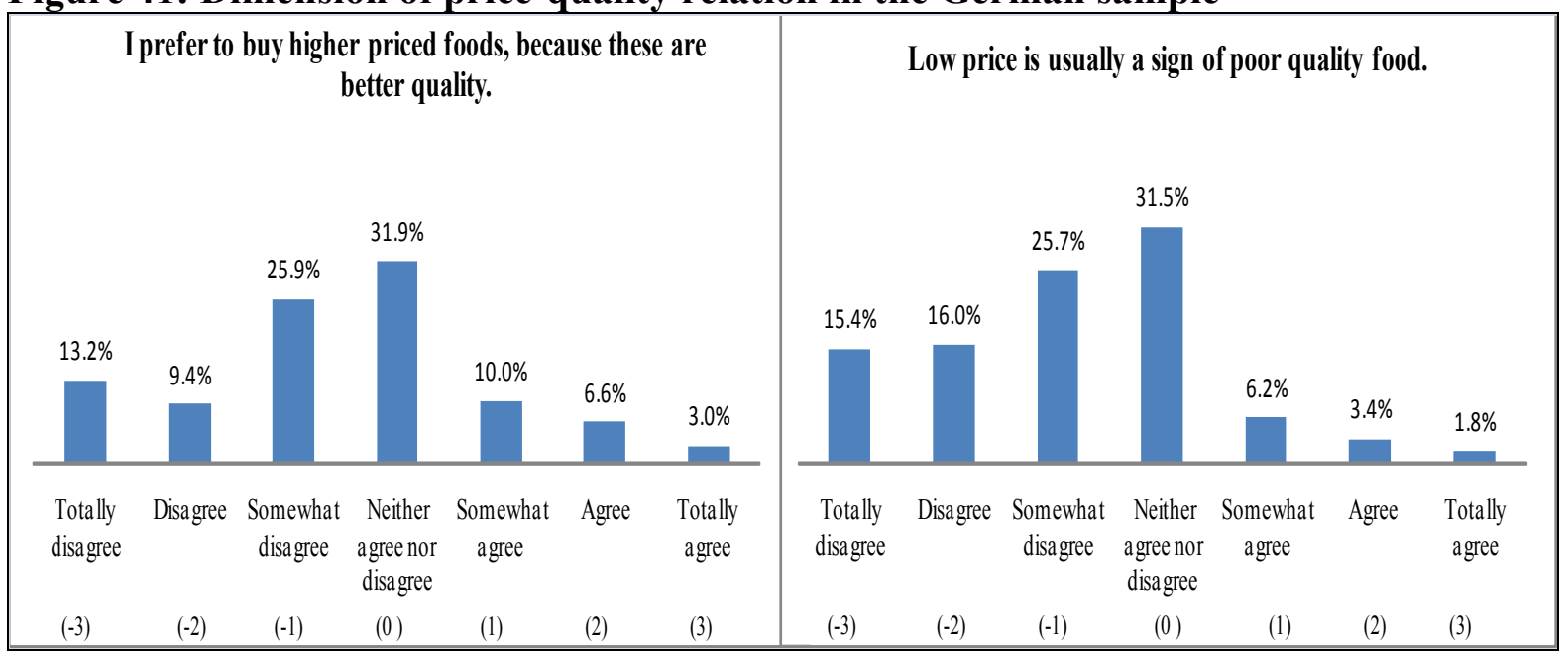

Source: Own estimation (2009).

\section{Mexican sample:}

Figure 42 displays the frequency of responses to the statement: "I prefer to buy higher priced foods, because these are better quality" as follows: $11.6 \%$ "totally disagree"; $10.8 \%$ "disagree"; 17.9\% "somewhat disagree"; 23.1\% "neither agree nor disagree"; similarly, 21.1\% "somewhat agree"; $10.1 \%$ "agree"; and 5.3\% "totally agree", respectively. As for the previous statement low prices are not associated with lacking of quality.

Regarding the statement: "Low price is usually a sign of poor quality food" the frequency of responses in Figure 42 are: 7.3\% "totally disagree"; 11.8\% "disagree"; 18.3 "somewhat disagree"; 22.7\% "neither agree nor disagree"; 16.6\% "somewhat agree"; 12\% "agree"; and $11.2 \%$ "totally agree," respectively (see Figure 42). In contrast to Germans, Mexicans provide more normally distributed responses. Moreover, since 21.1\% of Mexicans "somewhat agree", it seems that higher prices do seem to signal better quality, at least for a part of consumers. 
Figure 42: Dimension of price-quality relation in the Mexican sample

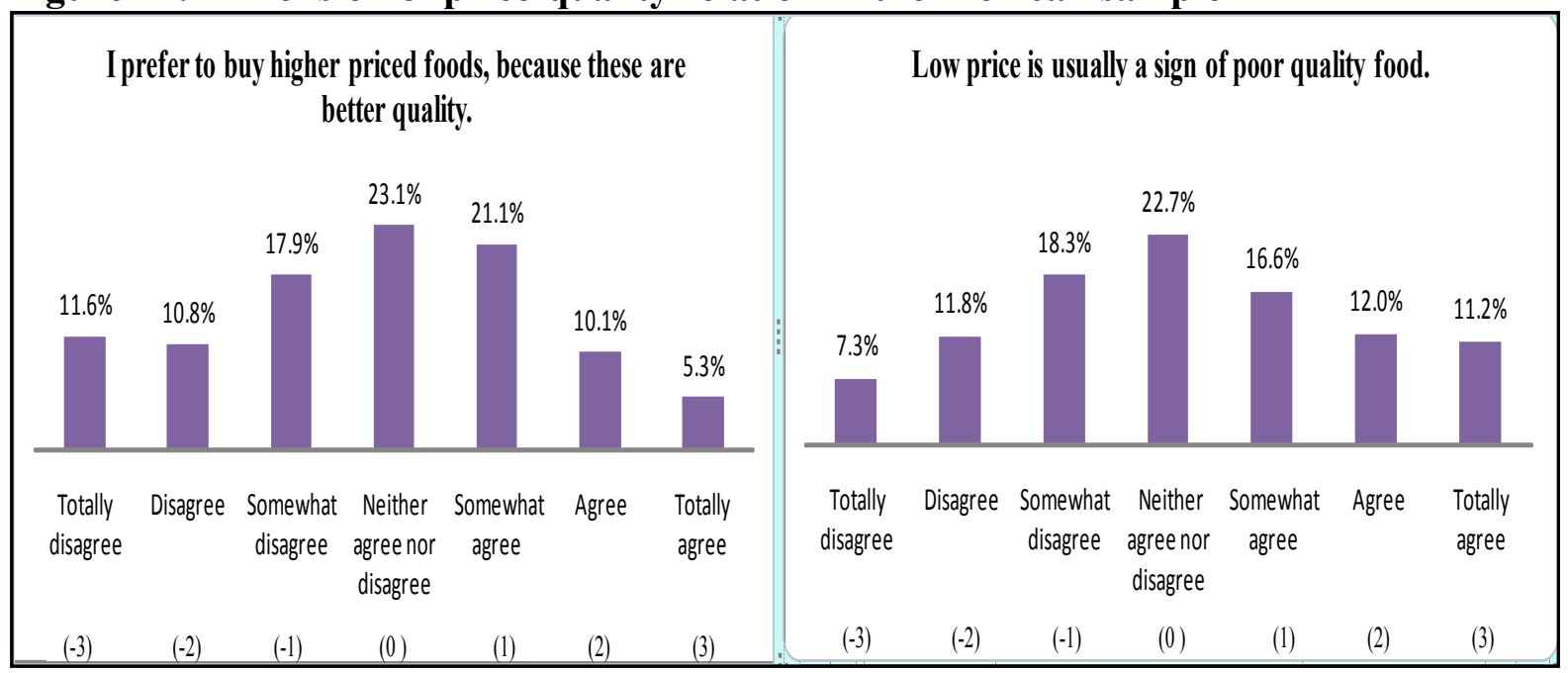

Source: Own estimation (2009).

\subsubsection{Price orientation}

\section{German sample:}

To measure the dimension of price orientation, the statement: "Before buying food, I always check the price" was used. Figure 43 indicates the frequency of responses which are: $1 \%$ "totally disagree"; similarly, $0.6 \%$ "disagree"; $4 \%$ "somewhat disagree"; $23.4 \%$ "neither agree nor disagree"; 18\% "somewhat agree"; 25.3\% "agree"; and 27.7\% "totally agree", respectively. The majority of responses are concentrated in the "somewhat-to-totally agree" range. Since the country is a rich one the responses indicate a high level of parsimony among Germans.

Regarding the statement: "The majority of our income goes toward the purchase of food". 10\% "totally disagree"; $11.6 \%$ "disagree"; $22.4 \%$ "somewhat disagree"; $34.5 \%$ "neither agree nor disagree"; $8.2 \%$ "somewhat agree"; similarly, 8\% "agree"; and 5.4\% "totally agree," respectively (see Figure 43). The higher number of responses placed in the "somewhat-tototally disagree" range in comparison to the opposite category confirm the high standard of living of the German population. 
Figure 43: Dimension of price orientation in the German sample

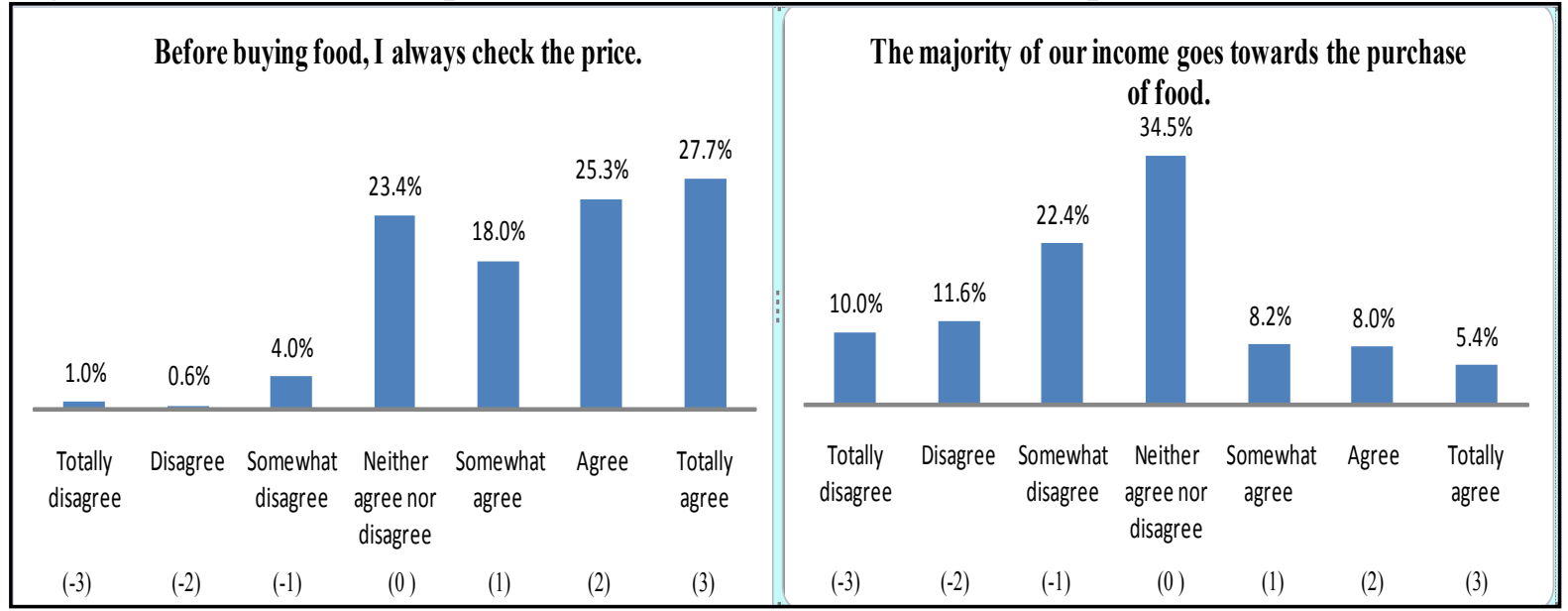

Source: Own estimation (2009).

\section{Mexican sample:}

The frequency of responses to the statement: "Before buying food, I always check the price". in Figure 44 is: $2.8 \%$ "totally disagree"; similarly, 2.6\% "disagree"; $4.7 \%$ "somewhat disagree"; 16.2\% "neither agree nor disagree"; 25.4\% "somewhat agree"; $24.5 \%$ "agree"; and similarly, 23.9\% "totally agree," respectively. The majority of responses are concentrated in the "somewhat to totally agree" range. Because of the lower income of Mexicans in comparison to Germans, these data are more easily explained by a higher level of penuriousness of resources rather than by a parsimony tendency as in the case of Germans.

The frequency of responses to the statement: "The majority of our income goes toward the purchase of food" is: 3\% "totally disagree"; 5.1\% "disagree"; $9.5 \%$ "somewhat disagree"; $15.4 \%$ "neither agree nor disagree"; 27\% "somewhat agree"; $21.1 \%$ "agree"; and 18.9\% "totally agree", respectively (see Figure 44). In comparison to the German sample, the findings are mirror-inverted: in fact, in the Mexican sample the higher number of responses is placed in the "somewhat-to-totally agree" range. This mirrors the lower standard of living of the Mexican population and endorses the response pattern of the previous statement. 
Figure 44: Dimension of price orientation in the Mexican sample

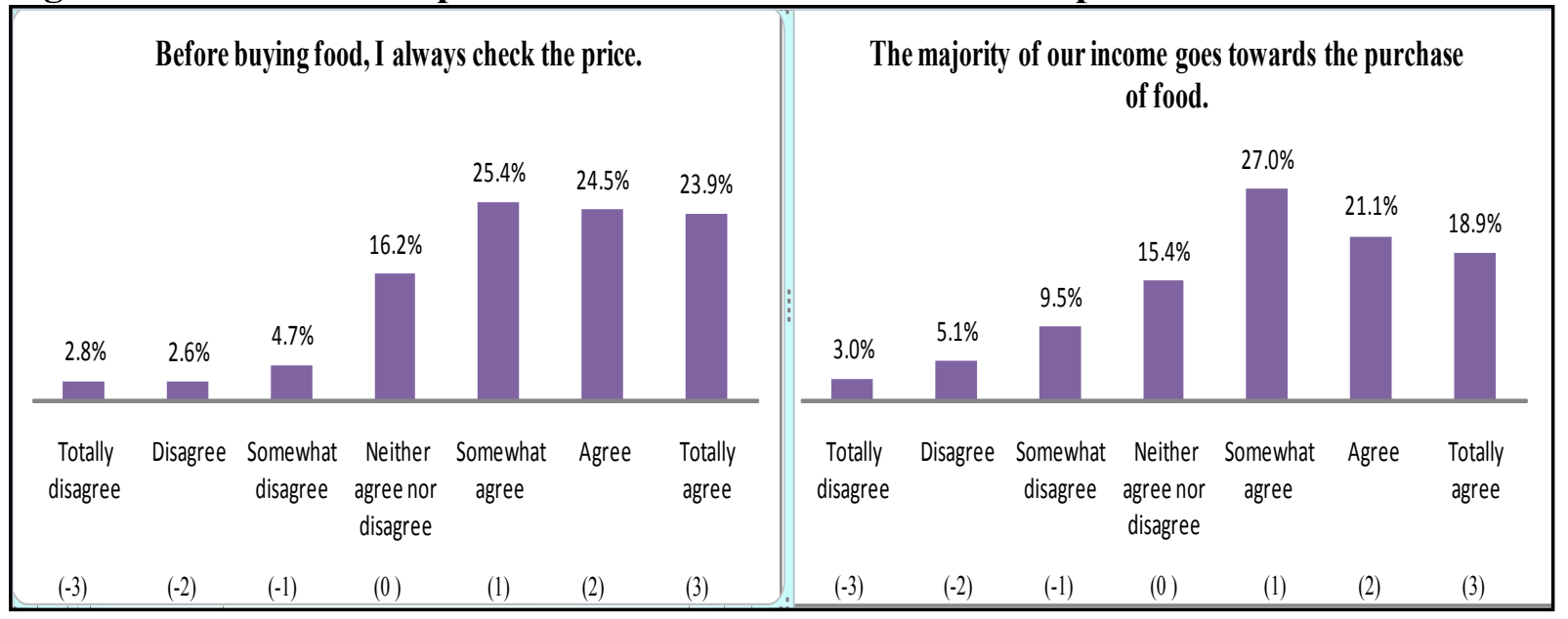

Source: Own estimation (2009).

\subsubsection{Consumer ethnocentrism}

\section{German sample:}

In measuring the dimension of consumer ethnocentrism, the statement: "I prefer fruit and vegetables from my home country" was used. Figure 45 displays the frequency of responses which is: $3.8 \%$ "totally disagree"; similarly, 3\% "disagree"; $8.6 \%$ "somewhat disagree"; $27.9 \%$ "neither agree nor disagree"; $14.2 \%$ "somewhat agree"; $22.4 \%$ "agree"; and 20.2\% "totally agree", respectively. The higher concentration of responses among Germans in the "somewhat-to-totally agree" area (56\%) indicates a high level of "ethnocentrism".

The frequency of responses to the statement: "I avoid products for which I do not know the country of origin" displayed in Figure 45 is: $8 \%$ "totally disagree"; $6.8 \%$ are "disagree"; 14.4\% "somewhat disagree"; 29.3\% "neither agree nor disagree"; 15.2\% "somewhat agree"; 13\% "agree"; and similarly, 13.4\% "totally agree," respectively. The normal distribution of the responses and the prevalence of responses in the "somewhat-to-totally agree" (41\%) range in contrast to the opposite category, shows a light degree of trustless toward products coming from outside Germany. 
Figure 45: Dimension of consumer ethnocentrism in the German sample

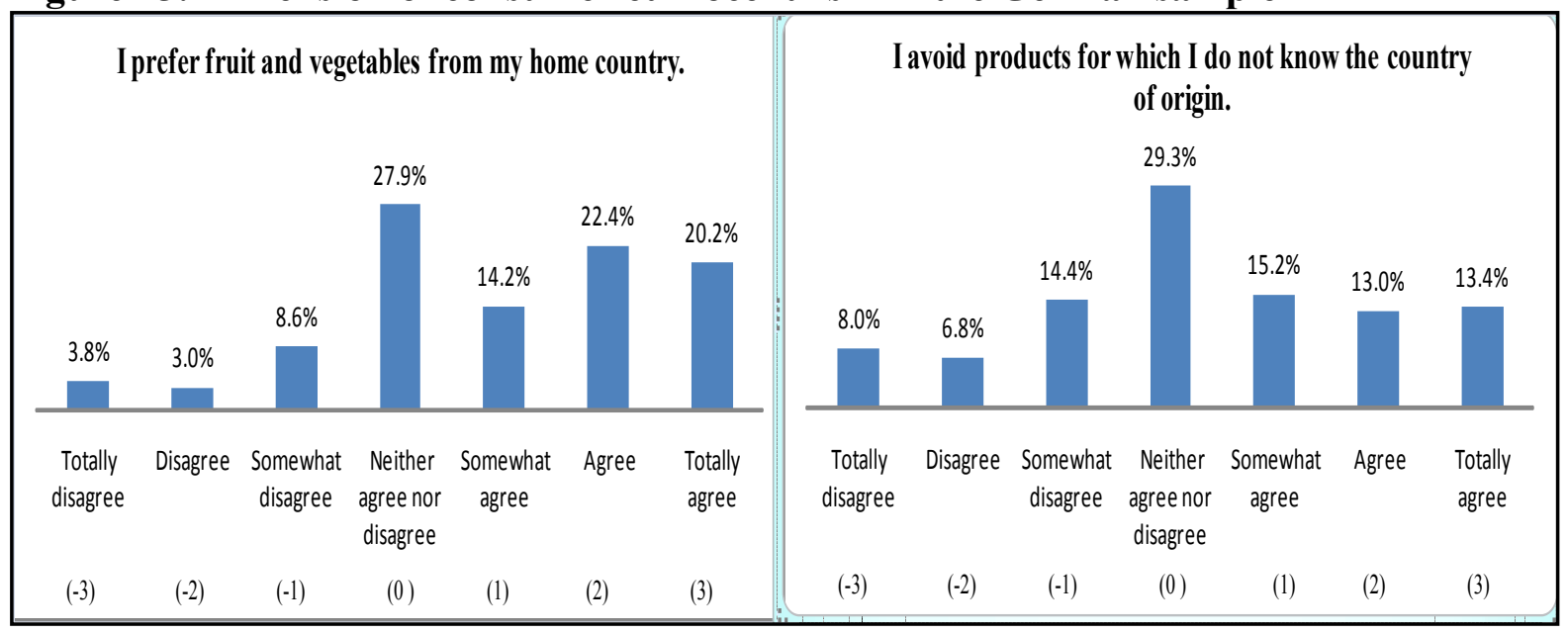

Source: Own estimation (2009).

\section{Mexican sample:}

Figure 46 provides an overview of the frequency of responses to the statement: "I prefer fruit and vegetables from my home country" as follows: $0.4 \%$ "totally disagree"; similarly, $0.8 \%$ "disagree"; 1.4\% "somewhat disagree"; $8.3 \%$ "neither agree nor disagree"; 10.7\% "somewhat agree"; $32.1 \%$ "agree"; and 46.4\% "totally agree", respectively. The higher concentration of responses among Mexicans in the "somewhat-to-totally agree" area (88\%) indicates a higher level of consumer ethnocentrism among the Mexicans than Germans.

The responses to the statement: "I avoid products for which I do not know the country of origin" also displayed in Figure 46 are as follow: 4.9\% "totally disagree"; similarly, 5.1\% "disagree"; 9.7\% "somewhat disagree"; 26.6\% "neither agree nor disagree"; 15.6\% "somewhat agree"; 18.1\% "agree"; similarly, 19.9\% "totally agree", respectively. The normal distribution of the responses and the prevalence of responses in the "somewhat-to-totally agree" range (53\%) in contrast to the opposite category, shows also among Mexicans a light tendency to disdain products coming from outside Mexico however it is higher than among the Germans. 
Figure 46: Dimension of consumer ethnocentrism in the Mexican sample

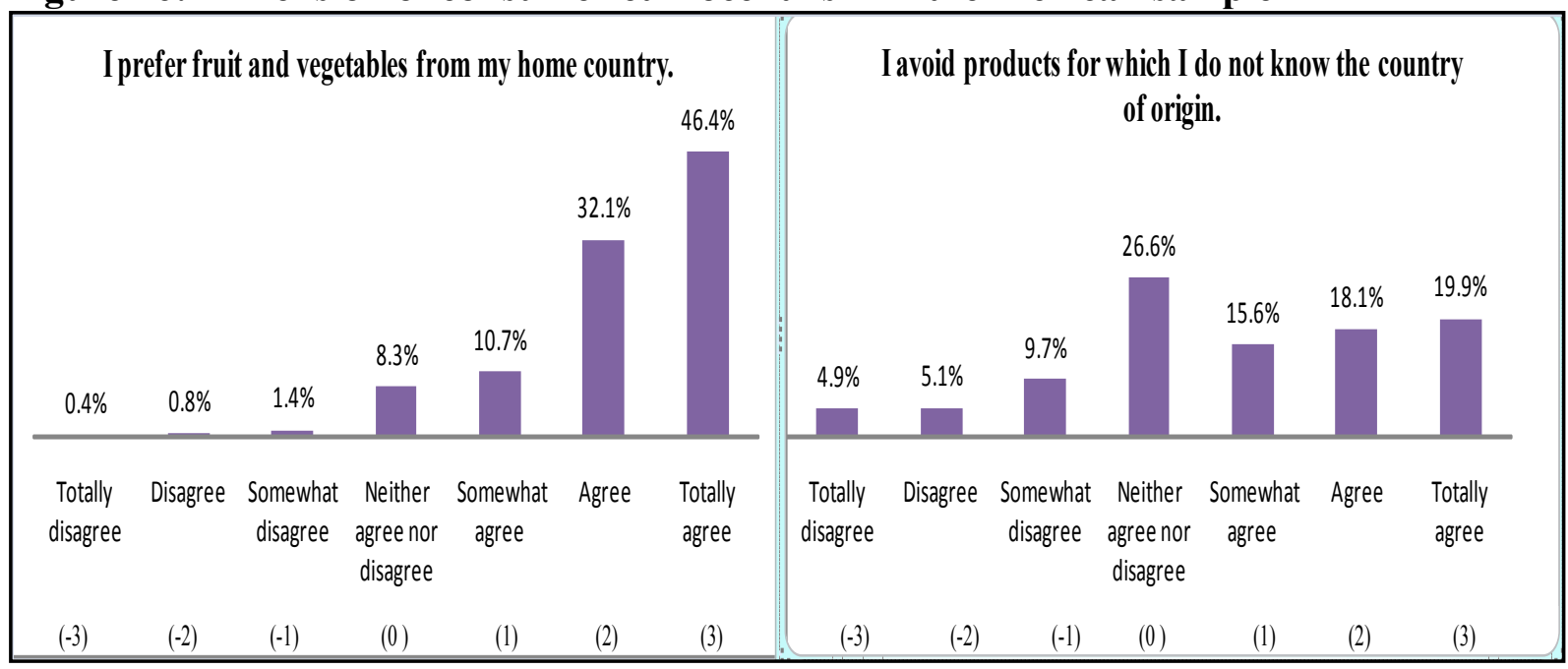

Source: Own estimation (2009).

\subsubsection{Malinchismo of food (preference for foreign)}

The measuring of the dimension of malinchismo of food (referred as the preference for foreign food products) was restricted to the Mexican sample. The statement: "Most of the time I have the impression that foreign products are better than Mexican products" was used. Figure 47 provides an overview of the frequency of responses described as follows: $12.8 \%$ "totally disagree"; 13.4\% "disagree"; 17.9\% "somewhat disagree"; $23.9 \%$ "neither agree nor disagree"; 16.4\% "somewhat agree"; 9.7\% "agree"; and 5.9\% "totally agree," respectively. In contrast to the responses provided within the dimension of ethnocentrism above described the higher concentration of responses in the "neither agree nor disagree-to-totally agree" area indicates a receptiveness for foreign goods and imitation of lifestyle as it was suggested by a market analysis for the food retailing sector by the USDA (2002). 
Figure 47: Dimension of malinchismo of food in the Mexican sample

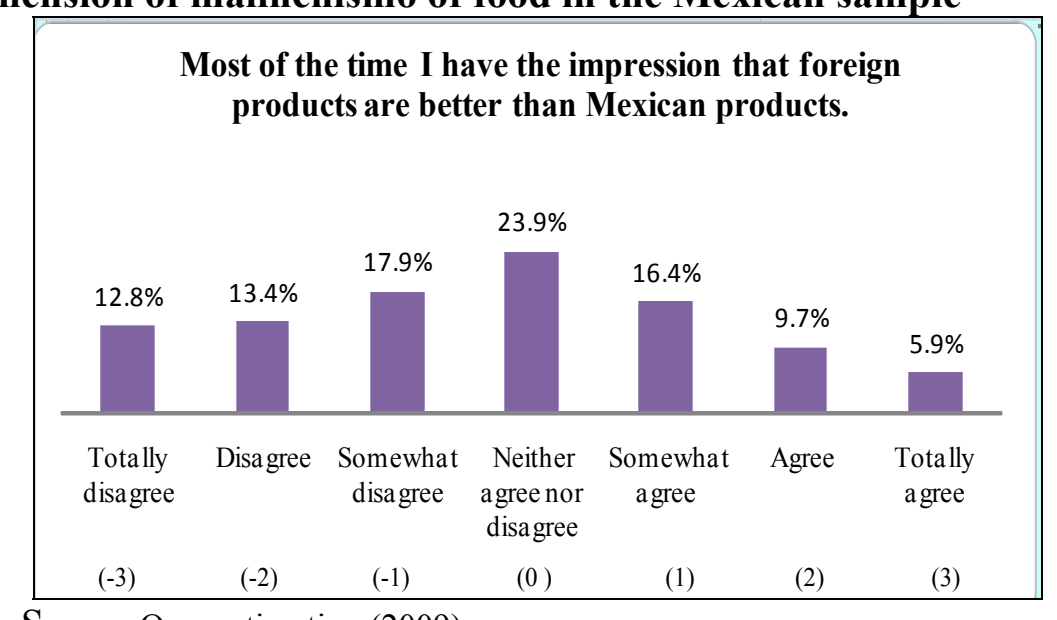

Source: Own estimation (2009).

\subsection{Frequency of responses of values in the German and Mexican samples}

To provide cultural differences, we asked about some attitudes in order to infer values between responses from the two countries compared Mexican and German respondents. In following will be displayed the most interesting frequencies of the responses provided for the statements regarding some attitudes in order to infer differences on values between the two national cultures.

\subsubsection{Technological progress/Technophobia}

\section{German sample:}

To measure the dimension of technological progress and technophobia, was used the statement: "I worry that technological progress will destroy our lives". resulted in the following distribution: $6.6 \%$ "totally disagree"; similarly, $6.2 \%$ "disagree"; $22.8 \%$ "somewhat disagree"; 34.7\% "neither agree nor disagree"; 12.2\% "somewhat agree"; 9.2\% "agree"; $6.8 \%$ "totally agree"; and $1.6 \%$ of the respondents did not provide a response and are therefore categorized as "missing values", respectively. 
The frequency of responses to the statement: "Computers and other electronic devices are funny" displayed in Figure 48 are described as follows: $1.8 \%$ "totally disagree"; $0.4 \%$ "disagree"; $2.4 \%$ "somewhat disagree"; $24.4 \%$ "neither agree nor disagree"; $17 \%$ "somewhat agree"; $23.6 \%$ "agree"; and 29.3\% "totally agree," respectively.

The higher concentration of the responses provided for the first statement in the "somewhat agree-neither agree nor disagree" area for the first statement and "neither agree nor disagreetotally agree" area for the second statement show a positive tendency to appreciate the advance of technology and enjoy technology this fact mirrors technophilia within the Germans.

\section{Figure 48: Dimension of technological progress/technophobia in the German sample}

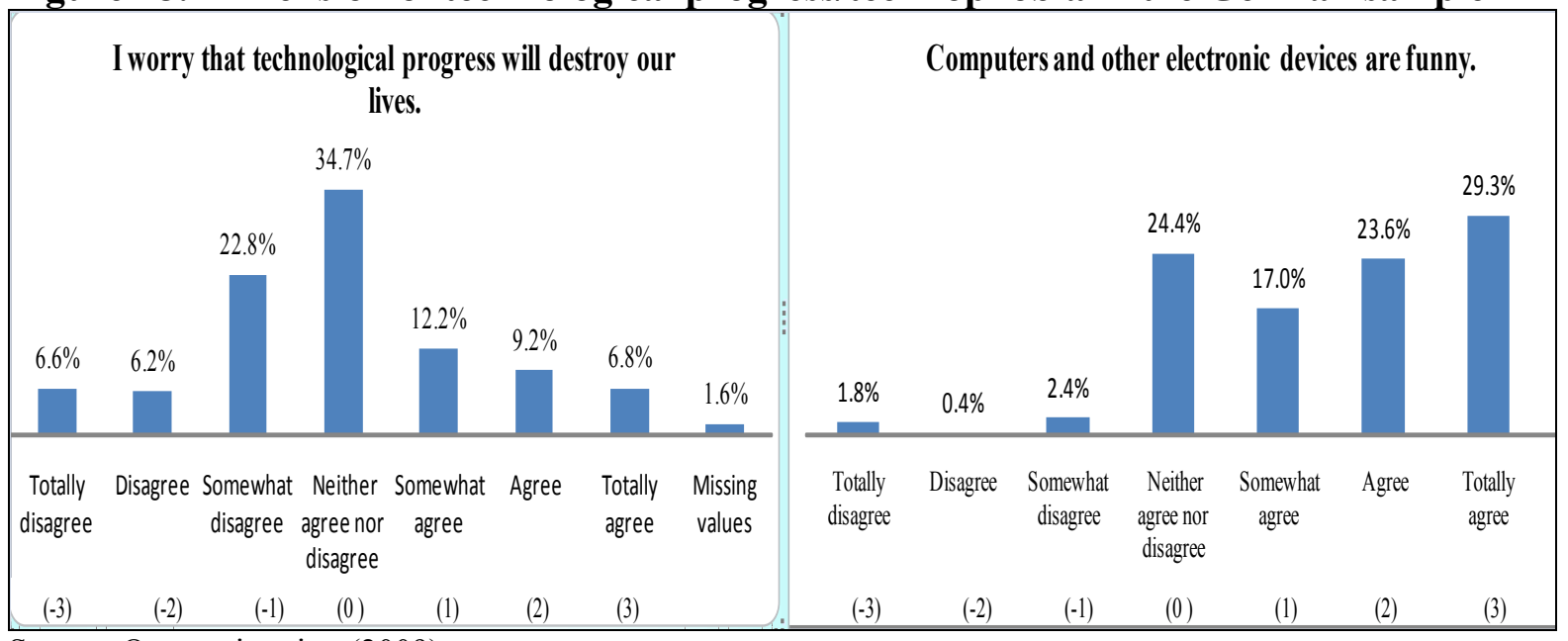

Source: Own estimation (2009).

\section{Mexican sample:}

The frequency of responses to the statement: "I worry that technological progress will destroy our lives". In Figure 49 is described as: 10.3\% "totally disagree"; 13.4\% "disagree"; 11.2\% "somewhat disagree"; $14.6 \%$ "neither agree nor disagree"; $18.1 \%$ "somewhat agree"; $15.8 \%$ "agree"; and similarly, $16.6 \%$ "totally agree," respectively. There is not a concentration of the responses in area this means that among the Mexican population there are different opinions about the technological progress.

The frequency of responses to the statement: "Computers and other electronic devices are funny". In Figure 49 is described as follows: 1\% "totally disagree"; $0.8 \%$ "disagree"; 1.6\% 
"somewhat disagree"; $8.9 \%$ "neither agree nor disagree"; $21.5 \%$ "somewhat agree"; 33.5\% "agree" and similarly $32.7 \%$ of the respondents "totally agree" respectively. Regarding enjoy of technology most of the responses are concentrated in the "neither agree nor disagree-totally agree" area. In contrast to Germans who show a tendency to "technophilia". Mexicans do not know if technological progress will destroy their life "technophobia" whereas enjoy technology "technophilia". This seems as a dualism however gender and age might be considered as demographic variables influencing this fact.

\section{Figure 49: Dimension of technological progress/technophobia in the Mexican sample}

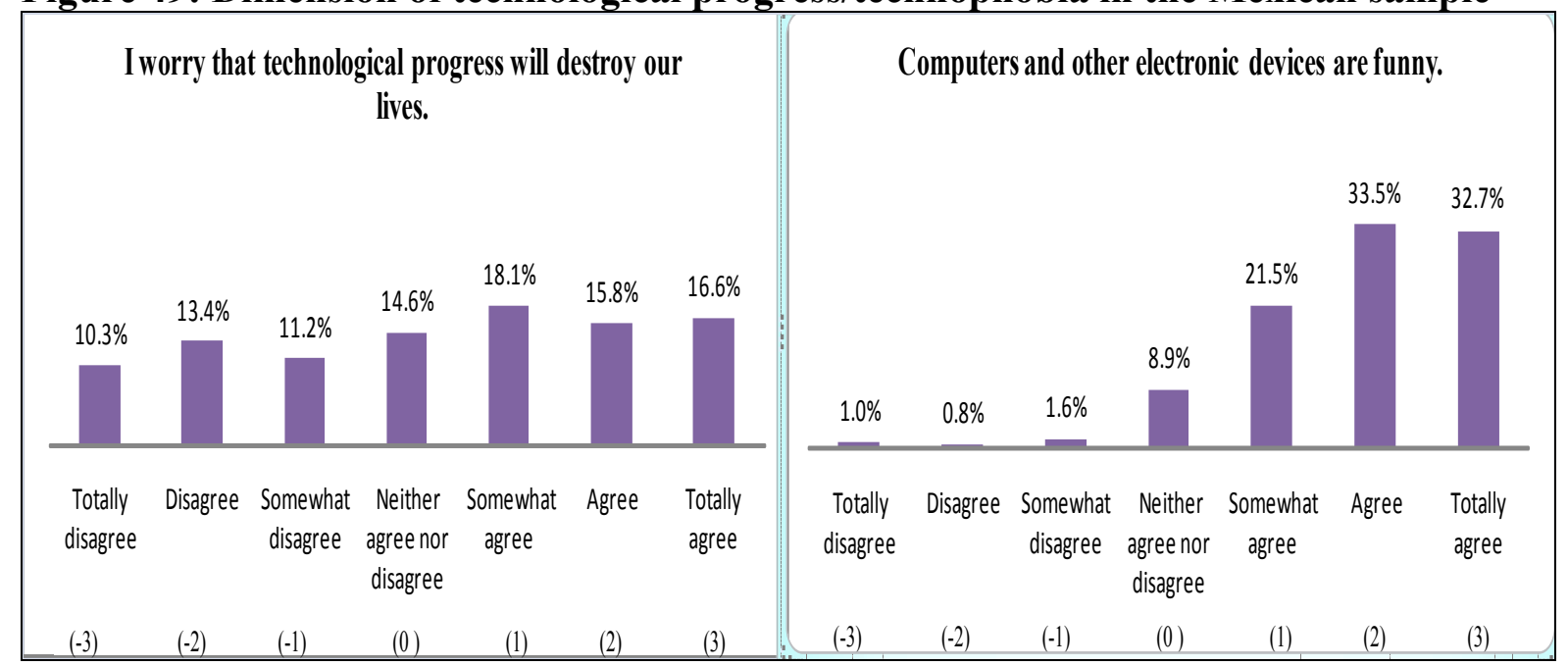

Source: Own estimation (2009).

\subsubsection{Fatalism}

\section{German Sample:}

To measure the dimension of fatalism, the statement: "We can't stop climate change". was used. Figure 50 shows the frequency of responses provided and is described in the following: $18 \%$ "totally disagree"; $15.2 \%$ "disagree"; 16.6\% "somewhat disagree"; most (30.7\%) "neither agree nor disagree"; 7.4\% "somewhat agree"; 5.6\% "agree"; similarly, 5\% "totally agree"; and $1.6 \%$ were categorized as "missing values", respectively.

The frequency of responses provided for the statement: "It will not change anything if I reduce the garbage I produce, because no one else cares about the environment". is also shown in Figure 50 and is described as follows: 12\% "totally disagree"; 13.2\% "disagree"; 
15.4\% "somewhat disagree"; most (28.3\%) "neither-nor"; $11.6 \%$ "somewhat agree"; 7\% "agree"; $11.4 \%$ "totally agree"; and 1.2\% were categorized as "missing values", respectively. The normal distribution of the responses and the prevalence of responses in the "neither nor disagree-totally disagree" for the two statements shown that Germans are concern with environment.

\section{Figure 50: Dimension of Fatalism in the German sample}

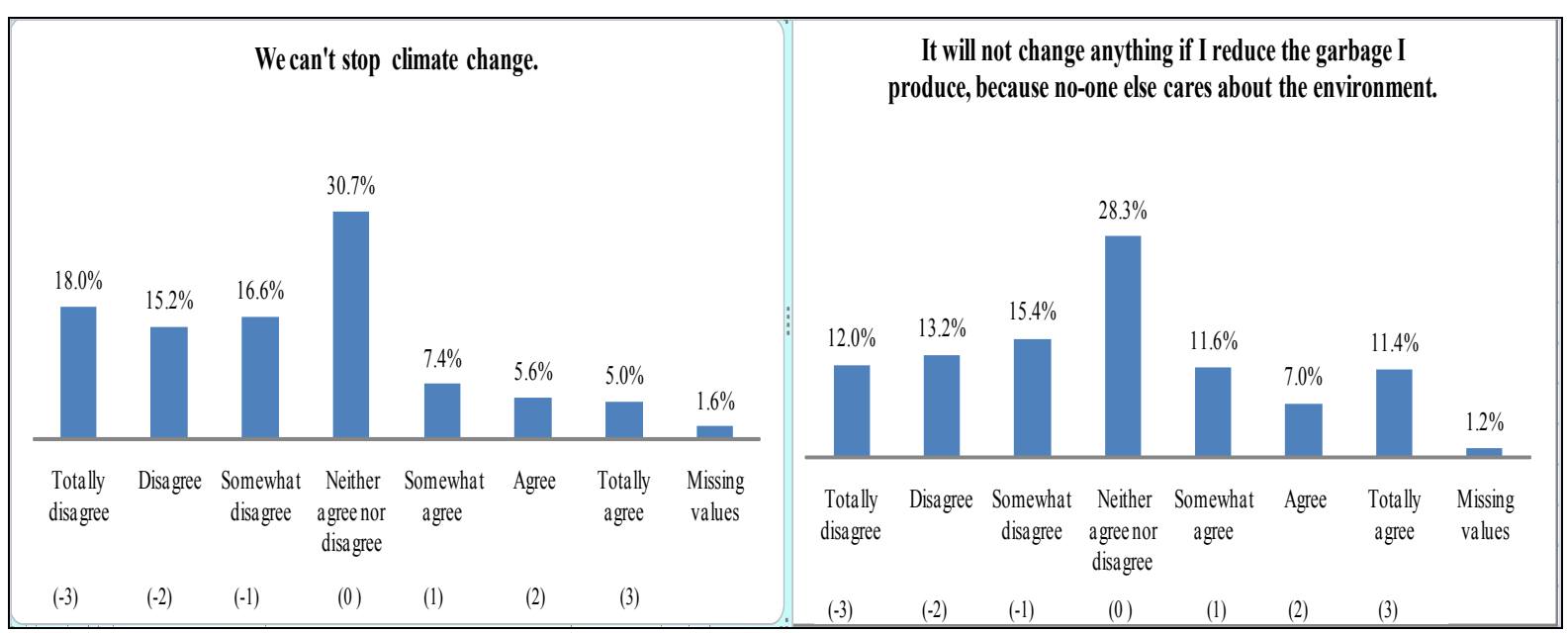

Source: Own estimation (2009).

\section{Mexican Sample:}

Regarding the frequency of responses to the statement: "We can't stop climate change". Figure 51 depicts the percentages, that is: most of the respondents $(37.3 \%)$ "totally disagree"; 20.3\% "disagree"; $15 \%$ "somewhat disagree"; 5.3\% "neither agree nor disagree"; $6.1 \%$ "somewhat agree"; 7.9\% "agree"; similarly, 8.1\% "totally agree" respectively.

The frequency of responses to the second statement: "It will not change anything if I reduce the garbage I produce, because no one else cares about the environment". Figure 51 is described as follows: most of the respondents (35.5\%) "totally disagree"; $19.9 \%$ "disagree"; 10.5\% "somewhat disagree"; $4.9 \%$ "neither agree nor disagree"; $8.9 \%$ "somewhat agree"; 7.7\% "agree"; and 12.6\% "totally agree", respectively. The higher concentration of responses in the "totally disagree-somewhat disagree" area for the two statement indicates that Mexicans are concern with environment similarly to Germans. 


\section{Figure 51: Dimension of Fatalism in the Mexican sample}

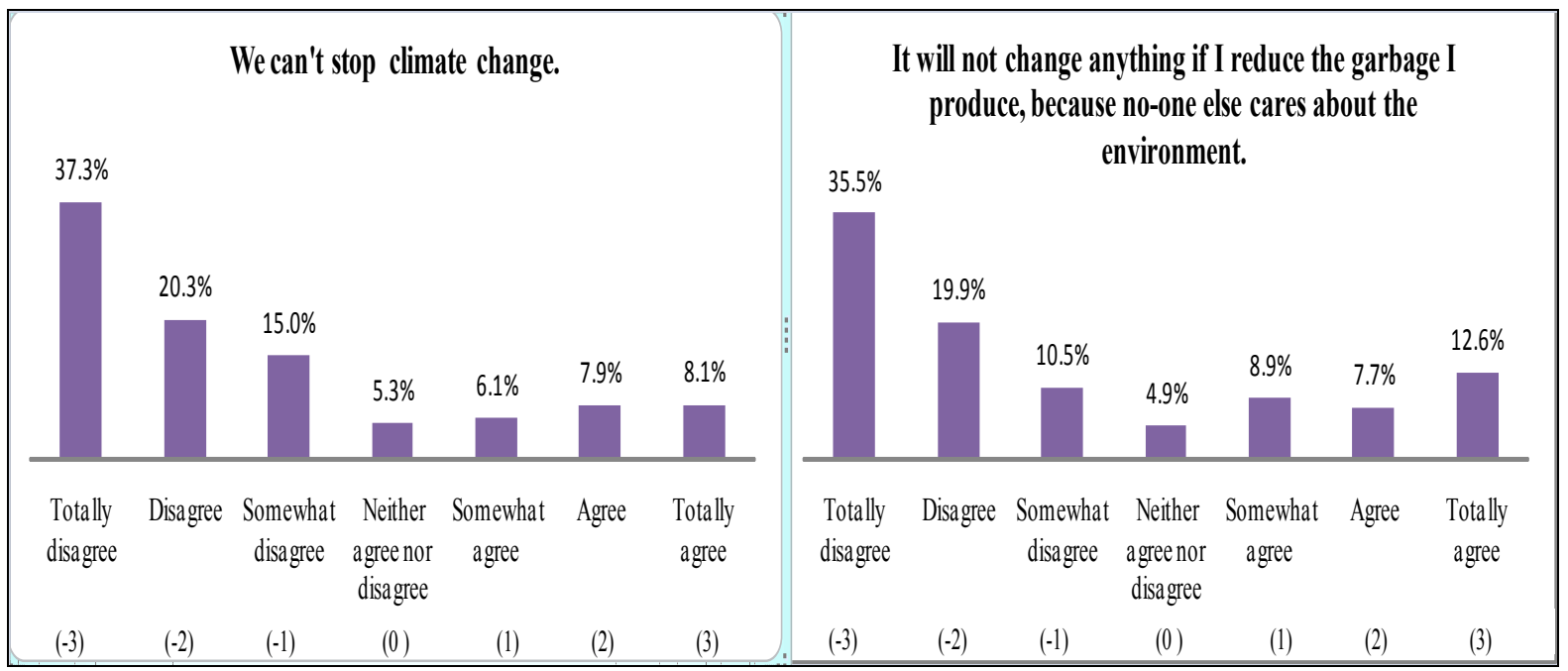

Source: Own estimation (2009).

\subsubsection{Fairness}

\section{German Sample:}

To measure the dimension of fairness, the statement: "Farmers are the basis of our wellbeing" was used. The frequency of responses in Figure 52 is described in the following: 2.6\% "totally disagree"; 4.4\% "disagree"; 11.2\% "somewhat disagree"; $41.3 \%$ "neither agree nor disagree"; 17\% "somewhat agree"; 11.4\% "agree"; 10.6\% "totally agree"; and 1.6\% are categorized as "missing values", respectively. The percentage of Germans believing than Farmers are the basis of well-being is higher due to the concentration of the responses provided within the categories of "somewhat agree-to-totally agree" in contrast to the responses within the opposite categories in the left side.

The frequency of responses provided for the statement: "Every individual is responsible for their own well-being in this country" in Figure 52 is described as: 9.8\% "totally disagree"; 17\% "disagree"; 22\% "somewhat disagree"; $32.1 \%$ "neither agree nor disagree"; $9.6 \%$ "somewhat agree"; $3.8 \%$ "agree"; $4.2 \%$ "totally agree"; and 1.6\% are categorized as "missing values," respectively. 
The normal distribution of the responses and the prevalence of responses in the "neither agree nor disagree-totally agree" range in contrast to the opposite category, show a positive attitude among the Germans to belief in the opportunities of everyone to have a good quality of life and improve their social class. This is explained by the fact that the national German culture scored low (31 points) in the Power distance index and higher in the dimension of individualism (63 points) in the measurement by Hofstede (2001). Similarly, the German culture tends to be in the dimension of egalitarianism according to Schwartz (2006).

\section{Figure 52: Dimension of fairness in the German sample}

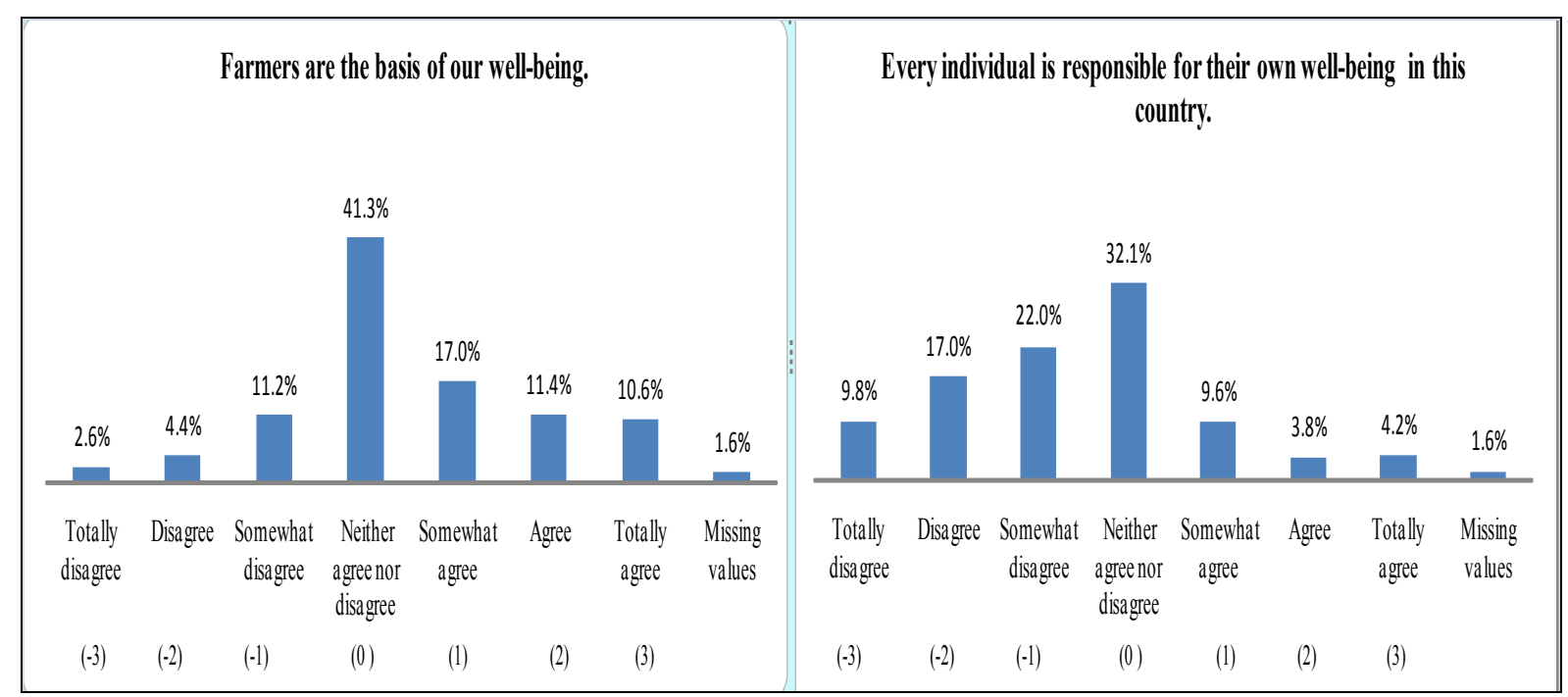

Source: Own estimation (2009).

\section{Mexican Sample:}

The frequency of responses to the statement "Farmers are the basis of our well-being" in Figure 53 is described as follows: $1.2 \%$ "totally disagree", $0.8 \%$ "disagree"; similarly, $2.6 \%$ "somewhat disagree"; $10.7 \%$ "neither agree nor disagree"; $23.1 \%$ "somewhat agree"; $29 \%$ “agree"; and 32.7\% "totally agree," respectively.

Figure 53 also depicts the frequency of responses to the statement: "Every individual is responsible for their own well-being in this country" described as follows: $31.6 \%$ "totally disagree"; similarly, 26.2\% “disagree"; 23.3\% "somewhat disagree"; similarly, $8.5 \%$ "neither agree nor disagree"; 6.1\% "somewhat agree"; 3\% "agree"; similarly, 1.2\% "totally agree"; and $0.2 \%$ are categorized as "missing values", respectively. In contrast to the German sample, 
the percentage of Mexicans recognizing farmers as basis of welfare is higher. Due to the percentage concentrated in the category of "somewhat agree-to totally agree".

Similarly to Germans, Mexicans do not agree whit the fact that every individual is responsible for their well-being due to the higher percentage of the responses concentrated within the categories of "somewhat disagree to totally disagree". This fact is also explained by the high score of the Mexican national culture in the index of power distance (81 points) by Hofstede (2001) and the dimension of hierarchy by Schwartz (2006).

\section{Figure 53: Dimension of fairness in the Mexican sample}

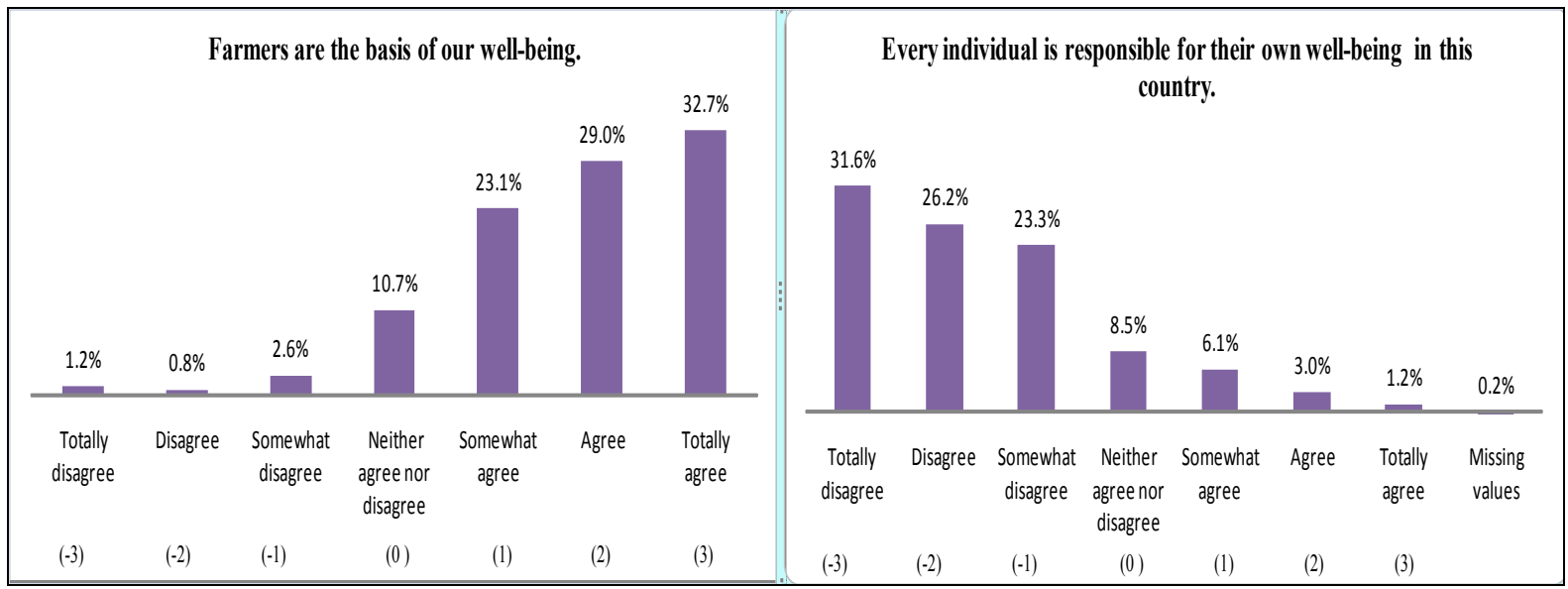

Source: Own estimation (2009).

\subsubsection{Materialism}

\section{German sample:}

In the measuring of the dimension of materialism, was used the statement: "It is important to me have a lot of money and expensive things". The frequency of responses in Figure 54 is described as follows: $17 \%$ "totally disagree"; $14.8 \%$ "disagree"; $24 \%$ "somewhat disagree"; 27.3\% "neither agree nor disagree"; $8.6 \%$ "somewhat agree"; 4\% "agree"; $3.2 \%$ "totally agree"; and $1.2 \%$ are categorized as "missing values", respectively. In the first statement the higher concentration of responses in the "neither agree nor disagree-to-totally disagree" area 
indicates that Germans reject accumulation of money and expensive things as important in life.

The frequency of responses to the statement: "I am unhappy because financially I can only afford very little" in Figure 54 is as follows: 4.6\% "totally disagree"; 9\% "disagree"; $12.2 \%$ "somewhat disagree"; $28.7 \%$ "neither agree nor disagree"; $12 \%$ "somewhat agree" and "agree"; $20.4 \%$ "totally agree"; and 1.2\% are categorized as "missing values", respectively (see Figure 54). However, the tendency to recognize financial reasons as source of unhappiness is not clear, due to the high concentration of the responses in the category of "neither agree nor disagree" as well as to high concentration in the category of "totally agree".

\section{Figure 54: Dimension of materialism in the German sample}

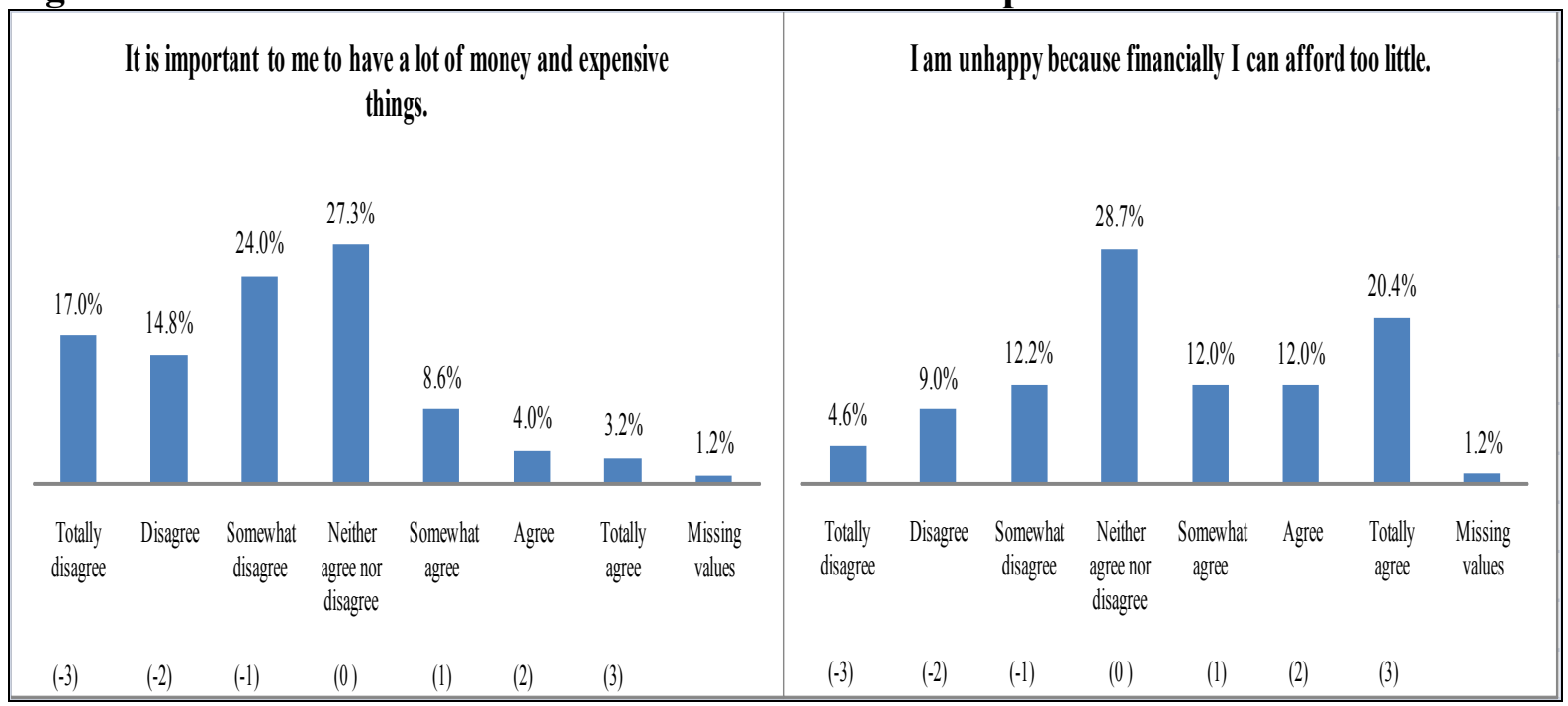

Source: Own estimation (2009).

\section{Mexican sample:}

Regarding the responses to the statement: "It is important to me to have a lot of money and expensive things". Figure 55 shows the frequency of responses and are described as: $20.9 \%$ "totally disagree"; 18.9\% "disagree"; 15.8\% "somewhat disagree"; 18.3\% "neither agree nor disagree"; $14.6 \%$ "somewhat agree"; $7.1 \%$ "agree"; and $4.3 \%$ "totally agree", respectively. Similarly to the German sample, most of the Mexicans recognize the the possession of expensive things and lot of money is not the most in important although the percentages concentrated in the "totally disagree-to-neither nor" area is higher than the provided by Germans. 
Figure 55 shows also the frequency of responses to the statement: "I am unhappy because financially I can afford too little" described in following: 7.3\% "totally disagree"; $14.2 \%$ "totally agree", "agree", and "disagree", respectively; 12.2\% "somewhat disagree;" 20.3\% "neither agree nor disagree"; and 17.6\% "somewhat agree" respectively. The percentages distributed between the "neither agree nor disagree-to-totally agree" area uncover the fact that Mexicans not recognize financial reasons as the ground for unhappiness however the distribution of the responses within the categories is different as the provided by Germans.

\section{Figure 55: Dimension of materialism in the Mexican sample}

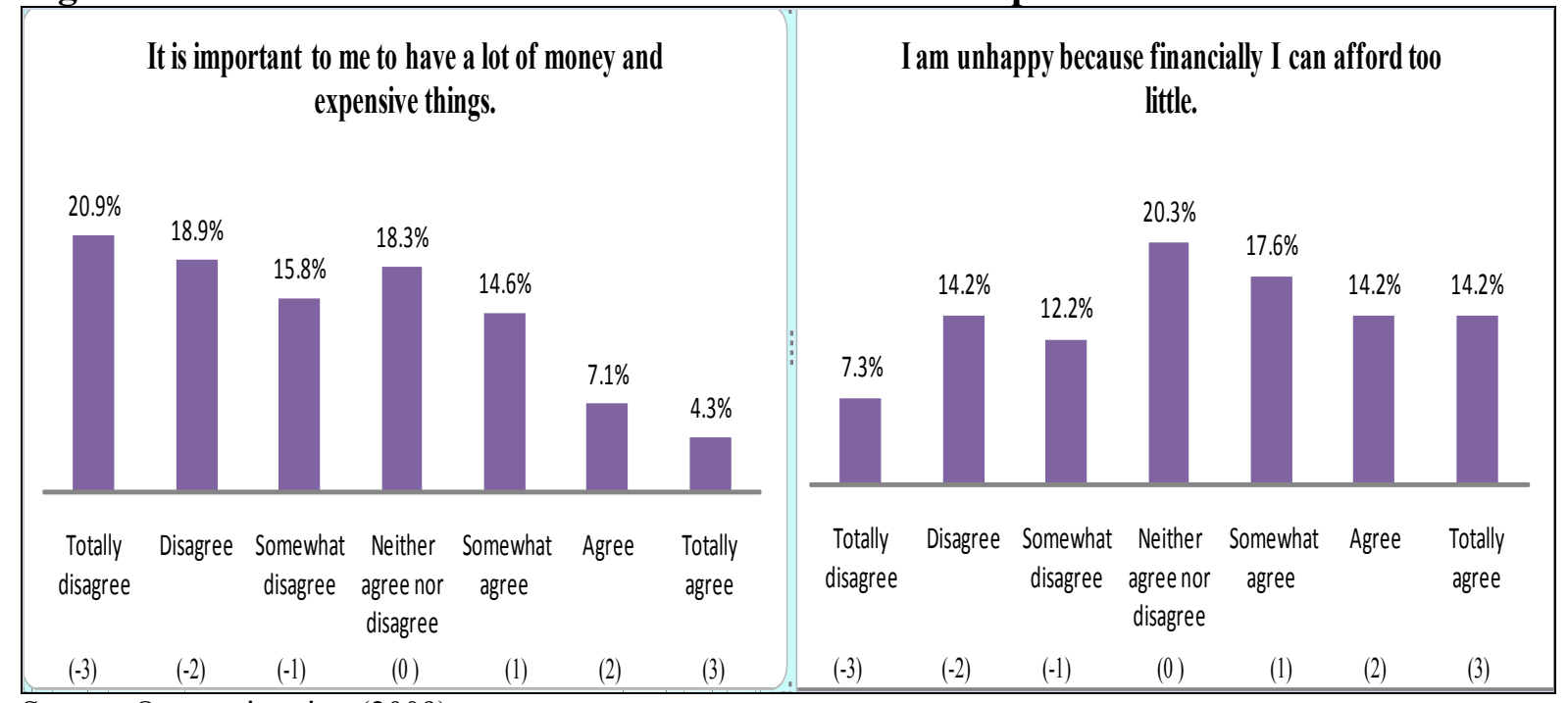

Source: Own estimation (2009).

\subsubsection{Variety in life/neophobia}

\section{German sample:}

In the measuring of the dimension of variety in life and neophobia was used the statement "I need variety in life". The frequency of responses in Figure 56 is described as: 3\% "totally disagree"; similarly, 3.8\% "disagree"; 13.8\% "somewhat disagree"; 37.9\% "neither agree nor disagree"; 20.4\% "somewhat agree"; 11.8\% "agree"; 7.8\% "totally agree"; and 1.6\% are categorized as "missing values" due to lack of response, respectively.

The frequency of responses to the statement: "Changes worry me" is: $6.2 \%$ "totally disagree"; 10.6\% "disagree"; $20.6 \%$ "somewhat disagree"; $37.3 \%$ "neither agree nor disagree"; 13\% 
"somewhat agree"; 4.8\% "agree"; and 6.4\% "totally agree", respectively (see Figure 56). The higher concentration of responses in the "neither agree nor disagree" category indicates a moderate tendency toward neophobia and neophilia within the Germans which in contrast show a preference of "status quo".

\section{Figure 56: Dimension of variety in life/neophobia in the German sample}

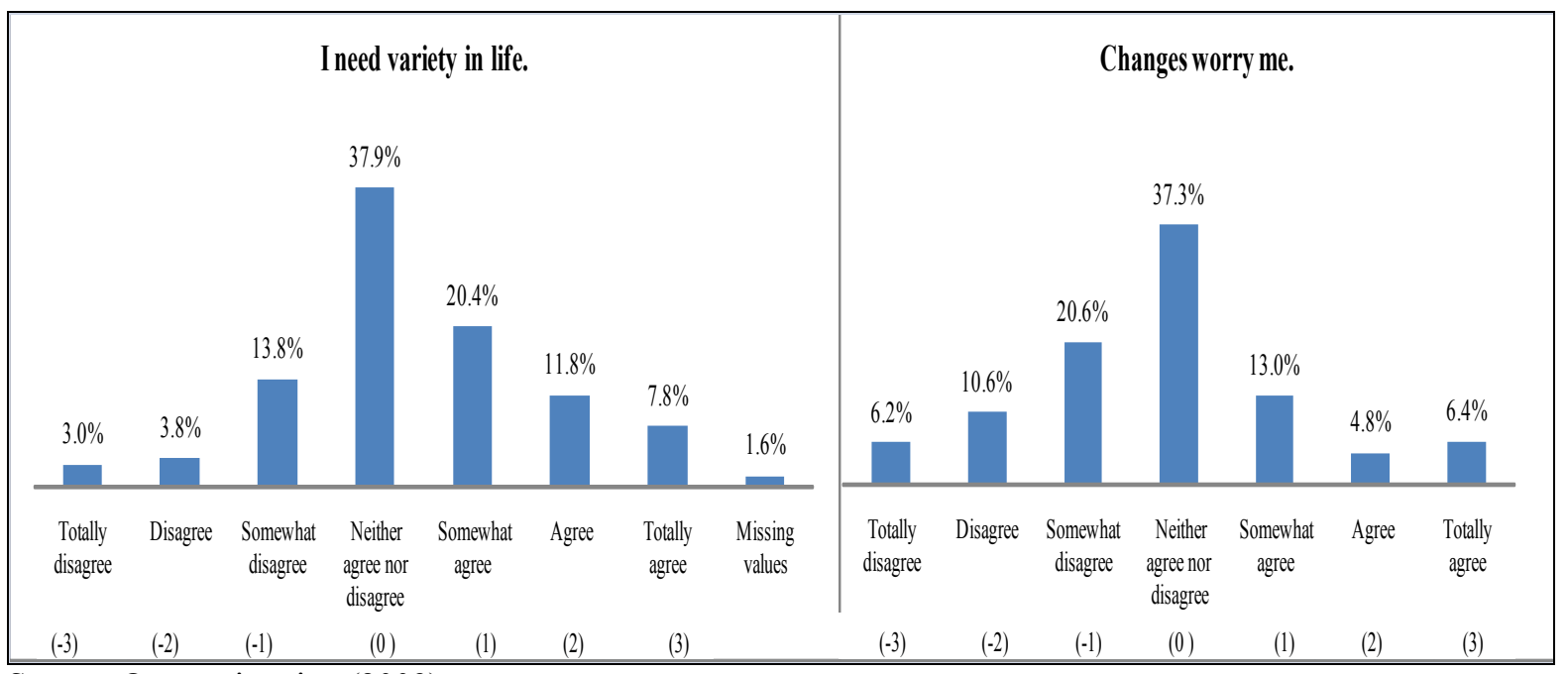

Source: Own estimation (2009).

\section{Mexican sample:}

Regarding the frequency of responses to the statement: "I need variety in life". Figure 57 describes the percentages as follows: $0.6 \%$ "totally disagree"; similarly, $1 \%$ "disagree"; $3 \%$ "somewhat disagree"; $11.6 \%$ "neither agree nor disagree;" $25.2 \%$ "somewhat agree"; $27.6 \%$ "agree"; $31 \%$ "totally agree", respectively. In contrast to the German sample, the high concentration of responses within the "neither agree nor disagree-totally agree" area show a more positive attitude toward trying new experiences, products, things, -neophilia- among the Mexicans.

The frequency of responses to the statement: "Changes worry me". In Figure 57 are described in the following: $12 \%$ "totally disagree"; $18.7 \%$ "disagree"; $13.2 \%$ "somewhat disagree"; 20.1\% "neither agree nor disagree"; 17.2\% "somewhat agree"; 9.7\% "agree"; and 9.1\% "totally agree," respectively. The variability of the responses provided for the second statement, shows an unclear tendency among the Mexicans about the worry by changes. As a 
result, -individual characteristics- personality, and some demographic variables such as gender, age, and incomes might explain this attitudes toward changes.

Figure 57: Dimension of variety in life/neophobia in the Mexican sample

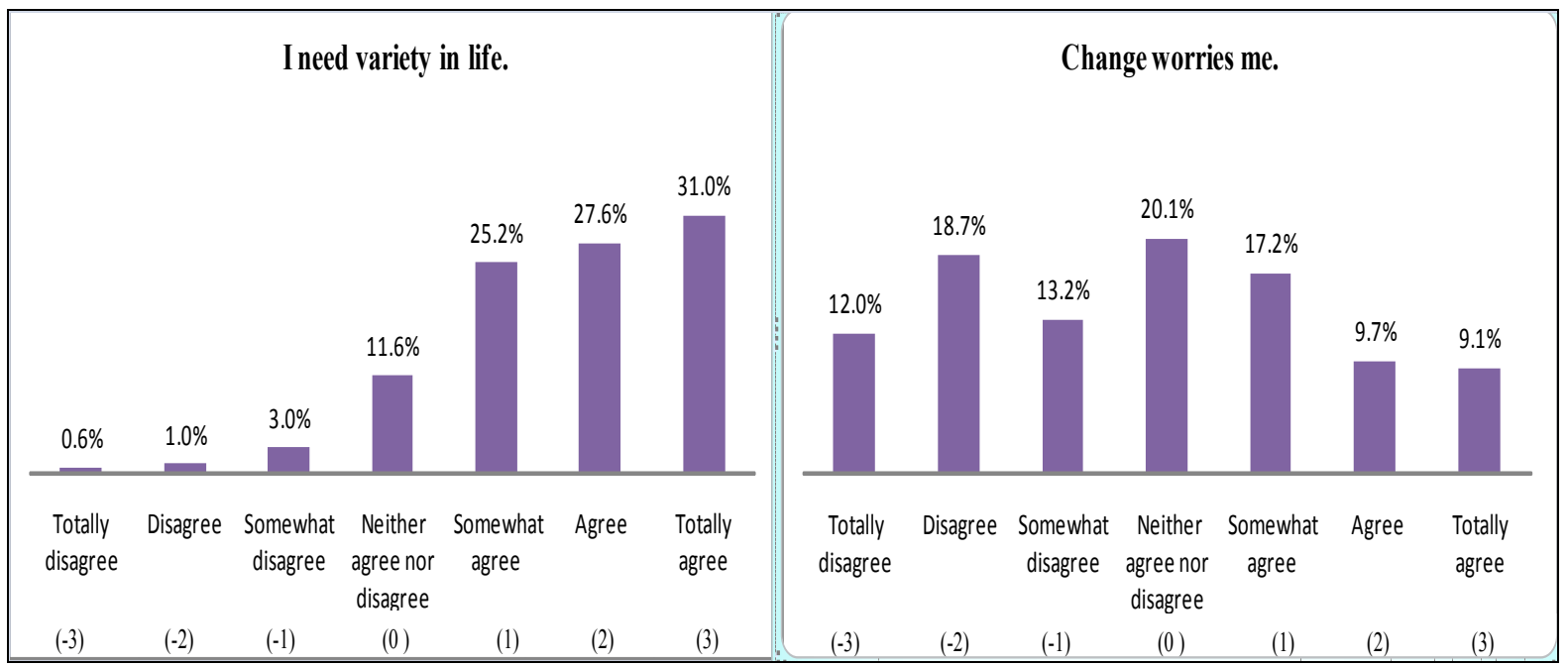

Source: Own estimation (2009).

\subsubsection{Hedonism}

\section{German sample:}

To measure the dimension of hedonism, was used the statement: "The most important thing in life is to have fun" Figure 58 displays the frequencies of the responses in the following described: $3.2 \%$ "totally disagree"; $6.2 \%$ "disagree"; $14 \%$ "somewhat disagree"; $33.5 \%$ "neither agree nor disagree"; 16.8\% "somewhat agree"; $14.4 \%$ "agree"; $10.4 \%$ "totally agree"; and $1.6 \%$ are categorized as "missing values" respectively.

The frequency of responses provided for the statement "For me the most important is to enjoy life and be pleasant to others" displayed in Figure 58 is described as: $10.8 \%$ "totally disagree"; 19.4\% "disagree"; 25\% "somewhat agree"; "somewhat disagree"; $31.5 \%$ "neither agree nor disagree"; $8.2 \%$ "somewhat agree"; $1.8 \%$ "agree" and 1.8\% "totally agree" respectively. The normal distribution of the responses and the prevalence of responses in the "somewhat agree-to-totally disagree" area in contrast to the opposite categories "somewhat 
agree to totally agree", shows a low attitude to enjoy life and be pleasant to other among the Germans.

The normal distribution of the responses and the prevalence of responses in the "somewhat agree-to-totally agree" range in contrast to the opposite categories shows among the Germans a tendency to behavior in a hedonistic way. This fact is linked with the high score of the German culture (63 points) in the dimension of individualism measured by Hofstede (2001).

\section{Figure 58: Dimension of hedonism in the German sample}

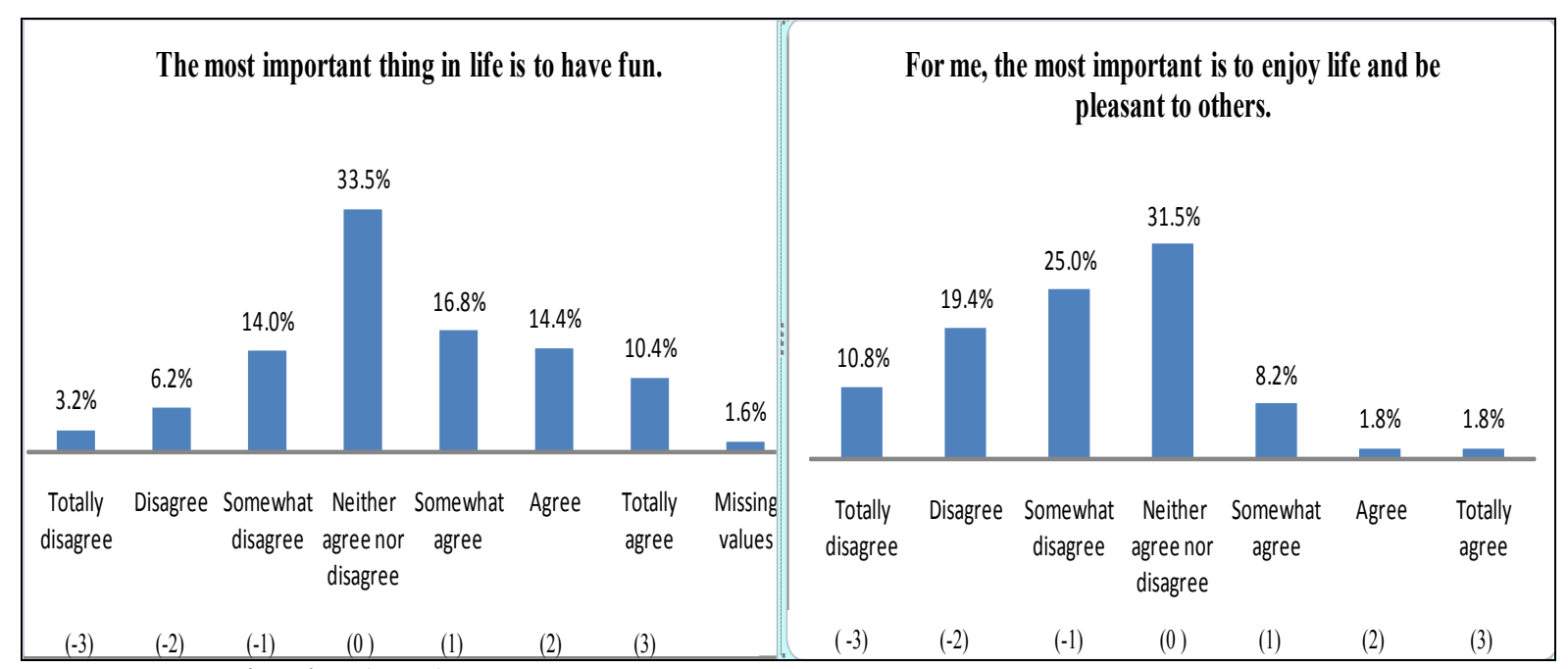

Source: Own estimation (2009).

\section{Mexican sample:}

Regarding the statement: "The most important thing in life is to have fun" in Figure 59 is described as follows: $8.1 \%$ "totally disagree"; 14.4\% "disagree"; 18.1\% "somewhat disagree"; 19.5\% "neither agree nor disagree"; $20.9 \%$ "somewhat agree"; $11 \%$ "agree"; $7.7 \%$ "totally agree"; $0.2 \%$ are "missing values". The higher concentration of responses in the "somewhat disagree-to-totally disagree" are indicates a similar hedonist attitudes among the Mexicans and Germans. In contrast to the percentage concentrated within the categories of "somewhat agree-to-totally agree". This is higher than the provided for the same range of categories among the Germans.

The frequency of responses to the statement: "For me the most important is to enjoy life and be pleasant to others" displayed in Figure 59 shows the frequency of responses described as: $20.7 \%$ "totally disagree"; $26.4 \%$ "disagree"; $25 \%$ "somewhat disagree"; $15.2 \%$ "neither agree 
nor disagree"; similarly, 8.1\% "somewhat agree"; 3.4\% "agree"; and 1.2\% "totally agree", respectively. Similarly to Germans the higher concentration of responses in the "totally disagree-to-somewhat agree" area in contrast to the opposite categories "somewhat agree-tototally agree" indicates a negative attitude to enjoy life and be pleasant to others among the Mexicans to than Germans.

Moreover the previous findings by Hosftede (2001) who refers the Mexican national culture as a collectivistic due to its low score (30 points) in the dimension of individualism and similarly findings provided by Schwartz (2006). Both Germans and Mexicans seem similarly hedonistic. According to the responses provided in both samples Mexicans seem more concern with fun than Germans. However, the meaning that the word "fun" and "enjoyment" have in German and Spanish languages might be considered in further interpretations even the meaning that each German and Mexican culture might give to the word "fun".

\section{Figure 59: Dimension of hedonism in the Mexican sample}

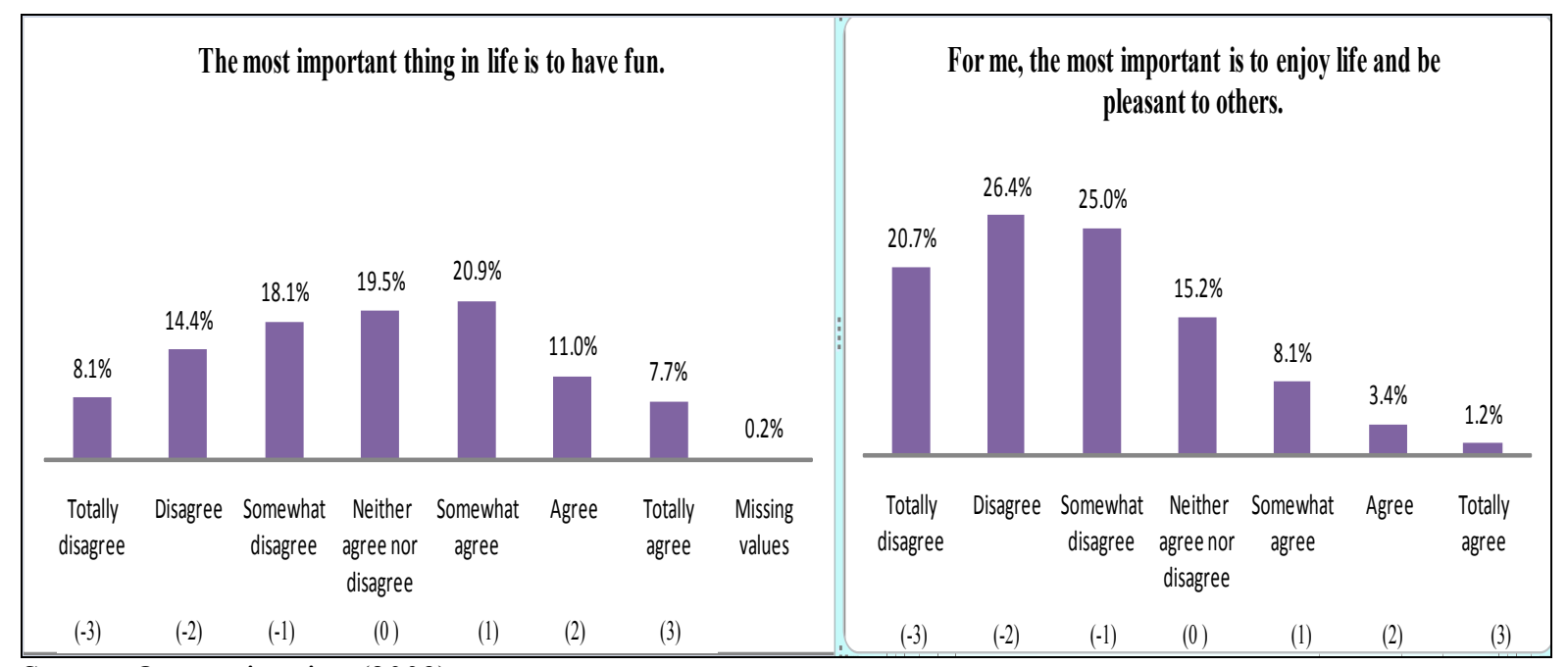

Source: Own estimation (2009). 


\subsubsection{Ethnocentrism}

\section{German sample:}

In the measuring of the dimension of ethnocentrism was used the statement: "I can well imagine living permanently abroad". Figure 60 shows the frequency of responses to the statement as following described: 13.6\% "somewhat disagree" and "totally disagree," respectively; 7.4\% "disagree"; 22.8\% "neither nor disagree"; $13.4 \%$ "somewhat agree"; similarly, 13.2\% "agree"; $14.6 \%$ "totally agree"; and 1.6\% are "missing values", respectively. The normal distribution of the responses and the prevalence of responses in the "somewhat disagree-to-totally agree" range in contrast to the opposite category, shows a moderate tendency of the Germans to leave the country permanently.

The frequency of responses to the statement: "I am firmly rooted to my country and its culture" shown in Figure 60 is described in the following: 5\% "totally disagree"; similarly, 4.6\% "disagree"; $8.8 \%$ "somewhat disagree"; 29.7\% "neither-nor disagree"; $21.8 \%$ "somewhat agree"; 17.8\% "agree"; 10.8\% "totally agree," respectively. Germans are also rooted to their country and culture, due to the higher percentage of responses provided within the categories of "somewhat agree-to-totally agree" area.

\section{Figure 60: Dimension of ethnocentrism in the German sample}

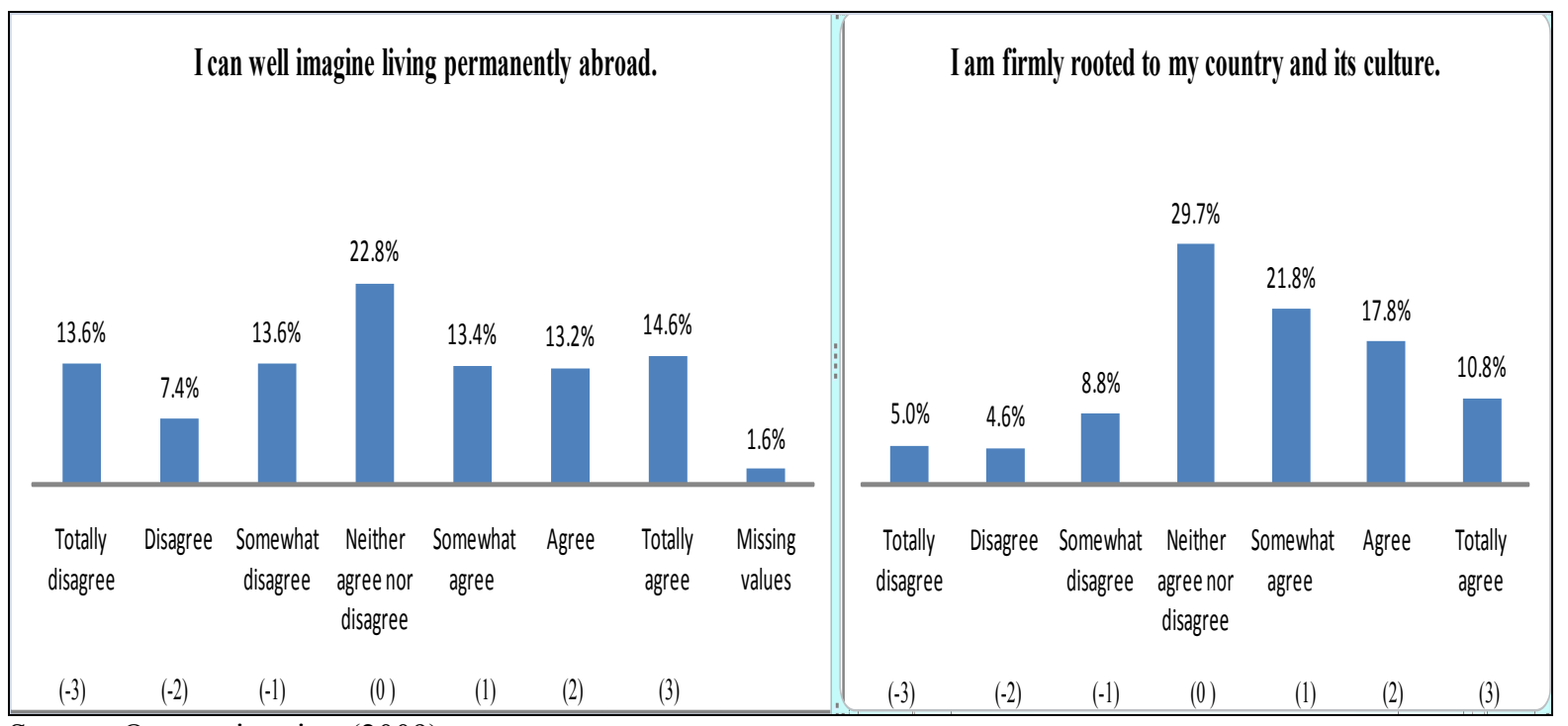

Source: Own estimation (2009). 


\section{Mexican sample:}

Regarding the statement: "I can well imagine living permanently abroad" displayed in Figure 61 is described in the following: 13\% "totally disagree"; $14.8 \%$ "disagree"; $12.4 \%$ "somewhat disagree"; 19.1\% "neither agree nor disagree"; 17.4\% "somewhat agree"; 9.7\% "agree"; and $13.6 \%$ "totally agree," respectively. Among the Mexicans the percentage of responses provided within the categories of "somewhat disagree-to-totally agree" is lower than the provided among Germans. All in all, these responses provided for the statement shows interesting insights at the cultural level due to although Mexico -developing country- and Germany -developed country- have different economical development both Germans and Mexico show similar attitudes toward leave permanently their home countries.

Figure 61 also displays the frequency of responses to the statement: "I am firmly rooted in my country and its culture" and is described as: 1.8\% "totally disagree"; similarly, $2.8 \%$ "disagree"; 3.9\% "somewhat disagree"; $13.8 \%$ "neither agree nor disagree"; $20.1 \%$ "somewhat agree"; 27.4\% "agree"; and 30.2\% "totally agree", respectively. Opposite to the responses provided for the previous statement. The percentage concentrated within the categories of "somewhat agree-to-totally agree" is higher among the Mexicans more so than Germans. This fact is also interesting at the cultural level.

Regarding the idea of living permanently in other country there is not a clear concentration of responses in one of the categories in both countries. However, the belief of being rooted to the country and culture is higher among the Mexicans than Germans. This suggests cultural differences in two different levels societal and individual linked with beliefs such as sense of belonging and embeddedness (Schwartz, 2006). In further researches the study of sociodemographic variables such as gender, age, incomes, and family size might be useful in the study of ethnocentric attitudes. 


\section{Figure 61: Dimension of ethnocentrism in the Mexican sample}

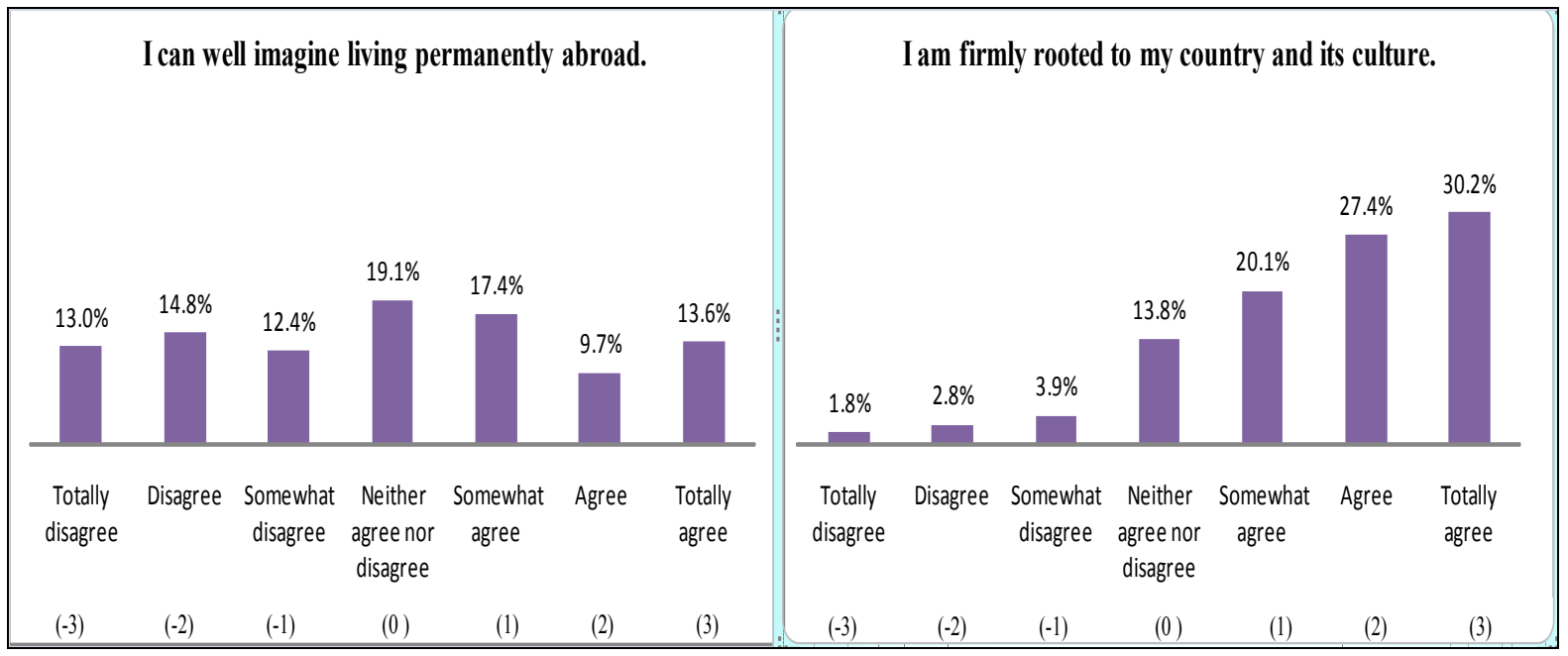

Source: Own estimation (2009).

\subsubsection{Malinchismo}

The measuring of the dimension of malinchismo was restricted to the Mexican sample with the statement: "I identify strongly with the lifestyle of other countries (e.g. U.S.A., Canada, Europe)". The frequency of responses displayed in Figure 62 is described in the following: $11.4 \%$ "totally disagree"; $8.7 \%$ "disagree"; $12.6 \%$ "somewhat disagree"; $23.3 \%$ "neither agree nor disagree"; 18.9\% "somewhat agree"; 14.4\% "agree"; 10.5\% "totally agree"; and $0.2 \%$ are categorized as "missing values", respectively. In contrast to the responses provided within the dimension of ethnocentrism above described the higher concentration of responses in the "neither agree nor disagree-to-totally agree" area indicates a receptiveness for foreign goods and imitation of lifestyle. 
Figure 62: Dimension of malinchismo in the Mexican sample

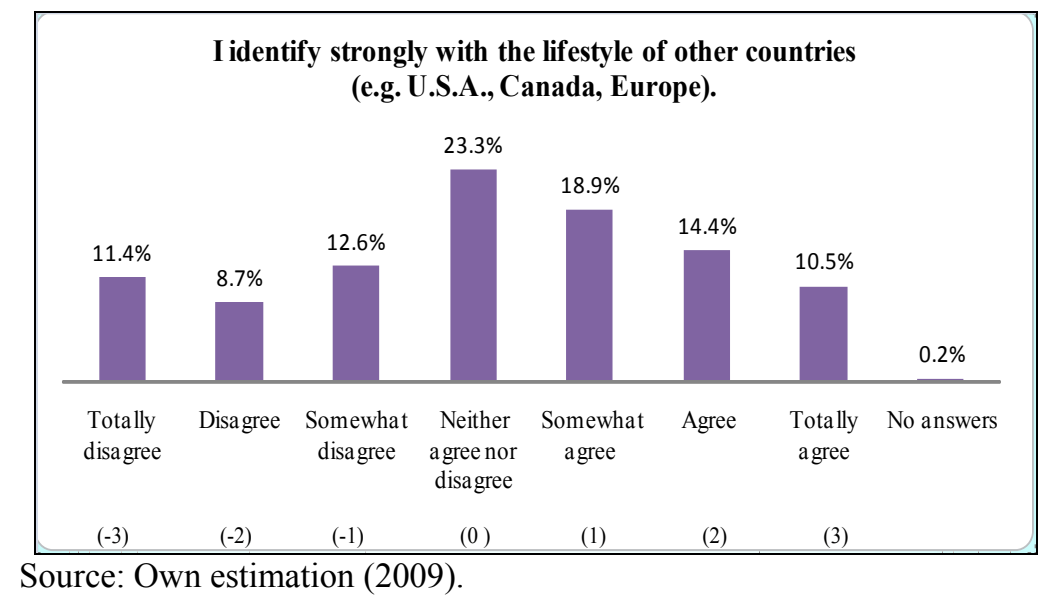

\subsection{Comparison of the average value priorities of groups of buyers and non-buyers of organic food in Germany and Mexico: dimensions of motives}

To establish a cross-country comparison of the motives and values in the purchase of organic food, the average value priorities of the groups were compared. The term average value priority of a group refers to "what the members of a group or nation have in common" (Mooij, 2004). Among the findings of the results, there are some interesting insights between the average value priorities of each group: German non-buyers, Mexican non-buyers, German consumers of organic food, and Mexican consumers of organic food; only high significant differences $(* * * p<0.001)$ are reported.

\subsubsection{Environmental concern/information}

As indicated in Figure 63, with respect to knowledge of the impact on the environment. The average value provided for German non-buyers $(\mathrm{M}=1.21)(\sigma=1.411)$ is slightly more positive than that of Mexican non-buyers $(\mathrm{M}=1.00)(\sigma=1.758)$, regarding the statement "It is difficult to know if the production of food has a negative impact on the environment". The average value for German consumers of organic food is slightly higher $(\mathrm{M}=1.52)(\sigma=1.078)$ than that of Mexicans $(\mathrm{M}=1.00)(\sigma=1.584)$ respectively. Mexicans consumers are not so insecure 
than German about negative impacts of food production on the environment. Especially German consumers of organic food who think they do not have enough information about the environmental aspects in the food production.

With respect to concern of production, -statement "I am careful to buy food products that have been produced in an environmentally friendly way".- The average value of the German non-buyers is $(\mathrm{M}=-0.19)(\sigma=1.637)$, compared to $(\mathrm{M}=0.87)(\sigma=1.405)$ for the Mexican non-buyers. German non-buyers appear to be less environmentally driven than Mexican nonbuyers. The average value for German consumers of organic food $(M=0.45)(\sigma=1.322)$ is lower than for the Mexicans $(M=1.32)(\sigma=1.268)$, respectively. Mexicans consumers of organic food show more an environmental orientation than Germans. The stage of the domestic market in both countries is useful to understand that Germans are more familiarized with the term "organic" and "organic assortment" because the mature stage of the market in Germany than Mexicans. Consequently Mexican consumers of organic food care more and because they are not involved or familiarized as Germans (see Figure 63).

\section{Figure 63: Environmental concern/information - average value priorities of group}

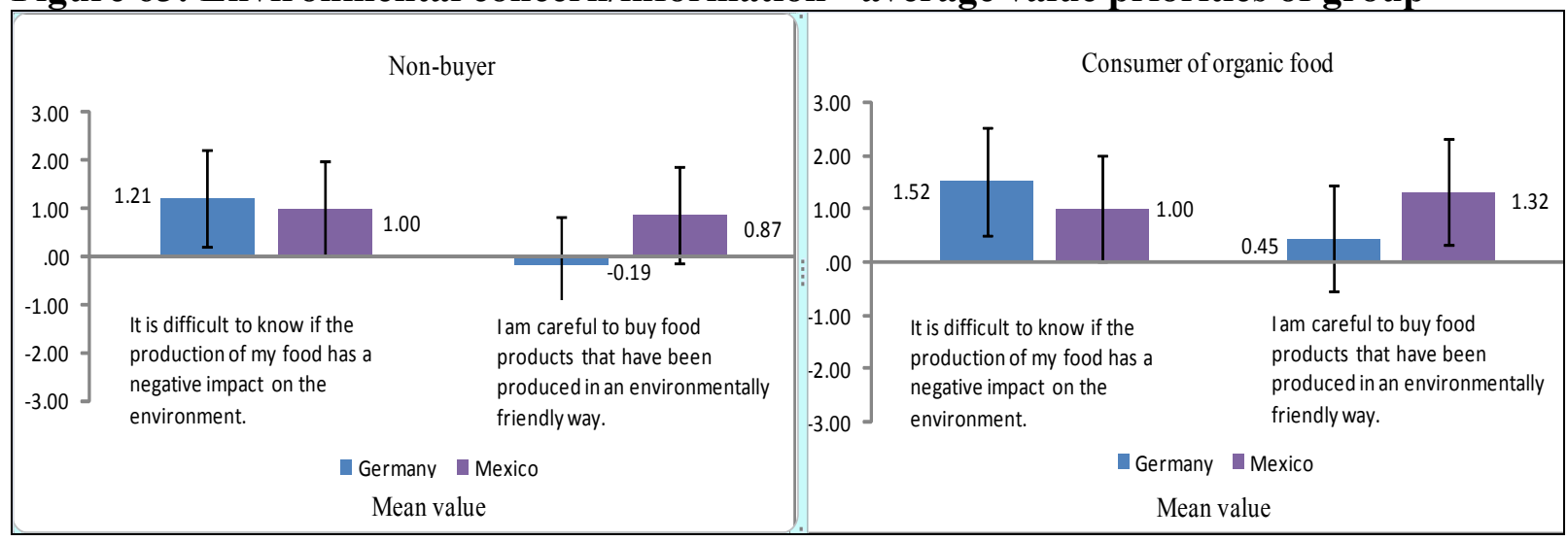

7-point Likert-type scale: (-3) strongly disagree, (-2) disagree, (-1) somewhat disagree, (0) neither agree nor disagree, (1) somewhat agree, (2) agree, and (3) strongly agree. "It is difficult to know if the production of food has a negative impact on the environment". F value $=7.114$. "I am careful to buy food products that have been produced in an environmentally friendly way". $F=50.963$.

\subsubsection{Information}

With regard to information provided on packaging -statement "I would like more information to be provided on the packaging”. Figure 64 displays the average value of $(M=0.63)$ $(\sigma=1.566)$ for German non-buyers which is slightly more positive than for Mexican non- 
buyers $(M=2.00)(\sigma=1.296)$. In relation to the desire to have more information provided on packaging, the average value for German consumers of organic food is lower $(\mathrm{M}=1.30)$ $(\sigma=1.373)$ than that of the Mexicans $(\mathrm{M}=2.16)(\sigma=1.296)$. Conversely, German consumers of organic food are more moderate than Mexicans regarding more information provided on packaging.

For the statement: "Before buying food, I carefully read the information on the packaging". The average values for German non-buyers are $(M=-0.39)(\sigma=1.610)$, whilst it is $(M=1.07)$ $(\sigma=1.401)$ for Mexican non-buyers. German non-buyers appear to be less careful or concerned with nutritional information than Mexican non-buyers.

With respect to carefully reading information on packages prior to purchase, the average value of German consumers of organic food $(\mathrm{M}=0.58)(\sigma=1.435)$ is lower than that of Mexicans $(M=1.48)(\sigma=1.210)$. Therefore, it is understood that German are less interested in information provided on packaging than Mexicans (see Figure 64). Within Mexicans differences are slightly whilst, within Germans consumers and non-buyers differences are more clear. Consequently Germans consumers of organic food are more concern with product information than non-buyers.

\section{Figure 64: Information - average value priorities of group}

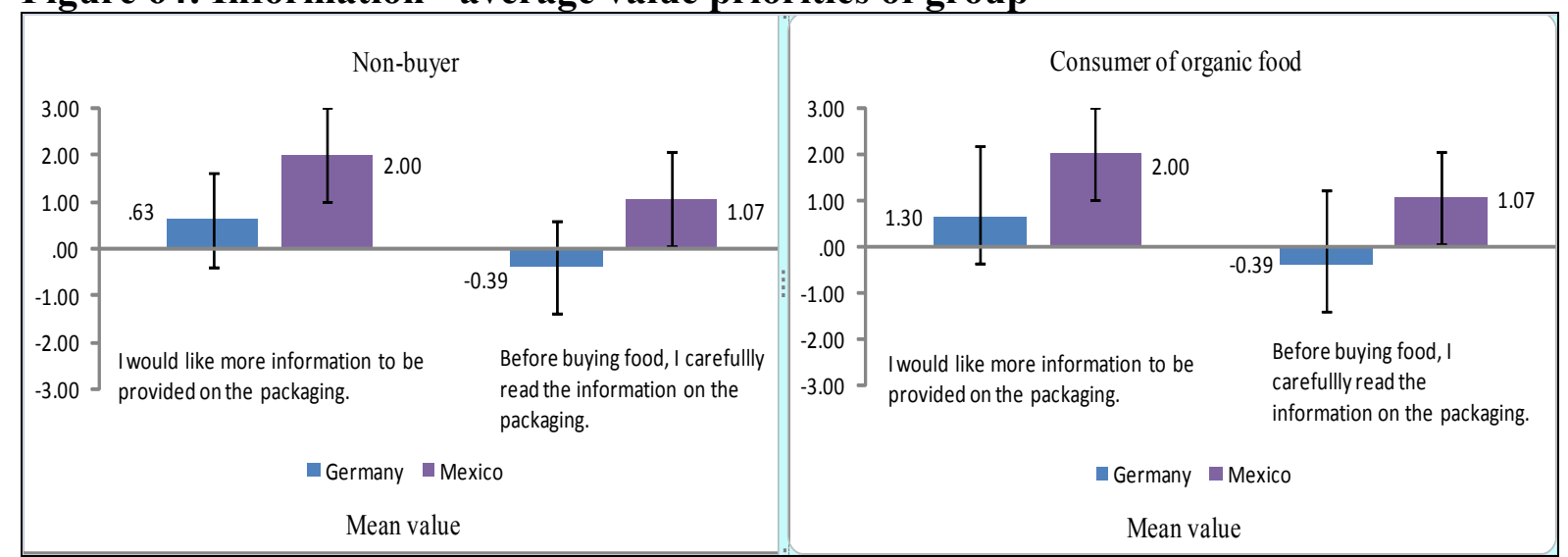

7-point Likert-type scale: (-3) strongly disagree, (-2) disagree, (-1) somewhat disagree, (0) neither agree nor disagree, (1) somewhat agree, (2) agree, and (3) strongly agree. "I would like more information to be provided on the packaging". F value $=68.042$. "Before buying food, I carefully read the information on the packaging". F $=78.313$. 


\subsubsection{Weight control}

As depicted in Figure 65, in relation to eating low-fat foods as much as possible, the average value for Mexican non-buyers $(\mathrm{M}=1.20)(\sigma=1.417)$, is more positive than that of German non-buyers $(\mathrm{M}=0.07)(\sigma=1.645)$. In relation to eating low-calories food, again the average value for Mexican non-buyers $(\mathrm{M}=0.94)(\sigma=1.452)$ is more positive than that of German nonbuyers $(\mathrm{M}=-0.22)(\sigma=1.542)$. German non-buyers tend to be more skeptical about weight control than Mexican non-buyers.

The average values of the consumers of organic food seem to be different in relation to eating low-fat foods. The average value for Mexicans $(\mathrm{M}=1.66)(\sigma=1.273)$ is more positive than for Germans $(\mathrm{M}=0.55)(\sigma=1.514)$. Thus, German consumers of organic food are more moderate than Mexicans regarding low-fat food in their diet. Mexicans tend to be more careful about low-calorie food whereby the average value for them $(\mathrm{M}=1.29)(\sigma=1.433)$ is higher than for Germans $(\mathrm{M}=0.28)(\sigma=1.552)$, respectively (see Figure 65). In both countries organic consumers are more aware of the obesity problems than non-buyers.

Figure 65: Weight control - average value priorities of group

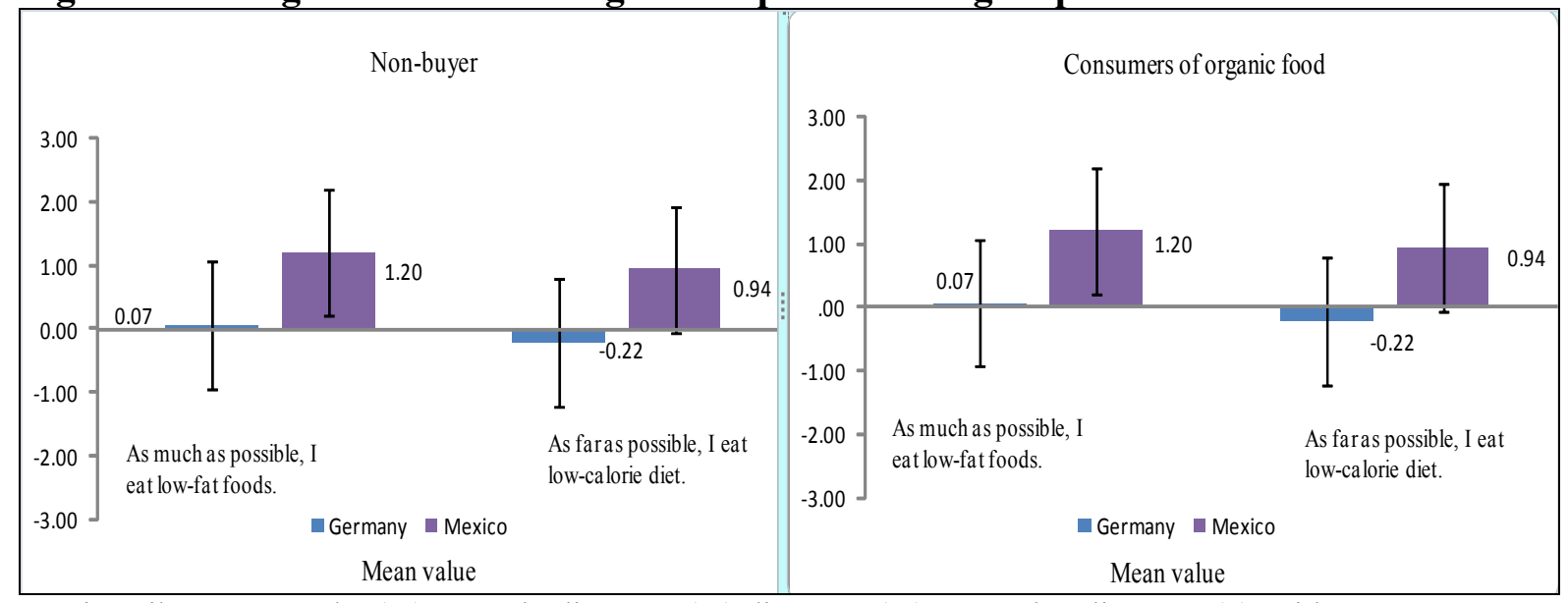

7-point Likert-type scale: (-3) strongly disagree, (-2) disagree, (-1) somewhat disagree, (0) neither agree nor disagree, (1) somewhat agree, (2) agree, and (3) strongly agree. "As much as possible, I eat low-fat foods". F value $=57.262$. "As far as possible, I eat a low-calorie diet". $F=49.989$. 


\subsection{4}

Healthy eating

With respect to forcing oneself to eat food that doesn't taste good but is healthy, Figure 66 displays the average value for German non-buyers in the negative $(M=-0.37)(\sigma=1.544)$; however, the average value for Mexican non-buyers is positive $(M=0.15)(\sigma=1.715)$. Concerning following a Healthy eating, but not being able to manage it, the average value of Mexican non-buyers is slightly more positive $(M=0.89)(\sigma=1.747)$ than that of German nonbuyers $(\mathrm{M}=0.66)(\sigma=1.598)$. Mexican non-buyers tend to have a healthier diet than German non-buyers.

With regard to forcing oneself to eat food that doesn't taste good but is healthy, the average values of the consumers of organic food seem slightly different. The average value for Mexicans $(\mathrm{M}=0.32)(\sigma=1.727)$ is slightly more positive compared to that of Germans $(\mathrm{M}=-0.37)(\sigma=1.544)$. Concerning following a healthy eating, but not being able to manage it, again the average value for Mexicans $(\mathrm{M}=0.56)(\sigma=1.800)$ is slightly higher than for Germans $(\mathrm{M}=0.42)(\sigma=1.496)$. In Mexico and especially in Germany, organic consumers care more their diet.

Figure 66: Healthy eating - average value priorities of group

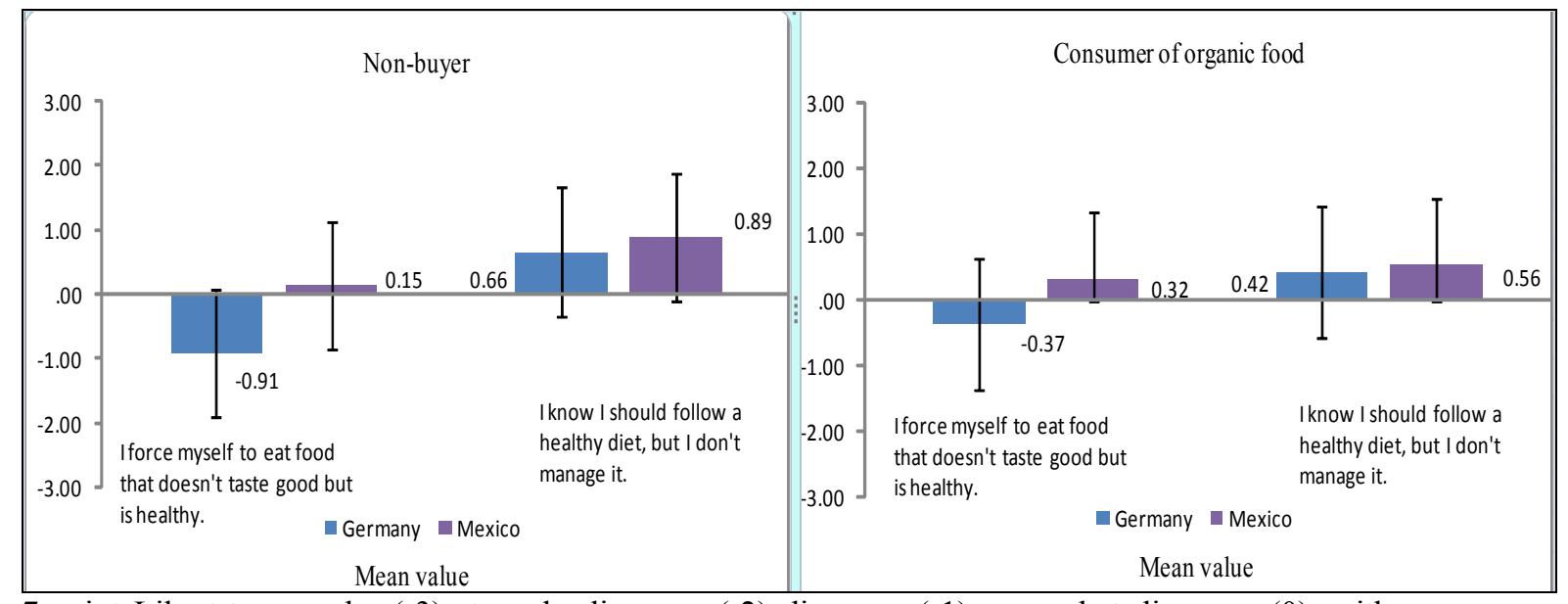

7-point Likert-type scale: (-3) strongly disagree, (-2) disagree, (-1) somewhat disagree, (0) neither agree nor disagree, (1) somewhat agree, (2) agree, and (3) strongly agree. "I force myself to eat food that doesn't taste good but is healthy". F value $=28.079$. "I know I should follow a Healthy eating, but I don't manage it". F $=3.609$. 


\subsubsection{Natural content}

Regarding assurance that no artificial flavours have been added to food, Figure 56 displays the average value for German non-buyers $(M=-0.08)(\sigma=1.648)$ which is negative, whereas that of Mexican non-buyers is positive $(M=1.02)(\sigma=1.569)$. Concerning avoidance of all products containing flavour enhancers, the average value for German non-buyers is negative $(\mathrm{M}=-0.47)(\sigma=1.500)$, whilst for Mexican non-buyers $(\mathrm{M}=0.79)(\sigma=1.516)$ is slightly higher on the positive side. German non-buyers tend to be less careful about artificial flavors than Mexican non-buyers.

The average values provided among the consumers of organic food seem slightly different. With respect to assurance that no artificial flavours have been added to food, the average value for Mexicans $(\mathrm{M}=1.43)(\sigma=1.346)$ is slightly more positive than for the Germans $(\mathrm{M}=0.74)(\sigma=1.442)$. Again, regarding avoidance of all products containing flavour enhancers, the average value for Mexicans $(M=1.29)(\sigma=1.404)$ is higher than for Germans $(\mathrm{M}=0.23)(\sigma=1.511)$. German and Mexican consumers tend to pay attention to natural content of food, for instance, that no artificial flavors have been added. However, Mexicansconsumers and non-buyers are more prudent than Germans (see Figure 67).

\section{Figure 67: Natural content - average value priorities of group}

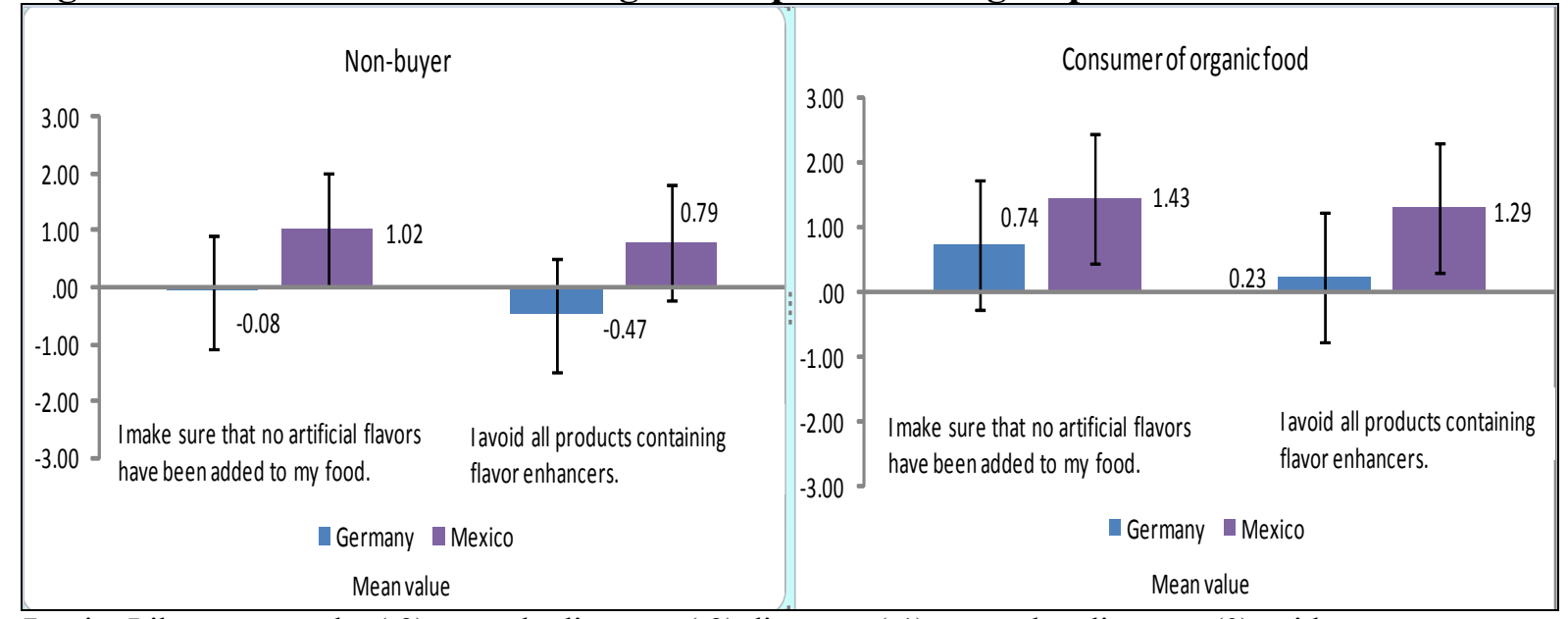

7-point Likert-type scale: (-3) strongly disagree, (-2) disagree, (-1) somewhat disagree, (0) neither agree nor disagree, (1) somewhat agree, (2) agree, and (3) strongly agree. "I make sure that no artificial flavours have been added to food". F value $=44.168$. "I avoid all products containing flavour enhancers". $\mathrm{F}=64.509$. 


\subsubsection{Free of Genetically Modified Organism}

Regarding purchasing of GM food, Mexicans non-buyers are fewer sceptics than Germans. Figure 68 displayed the average value provided for the statements "Sometimes I buy products that may contain genetically modified material". Mexican non-buyers $(\mathrm{M}=0.66)(\sigma=1.873)$ seems slightly more positive than for German non-buyers $(M=0.23)(\sigma=1.583)$. Concerning the purchasing of non-GM food -statement "I buy only non-genetically modified food products".- German negative average value provided $(\mathrm{M}=-0.55)(\sigma=1.750)$ whilst for Mexican non-buyers it is slightly positive $(\mathrm{M}=0.28)(\sigma=1.517)$. It can therefore be ascertained that German non-buyers tend to have more confidence in Genetically Modified Food than Mexican non-buyers.

The average values provided among the consumers of organic food appear slightly different. With regard to the purchasing of GM food -statement "Sometimes I buy products that may contain genetically modified material".- The average value for Mexicans $(M=0.74)(\sigma=1.624)$ is slightly more positive than that of the Germans $(M=0.08)(\sigma=1.506)$. Concerning the purchasing of non-GM food, again, the average value for Mexicans $(\mathrm{M}=0.76)(\sigma=1.511)$ is higher than for Germans $(\mathrm{M}=0.20)(\sigma=1.647)$. Therefore, it is apparent that there is a slight difference between German and Mexican consumers concerning Genetically Modified Food (see Figure 68). However, there is more skepticism to purchasing GM food among the Germans than Mexicans consumers and non-buyers. In both countries organic consumers are more aware of GMOs. 
Figure 68: Free of GMO - average value priorities of group

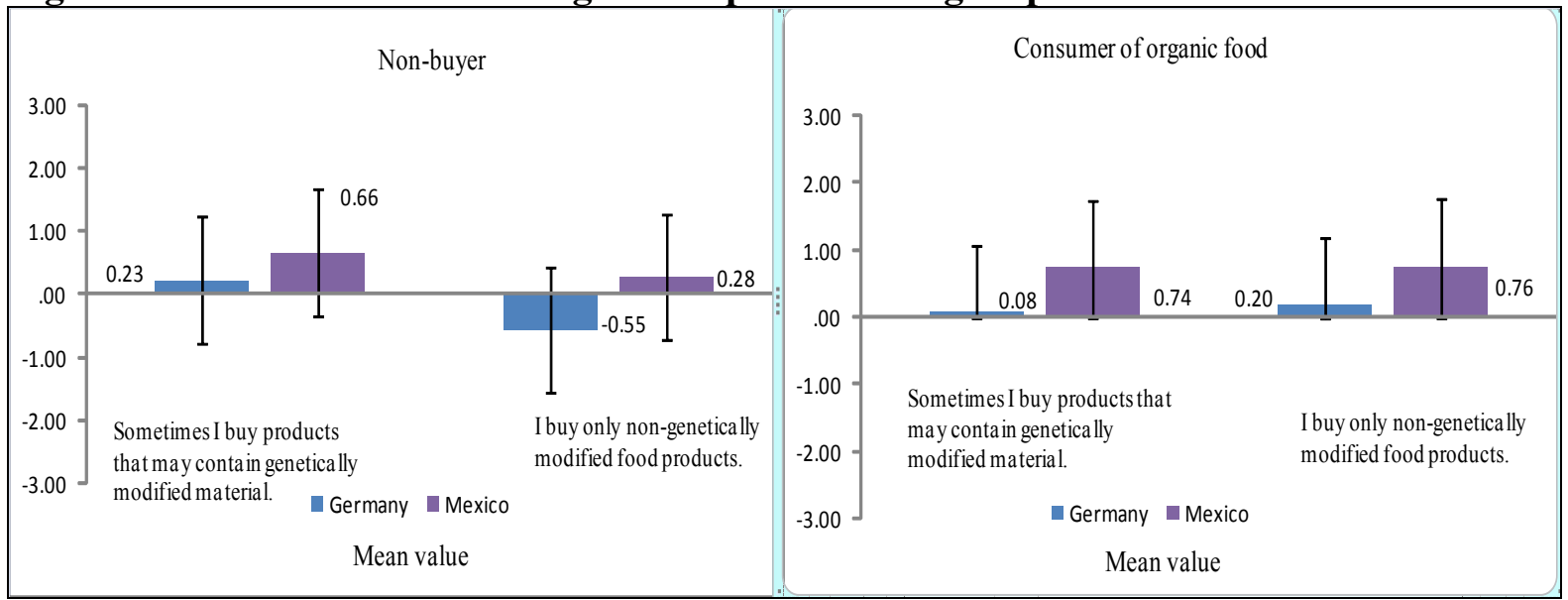

7-point Likert-type scale: (-3) strongly disagree, (-2) disagree, (-1) somewhat disagree, (0) neither agree nor disagree, (1) somewhat agree, (2) agree, and (3) strongly agree. "Sometimes I buy products that may contain genetically modified material". $\mathrm{F}$ value $=9.840$. "I buy only non-genetically modified food products" $\mathrm{F}=$ 27.791.

\subsubsection{Familiarity/neophobia}

Regarding inclination to buy well-known brands of food products, Figure 69 describes the average value for Mexican non-buyers as more positive $(M=1.87)(\sigma=1.236)$ than for German non-buyers $(\mathrm{M}=0.33)(\sigma=1.578)$. With respect to trying new -different- food, similarly the average value for German non-buyers is $(\mathrm{M}=0.56)(\sigma=1.391)$, and for Mexican non-buyers it is $(\mathrm{M}=1.25)(\sigma=1.364)$. Mexican non-buyers tend to purchase brands that are more familiar or are advertised than Germans and are also more willing to try new products or brands than German non-buyers who tend to remain moderate.

The average values of consumers of organic food appear slightly different. Regarding inclination to buy well-known brands of food products, the average value for Germans $(\mathrm{M}=0.36)(\sigma=1.380)$ is lower although positive than that of Mexicans $(\mathrm{M}=1.60)(\sigma=1.200)$. Concerning the average values related to purchasing only non-genetically modified food products, again, the average value for Germans $(\mathrm{M}=1.01)(\sigma=1.262)$ is lower than for the Mexicans $(\mathrm{M}=1.71)(\sigma=1.049)$. There is a difference between German and Mexican consumers concerning the purchasing of well-known brands and trying new brands or food products (see Figure 69). In both countries non-buyers of organic food demonstrate a higher degree of neophobia. 
Figure 69: Familiarity/neophobia - average value priorities of group

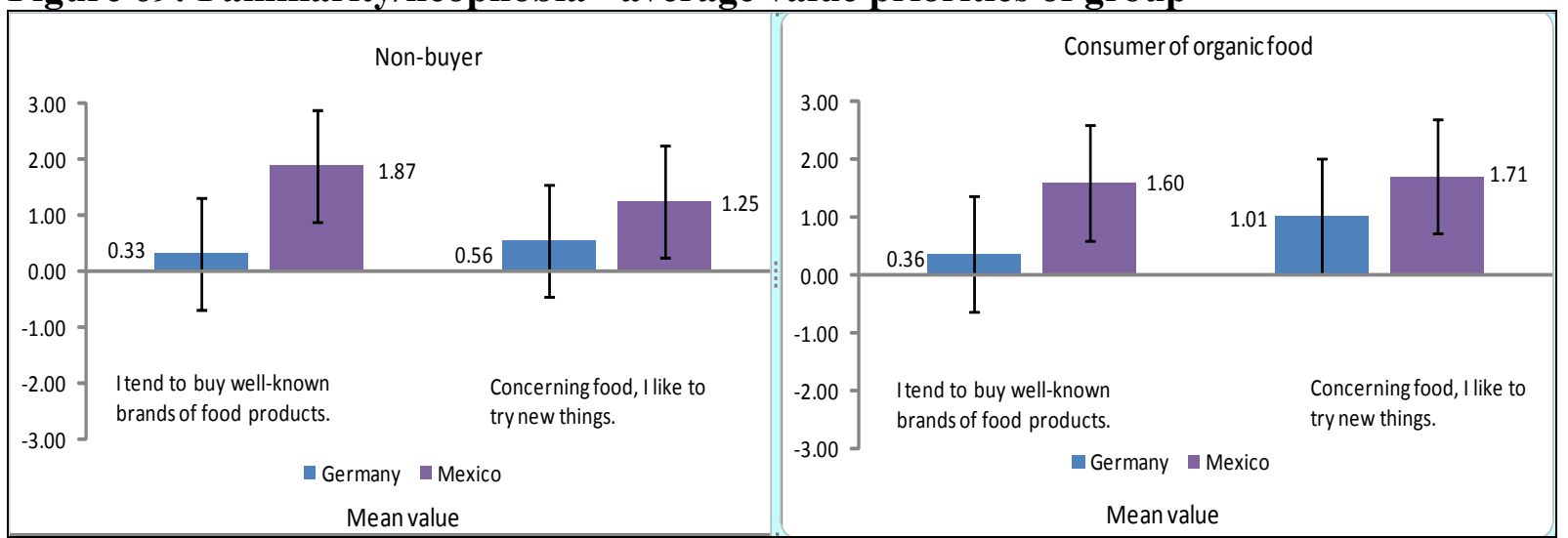

7-point Likert-type scale: (-3) strongly disagree, (-2) disagree, (-1) somewhat disagree, (0) neither agree nor disagree, (1) somewhat agree, (2) agree, and (3) strongly agree. "I tend to buy well-known brands of food products". F value $=89.688$. "Concerning food, I like to try new things". $\mathrm{F}=35.747$.

\subsubsection{Advertising}

Regarding frequently purchasing food because of packaging, the average value for German non-buyers $(\mathrm{M}=-0.72)(\sigma=1.658)$ is lower than for Mexican non-buyers $(\mathrm{M}=-0.37)$ $(\sigma=1.760)$. Concerning frequently purchasing food that has been advertised, again the average value for German non-buyers is negative $(M=-0.40)(\sigma=1.534)$ whilst for the Mexican nonbuyers it is positive $(M=0.78)(\sigma=1.465)$ (see Figure 70$)$. Therefore, German non-buyers tend to base their purchases less on packaging and advertising than Mexicans, whereas Mexicans are more willing to try new products or brands because advertising than German non-buyers.

The average values among consumers of organic food appear slightly different. Regarding frequency of purchasing food because of packaging, the average value for Germans $(\mathrm{M}=-0.43)(\sigma=1.479)$ is negatively lower than for Mexicans $(\mathrm{M}=-0.17)(\sigma=1.724)$. Concerning frequently purchasing food that has been advertised, again, the average value for Germans is negative $(\mathrm{M}=-0.22)(\sigma=1.382)$, whilst for Mexicans it is positive $(\mathrm{M}=0.99)$ $(\sigma=1.248)$. In respect to the purchasing of because advertising and packaging, there is a difference between German and Mexicans consumers of organic food (see Figure 70). Germans consumers and non-buyers are skeptic about the purchasing of food products because advertising and packaging. Among Mexicans non buyers are skeptic than consumers who show a positive tendency. 
Interestingly and surprisingly is the fact that because the ideological background of the organic movement, in both countries consumers of organic food are less advertising aware than non-buyers. However, the higher incomes of consumers of organic food are a demographic variable explaining their receptiveness to advertisement.

Figure 70: Advertising - average value priorities of group

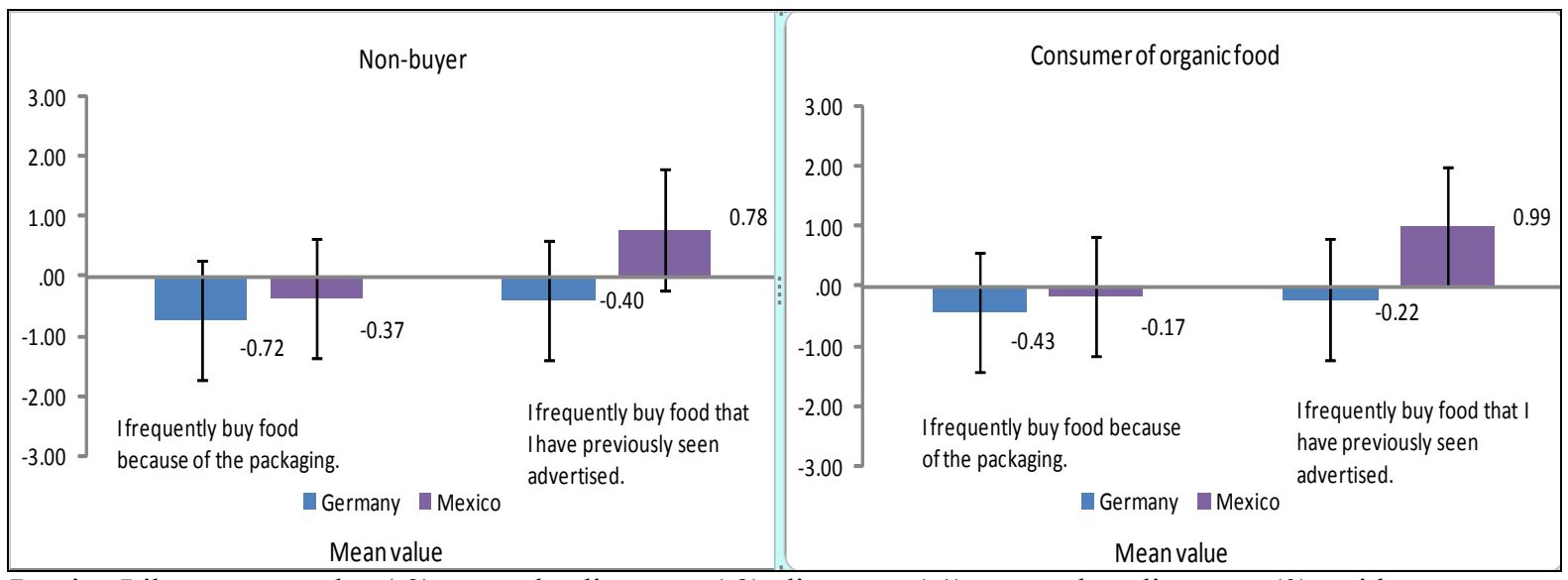

7-point Likert-type scale: (-3) strongly disagree, (-2) disagree, (-1) somewhat disagree, (0) neither agree nor disagree, (1) somewhat agree, (2) agree, and (3) strongly agree. "I frequently buy food because of the packaging". F value $=4.664$. "I frequently buy food that I have previously seen advertised". $F=61.897$.

\subsubsection{Shopping convenience}

Regarding food shopping as an unpleasant task, the average value for German non-buyers $(\mathrm{M}=-0.30)(\sigma=1.663)$ is less negative than for Mexican non-buyers $(\mathrm{M}=0.92)(\sigma=1.759)$. Concerning quick shopping food the average value for German non-buyers is more positive $(\mathrm{M}=0.93)(\sigma=1.581)$ than that of Mexican non-buyers $(\mathrm{M}=0.24)(\sigma=1.840)$. German nonbuyers appear to appreciate more shopping convenience than Mexicans. The average values are shown in Figure 71.

The average values provided among the consumers of organic food appear slightly different. Regarding viewing shopping for food as an unpleasant task, the average value for Germans $(\mathrm{M}=-0.39)(\sigma=1.516)$ is less negative than for Mexicans $(\mathrm{M}=-0.82)(\sigma=1.647)$. Concerning the quick purchasing food, the average value of Germans $(M=0.55)(\sigma=1.386)$ is more positive than that for Mexicans $(\mathrm{M}=0.33)(\sigma=1.703)$. Again, German consumers appear to appreciate more shopping convenience than Mexicans (see Figure 71). 
All in all, the differences between consumers and non-buyers are small in both countries. Shopping convenience in the purchase of organic food seems more appreciated by Germans than Mexicans.

Figure 71: Shopping convenience - average value priorities of group

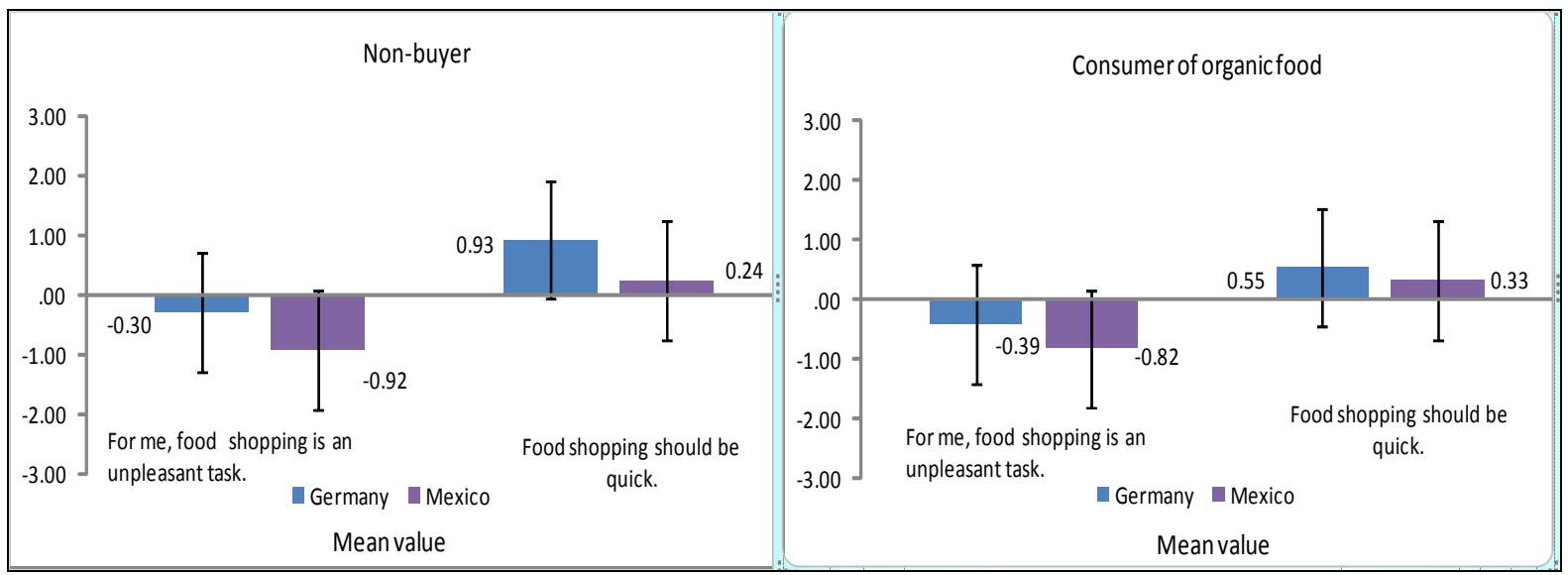

7-point Likert-type scale: (-3) strongly disagree, (-2) disagree, (-1) somewhat disagree, (0) neither agree nor disagree, (1) somewhat agree, (2) agree, and (3) strongly agree. "For me, food shopping is an unpleasant task". F value $=8.689$. "Food shopping should be quick". $F=8.377$.

\subsubsection{Cooking convenience}

Mexicans seem more involved with cooking than Germans; Figure 72 indicates the average value priorities of the belief that the growth of assortment of convenience food is beneficial. The average value for German non-buyers $(M=0.85)(\sigma=1.476)$ is more positive than for Mexican non-buyers $(\mathrm{M}=-0.08)(\sigma=1.706)$. Concerning easier preparation of meals, the average value for German non-buyers is slightly more positive $(M=1.01)(\sigma=1.497)$ than for Mexican non-buyers $(\mathrm{M}=0.84)(\sigma=1.629)$. German non-buyers tend to be more interested in the convenience of cooking than Mexicans.

The average values for consumers of organic food appear slightly different. Regarding the belief that the growth of assortment of convenience food is beneficial, the average value for the Germans is slightly similar $(M=0.49)(\sigma=1.480)$ than for Mexicans $(M=0.51)(\sigma=1.657)$. Concerning easier preparation of meals, again, the average value for Germans is slightly less positive $(M=0.56)(\sigma=1.438)$ than for Mexicans $(M=0.97)(\sigma=1.523)$. There seems to be a slight difference between German and Mexican consumers of organic food regarding cooking convenience (see Figure 72). 
Comparing consumers and non-buyers of organic food, differences between both countries are observed. Whereas for German consumers of organic food cooking convenience is not so important, the opposite is true for the Mexican population.

Figure 72: Cooking convenience - average value priorities of group

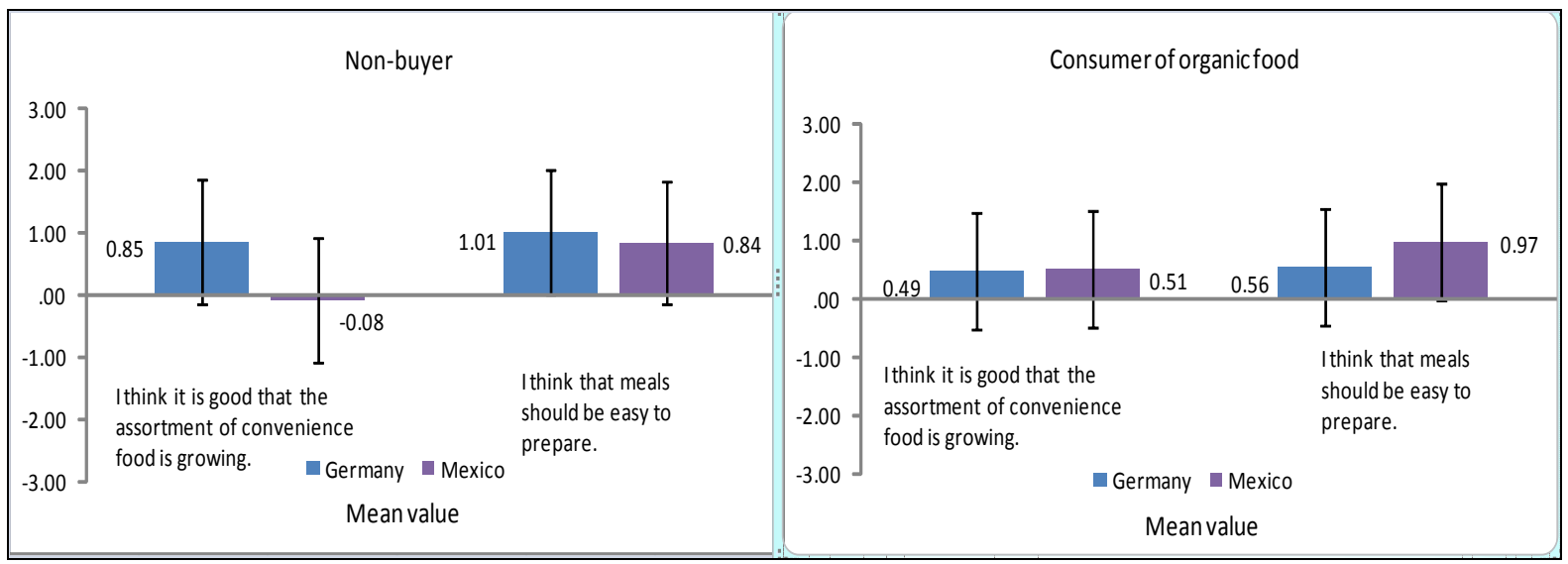

7-point Likert-type scale: (-3) strongly disagree, (-2) disagree, (-1) somewhat disagree, (0) neither agree nor disagree, (1) somewhat agree, (2) agree, and (3) strongly agree. "I think it is good that the assortment of convenience food is growing". F value $=14.263$. "I think that meals should be easy to prepare". $F=4.672$.

\subsubsection{Nostalgia}

Mexicans seem more available to purchase food because nostalgia than Germans the average values are showing in Figure 73. Regarding the purchasing of food because the evocation of the childhood. The average value for German non-buyers $(M=0.55)(\sigma=1.690)$ is less positive than for Mexican non-buyers $(\mathrm{M}=1.27)(\sigma=1.465)$.

Regarding the evocation of taste again the average value for Mexicans is more positive $(\mathrm{M}=1.53)(\sigma=1.484)$ than that for German non-buyers $(\mathrm{M}=0.90)(\sigma=1.592)$. Therefore, German non-buyers are less concerned with nostalgia than Mexicans.

The average values for the consumers of organic food seem slightly different. With respect to the purchasing of food because the remembering of childhood the average value for Germans $(\mathrm{M}=0.99)(\sigma=1.411)$ is slightly less positive than for Mexicans $(\mathrm{M}=1.08)(\sigma=1.431)$.

Concerning the purchasing of food because the evocation of taste again the average value for Germans $(\mathrm{M}=0.84)(\sigma=1.442)$ is less positive than that of Mexicans $(\mathrm{M}=1.48)(\sigma=1.395)$. 
Concerning nostalgia regarding food products, there are slight differences between German and Mexican consumers; however, with respect to sensory variables such as "taste" the difference is higher among Mexican consumers (see Figure 73). Again Mexicans tend to purchase food because nostalgia than Germans.

Figure 73: Nostalgia - average value priorities of group

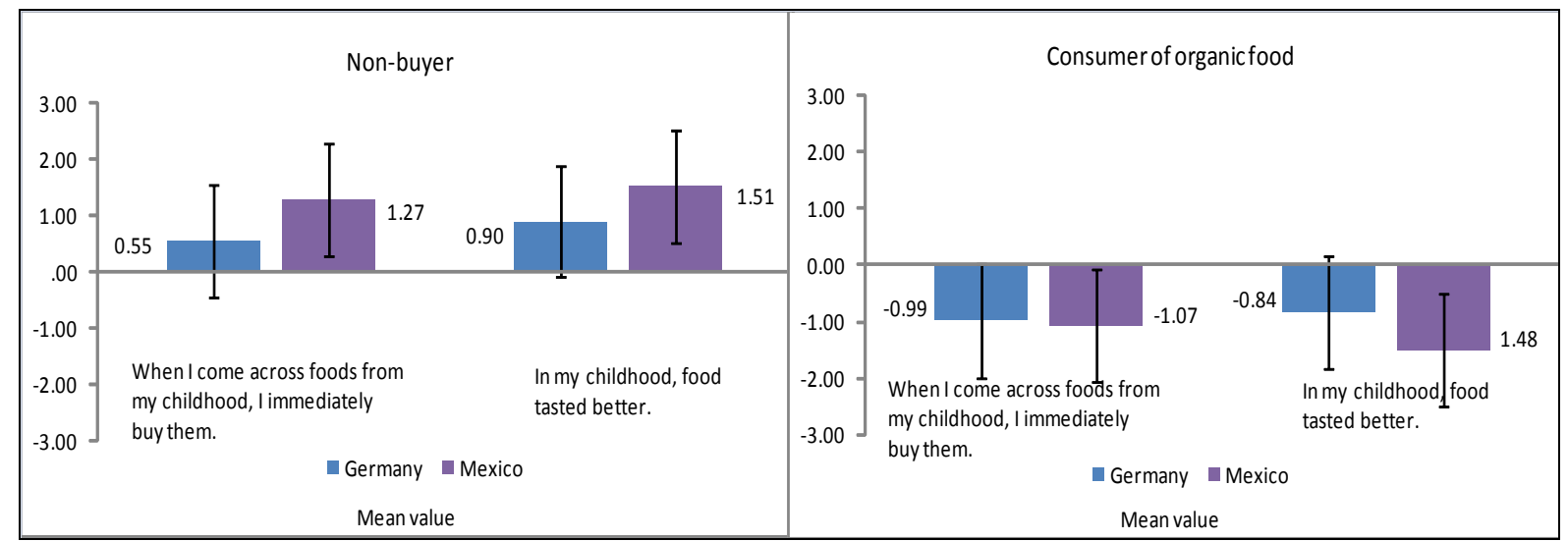

7-point Likert-type scale: (-3) strongly disagree, (-2) disagree, (-1) somewhat disagree, (0) neither agree nor disagree, (1) somewhat agree, (2) agree, and (3) strongly agree. "When I come across foods from my childhood, I immediately buy them". F value $=9.850$. "In my childhood, food tasted better". $\mathrm{F}=15.543$.

\subsubsection{Consumer ethnocentrism}

Figure 74 indicates average value priorities regarding avoidance of products for which the country of origin is not known. The average value for German non-buyers $(\mathrm{M}=-0.13)$ $(\sigma=1.680)$ is negative compared to Mexicans $(M=0.77)(\sigma=1.693)$. Regarding preference for home grown fruit and vegetables, the average value for German non-buyers is $(\mathrm{M}=0.56)$ $(\sigma=1.705)$, whilst the Mexican average value is more positive $(M=2.10)(\sigma=1.170)$. Mexican non-buyers have an ethnocentric attitude in their purchasing behavior than Germans.

Regarding avoidance of products for which the country of origin is not known, the average values provided among the consumers of organic food seems slightly different. The average value for Germans $(\mathrm{M}=0.66)(\sigma=1.656)$ is slightly less positive than that for Mexicans $(\mathrm{M}=0.76)(\sigma=1.678)$. Concerning preference for home grown fruit and vegetables, again, the average value for Germans $(\mathrm{M}=1.26)(\sigma=1.411)$ is less positive than for Mexicans $(\mathrm{M}=2.10)$ $(\sigma=1.170)$. German and Mexican consumers of organic food are characterized by a higher 
degree of ethnocentrism than non-buyers, and Mexican consumers of organic food, even nonbuyers, seem more attuned to consumer ethnocentrism than Germans (see Figure 74).

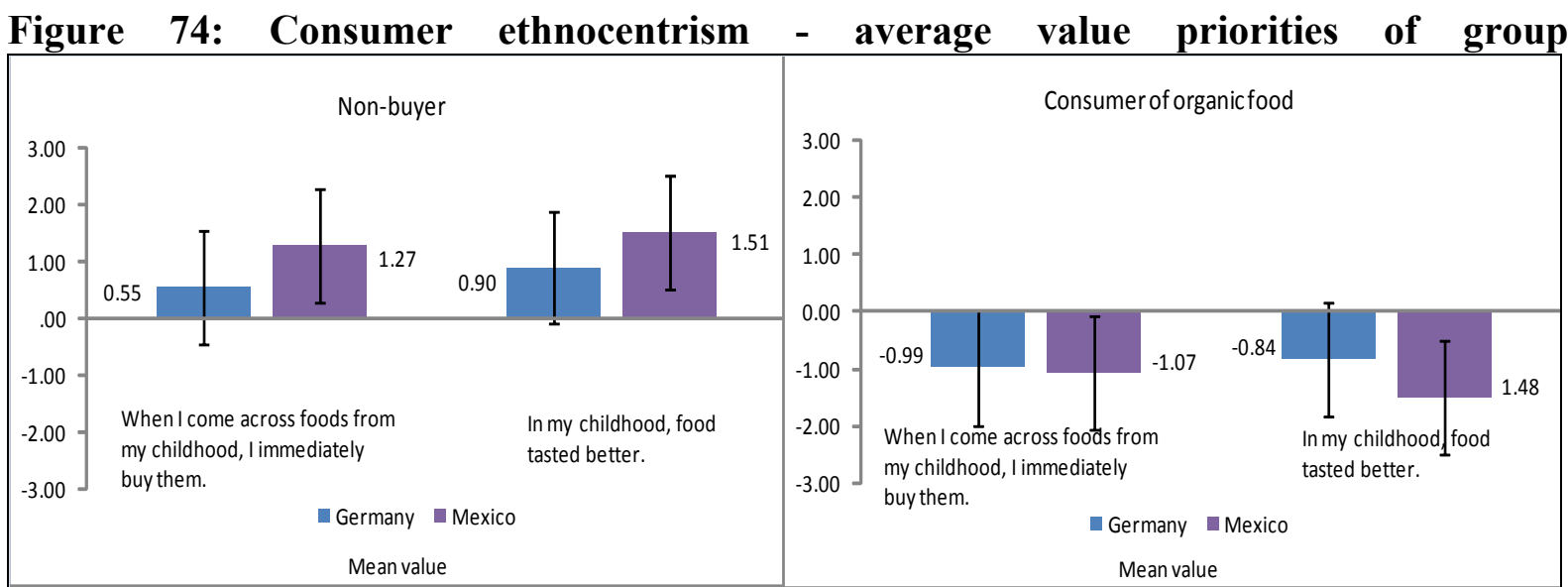

7-point Likert-type scale: (-3) strongly disagree, (-2) disagree, (-1) somewhat disagree, (0) neither agree nor disagree, (1) somewhat agree, (2) agree, and (3) strongly agree. "I avoid products for which I do not know the country of origin". F value $=15.932$. "I prefer fruits and vegetables from my home country". $\mathrm{F}=73.391$.

As Mooij (2004) mentioned culture is at different levels affecting human and consumer behaviour. Consequently some differences between Germans and Mexicans regarding motives to purchase food products were above displayed. Comparing the average values priorities by each group differences between the countries were uncover, as well as some affinities between consumers of organic food in both countries. These provide interestingly insights at the psychographic level in the purchasing of organic food for instance that Germans and Mexicans consumers of organic food are more aware about a healthy diet than non-buyers. Convenience in shopping is important for Germans and Mexicans however, for Germans most be quickly than for Mexicans who also seem less involved with cooking convenience which seem more important for Germans consumers of organic food than for Mexicans consumers of organic food. 


\subsection{Comparison of the average values priorities: dimensions of values}

In the basis of values influence the behaviour, belief and attitudes. In this section are provided the most interesting insights of the findings on values to uncover differences at the cultural level between Germans and Mexicans and within non-buyers and consumers of organic food in both countries.

\subsubsection{Technological progress/technophobia}

The average values for non-buyers in Figure 75 relates to the unconventional use of computers and other electronic devices. The average value for German non-buyers $(\mathrm{M}=1.33)$ $(\sigma=1.545)$ is less positive than that of Mexicans $(M=1.80)(\sigma=1.228)$. Regarding quality of life may be improved with technological progress, the average value for German non-buyers $(\mathrm{M}=0.73)(\sigma=1.327)$ again is less positive than that for Mexicans $(\mathrm{M}=1.86)(\sigma=1.162)$. German non-buyers seem more skeptics than Mexicans about technological progress.

The average values of the consumers of organic food seem slightly different. Regarding computers and other electronic devices, the average value for Germans $(M=1.56)(\sigma=1.205)$ is less positive than for Mexicans $(\mathrm{M}=1.81)(\sigma=1.123)$. Concerning technological progress that might improve quality of life, again, the average value for Germans $(M=0.90)(\sigma=1.220)$ is less positive than for Mexicans $(\mathrm{M}=1.84)(\sigma=1.059)$. German consumers of organic food show an optimistic tendency to technological progress -technophilia- than German nonbuyers. However, Mexican consumer and non-buyer groups seem insignificant and more optimistic than Germans about technological progress (see Figure 75). 
Figure 75: Technological progress - average value priorities of group

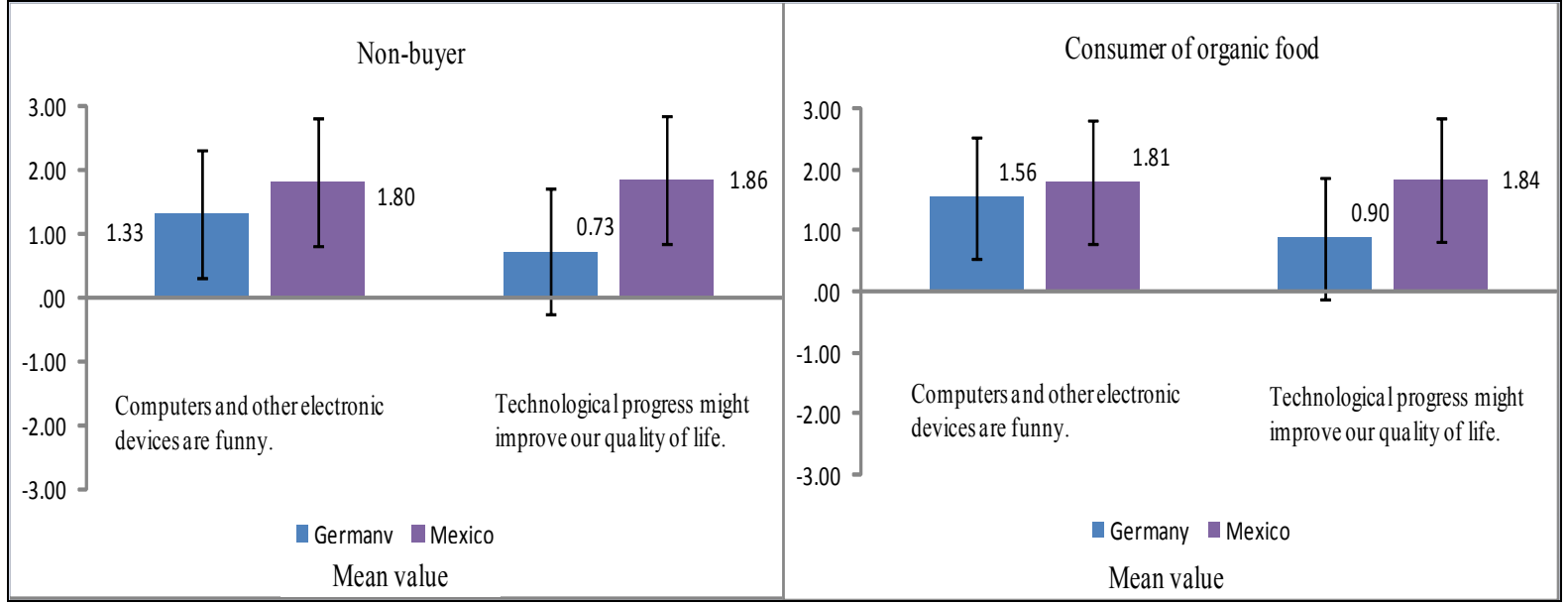

7-point Likert-type scale: (-3) strongly disagree, (-2) disagree, (-1) somewhat disagree, (0) neither agree nor disagree, (1) somewhat agree, (2) agree, and (3) strongly agree. "Computers and other electronic devices are funny". F value $=7.831$. "Technological progress might improve our quality of life". $F=62.961$.

\subsubsection{Fatalisms}

In relation to the inability to stop climate change the average value for German non-buyers $(\mathrm{M}=-0.44)(\sigma=1.670)$ is less negative than for Mexicans $(\mathrm{M}=-1.06)(\sigma=2.089)$. Similarly, the average value for German non-buyers $(M=0.13)(\sigma=1.835)$ is positive than for Mexicans $(\mathrm{M}=-0.76)(\sigma=2.245)$. Consequently, among the Germans non-buyers there are negative attitudes toward the reduction of garbage than Mexican that seem more optimistic to stop climate change and reduce garbage (see Figure 76).

The average values provided among the consumers of organic food seems different. Regarding inability to stop climate change, the average value for Germans $(\mathrm{M}=-0.89)$ $(\sigma=1.607)$ is less negative than for Mexicans $(\mathrm{M}=-1.35)(\sigma=1.886)$. Concerning care about the environment "It will not change anything if I reduce the garbage I produce, because noone else cares about the environment" the average values for Germans $(M=-0.45)(\sigma=1.701)$ is less negative than that of Mexicans $(\mathrm{M}=-1.12)(\sigma=2.104)$. German and Mexican consumers seem more optimistic than non-buyers regarding the care of environment and stop of climate change (see Figure 76). 
Figure 76: Fatalism - average value priorities of group

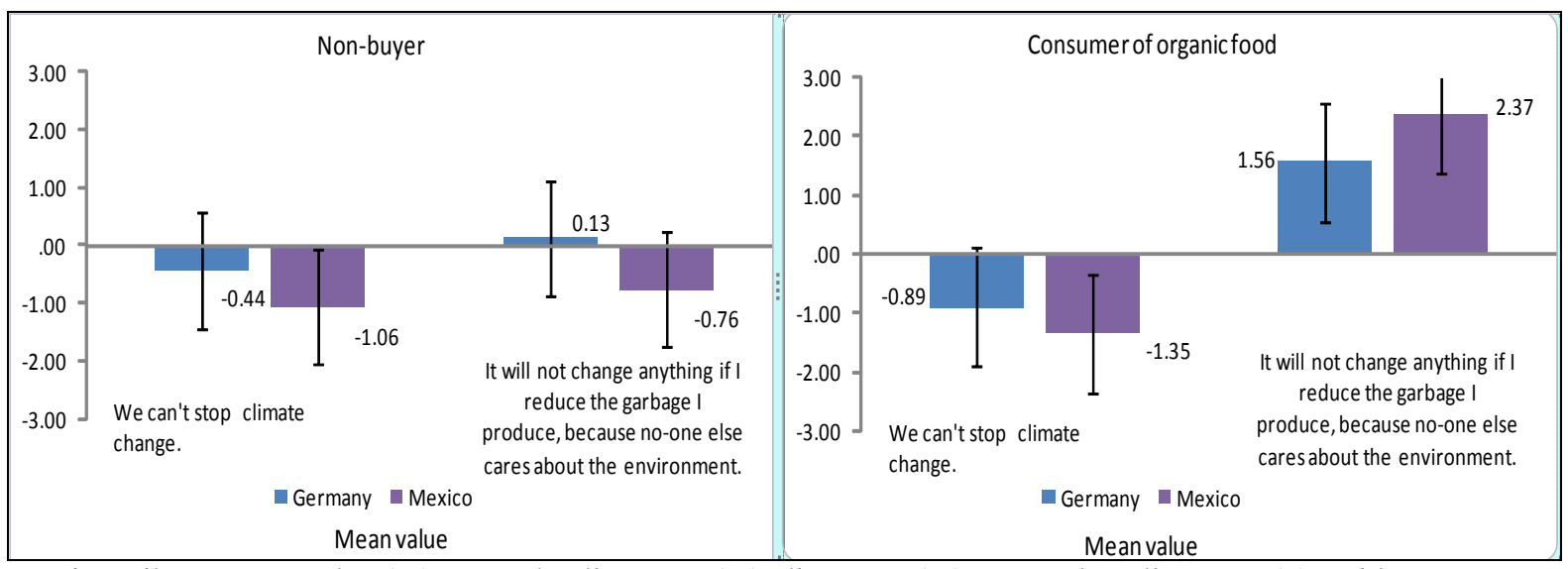

7-point Likert-type scale: (-3) strongly disagree, (-2) disagree, (-1) somewhat disagree, (0) neither agree nor disagree, (1) somewhat agree, (2) agree, and (3) strongly agree. "We can't stop climate change". F value = 10.610. "It will not change anything if I reduce the garbage I produce, because no-one else cares about the environment". $\mathrm{F}=17.258$.

\subsubsection{Fairness}

Regarding fairness, the average values provided for non-buyers in Figure 77 is related to the recognition of farmers as basis of their well-being. The average value for German non-buyers $(\mathrm{M}=0.46)(\sigma=1.416)$ is less positive than for Mexicans $(\mathrm{M}=1.70)(\sigma=1.335)$. Regarding every individual's responsibility for their own well-being, the average value for German non-buyers $(\mathrm{M}=0.57)(\sigma=1.472)$ is less positive than for Mexicans $(\mathrm{M}=1.48)(\sigma=1.497)$. Mexicans are more positive to fairness than Germans non-buyers.

The average values among consumers of organic food seem similar from that of non-buyers. Figure 77 also shows the average values concerning recognition of farmers as basis of their well-being. The average value for Germans $(M=0.43)(\sigma=1.374)$ is less positive than for Mexicans $(M=1.73)(\sigma=1.165)$. Concerning the average values related to every individual's responsibility for their own well-being, again, the average value for Germans $(\mathrm{M}=0.56)$ $(\sigma=1.450)$ is less positive than that of Mexicans $(\mathrm{M}=1.62)(\sigma=1.341)$. Regarding fairness, there seems to be differences between German and Mexican consumers and non-buyers. Mexicans seem more concern with fairness than Germans who seem more moderates. 
Figure 77: Fairness - average value priorities of group

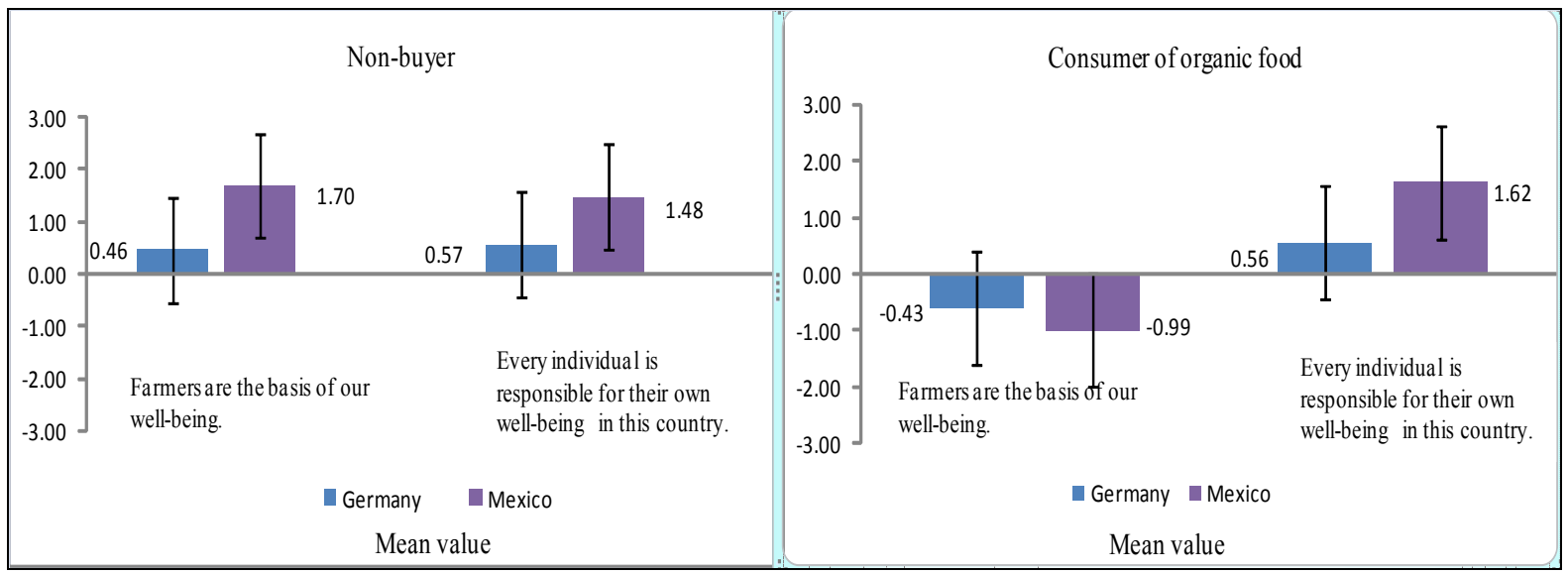

7-point Likert-type scale: (-3) strongly disagree, (-2) disagree, (-1) somewhat disagree, (0) neither agree nor disagree, (1) somewhat agree, (2) agree, and (3) strongly agree. "Farmers are the basis of our well-being". F value $=77.051$. "Every individual is responsible for their own well-being in this country". $\mathrm{F}=39.612$.

\subsubsection{Hedonism}

Regarding having fun as the most important thing in life, the average values provided for nonbuyers are displayed in Figure 78. The average values for German non-buyers $(\mathrm{M}=0.50)$ $(\sigma=1.535)$ is positive and negative for Mexicans $(\mathrm{M}=-0.77)(\sigma=1.937)$. With respect to the most important is to enjoy life and be pleasant to others. The average value for German nonbuyers $(M=0.74)(\sigma=1.358)$ is positive and slightly more positive for Mexicans $(M=1.25)$ $(\sigma=1.460)$.

The average values among consumers of organic food seem to differ with non-buyers. For the statement: "The most important thing in life is to have fun". The average value for Germans $(\mathrm{M}=0.33)(\sigma=1.450)$ is more positive than for Mexicans $(\mathrm{M}=-0.89)(\sigma=1.940)$. Concerning enjoying the life and be pleasant to others as the most important. The average value for Germans $(\mathrm{M}=0.86)(\sigma=1.261)$ is slightly less positive than that of Mexicans $(\mathrm{M}=1.19)$ $(\sigma=1.384)$. In Germany consumers appear to be slightly similar concerned with hedonism than non-buyers. While differences between Mexican consumers and non-buyers are slight (see Figure 78). However, cultural differences between Germans and Mexicans are uncovered. 
Figure 78: Hedonism - average value priorities of group

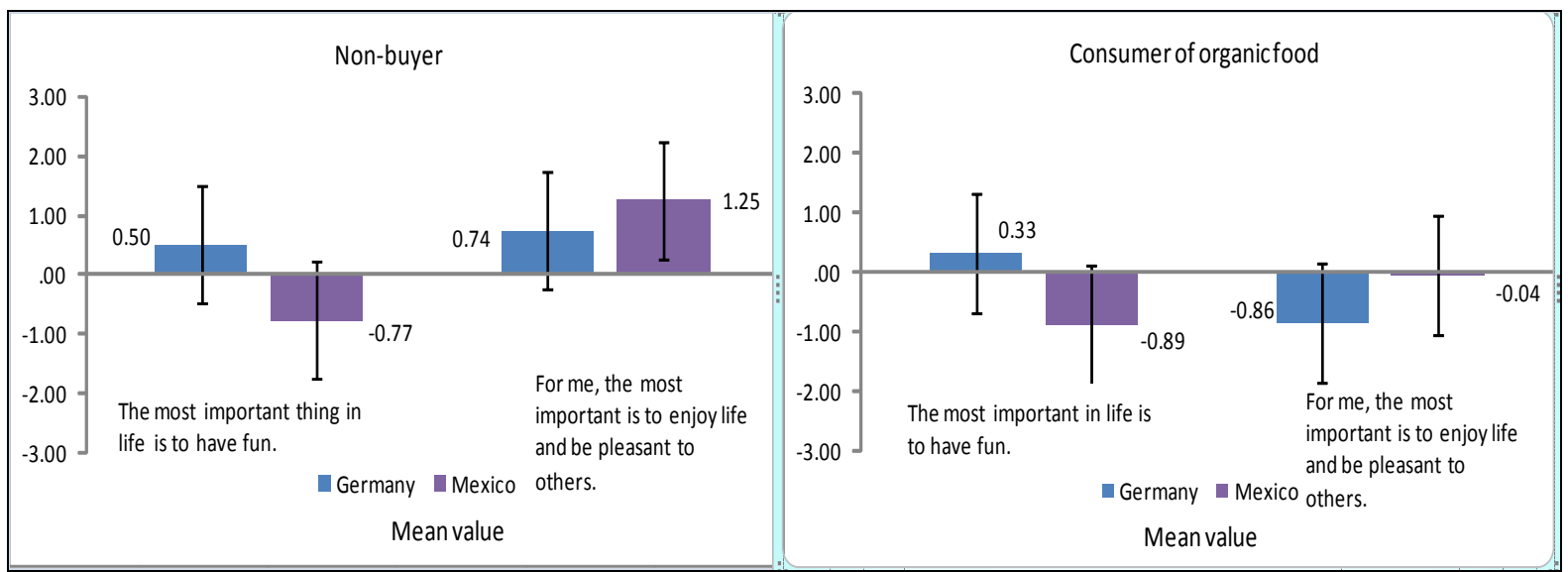

7-point Likert-type scale: (-3) strongly disagree, (-2) disagree, (-1) somewhat disagree, (0) neither agree nor disagree, (1) somewhat agree, (2) agree, and (3) strongly agree. "The most important thing in life is to have fun". $\mathrm{F}$ value $=43.604$. "For me, the most important is to enjoy life and be pleasant to others". $\mathrm{F}=8.106$.

\subsection{Factor analysis}

After the univariate and bivariate analyses described in the previous sections, Exploratory Factor Analysis were done using a principal component analysis with varimax rotation firstly to identify underlying constructs and secondly to reduce the number of variables in order to retain information as much as possible (Aaker et al., 2007).

Firstly, to determine cultural differences, the total samples in Germany (501) and Mexico (507) including consumers and non-buyers were analysed. The expected outcome of this action was to find differences between the two samples regarding motives to purchase organic food within groups, i.e. Mexican and German non-buyers and consumers of organic food, respectively; after that, differences of values between the two samples were analysed, as well as differences between consumers of organic food. The procedure to analyse the data is the following.

Ten separate exploratory factor analyses were carried out (principal component analysis with varimax rotation as a factor extraction procedure). The aim of this procedure was to check whether the factors for each sample would tend to group items together (Grunert et al., 1993) into the following dimensions: 1) motives and 2) values related to purchasing of food products in general, and related to purchasing of food products. 3) and 4) motives and values 
in the purchasing of organic food. The findings of the exploratory analyses (Table 27 to Table 32) included only factors with eingenvalue $>1$ (Aaker et al., 2007).

Firstly, the 39 items of the theoretical motives were factor analysed. Secondly, the number of the variables of values the 27 items of the theoretical values were factor analysed to find cultural differences or similarities between the two total samples.

\subsubsection{Exploratory factor analyses and construction of scales of motives related to purchasing of food products in general}

Below are presented the four separates Explorative Factor Analyses (EFA) loaded to determine the motives driving the purchasing of food products in general in Germany and Mexico. 1) the factors loaded for the motives of weight control and healthy eating, 2) the motives to purchase food products in general in Germany, 3) motives to purchase food products in general in Mexico; 4) values in Germany, 5) values in Mexico.

The constructs of "weight control" and "healthy eating" were separately analysed form the rest of the theoretical motives. The resulting factor solution accounts for $69.84 \%$ of the variance explained with a KMO value of 0.620 . The two factor loadings carried out for the German sample are shown in Table 27. For the Mexican sample the resulting factor solution accounts for $70.62 \%$ of the variance explained with a KMO value of 0.601 are displayed in Table 27.

Apart from the analysis of weight control and healthy eating five factors were loaded by the German sample, the resulting factor solution accounts for $63.40 \%$ of the variance explained with a KMO value of 0.853 , the factor loadings are shown in Table 28 . Only the first fourth were retained. The fifth factor was dropped because the lower reliability. The resulting factor solution for the Mexican sample; regards five factors loaded they accounts for $58.24 \%$ of the variance with a KMO value of 0.805 and are below described in Table 30. Similarly to the German sample, the fifth factor was dropped because the lower reliability.

The resulting factor solution accounts for $66.60 \%$ of the variance explained with a KMO value of 0.696 for the German sample; the factor loadings are shown in Table 31. Six factors were loaded and two of them were dropped because their low reliability. The resulting factor 
solution for the Mexican sample accounts for $60.30 \%$ of the variance with a KMO value of 0.734 .

\subsubsection{Exploratory factor analyses of weight control and healthy eating: German and Mexican total samples}

The factor -"weight control"- was the principal and explains $44.52 \%$ of the variance (Cronbach's $\alpha=0.79$ ). This factor consists of three items stressing the consumption of low calories, low fat food and forcing oneself to consume healthy food even though if it is not tasty food. The factor -Healthy- was the second one and explains $25.32 \%$ of the variance. The factor was omitted in further analysis because of its low reliability -internal consistency(Cronbach's $\alpha=0.42)$.

Table 27: Exploratory factor analyses of weight control and healthy motives related to purchase food products: German consumers and non-buyers of organic food.

\begin{tabular}{|l|l|l|r|r|r|}
\hline $\begin{array}{l}\text { Dimension } \\
\text { of motives }\end{array}$ & \multicolumn{1}{|c|}{ Construct } & \multicolumn{1}{|c|}{ Indicators (items) } & $\begin{array}{c}\text { Factor } \\
\text { loading }\end{array}$ & $\begin{array}{c}\text { Variance } \\
\text { explained } \\
\text { (\%) }\end{array}$ & $\begin{array}{c}\text { Cronbach's } \\
\text { Alpha }\end{array}$ \\
\hline $\begin{array}{l}\text { Weight } \\
\text { control }\end{array}$ & Weight control & $\begin{array}{l}\text { As much as possible, I eat a } \\
\text { low-calorie diet. }\end{array}$ & .887 & 44.52 & 0.790 \\
\cline { 2 - 6 } & Weight control & $\begin{array}{l}\text { As much as possible, I eat } \\
\text { low-fat foods. }\end{array}$ & .883 & & \\
\cline { 2 - 6 } & Healthy eating & $\begin{array}{l}\text { I force myself to eat food that } \\
\text { doesn't taste good but is } \\
\text { healthy. }\end{array}$ & .738 & & \\
\hline $\begin{array}{l}\text { Healthy } \\
\text { eating }\end{array}$ & Healthy eating & $\begin{array}{l}\text { I know I should follow a } \\
\text { healthy diet, but I don't } \\
\text { manage it. }\end{array}$ & .777 & 25.32 & \\
\hline
\end{tabular}

Source: Own elaboration (2010).

The two factor loadings are shown in Table 28. The first factor, "weight control" explains $41 \%$ of the variance. Similar to the German sample, the factor consists of three items stressing the consumption of low calories, low fat food and forcing oneself to eat healthy food which is not tasty (Cronbach's $\alpha=0.69$ ). The second factor, "healthy eating”, explains $29.55 \%$ of the variance and stresses positive in healthy diet and rarely being able to manage weight control (Cronbach's $\alpha=0.61$ ). Both were retained for further analysis. 
Table 28: Exploratory factor analyses for the weight control and healthy motives related to purchase food products: Mexican consumers and non-buyers of organic food

\begin{tabular}{|l|l|l|r|r|r|}
\hline $\begin{array}{l}\text { Dimension } \\
\text { of motives }\end{array}$ & \multicolumn{1}{|c|}{ Construct } & \multicolumn{1}{|c|}{ Indicators (items) } & $\begin{array}{c}\text { Factor } \\
\text { loading }\end{array}$ & $\begin{array}{c}\text { Variance } \\
\text { explained } \\
\text { (\%) }\end{array}$ & $\begin{array}{c}\text { Cronbach's } \\
\text { Alpha }\end{array}$ \\
\hline $\begin{array}{l}\text { Weight } \\
\text { control }\end{array}$ & Weight control & $\begin{array}{l}\text { As much as possible, I eat a } \\
\text { low-calorie diet. }\end{array}$ & 0.861 & 41.06 & 0.698 \\
\cline { 2 - 6 } & Weight control & $\begin{array}{l}\text { As much as possible, I eat low- } \\
\text { fat foods. }\end{array}$ & 0.848 & & \\
\cline { 2 - 6 } & Healthy eating & $\begin{array}{l}\text { I force myself to eat food that } \\
\text { doesn't taste good but is healthy. }\end{array}$ & 0.678 & & \\
\hline \multirow{2}{*}{$\begin{array}{l}\text { Healthy } \\
\text { eating }\end{array}$} & Healthy eating & $\begin{array}{l}\text { I know I should follow a healthy } \\
\text { diet, but I don't manage it. }\end{array}$ & 0.857 & 29.55 & 0.661 \\
\cline { 2 - 6 } & Weight control & I rarely weight myself. & 0.832 & & \\
\hline \multicolumn{2}{|l|}{ Total variance explained 70.62; KMO 0.601; Mexican Sample (n=507). } \\
\hline
\end{tabular}

Source: Own elaboration (2010).

\subsubsection{Exploratory factor analyses of the motives related to purchase of food products in general: German total sample}

The principal factor is entitled "High food involvement" and explains $28.74 \%$ of the variance (Cronbach's $\alpha=0.75$ ). The factor consists of 8 items stressing positive in a combination of the theoretical constructs of natural content, free of GM food, product information, pricequality orientation, and ethnocentrism.

The second factor labelled as "Product Information" explains $13.42 \%$ of the variance. The factor consists of 3 items stressing positive on product information concerning it availability on packaging about the shake to nature with their production, and animal welfare in the case of animal food products (Cronbach's $\alpha=0.65$ ).

The third factor labelled as "Convenience" explains $9.87 \%$ of the variance stressing quick availability, purchasing of food as unpleasant and easy to prepare, consists of 3 items. (Cronbach's $\alpha=0.65)$.

The fourth factor was labelled as "Advertising" pertaining to 2 items stressing positive on the receptiveness of attractive packaging and advertisements. The factor explains $5.76 \%$ of the variance (Cronbach's $\alpha=0.65$ ). The fifth factor- "Nostalgia”- explains 5.59\% of the variance, however, it was omitted from further analyses because of low reliability-internal consistency (Cronbach's $\alpha=0.29$ ). 
Table 29: Exploratory factor analyses of the motives related to purchase food products: German non-buyers and consumers of organic food

\begin{tabular}{|c|c|c|c|c|c|}
\hline $\begin{array}{l}\text { Dimension of } \\
\text { motives }\end{array}$ & Construct & Indicators (items) & $\begin{array}{l}\text { Factor } \\
\text { loading }\end{array}$ & $\begin{array}{l}\text { Variance } \\
\text { explained } \\
(\%)\end{array}$ & $\begin{array}{l}\text { Cronbach's } \\
\text { Alpha }\end{array}$ \\
\hline \multirow[t]{8}{*}{$\begin{array}{l}\text { High Food } \\
\text { involvement }\end{array}$} & Natural content & $\begin{array}{l}\text { I try to buy food that does not } \\
\text { contain artificial flavours. }\end{array}$ & 0.841 & 28.74 & 0.751 \\
\hline & $\begin{array}{l}\text { Environmental } \\
\text { concern/ } \\
\text { information }\end{array}$ & $\begin{array}{l}\text { I am careful to buy food that is } \\
\text { produced without disturbing the } \\
\text { natural balance. }\end{array}$ & 0.783 & & \\
\hline & $\begin{array}{l}\text { Consumer } \\
\text { ethnocentrism }\end{array}$ & $\begin{array}{l}\text { I avoid products for which I do } \\
\text { not know the country of origin. }\end{array}$ & 0.780 & & \\
\hline & Natural content & $\begin{array}{l}\text { I avoid all products containing } \\
\text { flavour enhancers. }\end{array}$ & 0.755 & & \\
\hline & Information & $\begin{array}{l}\text { Before buying food, I carefully } \\
\text { read information on the } \\
\text { package. }\end{array}$ & 0.747 & & \\
\hline & Free of GMO & $\begin{array}{l}\text { I buy only non-genetically } \\
\text { modified food products. }\end{array}$ & 0.727 & & \\
\hline & $\begin{array}{l}\text { Consumer } \\
\text { ethnocentrism }\end{array}$ & $\begin{array}{l}\text { I prefer fruit and vegetables } \\
\text { grown in my own country. }\end{array}$ & 0.604 & & \\
\hline & $\begin{array}{l}\text { Price-Quality } \\
\text { relation }\end{array}$ & $\begin{array}{l}\text { I prefer to buy higher priced } \\
\text { foods, because they are better } \\
\text { quality. }\end{array}$ & 0.560 & & \\
\hline \multirow[t]{3}{*}{$\begin{array}{l}\text { Product } \\
\text { Information }\end{array}$} & $\begin{array}{l}\text { Environmental } \\
\text { concern/ } \\
\text { information }\end{array}$ & $\begin{array}{l}\text { It is difficult to know if the } \\
\text { production of my food has a } \\
\text { negative impact on the } \\
\text { environment. }\end{array}$ & 0.813 & 13.42 & 0.659 \\
\hline & $\begin{array}{l}\text { Animal welfare/ } \\
\text { information }\end{array}$ & $\begin{array}{l}\text { Regarding dairy and meat } \\
\text { products, it is difficult to know } \\
\text { whether the animals have been } \\
\text { treated well. }\end{array}$ & 0.760 & & \\
\hline & Information & $\begin{array}{l}\text { I would like more information } \\
\text { to be provided on the } \\
\text { packaging. }\end{array}$ & 0.626 & & \\
\hline \multirow[t]{3}{*}{ Convenience } & $\begin{array}{l}\text { Shopping } \\
\text { convenience }\end{array}$ & Food shopping should be quick. & 0.775 & 9.87 & 0.656 \\
\hline & $\begin{array}{l}\text { Shopping } \\
\text { convenience }\end{array}$ & $\begin{array}{l}\text { For me, food shopping is an } \\
\text { unpleasant task. }\end{array}$ & 0.763 & & \\
\hline & $\begin{array}{l}\text { Cooking } \\
\text { convenience }\end{array}$ & $\begin{array}{l}\text { I think that meals should be } \\
\text { easy to prepare. }\end{array}$ & 0.720 & & \\
\hline \multirow[t]{2}{*}{ Advertising } & Advertising & $\begin{array}{l}\text { I frequently buy food because of } \\
\text { the packaging. }\end{array}$ & 0.844 & 5.76 & 0.650 \\
\hline & Advertising & $\begin{array}{l}\text { I frequently buy food that I have } \\
\text { previously seen advertised. }\end{array}$ & 0.806 & & \\
\hline \multirow[t]{2}{*}{ Nostalgia } & Nostalgia & $\begin{array}{l}\text { In my childhood, food tasted } \\
\text { better. }\end{array}$ & 0.782 & 5.59 & 0.299 \\
\hline & Nostalgia & $\begin{array}{l}\text { In the past, I had more } \\
\text { confidence in the quality of } \\
\text { food. }\end{array}$ & 0.765 & & \\
\hline
\end{tabular}

Source: Own elaboration (2010). 


\subsubsection{Exploratory factor analyses and construction of scales of motives related to purchase of food products in general: Mexican sample}

The main factor consists of 5 items explaining $21.4 \%$ of the variance. The factor is labelled as "Low involvement"; due to 2 items stress positive in the general disregard of ingredients in food, 2 other items stress positive in purchasing because of packaging and being able to quickly purchase food and one item stress positive in purchasing because advertising. (Cronbach's $\alpha=0.79)$.

The second factor is labelled as "High food involvement"; this factor is slightly similar to the one carried out in the German sample and explains $15.34 \%$ of the variance. However, the difference in the Mexican sample is that it consists of only 5 items that stressed positive in the concern regarding the naturalness of food ingredients, product information available on the packaging, and avoidance or distrust if the country of origin is unknown (Cronbach's $\alpha=0.72)$.

The third factor explains $8.74 \%$ of the variance was labelled as "Nostalgia" and is similar to the factor carried out in the German sample with the same label; however, within the Mexican sample, this factor consists of 3 items stressing high positive that taste and quality were better in the past (Cronbach's $\alpha=0.63)$.

The fourth factor "Product orientation" explains $6.72 \%$ of the variance consists of 3 items stressing positive in the purchasing of well-known brands, willing to purchase foreign products or brands, and the preference for fruits and vegetables from the home country. The first item refers "familiarity" due to the preference for well-known brands. The second and third items seem in a first view as contradictory because the preference for foreign products or brands but simultaneously the preference for fruits and vegetables produced in the country. However, the fact is not at all surprising due to on the one hand, the country is characterised as producer/exporter of fruits and vegetables. Consequently these are preferred because freshness whereas non-fresh food products imported or foreign might be preferred in the ground of more variety in processed products assortment that are not produced by the national food sector industry. i.e., soya milk, wine, cereals (Cronbach's $\alpha=0.56)$. Other fact useful to understand the apparently contradiction is the fact that Mexicans show neophilic and 
neophobic attitudes toward food products (see average value priorities of groups, and frequency for the dimension of Familiarity/Neophobia).

The fifth factor "Miscellaneous", is the weak factor, it explains $6 \%$ of the variance. The factor was omitted because of low reliability-internal consistency (Cronbach's $\alpha=0.44$ ). 
Table 30: Exploratory factor analyses for the motives related to purchase food products: Mexican consumers and non-buyers of organic food

\begin{tabular}{|c|c|c|c|c|c|}
\hline $\begin{array}{l}\text { Dimension of } \\
\text { motives }\end{array}$ & Construct & Indicators (items) & $\begin{array}{l}\text { Factor } \\
\text { loading }\end{array}$ & \begin{tabular}{|c|}
$\begin{array}{c}\text { Variance } \\
\text { explained } \\
(\%)\end{array}$ \\
\end{tabular} & $\begin{array}{l}\text { Cronbach's } \\
\text { Alpha }\end{array}$ \\
\hline \multirow[t]{5}{*}{$\begin{array}{l}\text { Low food } \\
\text { involvement }\end{array}$} & Natural content & $\begin{array}{l}\text { I eat what I like and rarely care } \\
\text { about the ingredients. }\end{array}$ & 0.760 & 21.40 & 0.79 \\
\hline & $\begin{array}{l}\text { Shopping } \\
\text { convenience }\end{array}$ & $\begin{array}{l}\text { For me, food shopping is an } \\
\text { unpleasant task. }\end{array}$ & 0.735 & & \\
\hline & Advertising & $\begin{array}{l}\text { I frequently buy food because of } \\
\text { the packaging. }\end{array}$ & 0.730 & & \\
\hline & Natural content & $\begin{array}{l}\text { I rarely check to see whether a } \\
\text { product contains preservatives. }\end{array}$ & 0.720 & & \\
\hline & $\begin{array}{l}\text { Shopping } \\
\text { convenience }\end{array}$ & Food shopping should be quick. & 0.662 & & \\
\hline \multirow[t]{5}{*}{$\begin{array}{l}\text { High food } \\
\text { involvement }\end{array}$} & Natural content & $\begin{array}{l}\text { I avoid all products containing } \\
\text { flavour enhancers. }\end{array}$ & 0.771 & 15.34 & 0.724 \\
\hline & $\begin{array}{l}\text { Environmental } \\
\text { concern/ } \\
\text { information } \\
\end{array}$ & $\begin{array}{l}\text { I am careful to buy food produced } \\
\text { in way that does not upset the } \\
\text { balance of nature. }\end{array}$ & 0.692 & & \\
\hline & Natural content & $\begin{array}{l}\text { I try to buy food that does not } \\
\text { contain artificial flavours. }\end{array}$ & 0.682 & & \\
\hline & Information & $\begin{array}{l}\text { Before buying food, I carefully } \\
\text { read the information on the } \\
\text { package. }\end{array}$ & 0.591 & & \\
\hline & $\begin{array}{l}\text { Consumer } \\
\text { ethnocentrism }\end{array}$ & $\begin{array}{l}\text { I avoid products for which I do } \\
\text { not know the country of origin. }\end{array}$ & 0.516 & & \\
\hline \multirow[t]{3}{*}{ Nostalgia } & Nostalgia & $\begin{array}{l}\text { In my childhood, food tasted } \\
\text { better. }\end{array}$ & 0.811 & 8.74 & 0.639 \\
\hline & Nostalgia & $\begin{array}{l}\text { Before, I had more confidence in } \\
\text { the quality of food. }\end{array}$ & 0.709 & & \\
\hline & Nostalgia & $\begin{array}{l}\text { When I come across foods from } \\
\text { my childhood, I immediately buy } \\
\text { them. }\end{array}$ & 0.622 & & \\
\hline \multirow[t]{3}{*}{$\begin{array}{l}\text { Product } \\
\text { orientation }\end{array}$} & $\begin{array}{l}\text { Familiarity/ } \\
\text { Neophobia }\end{array}$ & $\begin{array}{l}\text { I tend to buy well-known brands } \\
\text { of food products. }\end{array}$ & 0.733 & 6.72 & 0.566 \\
\hline & Malinchismo & $\begin{array}{l}\text { Many times I have the impression } \\
\text { that foreign food products are } \\
\text { better than Mexican products. }\end{array}$ & 0.679 & & \\
\hline & $\begin{array}{l}\text { Consumer } \\
\text { ethnocentrism }\end{array}$ & $\begin{array}{l}\text { I prefer fruit and vegetables from } \\
\text { my home country. }\end{array}$ & 0.647 & & \\
\hline \multirow[t]{2}{*}{ Miscellaneous } & Information & I feel well informed about food. & 0.733 & 6.01 & 0.44 \\
\hline & $\begin{array}{l}\text { Shopping } \\
\text { convenience }\end{array}$ & $\begin{array}{l}\text { I think it is beneficial that the } \\
\text { assortment of convenience food is } \\
\text { growing. }\end{array}$ & 0.693 & & \\
\hline
\end{tabular}

Source: Own elaboration (2010). 


\subsubsection{Exploratory factor analyses and construction of scales of values related to purchasing of food products in general: German sample}

The main factor explains $21.08 \%$ of the variance and is termed "Hedonism" due to the 3 items stressed positive in the goals of pursuit of individual interest i.e., fun, variety in life, and enjoyment (Cronbach's $\alpha=0.72$ ).

The second factor was labelled as "Fatalism" explains the $11.96 \%$ of the variance. The label of the factor was inspired by Rotter, (1966) (cited in Ross et al., 1983). According to Rotter (1966) "fatalism is a general expectation that outcomes of situations are determined by external forces (cited in Ross et al., 1983). The factor consists of 2 items stressed positive in the impossibility to avoid the destruction of the earth and the lost of natural resources (Cronbach's $\alpha=0.73$ ).

The third factor, "Unhappiness" explains the $9.95 \%$ of the variance. The factor consist of 4 items stressing positive on financial dissatisfaction, dislike for people displaying possessions, worry about changes, and hopelessness about outcomes of situation with at external locus of control -fatalism- with regards to environmental destruction (Cronbach's $\alpha=0.60$ ).

The fourth factor, "Self-esteem" explains $9.14 \%$ of the variance consists of 2 items from the theoretical construct of fairness and was labelled as "self-esteem" due to both items stress positive in the individual effort -self-sufficiency- to improve well-being. In this case the answers remarks an internal locus of control orientation within this dimension (Cronbach's $\alpha$ $=0.69)$.

The fifth factor, "Technophilia", explains $7.99 \%$ of the variance (Cronbach's $\alpha=0.48$ ) and the sixth factor "Ethnocentrism", explains $6.46 \%$ of the variance (Cronbach's $\alpha=0.47$ ). Both factors were omitted from further analysis because of the low reliability-internal consistency (Cronbach's $\alpha$ ). 
Table 31: Exploratory factor analyses of values related to purchasing of food: German sample

\begin{tabular}{|c|c|c|c|c|c|}
\hline $\begin{array}{l}\text { Dimension of } \\
\text { values }\end{array}$ & Construct & Indicators (items) & $\begin{array}{l}\text { Factor } \\
\text { loading }\end{array}$ & $\begin{array}{l}\text { Variance } \\
\text { explained } \\
(\%)\end{array}$ & $\begin{array}{l}\text { Cronbach' } \\
\text { s Alpha }\end{array}$ \\
\hline \multirow[t]{3}{*}{ Hedonism } & Hedonism & $\begin{array}{l}\text { The most important thing in } \\
\text { life is to have fun. }\end{array}$ & 0.790 & 21.08 & 0.724 \\
\hline & $\begin{array}{l}\text { Variety in life / } \\
\text { Neophobia }\end{array}$ & I need variety in life. & 0.770 & & \\
\hline & Hedonism & $\begin{array}{l}\text { For me, the most important is } \\
\text { to enjoy life and be pleasant } \\
\text { to others. }\end{array}$ & 0.741 & & \\
\hline \multirow[t]{2}{*}{ Fatalism } & Fatalism & $\begin{array}{l}\text { Lots of natural resources have } \\
\text { already been destroyed. }\end{array}$ & 0.855 & 11.96 & 0.736 \\
\hline & Fatalism & $\begin{array}{l}\text { If we continue in this way, we } \\
\text { will destroy the earth. }\end{array}$ & 0.839 & & \\
\hline \multirow[t]{4}{*}{ Unhappiness } & Materialism & $\begin{array}{l}\text { I am dissatisfied because } \\
\text { financially I can only afford } \\
\text { very little. }\end{array}$ & 0.700 & 9.95 & 0.602 \\
\hline & $\begin{array}{l}\text { Variety in life/ } \\
\text { Neophobia }\end{array}$ & Changes worry me. & 0.680 & & \\
\hline & Fatalism & $\begin{array}{l}\text { It will not change anything if I } \\
\text { reduce the garbage I produce, } \\
\text { because no one else cares } \\
\text { about the environment. }\end{array}$ & 0.673 & & \\
\hline & Anti-Materialism & $\begin{array}{l}\text { I do not like people who } \\
\text { openly display } \\
\text { possessions. }\end{array}$ & 0.603 & & \\
\hline \multirow[t]{2}{*}{ Self-esteem } & Fairness & $\begin{array}{l}\text { In this country, whoever } \\
\text { makes an effort can improve } \\
\text { their social class. }\end{array}$ & 0.851 & 9.14 & 0.694 \\
\hline & Fairness & $\begin{array}{l}\text { Every individual is } \\
\text { responsible for their own } \\
\text { well-being in this country. }\end{array}$ & 0.830 & & \\
\hline \multirow[t]{3}{*}{ Technophilia } & $\begin{array}{l}\text { Technological } \\
\text { progress/ } \\
\text { Technophobia }\end{array}$ & $\begin{array}{l}\text { Computers and other } \\
\text { electronic devices are funny. }\end{array}$ & 0.757 & 7.99 & 0.481 \\
\hline & $\begin{array}{l}\text { Technological } \\
\text { progress/ } \\
\text { Technophobia }\end{array}$ & $\begin{array}{l}\text { Technological progress might } \\
\text { improve our quality of life. }\end{array}$ & 0.635 & & \\
\hline & $\begin{array}{l}\text { Technological } \\
\text { progress/ } \\
\text { Technophobia }\end{array}$ & $\begin{array}{lll}\text { I'm not worried that } & \text { technological progress } & \text { will } \\
\text { destroy our lives. }\end{array}$ & 0.594 & & \\
\hline \multirow[t]{2}{*}{ Ethnocentrism } & Ethnocentrism & $\begin{array}{l}\text { I am firmly rooted in my } \\
\text { country and its culture. }\end{array}$ & 0.786 & 6.46 & 0.472 \\
\hline & Ethnocentrism & $\begin{array}{l}\text { I cannot imagine living } \\
\text { permanently abroad. }\end{array}$ & 0.771 & & \\
\hline
\end{tabular}

Source: Own elaboration (2010). 


\subsubsection{Exploratory factor analyses and construction of scales of values related to purchasing of food products in general: Mexican sample}

Similarly to the German sample, six factors were loaded. The factors of "Fatalism", "Selfesteem", "Technophilia", and "Ethnocentrism" (see Table 32). "Technophilia", and "Ethnocentrism" were dropped because their low realibility. However, as it was expected cultural differences are reflected by the factor loading which seem stronger by the German sample than the carried out by the Mexican and the different factors resulting below described.

The first factor is termed as "Living now" and explains $18.86 \%$ of the variance. The name was inspired in a similar finding reported in 1988 by Hofstede and Bond in a dimension named by the authors as short term orientation "where spending now is more important than saving for tomorrow" (cited in Mooij, 2004). With regards to our study the factor consists of 4 items stressing positive in fatalism and hedonistic theoretical dimensions. Fatalism regard the impossibility to stop the climate change and hopelessness to care about environment whereas the items stressing positive in hedonism regards fun as the important and neophobia due to the worry about changes (Cronbach's $\alpha=0.68$ ).

The second factor is labelled as "Materialism", explains $12.3 \%$ of the variance the 4 items factor stressed positive in the goals of the pursuit of individuals' own interest, and the identification with foreign lifestyles. In Mexico; most of the trends in fashion, innovative products and lifestyle are strongly promoted in advertising (Cronbach's $\alpha=0.66$ ).

The third factor, "Fatalism" explains $10.09 \%$ of the variance. The factor is similar to the one carried out by the German sample; however, in the Mexican sample the factor consists of 3 items stressing positive in hopelessness of the impossibility to avoid destruction of environment and natural resources (Cronbach's $\alpha=0.64)$.

The fourth factor is also similar as the one carried out by the German sample labelled as "Self-esteem"; among the Mexican sample the factor explains $7.32 \%$ of the variance. The factor consists of 2 items stressed positive in the self-sufficiency of everyone (Cronbach's $\alpha=0.57)$. 
The factors of "Technophilia" (Cronbach's $\alpha=0.37$ ), and "Ethnocentrism" (Cronbach's $\alpha=0.33$ ), were carried out among the Mexican sample similarly to the German sample, and in this case they were omitted from the analysis because of low reliability-internal consistency.

Table 32: Exploratory factor analysis of values related to purchasing of food: Mexican sample

\begin{tabular}{|c|c|c|c|c|c|}
\hline $\begin{array}{l}\text { Dimension } \\
\text { of values }\end{array}$ & Construct & Indicators (items) & $\begin{array}{l}\text { Factor } \\
\text { loading }\end{array}$ & $\begin{array}{l}\text { Variance } \\
\text { explained } \\
(\%)\end{array}$ & $\begin{array}{l}\text { Cronbach's } \\
\text { Alpha }\end{array}$ \\
\hline \multirow[t]{4}{*}{ Living Now } & Fatalism & We can't stop climate change. & 0.742 & 18.86 & 0.681 \\
\hline & Hedonism & $\begin{array}{l}\text { The most important thing in life is } \\
\text { to have fun. }\end{array}$ & 0.731 & & \\
\hline & Fatalism & $\begin{array}{l}\text { It will not change anything if I } \\
\text { reduce the garbage I produce, } \\
\text { because no one else cares about the } \\
\text { environment. }\end{array}$ & 0.715 & & \\
\hline & Neophobia & Changes worry me. & 0.581 & & \\
\hline \multirow[t]{4}{*}{$\begin{array}{l}\text { Materialis } \\
\text { m }\end{array}$} & Materialism & $\begin{array}{l}\text { I work so that I can afford some } \\
\text { luxuries in life. }\end{array}$ & 0.740 & 12.30 & 0.660 \\
\hline & Hedonism & $\begin{array}{l}\text { For me, most importantly is to } \\
\text { enjoy life and be pleasant to others. }\end{array}$ & 0.721 & & \\
\hline & Malinchismo & $\begin{array}{l}\text { I identify strongly with the lifestyle } \\
\text { of other countries (e.g. U.S.A., } \\
\text { Canada, and Europe). }\end{array}$ & 0.643 & & \\
\hline & Hedonism & $\begin{array}{l}\text { I'm not worried about the future; } \\
\text { most important is the present. }\end{array}$ & 0.542 & & \\
\hline \multirow[t]{3}{*}{ Fatalism } & Fatalism & $\begin{array}{l}\text { Lots of natural resources have } \\
\text { already been destroyed. }\end{array}$ & 0.825 & 10.09 & 0.645 \\
\hline & Fatalism & $\begin{array}{l}\text { In comparison to other countries, } \\
\text { we act irresponsibly toward our } \\
\text { natural resources. }\end{array}$ & 0.786 & & \\
\hline & Fatalism & $\begin{array}{l}\text { If we continue in this way, we will } \\
\text { destroy the earth. }\end{array}$ & 0.622 & & \\
\hline \multirow[t]{2}{*}{ Self-esteem } & Fairness & $\begin{array}{l}\text { In this country, whoever makes an } \\
\text { effort can improve their social } \\
\text { class. }\end{array}$ & 0.842 & 7.32 & 0.573 \\
\hline & Fairness & $\begin{array}{l}\text { Every individual is responsible for } \\
\text { their own well-being in this } \\
\text { country. }\end{array}$ & 0.753 & & \\
\hline \multirow[t]{3}{*}{$\begin{array}{l}\text { Technophili } \\
\text { a }\end{array}$} & $\begin{array}{l}\text { Technological } \\
\text { progress/ } \\
\text { techno-phobia }\end{array}$ & $\begin{array}{l}\text { Technological progress might } \\
\text { improve our quality of life. }\end{array}$ & 0.706 & 6.10 & 0.373 \\
\hline & $\begin{array}{l}\text { Technological } \\
\text { progress/ }\end{array}$ & $\begin{array}{l}\text { I'm not worried that technological } \\
\text { progress will destroy our lives. }\end{array}$ & 0.663 & & \\
\hline & techno-phobia & $\begin{array}{l}\text { Computers and other electronic } \\
\text { devices are funny. }\end{array}$ & 0.510 & & \\
\hline \multirow[t]{2}{*}{$\begin{array}{l}\text { Ethno- } \\
\text { centrism }\end{array}$} & Ethnocentrism & $\begin{array}{l}\text { I am firmly rooted in my country } \\
\text { and its culture. }\end{array}$ & 0.803 & & \\
\hline & Ethnocentrism & $\begin{array}{l}\text { I cannot imagine living } \\
\text { permanently abroad. }\end{array}$ & 0.654 & 5.61 & 0.331 \\
\hline
\end{tabular}




\subsubsection{Exploratory factor analysis of the motivations and values in the purchase of organic food}

In this section, the findings of common motives and values of consumers of organic food in both countries will be shown. For this reason both samples were split, and we concentrated only on consumers of organic food in both countries, i.e., 269 consumers in Germany and 257 in Mexico. The procedure for this data analysis is explained below:

Two separate exploratory factor analyses (EFA) using PCA with varimax rotation were carried out. Based on Kaiser's eigenvalue-greater-than-one-criterion, only factors with eigenvalue $>1$ were retained for further analysis.

Seven factors were carried out for the German sample, the resulting factor solution accounts for $67.32 \%$ of the variance with a $\mathrm{KMO}$ value of 0.754 for the motivations to purchase organic food. With respect to the Mexican sample five factors have been extracted and the resulting solution accounts for $64.31 \%$ of the variance with a KMO value of 0.828 . All in all, the common factors were two motives: traditional motives and weight control. The factor with high loadings and indicator reliability (Cronbach's Alpha $\geq 0.5$ ) were retained to further multivariate analysis.

With regards to values, five factors of values groupings have been extracted by the German sample, and the resulting factor solution accounts for $61.78 \%$ of the variance with a KMO value of 0.714 . Five factors were carried out for the Mexican sample. The resulting factor solution accounts for $65.42 \%$ of the variance with a $\mathrm{KMO}$ value of 0.680 ; only four factors were retained. All in all; one common factor "fatalism" was found in the two samples. Only factors with high loadings and indicator reliability (Cronbach's Alpha $\geq 0.5$ ) were retained to further analysis.

Among the Mexican and German consumers of organic food were uncover attitudes with an internal locus of control. In both samples, most of the values are with arrow in the same direction and feeling but different intensity. However the dimensions carried out seem as a combination of goals in the pursuit of individual and collective interests. The factors carried out and their statements are displayed in Table 35 for the German sample and in Table 36 for the Mexican sample, respectively. 
Table 33 and Table 34 display the factor of motivations to purchase organic food carried out for the German and the Mexican samples, respectively.

\section{8.2.1 Exploratory factor analysis of the motivations in the purchase of organic food in Germany}

The factor of "Traditional motives" consists of 6 items stressing positive on the theoretical motives of animal welfare, fairness for farmers, healthy, tasty, and environmental concern. These are motives commonly mentioned in the international literature as drivers of the purchasing of organic food. The factor explains $21.66 \%$ of the total variance and show high reliability (Cronbach's $\alpha=0.884$ ) (see Table 33).

The factor of "Convenience" explains $11.86 \%$ of the total variance and consists of 4 items from the theoretical constructs of convenience in cooking and convenience in purchasing. Among the statements within the factor seem that for Germans consumers of organic food convenience should be in twofold: purchasing and in cooking. This is similar to the previous findings by Grunert et al. (1993) in the FRL cross culturally measured in Europe (Cronbach's $\alpha=0.699)$.

The factor of "Weight control" explains $8.5 \%$ of the total variance and remarks the importance of the consumption of low calories and low fat foods, however, not only because of the desire of a trim figure and fitness, also as mentioned by Steptoe et al. (1995), the eating of low calories and fats is associated with a healthy diet (Cronbach's $\alpha=0.777$ ).

The factor of "Nostalgia" explains 7.77\% of the total variance. The items within the factor are associated with better taste and quality in the past and certain mistrust in the food which consumers are not familiar with (Cronbach's $\alpha=0.674)$.

The factor of "Quality orientation" explains $6.42 \%$ of the total variance. The two items within the factor remarks an association of higher prices with better quality (Cronbach's $\alpha=0.710)$. 
The factor of "Product information" explains 5.91\% of the total variance. The factor consists of items regarding environment concern and animal welfare and the few available information about the impact on the environment and the treatment to animal in the production of the food (Cronbach's $\alpha=0.628)$.

The last factor was "Neophilia". The factor explains 5.18\% of the total variance. The factor consists of items regarding affinity to try new products and the receptiveness to advertising (Cronbach's $\alpha=0.512$ ). All the factors were retained for further analysis. Below are described the factors carried out by the Mexican consumers of organic food. 
Table 33: Exploratory factor analysis for the motivations to purchase organic food in Germany

\begin{tabular}{|c|c|c|c|c|}
\hline $\begin{array}{c}\text { Dimension } \\
\text { of } \\
\text { Motivations }\end{array}$ & Indicators (items) ${ }^{\mathrm{a}}$ & $\begin{array}{l}\text { Factor } \\
\text { loading }\end{array}$ & $\begin{array}{c}\text { Variance } \\
\text { explained } \\
(\%)\end{array}$ & $\begin{array}{l}\text { Cronbach's } \\
\text { Alpha }\end{array}$ \\
\hline \multirow{6}{*}{$\begin{array}{l}\text { Traditional } \\
\text { motives }\end{array}$} & Organic produce is better for the environment. & 0.834 & 21.66 & 0.884 \\
\hline & $\begin{array}{l}\text { I buy organic products because I believe that it is a } \\
\text { better way of production for the animals. }\end{array}$ & 0.809 & & \\
\hline & Organic products are healthier than other products. & 0.804 & & \\
\hline & $\begin{array}{l}\text { Only with organic meat could I be sure that hormones } \\
\text { and antibiotics were not used. }\end{array}$ & 0.802 & & \\
\hline & Organic products taste better than other products. & 0.754 & & \\
\hline & Organic produce support small and medium farmers. & 0.692 & & \\
\hline \multirow[t]{4}{*}{ Convenience } & I think that meals should be easy to prepare. & 0.766 & & \\
\hline & Food shopping should be quick. & 0.715 & 11.86 & 0.699 \\
\hline & For me, food shopping is an unpleasant task. & 0.703 & & \\
\hline & $\begin{array}{l}\text { I think it is good that the assortment of convenience } \\
\text { food is growing. }\end{array}$ & 0.655 & & \\
\hline \multirow{3}{*}{$\begin{array}{l}\text { Weight } \\
\text { Control }\end{array}$} & As far as possible, I eat a low-calorie diet. & 0.926 & 8.50 & 0.777 \\
\hline & As much as possible, I eat low-fat foods. & 0.921 & & \\
\hline & $\begin{array}{l}\text { I force myself to eat food that doesn't taste good but is } \\
\text { healthy. }\end{array}$ & 0.536 & & \\
\hline \multirow[t]{3}{*}{ Nostalgia } & Before, I had more confidence in the quality of food. & 0.779 & 7.77 & 0.674 \\
\hline & In my childhood, food tasted better. & 0.754 & & \\
\hline & $\begin{array}{l}\text { I am very careful with food products that I do not } \\
\text { know. }\end{array}$ & 0.697 & & \\
\hline \multirow[t]{2}{*}{$\begin{array}{l}\text { Price quality } \\
\text { orientation }\end{array}$} & $\begin{array}{l}\text { I prefer to buy higher priced foods, because these are } \\
\text { better quality. }\end{array}$ & 0.844 & 6.42 & 0.71 \\
\hline & Low price is usually a sign of poor quality food. & 0.803 & & \\
\hline \multirow[t]{2}{*}{$\begin{array}{l}\text { Product } \\
\text { information }\end{array}$} & $\begin{array}{l}\text { It is difficult to know if the production of my food has } \\
\text { a negative impact on the environment. }\end{array}$ & 0.809 & 5.91 & 0.628 \\
\hline & $\begin{array}{l}\text { Regarding dairy and meat products, it is difficult to } \\
\text { know whether the animals have been treated well. }\end{array}$ & 0.782 & & \\
\hline \multirow{2}{*}{$\begin{array}{l}\text { Neophilia in } \\
\text { food }\end{array}$} & Concerning food, I like to try new things. & 0.818 & 5.18 & 0.512 \\
\hline & $\begin{array}{l}\text { I frequently buy food that I have previously seen } \\
\text { advertised. }\end{array}$ & 0.706 & & \\
\hline
\end{tabular}

Source: Own elaboration (2010). 


\section{8.2.2 Exploratory factor analysis of the motivations in the purchase of organic food in Mexico}

The identified motives to purchase organic food in Mexico displayed below in Table 34 . Similarly to the German sample the factor of "Traditional motives" was carried out. The factor consists of the same 6 items. The variance explain by the factor is $27 \%$ and with high reliability (Cronbach's $\alpha=0.820)$.

The dimension of food involvement among the consumers of organic food was discovering in two levels. The factor of "Low involvement" which consists of 5 items associated with the frequently purchasing because packaging, advertising, low interest in product information displayed, and purchasing or food as an unpleasant task. The factor explains $16.56 \%$ of the variance, and high reliability (Cronbach's $\alpha=0.815$ ).

In contrast, the factor of "High food involvement" consists of 4 items regarding the concern with Free GM Food, natural contents -absences of flavor enhancers, not artificial flavor-, and concern with environment. The factor explains $8.36 \%$ of the total variance and high reliability (Cronbach's $\alpha=0.759)$.

The factor of "Weight control" was carried out by the sample of Mexican consumers of organic food with two items regarding the consumption of low calories and low fat foods. The factor explains a variance of $6.34 \%$ and high reliability (Cronbach's $\alpha=0.778$ ).

The factor of "Consumer ethnocentrism" consist of only 1 item and because of the low satisfactory indicator reliability (Cronbach's Alpha $>0.4$ ) the factor was dropped.

The results of the EFA for the motives to purchase food products in general and organic food summarized in Table 33 and Table 34 show that consumers of organic products in Germany and Mexico differ in motives and values. In following, the factors of values carried out will be described. 
Table 34: Exploratory factor analysis for the motivations to purchase organic food in Mexico

\begin{tabular}{|c|c|c|c|c|}
\hline $\begin{array}{l}\text { Dimension of } \\
\text { Motivations }\end{array}$ & Indicators (items) $^{\mathrm{a}}$ & $\begin{array}{l}\text { Factor } \\
\text { loading }\end{array}$ & $\begin{array}{c}\text { Variance } \\
\text { explained } \\
(\%)\end{array}$ & $\begin{array}{c}\text { Cronbach's } \\
\text { Alpha }\end{array}$ \\
\hline \multirow{6}{*}{$\begin{array}{l}\text { Traditional } \\
\text { motives }\end{array}$} & Organic production is better for the environment. & 0.794 & 27.0 & 0.82 \\
\hline & Organic products are healthier than other products. & 0.749 & & \\
\hline & Organic products taste better than other products. & 0.741 & & \\
\hline & $\begin{array}{l}\text { Only with organic meat could I be sure that } \\
\text { hormones and antibiotics were not used. }\end{array}$ & 0.682 & & \\
\hline & $\begin{array}{l}\text { I buy organic products because I am convinced that } \\
\text { it is a better way of production for the animals. }\end{array}$ & 0.673 & & \\
\hline & $\begin{array}{l}\text { Organic production supports small and medium } \\
\text { farmers. }\end{array}$ & 0.510 & & \\
\hline \multirow[t]{5}{*}{$\begin{array}{l}\text { Low food } \\
\text { involvement }\end{array}$} & $\begin{array}{l}\text { I rarely check to see whether a product contains } \\
\text { preservatives. }\end{array}$ & 0.825 & 16.56 & 0.815 \\
\hline & I frequently buy food because of the packaging. & 0.817 & & \\
\hline & $\begin{array}{l}\text { I eat what I like and rarely care about the } \\
\text { ingredients. }\end{array}$ & 0.813 & & \\
\hline & For me, food shopping is an unpleasant task. & 0.716 & & \\
\hline & $\begin{array}{l}\text { I frequently buy food that I have previously seen } \\
\text { advertised. }\end{array}$ & 0.579 & & \\
\hline \multirow{4}{*}{$\begin{array}{l}\text { High food } \\
\text { involvement }\end{array}$} & I buy only non-genetically modified food products. & 0.721 & 8.36 & 0.759 \\
\hline & $\begin{array}{l}\text { I try to buy food that does not contain artificial } \\
\text { flavors. }\end{array}$ & 0.719 & & \\
\hline & I avoid all products containing flavor enhancers. & 0.701 & & \\
\hline & $\begin{array}{l}\text { I am careful to buy food produced in a way that } \\
\text { does not disturbs the balance of nature. }\end{array}$ & 0.650 & & \\
\hline \multirow{2}{*}{$\begin{array}{l}\text { Weight } \\
\text { control }\end{array}$} & As much as possible, I eat low-fat foods. & 0.816 & 6.34 & 0.778 \\
\hline & As much as possible, I eat a low-calorie diet. & 0.792 & & \\
\hline $\begin{array}{l}\text { Consumer } \\
\text { ethno- } \\
\text { centrism }\end{array}$ & $\begin{array}{l}\text { I prefer fruits and vegetables from my home } \\
\text { country. }\end{array}$ & 0.899 & 6.0 & ------ \\
\hline
\end{tabular}

Source: Own elaboration (2010). 


\section{8.2.3 Exploratory factor analyses and construction of index for the values to purchase organic food in the German sample}

The factor labeled as "Hedonism" is the main factor explaining $21.38 \%$ of the variance. It consists of 5 statements mirroring self-centered attitudes due to the conceptual meaning of 'fun' and 'enjoyment in life', the 'need for variety in life', 'accumulation' among the meanings. The factor shows a high reliability (Cronbach's $\alpha=0.748$ ).

The second main factor was labeled as "Fatalism", the factor consists of 2 statements from the theoretical construct of fatalism and explains $13.27 \%$ of the total variance. The definition of the value was provided in the precious EFA. The factor shows a high reliability (Cronbach's $\alpha=0.765$ ).

The factor of "Instrumentalism" consists of 4 statements mirroring an internal locus control among the attitudes. According to Ross et al. (1983) Instrumentalism is defined as the opposite to fatalism. This means, it is "a general expectations that outcomes are contingents on one's own behavior" (Ross et al., 1983). The factor explains $10.69 \%$ of the variance (Cronbach's $\alpha=0.575$ ).

The fourth factor "Self-made" consists of two statements with an internal locus of control describing 'self' as responsible firstly for fulfillment carrying out duties and secondly for effort some luxuries. The factor explains $9.22 \%$ of the variance (Cronbach's $\alpha=0.624$ ).

The last factor "Ethnocentrism" was dropped for the analysis in the basis of it low reliability. (Cronbach's $\alpha=0.466$ ). The factors of hedonism and fatalism were shared by the German total sample. Below are displayed and described the values carried out by the Mexican sample. 
Table 35: Exploratory factor analysis for values of German consumers of organic food

\begin{tabular}{|c|c|c|c|c|}
\hline $\begin{array}{c}\text { Dimension } \\
\text { of Values }\end{array}$ & Indicators (items) $)^{\mathbf{a}}$ & $\begin{array}{l}\text { Factor } \\
\text { loading }\end{array}$ & $\begin{array}{l}\text { Variance } \\
\text { explained } \\
(\%)\end{array}$ & $\begin{array}{c}\text { Cronbach's } \\
\text { Alpha }\end{array}$ \\
\hline \multirow[t]{5}{*}{ Hedonism } & $\begin{array}{l}\text { For me, it is most important to enjoy life and be } \\
\text { pleasant to others. }\end{array}$ & 0.779 & 21.38 & 0.748 \\
\hline & The most important thing in life is to have fun. & 0.727 & & \\
\hline & $\begin{array}{l}\text { It is important to me to have a lot of money and } \\
\text { expensive things. }\end{array}$ & 0.694 & & \\
\hline & I need variety in life. & 0.690 & & \\
\hline & Computers and other electronic devices are strange. & 0.560 & & \\
\hline \multirow[t]{2}{*}{ Fatalism } & If we continue in this way, we will destroy the earth. & 0.865 & 13.27 & 0.765 \\
\hline & $\begin{array}{l}\text { Lots of natural resources have already been } \\
\text { destroyed. }\end{array}$ & 0.855 & & \\
\hline \multirow[t]{4}{*}{$\begin{array}{l}\text { Instrumen- } \\
\text { talism }\end{array}$} & $\begin{array}{l}\text { It will not change anything if I reduce the garbage I } \\
\text { produce, because no one else cares about the } \\
\text { environment. }\end{array}$ & 0.722 & 10.69 & 0.575 \\
\hline & $\begin{array}{l}\text { I do not like people who openly show their } \\
\text { possessions. }\end{array}$ & 0.693 & & \\
\hline & $\begin{array}{l}\text { In comparison to other countries, we act irresponsibly } \\
\text { with our natural resources. }\end{array}$ & 0.585 & & \\
\hline & $\begin{array}{l}\text { I'm not worried about the future, most important is } \\
\text { the present. }\end{array}$ & 0.575 & & \\
\hline \multirow[t]{2}{*}{ Self-made } & $\begin{array}{l}\text { Fulfillment in life is only possible through carrying } \\
\text { out one's duty. }\end{array}$ & 0.811 & 9.22 & 0.624 \\
\hline & I work so that I can afford some luxuries in life. & 0.792 & & \\
\hline \multirow{2}{*}{$\begin{array}{l}\text { Ethno- } \\
\text { centrism }\end{array}$} & I am firmly rooted in my country and its culture. & 0.769 & & \\
\hline & I cannot imagine living permanently abroad. & 0.759 & 7.20 & 0.466 \\
\hline
\end{tabular}

Source: Own elaboration (2010). 


\subsubsection{Exploratory factor analyses and construction of index for the values to purchase organic food in the Mexican sample}

Among the consumers of organic food in Mexico five factors were carried out. The factor labeled as "Living now" is the main factor uncovering the same 4 statements of the factor carried out by the Mexican total sample. The factor explains $21 \%$ of the variance (Cronbach's $\alpha=0.713)$.

The factor of "Materialism" is similar to the factor labeled with the same name by the Mexican total sample; the difference is by two statements. The statement: changes worry me is by consumers of organic food in the component of living now. The statement of: computers and other electronic devices are funny become within this factor. The same statement among the German consumers of organic food become within the factor of "Hedonism". Consequently, this finding supposes the mirror of a cultural difference between German and Mexican consumers of organic food. The factor explain 16.04\% of the variance (Cronbach's $\alpha=0.638)$.

Similarly to the tree samples previously analyzed, among the Mexicans consumers of organic food was carried out the factor of "Fatalism". For this group, the factor consists of 2 statements and explains $11.22 \%$ of the variance (Cronbach's $\alpha=0.677$ ).

The factor of "Self-esteem" was also carried out among the Mexican consumers of organic food and consists of the two same statements building the factor among the Mexican total sample. For this group, the factor explains $7.79 \%$ of the variance (Cronbach's $\alpha=0.549$ ).

The statements of concern with nature and environmental resources with an external locus of control and motivational goals in the pursuit of collective interest built the factor of "Benevolence" the label was inspired in the dimension with the same name by Schwartz (1992). But not reliability was possible to be tested and because of it dropped from the analysis. 
Table 36: Exploratory factor analysis of values of Mexican consumers of organic food

\begin{tabular}{|c|c|c|c|c|}
\hline $\begin{array}{l}\text { Dimension } \\
\text { of Values }\end{array}$ & Indicators (items) ${ }^{a}$ & $\begin{array}{l}\text { Factor } \\
\text { loading }\end{array}$ & $\begin{array}{l}\text { Variance } \\
\text { explained } \\
(\%)\end{array}$ & $\begin{array}{l}\text { Cronbach's } \\
\text { Alpha }\end{array}$ \\
\hline \multirow[t]{4}{*}{ Living now } & We can't stop climate change. & 0.776 & 21 & 0.713 \\
\hline & The most important thing in life is to have fun. & 0.775 & & \\
\hline & $\begin{array}{l}\text { It will not change anything if I reduce the garbage I } \\
\text { produce, because no one else cares about the } \\
\text { environment. }\end{array}$ & 0.759 & & \\
\hline & Changes worry me. & 0.550 & & \\
\hline \multirow[t]{4}{*}{ Materialism } & I work so that I can afford some luxuries in life. & 0.748 & 16.04 & 0.638 \\
\hline & $\begin{array}{l}\text { I identify strongly with the lifestyle of other } \\
\text { countries (e.g. U.S.A., Canada, and Europe). }\end{array}$ & 0.693 & & \\
\hline & Computers and other electronic devices are strange. & 0.647 & & \\
\hline & $\begin{array}{l}\text { For me, it is most important to enjoy life and be } \\
\text { pleasant to others. }\end{array}$ & 0.623 & & \\
\hline \multirow[t]{2}{*}{ Fatalism } & $\begin{array}{l}\text { In comparison to other countries, we act } \\
\text { irresponsibly with our natural resources. }\end{array}$ & 0.853 & 11.22 & 0.677 \\
\hline & $\begin{array}{l}\text { Lots of natural resources have already been } \\
\text { destroyed. }\end{array}$ & 0.827 & & \\
\hline \multirow[t]{2}{*}{ Self-esteem } & $\begin{array}{l}\text { In this country, whoever makes an effort can } \\
\text { improve their social class. }\end{array}$ & 0.824 & 7.79 & 0.549 \\
\hline & $\begin{array}{l}\text { Every individual is responsible for their own well- } \\
\text { being in this country. }\end{array}$ & 0.792 & & \\
\hline Benevolence & $\begin{array}{l}\text { If we continue in this way, we will destroy the } \\
\text { earth. }\end{array}$ & 0.739 & 8.31 & ----- \\
\hline
\end{tabular}
Source: Own elaboration (2010).

Table 37 and Table 38 displayed below summarize the factors of motives to purchase general food products and organic food, the reliability test for the factors of motivations and values carried out among the German and Mexican total sample and the German and Mexican consumers of organic food, only the factors with Cronbach's alpha $\geq 0.5$ were retained for further analysis. After the EFA, simple lineal and multiple linear regression analysis were done and are in the next section described. 
Table 37: Dimension of motives related to the purchasing of food products and organic food in Germany and Mexico

\begin{tabular}{|c|c|c|c|c|}
\hline \multirow[b]{2}{*}{ Factor } & \multicolumn{2}{|c|}{ Food products } & \multicolumn{2}{|c|}{ Organic products } \\
\hline & $\begin{array}{c}\text { Germany } \\
(n=501)\end{array}$ & $\begin{array}{l}\text { Mexico } \\
(n=507)\end{array}$ & $\begin{array}{c}\text { Germany } \\
(n=269)\end{array}$ & $\begin{array}{l}\text { Mexico } \\
(n=263)\end{array}$ \\
\hline Nostalgia & (2 items) & (3 items) & (3 items) & \\
\hline Cronbach's $\alpha$ & 0.299 & 0.639 & 0.674 & \\
\hline Weight control & (3 items) & (3 items) & (3 items) & (2 items) \\
\hline Cronbach's $\alpha$ & 0.790 & 0.698 & 0.777 & 0.778 \\
\hline Healthy eating & (2 items) & (2 items) & & \\
\hline Cronbach's $\alpha$ & 0.420 & 0.661 & & \\
\hline Convenience & (3 items) & & (4 items) & \\
\hline Cronbach's $\alpha$ & 0.656 & & 0.699 & \\
\hline Traditional motives ${ }^{\mathrm{a}}$ & & & (7 items) & (6 items) \\
\hline Cronbach's $\alpha$ & & & 0.884 & 0.820 \\
\hline Advertising & (2 items) & & & \\
\hline Cronbach's $\alpha$ & 0.650 & & & \\
\hline High food involvement & (8 items) & (5 items) & & (4 items) \\
\hline Cronbach's $\alpha$ & 0.751 & 0.724 & & 0.759 \\
\hline Low food involvement & & (5 items) & & (5 items) \\
\hline Cronbach's $\alpha$ & & 0.790 & & 0.815 \\
\hline Product information & (3 items) & & (2 items) & \\
\hline Cronbach's $\alpha$ & 0.659 & & 0.628 & \\
\hline Product orientation & & (3 items) & & \\
\hline Cronbach's $\alpha$ & & 0.566 & & \\
\hline Price quality orientation & & & (2 items) & \\
\hline Cronbach's $\alpha$ & & & 0.710 & \\
\hline Neophilia & & & (2 items) & \\
\hline Cronbach's $\alpha$ & & & 0.512 & \\
\hline
\end{tabular}

${ }^{\mathrm{a}}$ among this dimension there are the motives of tasty, healthy, fairness, concern with environment, animal welfare.

Source: Own elaboration (2010). 
Table 38: Dimension of values related to the purchasing of food products and organic food in Germany and Mexico

\begin{tabular}{|c|c|c|c|c|}
\hline \multirow[b]{2}{*}{ Factor } & \multicolumn{2}{|c|}{ Food products } & \multicolumn{2}{|c|}{ Organic products } \\
\hline & $\begin{array}{l}\text { Germany } \\
(n=501)\end{array}$ & $\begin{array}{l}\text { Mexico } \\
(\mathrm{n}=\mathbf{5 0 7}) \\
\end{array}$ & $\begin{array}{c}\text { Germany } \\
(n=269)\end{array}$ & $\begin{array}{l}\text { Mexico } \\
(n=263) \\
\end{array}$ \\
\hline $\begin{array}{l}\text { Hedonism } \\
\text { Cronbach's } \alpha\end{array}$ & $\begin{array}{r}\text { (3 items) } \\
0.724 \\
\end{array}$ & & $\begin{array}{r}\text { (5 items) } \\
0.748\end{array}$ & \\
\hline $\begin{array}{l}\text { Fatalism } \\
\text { Cronbach's } \alpha\end{array}$ & $\begin{array}{r}(2 \text { items }) \\
0.736\end{array}$ & $\begin{array}{r}\text { (3 items) } \\
0.645\end{array}$ & $\begin{array}{r}(2 \text { items }) \\
0.765\end{array}$ & $\begin{array}{r}(2 \text { items }) \\
0.677\end{array}$ \\
\hline $\begin{array}{l}\text { Unhappiness } \\
\text { Cronbach's } \alpha\end{array}$ & $\begin{array}{r}(4 \text { items }) \\
0.602\end{array}$ & & & \\
\hline $\begin{array}{l}\text { Self-esteem } \\
\text { Cronbach's } \alpha\end{array}$ & $\begin{array}{r}\text { (2 items) } \\
0.694\end{array}$ & $\begin{array}{r}\text { (2 items) } \\
0.573\end{array}$ & & $\begin{array}{r}\text { (2 items) } \\
0.549\end{array}$ \\
\hline $\begin{array}{l}\text { Technophilia } \\
\text { Cronbach's } \alpha\end{array}$ & $\begin{array}{r}(3 \text { items }) \\
0.481 \\
\end{array}$ & $\begin{array}{r}\text { (3 items) } \\
0.373 \\
\end{array}$ & & \\
\hline $\begin{array}{l}\text { Ethnocentrism } \\
\text { Cronbach's } \alpha\end{array}$ & $\begin{array}{r}(2 \text { items }) \\
0.472\end{array}$ & $\begin{array}{r}(2 \text { items }) \\
0.331\end{array}$ & $\begin{array}{r}(2 \text { items }) \\
0.466\end{array}$ & \\
\hline $\begin{array}{l}\text { Living now } \\
\text { Cronbach's } \alpha\end{array}$ & & $\begin{array}{r}\text { (4 items) } \\
0.681\end{array}$ & & $\begin{array}{r}\text { (4 items) } \\
0.713\end{array}$ \\
\hline $\begin{array}{l}\text { Materialism } \\
\text { Cronbach's } \alpha\end{array}$ & & $\begin{array}{r}\text { (4 items) } \\
0.660\end{array}$ & & $\begin{array}{r}\text { (4 items) } \\
0.638\end{array}$ \\
\hline $\begin{array}{l}\text { Instrumentalism } \\
\text { Cronbach's } \alpha\end{array}$ & & & $\begin{array}{r}\text { (4 items) } \\
0.575\end{array}$ & \\
\hline $\begin{array}{l}\text { Self-made } \\
\text { Cronbach's } \alpha\end{array}$ & & & $\begin{array}{r}\text { (2 items) } \\
0.624\end{array}$ & \\
\hline
\end{tabular}

Source: Own elaboration (2010).

\subsubsection{Multiple regression analysis}

To understand the nature of the relationship between the motivations, values and buying behavior, Multiple Linear Regression Analysis (MLR) was used. As dependent or response variable (Y) the variable labeled as organic diet was entered. The variable is an index built with the responses provided to the frequency of eating organic bakery, organic vegetables, organic fruits, organic muesli, organic meat, organic eggs, and organic milk. Demographic variables such as age and size of the household were used as continuous independent variables and household incomes, gender, and education were used as non-continuous (categorical) variables. Firstly, a simple linear regression analysis with the common motives and values between the German and the Mexican samples of consumer of organic food were carried out. After that, a MLR analysis was estimated. 
Pearson correlation was used to measure the linear association between the set of independent variables -the factors of motives, values- and the dependent variable of organic diet in the purchasing of organic food. Only the factors with statistic significance were included in the subsequently analysis. Multiple Linear Regression analysis (MLR) test using stepwise method was employed. The MLR was estimated using the general form $\left(\mathrm{Y}=\alpha+\beta_{1} \mathrm{X}_{1}+\beta_{2} \mathrm{X}_{2}+\ldots \ldots+\right.$ $\left.\mathrm{BiX}_{\mathrm{i}}+\varepsilon\right)$. Stepwise method was selected because it is usefulness to understand the contribution of the previous independent variables introduced that another independent variables added (Statistics solutions, 2010). The contribution of each independent variable to the model was observed. The MLR analysis was done following the procedure by Field (2010).

\subsubsection{Common motives and values among the consumers of organic food in the German and Mexican samples: simple linear regression.}

To measure the linear association between the factors of motives and values -predictor variables- and organic diet -dependent variable- Pearson correlation coefficient was measured. The results of the test displayed in Table 39 indicate that "Traditional motives", "Weight control", and "Fatalism" were significantly associated with organic diet by the German sample. Whereas, “Traditional motives" was the only one predictor variable significantly associated with organic diet by the Mexican sample. To follow with the comparison a simple linear regression model was carried out.

Table 39: Relationships between the independent variables and organic diet in Germany

\begin{tabular}{|l|r|l|}
\hline & $\begin{array}{c}\text { German sample } \\
(\mathbf{n = 2 6 9 )}\end{array}$ & $\begin{array}{c}\text { Mexican sample } \\
(\mathbf{n = 2 6 3 )}\end{array}$ \\
\hline Independent variable $^{\mathrm{a}}$ & $\mathbf{p}$ value & p value \\
\hline $\mathrm{X} 1=$ Traditional motives & $0.398^{* *}$ & $0.265^{* *}$ \\
\hline $\mathrm{X} 2$ = Weight control & $0.159^{* *}$ & \\
\hline $\mathrm{X} 5$ = Fatalism & $0.176^{* *}$ & \\
\hline
\end{tabular}

a 7-point Likert-type scale from strongly agree to strongly disagree. $* * \mathrm{p} \leq 0.01$.

Source: Own elaboration (2010). 
The factor of "Traditional motives" is shared by individuals having an organic diet in Germany and Mexico. However, the relationship is higher among the German consumers than Mexicans. Thus, $H 1$ states: traditional motives have a more positive influence in the purchase of organic food in Germany than in Mexico.

To verify this, a simple linear regression was done. Table 40 shows the iteration between “Traditional motives” (predictor variable) and organic diet for the German and Mexican samples. The model is significant at the $1 \%(\mathrm{p}<.001)$ for the two samples. The amount of variance explained in organic diet by size is low. $14 \%\left(\mathrm{R}^{2}=0.148\right)$ and (adjusted $\left.\mathrm{R}^{2}=.145\right)$ for the German sample and $8 \%\left(\mathrm{R}^{2}=0.8\right)$ and (adjusted $\left.\mathrm{R}^{2}=.077\right)$ for the Mexican sample. The unstandardized beta value $(ß)$ of 0.393 shows that "Traditional motives" as predictor variable has more impact in the model for the German sample than the beta value $(ß)$ of 0.329 for the Mexican sample. The ' $\mathrm{t}$ ' value is the error level present in the model tanking organic diet as dependent variable and "Traditional motives" as independent variable. For the German sample the ' $t$ ' value (6.673) is higher than for the Mexican sample (4.706).

Table 40: Variance explained by the motivations to purchase organic food in Germany and Mexico

\begin{tabular}{|l|r|r|r|r|r|r|}
\hline & $\mathbf{R}^{\mathbf{2}}$ & $\begin{array}{c}\text { Adjusted } \\
\mathbf{R}^{\mathbf{2}}\end{array}$ & \multicolumn{1}{c|}{$\mathbf{p}$} & $\begin{array}{c}\text { Non } \\
\text { standardized } \\
\mathbf{B}\end{array}$ & $\mathbf{t}$ & \multicolumn{1}{c|}{$\mathbf{n}$} \\
\hline Germany & .148 & .145 & .000 & .393 & 6.673 & 258 \\
\hline Mexico & .081 & .077 & .000 & .329 & 4.706 & 254 \\
\hline
\end{tabular}

a. Predictors: (Constant). Traditional motives (7-point Likert-type scale from strongly agree to strongly disagree) b. Independent variable: organic diet. ${ }^{* * *} \mathrm{p} \leq 0.001 ; * * \mathrm{p} \leq 0.01 ;{ }^{*} \mathrm{p} \leq 0.05$.

Source: Own elaboration (2010).

The results clearly indicate a systematic association between traditional motives and the consumption of organic food in both countries. However, the predictive power of the model is reduced when "Traditional motives" is the only independent variables in the model. The factor of "Traditional motives" alone seems as a weak predictor. This fact suggests an improving of the model to gain understanding in the consumption of organic food. In the next section the improving of the total model for the German and the Mexican samples will be provided. 


\section{8.3.2 Motives and values in the purchase of organic food in Germany and Mexico: MLR}

After the finding of "Traditional motives" as weak predictor explaining it influencing in the purchase of organic food by individuals in Germany and Mexico the models were improved. MLR analysis test using stepwise method was employed to understand particular motives, values, and demographic variables influencing the purchase of organic food in each country. Pearson correlation was used to measure the linear association between the factors of motives, and values, and the statements -not carried out within the factors- used as independent variables; "Organic diet" was used as dependent variable. Only the variables with statistic significance were included in the subsequently analysis.

\subsection{Multiple regression of the German sample}

The correlation test indicates that apart from the factors of "Traditional motives", and "Weight control”, "Quality orientation” was statistically significant associated with organic diet as well as 8 statements from the theoretical constructs of: "Natural content", "Free of GMO", "Product information", "Price orientation". With regards to values the factor of "Fatalism" -shared value- the factors of the values of: "Hedonism" and "Self-made"- and 3 statements from the theoretical construct of "Fairness" were also high statistically significantly related. A total of twenty three independent variables displayed in Table 41 were included in the total model.

After the Pearson correlation test, we can postulate that apart from traditional motives, the motives of natural contents and product information have a higher positive influence on the purchase of organic food than the motives of "Price orientation", "Free of GMO", and the values. Thus, $\mathrm{H} 1 \mathrm{a}$ is: individuals are positive influenced by the factor of traditional motives, and the motives of natural contents and product information. $\mathrm{H} 1 \mathrm{~b}$ is: individuals are positive influenced by the values of "Hedonism", "Fatalism", "Self-made", and "Fairness" in the purchase of organic food. 
Table 41: Relationships between the independent variables and organic diet in Germany

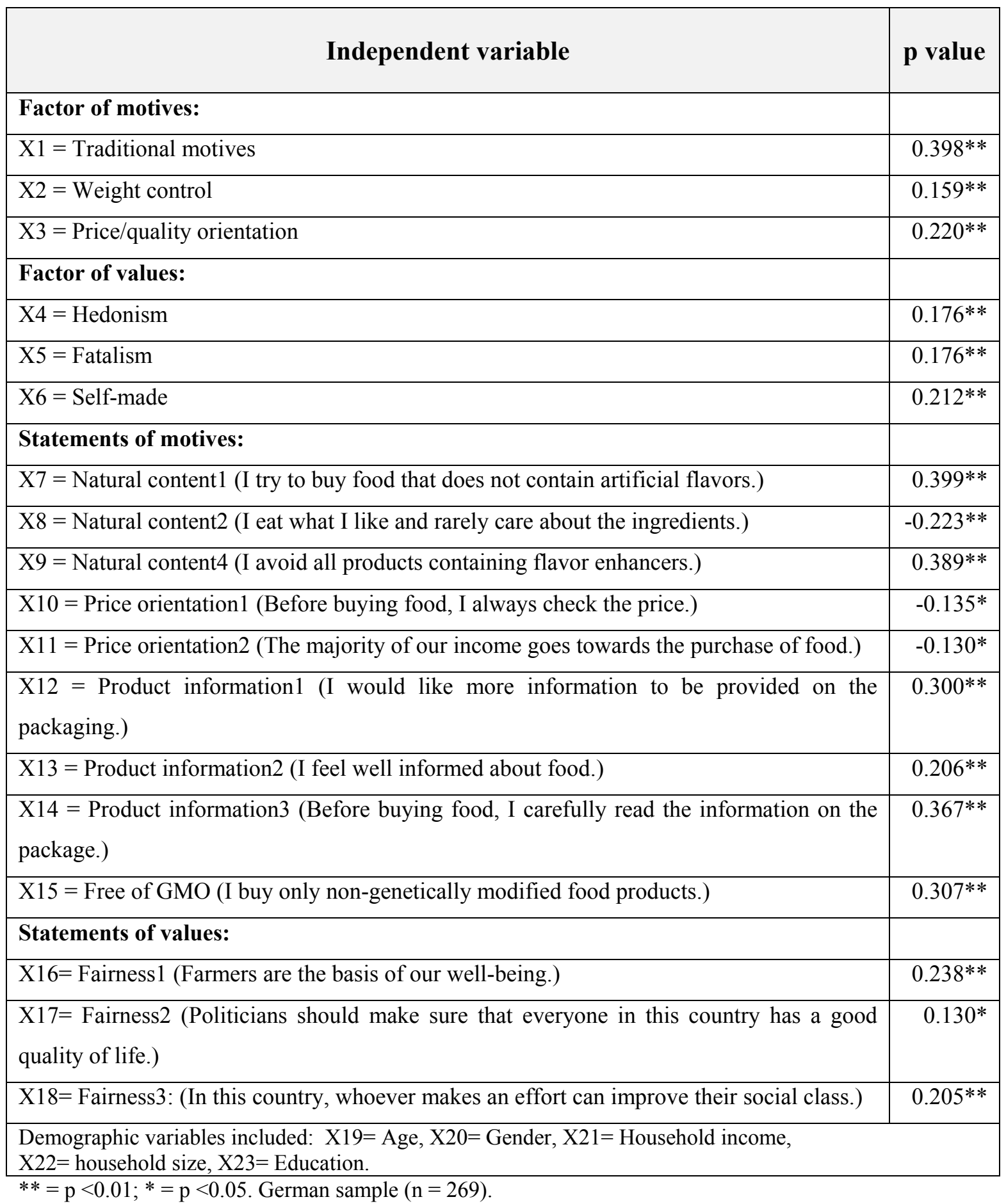

Source: own elaboration (2010). 
The total model consists of six independent variables explaining $31 \%$ of the variance. In the first iteration when only the variable of "Natural content1" (I try to buy food that does not contain artificial flavors) is used as predictor, the amount of variance explained is $18 \%$; $\mathrm{R}^{2}=0.184$ (adjusted $\mathrm{R}^{2}$ is 0.180 ). The amount of variance explained when the factor of “Traditional motives" was entered is $23 \% ; \mathrm{R}^{2}=0.235$ (Adjusted $\mathrm{R}^{2}=0.228$ ). The factor of "Traditional motives" improves the model by 5\%. The amount of variance explained when the factor "Hedonism" was entered is $26 \% ; \mathrm{R}^{2}=0.267$ (adjusted $\mathrm{R}^{2}$ is 0.257 ). The factor of "Hedonism" improves the model by $3 \%$. When the independent variable of "Natural content4" (I avoid all products containing flavor enhancers) was entered to the model the amount of variance explained increase to $28 \% ; \mathrm{R}^{2}=0.288$ (adjusted $\mathrm{R}^{2}$ is $=0.274$ ). "Natural content4" contributes the model $\mathrm{R}^{2}$ by $2 \%$. When the variable incomes was entered the amount of variance explained was $30 \% ; \mathrm{R}^{2}=0.306$ (adjusted $\mathrm{R}^{2}$ is 0.289 ) the variable improve the model by 2\%. When the variables "Fairness1" (Farmers are the basis of our well-being) was entered, the amount of variance explained was slightly improved to $31 \%, \mathrm{R}^{2}$ $=0.319$ (adjusted $\mathrm{R}^{2}$ is 0.299 ) the variable improves the model by $1 \%$. The total model displayed in Table 42 indicates that there were systematic associations between the independent variables with the dependent variable -organic diet. 
Table 42: Variance explained by factors in organic diet in Germany

\begin{tabular}{|l|r|r|r|r|r|}
\hline Model & \multicolumn{1}{|c|}{$\mathbf{R}$} & $\mathbf{R}^{\mathbf{2}}$ & Adjusted $\mathbf{R}^{\mathbf{2}}$ & \multicolumn{1}{|c|}{$\mathbf{F}$} & \multicolumn{2}{|c|}{$\mathbf{p}$} \\
\hline 1 & $.429 \mathrm{a}$ & .184 & .180 & 47.854 & .000 \\
\hline 2 & $.485 \mathrm{~b}$ & .235 & .228 & 32.462 & .000 \\
\hline 3 & $.485 \mathrm{c}$ & .267 & .257 & 25.519 & .000 \\
\hline 4 & $.536 \mathrm{~d}$ & .288 & .274 & 21.111 & .000 \\
\hline 5 & $.553 \mathrm{e}$ & .306 & .289 & 18.307 & .000 \\
\hline 6 & $.564 \mathrm{f}$ & .319 & .299 & 16.128 & \\
\hline
\end{tabular}

a. Predictors: (Constant). Natural content1.

b. Predictors: (Constant). Natural content1, Traditional motives.

c. Predictors: (Constant). Natural content1, Traditional motives, Hedonism.

d. Predictors: (Constant). Natural content1, Traditional motives, Hedonism, Natural content4

e. Predictors: (Constant). Natural content1, Traditional motives, Hedonism, Natural content4, Incomes

f. Predictors: (Constant). Natural content1, Traditional motives, Hedonism, Natural content4, Incomes, Fairness 1.

g. independent variable: Organic diet. German sample $(n=269)$.

Source: Own elaboration (2010).

The total model significantly improves the ability to predict the outcome variable. For the first model the value of $F$ is 47.85 . For the sixth model the value of $F$ decrease to 16.12 . The total model was significant at the 1 percent level $(* * * p<.001)$ this means, there was a significant multivariate effect for the independent and dependent variables (see Table 42).

To answer the question: which motives and values have the greater influence in the purchase of organic food in Germany? The beta values ( $(3)$ were compared. The dominant factors in the consumption of organic food are given in Table 43.

The beta value ( $($ ) indicated that "Incomes" and "Traditional motives" are the main factor to provide understanding in the purchase of organic food. "Incomes" is the strongest predictor with more impact in the total model with beta value (B) 0.315 and significant at the 10 percent level $(* * \mathrm{p} \leq 0.01)$. The factor of "Traditional motives" is the second stronger predictor with more impact in the model beta value $(ß)$ of 0.233 and significant at the 1 percent level $\left({ }^{* * *} \mathrm{p} \leq 0.001\right)$. "Hedonism" is the third variable with a beta value $(ß)$ of 0.151 and significant at the 5 percent level $(* \mathrm{p} \leq 0.05)$; the factor of "Hedonism" explain more so than the statement 
of "Natural content 1 " with a beta value $(\beta)$ of 0.148 and significant at the 10 percent level $(* \mathrm{p} \leq 0.01)$. The statements of "Natural content4" beta value (B) of 0.116 and "Fairness1" beta value (B) of 0.094 were not significant predictors of organic consumption. This is confirmed by the the ' $t$ ' value (the error level) due to the error level decrease not only because the addition of "Hedonism", but also because of the iteration or the joint contribution of "Natural content4”, “Incomes”, "Fairness1", ”Natural content4”, and "Fairness1" are not significant. And incomes was significant at the 10 percent level $(* * p<.01)$.

The findings of the results suggest a combination of different motivations driving the consumption of organic food in Germany. In fact, "Incomes" have the more positive influence in the purchasing of organic food among German consumers of organic food follow by the factor of "Traditional motives" which define organic food as healthier, fairness for farmers, concern with environment, tasty and the concern for "Natural content" in food. The variable of "Hedonism" seems also driving the purchasing of organic food in the country, more so than the variables of natural content and fairness which seem as weak predictors.

Table 43: Factors predicting organic diet in Germany

\begin{tabular}{|c|c|c|c|}
\hline Model & 及 & $\mathbf{t}$ & Sig. \\
\hline (Constant) & 1.654 & 17.795 & .000 \\
\hline Natural content $1^{\mathrm{a}}$ & .148 & 2.602 & .010 \\
\hline Traditional motives ${ }^{a}$ & .233 & 3.477 & .001 \\
\hline Hedonism $^{\text {a }}$ & .151 & 2.436 & .016 \\
\hline Natural content $4^{\text {a }}$ & .116 & 2.196 & .029 \\
\hline Household incomes & .315 & 2.617 & .010 \\
\hline Fairness $1^{\mathrm{a}}$ & .094 & 1.985 & .048 \\
\hline
\end{tabular}

Dependent variable: Organic diet. ${ }^{a}$ 7-point Likert-type scale from strongly agree to strongly disagree. ${ }^{* * *} \mathrm{p} \leq 0.001 ; * * \mathrm{p} \leq 0.01 ;{ }^{*} \mathrm{p} \leq 0.05$. German sample $(\mathrm{n}=269)$.

Source: Own elaboration (2010). 


\subsection{Multiple regression of the the Mexican sample}

To find linear association between the motives and values -independent variable- and "Organic diet" -dependent variable- within the Mexican sample. Pearson correlation coefficient was tested. The results displayed in Table 44 indicate that apart from the factor of "Traditional motives", the factor of "High involvement"; 4 statements of the theoretical construct of motives: "Nostalgia3", "Price orientation1", "Product information2", and "Convenience in cooking1". With regards to the factors of values "Living now", "Materialism", and "Self-esteem" and 4 statements of the theoretical constructs of "Hedonism2”, “Anti-hedonism”, “Ethnocentrism2”, and "Fairness1” were significantly related to "Organic diet". 
Table 44: Relationships between the independent variables and organic diet in Mexico

\begin{tabular}{|l|r|}
\hline Independent variable & p value \\
\hline Factors of motives: & \\
\hline X1 = Traditional motives & $0.265^{* *}$ \\
\hline X2 = High food involvement & $0.336^{* *}$ \\
\hline Factors of values: & \\
\hline X3 = Living now & $0.219^{* *}$ \\
\hline X4 = Materialism & $0.132^{*}$ \\
\hline X5 = Self-esteem & $0.144^{*}$ \\
\hline Statement of variables of motives: & $0.138^{*}$ \\
\hline X6 = Nostalgia3 (In my childhood, food tasted better) & $0.144^{*}$ \\
\hline X7 = Price orientation1 (The majority of our income goes towards the purchase of food). & $0.244^{* *}$ \\
\hline X8 = Product Information2 (I feel me well informed about food) & $0.128^{*}$ \\
\hline X9 = Convenience in cooking1 (I think it is good that the assortment of convenience food is \\
growing) & \\
\hline Statement of variables of values: & \\
\hline X10 = Hedonism2 (I'm not worried about future, most important is the present) & $0.212^{* *}$ \\
\hline X11 = Anti hedonism (Fulfillment in life is only possible through carrying out one's duty) & $0.229^{* *}$ \\
\hline X12 = Ethnocentrism2 (I am firmly rooted to my country and its culture) & $0.186^{* *}$ \\
\hline X13 = Fairness1 (Farmers are the basis of our well-being) & $0.135^{*}$ \\
\hline Demographic variables included: X14= Age, X15= Gender, X16= Household incomes, \\
X17= household size, X18= Education. & \\
\hline
\end{tabular}

${ }^{* *} \mathrm{p} \leq 0.01 ; * * \mathrm{p} \leq 0.05$. Mexican sample $(\mathrm{n}=263)$.

Source: Own elaboration (2010).

After Pearson correlation test two hypotheses were stated for the total model. $H 1 a$ is: individuals are positive influenced by the "Traditional motives", "High involvement", and "Product information" to purchase organic food. $H 1 b$ is: individuals are positive influenced by the values of "Living now”, "Materialism", "Self-esteem”, “Hedonism2”, "Antihedonism”, "Ethnocentrism2", and "Fairness1" to purchase organic food.

Using stepwise regression method a multiple linear regression (MLR) tests were estimated to find the motives and values related with organic diet in Mexico. The total model was entered by 4 independent variables explaining $31 \%$ of the variance. In the first iteration when only the 
factor of "High involvement" is used as predictor the amount of variance explained was $14 \%$ $\mathrm{R}^{2}=0.149$ (adjusted $\mathrm{R}^{2}$ is 0.146 ). When the variable "Traditional motives" was entered the amount of variance explained by the factor is $22 \% \mathrm{R}^{2}=0.229$ (adjusted $\mathrm{R}^{2}$ is 0.223 ). The variable improved the model by $8 \%$. When the variable "Size of household" was entered the amount of variance explained was $29 \%$. The by $\mathrm{R}^{2}=0.291$ (adjusted $\mathrm{R}^{2}$ is 0.282 ) the variable improve the model by $7 \%$. When the variable "Living now" was entered to the model the amount of variance explained in the total model was $31 \% ; \mathrm{R}^{2}=0.315$ (adjusted $\mathrm{R}^{2}$ is $=0.304$ ) the variable improved the $\mathrm{R}^{2}$ by $2 \%$. The total model summarized in Table 45 indicates that for the Mexican sample, there were systematic associations between the independent variables and the dependent variable of organic diet.

Table 45: Variance explained by factors in organic diet in Mexico

\begin{tabular}{|l|r|r|r|r|r|}
\hline Model & $\mathbf{R}$ & $\mathbf{R}^{2}$ & Adjusted $\mathbf{R}^{\mathbf{2}}$ & \multicolumn{1}{c|}{$\mathbf{F}$} & \multicolumn{1}{c|}{$\mathbf{p}$} \\
\hline 1 & $.386 \mathrm{a}$ & .149 & .146 & 43.921 & $.000^{\mathrm{a}}$ \\
\hline 2 & $.478 \mathrm{~b}$ & .229 & .223 & 37.060 & $.000^{\mathrm{b}}$ \\
\hline 3 & $.539 \mathrm{c}$ & .291 & .282 & 34.019 & $.000^{\mathrm{c}}$ \\
\hline 4 & $.561 \mathrm{~d}$ & .315 & .304 & 28.461 & $.000^{\mathrm{d}}$ \\
\hline
\end{tabular}

a. Predictors: (Constant). High food involvement

b. Predictors: (Constant). High food involvement, Traditional motives

c. Predictors: (Constant). High food involvement, Traditional motives, size of the household

d. Predictors: (Constant). High food involvement, Traditional motives, size of the household, Living now

e. Predictors: (Constant). Organic diet. Mexican sample $(n=263)$.

Source: Own elaboration (2010).

The final model significantly improves the ability to predict the outcome variable due to for the first predictor -"High food involvement"- the value of $\mathrm{F}$ is 43.921. For the fourth variable "Living now" the value of $\mathrm{F}$ decreases to 28.461. Table 45 shows that the model is significant at the 1 percent level $(* * * p<.001)$ this means, there was a significant multivariate effect for the independent and dependent variables.

As in the German total model, to answer the question: which motives and values have the greater influence in the purchase of organic food in Mexico? Beta values ( $($ ) were compared. The dominant factors in the consumption of organic food are given in Table 45 the beta value (ß) indicated that high involvement and traditional motives are the stronger predictors in the 
understating of the motives influencing the purchase of organic food in Mexico. "High food involvement" was the independent variable with the more impact in the total model of beta value (B) 0.384 . The factor of "Traditional motives" is the second independent variable with more impact in the model beta value ( $(3)$ of 0.348 both were significant at the 1 percent level $\left(*^{* *} \mathrm{p}<.001\right)$. The variables of "Size of household" beta value (ß) of 0.145 and "Living now" beta value $(B)$ of 0.186 were not significant predictors of organic consumption. This is confirmed by the the ' $t$ ' value (the error level) due to the error level decrease not only because the addition of "Traditional motives", but also because of the iteration or the joint contribution of "Size of household" and "Living now". This implies that the contribution of size of household and living now, beta value $(ß)$ of to the model is decreased both were also significant at the 1 percent level $(* * * p<.001)$.

Table 46: Factors of motives and values predicting an organic diet in Mexico

\begin{tabular}{|l|r|r|r|}
\hline \multicolumn{1}{|c|}{ Model } & \multicolumn{1}{c|}{ 制|} & \multicolumn{1}{c|}{ Sig. } \\
\hline (Constant) & 1.715 & 11.111 & .000 \\
\hline High food involvement & .384 & 6.172 & .000 \\
\hline Traditional motives & .348 & 5.716 & .000 \\
\hline Size of the household & .145 & 4.241 & .000 \\
\hline Living now & .186 & 2.941 & .004 \\
\hline
\end{tabular}

Dependent variable: Organic diet. ${ }^{* * *} \mathrm{p}<.001$. 7-point Likert-type scale from strongly agree to strongly disagree. Mexican sample $(\mathrm{n}=263)$.

Source: Own elaboration (2010).

\subsubsection{Final discussion}

As Mooij (2004) suggests, culture determines patterns of behavior of everyday life and consumer behavior is not an exception. As expected among the findings of this research, differences and similarities of theoretical values and motives driving the purchase of food were found among the four groups compared. As literature in cross-cultural comparative research suggest, comparing the average values of priorities of groups with cultural differences was more observable between the two countries among consumers and non-buyers of organic food. In general, Germans were more skeptical than Mexicans who as we expected 
because of their positive attitudes and sense of social desirability seem more optimistic. Interestingly, both Germans and Mexicans show a positive attitude toward technological changes- 'technophilia'. Mexicans are more 'fatalistic' than Germans which means that Mexicans tend to believe more in an external focus of control over events in life than Germans. Germans show a more 'hedonistic' attitude than Mexicans regarding fun, but Mexicans seem more aware of enjoyment than Germans; this probably arose in this research because of the differences in meaning of words in the Spanish and German languages.

With the multivariate analysis methods -factor analysis- the 37 theoretical motives postulated were reduced to 12 variables; the reduction of the statement of the 8 theoretical values better indicates differences among the 11 values carried out among the four groups under comparison. The findings of the results provide insights into the relation of similarities and differences between and within the cultural comparison.

\section{$>$ Cultural similarities of motives (between-comparison)}

Among the similarities, there is the fact that German and Mexican groups seem committed to low fat/calorie diets as a motivation to purchase organic food and food products in general, due to the factor of "Weight control" that was commonly displayed by the four groups. Germans and Mexicans again seem committed to their health. In fact, the factor "Healthy eating" was shared in the German and Mexican total samples. Among the consumers of organic food, "Healthy" was associated with organic food and was included within the factor of "Traditional motives". This factor was established for German and Mexican consumers of organic food and included motives relating to fair prices, healthy, tasty, animal concern, and concern with the environment. All the motives seem important to consumers of organic food in both countries. This fact is not surprising, as these are the most common motives reported in the international literature review. Thus, their existence among the German and Mexican consumers of organic food was confirmed.

\section{$>$ Cultural differences of motives (between-comparison)}

Some differences among the four groups are with regard to "Nostalgia"; this factor was shared in the German and Mexican total samples, specifically with German consumers of organic food but not for Mexican consumers of organic food. This is interesting, because the absence of nostalgia might be supposed to relate to the receptiveness of innovative food 
among the Mexican consumers of organic food; however, no one factor in this regard was established. In contrast, among the German consumers of organic food the existence of the factor "Nostalgia" might lead one to suppose that receptiveness to trying new products or new things is low. However, consumers within this group seem to have neophilic attitudes contrary to expectations. This might suppose the existence of duality in preferences for new products but also for traditional products among the consumers of organic food in both countries.

Another cultural difference among Germans and Mexicans was uncovered within the factor of "High food involvement". Although this factor was evident in the German total sample, the Mexican total sample and the sample of Mexican consumers of organic food, the statements within this factor among the Mexican groups seem similar; however, in the German sample, more concern with methods of the production of food, information, preference for home country produce, and concern with free of GMO food was found. The factor of "Low food involvement" was only evident in the two Mexican groups but not in the German groups which is not surprising due to the fact that Germans seem more knowledgeable about the production of food. This fact becomes clear with the factor "Product information" that was evident in the two German groups but not by the Mexicans.

The factor "Convenience" was common to the two German groups but not to the Mexican groups. This fact points out an interesting cultural difference, because Germans seem to associate convenience with the two factors regarding cooking and shopping; whereas, Mexicans seem to associate convenience with shopping only. Among Mexicans, the statements regarding "Shopping convenience" were perceived as factors of "Low involvement"; however, no one statement regarding "Cooking convenience" was evident for any one of the groups. This factor provides differences related to the stage of the market level, national supply chain structure, or at the cultural level regarding habits or rituals of eating; for instance, as Grunert et al. (1993) pointed out, eating as a social activity with friends or family or as a biological need to satisfy.

\section{$>$ Cultural differences of motives (within-comparison)}

Cultural differences within the groups were found; for instance, German consumers and nonbuyers. The factor of “Advertising” was established only for the German total, as these statements referred to purchasing of food because of the receptiveness of advertising. 
Conversely, the factor "Price quality orientation" was carried out only for German consumers of organic food. This factor highlights an association with price as a characteristic of quality.

Differences between Mexican consumers and non-buyers related to the factor "Product orientation" were found in the Mexican total sample but not in the group of Mexican consumers of organic food. Among this factor an orientation to purchasing imported products was discovered, but simultaneously, home country produce and well-known brands were also evident. Regarding organic food consumption, this is interesting, because it provides insight into the receptiveness of purchasing food because of its market positioning.

These findings provide empirical evidence to postulate the existence of cultural differences in the purchase of food products-general and organic food. However, how and the level of influence on consumption of food products determined by these motives implies further research. More differences are related to values.

\section{$>$ Cultural similarities of values (between country comparison)}

The factor of the "Fatalism" value provided another similarity among the four groups. In fact, Germans and Mexicans show a shared attitude toward the acceptance of outcomes of situations as determined by external forces.

\section{$>$ Cultural differences of values (between country comparison)}

The factor of "Ethnocentrism" was shared by the two German groups and the Mexican groups in total but not by Mexican consumers of organic food. This fact is interesting due to the receptiveness of foreign products which was uncovered among Mexican consumers.

The factor of "Self-esteem" was shared by three groups: the two Mexican groups and the total sample of Germans but not by German consumers of organic food. However, within this group the factor "Self-made" was uncovered. Both factors might be postulated as a similar psychological direction but with stronger intensity. Similarly, the factor "Instrumentalism" was uncovered only among the German consumers of organic food; this factor is postulated as being opposite to the value of fatalism. In fact, whilst "Fatalism" is a directional force in accepting the outcome of situations as something determined by external forces, "Instrumentalism" is a belief in an internal focus of control. This means that the outcome of a 
situation is considered contingent on one's own behavior (Ross et al., 1983). Thus, the existence of both values - "Instrumentalism" and "Self-made"- point out a stronger internal focus of control among the consumers of organic food in Germany than within the other groups, including individuals sharing their national cultural background.

The factors of "Hedonism" and "Unhappiness" were shared by the two groups of Germans which means that German consumers of organic food and non-buyers seem to prioritize goals in the pursuit of individual interests. Whilst the factor "Hedonism" highlights enjoyment and fun, the factor of unhappiness predisposes a negative attitude toward financial dissatisfaction, worry about changes, disregard for the environment and dislike of the display of possessions by others.

Among the Mexicans, the factors of "Living now" and "Materialism" were shared by both groups, consumers of organic food and non-buyers. Both values show a prioritization of goals in the pursuit of individual interests but in a different direction and probably with different needs as those associated with the values of hedonist and unhappiness carried out among the German samples. Although these cultural differences between Germans and Mexicans provide interesting insights, an in-depth understanding of them was not included in the objectives of this research.

\section{$>$ Cultural differences of values (within-country comparison)}

Although the factor "Technophilia" was not reliable, the fact that it was shared by Germans and Mexicans in total but not by German and Mexican consumers of organic food is noted with the aim to show more than between-country differences and within-country differences, i.e. German non-buyers and consumers and Mexican non-buyers and consumers of organic food.

The next step of the multivariate analysis consists firstly of a simple linear regression analysis to search the relationship between organic diet and the factor of "Traditional motives". When this factor is used as a unique variable to provide understanding of purchasing organic food, it is a weak predictor; however, when the factor is entered into a multidimensional model, the results provided in the MLR indicates that the variable significantly improves the total model and contributes to the understanding of the purchasing of organic food in each country. 
The findings of the total models measured by MLR suggest that a multidimensional model provides better understanding about the motives and values influencing the consumption of organic food. In both countries "Traditional motives" which is a factor that incorporates healthy, tasty, better for environment, animal welfare, and better prices for farmers (fairness) was a stronger predictor. These confirm the existence of frequent motives reported by the literature review in Germany and Mexico; however, some peculiarities of the German and Mexican models were found. Firstly, individuals in Mexico are motivated to consume organic food because of their "High involvement" in issues related to food production such as free of GM food, natural content, and the shake of natural balance because of their production; and secondly, they are motivated by "Traditional motives", as indicated by the demographic variable and the size of household. With regard to Germany, household income was the first variable influencing the purchase of organic food, i.e. the "Traditional motives". This fact pointed out differences more so than at the demographic level, a combination of differences at the cultural level as well as at the stage of the market level. The findings are not surprising due to the approach of "Most different system design" used in the comparison. 


\section{Discussions and conclusions}

To provide an understanding about cultural differences associated with the purchase of organic food in two separate countries that have dissimilar national cultural backgrounds, the main insights on differences and similarities between the values and motives of German and Mexican non-buyers and consumers of organic food compared cross-culturally are discussed in this Chapter divided into three sections. Firstly, the main findings, secondly, the limitations of the research, and finally, guidelines and suggestions for further research are highlighted.

\subsection{Main findings on cross-cultural motives and values}

In line with the findings of the results displayed in Chapter 7, our discussion begins with the dimension of "Animal welfare". This dimension was included in the study, because it was frequently mentioned as a motive to purchase organic food in international literature in European countries including Germany. In this regard, within the factor of "Traditional motives" Table 33 and Table 34 show that German consumers of organic food are more concerned with the issue of animal welfare than Mexicans. Some reasons that help us to understand the low concern of individuals purchasing organic food in Mexico with this terminology are the following facts: firstly, the absence of food scandals related to the national food sector industry. Secondly, the country is characterized as a fresh fruit and vegetable exporter; consequently, there is in general terms trustworthiness of food safety. Also in general terms, the Mexican population does not concern itself with how food is produced, i.e. animal produce. On the contrary, because of the food scandals (BSE, Nitrogen scandal, for example) of the 1990s in several countries within the European Union (and especially in Germany), consumers have changed their attitudes and trustworthiness towards the food industry sector, which precipitated the increase in demand of organic food (Frentrup $\&$ Theuvsen, 2006; Schulze et al., 2008). This study provides enough evidence to support the argument that the concern with "Animal welfare" is linked to two factors: on the one hand, demand for process-related information on the production of food; and on the other, the stage of development of the organic food industry sector on a national basis, i.e. the mature stage of the German market versus the seed stage of the Mexican market. 
Regarding process-related information-product information and consumer environmental concern (labeled as environmental concern/information), respondents in the German sample seem more concerned than Mexicans. Again, historical facts are useful in understanding this difference. As mentioned in Chapter 2, organic farming in Germany was established during the 1970s as part of the environmental movement, whereas in Mexico it was established during the 1990s within an economical scheme that supported export-oriented produce. These historical reasons explain why the German population is still more concerned with environmental issues such as sustainable development and sustainable farming methods and the state of nature by the production of food than the Mexican population where discussion on these issues has just recently begun. However, this belated initiation of discussions does not mean that Mexicans are not interested in them.

The relevance of "Product information" regarding nutrition (labeled Information) is less widely ascertained in Mexico than in Germany. As our findings have shown, Mexicans would like to have more information than Germans. Hence, it still remains unclear whether Germans are less involved with food issues, or, as is even more plausible, they perceive themselves as already informed enough concerning food products.

German consumers of organic food also seem more concerned with "Natural content" in food products than non-consumers. This is not surprising according to findings reported by Baker et al. (2004) in their comparative study of German and English consumers of organic food. As the authors mentioned, perceptual orientations about the belief in nature, absence of pesticides and chemicals, absence of genetically modified material, avoidance of unnatural contents, health-related concerns, and well-being were stronger among the Germans than the English.

Our findings clearly show less involvement of German non-buyers concerning the dimension of "Natural content" than German consumers of organic food. Interestingly, natural content seems to be a stronger predictor associated with the purchase of organic food, whereas among the Mexican sample the statements were carried out within the factors labeled as "High food involvement" because the care about natural contents, however, within the factor "Low food involvement" there were the low care about natural contents All in all, "High food involvement" was a stronger predictor associated with the purchase of organic food in Mexico. These facts also provide interesting insights regarding trends on 'Clean label' food. The original initiative took place in the United Kingdom. The term refers to the non use of 
additives such as colors, preservatives, antioxidants, acidity regulators, flavor enhancers, etc. In Germany, 'Clean label' products remain more as a niche of the market, especially of interest by health-conscious consumers who probably have similarities with consumers of organic food (Decision News Media, 2007; Food Technologie Magazin, 2009).

In the case of Mexico, different factors have been identified which might influence the attitudes of Mexican consumers and non-buyers of organic food with respect to "Natural contents”. Firstly, similarly to the dimension of animal welfare, the stage of development of food industry sectors and the absence of food scandals might positively influence the trustworthiness of food safety. Secondly, the limited knowledge on food product information and food process-related information might help us to understand this fact. The common belief that small-scale producers do not use agrochemicals, fertilizers, and pesticides by most of the Mexican population is a reason to trust in the naturalness of food.

With regard to "Healthy eating", it is interesting that though Germans feel they are well informed about food, they are conscious of their poor management of a healthy diet. Conversely, Mexicans do not believe that they are well informed, but similar to Germans, they have the impression that they do not manage a healthy eating lifestyle. All in all, Mexican and German consumers of organic food appreciate organic food as healthy. However, it is interesting that Germans indicate an association between taste and healthy food. However, more Germans than Mexicans claim that they do not forsake tasty food for healthy food. Hence, our findings confirm previous results of Steptoe et al. (1995) who regard a "relation between taste and fatty food which makes taste an important variable in the selection of food".

Unfortunately little information was provided by the construct of "Free of GMO" in our study. In Mexico both consumers and non-buyers have a positive attitude toward the purchasing of GM food, which, in our opinion, is mainly due to little product information and low consumer knowledge about this issue. According to a survey conducted by Greenpeace in Mexico in 2005, 96.5\% of consumers neither could explain the term 'GM food' nor had ever eaten any GM food. What is more, $98 \%$ of the respondents claimed that producers should provide information on packaging if GM food is included in the content (Greenpeace, 2005). Germans display more concern with the production of GM food; however, in this research there was not enough information to reflect cultural differences. 
Responses provided in the Mexican and German samples show slight differences within the dimension of "Familiarity" on the grounds of the dual dimension neophobia/neophilia. The most interesting result is that German consumers of organic food show more neophilic behavioral traits. This shows that the latter are more responsive to innovative food offerings (tastes, products, experiences, etc.) than German non-buyers.

Other interesting insights can be drawn with respect to "Advertising". In general terms, German consumers of organic food are more skeptical about advertising than non-buyers, whereas Mexicans tend to be more receptive to purchasing as a result of advertising without any distinction between consumers of organic food and non-buyers.

Moreover, within the stage of the food sector industry and the national food supply chain, "Convenience" is a useful dimension in discerning cultural differences, although the idea of differentiating between convenience in cooking and in purchasing was postulated. Among the findings there is empirical evidence that convenience at least for Germans includes both purchasing and cooking. There is an overall appreciation of easy-to-prepare meals. Probably this fact is linked with lifestyle or time available for shopping for food products. This explains the preference for purchasing at discounter retailers which are "a good option" for "smart shopping" because of their especial assortments, good prices, and convenient locations. The factor of convenience does not seem to influence the purchase of food including organic by Mexicans. The statement: for me, food shopping is an unpleasant task was within the factor of low food involvement in the two groups: total sample and consumers of organic food. Convenience in cooking seems to go along the same line. Concerning the growing assortment of convenience food, however, Mexican non-buyers show a few concerns. Two reasons could be proposed for this fact: either they are more skeptical because of financial constraints (convenience foods are generally more expensive than traditional) or, as endorsed by the study of Padilla \& Perez (2006), they have some problems with accepting this kind of food due to the fact that they are accustomed to purchasing non-processed, fresh and seasonal food.

In the total sample "Nostalgia" also indicates differences between Mexican and German consumers of organic food. In fact, the evocation of past experiences in childhood -nostalgiais higher among Mexican respondents except for Mexican consumers of organic food. This confirms the finding by Mines \& Nichols (2005) in their study of "nostalgia markets" with respect to Mexican migrants living in the U.S.A. 
"Price orientation" is a variable surveyed in both countries. In general terms the Mexican market is characterized as price-sensible (USDA, 2002), whereas the German market is characterized as price-oriented (KPMG, 2006). It seems therefore that both Germans and Mexicans are price-oriented. However, the reasons are different at the demographic and psychographic levels. When the mean value was compared, German non-buyers seem more 'price oriented' than Mexican non-buyers. Whereas in Mexico "the majority of income goes towards the purchase of food" (both for consumers of organic food and non-buyers, cf. Mexican market as price-sensible, USDA, 2002), this economical aspect does not concern Germans at all. In consideration of the foregoing, it is interesting to note that price orientation was detected as a dimension that transversely concerns all surveyed groups in both countries (cf. study of KPMG, 2006 on price orientation within the German market). Hence, both Germans and Mexicans carefully check prices while shopping. However, when the variables were multivariate analyzed-factor analysis and multiple linear regression-not one statements were carried out among the Germans and Mexican samples. Notwithstanding when the variable "Price quality relation" was analyzed, high prices were associated with better quality by German consumers of organic food within the factor of high food involvement among the German total sample. Future research on relevant associations with price in both countries could be studied further.

Germans and Mexicans both seem 'ethnocentrically oriented' in terms of consumer behavior (dimension of consumer ethnocentrism). However, this attitude is more predominant among Mexican consumers of organic food than Germans, although the four groups show preference of purchasing food produced in their home country. Furthermore, our study shows that within the dimension of consumer ethnocentrism the preference for fruits and vegetables from one's own country transversely relates to all categories of consumers in both countries.

Emphasis on consumer ethnocentrism related to purchasing of organic food uncovered a paradox. On the one hand there is a preference for food of national origin and profitability for farmers-fair price. On the other hand, there is the need for variety and neophilia of food stressors in demands for "exotic", "different", or "trying something new (things/experiences)". Consequently, products of foreign origin have enlarged assortment of food in both countries. This fact is not exclusive to Germany and Mexico; however, the question about how much miles have to be transported food products? is regarded especially 
stressful on the principles of environmental concern and sustainability of organic produce by the highly involved consumer of organic food.

All in all, 22 theoretical motives related to purchasing of organic food were postulated. After factor analyses they were reduced to 7 motives in Germany and 4 in Mexico. Six of them which are the most frequently mentioned in the literature were included in the factor of traditional motives: tasty, healthy, fairness for farmers, concern with environmental and animal welfare. This factor was the only one commonly shared by consumers of organic food in Germany and Mexico, and it seems that it is a stronger predictor in the understanding of the purchase of organic food across these countries. Although weight control was shared by the four groups of consumers, this dimension was only significant for the German consumers of organic food but not for the Mexicans. Moreover, this factor was a weak predictor in explaining the total German model.

Regarding the findings on values, the following provides an examination of the main differences between German and Mexican cultures that was undertaken in this study. Interestingly, the fact that "Technophilia" measured with the dimension of Technological progress/Technophobia was transversely portrayed among the Germans and Mexicans but weak among the four groups.

Germans and Mexicans tend to be 'fatalistic'. Among the four groups of both countries there is an attitude of acceptance of the outcomes of situations as something determined by external forces (Rotter, 1966 cited in Ross et al., 1983); for instance, the impossibility of avoiding the destruction of the environment and natural resources. Furthermore, as detected in previous studies on the consumption of organic food, our findings show a difference between both countries. In general terms Mexicans tend to be more fatalistic than Germans; however, Mexican organic consumers tend to be a few less fatalistic than Mexican non-buyers.

Interestingly, differences were found between Germans and Mexicans with regard to "Selfesteem" (theoretical construct of "Fairness"). However, German consumers of organic food seem to be heading in a similar direction and philosophy but with a different intensity toward the value of "Self-made". The value of self-made does not seem to influence the purchase of organic food. 
"Hedonism" is a dimension providing interesting insights on cultural differences. Germans seem more hedonistic than Mexicans, since Mexicans remain more skeptical about hedonistic behavior than Germans, but when looking at other values among Mexican respondents, the values of materialism seem to be driving them in the same direction, that is: prioritizing of their goals in the pursuit of individualistic rather than collective interests. The findings of the German sample are not surprising as in 2001 Hofstede reported the German national culture as highly individualistic (63 points). However, it is surprising that the results of this study of the Mexican sample did not entirely correspond with the foregoing author's characterization of the Mexican national culture as collectivistic although more individualistic in comparison with other countries in Latin America. According to our findings regarding this dimension, there is now a tendency for Mexicans to lean toward individualization which emerged in the two groups: non-consumers and consumers of organic food.

The value of "Living now" uncovered within the Mexican consumers of organic food seems similar to the dimension labeled as short-term orientation discovered in 1988 by Hofstede and Bond (cited in Mooij, 2004). According to them, the dimension of short-term orientation was defined as "spending now is more important than saving for tomorrow." This similarity is compared in this study due to the value that seems to be an external focus of control and enjoyment. On the other side, the value of "Materialism”, carried out within the same groupMexican consumers of organic food seems to be an internal focus of control and hedonistically oriented. This fact is interesting due to the apparent contradiction of a combination of fatalism, enjoyment, and the need to be pleasant to others; these statements are the same as the dimension of hedonism among the German consumers of organic food. Consequently, a kind of hedonism might be postulated among the Mexican consumers of organic food but with a different intensity than the German consumers of organic food.

Within the findings of cross-cultural values, both Germans and Mexicans prioritize goals in the pursuit of individual interests. Germans seem more hedonistic, whereas living now seems important for Mexicans. Even more so, "Ethnocentrism" and "Technophilia" are common values shared by Germans and Mexicans but with low significance. Self-esteem was shared by German non-buyers and Mexicans with the exception of German consumers of organic food where this value was more intense and then became as self-made. Fatalism was shared by the four groups; this might suppose the existence of a universal tendency. However, it was 
not significantly correlated to the purchase of organic food. The value of "Self-esteem" might suppose no difference between the Mexican non-buyers and the Mexican consumers of organic food. However, on the basis that self-made and self-esteem have the same emotional origin and the same arrow direction but with a different intensity, this might suppose the existence of differences between German non-buyers and German consumers of organic food.

Although within the factor carried out on traditional motives, there is a combination of pursuing a collective interest-concern with environment, animal welfare, and fair payments for farmers, and individual interest-tasty and healthy, individualistic goals such as pleasure and enjoyment-hedonistic orientation were uncovered. The tendency to satisfy individual needs instead of collective needs-fair payments, concern with environment, is revealed among the values of consumers of organic food in both countries. These provide twofold insights: there is an apparent predominance of egoistic motives driving the purchase of organic food (Magnusson et al., 2003) in Germany and in Mexico. In relation to the findings reporting collective benefits such as fair trade, political motives, environmental concern, animal welfare concern, ethical acts (see Table 13 and Table 14 in Chapter 5) by early studies on the consumption of organic food, there is enough evidence to postulate the reduction of their importance and the gaining of individual benefits among the findings of values in our study. Figure 79 summarizes the main findings of this comparison. 
Figure 79: Motivations and values related to the purchasing of food products and organic food in Germany and Mexico

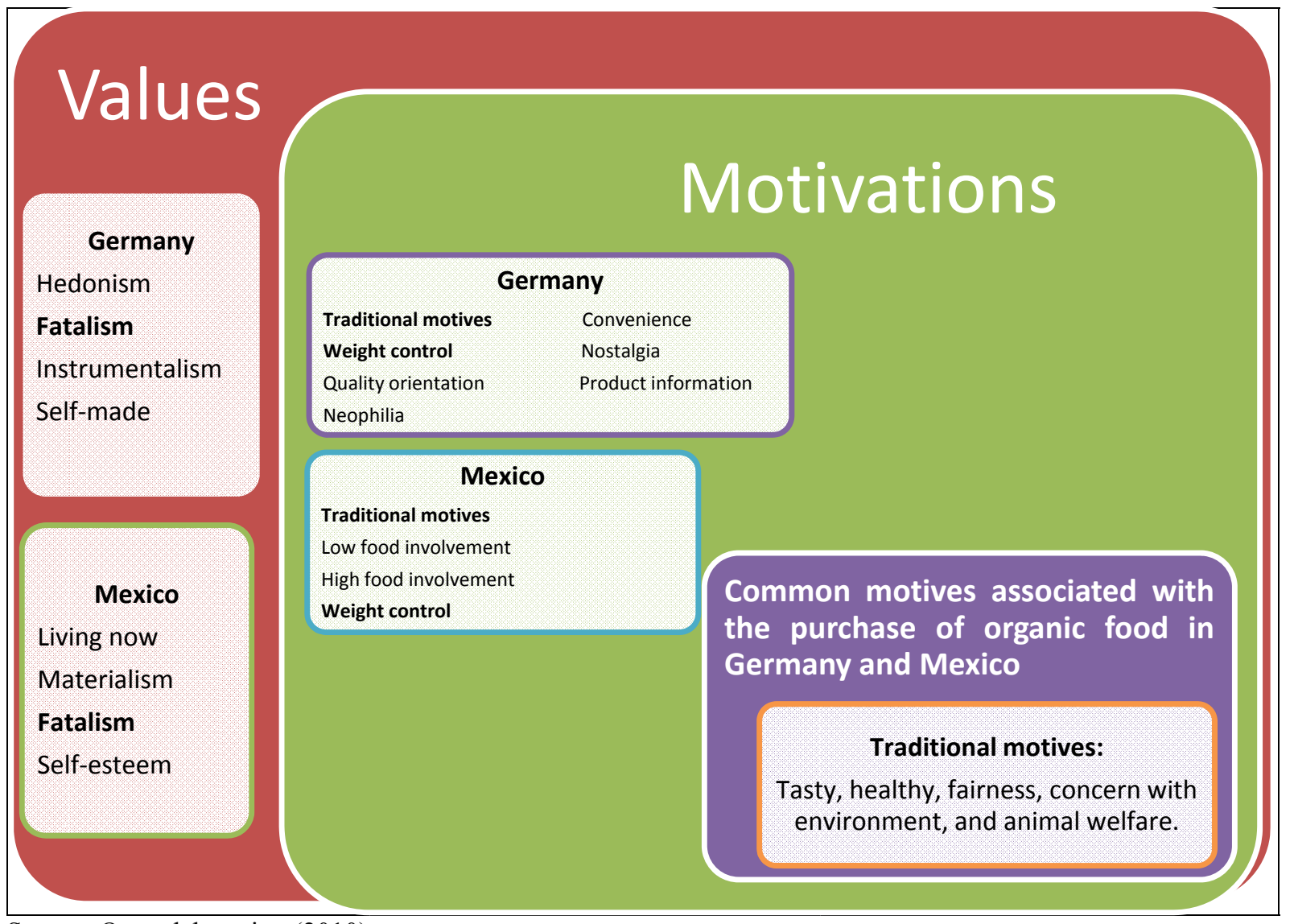

Source: Own elaboration (2010).

\subsection{Framework}

This section expands on the main findings of this study placing them in a contextual landscape to enhance further empirical research. The conceptual framework that has been introduced herein focuses on individual consumption patterns interpreted within the two mainstream theories of organic produce, i.e. conventionalization and social embeddedness. As already mentioned at the beginning of this dissertation, conventionalization refers to "the appropriation by agribusiness firms of the most lucrative segments of organic commodity chains, abandoning the more sustainable agronomic and marketing practices associated with organic agriculture" (Guthman, 2004). Consequently, the phenomena of conventionalization have been studied alongside the food supply chain emphasizing production and marketing. This research provides empirical evidence of purchasing organic food in relation to 
conventionalization in Germany which is twofold: shopping convenience-purchasing factor and cooking convenience-personal factors.

On the one hand, many scholars have provided empirical evidence about conventionalization of organic farming in developed countries, most of them with mature markets for organic food. (Best, 2008; Darnhofer 2006; De with \& Verhoog, 2007; Guthman, 2004; Hall \& Mogyorody, 2001; Kratochvil \&Leitner, 2005; Lockie \& Halpin, 2005; Padel \& Midmore, 2005). In this regard in Germany Best (2008) distinguishes between two kinds of organic farmers: the pioneers who know the principles and are committed to growing organic produce and the converted farmers who exhibit higher specialization in crops, large sized farms, and avoidance of traditional channels such as direct marketing.

These changes in organic produce in the German market structure display the coexistence of two parallel segments attracting different target groups who are not completely separate. Thus, different marketing strategies should be developed for each target group. In fact, similar to the typology of organic producers by Best (2008), it is possible to postulate the existence of consumers committed to organic farming and non-committed consumers who are disconnected from its origin and principles, because their preferences relate more to personal factors-hedonistic assets such as "food safety", "fashionable", "enjoyable", and "convenient" rather than collectivistic matters such as "environmental concern", "animal welfare", "fair price", "social justice" and "sustainability" related to the pioneering or committed consumers.

Since the early 2000s the total turnover of organic food has increased by approximately $4 \%$ annually by conventional food retailers, while the total turnover by direct marketing and health food shops has proportionately decreased. According to the results of the German sample, there is enough evidence to conclude that "Convenience" is the main purchase factor motivating the migration of consumers from traditional channels to conventional food retailers.

The findings of this research provide empirical evidence on "Shopping convenience" and "Cooking convenience" as preferences for purchasing organic food that is encouraging the conventionalization process. In fact, "Shopping convenience” has been a successful marketing strategy by conventional food retailers that has strengthened concentration of demand on this 
marketing channel with implications related to organic farming produce. In the words of Brand et al. (2004), "Organic food is marketed without any organic context."

Moreover, with respect to shopping convenience as a marketing strategy by conventional food retailers, the findings reveal personal preferences-hedonistic assets for cooking convenience and convenience food considered when consumers purchase organic food and this is a negative aspect about marketing of organic food because it is opposite to the principals of organic farming. In fact, personal preferences mirror individual differences more than goals in the pursuit of collective interest. As was provided in Chapter 4, measuring values is useful in ascertaining cultural differences because of their relevance at the individual and societal level.

Within the findings of our research on values, a mixed dimension was uncovered "Traditional motives" which consists of the theoretical dimensions labeled as: healthy, tasty, fair price, animal welfare, environmental concern. The dimension of "Traditional motives" mirrors concerns regarding collective interests such as: environment, animal benefits, and farmers; and individual interests such as health and taste among the consumers of organic food in both countries. The findings are interesting not only because they provide evidence of commonalities among consumers of organic food but also differences within their national cultures; furthermore, they also provide evidence of mixed goals in the pursuit of collectivistic and individualistic (hedonistic) interests.

All in all, the findings of hedonistic values and "Traditional motives" relating to animal welfare, fair payments for farmers and concern with the environment allow us to propose a mix of the principles of organic farming: health, ecology, fairness, care enhancing “environmental concern", "environmentally friendly", "fair price”, "better for farmers", “food quality" and "food security" as guidelines in the design of marketing strategies to strengthen the term "organic" among committed consumers and disseminate it among converted consumers who prioritize convenience/hedonistic attributes probably without knowledge and depth of understanding of the origins and principles of organic (IFOAM, 2010).

In this regard, the theory of social embeddedness recalls the importance of social relations to economic exchange processes (Granovetter, 1985) even as applied to food consumption; it deals with "all local social relations of consumption based on trust relations between producers and consumers" (Winter, 2003). Many scholars who have surveyed the organic 
market claim that improved regional localness of these relations "might strengthen the environmental and social profile and therefore the meaning of organic" (Kratochvil \& Leitner, 2005). Local embeddedness which is a refining of "social embeddedness" in a local context (Winter, 2003) which has been proposed recently for direct marketing to overcome conventionalization (Kratochvil \& Leitner, 2005; Seyfang, 2005). However, this is restricted to the spatial condition of localness.

Throughout this dissertation research has revealed complex motivational and value-related dimensions among consumers of organic food both in Germany and Mexico. Although some of these dimensions differ between countries, for instance convenience in purchasing, some commonalities have been detected that were derived from the findings of this study. As will be seen, they entail both principles of the conventionalization and embeddedness theories. For this reason they have been placed under the theoretical umbrella of "convenience-driving Embeddedness (CdE)" (see Figure 80).

The core idea of the CdE is that organic food exchanged within the trustworthy relationship between producers and consumers "are wrapped" by the cultural dimensions (motives and values) detected in our study. Thanks to these dimensions, the consumption of organic food acquires a "new meaning" (Winter 2003) by direct marketing within a local context redefining the words by Brand et al. (2004) of "organic with(out) an organic context".

\section{Figure 80: Convenience-driving Embeddedness (CdE)}

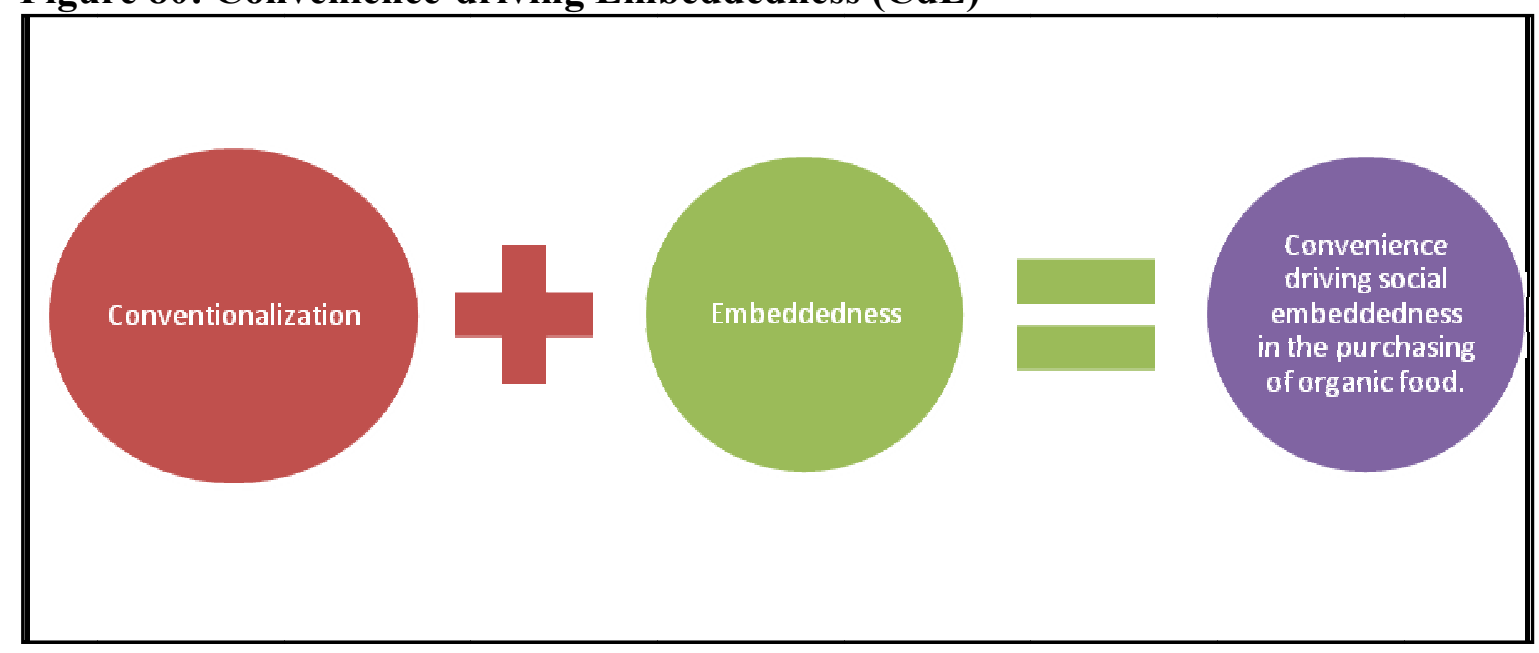

Source: Own elaboration (2010). 
What's more, since quality is a socially constructed concept (ibid.), direct marketers of organic food might build upon the development of quality-related notions. In doing so, they not only should integrate the values of natural enjoyment enhancing hedonism, love for nature, and variety in life detected in our study but also the motives of shopping convenience and cooking convenience that concerns consumers of organic food in both countries.

Transference into practice could be developed through innovative CdE marketing strategies as follows:

- Conventional direct selling, one-to-many

- Online direct selling, many-to-many

- Offline direct selling, many-to-many

Conventional direct selling, one-to-many: In Germany, although direct selling at weekly markets already exists, only a few organic farmers offer ready-to-eat organic food. However, as detected at specialized meetings (Albers, 2008) and even at weekly markets, consumers appreciate being able to purchase organic food that is almost ready to eat and can be prepared quickly and easily.

However, we do not think that embeddedness of the organic market uniquely means trustworthy relations which take place exclusively in rural (vulnerable) areas. This view is also endorsed by other scholars who label this unilateral position as "defensive localism" (Winter 2003). This could translate into a "conservative celebration of the local" and thus nearing a nothing-but-closed parochialism (ibid.).

Based on these factors, we will attempt to trace some marketing strategies based on social marketing defined by Kotler \& Zaltman (1971) as "the design, implementation, and control of programs calculated to influence the acceptability of social ideas and involving considerations of product planning, pricing, communication, distribution, and marketing research". For this reason, the next two strategies refer to an extra-rural context.

Online direct selling, many-to-many: A growing number of organic platforms could become the venue for trustworthy relations between producers and consumers. In Germany, for 
instance, this type of promotion is gathering momentum (cf. Utopia.de; naturkost.de, ecoworld.com.de, biobay, vinculando.org).

Offline direct selling, many-to-many: The novel traits of organic consumers could be exploited further to design new encounter possibilities-places between producers and consumers. The creation of direct selling fairs where producers of organic products and consumers-groups, communities of consumers can meet could help strengthen face-to-face interaction. Besides, such a venue could help disseminate information on the principles and origin of the organic movement which, as our study has shown, are rooted both in Germany and Mexico (i.e. www.terrae-it.eu).

The different interpretations of the meaning of organic food purchasing correspond to personal needs, desires and wants of the consumers, thus marketing strategies are designed according to the food retailing channel-organic farmers and conventional food retailers trying to fulfill these needs by positioning their facilities to meet these objectives.

The utilization of social marketing seems useful in repositioning "organic in an organic context" due to organic farming having its roots in a social movement. In Germany, the concentration of demand on conventional food retailers and conventionalization was a consequence of organic marketing without a social context; however, social marketing seems useful in socializing and repositioning the term "organic" more with convenience and localness within the "global village" because of electronic mass media. Whereas in Mexico the German experience of conventionalization of produce and marketing -concentration of demand on conventional food retailers- should be pondered within the development of the domestic market.

\subsection{Limitations}

As in other research projects in social sciences and comparative studies, some problems and limitations regarding methodology and sampling aspects were encountered in the research for this study as described below. 
Among the methodological aspects, one of the main problems encountered was the construct of equivalence. On the basis of the literature review on motives to purchase organic food, difficulties arose regarding measuring some of them in Mexico which resulted in complications in establishing the comparison especially related to meanings of words; for instance, measuring the dimension of "Animal welfare" and its concept in the Spanish language. The translation equivalencies, word meanings, and meanings in use were of great concern to the authors, especially since only a small percentage of the Mexican population understands the concept of "Animal welfare”. However, as mentioned above, it was reasoned that because of the absence of food scandals in the national food sector industry in Mexico, this would result in a positive influence on the trustworthiness of Mexicans regarding food safety even though there seems to be a prevalence of misinformation.

Moreover, besides problems with the measurement of single-item scales of "Animal welfare", other problems in measuring were experienced with regard to "Fair price", "Malinchismo" and "Free of GMO" among the Mexican sample. This is probably because of the lack of knowledge about these terms. Thus, improving the dimension of "Animal welfare", "Malinchismo", "Free of GMO” (GM free food) is suggested.

Some problems with the measurement of the construct of "Free of GMO" were also detected within the German sample. Thus, improvement in measuring this construct is suggested in further research in this country.

Both Germans and Mexicans carefully look at prices while shopping. Although literature suggests that Germans and Mexicans are price-oriented. The Mexican market was characterized as price-sensible (USDA, 2002) and the German market as price-oriented (KPMG, 2006). However, not enough relevant findings were discovered in this study for the construct of "Price orientation" surveyed in both countries. This fact suggests that the measurement of this dimension should be improved.

Although it was preconceived that the dimension of "Variety in life (Neophilia/Neophobia)" would have interesting cultural differences, no meaningful differences were found for this construct which is a dual measuring dimension within-country (groups in any countries). All in all, further research on this dimension is suggested to provide more insights about the need for variety among consumers of organic food in the two countries. 
In the Mexican sample some problems were also detected in the sampling. Although the online collection of data was useful in reducing social desirability, the representation of the Mexican population was restricted due to the underrepresentation of respondents with low household incomes. Thus, the inclusion of respondents from this income level would have been useful. This is directly related to the concentration of marketing channels of organic food in Mexico City; therefore, it was determined that the inclusion of respondents from other cities would improve the national sample representation.

Although with the improvement of measurement scales and even the use of other research methods in order to reduce the problem of social desirability of responses, positive attitudes and the collectivistic characteristic of the national culture resulted in more difficultly in the measurement of psychographics using only quantitative methods. Consequently, the restricted use of quantitative methods is recognized as a limitation of this research.

\subsection{Guidelines for further research}

In this cross cultural comparison, the focus was on the search for differences and similarities of values and motives in purchasing organic food in Germany and Mexico. However, determination of societal variables or dimensions would have been useful in establishing typologies of consumers. According to Mooij (2004), typologies of consumers are used in marketing and advertising to create messages for imaginary consumers (i.e. post-materialism, conservative) who are not necessarily culturally similar. Whereas in the search for similarities, the level of cultural variables is used in market segmentation (e.g. lifestyle, occasional, heavy consumers), search for similarities and cluster analysis is suggested to determine new market segments.

Structural Equation Models (SEM) would seem as a useful alternative method to measure the influence of values, motivations, and demographics to determine their influence on the purchase of organic food. Even with the usefulness of quantitative research approaches to provide evidence in cross-cultural comparative studies, qualitative research might be inadequately represented, thus it is suggested that qualitative research approaches should be 
used to provide in-depth understanding of psychographics and in the study of consumer behavior.

Regarding further studies of psychographics, it is difficult to measure what exactly is in a consumer's mind; and the borders between values, beliefs, attitudes, and motives are not clear. The design of hybrid models and measurements might be useful to uncover different psychographics to provide further insights into the purchasing of food products and organic food.

With regard to the motives to purchase food products in general and organic food specifically, the inclusion of trustworthiness and attitudes toward the food sector industry and food security is suggested particularly in Mexico.

All in all, further studies on values influencing the purchasing of organic food are suggested. Finally, though most of the international literature on earlier studies mentions concerns by consumers of organic food with the environment, fairness, and local development, their values influencing the purchasing of organic food have been studied (De Wit \& Verhoog, 2007; Grunert \& Juhl, 1995; Honkanen et al., 2006). However, the dominant direction of the values carried out in our study uncovered egoistic motives in the consumption of organic food as reported in 2003 by Magnusson et al. Thus, after these findings, further study could be addressed to provide an in-depth understanding of the values of consumers of organic food. This fact is suggested for twofold reasons: to contribute more evidence about changes or preservation of the values and motives reported by first consumers-organic pioneers in Germany, and values reported by consumers in the 2000s after the conventionalization process. This knowledge would be useful in the prediction of consumer behavior and trends in consumption by modern and/or future target groups.

The relationships between demographic variables and values among consumers of organic food might also be useful in providing an interpretation of organic consumer behavior. And differences in values between consumers and non-buyers of organic food would be another interesting line of research among non-buyers to further understand the demographic factors limiting the purchase of organic food and differences at the psychographic level between them (i.e. the value of self-esteem within the German total sample and the value of self-made within the German consumers of organic food). Hierarchical values could be used to uncover 
potential consumers who might be sensitive to environmental or fairness issues and potentially receptive to purchasing ethical products. Therefore, it is advisable to consider the usefulness of psychographics to uncover cultural and target differences. 


\section{References}

Aaker, D. A., Kumar, V., \& Day, G. S. (2007). Marketing research, (9 ed.). Hoboken, NJ: Wiley.

Aertsens, J., Verbeke, W., Mondelaers, K., \& van Huylenbroeck G. (2009) Personal determinants of organic food consumption: a review. British food Journal, 111(10), 1140$\begin{array}{llllll}\text { 1167. } & \text { Retrieved } & \text { February } & 3, & 2010 & \text { from }\end{array}$ www.emeraldinsight.com/10.1108/00070700910992961.

Albers, E. (2008). Hofladen, Pferdepension, Partyservice und Co. Die Vielfalt der zusätzlichen Einkommensfelder für Landwirte. Presentation held at the Conference on farm tourism, November, 11/12 2008, organized by Netzwerk ländlicher Raum, Weiden (Germany).

Allen, P., \& Kovach, M. (2000). The capitalist composition of organic: The potential of markets in fulfilling the promise of organic agriculture. Agriculture and Human Values, 17(3), 221-232. Retrieved August 15, 2009 from http://dx.doi.org/10.1023/A:1007640506965.

Altieri, M. A. (2002). Agroecology: the science of natural resource management for poor farmers in marginal environments. Agriculture, Ecosystems \& Environment, 93(1-3), 1-24. Retrieved August 1, 2009 from http://www.sciencedirect.com/science/article/B6T3Y46H4D3V-2/2/599f34c2817cf41calbdf7ae269f7378.

Ares, G., \& Gámbaro, A. (2007). Influence of gender, age and motives underlying food choice on perceived healthiness and willingness to try functional foods. Appetite, 49(1), 148158. Retrived February 9, 2008 from http://www.sciencedirect.com/science/article/B6WB24MYMFN1-1/2/b707e8d840b226fc16288d4a8a4fc51f.

Arnould, E., Price, L., \& Zhinkan, G. (2004). Consumers, McGraw-Hill, (2 ed.) U.S.A.

Bagozzi, R. P., \& Yi, Y. (1988). On the Evaluation of Structural Equation Models," Journal of the Academy of Marketing Science, 16(1), 74-94. 
Baker, S., Thompson K.E., and Engelken, J. (2004). Mapping the values driving organic food choice. European Journal of Marketing 38(8), 995-1012. Retrieved February 6, 2008 from www.emeraldinsight.com/10.1108/03090560410539131.

Bahrdt, K., Ludwig, J., \& Nierenköther, J. (2003). Bio-Supermärkte in Deutschland -Chancen und Entwicklungen 2003. Frankfurt a. M.: Unternehmensberatung Synergie.

Blackwell, R. D., Miniard, P. W., \& Engel, J. F. (2006). Consumer behavior (10. ed., Student ed.). Mason, Ohio. (ed.)Thompson, South-Western.

Blythe, J. (2008). Consumer behaviour. London [ed.]: Thompson.

Brand, K.W., Engel, A., Kropp, C., Spiller A. \& Ulmer H. (2004): Von der Agrarwende zur Konsumwende? Beitrag zum Statusseminar Sozial-ökologische Forschung des BMBF Kompetenznetzwerk zur Agrar-und Ernährungsforschung am 21-22.06.2004 in Berlin.

Bredahl, L. (2001). Determinants of Consumer Attitudes and Purchase Intentions With Regard to Genetically Modified Food - Results of a Cross-National Survey. Journal of Consumer Policy, 24(1), 23-61. Retrieved May 10, 2009 from http://www.springerlink.com/content/10r44p1068m212x9/.

Best, H. (2008) Organic agriculture and the conventionalization hypothesis: A case study from West Germany. Agriculture and Human Values 25, 95-106. Retrieved February 23, 2010 from www.springerlink.com/index/w886p8nj775g49gw.pdf.

Biovista (2010). Bio-Fachhandel 2009. Sortimentstrends und Marktentwicklung. Presentation held at Biofach 2010 Nürnberg 17.02.2010.

Breithor, T., Breitkopf, S., Horbert C., Humbach, C., Pietersen, F., \& Vortmann, A., (2001). Status Quo and prospects in the German food market. KPMG Germany. Retrieved April 17, 2007 from http://www.ehi.org/en/geschaeftsbereiche/publishing.

Bruhn, M. (2001). Verbrauchereinstellungen zu Bioprodukten-Der Einfluss der BSE-Krise 2000/2001. Kiel. Retrieved February 3, 2007 from http://www.unikiel.de/agrarmarketing/VERSION5.PDF. 
Bruhn, M. (2002). Die Nachfrage nach Bioprodukten Eine Langzeitstudie unter besonderer Berückssichtigung von Verbrauchereinstellungen. Peter Lang Europäischer Verlag der Wissenschaften Frankfurt am Main, Germany.

Bolten, J., Kennerknecht, R., \& Spiller, A. (2006). Perspectives of small retailers in the organic market: Customer satisfaction and customer enthusiast. 1-12. In: 98th EAAE Conference Marketing dynamics within the global trading system. Chania Crete, Greece.

Campbell, H. \& Liepins, R. (2001). Naming Organics: Understanding organic standards in New Zealand as a discursive field. Sociologia Ruralis 41(1), 21-39.

Cicia, G., Del Guidice, T., \& Scarpa R., (2002). Consumer perception of quality in organic food a random utility model under preferences heterogeneity and choice correlation from rank-orderings. British Food Journal 104 (3/4/5), 200-213. Retrieved January 16, 2008 from www.emeraldinsight.com/10.1108/00070700210425660.

Chinnici, G., D’Amico M., \& Pecorino, B. (2002). A multivariate statistical analysis on the consumers of organic products. British Food Journal 104(3/4/5), 187-199. Retrieved January 16, 2008 from www.emeraldinsight.com/10.1108/00070700210425651.

Chryssohoidis, G. M., \& Krystallis, A. (2005). Organic consumers' personal values research: Testing and validating the list of values (LOV) scale and implementing a value-based segmentation task. Food Quality and Preference, 16(7), 585-599. Retrieved July 12, 2009 from www.sciencedirect.com/science/article/B6T6T-4GDSDW31/2/0e18a9d5cfdfb2d36b5f835d7321e424.

Darnhofer, I. (2006). Organic farming between professionalization and conventionalisation The need for a more discerning view off farmers practices. Paper presented at Joint Organic Congress. Odense, Denmark, May 30-31, 2006. //www.orprints.org/7390/.

Davies, A., Titterington, A., \& Cochrane, C. (1995). Who buys organic food? A profile of the purchasers of organic food in Northern Ireland. British Food Journal, 97(10), 17-23. Retrieved June 13, 2007 from www.emeraldinsight.com/10.1108/00070709510104303.

Dawson, J. (2006). Retail trends in Europe Retailing in the 21st century, 41-58. Berlin (Ed.) Springer. 
DeMuth, S. (1993). Defining Community Supported Agriculture (CSA). Retrieved in July, 15, 2009 from http://www.nal.usda.gov/afsic/csa/csadef.htm.

De Wit, J. \& Verhoog, H. (2007). Organic values and the conventionalization of organic agriculture. NJAS Wageningen Journal of Life Sciences, 54(4), 449-462. Retrieved in January 30, 2010 from http://www.sciencedirect.com/science/article/B94T2-4WFBS67B/2/5248fbdd9e253cb48b6b16f60e5083e4.

Dibbern, J., \& Chin, W. W. (2005). Multi-Group Comparison: Testing a PLS Model on the Sourcing of Application Software Services across Germany and the U.S.A. Using a Permutation Based Algorithm. In Friedhelm Bliemel (Eds), Manual of PLS-Path modelling. Stuttgart, Schäffer-Poeschel.

Eberl, M. (2010). An application of PLS in multi-group analysis. The need for differentiated corporate-level marketing in the mobile communications industry. Handbook of partial least squares, 487-514.

Eertmans, A., Victoir, A., Notelaers, G., Vansant, G., \& Van den Bergh, O. (2006). The Food Choice Questionnaire: Factorial invariant over western urban populations? Food Quality and Preference, 17(5), 344-352. Retrieved April 5, 2007 from http://www.sciencedirect.com/science/article/B6T6T-4G9Y4HT1/2/f8d8cebe8a899bb652d8fa638052e40c.

Field, A. P., (2008). Multiple regression using SPSS/PASW. C8057 (Research methods in psychology): Multiple regression. Retrieved August 13, 2010 from www.statisticshell.com/multireg.pdf

Frentrup, M., \& Theuvsen, L. (2006). Transparency in Supply Chains: Is Trust a Limiting Factor? Paper prepared for presentation at the 99th EAAE Seminar 'Trust and Risk in Business Networks, Bonn, Germany. February 8-10, 2006. Retrieved June 7, 2010 from http://ageconsearch.umn.edu/bitstream/7725/1/sp06ma07.pdf.

Fotopoulos, C., Krystallis, A., Vassallo, M., \& Pagiaslis, A. (2009). Food Choice Questionnaire (FCQ) revisited. Suggestions for the development of an enhanced general food motivation model. Appetite, 52(1), 199-208. Retrieved April 25, 2009 from 
http://www.sciencedirect.com/science/article/B6WB2-4THSWY92/2/3a0976004add083525be12b3822d4095.

Fotopoulus, C., \& Krystallis, A. (2002). Purchasing motives and profile of the Greek organic consumers: a country wide survey. British Food Journal, 104(9), 730-765. Retrieved April 28, 2008 from www.emeraldinsight.com/10.1108/00070700210425697.

Garcia C., Fearne, A. \& Wood, L. (2010). The role of involvement attention paid by supermarket shoppers to organic products. Journal of Innovation Economics, 5(1), 127-144. Retrieved May 12, 2010 from http://www.cairn.info/revue-journal-of-innovation-economics2010-1-page-127.htm

Gerlach S. \& Spiller, A. (2006). Bio-Fachhandel: Auslaufmodell oder Wertebasis der Branche? In: Brand, K.-W. (Hrsg.): Die neue Dynamik des Bio-Markts. München, 129-144.

Gerlach, S., Kennerknecht, R.,\& Spiller, A. (2005). Die Zukunft des Großhandels in der BioWertschöpfungskette. Georg-August Universität Göttingen, Institute für Agrarökonomie, Diskussionspaper No. 13.

Gil, J. M., Gracia, A., \& Sánchez, M. (2000). Market segmentation and willingness to pay for organic products in Spain. The International Food and Agribusiness Management Review, 3(2), 207-226. Retrieved February 3, 2008 from www.sciencedirect.com/science/article/B6W4N-44PX45R5/2/b9bda7719d338ccfe778177b4eb54ae6.

Gómez Cruz, M. A., Schwentesius Rinderman, R., Gómez Tovar, L., Ortigoza Rufino, J., \& Nelson, E. (2009). Latin America Country Report Mexico. In Willer, H., \& Yussefi, M. The World of Organic Agriculture - Statistics and Emerging Trends 2009, (216-218): International Federation of Organic Agriculture Movements IFOAM, DE-Bonn and Research Institute of Organic Agriculture, FiBL, CH-Frick.

Gómez Cruz, M. A., Schwentesius Rinderman, R., Meraz Alvarado, M.A., Lobato Garcia, A., \& Gómez Tovar. L. (2005a). Agricultura, Apicultura y Ganaderías Orgánicas de México Situación, Retos y Tendencias. CIESTAAM-UACh, Chapingo, Estado de México. 
Gómez Tovar, L., Martin, L., Gómez Cruz, M. A., \& Mutersbaugh, T. (2005b). Certified organic agriculture in Mexico: Market connections and certification practices in large and small producers. Journal of Rural Studies, 21(4), 461-474. Retrieved March 23, 2008 from http://www.sciencedirect.com/science/article/B6VD9-4HK669R1/2/3f3eddc03e17c652ac0c0a7c88a77c29.

Götz, O., \& Gobbers, K. (2004). “Analyse von Strukturgleichungsmodellen mit Hilfe der Partial-Least-Squares-(PLS)-Methode,” Die Betriebswirtschaft, 64(6), 714-738.

Granovetter, M. (1985). Economic Action and Social Structure: The Problem of Embeddedness. The American Journal of Sociology, 91(3), 481-510. Retrieved September 01, 2007 from http://www.jstor.org/stable/2780199.

Greenpeace (2005). Guia roja y verde de alimentos transgénicos . Retrieved April 28, 2010 from http://www.greenpeace.org/raw/content/mexico/prensa/reports/copy-of-gu-a-roja-yverde-de-a.pdf.

Grunert, K. G., Harmsen, H, Hartvig Larsen, H., SØrensen E., \& Bisp, S. In Wierenga, B., Tilburg, A. v., \& Grunert, K. G. (1997). Agricultural marketing and consumer behavior in a changing world. Boston (ed.) Kluwer Acad. Publ.

Grunert, S. C., \& Juhl, H. J. (1995). Values, environmental attitudes, and buying of organic foods. Journal of Economic Psychology, 16(1), 39-62. Retrieved January 14, 2007 from http://www.sciencedirect.com/science/article/B6V8H-3Y5FPW5-

13/2/1dfefd2d4cbe0c4a040c4739713ca687.

Grunert, K. G., BrunsÃ,, K., \& Bisp, S. R. (1993). Food-related life style: Development of a cross-culturally valid instrument for market surveillance. Retrieved May 8, 2010 from http://research.asb.dk/fbspretrieve/88/wp12.pdf.

Grunert, S. C., \& Scherhorn, G. (1990). Consumer values in West Germany underlying dimensions and cross-cultural comparison with North America. Journal of Business Research, 20(2), 97-107. Retrived May 15, 2009 from http://www.sciencedirect.com/science/article/B6V7S-460P4YD28/2/5e873f6d7570b39f6f888b61c96d1fbf. 
Guthman, J. (2000). Raising organic: An agro-ecological assessment of grower practices in California. Agriculture and Human Values, 17(3), 257-266. Retrieved August 10, 2007 from http:/www.springerlink.com/content/w576248x30542340/.

Guthman, J. (2004). The Trouble with 'Organic Lite' in California: a Rejoinder to the 'Conventionalisation' Debate. Sociologia Ruralis, 44, 301-316. Retrieved August 10, 2008 from http://www.ingentaconnect.com/content/bpl/soru/2004/00000044/00000003/art00004.

Haccius, M. \& Lünzer, I. (2000). Organic agriculture in Germany. 109-128. Retrieved 10 August 2007 from http://www.organic-europe.net.

Haccius, M., (2005). Does "the" organic consumer exist? Proceedings of the 1st scientific FQH conference, Frick 2005. Retrieved September 2, 2007 from www.organicfqhresearch.org.

Haccius, M. (2009). Konsequent kundenorientiert und nachhaltig Bio-Lebensmittel vermarkten. Bad Düben, 12th November, 2009. Retrieved April 20, 2010 from http://www.bund-sachsen.de/doc/agrarwende/jubilaeum/04.pdf.

Hall, A., \& Mogyorody, V. (2001). Organic Farmers in Ontario: An Examination of the Conventionalization Argument. Sociologia Ruralis, 41(4), 399-322. Retrieved April 28, 2008 from http://www3.interscience.wiley.com/journal/119019625/abstract.

Hamm. U., Gronefeld, F., \& Halpin, D. (2002). Analysis of the European market for organic food. Organic Marketing Initiatives and Rural Development (OMIaRD) Vol. 1. University of Wales Aberystwyth. UK.

Hamm, U. \& Rippin, M. (2007). Umsätze und Umsatzanteile für Öko-Lebensmittel in Deutschland nach Absatzebenen. Biopress, 51(7), 63.

Hansmann, K.-W., \& Ringle, C. M. (2004). Smart PLS user's guide. Hamburg. University Hamburg.

Harkness, J. A., van de Vijver, F. J. R., \& Mohler, P. PH. (2003). Cross-cultural survey methods. Wiley United States of America. 
Harper, G. \& Makautoni, A. (2002). Consumer perception of organic food production and farm animal welfare. British Food Journal, 104(3), 287-299. Retrieved January 15, 2008 from www.emeraldinsight.com/10.1108/00070700210425723.

Hawkins, D. I., Best, R. J., Coney, K. A., \& Koch, E. C. (2004). Consumer behavior. Building marketing strategy (9 ed.). Boston, Mass. [ed.]: McGraw-Hill/Irwin.

Hofstede, G. (2001). Culture's consequences. Comparing values, behaviors, institutions and organizations across nations (2 ed.). Thousand Oaks, Calif. [ed.]: Sage Publ.

Honkanen, P., Verplanken, B., \& Olsen, S. O. (2006). Ethical values and motives driving organic food choice. Journal of Consumer Behaviour, 5(5), 420-430. Retrieved January 26, 2008 from http://www3.interscience.wiley.com/journal/113390868/abstract.

Homburg, C., \& Giering, A. (1996). Konzeptualisierung und Operationalisierung komplexer Konstrukte - Ein Leitfaden für die Marketingforschung. Marketing - Zeitschrift für Forschung und Praxis, 18(1), 5-24.

Hoyer, W. D., \& MacInnis, D. J. (2007). Consumer behavior (4 ed.). Boston, Mass. [Ed.]: Houghton Mifflin.

Hughner, R. S., McDonagh, P., Prothero, A., II, C. J. S., \& Stanton, J. (2007). Who are organic food consumers? A compilation and review of why people purchase organic food. Journal of Consumer Behaviour, 6(2-3), 94-110. Retrieved August 10, 2008 from http://www3.interscience.wiley.com/journal/114265522/abstract.

Jonas, A., \& Roosen, J. (2005). Private Labels for premium products the example of organic food. International Journal of Retailing and Distribution Management, 33(8), 636-653. Retrieved April 25, 2010 from www.emeraldinsight.com/10.1108/09590550510608412.

Stuart Mill, J. (1843) A System of Logic, Ratiocinative and Inductive, Being a Connected View of the Principles of Evidence, and the Methods of Scientific Investigation.

Johnston, J., \& Baker, L. (2005). Eating Outside the Box: FoodShare's Good Food Box and the Challenge of Scale. Agriculture and Human Values, 22, 313-325. Retrived May 19, 2009 from http://www.ingentaconnect.com/content/klu/ahum/2005/00000022/00000003/00006048. 
Kirwan, J. (2006). The interpersonal world of direct marketing: Examining conventions of quality at UK farmers' markets. Journal of Rural Studies, 22(3), 301-312. Retrieved in March 13, 2007 from http://www.sciencedirect.com/science/article/B6VD9-4HCDJR41/2/741c37e0166d6fb99895a9c425b7b873.

Kinsella, K., \& He, W. (2009). An aging world 2008: International population reports. Issued June 2009. US Census Bureau. Retrieved September, 2009 from http://www.census.gov/prod/2009pubs/p95-09-1.pdf.

Kloppenburg, J., Hendrickson, J., \& Stevenson, G. W. (1996). Coming into the foodshed. Agriculture and Human Values, 13(3), 33-42. Retrieved May 25, 2009 from http://www.springerlink.com/content/u1pn44q884603t70/.

KPMG \& EHI, (2006). Status quo und Perspektiven im deutschen Lebensmittel-einzelhandel 2006. KPMG. Germany. Retrieved June 272007 from www.kpmg.de/Themen/3605.htm.

Kratochvil, R. \& Leitner, H. (2005). The "Trap of conventionalisation": organic farming between vision and reality. In: XXI Congress of the ESRS. Keszthely. Hungary. Retrieved May $24 \quad 2007$ from http://www-dev.boku.ac.at/fileadmin/_PFBioLandwirtschaft/pubs/Sozokon/2005_Leitner_ESRS.pdf.

Kreuzer, K. (1996). Bio-Vermarktung Vermarktungswege für Lebensmittel aus ökologischer Erzeugung. Darmstadt. Germany.

Kotler, P. \& Zaltman, G. (1971). Social Marketing: An Approach to Planned Social Change. The Journal of Marketing, 35(3), 3-12. Retrieved May 20, 2010 from http://www.jstor.org/stable/1249783.

Lernoud, A. P., \& Piovano, M. (2006). Latin America Country reports. In Willer, H. \& Yussefi, M. (Eds.) The world of organic agriculture statistics and emerging trends 2006. IFOAM \& FiBL. Germany-Switzerland.

Lindeman, M., \& Väänänen, M. (2000). Measurement of ethical food choice motives. Appetite, 34(1), 55-59. Retrieved February 22, 2008 from http://www.sciencedirect.com/science/article/B6WB2-45FK7D32S/2/328dbaa39fe08450df5ae83825c32150. 
Lindeman, M., \& Verkasalo, M. (2005). Measuring Values with the Short Schwartz's Value Survey. Journal of personality assessment, 85(2), 170-178.

Lockie, S., Lyons, K., Lawrence, G., \& Grice, J. (2004). Choosing organics: a path analysis of factors underlying the selection of organic food among Australian consumers. Appetite, 43(2), 135-146. $\quad$ Retrieved $\quad$ September $\quad 12, \quad 2009$ from http://www.sciencedirect.com/science/article/B6WB2-4D99BRV1/2/829973b28dfbb9306ff0a94a37070f2f.

Lockie, S., Lyons, K., Lawrence, G., \& Mummery, K. (2002). Eating 'Green': Motivations behind organic food consumption in Australia. Sociologia Ruralis, 42(1), 23-40. Retrieved August 3, 2007 from http://www.lib.cau.edu.cn/zjy/a059.pdf.

Lockie, S.\& Halpin, D. (2005). The 'Conventionalisation' Thesis Reconsidered: Structural and Ideological Transformation of Australian Organic Agriculture. Sociologia Ruralis, 45 (4), $\begin{array}{lllll}\text { 1-24. } & \text { Retrieved } & \text { March, } & 15 & 2007\end{array}$ from http://www.ingentaconnect.com/content/bpl/soru/2005/00000045/00000004/art00002.

Lopez, R. E. (2005). Distribución de Niveles Socio-económicos en el México Urbano. Asociación Mexicana de Agencias de Investgación de Mercado (AMAI). Retrived August 12, 2008 from www.amai.org/niveles.php.

Magistris, T., \& Gracia, A. (2008). The decision to buy organic food products in Southern Italy. British Food Journal, 110(9), 929-947. Retrieved November 14, 2008 from www.emeraldinsight.com/10.1108/00070700810900620.

Magnusson, M. K., Arvola, A., Hursti, U.-K. K., Åberg, L., \& Sjödén, P.-O. (2003). Choice of organic foods is related to perceived consequences for human health and to environmentally friendly behaviour. Appetite, 40(2), 109-117. Retrieved June 13, 2007 from linkinghub.elsevier.com/retrieve/pii/S0195666303000023.

Michelsen, J. (1999). The European market for organic products. Growth and development. Stuttgart: Inst. für Landwirtschaftliche Betriebslehre. Retrieved July 18, 2007 from http://orgprints.org/8486/. 
Mines, R., \& Nichols, S. (2005). The Mexican Mercado Paisano. A framework to study its development potential in migrant-dependent communities. DRAFT. Retrieved February 10, 2010 from http://geocommunities.org/metaPage/lib/Merc-Paisano-Paper-12-05.pdf.

Mills, M., van de Bunt, G. G., \& de Bruijn, J. (2006). Comparative research persistent problems and promising solutions. International Sociology 21(5), 619-631. Retrived 11, September, 2009 from http://iss.sagepub.com/cgi/content/abstract/21/5/619.

Mooij, M. K. d. (2004). Consumer behavior and culture. Consequences for global marketing and advertising. Thousand Oaks, Calif. [Ed.]: SAGE Publ.

Mowen, J. C. \& M. Minor, (2001). Consumer Behavior: A Framework, Prentice Hall, Inc. U.S.A.

Nelson, E., Gómez Tovar, L., Schwentesius Rindermann, R., \& Gómez Cruz, M. A. (2009). Participatory organic certification in Mexico: an alternative approach to maintaining the integrity of the organic label. Agriculture and Human Values (2010), 27, 227-237. Retrieved July 1, 2009 from http://www.springerlink.com/content/q185516276x46211/.

Nguyen, T. D., Nguyen, T. T. M., \& Barrett, N. J. (2008). Consumer ethnocentrism, cultural sensitivity, and intention to purchase local products-evidence from Vietnam. Journal of Consumer Behaviour, 7(1), 88-100. Retrieved August 23, 2009 from http://www3.interscience.wiley.com/cgi-bin/fulltext/117908133/PDFSTART.

Niessen, J. And Hamm, U. (2008). Identifying the gap between stated and actual buying behaviour on organic products based on consumer panel data. 16th IFOAM Organic World Congress, Modena, Italy, June 16-20, 2008. Retrieved April, 122010 from http://orgprints.org/11998.

Organic Trade Association. (2004). OTA Market overview Mexican Organic Market. Retrieved August 52007 from www.ota.com/pics/documents/mexicanmarketoverview.pdf.

O'Shaughnessy, J. (1992). Explaining buyer behavior. Central concepts and philosophy of science issues. New York [Ed.]: Oxford Univ. Press. 
Padel, S., \& Foster, C. (2005). Exploring the gap between attitudes and behaviour Understanding why consumers buy or do not buy organic food. British Food Journal, 107(8), 606-625. Retrieved $\quad$ September $20, \quad 2008$ from www.emeraldinsight.com/10.1108/00070700510611002.

Padel, S., \& Midmore, P. (2005). The development of the European market for organic products: insights from a Delphi study. British Food Journal, 107 (8), 626-647. Retrieved $\begin{array}{llll}\text { September } & 20, & 2008 & \text { from }\end{array}$ www.emeraldinsight.com/Insight/viewContentItem.do? contentType=Article\&contentId $=1514$ 927.

Padilla, Bernal. L. E., \& Perez Veyna, O. (2006). Tipificación del consumidor potencial de frutas y hortalizas organicas en el Mercado local y regional. Problemas del Desarrollo, 37(146), 169-87. $\quad$ Retrieved February 20, 2008 from http://www.journals.unam.mx/index.php/pde/article/view/7624.

Pollard, T. M., Steptoe, A., \& Wardle, J. (1998). Motives underlying healthy eating: using the food choice questionnaire to explain variation in dietary intake. Journal of Biosocial Science, 30(02), 165-179. $\quad$ Retrieved October 29, 2008 from http://journals.cambridge.org/action/displayAbstract?fromPage=online\&aid=10113\&fulltextT ype $=$ RA\& fileId $=$ S0021932098001655.

Prescott, J., Young, O., O'Neill, L., Yau, N. J. N., \& Stevens, R. (2002). Motives for food choice: a comparison of consumers from Japan, Taiwan, Malaysia and New Zealand. Food Quality and Preference, 13(7-8), 489-495. Retrieved April 5, 2007 from http://www.sciencedirect.com/science/article/B6T6T-468D8FX6/2/62837005d8e3195ee868b6773268692c.

Przeworski, A., \& Teune, H. (1970). The Logic of Comparative Social Inquiry, New York, London, Toronto, Sidney: Wiley-Interscience.

Radman, M. (2005). Consumer consumption and perception of organic products in Croatia. British Food Journal, 107(4), 263-273. Retrieved April 18, 2008 from www.emeraldinsight.com/10.1108/00070700510589530. 
Reid, M., Grunert, K., Li, E., \& Bruwer, J. (2000). Food-Related Life Styles in Australia: application of the MAPP Food-Related Life Styles instruments in an Australian Context. ANZMAC 2000 Visionary Marketing for the 21st Century: Facing the Challenge. Retrieved $\begin{array}{llll}\text { March } & 12, & 2009 & \text { from }\end{array}$ smib.vuw.ac.nz:8081/www/ANZMAC2000/CDsite/papers/qr/Reid2.PDF.

Reynolds T. \& Gutman, J. (1988). Laddering theory, method, analysis, and interpretation. Journal of Advertising Research, 28 (February/March), 11-31.

Richter, T., \& Hempfling, G. (2003). Supermarket Study 2002 Organic products in European supermarkets. FiBL. Frick. Switzerland.

Roddy, G., Cowan, C., \& Hutchinson, G. (1994). Organic food: a description of the Irish market. British Food Journal, 96(4), 3-10. Retrieved February 3, 2007 from www.emeraldinsight.com/10.1108/00070709410060998.

Roddy, G., Cowan, C. A., \& Hutchinson, G. (1996). Consumer attitudes and behaviour to organic foods in Ireland. Journal of International Consumer Marketing, 9(2), 41 - 63.

Roininen, K. (2001). Evaluation of food choice behavior: development and validation of health and taste attitude scales. University of Helsinki. Retrieved September 20, 2008 from ethesis.helsinki.fi/julkaisut/maa/elint/vk/roininen/evaluati.pdf.

Roitner-Schobesberger, B., Darnhofer, I., Somsook, S., \& Vogl, C. R. (2008). Consumer perceptions of organic foods in Bangkok, Thailand. Food policy, 33(2), 112-121. Retrieved September 2008 from http://www.sciencedirect.com/science/article/B6VCB-4R46FFX1/2/051876924d0041411f5cd23c1aea3a2e.

Ross, C. E., \& Mirowsky, J. (1984). Socially-Desirable Response and Acquiescence in a Cross-Cultural Survey of Mental Health. Journal of Health and Social Behavior, 25(2), 189197. Retrieved April 2, 2009 from http://www.jstor.org/stable/2136668.

Ross, C. E., Mirowsky, J., \& Cockerham, W. C. (1983). Social class, Mexican culture, and fatalism: Their effects on psychological distress. American Journal of Community Psychology, 11(4), 383-399. Retrieved April 28, 2009 from http://www.springerlink.com/content/t81667550q745713/. 
Scheibehenne, B., Miesler, L., \& Todd, P. M. (2007). Fast and frugal food choices: Uncovering individual decision heuristics. Appetite, 49(3), 578-589. Retrieved May 9, 2008 from http://www.sciencedirect.com/science/article/B6WB2-4NDDKVF2/2/0b84ea7c28ab386c7fe78495ca2b624a.

Schiffman, L. G., \& Kanuk, L. L. (2004). Consumer behavior (8 ed). Upper Saddle River, NJ [Ed.]: Pearson Education International.

Schiffman, L. G., Kanuk, L. L., \& Hansen, H. v. (2008). Consumer behaviour. A European outlook. Harlow (Ed.) Financial Times Prentice Hall.

Schifferstein, H. N. J., \& Oude Ophuis, P. A. M. (1998). Health-related determinants of organic food consumption in The Netherlands. Food Quality and Preference, 9(3), 119-133. Retrieved February 2, 2008 from http://www.sciencedirect.com/science/article/B6T6T3T2G1S4-C/2/e2ca52f88af493265044820ab7eb1c6f.

Schultz, I., Stiess, I., \& Birzle-Harder, B. (2003). New target groups in the organic food market (Bio-Pro). ISOE. Germany. Retrieved March 18, 2008 from www.isoe.de/english/projects/biopro.htm.

Schulze, H., Albermeier, F., Gawron, J.C., Spiller, A. \& Theuvsen, L. (2008). Heterogeneity in the Evaluation of Quality Assurance Systems: The International Food Standard (IFS) in European Agribusiness. International Food and Agribusiness Management Review 11(3). 99139. Retrieved June 6, 2010 from http://ageconsearch.umn.edu/bitstream/53727/2/20081010_Formatted.pdf.

Schwartz, S.H. (1992). 'Universals in the content and structure of values: Theoretical advance and empirical tests in 20 countries'. In: M. Zanna (Ed.). Advances in Experimental Social Psychology, 25, 1-65. San Diego, CA: Academic Press.

Schwartz, S.H. (2006). A Theory of Cultural Value Orientations: Explication and Applications. Comparative Sociology, 5, 137-182. Retrieved November 10, 2008 from http://www.ingentaconnect.com/content/brill/comps/2006/00000005/F0020002/art00003. 
Schwentesius, Rinderman R. \& Gómez Cruz, M.A. (2006). "Supermercados y pequeños productores hortofrutícolas en México". Comercio Exterior, 56(3), 205-218. Retrieved April 5, 2007 from http://revistas.bancomext.gob.mx/rce/magazines/89/3/Gomez-Schventesius.pdf.

Seyfang, G. (2005). Local Organic Food: The social implications of sustainable consumption. Centre for Social and Economic Research on the Global Environment (CSERGE).University of East Anglia. Norwich. UK. Retrieved March 13, 2007 from www.uea.ac.uk/env/cserge/pub/wp/edm/edm_2004_09.pdf.

Sirieix, L., Abreu, S., Watanabe, M.A., \& Kledal P. (2007). Comparing organic urban consumers in developing countries: First results in Brazil and France. Working Paper presented to the AIEA2 and SOBER International Conference, Londriwna, Parana, Brazil 2227 July 2007. Retrieved April 14, 2008 from http://www.montpellier.inra.fr/moisa/bartoli/download/moisa2007_pdf/WP_4-2007.pdf.

Sirieix, L., \& Schaer, B. (2000). A cross-cultural research on consumer's attitudes and behaviors towards organic and local foods. Retrieved March 25, 2008 from http://marketing.byu.edu/htmlpages/ccrs/proceedings99/sirieixschaer.htm.

Solomon, M. R., Bamossy, G. J., \& Askegaard, S. R. (2000). Consumer behaviour. A European perspective. Harlow (Ed.) Prentice Hall Europe.

Solomon, M. R. (2006). Consumer behavior. Buying, having, and being (7 ed.). Upper Saddle River, NJ: Pearson Prentice-Hall.

Spiller, A. (2006). Zielgruppen im Markt für Bio-Lebensmittel: Ein Forschungsüberblick. Diskussionsbeitrag 0608. Georg-August Universität Göttingen.

Squires, L., Juric, B., \& Cornwell, B. T. B. (2001). Level of market development and intensity of organic food consumption: Cross cultural study of Denmark and New Zealand customers. Journal of Consumer Marketing, 18(5), 392-409. Retrieved February 21, 2007 from www.emeraldinsight.com/10.1108/07363760110398754.

Stagl, S. (2002). Local Organic Food Markets: Potentials and Limitations for Contributing to Sustainable Development. Empirica, 29(2), 145-162. Retrieved June 5, 2009 from: http://www.springerlink.com/content/yctc46v63uy9vxvd/. 
Stockebrand, N. \& Spiller, A. (2007). Regional marketing for organic retailers: Status quo and capabilities, in: Proceedings of the 1st IFOAM International Conference on Marketing of Organic and Regional Values, 26.-28. August 2007, Schwäbisch Hall, Germany.

Steptoe, A., Pollard, T. M., \& Wardle, J. (1995). Development of a Measure of the Motives Underlying the Selection of Food: the Food Choice Questionnaire. Appetite, 25(3), 267-284. Retrieved February 22, 2008 from http://www.sciencedirect.com/science/article/B6WB245R7GG5-H/2/8201a3f43fff3f530458eef823fcc0bf.

Tarkiainen, A., \& Sundqvist, S. (2005). Subjective norms, attitudes, and intentions of finish consumers in buying organic food. British Food Journal, 107(11), 808-822. Retrieved July 12, 2007 from www.emeraldinsight.com/10.1108/00070700510629760.

USDA (2002). Mexico Proves a Resilient, Robust Market for U.S. Exports. AgExporter, 4-7. Retrieved March 12, 2007 from www.fas.usda.gov/info/agexporter/2002/Jan/page4-7.pdf.

Von Alvensleben, R. (1998). "Ecological aspects of food demand: the case of organic food in Germany", AIR-CAT 4th Plenary Meeting: Health, Ecological and Safety Aspects in Food Choice, 4(1), 68-79. Retrieved January 10, 2010 from www.unikiel.de/agrarmarketing/Lehrstuhl/econ.pdf.

Wier, M., O'Doherty Jensen, K., Andersen, L. M., \& Millock, K. (2008). The character of demand in mature organic food markets: Great Britain and Denmark compared. Food policy, 33(5), 406-421. $\quad$ Retrieved January $9, \quad 2009$ from http://www.sciencedirect.com/science/article/B6VCB-4RV7H121/2/df9a36bd6b8a73619023c8ba21b27ee5.

Wier, M. \& Calverley, C. (2002). Market potential for organic food in Europe. British Food Journal, 104(1), 45-62. Retrieved February 10, 2008 from www.emeraldinsight.com/10.1108/00070700210418749.

Willer, H., \& Yussefi, M. (2009). The World of Organic Agriculture - Statistics and Emerging Trends 2009: International Federation of Organic Agriculture Movements IFOAM, DE-Bonn and Research Institute of Organic Agriculture, FiBL, CH-Frick. 
Willer, H., \& Yussefi, M. (2007). The World of Organic Agriculture - Statistics and Emerging Trends 2007: International Federation of Organic Agriculture Movements IFOAM, DE-Bonn and Research Institute of Organic Agriculture, FiBL, CH-Frick. Retrieved April 20, 2008 from http://www.orgprints.org/10506.

Willer, H. \& Yussefi, M. (2006). The world of organic agriculture statistics and emerging trends 2006. IFOAM \& FiBL. Germany-Switzerland.

Willer, H. \& Yussefi, M. (2004). The world of organic agriculture statistics and emerging trends 2004. IFOAM \& FiBL. Germany-Switzerland. Retrieved April 20, 2008 from http://aaoch.cl/files/world-of-organic-agriculture-2008.pdf.

Willer, H. \& Yussefi, M. (2002). The world of organic agriculture 2002 statistics and future prospects. IFOAM \& FiBL. Germany-Switzerland. Retrieved from July 30, 2007. http://www.soel.de/inhalte/publikationen/s_74_04.pdf

Willer, H. \& Yussefi, M. (2001). The world of organic agriculture 2001 statistics and future prospects. IFOAM \& FiBL. Germany-Switzerland. Retrieved from July 28, 2007. http://www.soel.de/inhalte/publikationen/s_74_03.pdf

Willer, H. \& Yussefi, M. (2000). The world of organic agriculture statistics and future prospects. IFOAM \& FiBL. Germany-Switzerland. Retrieved from July 25, 2007. http://www.soel.de/inhalte/publikationen/s_74_02.pdf

Winter, M. (2003). Embeddedness, the new food economy and defensive localism. Journal of Rural Studies. 19 (1) 23-32. Retrieved August 17, 2007 from http://www.sciencedirect.com/science/article/B6VD9-47CR73B-

2/2/d1bcede1181e8ce7dd02e6172504a0e1.

Wippermann, K., \& Wipperman C. (2006). Bio-Käufer in den Sinus Milieu. Bio verlag und Sinus Sociovision. Germany.

Worner, F., \& Meier-Ploeger, A. (1999). What the consumer says. Ecology and farming, 20(January-April), 14-15.

Wright, R. (2009). Consumer behaviour. Thompson, (1 ed.) U.K. 
Zanoli, R., \& Naspetti, S. (2002). Consumer motivations in the purchase of organic food. A Means-end approach. British Food Journal, 104(8), 643-653. Retrieved October 10, 2007 from www.emeraldinsight.com/10.1108/00070700210425930.

\section{Internet resources:}

http://www.alnatura.de retrieved August 17, 2007.

http://www.biobay.de retrieved May 30, 2010.

http://www.bio-siegel.de/fileadmin/user_upload/Dokumente/Broschueren/Bio-

Siegel_at_a_glance_01.pdf retrieved 11 April, 2010.

http://www.europeansocialsurvey.org/ retrieved 15 May, 2009.

http://www.foodnavigator.com/content/ retrieved 12 August, 2009.

http://www.geert-hofstede.com/hofstede_mexico.shtml retrieved 20 December, 2009.

http://www.harnish.com retrieved 18 April, 2010.

http://www.ifoam.org retrieved May 30, 2010.

http://www.naturkost.de retrieved 11 April, 2010.

http://www.ecoworld.de retrieved May 30, 2010.

http://www.oekolandbau.de retrieved August 19, 2007.

http://www.statisticssolutions.com retrieved July 20, 2010.

http://www.terrae-it.eu retrieved May 30, 2010.

http://www.utopia.de/ retrieved May 30, 2010.

http://www.vinculado.org retrieved June 5, 2007.

http://www.zmp.de retrieved August 12, 2007. 
Appendix 


\section{Appendix 1: First pre-testing survey in Mexico (Spanish version)}

Muchas personas hablan sobre los productos orgánicos, pero el significado es (en muchas ocasiones) diferente, ¿Cómo describiría a los productos orgánicos. Por favor escriba tres aspectos?

1.

2.

3.

1. Ahora, queremos saber si ¿alguna vez ha comprado productos orgánicos?

$\mathrm{Si}$

2. Por favor diga, ¿con que frecuencia compra productos orgánicos por semana en las siguientes tiendas?(Marque solo un recuadro por línea)

\begin{tabular}{|l|l|l|l|l|l|}
\hline & $\begin{array}{c}\text { Menos de } \\
\text { una vez a la } \\
\text { semana }\end{array}$ & $\begin{array}{c}\text { Una vez a la } \\
\text { semana }\end{array}$ & $\begin{array}{c}\text { Dos veces a } \\
\text { la semana }\end{array}$ & $\begin{array}{c}\text { Tres veces } \\
\text { a la semana }\end{array}$ & $\begin{array}{c}\text { Mas de tres } \\
\text { veces a la } \\
\text { semana }\end{array}$ \\
\hline $\begin{array}{l}\text { Hipermercados (ej. Wal-Mart } \\
\text { Supercenter, Comercial } \\
\text { Mexicana) }\end{array}$ & & & & & \\
\hline $\begin{array}{l}\text { Supermercados (ej. Sumesa, } \\
\text { Superama) }\end{array}$ & & & & & \\
\hline $\begin{array}{l}\text { Tiendas de autoservicio (ej. } \\
\text { Oxxo) }\end{array}$ & & & & & \\
\hline $\begin{array}{l}\text { Members clubs (ej. Costo, } \\
\text { Sams Club) }\end{array}$ & & & & & \\
\hline Mercados tradicionales & & & & & \\
\hline Mercados semanales & & & & & \\
\hline Fruterías & & & & & \\
\hline Tiendas especializadas & & & & & \\
\hline Directamente del productor & & & & & \\
\hline
\end{tabular}


En esta sección queremos saber porque usted compra en esta tienda.

3. Otros compradores mencionaron las siguientes razones por las que compran en esta tienda. Que tan de acuerdo estaría con ellos. (Por favor marque un recuadro por línea).

Yo compro en esta tienda por que...

\begin{tabular}{|l|l|l|l|l|l|}
\hline & $\begin{array}{l}\text { Totalmente } \\
\text { de acuerdo }\end{array}$ & De acuerdo & $\begin{array}{l}\text { Ni acuerdo ni } \\
\text { desacuerdo }\end{array}$ & Desacuerdo & $\begin{array}{l}\text { Totamente } \\
\text { desacuerdo }\end{array}$ \\
\hline $\begin{array}{l}{[1]^{*} \text { Los trabajadores me brindan }} \\
\text { información sobre los productos } \\
\text { orgánicos. }\end{array}$ & & & & & \\
\hline $\begin{array}{l}\text { La alta calidad de los productos } \\
\text { orgánicos. }\end{array}$ & & & & & \\
\hline $\begin{array}{l}\text { Siempre hay promociones especiales } \\
\text { para productos orgánicos. }\end{array}$ & & & & & \\
\hline $\begin{array}{l}\text { Hay información y anuncios acerca } \\
\text { del productor de los productos } \\
\text { orgánicos que son vendidos aquí. }\end{array}$ & & & & & \\
\hline $\begin{array}{l}\text { Encuentro un surtido de productos } \\
\text { que no lo encuentro fácilmente en } \\
\text { otras tiendas. }\end{array}$ & & & & & \\
\hline Por la frescura de la comida orgánica. & & & & & \\
\hline Esta cerca de mi casa. & & & & & \\
\hline $\begin{array}{l}\text { Encuentro muy buenas promociones } \\
\text { en productos orgánicos. }\end{array}$ & & & & & \\
\hline $\begin{array}{l}\text { Los precios son realmente buenos en } \\
\text { comparación con otras tiendas. }\end{array}$ & & & & & \\
\hline $\begin{array}{l}\text { Comprar aquí es una garantía de } \\
\text { calidad. }\end{array}$ & & & & & \\
\hline $\begin{array}{l}\text { Es famoso por la frescura de los } \\
\text { productos orgánicos en todas las } \\
\text { temporadas. }\end{array}$ & & & & & \\
\hline $\begin{array}{l}\text { Tiene una gran variedad de productos } \\
\text { orgánicos. }\end{array}$ & & & & & \\
\hline 1$]$ * Esta pregunta será solo para supermercados. & & & & \\
\hline
\end{tabular}


4. Ahora queremos saber su evaluación a esta tienda en los siguientes aspectos (Por favor marque un recuadro por línea).

\begin{tabular}{|l|l|l|l|l|l|}
\hline & $\begin{array}{c}\text { Muy } \\
\text { Bueno }\end{array}$ & Bueno & Medio & Mal & Muy mal \\
\hline $\begin{array}{l}\text { Ayuda del personal } \\
\text { Servicio }\end{array}$ & & & & & \\
\hline $\begin{array}{l}\text { Personal amigable } \\
\text { Calidad de los productos }\end{array}$ & & & & & \\
\hline $\begin{array}{l}\text { Apariencia de la tienda } \\
\text { Atmósfera }\end{array}$ & & & & & \\
\hline
\end{tabular}


En esta sección queremos saber porqué compra productos orgánicos

5. ¿Qué tanto esta de acuerdo con las siguientes oraciones? (Por favor marque un recuadro por línea)

Es importante para mí que la comida orgánica...

\begin{tabular}{|c|c|c|c|c|c|}
\hline & $\begin{array}{l}\text { Nada } \\
\text { importante }\end{array}$ & $\begin{array}{l}\text { Poco } \\
\text { importante }\end{array}$ & $\begin{array}{l}\text { Moderada- } \\
\text { mente } \\
\text { importante }\end{array}$ & Importante & $\begin{array}{l}\text { Muy } \\
\text { importante }\end{array}$ \\
\hline \multicolumn{6}{|l|}{$\begin{array}{l}\text { Haya sido producida de tal forma } \\
\text { que los animales no hayan sufrido } \\
\text { dolor. }\end{array}$} \\
\hline \multicolumn{6}{|l|}{ No sea cara. } \\
\hline \multicolumn{6}{|l|}{$\begin{array}{l}\text { No se necesita mucho tiempo para } \\
\text { prepararla. }\end{array}$} \\
\hline \multicolumn{6}{|l|}{ Sea de confianza. } \\
\hline \multicolumn{6}{|l|}{$\begin{array}{l}\text { Sea fácil de conseguir en las tiendas y } \\
\text { supermercados. }\end{array}$} \\
\hline \multicolumn{6}{|l|}{$\begin{array}{l}\text { Haya sido producido de tal manera } \\
\text { que los derechos de los animales son } \\
\text { respetados. }\end{array}$} \\
\hline \multicolumn{6}{|l|}{ Me ayuda a controlar mi peso. } \\
\hline \multicolumn{6}{|l|}{ Vale mi dinero. } \\
\hline \multicolumn{6}{|l|}{ Se vea bien. } \\
\hline \multicolumn{6}{|l|}{$\begin{array}{l}\text { Sea recomendada por las personas } \\
\text { que conozco. }\end{array}$} \\
\hline \multicolumn{6}{|l|}{ Sea fácil de cocinar. } \\
\hline \multicolumn{6}{|l|}{$\begin{array}{l}\text { Haya sido producido de tal manera } \\
\text { que el equilibrio de la naturaleza no } \\
\text { haya sido alterado. }\end{array}$} \\
\hline \multicolumn{6}{|l|}{ Sea buena para mi. } \\
\hline \multicolumn{6}{|l|}{$\begin{array}{l}\text { No esta relacionada con la } \\
\text { contaminación. }\end{array}$} \\
\hline \multicolumn{6}{|l|}{ Haya sido producida localmente. } \\
\hline \multicolumn{6}{|l|}{ No contiene aditivos. } \\
\hline \multicolumn{6}{|l|}{$\begin{array}{l}\text { Proviene de un país donde los } \\
\text { derechos humanos son respetados. }\end{array}$} \\
\hline \multicolumn{6}{|l|}{$\begin{array}{l}\text { Puede comprarse cerca de donde } \\
\text { vivo o trabajo. }\end{array}$} \\
\hline \multicolumn{6}{|l|}{ Sea baja en calorías. } \\
\hline \multicolumn{6}{|l|}{$\begin{array}{l}\text { No sea solo un producto, es una } \\
\text { forma de vida. }\end{array}$} \\
\hline \multicolumn{6}{|l|}{ Contiene ingredientes naturales. } \\
\hline \multicolumn{6}{|l|}{$\begin{array}{l}\text { Haya sido producida de manera } \\
\text { amigable con el medio ambiente. }\end{array}$} \\
\hline \\
\hline \multicolumn{6}{|l|}{ Tenga buen sabor. } \\
\hline \multicolumn{6}{|l|}{$\begin{array}{l}\text { Haya sido producida de tal manera } \\
\text { que no contraste con mis valores } \\
\text { políticos. }\end{array}$} \\
\hline \multicolumn{6}{|l|}{$\begin{array}{l}\text { Este libre de organismos } \\
\text { modificados genéticamente. }\end{array}$} \\
\hline Me mantenga saludable. & & & & & \\
\hline Sea lo que usualmente como. & & & & & \\
\hline
\end{tabular}


6. ¿Qué tanto concuerda con las siguientes frases? (Por favor marque un recuadro por línea)

\begin{tabular}{|c|c|c|c|c|c|}
\hline & $\begin{array}{l}\text { Nada } \\
\text { importante }\end{array}$ & $\begin{array}{l}\text { Poco } \\
\text { Importante }\end{array}$ & $\begin{array}{c}\text { Ni importante } \\
\text { ni no } \\
\text { importante }\end{array}$ & Importante & $\begin{array}{l}\text { Totalmente } \\
\text { importante }\end{array}$ \\
\hline \multicolumn{6}{|l|}{$\begin{array}{l}\text { Disfrutar la vida (comida, pareja, } \\
\text { descanso, tiempo libre). }\end{array}$} \\
\hline \multicolumn{6}{|l|}{$\begin{array}{l}\text { Una vida diversa (llena de retos, } \\
\text { novedades y cambios). }\end{array}$} \\
\hline \multicolumn{6}{|l|}{$\begin{array}{l}\text { Sentido de pertenencia (sentir que } \\
\text { otrso se preocupan por mi). }\end{array}$} \\
\hline \multicolumn{6}{|l|}{$\begin{array}{l}\text { Protección al medio ambiente } \\
\text { (preservación de la naturaleza). }\end{array}$} \\
\hline \multicolumn{6}{|l|}{$\begin{array}{l}\text { Ayuda (cooperar para el bienestar de } \\
\text { los demás). }\end{array}$} \\
\hline \multicolumn{6}{|l|}{$\begin{array}{l}\text { Una vida emocionante (experiencias } \\
\text { estimulantes). }\end{array}$} \\
\hline \multicolumn{6}{|l|}{ Creatividad (originilidad, imaginación). } \\
\hline \multicolumn{6}{|l|}{ Placer (gratificación de los deseos). } \\
\hline \multicolumn{6}{|l|}{$\begin{array}{l}\text { Pensamiento amplio (tolerar las } \\
\text { diferencias de ideas y creencias). }\end{array}$} \\
\hline \multicolumn{6}{|l|}{ Honestidad (genuini, sincero). } \\
\hline $\begin{array}{l}\text { Saludable (no estar enfermo ni física ni } \\
\text { mentalmente). }\end{array}$ & & & & & \\
\hline
\end{tabular}

Finalmente, por favor proporcione nos algunos datos personales

7.¿Cuando nació? 19

8.¿Donde creció?

\begin{tabular}{|l|l|}
\hline Area rural & Area urbana \\
\hline & \\
\hline
\end{tabular}

9. ¿Donde vive ahora?

\begin{tabular}{|l|l|}
\hline Area rural & Area urbana \\
\hline & \\
\hline
\end{tabular}


10. ¿Nos podría decir cuanto gasta en comida a la semana? (Por favor marque un recuadro)

\begin{tabular}{|l|c|c|c|c|}
\hline Menos de 500 & $500-700$ & $701-900$ & $901-1200$ & Mas de 1 200 \\
\hline & & & & \\
\hline
\end{tabular}

11. ¿Nos podría decir aproximadamente cual es su ingreso total mensual en MX\$? (por favor marque un recuadro)

\begin{tabular}{|c|c|c|c|c|c|}
\hline $\begin{array}{c}\text { Menos de } \\
2700\end{array}$ & $\begin{array}{c}2801- \\
6800\end{array}$ & $\begin{array}{c}6801- \\
11600\end{array}$ & $\begin{array}{c}11601- \\
34999\end{array}$ & $\begin{array}{c}35000- \\
84499\end{array}$ & $\begin{array}{c}\text { Mas de } \\
85000\end{array}$ \\
\hline & & & & & \\
\hline
\end{tabular}

12. ¿Cual es su nivel de escolaridad máxima que haya terminado? (Por favor marque un recuadro)

\begin{tabular}{|l|l|l|l|l|l|l|l|}
\hline $\begin{array}{l}\text { Escuela } \\
\text { Primaria }\end{array}$ & Secundaria & $\begin{array}{l}\text { Secundaria } \\
\text { técnica }\end{array}$ & Preparatoria & Universidad & Maestría & Doctorado & Otro \\
\hline & & & & & & & \\
\hline
\end{tabular}

13. ¿Usted vive con? Yo vivo....

\begin{tabular}{|c|c|c|c|c|c|}
\hline Solo & $\begin{array}{c}\text { Con mi } \\
\text { pareja }\end{array}$ & $\begin{array}{c}\text { Con mi pareja e } \\
\text { hijos }\end{array}$ & $\begin{array}{c}\text { Padre/ Madre } \\
\text { soltero(a) con } \\
\text { hijos }\end{array}$ & $\begin{array}{c}\text { En un } \\
\text { departamento } \\
\text { (con amigos) }\end{array}$ & $\begin{array}{c}\text { Con mis } \\
\text { padres }\end{array}$ \\
\hline & & & & & \\
\hline
\end{tabular}

14. ¿Cuantas personas viven en su casa?

15. ¿Cuantos niños viven en su casa?

16. Género

\begin{tabular}{|l|l|}
\hline Mujer & Hombre \\
\hline & \\
\hline
\end{tabular}




\section{Appendix 2: First pre-testing survey in Mexico (English Version version)}

Motives to buy organic food products

A project of the University of Göttingen in Germany

Dear consumers, since the earliest 1990s the consumption of organic food products has increased in several countries. In this survey we want to know why and where consumers buy organic food products in Germany and Mexico, your opinion about some values and food buying behaviour.

The questionnaire will take around 20 minutes you will help us a lot if you take part in the survey all the information that you give as will be confidential and when you will finish you get a small present.

Thank you very much for your cooperation.

If you have questions, please feel free to contact us. 
1. Many people talk about organic products, but the meaning is often different. How would you describe organic products? Please give three aspects.

1.

2.

3.

2. Now, we want to know if you have ever bought organic products?

Yes

Not

3. Please say us, how often do you buy organic food products per week in the following stores? (check one box per line)

\begin{tabular}{|l|l|l|l|l|l|}
\hline & $\begin{array}{c}\text { Less than } \\
\text { once per } \\
\text { week }\end{array}$ & $\begin{array}{c}\text { Once per } \\
\text { week }\end{array}$ & $\begin{array}{c}\text { Two times } \\
\text { per week }\end{array}$ & $\begin{array}{c}\text { Three times } \\
\text { per week }\end{array}$ & $\begin{array}{c}\text { More than } \\
\text { three times } \\
\text { per week }\end{array}$ \\
\hline $\begin{array}{l}\text { Hypermarkets (i.e. Wal- } \\
\text { Mart Supercenter, } \\
\text { Comercial Mexicana) }\end{array}$ & & & & \\
\hline $\begin{array}{l}\text { Supermarket (i.e. } \\
\text { Sumesa, Superama) }\end{array}$ & & & & \\
\hline $\begin{array}{l}\text { Self-service stores (i.e. } \\
\text { Oxxo) } \\
\text { Members clubs (i.e. } \\
\text { Costo, Sams Club) }\end{array}$ & & & & & \\
\hline Traditional Markets & & & & & \\
\hline Weekly markets & & & & & \\
\hline Fruit shops & & & & & \\
\hline Specialized stores & & & & & \\
\hline Direct seller & & & & & \\
\hline
\end{tabular}


In this section we want to know why you buy in this store.

4. Below are some mentioned reason because other buyers buy in this store read these statements and say how you agree with them (please check a box per line).

I buy in this store because...

\begin{tabular}{|c|c|c|c|c|c|}
\hline & \multirow[t]{2}{*}{$\begin{array}{l}\text { Strongly } \\
\text { agree }\end{array}$} & \multirow[t]{2}{*}{ Agree } & \multirow{2}{*}{$\begin{array}{l}\text { Neither } \\
\text { disagree } \\
\text { nor agree }\end{array}$} & \multirow[t]{2}{*}{ Disagree } & \multirow[t]{2}{*}{$\begin{array}{l}\text { Strongly } \\
\text { disagree }\end{array}$} \\
\hline & & & & & \\
\hline $\begin{array}{l}{[1]^{*} \text { Employees can give m }} \\
\text { lot of information about } \\
\text { organic products }\end{array}$ & & & & & \\
\hline $\begin{array}{l}\text { The high quality of organic } \\
\text { products }\end{array}$ & & & & & \\
\hline $\begin{array}{l}\text { There are always special } \\
\text { promotions for organic } \\
\text { products }\end{array}$ & & & & & \\
\hline $\begin{array}{l}\text { There are information and } \\
\text { advice about who produce } \\
\text { the organic products whic } \\
\text { are sold here }\end{array}$ & & & & & \\
\hline $\begin{array}{l}\text { I find an assortment of } \\
\text { organic product that I not } \\
\text { easily find in other stores }\end{array}$ & & & & & \\
\hline $\begin{array}{l}\text { Of the freshness of organic } \\
\text { food }\end{array}$ & & & & & \\
\hline It is near to my home & & & & & \\
\hline $\begin{array}{l}\text { I find really good } \\
\text { promotions for organic } \\
\text { products }\end{array}$ & & & & & \\
\hline $\begin{array}{l}\text { The prices are really good } \\
\text { than in other stores }\end{array}$ & & & & & \\
\hline $\begin{array}{l}\text { To buy here is a guarantee } \\
\text { of quality }\end{array}$ & & & & & \\
\hline $\begin{array}{l}\text { It is famous for the } \\
\text { freshness of organic } \\
\text { products in all the seasons }\end{array}$ & & & & & \\
\hline $\begin{array}{l}\text { Has a big assortment of } \\
\text { organic products }\end{array}$ & & & & & \\
\hline
\end{tabular}


5. Now we want to know how you assess this store in the next aspects (please check one box per line).

\begin{tabular}{|l|l|l|l|l|l|}
\hline & Very good & Good & Medium & Bad & Very bad \\
\hline Helpful staff & & & & & \\
\hline Service & & & & \\
\hline Friendly staff & & & & \\
\hline $\begin{array}{l}\text { Quality of the } \\
\text { products }\end{array}$ & & & & \\
\hline $\begin{array}{l}\text { Attractiveness } \\
\text { of the store }\end{array}$ & & & & \\
\hline Atmosphere & & & & \\
\hline
\end{tabular}


In this section we want to know why you buy organic products

6. How do you agree with the next statements? (please check a box per line)

It is important to me that organic food...

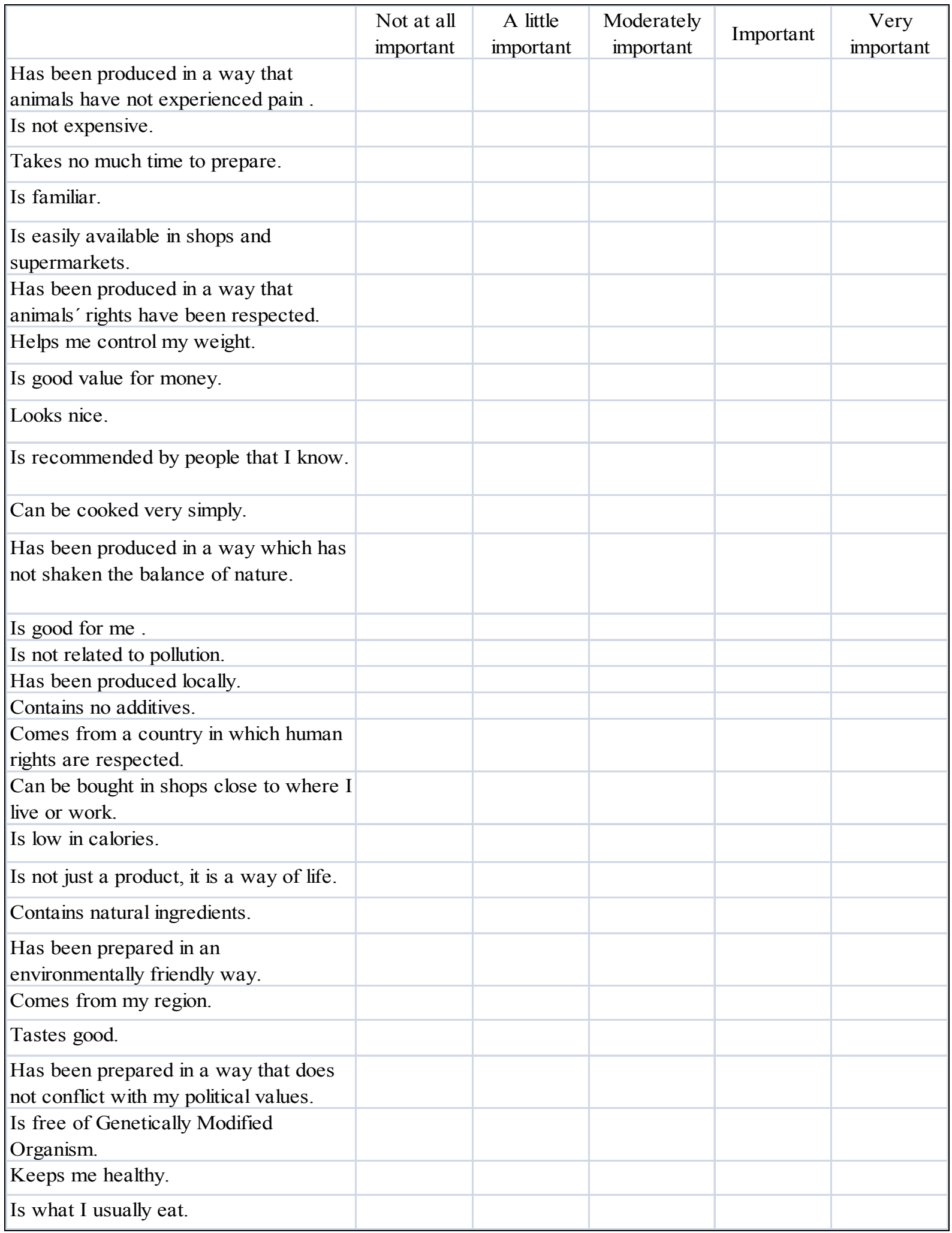


7. How do you agree with these statements? (please check a box per line)

\begin{tabular}{|c|c|c|c|c|c|}
\hline & $\begin{array}{l}\text { Not at all } \\
\text { important }\end{array}$ & $\begin{array}{c}\text { A little } \\
\text { important }\end{array}$ & $\begin{array}{l}\text { Moderately } \\
\text { important }\end{array}$ & Important & $\begin{array}{c}\text { Very } \\
\text { important }\end{array}$ \\
\hline \multicolumn{6}{|l|}{$\begin{array}{l}\text { Enjoying Life (Enjoying food, } \\
\text { sex, leisure). }\end{array}$} \\
\hline \multicolumn{6}{|l|}{$\begin{array}{l}\text { A varied life (filled with } \\
\text { challenges, novelty, and } \\
\text { changes). }\end{array}$} \\
\hline \multicolumn{6}{|l|}{$\begin{array}{l}\text { Sense of belonging (feeling } \\
\text { that others care about me). }\end{array}$} \\
\hline \multicolumn{6}{|l|}{$\begin{array}{l}\text { Freedom (of action and } \\
\text { thought). }\end{array}$} \\
\hline \multicolumn{6}{|l|}{$\begin{array}{l}\text { Protecting the environment } \\
\text { (preserving nature). }\end{array}$} \\
\hline \multicolumn{6}{|l|}{$\begin{array}{l}\text { Helpful (working for the } \\
\text { welfare of others). }\end{array}$} \\
\hline \multicolumn{6}{|l|}{$\begin{array}{l}\text { An exciting life (stimulating } \\
\text { experiences). }\end{array}$} \\
\hline \multicolumn{6}{|l|}{$\begin{array}{l}\text { Creativity (uniqueness and } \\
\text { imagination). }\end{array}$} \\
\hline \multicolumn{6}{|l|}{$\begin{array}{l}\text { Pleasure (gratification of } \\
\text { desires). }\end{array}$} \\
\hline \multicolumn{6}{|l|}{$\begin{array}{l}\text { Broad-Minded (tolerant of } \\
\text { different ideas and beliefs). }\end{array}$} \\
\hline \multicolumn{6}{|l|}{$\begin{array}{l}\text { Broad-Minded (tolerant of } \\
\text { different ideas and beliefs). }\end{array}$} \\
\hline $\begin{array}{l}\text { Broad-Minded (tolerant of } \\
\text { different ideas and beliefs). }\end{array}$ & & & & & \\
\hline
\end{tabular}

Finally, please give us some personal information:

Gender:

\begin{tabular}{|l|l|}
\hline Woman & Man \\
\hline & \\
\hline
\end{tabular}

8. When have you born? 19 
9. Where did you grow up?

\begin{tabular}{|l|l|}
\hline Farming Area & Urban area \\
\hline & \\
\hline
\end{tabular}

10. Where do you live now?

\begin{tabular}{|l|l|}
\hline Farming Area & Urban area \\
\hline & \\
\hline
\end{tabular}

11. Could you say how many do you spend per week in food? (Please check one box)

\begin{tabular}{|l|l|l|l|l|}
\hline $\begin{array}{l}\text { Less than } \\
500\end{array}$ & $500-700$ & $701-900$ & $901-1200$ & $\begin{array}{l}\text { More than } \\
1200\end{array}$ \\
\hline & & & & \\
\hline
\end{tabular}

12. Could you say what is approximately your total household net incomes monthly in MX\$? (Please check one box)

\begin{tabular}{|l|l|l|l|l|l|}
\hline $\begin{array}{l}\text { Less than } \\
2700\end{array}$ & $\begin{array}{l}2801- \\
6800\end{array}$ & $\begin{array}{l}6801- \\
11600\end{array}$ & $\begin{array}{l}11601- \\
34999\end{array}$ & $\begin{array}{l}35000- \\
84499\end{array}$ & $\begin{array}{l}\text { More than } \\
85000\end{array}$ \\
\hline & & & & & \\
\hline
\end{tabular}

13. What is the highest level of formal education you have completed? (Please check one box)

\begin{tabular}{|l|l|l|l|l|l|l|l|}
\hline Elementary & $\begin{array}{l}\text { High } \\
\text { school }\end{array}$ & $\begin{array}{l}\text { Technical } \\
\text { high } \\
\text { school }\end{array}$ & $\begin{array}{l}\text { Prepara } \\
\text { tory } \\
\text { school }\end{array}$ & Bachelor & Master & PhD & Other \\
\hline & & & & & & & \\
\hline
\end{tabular}


14. Do you live with? I live ....

15.

\begin{tabular}{|c|c|c|c|c|c|}
\hline Alone & $\begin{array}{c}\text { With my } \\
\text { partner }\end{array}$ & $\begin{array}{c}\text { With my partner } \\
\text { and children }\end{array}$ & $\begin{array}{c}\text { Single parent } \\
\text { with children }\end{array}$ & $\begin{array}{c}\text { In a flat } \\
\text { (with friend) }\end{array}$ & $\begin{array}{c}\text { With my } \\
\text { parents }\end{array}$ \\
\hline & & & & & \\
\hline
\end{tabular}

16. How many people are living at home?

17. How many children are living at home? 


\section{Appendix 3: Results of the pre-test in Mexico of motives driving the purchasing of organic food}

\begin{tabular}{|c|c|c|c|c|c|c|c|c|}
\hline Statements & $\begin{array}{l}\text { Not at all } \\
\text { important }\end{array}$ & $\begin{array}{c}\text { A little } \\
\text { important }\end{array}$ & $\begin{array}{c}\text { Moderately } \\
\text { important }\end{array}$ & Important & $\begin{array}{c}\text { Very } \\
\text { important }\end{array}$ & $\%$ & $\mu$ & $\sigma$ \\
\hline \multicolumn{9}{|l|}{ Animal welfare: } \\
\hline Has been produced in a way that animals have not experienced pain & 1.9 & 2.7 & 8.2 & 12.1 & 75.1 & 100 & 1.56 & .90 \\
\hline Has been produced in a way that animals' rights have been respected & 1.9 & 1.9 & 8.9 & 14.0 & 72.8 & 99.6 & 1.54 & .88 \\
\hline \multicolumn{9}{|l|}{ National concern: } \\
\hline Are profitable for Mexican farmers & 1.6 & 3.9 & 5.8 & 10.5 & 77.8 & 99.6 & 1.60 & .88 \\
\hline Has been produced in in Mexico & 3.1 & 2.7 & 10.1 & 17.5 & 66.1 & 99.6 & 1.41 & .99 \\
\hline \multicolumn{9}{|l|}{ Lifestyle: } \\
\hline Organic food is not just a product is a way of life & 7.0 & 6.6 & 13.6 & 16.7 & 55.6 & 99.6 & 1.08 & 1.26 \\
\hline Has been recommended by people that I well-know & 12.1 & 12.5 & 24.5 & 16.3 & 34.2 & 99.6 & .48 & 1.39 \\
\hline \multicolumn{9}{|l|}{ Weight control: } \\
\hline Helps me to control my weight & 13.6 & 11.3 & 16.3 & 13.2 & 45.5 & 100 & .66 & 1.48 \\
\hline Is low in calories & 12.5 & 13.6 & 22.6 & 16.7 & 34.6 & 100 & .47 & 1.40 \\
\hline \multicolumn{9}{|l|}{ Sensory appeal: } \\
\hline Looks nice & 3.9 & 7.8 & 13.6 & 19.1 & 55.6 & 100 & 1.15 & 1.16 \\
\hline Taste good & & 2.7 & 5.4 & 14.0 & 77.0 & 99.2 & 1.67 & .71 \\
\hline \multicolumn{9}{|l|}{ Healthy eating: } \\
\hline Keeps me healthy & .8 & 1.2 & 1.2 & 10.1 & 86.8 & 100 & 1.81 & .59 \\
\hline Are good for me & .8 & 1.2 & 1.9 & 8.9 & 87.2 & 100 & 1.81 & .60 \\
\hline \multicolumn{9}{|l|}{ Environmental concern: } \\
\hline Has been transported with a low environmental cost & 1.9 & 1.6 & 6.2 & 11.7 & 77.0 & 98.4 & 1.63 & .83 \\
\hline Has been produced in a way which has not shaken the balance of nature & 1.6 & 3.1 & 3.9 & 10.5 & 80.9 & 100 & 1.66 & .82 \\
\hline \multicolumn{9}{|l|}{ Natural content: } \\
\hline Contains no additives: & .8 & .8 & 3.1 & 5.8 & 89.5 & 100 & 1.82 & .59 \\
\hline Contains natural ingredients & 2.7 & 2.3 & 2.3 & 7.0 & 84.8 & 99.2 & 1.70 & .85 \\
\hline \multicolumn{9}{|l|}{ Free of GM0: } \\
\hline Is free from GMO (Genetically Modified Organism) & 5.1 & 4.3 & 7.4 & 8.2 & 74.7 & 99.6 & 1.44 & 1.13 \\
\hline \multicolumn{9}{|l|}{ Political concern: } \\
\hline Comes from a country in which human rights are respected & 3.1 & 3.5 & 9.7 & 11.7 & 72.0 & 100 & 1.46 & 1.02 \\
\hline Has been prepared in a way that does not conflict with my political values & 5.4 & 4.7 & 21.0 & 12.8 & 54.5 & 98.4 & 1.08 & 1.21 \\
\hline \multicolumn{9}{|l|}{ Familiarity: } \\
\hline Is a product or brands that I know & 15.6 & 12.5 & 25.7 & 18.7 & 27.2 & 99.6 & .30 & 1.40 \\
\hline Is what I usually eat & 2.7 & 3.5 & 6.6 & 19.5 & 67.7 & 100 & 1.46 & .96 \\
\hline \multicolumn{9}{|l|}{ Cooking convenience: } \\
\hline Takes not too much time to prepare & 13.6 & 12.8 & 24.1 & 17.1 & 31.9 & 99.6 & .41 & 1.40 \\
\hline Can be cooked very simply & 11.7 & 10.1 & 22.6 & 15.6 & 39.7 & 99.6 & .62 & 1.40 \\
\hline \multicolumn{9}{|l|}{ Shopping convenience: } \\
\hline Are easily available in shops and supermarkets & .8 & 1.6 & 6.6 & 16.7 & 73.5 & 99 & 1.62 & .75 \\
\hline Can be bought in shops close to where I live or work & .4 & 3.1 & 6.2 & 18.7 & 71.6 & 100 & 1.58 & .78 \\
\hline \multicolumn{9}{|l|}{ Organic label: } \\
\hline Has an organic labelling & 3.1 & 3.1 & 8.9 & 15.2 & 69.6 & 100 & 1.45 & 1.00 \\
\hline Has been certified by an agency that I well-know & 4.7 & 5.8 & 23.0 & 21.8 & 44.0 & 99 & .95 & 1.16 \\
\hline \multicolumn{9}{|l|}{ Price orientation: } \\
\hline Are good value for money & 1.6 & .8 & 7.0 & 14.4 & 76.3 & 100 & 1.63 & .78 \\
\hline Are not expensive & .8 & 3.1 & 16.0 & 24.1 & 55.3 & 99 & 1.31 & .91 \\
\hline
\end{tabular}




\section{Appendix 4: Results of the pre-test in Mexico section of values}

\begin{tabular}{|c|c|c|c|c|c|c|c|c|}
\hline Statements & $\begin{array}{l}\text { Not at all } \\
\text { important }\end{array}$ & $\begin{array}{c}\text { A little } \\
\text { important }\end{array}$ & \begin{tabular}{|c|} 
Moderately \\
important
\end{tabular} & Important & \begin{tabular}{|c|} 
Very \\
important \\
\end{tabular} & $\%$ & $\mu$ & $\sigma$ \\
\hline \multicolumn{9}{|l|}{ Hedonism: } \\
\hline Enjoying Life (Enjoying food, sex, leisure) & 1.2 & 1.2 & 3.9 & 12.5 & 81.3 & 100 & 1.72 & .70 \\
\hline Pleasure (gratification of desires) & 2.3 & 1.9 & 14.4 & 26.8 & 54.5 & 100 & 1.29 & .95 \\
\hline \multicolumn{9}{|l|}{ Stimulous: } \\
\hline A varied life (filled with challenges, novelty, and changes) & 1.9 & 1.2 & 8.6 & 24.5 & 63.8 & 100 & 1.47 & .85 \\
\hline An exciting life (stimulating experiences) & 1.6 & 3.5 & 8.2 & 26.5 & 60.3 & 100 & 1.40 & .90 \\
\hline \multicolumn{9}{|l|}{ Security: } \\
\hline Sense of belonging (feeling that others care about me) & 3.5 & 3.9 & 14.4 & 27.2 & 51.0 & 100 & 1.18 & 1.05 \\
\hline Healthy (not being sick physically or mentally) & .8 & 1.6 & .4 & 17.1 & 80.2 & 100 & 1.74 & .62 \\
\hline \multicolumn{9}{|l|}{ Self-direction: } \\
\hline Freedom (of action and thought) & .8 & 1.2 & .4 & 9.7 & 87.9 & 100 & 1.83 & .56 \\
\hline Creativity (uniqueness and imagination) & .8 & 1.2 & 9.7 & 20.2 & 68.1 & 100 & 1.54 & .78 \\
\hline \multicolumn{9}{|l|}{ Universalism: } \\
\hline Protecting the environment (preserving nature) & .4 & .8 & 2.3 & 14.0 & 82.5 & 100 & 1.77 & .56 \\
\hline Broad-Minded (tolerant of different ideas and beliefs) & .8 & 1.2 & 2.7 & 13.2 & 82.1 & 100 & 1.75 & .64 \\
\hline \multicolumn{9}{|l|}{ Benevolence: } \\
\hline Helpful (working for the welfare of others) & 1.2 & .8 & 6.6 & 16.7 & 74.7 & 100 & 1.63 & .74 \\
\hline Honest (genuine, sincere) & .8 & 1.2 & 1.2 & 11.7 & 85.2 & 100 & 1.79 & .59 \\
\hline
\end{tabular}




\section{Appendix 5: Second pre-testing survey in Germany (German version)}

Zunächst einmal: Wer ist bei Ihnen für den Lebensmitteleinkauf zuständig?

\begin{tabular}{|c|c|c|c|c|c|}
\hline $\begin{array}{c}\text { Zuständigkeit } \\
\text { Lebensmittelkauf }\end{array}$ & Nur ich & $\begin{array}{l}\text { Überwiegend } \\
\text { ich }\end{array}$ & $\begin{array}{c}\text { Mein(e) } \\
\text { Partner(in)/ } \\
\text { Mitbewohner } \\
\text { (in) und ich } \\
\text { zu etwa } \\
\text { gleichen } \\
\text { Teilen }\end{array}$ & $\begin{array}{c}\text { Überwiegend } \\
\text { jemand } \\
\text { anderes }\end{array}$ & $\begin{array}{l}\text { Ausschließlich } \\
\text { jemand anderes }\end{array}$ \\
\hline & & & & & \\
\hline
\end{tabular}

2. Bitte geben Sie Ihr Geschlecht an.

\begin{tabular}{|l|l|}
\hline Männlich & Weiblich \\
\hline & \\
\hline
\end{tabular}

3. In welchem Bundesland leben Sie?

\begin{tabular}{|l|l|}
\hline Bundesland & \\
\hline Baden- & \\
Württemberg & \\
\hline Bayern & \\
\hline Bremen & \\
\hline Hamburg & \\
\hline Niedersachsen & \\
\hline Schleswig-Holstein & \\
\hline Hessen & \\
\hline Nordrhein- & \\
\hline Westfalen & \\
\hline Rheinland-Pfalz & \\
\hline Saarland & \\
\hline Berlin & \\
\hline Brandenburg & \\
\hline Mecklenburg- & \\
\hline Vorpommern & \\
\hline Sachsen & \\
\hline Sachsen-Anhalt & \\
\hline Thüringen & \\
\hline
\end{tabular}


4. In welchem Jahr sind Sie geboren?

5. Können Sie ungefähr sagen, wie hoch ihr monatliches Netto-Haushaltseinkommen (Einkommen, das Ihrem gesamten Haushalt pro Monat zur Verfügung steht) ist? Bitte kreuzen Sie das Entsprechende an!

\begin{tabular}{|l|l|l|l|l|l|l|l|}
\hline$<900 €$ & $900-$ & $1.500-$ & $2.000-$ & $3.200-$ & $4.500-$ & & Keine \\
& $1.499 €$ & $1.999 €$ & $3.199 €$ & $4.499 €$ & $5.499 €$ & $>5.500$ & Angabe \\
\hline
\end{tabular}

6. Wie häufig kaufen Sie Lebensmittel in den folgenden Geschäften?

\begin{tabular}{|c|c|c|c|c|c|c|}
\hline & $\begin{array}{l}\text { Mehrmals } \\
\text { pro Woche }\end{array}$ & $\begin{array}{l}\text { Ca. } 1 \text { Mal } \\
\text { pro Woche }\end{array}$ & $\begin{array}{l}\text { Ca. alle } 2 \\
\text { Wochen }\end{array}$ & $\begin{array}{l}\text { Ca. } 1 \text { Mal } \\
\text { im Monat }\end{array}$ & $\begin{array}{l}\text { Weniger als } \\
1 \text { Mal im } \\
\text { Monat }\end{array}$ & (Fast) nie \\
\hline $\begin{array}{l}\text { Große Supermärkte } \\
\text { (wie Real oder } \\
\text { Kaufland) }\end{array}$ & & & & & & \\
\hline $\begin{array}{l}\text { Supermärkte (Edeka, } \\
\text { Rewe) }\end{array}$ & & & & & & \\
\hline Discounter (Aldi, Lidl) & & & & & & \\
\hline Wochenmarkt & & & & & & \\
\hline Obst- und & & & & & & \\
\hline Bio-Supermärkte & & & & & & \\
\hline $\begin{array}{l}\text { Bioläden/ } \\
\text { Naturkostfachgeschäft }\end{array}$ & & & & & & \\
\hline Direkt beim Landwirt & & & & & & \\
\hline
\end{tabular}


7. Nun geht es um die Aspekte, auf die Sie beim Lebensmitteleinkauf achten. Bitte kreuzen Sie jeweils an, ob die nachstehenden Fragen auf Sie zutreffen, oder nicht!

\begin{tabular}{|c|c|c|c|c|c|c|c|}
\hline & $\begin{array}{c}\text { Trifft voll } \\
\text { und ganz } \mathrm{zu}\end{array}$ & Trifft zu & $\begin{array}{c}\text { Trifft eher } \\
\mathrm{zu}\end{array}$ & Teils/ teils & $\begin{array}{l}\text { Trifft eher } \\
\text { nicht zu }\end{array}$ & $\begin{array}{c}\text { Trifft nicht } \\
\mathrm{zu}\end{array}$ & $\begin{array}{c}\text { Trifft } \\
\text { überhaupt } \\
\text { nicht zu }\end{array}$ \\
\hline $\begin{array}{l}\text { Ich habe keine Zeit, im Geschäft lange nach } \\
\text { den verschiedenen Lebensmitteln zu suchen. }\end{array}$ & & & & & & & \\
\hline $\begin{array}{l}\text { Ich möchte mit meinem Lebensmitteleinkauf } \\
\text { die Bauern/Erzeuger der Rohprodukte } \\
\text { unterstützen. }\end{array}$ & & & & & & & \\
\hline $\begin{array}{l}\text { Ich achte darauf, nur Gentechnik-freie } \\
\text { Lebensmittel zu kaufen. }\end{array}$ & & & & & & & \\
\hline $\begin{array}{l}\text { Ich achte beim Lebensmittelkauf darauf, dass } \\
\text { die Produkte umweltfreundlich produziert } \\
\text { wurden. }\end{array}$ & & & & & & & \\
\hline $\begin{array}{l}\text { Ich achte beim Einkauf von Milch- und } \\
\text { Fleischprodukten darauf, dass die } \\
\text { Tierschutzbestimmungen eingehalten wurden. }\end{array}$ & & & & & & & \\
\hline $\begin{array}{l}\text { Bei ausländischen Lebensmitteln achte ich } \\
\text { darauf, dass ein Fair-Trade Siegel drauf ist. }\end{array}$ & & & & & & & \\
\hline $\begin{array}{l}\text { Ich prüfe sehr genau die } \\
\text { Produktinformationen auf der Verpackung, } \\
\text { bevor ich etwas kaufe. }\end{array}$ & & & & & & & \\
\hline $\begin{array}{l}\text { Der Lebensmitteleinkauf muss bei mir schnell } \\
\text { gehen. }\end{array}$ & & & & & & & \\
\hline $\begin{array}{l}\text { Ich halte mich beim Lebensmittelkauf an } \\
\text { bekannte Produkte und Marken. }\end{array}$ & & & & & & & \\
\hline $\begin{array}{l}\text { Lebensmittelverpackungen enthalten meist } \\
\text { viel zu wenig Informationen über das } \\
\text { Produkt. }\end{array}$ & & & & & & & \\
\hline $\begin{array}{l}\text { Ich achte beim Lebensmitteleinkauf vor allem } \\
\text { auf den Preis. }\end{array}$ & & & & & & & \\
\hline $\begin{array}{l}\text { Ich achte beim Einkauf darauf, dass die } \\
\text { Lebensmittel keine Zusatzstoffe beinhalten. }\end{array}$ & & & & & & & \\
\hline $\begin{array}{l}\text { Günstig Lebensmittel eingekauft zu haben gibt } \\
\text { mir ein gutes Gefühl. }\end{array}$ & & & & & & & \\
\hline $\begin{array}{l}\text { Ich achte darauf, dass von dem Geld, das ich } \\
\text { für Lebensmittel ausgebe, auch genug bei den } \\
\text { Bauern ankommt. }\end{array}$ & & & & & & & \\
\hline Ich kaufe gern Lebensmittel ein. & & & & & & & \\
\hline $\begin{array}{l}\text { Ich achte beim Kauf frischer Lebensmittel vor } \\
\text { allem auf den Geruch. }\end{array}$ & & & & & & & \\
\hline $\begin{array}{l}\text { Es ist schwer, beim Kauf von Milch- und } \\
\text { Fleischprodukten zu erkennen, ob die Tiere } \\
\text { artgerecht gehalten wurden. }\end{array}$ & & & & & & & \\
\hline $\begin{array}{l}\text { Beim Lebensmitteleinkauf probiere ich gerne } \\
\text { Neues aus. }\end{array}$ & & & & & & & \\
\hline $\begin{array}{l}\text { Ich achte beim Lebensmittelkauf sehr genau } \\
\text { darauf, woher die Produkte kommen. }\end{array}$ & & & & & & & \\
\hline $\begin{array}{l}\text { Ich kaufe häufig Lebensmittel wegen der } \\
\text { besonderen Verpackung. }\end{array}$ & & & & & & & \\
\hline $\begin{array}{l}\text { Es ist schwer, beim Lebensmitteleinkauf zu } \\
\text { erkennen, ob die Umwelt bei der Produktion } \\
\text { geschont wurde. }\end{array}$ & & & & & & & \\
\hline $\begin{array}{l}\text { Ich achte beim Lebensmitteleinkauf auf eine } \\
\text { umweltfreundliche Verpackung. }\end{array}$ & & & & & & & \\
\hline $\begin{array}{l}\text { Früher hatte ich mehr Vertrauen in die } \\
\text { Qualität von Lebensmitteln. }\end{array}$ & & & & & & & \\
\hline $\begin{array}{l}\text { Der Preis sagt auch bei Lebensmitteln etwas } \\
\text { über die Qualität aus. }\end{array}$ & & & & & & & \\
\hline Ich bevorzuge Produkte aus Deutschland. & & & & & & & \\
\hline $\begin{array}{l}\text { Ich achte beim Kauf frischer Lebensmittel vor } \\
\text { allem auf das Aussehen. }\end{array}$ & & & & & & & \\
\hline $\begin{array}{l}\text { Wenn ich Lebensmittel wiederentdecke, die } \\
\text { ich als Kind gegessen habe, muss ich einfach } \\
\text { zuschlagen. }\end{array}$ & & & & & & & \\
\hline $\begin{array}{l}\text { Ich kaufe in den Lebensmittelgeschäften, die } \\
\text { am nächsten an meiner Wohnung / meinem } \\
\text { Arbeitsplatz sind. }\end{array}$ & & & & & & & \\
\hline
\end{tabular}


8. Nun noch einige Fragen zu Ihrem Kochverhalten. Bitte bewerten Sie, ob die folgenden Statements auf Sie zutreffen!

\begin{tabular}{|c|c|c|c|c|c|c|c|}
\hline & $\begin{array}{l}\text { Trifft voll } \\
\text { und ganz zu }\end{array}$ & Trifft zu & $\begin{array}{l}\text { Trifft eher } \\
\mathrm{zu}\end{array}$ & Teils/ teils & $\begin{array}{l}\text { Trifft eher } \\
\text { nicht } \mathrm{zu}\end{array}$ & $\begin{array}{l}\text { Trifft nicht } \\
\mathrm{zu}\end{array}$ & $\begin{array}{l}\text { Trifft } \\
\text { überhaupt } \\
\text { nicht zu }\end{array}$ \\
\hline $\begin{array}{l}\text { Lebensmittel müssen vor } \\
\text { allem schnell zuzubereiten } \\
\text { sein. }\end{array}$ & & & & & & & \\
\hline Ich koche gern. & & & & & & & \\
\hline Ich liebe Kochbücher. & & & & & & & \\
\hline $\begin{array}{l}\text { Ich probiere gerne neue } \\
\text { Kochrezepte aus. }\end{array}$ & & & & & & & \\
\hline $\begin{array}{l}\text { Lebensmittel müssen für } \\
\text { mich einfach zuzubereiten } \\
\text { sein. }\end{array}$ & & & & & & & \\
\hline $\begin{array}{l}\text { Komplizierte } \\
\text { Kochrezepte schrecken } \\
\text { mich ab. }\end{array}$ & & & & & & & \\
\hline
\end{tabular}

9. Bitte geben Sie auch Ihre Einschätzung zu den folgenden Aussagen zur Ernährung ab:

\begin{tabular}{|c|c|c|c|c|c|c|c|}
\hline & $\begin{array}{c}\text { Trifft voll } \\
\text { und ganz zu }\end{array}$ & Trifft zu & $\begin{array}{c}\text { Trifft eher } \\
\mathrm{zu}\end{array}$ & Teils/ teils & $\begin{array}{l}\text { Trifft eher } \\
\text { nicht } \mathrm{zu}\end{array}$ & $\begin{array}{c}\text { Trifft nicht } \\
\mathrm{zu}\end{array}$ & \begin{tabular}{|l|} 
Trifft \\
überhaupt \\
nicht zu \\
\end{tabular} \\
\hline $\begin{array}{l}\text { Eiweißreiche Produkte sind } \\
\text { wichtig für meine Ernährung. }\end{array}$ & & & & & & & \\
\hline $\begin{array}{l}\text { Ich ernähre mich möglichst } \\
\text { ballaststoffreich. }\end{array}$ & & & & & & & \\
\hline $\begin{array}{l}\text { Ich achte darauf, vor allem } \\
\text { Nahrungsmittel mit vielen } \\
\text { Vitaminen und } \\
\text { Mineralstoffen zu essen. }\end{array}$ & & & & & & & \\
\hline $\begin{array}{l}\text { In meiner Kindheit hatten } \\
\text { Lebensmittel noch einen viel } \\
\text { besseren Geschmack. }\end{array}$ & & & & & & & \\
\hline $\begin{array}{l}\text { Es ist mir wichtig, mein } \\
\text { Gewicht zu halten. }\end{array}$ & & & & & & & \\
\hline $\begin{array}{l}\text { Lebensmittel müssen vor } \\
\text { allem gut schmecken. }\end{array}$ & & & & & & & \\
\hline Ich ernähre mich gesund. & & & & & & & \\
\hline $\begin{array}{l}\text { Lebensmittel müssen für } \\
\text { mich möglichst } \\
\text { naturbelassen sein. }\end{array}$ & & & & & & & \\
\hline $\begin{array}{l}\text { Ich ernähre mich möglichst } \\
\text { fettarm. }\end{array}$ & & & & & & & \\
\hline $\begin{array}{l}\text { Ich ernähre mich möglichst } \\
\text { kalorienarm. }\end{array}$ & & & & & & & \\
\hline $\begin{array}{l}\text { Ich esse was mir schmeckt, } \\
\text { auf Inhaltsstoffe achte ich } \\
\text { kaum. }\end{array}$ & & & & & & & \\
\hline $\begin{array}{l}\text { Geschmacksverstärker finde } \\
\text { ich fürchterlich. }\end{array}$ & & & & & & & \\
\hline
\end{tabular}


10. Viele Menschen sprechen über Bio-Produkte, aber die Vorstellungen davon können sehr unterschiedlich sein. Daher wüssten wir zunächst gern, was Sie mit Bio-Lebensmitteln verbinden? (Bitte nennen Sie drei Dinge, die Bio-Lebensmittel auszeichnen).
A:
B:
$\mathrm{C}$ :

11. Welche der unten gezeigten Bio-Zeichen (Gütesiegel) bzw. Qualitätsprogramme sind Ihnen bekannt? Bitte klicken Sie die Qualitätszeichen an, die Sie kennen.

1

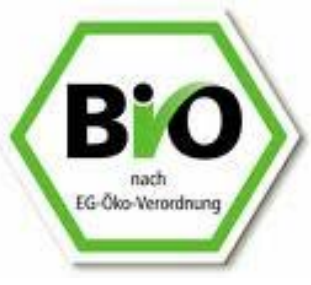

4

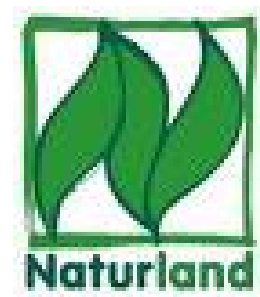

2

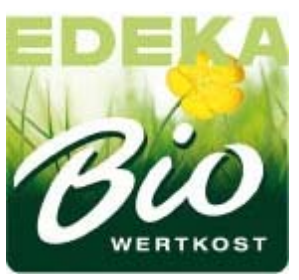

5

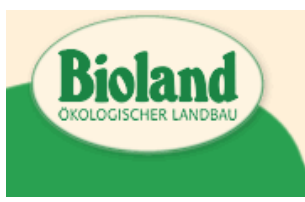

3

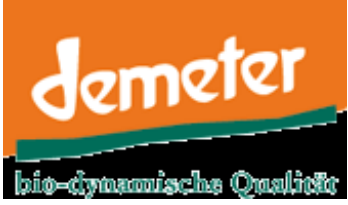

6

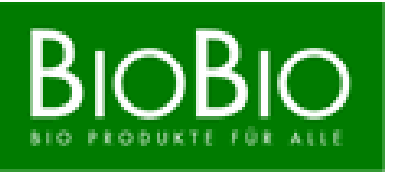

11. Haben Sie schon einmal Bio-Lebensmittel gekauft (Produkte mit einem der oben gezeigten Label oder aus sonstiger ökologischer Erzeugung)?

$\mathrm{Ja}$

Nein

12. Können Sie in etwa sagen, wie lange Sie bereits Bio-Lebensmittel kaufen?

Ich kaufe Bio-Produkten seit...

\begin{tabular}{|c|c|c|c|c|c|c|}
\hline $\begin{array}{c}\text {... weniger } \\
\text { als einem } \\
\text { Jahr }\end{array}$ & $\begin{array}{l}\ldots .1-2 \\
\text { Jahren }\end{array}$ & $\begin{array}{l}\ldots .2-3 \\
\text { Jahren }\end{array}$ & $\begin{array}{l}\ldots .3-4 \\
\text { Jahren }\end{array}$ & $\begin{array}{l}\ldots .4-5 \\
\text { Jahren }\end{array}$ & $\begin{array}{c}\text {...5-6 } \\
\text { Jahren }\end{array}$ & $\begin{array}{c}\text {...mehr als } 6 \\
\text { Jahren }\end{array}$ \\
\hline & & & & & & \\
\hline
\end{tabular}


13. Wie häufig kaufen Sie folgende Lebensmittel(-gruppen) in Form von Bioprodukten?

\begin{tabular}{|l|l|l|l|l|l|l|}
\hline & Immer & $\begin{array}{l}\text { (Fast) } \\
\text { immer }\end{array}$ & Häufig & Gelegentlich & Selten & Nie \\
\hline $\begin{array}{l}\text { Eier } \\
\text { Fleisch und } \\
\text { Wurstwaren }\end{array}$ & & & & & & \\
\hline Brot und Backwaren & & & & & & \\
\hline Obst & & & & & & \\
\hline $\begin{array}{l}\text { Getreideprodukte } \\
\text { z.B. Muesli, }\end{array}$ & & & & & & \\
\hline $\begin{array}{l}\text { Gemüse } \\
\text { Milch und } \\
\text { Milchprodukte (inkl. }\end{array}$ & & & & & & \\
\hline
\end{tabular}

14. Vielen Dank für Ihre bisherigen Angaben! Für unsere Forschungsarbeiten ist es wichtig, auch etwas über die hinter dem Kaufverhalten stehenden, etwas losgelösten Werte und Prinzipien, die Ihnen im Leben wichtig sind, zu erfahren.

Im Folgenden sehen Sie verschiedene solcher Prinzipien und Werte, die im Leben eine Rolle spielen können. Bitte bewerten Sie, von wie großer Bedeutung diese für Sie ganz persönlich sind.

\begin{tabular}{|c|c|c|c|c|c|c|c|}
\hline & $\begin{array}{c}\text { Trifft voll } \\
\text { und ganz zu }\end{array}$ & Trifft zu & $\begin{array}{c}\text { Trifft eher } \\
\mathrm{zu}\end{array}$ & Teils/ teils & $\begin{array}{l}\text { Trifft eher } \\
\text { nicht zu }\end{array}$ & $\begin{array}{c}\text { Trifft nicht } \\
\mathrm{zu}\end{array}$ & $\begin{array}{c}\text { Trifft } \\
\text { überhaupt } \\
\text { nicht zu }\end{array}$ \\
\hline \multicolumn{8}{|l|}{ Zugehörigkeit, Geborgenheit } \\
\hline \multicolumn{8}{|l|}{ Sichere Lebensumstände } \\
\hline \multicolumn{8}{|l|}{ Spaß im Leben } \\
\hline \multicolumn{8}{|c|}{ Enge Beziehungen zu anderen Menschen } \\
\hline \multicolumn{8}{|c|}{ Die Welt und das Leben genießen } \\
\hline \multicolumn{8}{|c|}{ Anerkannt und respektiert werden } \\
\hline \multicolumn{8}{|c|}{ Leistungsfähig sein, etwas erreichen } \\
\hline \multicolumn{8}{|c|}{ Selbstentfaltung, Weiterentwicklung } \\
\hline \multicolumn{8}{|c|}{ Ein aufregendes, abwechslungsreiches Leben } \\
\hline \multicolumn{8}{|c|}{ Gesellschaftliche Gerechtigkeit } \\
\hline \multicolumn{8}{|c|}{ Respekt gegenüber Mitgeschöpfen } \\
\hline \multicolumn{8}{|c|}{ Traditionen bewahren } \\
\hline \multicolumn{8}{|l|}{ Viel Geld verdienen } \\
\hline \multicolumn{8}{|c|}{ Hilfsbereitschaft gegenüber bedürftigen Mitmenschen } \\
\hline Umweltschutz & & & & & & & \\
\hline Ein Beruf, der mich ausfüllt & & & & & & & \\
\hline
\end{tabular}


15. Kreuzen Sie bitte auf der folgenden Liste diejenigen zwei Werte an, die Ihnen im Leben am wichtigsten sind.

\begin{tabular}{|l|l|l|}
\hline & Important & Not important \\
\hline Respect for fellow humans & & \\
\hline A job which fulfils me & & \\
\hline Self-fulfillment and self-development & & \\
\hline An exciting, varied life & & \\
\hline To perform well and achieve something & & \\
\hline Maintain traditions & & \\
\hline Social justice & & \\
\hline Readiness to help people in need & & \\
\hline Feeling of security and belonging & & \\
\hline Secure environment & & \\
\hline Environmental protection & & \\
\hline Enjoying the world and life & & \\
\hline Earn a lot of money & & \\
\hline Close relationships with other people & & \\
\hline To be recognised and respected & & \\
\hline
\end{tabular}

16. Nun haben Sie es fast geschafft! Wir haben nur noch einige Fragen zu Ihrer Person! Zunächst: Wo sind Sie aufgewachsen?

\begin{tabular}{|l|l|l|l|l|l|}
\hline & In einem & In einer & In einer & In einer & In einer \\
& größeren & kleinen Stadt & größeren Stadt & Großstadt & Großstadt mit \\
In einem kleinen & Dorf $(501-$ & $(5.001-$ & $(20.001-$ & $(100.001-$ & mehr als \\
Dorf (weniger als & 5.000 & 20.000 & 100.000 & 500.000 & 500.000 \\
500 Einwohner) & Einwohner $)$ & Einwohner $)$ & Einwohner $)$ & Einwohner $)$ & Einwohnern \\
\hline & & & & & \\
& & & & & \\
\hline
\end{tabular}

17. Und wo wohnen Sie zurzeit?

\begin{tabular}{|l|l|l|l|l|l|}
\hline & In einem & In einer & In einer & In einer & In einer \\
& größeren & kleinen Stadt & größeren Stadt & Großstadt & Großstadt mit \\
In einem kleinen & Dorf $(501-$ & $(5.001-$ & $(20.001-$ & $(100.001-$ & mehr als \\
Dorf (weniger als & 5.000 & 20.000 & 100.000 & 500.000 & 500.000 \\
500 Einwohner) & Einwohner) & Einwohner $)$ & Einwohner $)$ & Einwohner $)$ & Einwohnern \\
\hline & & & & & \\
& & & & & \\
\hline
\end{tabular}


18. Welchen Bildungsabschluss haben Sie?

\begin{tabular}{|c|c|c|c|c|c|}
\hline $\begin{array}{c}\text { Noch in der } \\
\text { Schule }\end{array}$ & $\begin{array}{c}\text { Volksschul- } \\
/ \\
\text { Hauptschula } \\
\text { bschluss }\end{array}$ & $\begin{array}{c}\text { Abschluss } \\
\text { der } \\
\text { Polytechnisc } \\
\text { hen } \\
\text { Oberschule }\end{array}$ & $\begin{array}{c}\text { Realschul- } \\
\text { oder gleich- } \\
\text { wertiger } \\
\text { Abschluss }\end{array}$ & $\begin{array}{c}\text { (Fach-) } \\
\text { Hochschulre } \\
\text { ife/ Abitur }\end{array}$ & $\begin{array}{c}\text { Kein } \\
\text { Abschluss }\end{array}$ \\
\hline & & & & & \\
\hline
\end{tabular}

19. Wie viele Personen wohnen in Ihrem Haushalt (inklusive Ihnen selbst)?

20. Wie wohnen Sie?

\begin{tabular}{|c|c|c|c|c|c|c|c|c|}
\hline Allein & $\begin{array}{l}\text { Allein, } \\
\text { Kinder sind } \\
\text { ausgezogen }\end{array}$ & $\begin{array}{l}\text { Mit } \\
\text { Partner(in) } \\
\text { und Kindern }\end{array}$ & \begin{tabular}{|l} 
Mit \\
Partner(in) \\
ohne Kinder
\end{tabular} & $\begin{array}{l}\text { Mit } \\
\text { Partner(in), } \\
\text { Kinder sind } \\
\text { ausgezogen }\end{array}$ & $\begin{array}{l}\text { Alleiner- } \\
\text { ziehend }\end{array}$ & $\begin{array}{l}\text { In einer Wohn- } \\
\text { gemeinschaft }\end{array}$ & \begin{tabular}{|l|} 
Bei den \\
Eltern \\
\end{tabular} & $\begin{array}{l}\text { Bei den } \\
\text { Kindern }\end{array}$ \\
\hline & & & & & & & & \\
\hline
\end{tabular}

21. Wie viele Kinder (unter 18 Jahren) wohnen in Ihrem Haushalt?

22. Können Sie ungefähr sagen, wie hoch ihr monatliches Netto-Haushaltseinkommen (Einkommen, das Ihrem gesamten Haushalt pro Monat zur Verfügung steht) ist? Bitte kreuzen Sie das Entsprechende an!

\begin{tabular}{|l|l|l|l|l|l|l|l|}
\hline$<900 €$ & $1.499 €$ & $1.500-$ & $2.000-$ & $3.200-$ & $4.500-$ & & No \\
& $1.999 €$ & $3.199 €$ & $4.499 €$ & $5.499 €$ & $>5.500$ & answer \\
\hline & & & & & & & \\
\hline
\end{tabular}

23. Können Sie ungefähr sagen, wie viel Ihr monatliches Netto-Haushaltseinkommen beträgt (Einkommen, das Ihnen pro Monat zur Verfügung steht)? Bitte kreuzen Sie das Entsprechende an!

\begin{tabular}{|l|l|l|l|l|l|l|l|}
\hline$<900 €$ & $\begin{array}{l}900- \\
1.499 €\end{array}$ & $\begin{array}{l}1.500- \\
1.999 €\end{array}$ & $\begin{array}{l}2.000- \\
3.199 €\end{array}$ & $\begin{array}{l}3.200- \\
4.499 €\end{array}$ & $\begin{array}{l}4.500- \\
5.499 €\end{array}$ & $>5.500$ & $\begin{array}{l}\text { No } \\
\text { answer }\end{array}$ \\
\hline & & & & & & & \\
\hline
\end{tabular}

24. Können Sie auch in etwa schätzen, wie viel Sie pro Woche für Lebensmittel ausgeben? 


\section{Appendix 6: Second pre-test in Germany (Enlgish version)}

1. Firstly, who is responsible for the purchasing of food?

\begin{tabular}{|c|c|c|c|c|}
\hline Just me & $\begin{array}{c}\text { Predominantly } \\
\text { me }\end{array}$ & $\begin{array}{c}\text { My partner(s)/ roommate } \\
\text { and me more or less } \\
\text { equally }\end{array}$ & $\begin{array}{c}\text { Mostly } \\
\text { someone else }\end{array}$ & $\begin{array}{c}\text { Only } \\
\text { someone else }\end{array}$ \\
\hline & & & & \\
\hline
\end{tabular}

2. What is your Gender?

Woman

Man

3. In wich federal state do you live?

\begin{tabular}{|c|c|}
\hline $\begin{array}{l}\text { Baden- } \\
\text { Württemberg }\end{array}$ & \\
\hline Bayern & \\
\hline Bremen & \\
\hline Hamburg & \\
\hline Niedersachsen & \\
\hline Schleswig-Holstein & \\
\hline Hessen & \\
\hline $\begin{array}{l}\text { Nordrhein- } \\
\text { Westfalen }\end{array}$ & \\
\hline Rheinland-Pfalz & \\
\hline Saarland & \\
\hline Berlin & \\
\hline Brandenburg & \\
\hline $\begin{array}{l}\text { Mecklenburg- } \\
\text { Vorpommern }\end{array}$ & \\
\hline Sachsen & \\
\hline Sachsen-Anhalt & \\
\hline Thüringen & \\
\hline
\end{tabular}

4. When have you born? 19 
5. Could you say what is approximately your total household net incomes monthly in $€$ ? (Please check one box)

\begin{tabular}{|l|l|l|l|l|l|l|l|}
\hline$<900 €$ & $900-$ & $1.500-$ & $2.000-$ & $3.200-$ & $4.500-$ & & No \\
& $1.499 €$ & $1.999 €$ & $3.199 €$ & $4.499 €$ & $5.499 €$ & $>5.500$ & answer \\
\hline
\end{tabular}

6. How often do you buy organic food products per week in the following stores?

\begin{tabular}{|l|l|l|l|l|l|l|}
\hline & $\begin{array}{l}\text { Several } \\
\text { times per } \\
\text { week }\end{array}$ & $\begin{array}{l}\text { About once } \\
\text { per week }\end{array}$ & $\begin{array}{l}\text { About twice } \\
\text { per week }\end{array}$ & $\begin{array}{l}\text { About once } \\
\text { per month }\end{array}$ & $\begin{array}{l}\text { Less than } \\
\text { once per } \\
\text { month }\end{array}$ & $\begin{array}{l}\text { Almost } \\
\text { never }\end{array}$ \\
\hline $\begin{array}{l}\text { Hypermarket (Real or } \\
\text { Kaufland) } \\
\text { Supermarket (Edeka, } \\
\text { Rewe) }\end{array}$ & & & & & \\
\hline $\begin{array}{l}\text { Discounter (Aldi, Lidl) } \\
\text { Week market }\end{array}$ & & & & & \\
\hline Fruit shop & & & & & \\
\hline $\begin{array}{l}\text { Organic supermarket } \\
\text { Organic shop/ Health } \\
\text { shop }\end{array}$ & & & & & \\
\hline Direct marketing & & & & & \\
\hline
\end{tabular}


7. Now there is a list of issues about the purchasing of food. Please check a box that you better agree with them.

\begin{tabular}{|c|c|c|c|c|c|c|c|}
\hline & $\begin{array}{l}\text { Strongly } \\
\text { disagree }\end{array}$ & Disagree & $\begin{array}{l}\text { Somewhat } \\
\text { disagree }\end{array}$ & $\begin{array}{l}\text { Nether } \\
\text { agree or } \\
\text { disagree }\end{array}$ & $\begin{array}{c}\text { Somewhat } \\
\text { agree }\end{array}$ & Agree & Strongly agree \\
\hline \multicolumn{8}{|l|}{ I have no time to look for food products in different stores. } \\
\hline \multicolumn{8}{|l|}{ I want to support the producers of raw / farmers with my food purchasing. } \\
\hline \multicolumn{8}{|l|}{ I am caferul to buy only non-genetically modified food products. } \\
\hline \multicolumn{8}{|c|}{ I pay attention to buy meat and dairy products that the animal welfare provisions have been complied. } \\
\hline \multicolumn{8}{|c|}{ For foreign food products I make sure that shown a fair trade label. } \\
\hline \multicolumn{8}{|l|}{ Before buying food, I carefully read the information on the package. } \\
\hline \multicolumn{8}{|l|}{ Food shopping should be quick. } \\
\hline \multicolumn{8}{|l|}{ I tend to buy well-known brands of food products. } \\
\hline \multicolumn{8}{|l|}{ Food packages usually contain too little information about the product. } \\
\hline \multicolumn{8}{|l|}{ Before buying food, I always check the price. } \\
\hline \multicolumn{8}{|l|}{ I make sure that the food I eat contains no additives. } \\
\hline \multicolumn{8}{|c|}{ Buying low priced food gives me a good feeling. } \\
\hline \multicolumn{8}{|c|}{ I am careful about farmers are paid enough from the money that I spend on food. } \\
\hline \multicolumn{8}{|c|}{ I like to buy food products. } \\
\hline \multicolumn{8}{|c|}{ I am careful to buy fresh food in particular on the smell. } \\
\hline \multicolumn{8}{|c|}{ Regarding dairy and meat products, it is difficult to know whether the animals have been treated well. } \\
\hline \multicolumn{8}{|c|}{ Regarding food products I like trying out something new. } \\
\hline \multicolumn{8}{|l|}{ I am careful about the origin of food products. } \\
\hline \multicolumn{8}{|c|}{ I frequently buy food because of the special packaging. } \\
\hline \multicolumn{8}{|c|}{ It is difficult to know if the production of my food has a negative impact on the environment. } \\
\hline \multicolumn{8}{|c|}{ I am careful to buy food products with environmentally friendly packaging. } \\
\hline \multicolumn{8}{|l|}{ Before, I had more confidence in the quality of food. } \\
\hline \multicolumn{8}{|l|}{ The price say about the quality of food. } \\
\hline \multicolumn{8}{|l|}{ I prefer fruit and vegetables from Germany. } \\
\hline \multicolumn{8}{|l|}{ I am careful to buy fresh food in particular on the appereance. } \\
\hline \multicolumn{8}{|l|}{ When I come across foods from my childhood, I immediately buy them. } \\
\hline I buy food products from shops in the vicinity of my house or office. & & & & & & & \\
\hline
\end{tabular}

\section{Now a few questions about your cooking behaviour please check a box that you better} agree with.

\begin{tabular}{|c|c|c|c|c|c|c|c|}
\hline & $\begin{array}{l}\text { Strongly } \\
\text { disagree }\end{array}$ & Disagree & $\begin{array}{c}\text { Somewhat } \\
\text { disagree }\end{array}$ & $\begin{array}{l}\text { Neither } \\
\text { agree or } \\
\text { disagree }\end{array}$ & $\begin{array}{c}\text { Somewhat } \\
\text { agree }\end{array}$ & Agree & Strongly agree \\
\hline \multicolumn{8}{|l|}{ think that meals should be fast to prepare. } \\
\hline \multicolumn{8}{|l|}{ like to cook. } \\
\hline \multicolumn{8}{|l|}{ I like cookbooks. } \\
\hline \multicolumn{8}{|l|}{ I like to try new recipes. } \\
\hline think that meals should be easy to prepare. & & & & & & & \\
\hline
\end{tabular}


9. Many people talk about organic products, but the meaning is often different. How would you describe organic products? (Please give three aspects).
A:
B:
$\mathrm{C}$ :

10. Which of the organic label (certification) shown below do you? Please check all that you know.

1
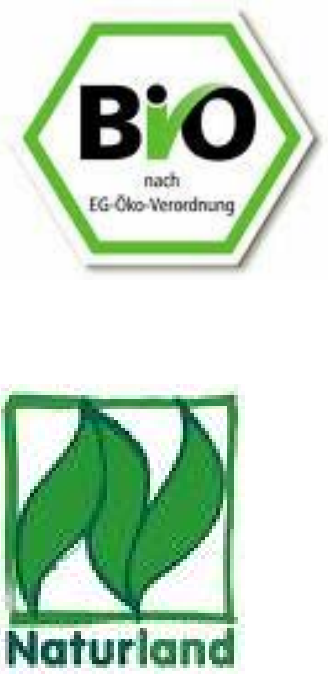

2
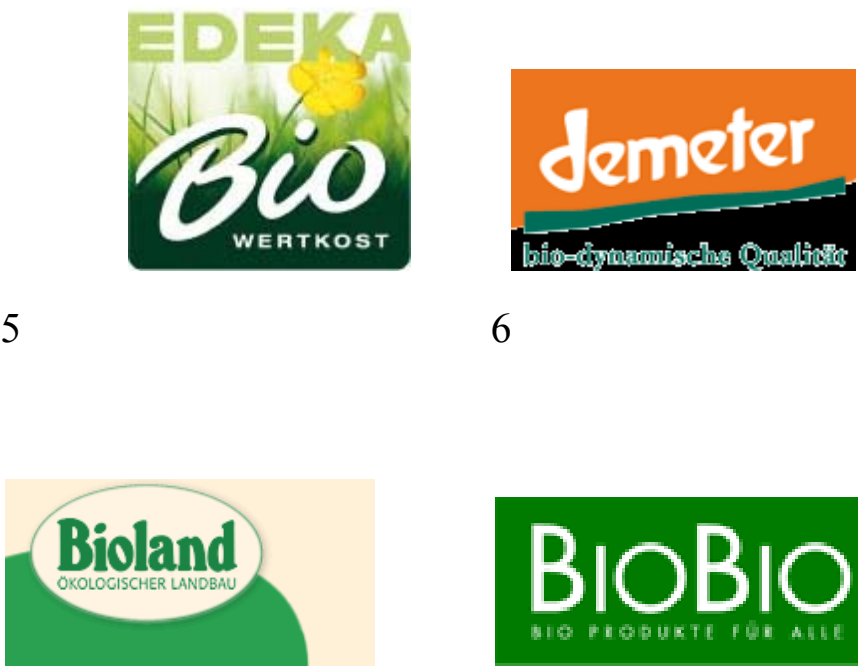

6

\section{BIOBIO}

11. Have you ever purchased organic food? (Products with someone of the label showed above?

Yes

No

12. How much time do you have purchasing organic products?

\begin{tabular}{|c|c|l|l|l|l|l|}
\hline $\begin{array}{c}\text { Less than } \\
\text { one year }\end{array}$ & $1-2$ Years & 2-3 Years & 3-4 Years & 4-5 Years & 5-6 Years & $\begin{array}{c}\text { More than 6 } \\
\text { Years }\end{array}$ \\
\hline & & & & & & \\
\hline
\end{tabular}


13. How often do you buy the following nutritional groups in organic quality?

\begin{tabular}{|l|l|l|l|l|l|l|}
\hline & Always & $\begin{array}{c}\text { Almost } \\
\text { always }\end{array}$ & Frequently & Ocasionally & Rarely & Never \\
\hline $\begin{array}{l}\text { Eggs } \\
\text { products }\end{array}$ & & & & & \\
\hline Bread and pastries & & & & & \\
\hline Fruits & & & & & \\
\hline $\begin{array}{l}\text { Cereals an muesli } \\
\text { Vegetables }\end{array}$ & & & & & \\
\hline $\begin{array}{l}\text { Milk and Dairy } \\
\text { products }\end{array}$ & & & & & \\
\hline
\end{tabular}

Thank you very much for your previous answers, for our research on consumer behaviour, it is important to know what of the followings values and principal in life are important to you.

14. There are below different principals and values that might play a role in life. Please score them according to the importance that these have in your life.

\begin{tabular}{|c|c|c|c|c|c|c|c|}
\hline & $\begin{array}{l}\text { Extremely } \\
\text { important }\end{array}$ & $\begin{array}{l}\text { Very } \\
\text { important }\end{array}$ & $\begin{array}{l}\text { Moderately } \\
\text { important }\end{array}$ & Neutral & $\begin{array}{l}\text { Low } \\
\text { importance }\end{array}$ & $\begin{array}{l}\text { Not at all } \\
\text { important }\end{array}$ & $\begin{array}{l}\text { Contrary to } \\
\text { my viewpoint }\end{array}$ \\
\hline \multicolumn{8}{|c|}{ Feeling of security and belonging } \\
\hline \multicolumn{8}{|c|}{ Close relationships with other people } \\
\hline \multicolumn{8}{|l|}{ Secure environment } \\
\hline \multicolumn{8}{|l|}{ Enjoying life } \\
\hline \multicolumn{8}{|c|}{ Enjoying the world and life } \\
\hline \multicolumn{8}{|c|}{ To be recognised and respected } \\
\hline \multicolumn{8}{|c|}{ To perform well and achieve something } \\
\hline \multicolumn{8}{|c|}{ Self-fulfillment and self-development } \\
\hline \multicolumn{8}{|c|}{ An exciting, varied life } \\
\hline \multicolumn{8}{|l|}{ Social justice } \\
\hline \multicolumn{8}{|c|}{ Respect for fellow humans } \\
\hline \multicolumn{8}{|l|}{ Maintain traditions } \\
\hline Earn a lot of money & & & & & & & \\
\hline
\end{tabular}


15. Please check two values that you consider are the main important in your life

\begin{tabular}{|l|l|l|}
\hline & Important & Not important \\
\hline Respect for fellow humans & & \\
\hline A job which fulfils me & & \\
\hline Self-fulfillment and self-development & & \\
\hline An exciting, varied life & & \\
\hline To perform well and achieve something & & \\
\hline Maintain traditions & & \\
\hline Social justice & & \\
\hline Readiness to help people in need & & \\
\hline Feeling of security and belonging & & \\
\hline Secure environment & & \\
Environmental protection & & \\
\hline Enjoying the world and life & & \\
\hline Earn a lot of money & & \\
\hline Close relationships with other people & & \\
\hline To be recognised and respected & & \\
\hline
\end{tabular}

16. Where did you grow up?

\begin{tabular}{|c|c|c|c|c|c|}
\hline $\begin{array}{l}\text { In a small village } \\
\text { (less than } 500 \\
\text { habitants) }\end{array}$ & $\begin{array}{l}\text { In a bigger } \\
\text { village ( } 501 \\
-5,000 \\
\text { habitants) }\end{array}$ & $\begin{array}{l}\text { In a small city } \\
(5,001- \\
20,000 \\
\text { habitants })\end{array}$ & $\begin{array}{l}\text { In a bigger city } \\
(20,001- \\
100,000 \\
\text { habitants })\end{array}$ & $\begin{array}{l}\text { In a } \\
\text { metropoli } \\
(100,001- \\
500,000 \\
\text { habitants })\end{array}$ & $\begin{array}{l}\text { In a } \\
\text { metropoli } \\
\text { more than } \\
500.000 \\
\text { habitants }\end{array}$ \\
\hline & & & & & \\
\hline
\end{tabular}

17. Where do you live now?

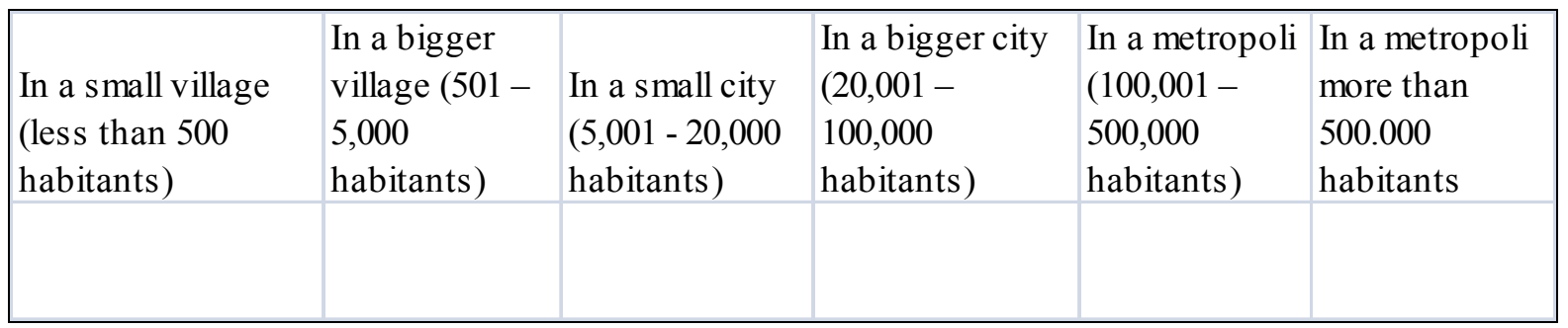

18. What is the highest level of formal education you have completed? (Please check one box)

\begin{tabular}{|c|c|c|c|c|c|c|}
\hline $\begin{array}{c}\text { Basic school } \\
\text { leaving } \\
\text { certificate }\end{array}$ & $\begin{array}{c}\text { Advanced } \\
\text { Vocational } \\
\text { Certificate }\end{array}$ & A-level & $\begin{array}{c}\text { University } \\
\text { degree }\end{array}$ & PhD & $\begin{array}{c}\text { Still at } \\
\text { school }\end{array}$ & $\begin{array}{c}\text { No qualification } \\
\text { (as yet) }\end{array}$ \\
\hline
\end{tabular}


19. How many people are living at home?

20. Do you live with? I live ....

\begin{tabular}{|c|c|c|c|c|c|c|c|c|}
\hline Alone & $\begin{array}{c}\text { Alone, the } \\
\text { children } \\
\text { moved }\end{array}$ & $\begin{array}{l}\text { With couple } \\
\text { and children }\end{array}$ & $\begin{array}{c}\text { With couple } \\
\text { and without } \\
\text { children }\end{array}$ & $\begin{array}{c}\text { With couple, } \\
\text { the children } \\
\text { moved }\end{array}$ & Single parent & $\begin{array}{l}\text { In a sharing } \\
\text { appartment }\end{array}$ & With parents & With children \\
\hline & & & & & & & & \\
\hline
\end{tabular}

21. How many children are living at home?

22. Could you say us what is approximately the total household net incomes monthly in $€$ ? (Please check one box)

\begin{tabular}{|l|l|l|l|l|l|l|l|}
\hline$<900 €$ & $900-$ & $1.500-$ & $2.000-$ & $3.200-$ & $4.500-$ & & No \\
& $1.499 €$ & $1.999 €$ & $3.199 €$ & $4.499 €$ & $5.499 €$ & $>5.500$ & answer \\
\hline
\end{tabular}

23. Could you say us what is approximately your disposal incomes monthly in $€$ ? (Please check one box)

\begin{tabular}{|l|l|l|l|l|l|l|l|}
\hline$<900 €$ & $900-$ & $1.500-$ & $2.000-$ & $3.200-$ & $4.500-$ & & No \\
& $1.499 €$ & $1.999 €$ & $3.199 €$ & $4.499 €$ & $5.499 €$ & $>5.500$ & answer \\
\hline
\end{tabular}

24. Can you estimate how much do you spend per week in the purchasing of food? 
Appendix 7: Results of the pre-test in Germany of motives driving the choice of food

\begin{tabular}{|c|c|c|c|c|c|c|c|c|c|c|}
\hline & $\begin{array}{l}\text { Strongly } \\
\text { disagree }\end{array}$ & Disagree & $\begin{array}{c}\text { Somerhat } \\
\text { disagree }\end{array}$ & $\begin{array}{c}\text { Neither agree } \\
\text { or disagree }\end{array}$ & $\begin{array}{c}\text { Somerhat } \\
\text { agree }\end{array}$ & Agree & Strongly agree & Total $\%$ & $\mu$ & $\sigma$ \\
\hline \multicolumn{11}{|l|}{ Natural content/Free of GM0: } \\
\hline I try to eat food that contains no additives. & 3.0 & 4.5 & 3.0 & 13.6 & 12.1 & 31.8 & 22.7 & 90.9 & 1.4 & 1.6 \\
\hline For me, food must be as natural as possible. & & & 16.7 & 15.2 & 4.5 & 21.2 & 33.3 & 90.9 & 1.4 & 1.6 \\
\hline I eat what tastes good, I rarely consider the ingredients. & & & 18.2 & 24.2 & 10.6 & 21.2 & 16.7 & 90.9 & 9 & 1.4 \\
\hline I find flavour-enhancers awfil. & & & 13.6 & 12.1 & 3.0 & 34.8 & 27.3 & 90.9 & 1.6 & 1.4 \\
\hline I am caferul to buy only non-genetically modified food products. & 3.0 & 3.0 & 3.0 & 18.2 & 22.7 & 22.7 & 18.2 & 90.9 & 1.2 & 1.5 \\
\hline \multicolumn{11}{|l|}{ Shopping convenience: } \\
\hline I have no time to book for food products in different stores. & 6.1 & 6.1 & 6.1 & 25.8 & 19.7 & 16.7 & 10.6 & 90.9 & .5 & 1.6 \\
\hline Food shopping should be quick. & 3.0 & 6.1 & 15.2 & 24.2 & 15.2 & 16.7 & 10.6 & 90.9 & .5 & 1.6 \\
\hline I like to buy food products. & 1.5 & & 4.5 & 12.1 & 18.2 & 25.8 & 28.8 & 90.9 & 1.6 & 1.3 \\
\hline I buy food products from shops in the vicinity of my house or office. & 1.5 & & 6.1 & 24.2 & 24.2 & 19.7 & 15,2 & 90.9 & 1.1 & 1.3 \\
\hline \multicolumn{11}{|l|}{ Familiarity: } \\
\hline I tend to buy well-known brands of food products. & 3.0 & 1.5 & 1.5 & 21.2 & 22.7 & 27.3 & 13.6 & 90.9 & 1.2 & 1.4 \\
\hline Regarding food products I like trying out something new. & 3.0 & 1.5 & 3.0 & 19.7 & 19.7 & 22.7 & 21.2 & 90.9 & 1.3 & 1.5 \\
\hline \multicolumn{11}{|l|}{ Fair price: } \\
\hline I want to support the producers of raw / farmers with my food purchasing. & 3.0 & 6.1 & & 24.2 & 15.2 & 25.8 & 16.7 & 90.9 & 1.1 & 1.6 \\
\hline I am carefil about farmers are paid enough from the money that I spend on food. & 3.0 & 3.0 & 10.6 & 12.1 & 18.2 & 27.3 & 16.7 & 90.9 & 1.1 & 1.6 \\
\hline For foreign food products I make sure that shown a fair trade label. & 4.5 & 4.5 & 6.1 & 16.7 & 15.2 & 31.8 & 12.1 & 90.9 & 1.0 & 1.6 \\
\hline \multicolumn{11}{|l|}{ Price orientation/Price_Quality relationship: } \\
\hline Buying low priced food gives me a good feeling. & 1.5 & 1.5 & 4.5 & 22.7 & 25.8 & 21.2 & 13.6 & 90.9 & 1.1 & 1.3 \\
\hline Before buying food, I always check the price. & 3.0 & 3.0 & 3.0 & 30.3 & 19.7 & 18.2 & 13.6 & 90.9 & 9 & 1.5 \\
\hline The price say about the quality of food. & 4.5 & 1.5 & 3.0 & 21.2 & 28.8 & 16.7 & 15.2 & 90.9 & 1.0 & 1.5 \\
\hline \multicolumn{11}{|l|}{ Nostalgia: } \\
\hline When I was a child, food tasted much better. & & & 16.7 & 22.7 & 10.6 & 16.7 & 24.2 & 90.9 & 1.1 & 1.5 \\
\hline When I come across foods from my childhood, I immediately buy them. & 1.5 & & 6.1 & 22.7 & 21.2 & 19.7 & 19.7 & 90.9 & 1.2 & 1.4 \\
\hline Before, I had more confidence in the quality of food. & 6.1 & 1.5 & 4.5 & 25.8 & 18.2 & 18.2 & 16.7 & 90.9 & 9 & 1.6 \\
\hline \multicolumn{11}{|l|}{ Information/ Advertising: } \\
\hline Before buying food, I carefilly read the information on the package. & 1.5 & 1.5 & 6.1 & 13.6 & 28.8 & 18.2 & 21.2 & 90.9 & 1.3 & 1.4 \\
\hline Food packages usually contain too little information about the product. & 3.0 & 1.5 & 3.0 & 24.2 & 13.6 & 19.7 & 25.8 & 90.9 & 1.3 & 1.5 \\
\hline I frequently buy food because of the special packaging. & 6.1 & 6.1 & 19.7 & 21.2 & 12.1 & 10.6 & 15.2 & 90.9 & 3 & 1.8 \\
\hline \multicolumn{11}{|l|}{ Sensory appeal: } \\
\hline I am carefil to buy fresh food in particular on the smell. & 3.0 & & 1.5 & 25.8 & 18.2 & 19.7 & 22.7 & 90.9 & 1.3 & 1.4 \\
\hline I am carefil to buy fresh food in particular on the appereance. & 1.5 & & 1.5 & 9.1 & 24.2 & 30.3 & 24.2 & 90.9 & 1.7 & 1.2 \\
\hline Food must above all taste good & & & 30.3 & 12.1 & 1.5 & 30.3 & 16.7 & 90.9 & 9 & 1.6 \\
\hline \multicolumn{11}{|l|}{ Consumer ethnocentrism: } \\
\hline Before to buy I am carefil about the origin of food products. & 1.5 & 3.0 & 1.5 & 21.2 & 25.8 & 22.7 & 15.2 & 90.9 & 1.2 & 1.3 \\
\hline I prefer fruit and vegetables from Germany. & 1.5 & & 7.6 & 15.2 & 21.2 & 25.8 & 19.7 & 90.9 & 1.3 & 1.4 \\
\hline \multicolumn{11}{|l|}{ Cooking involvement: } \\
\hline I like to try new recipes. & & & 18.2 & 10.6 & 3.0 & 25.8 & 33.3 & 90.9 & 1.5 & 1.6 \\
\hline \begin{tabular}{|l|} 
I like to cook. \\
\end{tabular} & & & 15.2 & 10.6 & 1.5 & 33.3 & 30.3 & 90.9 & 1.6 & 1.5 \\
\hline I like cookbooks. & & & 9.1 & 18.2 & 9.1 & 24.2 & 30.3 & 90.9 & & \\
\hline Complicated recipes fight me. & & & 10.6 & 15.2 & 16.7 & 22.7 & 25.8 & 90.9 & 1.4 & 1.4 \\
\hline \multicolumn{11}{|l|}{ Cooking convenience: } \\
\hline I think that meal should be fast to prepare. & & & 27.3 & 21.2 & 6.1 & 21.2 & 15.2 & 90.9 & .7 & 1.5 \\
\hline I think that meals should be easy to prepare. & & & 28.8 & 16.7 & 6.1 & 19.7 & 19.7 & 90.9 & .8 & 1.6 \\
\hline \multicolumn{11}{|l|}{ Weight control: } \\
\hline It is important to me not to put on weight. & & & 13.6 & 12.1 & 3.0 & 25.8 & 36.4 & 90.9 & 1.7 & 1.5 \\
\hline I eat as few calories as possible in my diet & & & 15.2 & 19.7 & 10.6 & 19.7 & 25.8 & 90.9 & 1.2 & 1.5 \\
\hline I eat as low-fat as possible in my diet & & & 7.6 & 12,1 & 12.1 & 22.7 & 36.4 & 90.9 & 1.8 & 1.3 \\
\hline \multicolumn{11}{|l|}{ Healthy eating: } \\
\hline I try to eat food containing lots of vitamins and minerals. & & & 21.2 & 7.6 & 6.1 & 22.7 & 33.3 & 90.9 & 1.4 & 1.6 \\
\hline I eat as much protein as possible in my diet & & & 18.2 & 22.7 & 12.1 & 18.2 & 19.7 & 90.9 & 1.0 & 1.5 \\
\hline I eat healthily & & & 13.6 & 18.2 & 1.5 & 24.2 & 33,3 & 90.9 & 1.5 & 1.5 \\
\hline I eat as much fibre as possible in my diet & & & 21.2 & 18.2 & 9.1 & 10.6 & 31.8 & 90.9 & 1.2 & 1.6 \\
\hline Environmental concern: & & & & & & & & & & \\
\hline I am carefiu to buy food products with environmentally friendly packaging. & 3.0 & 1.5 & 6.1 & 18.2 & 16.7 & 30,3 & 15.2 & 90.9 & 1.2 & 1.5 \\
\hline It i difficult to know if the production of my food has a negative impact on the environment. & 1.5 & & 1.5 & 18.2 & 19.7 & 31.8 & 18.2 & 90.9 & 1.5 & 1.2 \\
\hline I am carefiul to buy food produced in an enviromentally friendly way. & 3.0 & 1.5 & 4.5 & 19.7 & 12.1 & 33.3 & 16.7 & 90.9 & 1.2 & 1.5 \\
\hline Animal welfare: & & & & & & & & & & \\
\hline Regarding dairy and meat products, it is difficult to know whether the animals have been treated well. & 1.5 & & 4.5 & 22.7 & 13.6 & 24.2 & 24.2 & 90.9 & 1.4 & 1.4 \\
\hline I pay attention to buy meat and dairy products that the animal welfare provisions have been complied. & 3.0 & 3.0 & 7.6 & 13.6 & 19.7 & 25.8 & 18.2 & 90.9 & 1.1 & 1.6 \\
\hline
\end{tabular}




\section{Appendix 8: Results of the pre-test in Germany section of values}

\begin{tabular}{|c|c|c|c|c|c|c|c|c|c|c|}
\hline & $\begin{array}{l}\text { Extremely } \\
\text { important }\end{array}$ & $\begin{array}{c}\text { Very } \\
\text { important }\end{array}$ & $\begin{array}{c}\text { Moderately } \\
\text { important }\end{array}$ & Neutral & $\begin{array}{c}\text { Low } \\
\text { importance }\end{array}$ & $\begin{array}{l}\text { Not at all } \\
\text { important }\end{array}$ & $\begin{array}{l}\text { Contrary } \\
\text { to my } \\
\text { viewpoint }\end{array}$ & $\begin{array}{c}\text { Total } \\
\%\end{array}$ & $\mu$ & $\boldsymbol{\sigma}$ \\
\hline \multicolumn{11}{|l|}{ Security: } \\
\hline Feeling of security and belonging & 24,2 & 39,4 & 16,7 & 6,1 & 1,5 & 1,5 & 89,4 & & $-1,8$ & 1,1 \\
\hline Close relationships with other people & 27,3 & 31,8 & 16,7 & 10,6 & 1,5 & & 1,5 & 89,4 & $-2,0$ & 1,1 \\
\hline Secure environment & 30,3 & 37,9 & 16,7 & 3,0 & & & 1,5 & 89,4 & $-1,9$ & 1,1 \\
\hline \multicolumn{11}{|l|}{ Tradition: } \\
\hline Maintain traditions & 16,7 & 33,3 & 16,7 & 15,2 & 3,0 & 3,0 & 1,5 & 89,4 & $-1,3$ & 1,4 \\
\hline \multicolumn{11}{|l|}{ Power/achivement: } \\
\hline To be recognised and respected & 22,7 & 40,9 & 13,6 & 9,1 & 1,5 & & 1,5 & 89,4 & $-1,8$ & 1,2 \\
\hline Earn a lot of money & 18,2 & 22,7 & $\mathbf{3 0 , 3}$ & 10,6 & 4,5 & 1,5 & 1,5 & 89,4 & $-1,3$ & 1,3 \\
\hline To perform well and achieve something & 21,2 & 33,3 & 25,8 & 7,6 & & & 1,5 & 89,4 & $-1,7$ & 1,1 \\
\hline \multicolumn{11}{|l|}{ Self-direction: } \\
\hline Self-fulfillment and self-development & 24,2 & 37,9 & 13,6 & 12,1 & & & 1,5 & 89,4 & $-1,8$ & 1,2 \\
\hline A job which fulfils me & 27,3 & 31,8 & 16,7 & 12,1 & & & 1,5 & 89,4 & $-1,8$ & 1,2 \\
\hline \multicolumn{11}{|l|}{ Stimulation: } \\
\hline An exciting, varied life & 13,6 & $\mathbf{3 0 , 3}$ & 25,8 & 15,2 & 1,5 & 1,5 & 1,5 & 89,4 & $-1,3$ & 1,2 \\
\hline \multicolumn{11}{|l|}{ Hedonis m: } \\
\hline Enjoying life & 27,3 & 37,9 & 16,7 & 6,1 & & & 1,5 & 89,4 & $-1,7$ & 1,2 \\
\hline \multicolumn{11}{|l|}{ Univers alis m: } \\
\hline \multicolumn{11}{|l|}{ 1. Human life oriented: } \\
\hline Social justice & 19,7 & 39,4 & 18,2 & 9,1 & 1,5 & & 1,5 & 89,4 & $-1,7$ & 1,2 \\
\hline Respect for fellow humans & 31,8 & 25,8 & 21,2 & 9,1 & & & 1,5 & 89,4 & $-1,8$ & 1,2 \\
\hline Readiness to help people in need & 24,2 & 25,8 & 18,2 & 18,2 & 1,5 & & 1,5 & 89,4 & $-1,5$ & 1,3 \\
\hline \multicolumn{11}{|l|}{ 2. Unity with nature: } \\
\hline Enjoying the world and life & 24,2 & 31,8 & 22,7 & 7,6 & 1,5 & & 1,5 & 89,4 & $-1,7$ & 1,2 \\
\hline Environmental protection & 22,7 & 28,8 & 24,2 & 9,1 & 3,0 & & 1,5 & 89,4 & $-1,6$ & 1,2 \\
\hline
\end{tabular}




\section{Appendix 9: Cross cultural survey (German vesion)}

1. Zunächst einmal: Wer ist bei Ihnen für den Lebensmitteleinkauf zuständig?

\begin{tabular}{|c|c|c|c|c|c|}
\hline $\begin{array}{l}\text { Zuständigkeit } \\
\text { Lebensmittelk } \\
\text { auf }\end{array}$ & Nur ich & $\begin{array}{l}\text { Überwiegen } \\
d \text { ich }\end{array}$ & $\begin{array}{l}\text { Mein(e) } \\
\text { Partner(in)/ } \\
\text { Mitbewohner } \\
\text { (in) und ich } \\
\text { zu etwa } \\
\text { gleichen } \\
\text { Teilen }\end{array}$ & $\begin{array}{l}\text { Überwiegend } \\
\text { jemand } \\
\text { anderes }\end{array}$ & $\begin{array}{l}\text { Ausschließlich } \\
\text { jemand anderes }\end{array}$ \\
\hline
\end{tabular}

2. Bitte geben Sie Ihr Geschlecht an.

$$
\text { Männlich }
$$

Weiblich

3. In welchem Bundesland leben Sie?

\begin{tabular}{|l|l|}
\hline Bundesland & \\
\hline Baden- & \\
Württemberg & \\
\hline Bayern & \\
\hline Bremen & \\
\hline Hamburg & \\
\hline Niedersachsen & \\
\hline Schleswig-Holstein & \\
\hline Hessen & \\
\hline Nordrhein- & \\
Westfalen & \\
\hline Rheinland-Pfalz & \\
\hline Saarland & \\
\hline Berlin & \\
\hline Brandenburg & \\
\hline Mecklenburg- & \\
\hline Vorpommern & \\
\hline Sachsen & \\
\hline Sachsen-Anhalt & \\
\hline Thüringen & \\
\hline
\end{tabular}

4. In welchem Jahr sind Sie geboren? 
5. Können Sie ungefähr sagen, wie hoch ihr monatliches Netto-Haushaltseinkommen (Einkommen, das Ihrem gesamten Haushalt pro Monat zur Verfügung steht) ist? Bitte kreuzen Sie das Entsprechende an!

\begin{tabular}{|c|c|c|c|c|c|c|c|}
\hline$<900 €$ & $900-$ & $1.500-$ & $2.000-$ & $3.200-$ & $4.500-$ & $>5.500$ & $\begin{array}{c}\text { Keine } \\
\text { Angabe }\end{array}$ \\
\hline & & & & & & & \\
\hline & & & & & & & \\
\hline
\end{tabular}

6. Welchen Bildungsabschluss haben Sie?

\begin{tabular}{|c|c|c|c|c|c|c|}
\hline $\begin{array}{c}\text { Bildungsabs } \\
\text { chluss }\end{array}$ & $\begin{array}{c}\text { Noch in der } \\
\text { Schule }\end{array}$ & $\begin{array}{c}\text { Volksschul-/ } \\
\text { Hauptschulab } \\
\text { schluss }\end{array}$ & $\begin{array}{c}\text { Abschluss } \\
\text { der } \\
\text { Polytechnisc } \\
\text { hen } \\
\text { Oberschule }\end{array}$ & $\begin{array}{c}\text { Realschul- } \\
\text { oder } \\
\text { gleichwertig } \\
\text { er } \\
\text { Abschluss }\end{array}$ & $\begin{array}{c}\text { (Fach-) } \\
\text { Hochschulre } \\
\text { ife/ Abitur }\end{array}$ & $\begin{array}{c}\text { Kein } \\
\text { Abschluss }\end{array}$ \\
\hline & & & & & & \\
\hline
\end{tabular}

7. Welchen berufsbildenden Abschluss haben Sie?

\begin{tabular}{|c|c|c|c|c|c|c|}
\hline $\begin{array}{c}\text { Berufs- } \\
\text { abschluss }\end{array}$ & $\begin{array}{c}\text { Lehr- } \\
\text { ausbildung }\end{array}$ & $\begin{array}{c}\text { Meister-/ } \\
\text { Technikerau } \\
\text { sbildung/ } \\
\text { Fachschul- } \\
\text { abschluss }\end{array}$ & $\begin{array}{c}\text { Verwaltungs- } \\
\text { fachhoch- } \\
\text { schule }\end{array}$ & $\begin{array}{c}\text { Fachhoch- } \\
\text { schulabschluss }\end{array}$ & $\begin{array}{c}\text { Hochschul- } \\
\text { abschluss/ } \\
\text { Promotion }\end{array}$ & $\begin{array}{c}\text { Kein } \\
\text { Abschluss }\end{array}$ \\
\hline & & & & & & \\
\hline
\end{tabular}

8. Wie häufig kaufen Sie Lebensmittel in den folgenden Geschäften?

\begin{tabular}{|c|c|c|c|c|c|c|}
\hline & $\begin{array}{l}\text { Mehrmals } \\
\text { pro Woche }\end{array}$ & $\begin{array}{l}\text { Ca. } 1 \text { Mal } \\
\text { pro Woche }\end{array}$ & $\begin{array}{l}\text { Ca. alle } 2 \\
\text { Wochen }\end{array}$ & $\begin{array}{l}\text { Ca. } 1 \text { Mal } \\
\text { im Monat }\end{array}$ & $\begin{array}{c}\text { Weniger als } \\
1 \text { Mal im } \\
\text { Monat }\end{array}$ & (Fast) nie \\
\hline \multicolumn{7}{|l|}{$\begin{array}{l}\text { Große Supermärkte } \\
\text { (wie Real oder } \\
\text { Kaufland) }\end{array}$} \\
\hline \multicolumn{7}{|l|}{$\begin{array}{l}\text { Supermärkte (Edeka, } \\
\text { Rewe) }\end{array}$} \\
\hline \multicolumn{7}{|l|}{ Discounter (Aldi, Lidl) } \\
\hline \multicolumn{7}{|l|}{ Wochenmarkt } \\
\hline \multicolumn{7}{|l|}{ Obst- und Gemüseladen } \\
\hline \multicolumn{7}{|l|}{ Bio-Supermärkte } \\
\hline \multicolumn{7}{|l|}{$\begin{array}{l}\text { Bioläden/ } \\
\text { Naturkostfachgeschäft }\end{array}$} \\
\hline Direkt beim Landwirt & & & & & & \\
\hline
\end{tabular}


9. Viele Menschen sprechen über Bio-Produkte, aber die Vorstellungen davon können sehr unterschiedlich sein. Daher wüssten wir zunächst gern, was Sie mit Bio-Lebensmitteln verbinden? (Bitte nennen Sie drei Dinge, die Bio-Lebensmittel auszeichnen).
A:
B:
$\mathrm{C}$ :

10. Welche der unten gezeigten Bio-Zeichen (Gütesiegel) bzw. Qualitätsprogramme sind Ihnen bekannt? Bitte klicken Sie die Qualitätszeichen an, die Sie kennen.

1

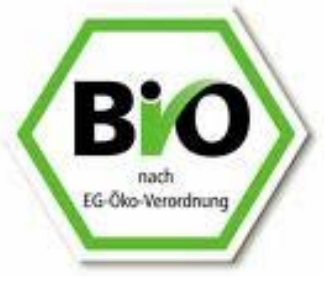

4

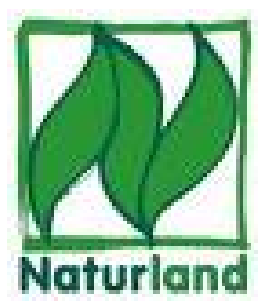

2

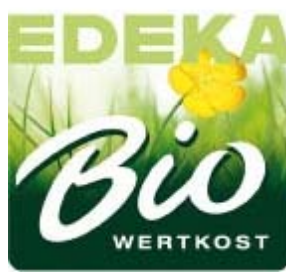

5

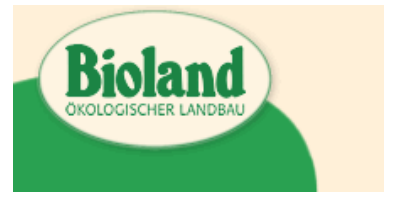

3

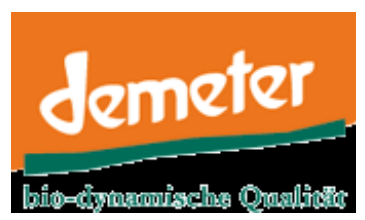

6

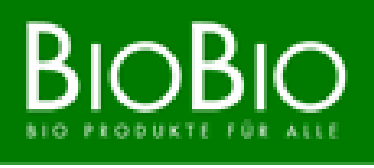

11. Haben Sie schon einmal Bio-Lebensmittel gekauft (Produkte mit einem der oben gezeigten Label oder aus sonstiger ökologischer Erzeugung)?

$\mathrm{Ja}$

Nein 
12. Können Sie in etwa sagen, wie lange Sie bereits Bio-Lebensmittel kaufen?

Ich kaufe Bio-Produkten seit...

\begin{tabular}{|c|c|c|c|c|c|c|}
\hline $\begin{array}{c}\text { a.weniger } \\
\text { Jahr }\end{array}$ & Jahren & Jahren & $\begin{array}{c}\ldots 3-4 \\
\text { Jahren }\end{array}$ & $\begin{array}{c}\ldots 4-5 \\
\text { Jahren }\end{array}$ & $\begin{array}{c}\ldots 5-6 \\
\text { Jahren }\end{array}$ & $\begin{array}{c}\ldots \text { mehr als 6 } \\
\text { Jahren }\end{array}$ \\
\hline & & & & & & \\
\hline
\end{tabular}

13. Wie häufig kaufen Sie folgende Lebensmittel(-gruppen) in Form von Bioprodukten?

\begin{tabular}{|c|c|c|c|c|c|c|}
\hline & Immer & $\begin{array}{l}\text { (Fast) } \\
\text { immer }\end{array}$ & Häufig & Gelegentlich & Selten & $\mathrm{Nie}$ \\
\hline Eier & & & & & & \\
\hline $\begin{array}{l}\text { Fleisch und } \\
\text { Wurstwaren }\end{array}$ & & & & & & \\
\hline Brot und Backwaren & & & & & & \\
\hline Obst & & & & & & \\
\hline $\begin{array}{l}\text { Getreideprodukte } \\
\text { (z.B. Muesli, }\end{array}$ & & & & & & \\
\hline Gemüse & & & & & & \\
\hline $\begin{array}{l}\text { Milch und } \\
\text { Milchprodukte (inkl. }\end{array}$ & & & & & & \\
\hline
\end{tabular}

14. Im Folgenden geht es um die Besonderheiten von Bioprodukten. Bitte bewerten Sie auch hier!

\begin{tabular}{|c|c|c|c|c|c|c|c|}
\hline & $\begin{array}{l}\text { Trifft voll } \\
\text { und ganz } \\
\text { zu }\end{array}$ & Trifft zu & $\begin{array}{l}\text { Trifft } \\
\text { eher zu }\end{array}$ & $\begin{array}{c}\text { Teils/ } \\
\text { teils }\end{array}$ & $\begin{array}{l}\begin{array}{l}\text { Trifft } \\
\text { eher } \\
\text { nicht zu }\end{array} \\
\end{array}$ & $\begin{array}{c}\text { Trifft } \\
\text { nicht zu }\end{array}$ & \begin{tabular}{|l|} 
Trifft \\
überhaupt \\
nicht zu
\end{tabular} \\
\hline $\begin{array}{l}\text { Ich kaufe Bioprodukte, weil ich überzeugt bin, } \\
\text { dass es den Tieren in dieser Produktionsform } \\
\text { besser geht. }\end{array}$ & & & & & & & \\
\hline $\begin{array}{l}\text { Nur bei Bio-Fleisch kann ich sicher sein, dass } \\
\text { keine Antibiotika eingesetzt wurden. }\end{array}$ & & & & & & & \\
\hline $\begin{array}{l}\text { Bio-Bauern verdienen mehr mit ihren Produkter } \\
\text { als andere Bauern. }\end{array}$ & & & & & & & \\
\hline $\begin{array}{l}\text { Die Bio-Produktion fördert vor allem kleine und } \\
\text { mittlere Bauern. }\end{array}$ & & & & & & & \\
\hline $\begin{array}{l}\text { Bio-Produkte schmecken besser. } \\
\text { Bio-Produkte sind gesünder als andere } \\
\text { Lebensmittel. }\end{array}$ & & & & & & & \\
\hline Bio-Produktion ist besser für die Umwelt. & & & & & & & \\
\hline $\begin{array}{l}\text { Ich glaube nicht, dass es den Tieren in der Bio- } \\
\text { Produktion besser geht. }\end{array}$ & & & & & & & \\
\hline Bio-Produkte sind mir einfach zu teuer. & & & & & & & \\
\hline $\begin{array}{l}\text { Bio-Produkte haben zwar eine bessere Qualität } \\
\text { als andere Lebensmittel, sind mir aber zu teuer. }\end{array}$ & & & & & & & \\
\hline $\begin{array}{l}\text { Ich glaube nicht, dass die Bio-Produktion besser } \\
\text { für die Umwelt ist. }\end{array}$ & & & & & & & \\
\hline $\begin{array}{l}\text { Bio-Produkte sind nicht gesünder als andere } \\
\text { Lebensmittel. }\end{array}$ & & & & & & & \\
\hline $\begin{array}{l}\text { In Deutschland werden keine Antibiotika in der } \\
\text { Fleischproduktion eingesetzt. }\end{array}$ & & & & & & & \\
\hline
\end{tabular}


15. Nun geht es um die Aspekte, die beim Lebensmitteleinkauf eine Rolle spielen können. Bitte kreuzen Sie jeweils an, ob die nachstehenden Fragen auf Sie zutreffen, oder nicht!

\begin{tabular}{|c|c|c|c|c|c|c|c|}
\hline & $\begin{array}{c}\text { Trifft voll } \\
\text { und ganz zu }\end{array}$ & Trifft zu & $\begin{array}{c}\text { Trifft eher } \\
\mathrm{zu}\end{array}$ & Teils/ teils & $\begin{array}{c}\text { Trifft eher } \\
\text { nicht zu }\end{array}$ & $\begin{array}{c}\text { Trifft nicht } \\
\mathrm{zu}\end{array}$ & \begin{tabular}{|l} 
Trifft \\
überhaupt \\
nicht zu
\end{tabular} \\
\hline $\begin{array}{l}\text { Ich achte darauf, dass kein } \\
\text { künstlichen Aromastoffe in } \\
\text { den Lebensmitteln sind. }\end{array}$ & & & & & & & \\
\hline $\begin{array}{l}\text { Ich vermeide Produkte, bei } \\
\text { denen ich nicht genau } \\
\text { erkennen kann, aus } \\
\text { welchem Land sie kommen }\end{array}$ & & & & & & & \\
\hline $\begin{array}{l}\text { Ich kaufe in den } \\
\text { Lebensmittelgeschäften, di } \\
\text { am nächsten an meiner } \\
\text { Wohnung / meinem } \\
\text { Arbeitsplatz sind. }\end{array}$ & & & & & & & \\
\hline $\begin{array}{l}\text { Ich halte mich beim } \\
\text { Lebensmittelkauf an } \\
\text { bekannte Marken. }\end{array}$ & & & & & & & \\
\hline $\begin{array}{l}\text { Ich glaube, dass von dem } \\
\text { Geld, das ich für } \\
\text { Lebensmittel ausgebe, } \\
\text { genug bei den Bauern } \\
\text { ankommt. }\end{array}$ & & & & & & & \\
\hline $\begin{array}{l}\text { Ich achte beim } \\
\text { Lebensmittelkauf darauf, } \\
\text { dass die Produkte } \\
\text { umweltfreundlich produzier } \\
\text { wurden. }\end{array}$ & & & & & & & \\
\hline $\begin{array}{l}\text { Wenn ich Lebensmittel } \\
\text { wiederentdecke, die ich als } \\
\text { Kind gegessen habe muss } \\
\text { ich einfach zugreifen. }\end{array}$ & & & & & & & \\
\hline $\begin{array}{l}\text { Günstig Lebensmittel } \\
\text { eingekauft zu haben gibt m } \\
\text { ein gutes Gefühl. }\end{array}$ & & & & & & & \\
\hline $\begin{array}{l}\text { Ich bevorzuge Obst und } \\
\text { Gemüse aus Deutschland. }\end{array}$ & & & & & & & \\
\hline $\begin{array}{l}\text { Es ist schwer, beim Kauf } \\
\text { von Milch- und } \\
\text { Fleischprodukten zu } \\
\text { erkennen, ob die Tiere } \\
\text { artgerecht gehalten wurder }\end{array}$ & & & & & & & \\
\hline $\begin{array}{l}\text { Ich kaufe Produkte mit den } \\
\text { Fair-Trade Siegel. }\end{array}$ & & & & & & & \\
\hline $\begin{array}{l}\text { Ich achte beim } \\
\text { Lebensmitteleinkauf auf } \\
\text { eine umweltfreundliche } \\
\text { Verpackung. }\end{array}$ & & & & & & & \\
\hline $\begin{array}{l}\text { Ich achte beim } \\
\text { Lebensmitteleinkauf vor } \\
\text { allem auf den Preis }\end{array}$ & & & & & & & \\
\hline
\end{tabular}


16. Auch im Folgenden geht es nochmals um Aspekte des Lebensmitteleinkaufs. Bitte kreuzen Sie auch hier an, ob die verschiedenen Aussagen auf Sie zutreffen!

\begin{tabular}{|c|c|c|c|c|c|c|c|}
\hline & $\begin{array}{c}\text { Trifft voll } \\
\text { und ganz zu }\end{array}$ & Trifft zu & $\begin{array}{c}\text { Trifft eher } \\
\text { zu }\end{array}$ & Teils/ teils & $\begin{array}{l}\text { Trifft eher } \\
\text { nicht zu }\end{array}$ & $\begin{array}{c}\text { Trifft nicht } \\
\mathrm{zu}\end{array}$ & $\begin{array}{c}\text { Trifft } \\
\text { überhaupt } \\
\text { nicht zu }\end{array}$ \\
\hline $\begin{array}{l}\text { Ich kaufe auch mal Produkte } \\
\text { von denen ich nicht genau } \\
\text { weiß, ob sie Gentechnik } \\
\text { enthalten. }\end{array}$ & & & & & & & \\
\hline $\begin{array}{l}\text { Ich achte selten darauf, ob in } \\
\text { einem Produkt } \\
\text { Konservierungsstoffe sind. }\end{array}$ & & & & & & & \\
\hline $\begin{array}{l}\text { Der Lebensmitteleinkauf muss } \\
\text { bei mir schnell gehen. }\end{array}$ & & & & & & & \\
\hline $\begin{array}{l}\text { Ich habe schon häufig } \\
\text { Lebensmittel einfach wegen } \\
\text { der auffälligen Verpackung } \\
\text { gekauft. }\end{array}$ & & & & & & & \\
\hline $\begin{array}{l}\text { Ich fühle mich gut über } \\
\text { Lebensmittel informiert. }\end{array}$ & & & & & & & \\
\hline $\begin{array}{l}\text { Früher hatte ich mehr } \\
\text { Vertrauen in die Qualität von } \\
\text { Lebensmitteln. }\end{array}$ & & & & & & & \\
\hline $\begin{array}{l}\text { Der Lebensmitteleinkauf ist } \\
\text { eine lästige Pflicht für mich. }\end{array}$ & & & & & & & \\
\hline $\begin{array}{l}\text { Ich prüfe sehr genau die } \\
\text { Produktinformationen auf der } \\
\text { Verpackung, bevor ich etwas } \\
\text { kaufe. }\end{array}$ & & & & & & & \\
\hline $\begin{array}{l}\text { Ich würde mir mehr } \\
\text { Informationen auf den } \\
\text { Lebensmittelverpackungen } \\
\text { wünschen. }\end{array}$ & & & & & & & \\
\hline $\begin{array}{l}\text { Beim Lebensmitteleinkauf } \\
\text { probiere ich gerne Neues aus. }\end{array}$ & & & & & & & \\
\hline $\begin{array}{l}\text { Obst und Gemüse müssen } \\
\text { perfekt aussehen, ich schaue } \\
\text { da sehr genau hin. }\end{array}$ & & & & & & & \\
\hline $\begin{array}{l}\text { Ich kaufe lieber höherpreisige } \\
\text { Lebensmittel, weil diese eine } \\
\text { bessere Qualität haben. }\end{array}$ & & & & & & & \\
\hline $\begin{array}{l}\text { Ich verzichte vollständig auf } \\
\text { Geschmacksverstärker. }\end{array}$ & & & & & & & \\
\hline $\begin{array}{l}\text { Es ist schwer, beim } \\
\text { Lebensmitteleinkauf zu } \\
\text { erkennen, ob die Umwelt bei } \\
\text { der Produktion geschont } \\
\text { wurde. }\end{array}$ & & & & & & & \\
\hline $\begin{array}{l}\text { Ich spare eher mal bei } \\
\text { Lebensmitteln als auf etwas } \\
\text { anderes zu verzichten. }\end{array}$ & & & & & & & \\
\hline $\begin{array}{l}\text { In meiner Kindheit hatten } \\
\text { Lebensmittel noch einen viel } \\
\text { besseren Geschmack. }\end{array}$ & & & & & & & \\
\hline $\begin{array}{l}\text { Ich esse was mir schmeckt, auf } \\
\text { Inhaltsstoffe achte ich kaum. }\end{array}$ & & & & & & & \\
\hline $\begin{array}{l}\text { Ich kaufe häufig Produkte, die } \\
\text { ich zuvor in der Werbung } \\
\text { gesehen habe. }\end{array}$ & & & & & & & \\
\hline $\begin{array}{l}\text { Bei Lebensmitteln die ich noch } \\
\text { nicht kenne bin ich sehr } \\
\text { vorsichtig. }\end{array}$ & & & & & & & \\
\hline $\begin{array}{l}\text { Ich kaufe nur Gentechnik-freie } \\
\text { Lebensmittel. }\end{array}$ & & & & & & & \\
\hline $\begin{array}{l}\text { Ein niedriger Preis ist } \\
\text { meistens ein Zeichen für } \\
\text { Lebensmittel von geringer } \\
\text { Qualität. }\end{array}$ & & & & & & & \\
\hline
\end{tabular}


17. Bitte geben Sie auch Ihre Einschätzung zu den folgenden Aussagen zur Ernährung ab:

\begin{tabular}{|c|c|c|c|c|c|c|c|}
\hline & $\begin{array}{l}\text { Trifft voll } \\
\text { und ganz zu }\end{array}$ & Trifft zu & $\begin{array}{c}\text { Trifft eher } \\
\mathrm{zu}\end{array}$ & Teils/ teils & $\begin{array}{c}\text { Trifft eher } \\
\text { nicht } \mathrm{zu}\end{array}$ & $\begin{array}{c}\text { Trifft nicht } \\
\mathrm{zu}\end{array}$ & $\begin{array}{l}\text { Trifft } \\
\text { überhaupt } \\
\text { nicht zu }\end{array}$ \\
\hline \multicolumn{8}{|l|}{$\begin{array}{l}\text { Ich ernähre mich möglichst } \\
\text { fettarm. }\end{array}$} \\
\hline \multicolumn{8}{|l|}{$\begin{array}{l}\text { Ich kontrolliere selten mein } \\
\text { Gewicht. }\end{array}$} \\
\hline \multicolumn{8}{|l|}{$\begin{array}{l}\text { Ich ernähre mich möglichst } \\
\text { kalorienarm. }\end{array}$} \\
\hline \multicolumn{8}{|l|}{$\begin{array}{l}\text { Eigentlich müsste ich mich } \\
\text { gesünder ernähren, aber ich } \\
\text { schaffe es einfach nicht. }\end{array}$} \\
\hline \multicolumn{8}{|l|}{$\begin{array}{l}\text { Ich zwinge mich, auch } \\
\text { Lebensmittel zu essen, die } \\
\text { nicht so gut schmecken, } \\
\text { aber gesund sind. }\end{array}$} \\
\hline \multicolumn{8}{|l|}{$\begin{array}{l}\text { Lebensmittel müssen für } \\
\text { mich einfach zuzubereiten } \\
\text { sein. }\end{array}$} \\
\hline $\begin{array}{l}\text { Ich finde es gut, dass die } \\
\text { Auswahl an Tiefkühl- und } \\
\text { Fertigprodukten immer } \\
\text { größer wird. }\end{array}$ & & & & & & & \\
\hline
\end{tabular}

18. Können Sie in etwa angeben, wie häufig Sie beim Kauf der verschiedenen Lebensmittel daran denken, dass diese Chemikalien enthalten könnten?

\begin{tabular}{|c|c|c|c|c|c|c|c|}
\hline & Immer & $\begin{array}{l}\text { (Fast) } \\
\text { immer }\end{array}$ & Häufig & Gelegentlich & Selten & Nie & $\begin{array}{l}\text { Esse/trinke } \\
\text { ich nicht }\end{array}$ \\
\hline Brot und Backwaren & & & & & & & \\
\hline $\begin{array}{l}\text { Getreideprodukte } \\
\text { (z.B. Muesli, } \\
\text { Nudeln, Reis) }\end{array}$ & & & & & & & \\
\hline Obst & & & & & & & \\
\hline Gemüse & & & & & & & \\
\hline $\begin{array}{l}\text { Milch und } \\
\text { Milchprodukte (inkl. } \\
\text { Käse, Joghurt) }\end{array}$ & & & & & & & \\
\hline Wurstwaren & & & & & & & \\
\hline Eier & & & & & & & \\
\hline
\end{tabular}


19. Im Folgenden haben wir nun zunächst einige Aussagen von Menschen zu ihren Lebenseinstellungen und Meinungen zu aktuellen Problemen zusammengestellt. Bitte lesen Sie diese durch und kreuzen Sie an, ob diese auch für Sie zutreffen!

\begin{tabular}{|c|c|c|c|c|c|c|c|}
\hline & $\begin{array}{l}\text { Trifft voll und } \\
\text { ganz zu }\end{array}$ & Trifft zu & $\begin{array}{c}\text { Trifft eher } \\
\text { zu }\end{array}$ & Teils/teils & \begin{tabular}{|l} 
Trifft eher \\
nicht zu
\end{tabular} & $\begin{array}{l}\text { Trifft nicht } \\
\text { zu }\end{array}$ & \begin{tabular}{|l|} 
Trifft \\
überhaupt
\end{tabular} \\
\hline $\begin{array}{l}\text { Computer und andere moderne } \\
\text { elektronische Geräte machen mir e } \\
\text { Spaß. }\end{array}$ & & & & & & & \\
\hline $\begin{array}{l}\text { Wenn ich genügend Geld hätte wür } \\
\text { nie mehr arbeiten. }\end{array}$ & & & & & & & \\
\hline Veränderungen beunruhigen mich. & & & & & & & \\
\hline $\begin{array}{l}\text { Es ist wichtig für mich, viel Geld zu } \\
\text { haben und teure Dinge zu besitzen. }\end{array}$ & & & & & & & \\
\hline $\begin{array}{l}\text { Die Politik muss sich darum kümm } \\
\text { dass jeder in diesem Land gut leber } \\
\text { kann. }\end{array}$ & & & & & & & \\
\hline $\begin{array}{l}\text { Es bringt nichts, wenn wir weniger } \\
\text { produzieren: andere nehmen nicht } \\
\text { Rücksicht. }\end{array}$ & & & & & & & \\
\hline $\begin{array}{l}\text { Ich mache mir keine Sorgen um di } \\
\text { Zukunft, das Jetzt ist entscheidend. }\end{array}$ & & & & & & & \\
\hline $\begin{array}{l}\text { Im Vergleich zu anderen Ländern } \\
\text { wir in Deutschland sehr unverantw } \\
\text { mit unseren Ressourcen um. }\end{array}$ & & & & & & & \\
\hline $\begin{array}{l}\text { Ich mag Leute nicht, die offen zeig } \\
\text { was sie besitzen. }\end{array}$ & & & & & & & \\
\hline $\begin{array}{l}\text { Ich bin unzufrieden, weil ich mir fin } \\
\text { zu wenig leisten kann. }\end{array}$ & & & & & & & \\
\hline $\begin{array}{l}\text { Der technische Fortschritt macht ff } \\
\text { mich das Leben lebenswert. }\end{array}$ & & & & & & & \\
\hline $\begin{array}{l}\text { Jeder ist selbst dafür verantwortlicl } \\
\text { gut er in diesem Land lebt. }\end{array}$ & & & & & & & \\
\hline $\begin{array}{l}\text { Lebenserfüllung ist nur durch } \\
\text { Pflichterfüllung möglich. }\end{array}$ & & & & & & & \\
\hline $\begin{array}{l}\text { Wenn wir so weitermachen wie bis } \\
\text { werden wir die Erde zerstören. }\end{array}$ & & & & & & & \\
\hline Ich brauche viel Abwechslung im I & & & & & & & \\
\hline $\begin{array}{l}\text { Meine Devise ist: Genießen und } \\
\text { möglichst angenehm leben. }\end{array}$ & & & & & & & \\
\hline $\begin{array}{l}\text { Ich fühle mich fest in meinem Land } \\
\text { seiner Kultur verwurzelt. }\end{array}$ & & & & & & & \\
\hline $\begin{array}{l}\text { Spaß zu haben ist das Wichtigste ir } \\
\text { Leben. }\end{array}$ & & & & & & & \\
\hline $\begin{array}{l}\text { Es sind schon viel zu viele natürlich } \\
\text { Ressourcen zerstört worden. }\end{array}$ & & & & & & & \\
\hline $\begin{array}{l}\text { Ich arbeite gern, um mir einiges lei } \\
\text { zu könnnen. }\end{array}$ & & & & & & & \\
\hline $\begin{array}{l}\text { Ich kann mir gut vorstellen, auf Da } \\
\text { Ausland zu leben. }\end{array}$ & & & & & & & \\
\hline $\begin{array}{l}\text { Am Klimawandel können wir nicht } \\
\text { ändern. }\end{array}$ & & & & & & & \\
\hline $\begin{array}{l}\text { Jeder der sich anstrengt kann sich } \\
\text { hocharbeiten. }\end{array}$ & & & & & & & \\
\hline $\begin{array}{l}\text { Ich fürchte, dass der technische } \\
\text { Fortschritt unser Leben zerstört. }\end{array}$ & & & & & & & \\
\hline $\begin{array}{l}\text { Die Bauern sind die Basis unseres } \\
\text { Wohlstands. }\end{array}$ & & & & & & & \\
\hline
\end{tabular}


20. Nun haben Sie es fast geschafft! Wir haben nur noch einige Fragen zu Ihrer Person! Zunächst: Wo sind Sie aufgewachsen?

\begin{tabular}{|l|l|l|l|l|l|}
\hline & In einem & In einer kleinen & In einer & In einer & In einer \\
größeren Stadt & Großstadt & Großstadt mit \\
In einem kleinen & größeren Dorf & Stadt (5.001 - & $(20.001-$ & $(100.001-$ & mehr als \\
500 Einwohiger als & $(501-5.000$ & 20.000 & 100.000 & 500.000 & 500.000 \\
\hline & Einwohner) & Einwohner) & Einwohner $)$ & Einwohner $)$ & Einwohnern \\
\hline & & & & & \\
\hline
\end{tabular}

21. Und wo wohnen Sie zurzeit?

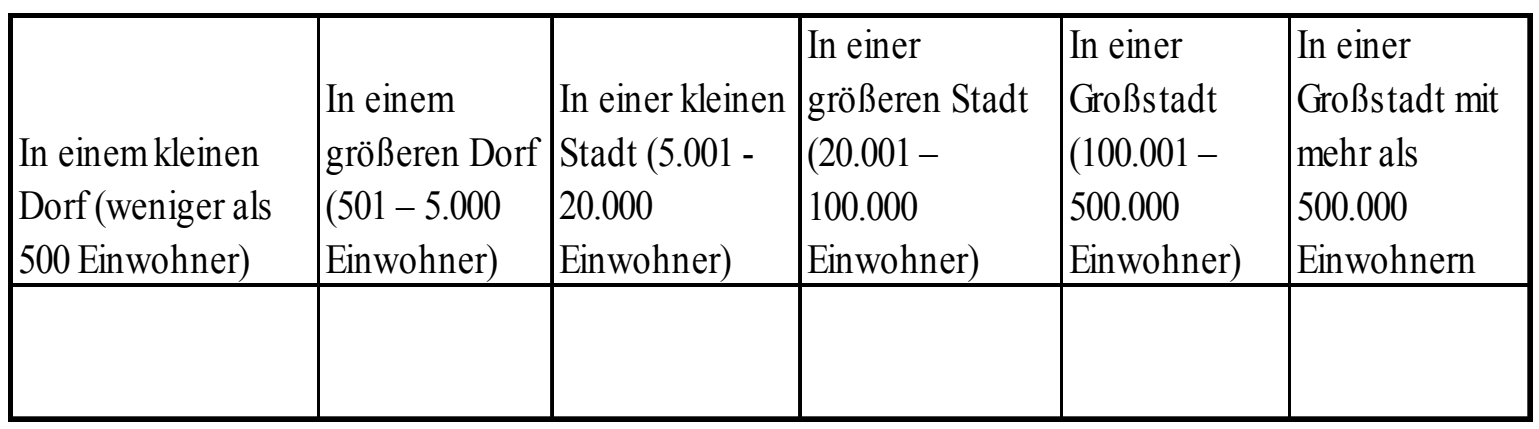

22. Wie viele Personen wohnen in Ihrem Haushalt (inklusive Ihnen selbst)?

23. Wie wohnen Sie?

\begin{tabular}{|c|c|c|c|c|c|c|c|c|}
\hline Allein & $\begin{array}{l}\text { Allein, } \\
\text { Kinder sind } \\
\text { ausgezogen }\end{array}$ & $\begin{array}{l}\text { Mit } \\
\text { Partner(in) } \\
\text { und Kindern }\end{array}$ & $\begin{array}{l}\text { Mit } \\
\text { Partner(in) } \\
\text { ohne Kinder }\end{array}$ & $\begin{array}{l}\text { Mit } \\
\text { Partner(in), } \\
\text { Kinder sind } \\
\text { ausgezogen }\end{array}$ & $\begin{array}{l}\text { Alleiner- } \\
\text { ziehend }\end{array}$ & $\begin{array}{l}\text { In einer Wohn- } \\
\text { gemeinschaft }\end{array}$ & $\begin{array}{l}\text { Bei den } \\
\text { Eltern }\end{array}$ & $\begin{array}{l}\text { Bei den } \\
\text { Kindern }\end{array}$ \\
\hline & & & & & & & & \\
\hline
\end{tabular}

24. Wie viele Kinder (unter 18 Jahren) wohnen in Ihrem Haushalt?

25. Können Sie auch in etwa schätzen, wie viel Sie pro Woche für Lebensmittel ausgeben? 


\section{Appendix 10: Cross cultural survey in Mexico (Spanish version)}

1. ¿Quién es responsable de la compra de alimentos en su hogar?

\begin{tabular}{|c|c|c|c|c|}
\hline Solamente yo & $\begin{array}{c}\text { Yo la mayor } \\
\text { parte del } \\
\text { tiempo }\end{array}$ & $\begin{array}{c}\text { Yo y mi pareja/ amigos(as)/las } \\
\text { personas con quien vivo }\end{array}$ & $\begin{array}{c}\text { La mayor parte } \\
\text { del tiempo alguien } \\
\text { más }\end{array}$ & $\begin{array}{c}\text { Exclusivamente } \\
\text { alguien más }\end{array}$ \\
\hline & & & & \\
\hline
\end{tabular}

\section{Género:}

Masculino

Femenino

3. ¿En dónde vive?

Distrito Federal___ Otras ciudades

4. ¿Cuántas veces compra alimentos en los siguientes establecimientos?

\begin{tabular}{|l|l|l|l|l|l|l|}
\hline & $\begin{array}{c}\text { Cerca de } \\
\text { dos veces } \\
\text { por semana }\end{array}$ & $\begin{array}{c}\text { Cerca de } \\
\text { una vez por } \\
\text { semana }\end{array}$ & $\begin{array}{c}\text { Menos de } \\
\text { una vez por } \\
\text { semana }\end{array}$ & $\begin{array}{c}\text { Una vez al } \\
\text { mes }\end{array}$ & $\begin{array}{c}\text { Menos de } \\
\text { una vez al } \\
\text { mes }\end{array}$ & Casi nunca \\
\hline $\begin{array}{l}\text { Mypermercados (ej. Wal } \\
\text { Comercial Mexicana, } \\
\text { Chedraui, Soriana) }\end{array}$ & & & & & & \\
\hline $\begin{array}{l}\text { Supermercados (ej. } \\
\text { Sumesa, Superama) }\end{array}$ & & & & & & \\
\hline Mercados tradicionales & & & & & & \\
\hline Tianguis & & & & & & \\
\hline $\begin{array}{l}\text { Tiendas especializadas } \\
\text { (ej. panadería, } \\
\text { carnicería, pescadería, } \\
\text { verdulería) }\end{array}$ & & & & & & \\
\hline $\begin{array}{l}\text { Tiendas de productos } \\
\text { orgánicos }\end{array}$ & & & & & & \\
\hline Tianguis orgánicos & & & & & & \\
\hline
\end{tabular}


5. A continuación hay una serie de preguntas relacionadas únicamente con los productos orgánicos. Todos hablan sobre los productos orgánicos pero el significado muchas veces es diferente. ¿Cómo describiría usted los productos orgánicos? (por favor mencione tres aspectos ó características).
A:
B:
C:

6. De las siguientes etiquetas orgánicas ¿Cuáles conoce?
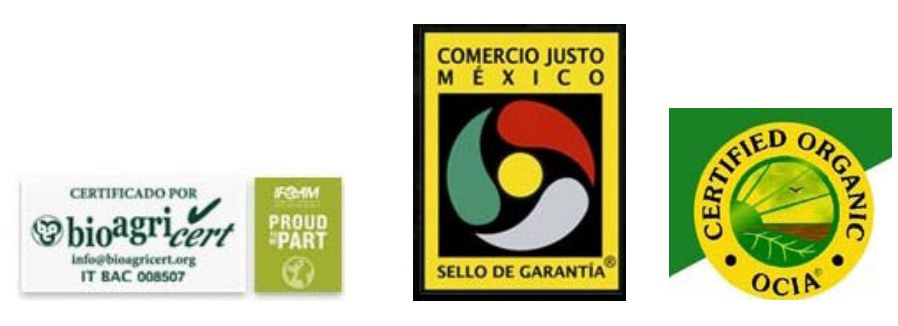

5

6

7
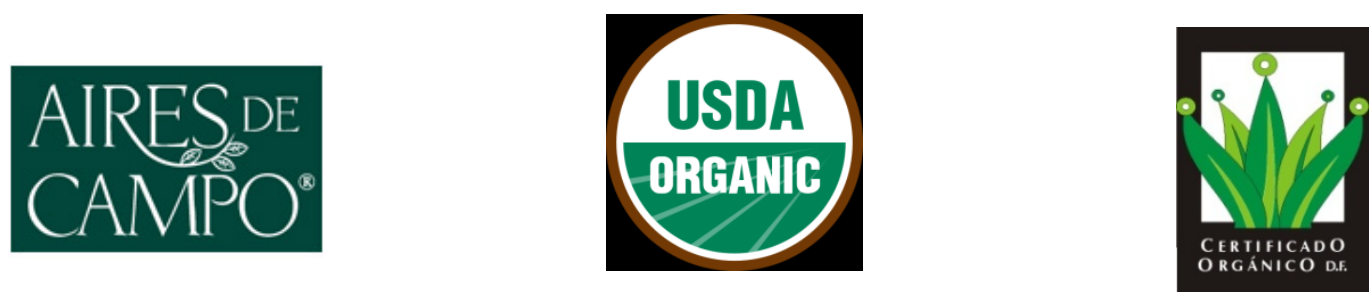

7. ¿Ha comprado productos orgánicos con alguna de las etiqueta anteriores o que provengan de algún tipo de cultivo alternativo?

$\mathrm{Si}$

No

8. Aproximadamente ¿Cuánto tiempo lleva comprando productos orgánicos?

\begin{tabular}{|c|c|c|c|c|c|}
\hline $\begin{array}{c}\text { Menos de un } \\
\text { año }\end{array}$ & $1-2$ años & $2-3$ años & $3-4$ años & $4-5$ años & $\begin{array}{c}\text { Más de 5 } \\
\text { años }\end{array}$ \\
\hline & & & & & \\
\hline
\end{tabular}


9. ¿Cómo conocio la existencia de los productos orgánicos?

\begin{tabular}{|c|c|c|c|c|c|c|}
\hline $\begin{array}{c}\text { Por un } \\
\text { amigo/a, } \\
\text { conocido/a }\end{array}$ & $\begin{array}{c}\text { Por un } \\
\text { familiar }\end{array}$ & $\begin{array}{c}\text { Cuando } \\
\text { anduve / viví } \\
\text { en el } \\
\text { extranjero }\end{array}$ & $\begin{array}{c}\text { Escuche } \\
\text { alguna noticia } \\
\text { en la radio ó } \\
\text { tv }\end{array}$ & $\begin{array}{c}\text { Escuche una } \\
\text { platica / } \\
\text { conferencia }\end{array}$ & $\begin{array}{c}\text { Ví algún } \\
\text { anuncio } \\
\text { publicitario }\end{array}$ & $\begin{array}{c}\text { Por alguna } \\
\text { degustación }\end{array}$ \\
\hline & & & & & \\
\hline
\end{tabular}

10. ¿Con qué frecuencia compra los siguientes grupos de alimentos con calidad orgánica?

\begin{tabular}{|c|c|c|c|c|c|c|c|}
\hline & Siempre & $\begin{array}{c}\text { Casi } \\
\text { siempre }\end{array}$ & $\begin{array}{l}\text { Frequente- } \\
\text { mente }\end{array}$ & Algunas veces & Rara vez & Nunca & $\begin{array}{l}\text { No } \\
\text { como/no } \\
\text { tomo/no } \\
\text { consumo }\end{array}$ \\
\hline $\begin{array}{l}\text { Panadería y } \\
\text { biscochería orgánica }\end{array}$ & & & & & & & \\
\hline $\begin{array}{l}\text { Cereales, granola } \\
\text { orgánica }\end{array}$ & & & & & & & \\
\hline Frutas orgánicas & & & & & & & \\
\hline Verduras orgánicas & & & & & & & \\
\hline $\begin{array}{l}\text { Leche y productos } \\
\text { lácteos (queso, } \\
\text { yogurth) orgánicos }\end{array}$ & & & & & & & \\
\hline $\begin{array}{l}\text { Carne y embutidos } \\
\text { orgánicos }\end{array}$ & & & & & & & \\
\hline Huevo orgánico & & & & & & & \\
\hline
\end{tabular}


11. En relación con la producción orgánica ¿Qué tanto concuerda con las siguientes frases?

\begin{tabular}{|c|c|c|c|c|c|c|c|}
\hline & $\begin{array}{l}\text { Muy de } \\
\text { acuerdo }\end{array}$ & De acuerdo & $\begin{array}{l}\text { Algo de } \\
\text { acuerdo }\end{array}$ & $\begin{array}{c}\text { Nide } \\
\text { acuerdo ni } \\
\text { en } \\
\text { desacuerdo }\end{array}$ & $\begin{array}{c}\text { Algo en } \\
\text { desacuerdo }\end{array}$ & $\begin{array}{c}\text { En } \\
\text { desacuerdo }\end{array}$ & $\begin{array}{c}\text { Muy en } \\
\text { desacuerdo }\end{array}$ \\
\hline $\begin{array}{l}\text { Compro productos orgánicos de } \\
\text { origen animal porque estoy } \\
\text { convencido/a de que las formas } \\
\text { de producción son mejores. }\end{array}$ & & & & & & & \\
\hline $\begin{array}{l}\text { Solo con la carne orgánica puedo } \\
\text { estar seguro/a que los animales } \\
\text { han sido tratados sin hormonas y } \\
\text { antibióticos. }\end{array}$ & & & & & & & \\
\hline $\begin{array}{l}\text { La producción orgánica es mejor } \\
\text { para el medio ambiente. }\end{array}$ & & & & & & & \\
\hline $\begin{array}{l}\text { Los productores orgánicos ganan } \\
\text { más dinero que otros } \\
\text { productores. }\end{array}$ & & & & & & & \\
\hline $\begin{array}{l}\text { Los productos orgánicos tienen } \\
\text { mejor sabor. }\end{array}$ & & & & & & & \\
\hline $\begin{array}{l}\text { Los productos orgánicos son } \\
\text { más saludables que otros } \\
\text { productos. }\end{array}$ & & & & & & & \\
\hline $\begin{array}{l}\text { La producción orgánica favorece } \\
\text { a los pequeños y medianos } \\
\text { productores. }\end{array}$ & & & & & & & \\
\hline $\begin{array}{l}\text { Los productos orgánicos son } \\
\text { sencillamente demasiado caros. }\end{array}$ & & & & & & & \\
\hline $\begin{array}{l}\text { No creo que los animales tengan } \\
\text { un mejor trato en la producción } \\
\text { orgánica. }\end{array}$ & & & & & & & \\
\hline
\end{tabular}


12. Compra de alimentos A continuación hay una seríe de frases relacionadas con la compra de alimentos. De acuerdo a sus críterios de compra de alimentos señale ¿Qué tanto concuerda con cada una de ellas?

\begin{tabular}{|c|c|c|c|c|c|c|c|}
\hline & $\begin{array}{l}\text { Muy de } \\
\text { acuerdo }\end{array}$ & De acuerdo & $\begin{array}{l}\text { Algo de } \\
\text { acuerdo }\end{array}$ & \begin{tabular}{|c} 
Ni de \\
acuerdo ni \\
en \\
desacuerdo
\end{tabular} & $\begin{array}{l}\text { Algo en } \\
\text { desacuerdo }\end{array}$ & En desacuerdo & $\begin{array}{c}\text { Muy en } \\
\text { desacuerdo }\end{array}$ \\
\hline \multicolumn{8}{|l|}{$\begin{array}{l}\text { Busco alimentos que no contengan ningún aroma } \\
\text { artificial. }\end{array}$} \\
\hline \multicolumn{8}{|l|}{$\begin{array}{l}\text { Prefiero comprar alimentos caros porque estos } \\
\text { tienen una mejor calidad. }\end{array}$} \\
\hline \multicolumn{8}{|l|}{$\begin{array}{l}\text { Cuando encuentro alimentos que comía cuando } \\
\text { era niño/a los compro de inmediato. }\end{array}$} \\
\hline \multicolumn{8}{|l|}{$\begin{array}{l}\text { Cuando compro alimentos antes que nada me } \\
\text { fijo en el precio. }\end{array}$} \\
\hline \multicolumn{8}{|l|}{ Prefiero frutas y verduras mexicanas. } \\
\hline \multicolumn{8}{|l|}{$\begin{array}{l}\text { Compro los alimentos en la tiendas que estan } \\
\text { cerca de mi casa ú oficina. }\end{array}$} \\
\hline \multicolumn{8}{|l|}{ Compro marcas conocidas de alimentos. } \\
\hline \multicolumn{8}{|l|}{$\begin{array}{l}\text { Compro alimentos con etiqueta de comercio } \\
\text { justo. }\end{array}$} \\
\hline \multicolumn{8}{|l|}{$\begin{array}{l}\text { Es díficil saber si se ha afectado el medio } \\
\text { ambiente con la producción de alimentos. }\end{array}$} \\
\hline \multicolumn{8}{|l|}{$\begin{array}{l}\text { A veces compro productos que no estoy seguro/a } \\
\text { si han sido géneticamente modificados. }\end{array}$} \\
\hline \multicolumn{8}{|l|}{$\begin{array}{l}\text { Muchas veces me parece que los productos que } \\
\text { provienen del extranjero son mejores que los } \\
\text { hechos en México. }\end{array}$} \\
\hline \multicolumn{8}{|l|}{ Las compras de alimentos deben ser rápidas. } \\
\hline \multicolumn{8}{|l|}{$\begin{array}{l}\text { Soy muy ciudadoso/a con los alimentos que no } \\
\text { conozco. }\end{array}$} \\
\hline \multicolumn{8}{|l|}{$\begin{array}{l}\text { En productos lácteos y cárnicos es díficil saber si } \\
\text { se ha procurado el bienestar de los animales. }\end{array}$} \\
\hline \multicolumn{8}{|l|}{$\begin{array}{l}\text { Frecuentemente compro alimentos } \\
\text { sencillamente por el empaque. }\end{array}$} \\
\hline \multicolumn{8}{|l|}{$\begin{array}{l}\text { La mayor parte de nuestros ingresos esta } \\
\text { destinada a la compra de alimentos. }\end{array}$} \\
\hline \multicolumn{8}{|l|}{$\begin{array}{l}\text { Compro solamente alimentos libres de } \\
\text { transgénicos. }\end{array}$} \\
\hline \multicolumn{8}{|l|}{$\begin{array}{l}\text { Como lo que a mi ma agrada y rara vez me fijo en } \\
\text { los ingredientes que contiene. }\end{array}$} \\
\hline \multicolumn{8}{|l|}{ Para mi es tedioso comprar alimentos. } \\
\hline \multicolumn{8}{|l|}{$\begin{array}{l}\text { En cuanto alimentos, me gusta probar cosas } \\
\text { nuevas. }\end{array}$} \\
\hline \multicolumn{8}{|l|}{$\begin{array}{l}\text { Rara vez me fijo si los productos contienen } \\
\text { conservadores. }\end{array}$} \\
\hline \multicolumn{8}{|l|}{$\begin{array}{l}\text { Me gustaría que los empaques de alimentos } \\
\text { contuvieran más información. }\end{array}$} \\
\hline \multicolumn{8}{|l|}{$\begin{array}{l}\text { Evito los productos que contienen saborizantes } \\
\text { artificiales. }\end{array}$} \\
\hline \multicolumn{8}{|l|}{$\begin{array}{l}\text { Me siento bien informado/a respecto a los } \\
\text { alimentos. }\end{array}$} \\
\hline \multicolumn{8}{|l|}{$\begin{array}{l}\text { Me parece bien que la variedad de alimentos } \\
\text { procesados vayan en aumento. }\end{array}$} \\
\hline \multicolumn{8}{|l|}{$\begin{array}{l}\text { Antes confiaba más en la calidad de los } \\
\text { alimentos. }\end{array}$} \\
\hline \multicolumn{8}{|l|}{$\begin{array}{l}\text { Las frutas y verduras deben tener una apariencia } \\
\text { perfecta, las examino muy cuidadosamente. }\end{array}$} \\
\hline \multicolumn{8}{|l|}{$\begin{array}{l}\text { Evito productos cuyo país de procedencia } \\
\text { desconozco. }\end{array}$} \\
\hline $\begin{array}{l}\text { Frecuentemente compro los alimentos que he } \\
\text { visto anteriormente anunciados. }\end{array}$ & & & & & & & \\
\hline $\begin{array}{l}\text { La mayoría de las veces un precio bajo es una } \\
\text { señal de baja la calidad de los alimentos. }\end{array}$ & & & & & & & \\
\hline $\begin{array}{l}\text { En mi infancia, los alimentos tenian un mejor } \\
\text { sabor. }\end{array}$ & & & & & & & \\
\hline $\begin{array}{l}\text { Me fijo en que los alimentos sean producidos de } \\
\text { forma que no contaminen el medio ambiente. }\end{array}$ & & & & & & & \\
\hline $\begin{array}{l}\text { Antes de comprar alimentos leo cuidadosamente } \\
\text { la información en el empaque. }\end{array}$ & & & & & & & \\
\hline $\begin{array}{l}\text { Para mi, los alimentos deben ser fáciles de } \\
\text { preparar. }\end{array}$ & & & & & & & \\
\hline
\end{tabular}


13. Alimentación Considerando su alimentación ¿Qué tanto concuerda con las siguientes frases?

\begin{tabular}{|c|c|c|c|c|c|c|c|}
\hline & $\begin{array}{l}\text { Muy de } \\
\text { acuerdo }\end{array}$ & De acuerdo & $\begin{array}{l}\text { Algo de } \\
\text { acuerdo }\end{array}$ & \begin{tabular}{|c} 
Nide \\
acuerdo ni \\
en \\
desacuerdo \\
\end{tabular} & $\begin{array}{c}\text { Algo en } \\
\text { desacuerdo }\end{array}$ & $\begin{array}{c}\text { En } \\
\text { desacuerdo }\end{array}$ & $\begin{array}{c}\text { Muy en } \\
\text { desacuerdo }\end{array}$ \\
\hline $\begin{array}{l}\text { En la medidad de lo } \\
\text { posible como alimentos } \\
\text { bajos en grasas. }\end{array}$ & & & & & & & \\
\hline $\begin{array}{l}\text { Rara vez controlo mi } \\
\text { peso. }\end{array}$ & & & & & & & \\
\hline $\begin{array}{l}\text { Me esfuerzo por comer } \\
\text { alimentos que no saben } \\
\text { bien pero son saludables }\end{array}$ & & & & & & & \\
\hline $\begin{array}{l}\text { En la medida de lo } \\
\text { posible como alimentos } \\
\text { bajos en calorías. }\end{array}$ & & & & & & & \\
\hline $\begin{array}{l}\text { En realidad debería } \\
\text { alimentarme sanamente } \\
\text { pero no lo logro. }\end{array}$ & & & & & & & \\
\hline
\end{tabular}

14. De acuerdo a su compra de los siguientes grupos de alimentos ¿Con qué frecuencia piensa en que estos pueden contener químicos? (si no consume productos de alguno de los grupos de alimentos por favor marque la opción No como/ No tomo/No consumo)

\begin{tabular}{|l|l|l|l|l|l|l|l|}
\hline & Siempre & $\begin{array}{c}\text { Casi } \\
\text { siempre }\end{array}$ & $\begin{array}{c}\text { Frequente- } \\
\text { mente }\end{array}$ & Algunas veces & Rara vez & Nunca & $\begin{array}{l}\text { No } \\
\text { como/no } \\
\text { tomo/no } \\
\text { consumo }\end{array}$ \\
\hline $\begin{array}{l}\text { Panadería y } \\
\text { biscochería orgánica }\end{array}$ & & & & & & & \\
\hline $\begin{array}{l}\text { Cereales, granola } \\
\text { orgánica }\end{array}$ & & & & & & & \\
\hline Frutas orgánicas
\end{tabular}


15. A continuación hay una serie de frases relacionadas con actitudes y algunos temas de actualidad, indique ¿Qué tan de acuerdo o desacuerdo esta con cada una de ellas?

\begin{tabular}{|c|c|c|c|c|c|c|c|}
\hline & $\begin{array}{l}\text { Muy de } \\
\text { acuerdo }\end{array}$ & De acuerdo & $\begin{array}{l}\text { Algo de } \\
\text { acuerdo }\end{array}$ & \begin{tabular}{|c|} 
Ni de \\
acuerdo ni \\
en \\
desacuerdo
\end{tabular} & $\begin{array}{c}\text { Algo en } \\
\text { desacuerdo }\end{array}$ & $\begin{array}{c}\text { En } \\
\text { desacuerdo }\end{array}$ & $\begin{array}{c}\text { Muy en } \\
\text { desacuerdo }\end{array}$ \\
\hline $\begin{array}{l}\text { Las computadoras y otros } \\
\text { aparatos electrónicos son } \\
\text { divertidos. }\end{array}$ & & & & & & & \\
\hline $\begin{array}{l}\text { No se puede hacer nada contra el } \\
\text { cambio climático. }\end{array}$ & & & & & & & \\
\hline Lo más importante es consentirse. & & & & & & & \\
\hline $\begin{array}{l}\text { Los agricultores y campesinos son } \\
\text { la base de nuestro bienestar. }\end{array}$ & & & & & & & \\
\hline $\begin{array}{l}\text { Es importante tener mucho dinero } \\
\text { y poseer cosas caras. }\end{array}$ & & & & & & & \\
\hline $\begin{array}{l}\text { Me puedo imaginar viviendo para } \\
\text { siempre en el extranjero. }\end{array}$ & & & & & & & \\
\hline $\begin{array}{l}\text { Estoy insatisfecho porque } \\
\text { económicamente me puedo } \\
\text { permitir muy poco. }\end{array}$ & & & & & & & \\
\hline Necesito variedad en la vida. & & & & & & & \\
\hline $\begin{array}{l}\text { Si continúamos como ahora, } \\
\text { destruiremos la tierra (nuestra } \\
\text { fuente de recursos). }\end{array}$ & & & & & & & \\
\hline $\begin{array}{l}\text { Temo que los avances } \\
\text { tecnológicos destruyan nuestra } \\
\text { vida. }\end{array}$ & & & & & & & \\
\hline $\begin{array}{l}\text { Las autoridades y el gobierno } \\
\text { deberían preocuparse por que } \\
\text { todos/as en este país podamos } \\
\text { vivir bien. }\end{array}$ & & & & & & & \\
\hline $\begin{array}{l}\text { La realización en la vida solo es } \\
\text { posible mediante el cumplimiento } \\
\text { de los deberes. }\end{array}$ & & & & & & & \\
\hline $\begin{array}{l}\text { Me siento fuertemente arraigado/a } \\
\text { a mi país y su cultura. }\end{array}$ & & & & & & & \\
\hline $\begin{array}{l}\text { Si produzco menos basura no } \\
\text { cambia la situación, porque a otras } \\
\text { personas no les interesa el medio } \\
\text { ambiente. }\end{array}$ & & & & & & & \\
\hline Los cambios me perturban. & & & & & & & \\
\hline $\begin{array}{l}\text { El futuro no me procupa, lo } \\
\text { principal es el presente. }\end{array}$ & & & & & & & \\
\hline Trabajo para darme algunos lujos. & & & & & & & \\
\hline $\begin{array}{l}\text { En este país cualquiera que se } \\
\text { esfuerce puede ascender de clase } \\
\text { social. }\end{array}$ & & & & & & & \\
\hline $\begin{array}{l}\text { En comparación con otros paises } \\
\text { en México somos muy } \\
\text { irresponsables con nuestros } \\
\text { recursos naturales. }\end{array}$ & & & & & & & \\
\hline $\begin{array}{l}\text { La diversión es lo más importante } \\
\text { en la vida. }\end{array}$ & & & & & & & \\
\hline $\begin{array}{l}\text { Los avances tecnológicos pueden } \\
\text { mejorar nuestra calidad de vida. }\end{array}$ & & & & & & & \\
\hline $\begin{array}{l}\text { Cada uno/a es responsable de que } \\
\text { tan bien vive en este país. }\end{array}$ & & & & & & & \\
\hline $\begin{array}{l}\text { No me gusta la gente que muestra } \\
\text { sus pertenencias abiertamente. }\end{array}$ & & & & & & & \\
\hline $\begin{array}{l}\text { Me siento fuertemente identificado } \\
\text { con el estilo de vida de otros } \\
\text { países (ej. Estados Unidos, } \\
\text { Canada, Europa). }\end{array}$ & & & & & & & \\
\hline $\begin{array}{l}\text { Se han destruido ya muchos } \\
\text { recursos naturales. }\end{array}$ & & & & & & & \\
\hline
\end{tabular}


16. ¿En qué año nació? 19

17. ¿En dondé creció usted?
Cuidad
Provincia
Suburbio

18. ¿Cuál es el máximo nivel de educación formal que ha recibido?

\begin{tabular}{|l|l|l|l|l|l|l|}
\hline Primaria & Secundaria & Preparatoria & Universidad & Maestria & Doctorado & Sin estudios \\
\hline & & & & & & \\
\hline
\end{tabular}

19. ¿Cuántas personas viven en su casa incluyendose usted?

20. ¿Con quién(es) vive? Yo vivo...

\begin{tabular}{|c|c|c|c|c|c|c|}
\hline Solo/a & $\begin{array}{c}\text { Solo/a (los } \\
\text { hijos se han } \\
\text { mudado) }\end{array}$ & $\begin{array}{c}\text { Con pareja e } \\
\text { hijos }\end{array}$ & $\begin{array}{c}\text { Con pareja } \\
\text { sin hijos (los } \\
\text { hijos se han } \\
\text { mudado) }\end{array}$ & $\begin{array}{c}\text { Sin pareja con } \\
\text { hijos }\end{array}$ & $\begin{array}{c}\text { Comparto } \\
\text { vivienda }\end{array}$ & $\begin{array}{c}\text { Con mis } \\
\text { padres }\end{array}$ \\
\hline & & & & & \\
\hline
\end{tabular}

21. ¿Cuántos menores de edad viven en su casa?

22. Aproximadamente ¿Cuál es el total de los ingresos netos en su hogar al mes? (los ingresos netos son el total de todos los ingresos al hogar en el periodo de un mes)

\begin{tabular}{|c|c|c|c|c|}
\hline $\begin{array}{c}\text { Menos de } \\
6,800 \$ \mathrm{Mx}\end{array}$ & $6,801-11,600$ & $11,601-$ & $\begin{array}{c}35,000- \\
8 \mathrm{Mx}\end{array}$ & $\begin{array}{c}\text { Mas de } \\
34,999 \$ \mathrm{Mx}\end{array}$ \\
\hline & & & & \\
& & & & \\
\hline
\end{tabular}

23. Aproximadamente ¿A cuánto asciende su gasto semanal en alimentos en $\$ M x$ ? 


\section{Appendix 11: Cross cultural survey (English version)}

1. Firstly, who is responsible for the purchasing of food?

\begin{tabular}{|c|c|c|c|c|}
\hline Just me & $\begin{array}{c}\text { Predominantly } \\
\text { me }\end{array}$ & $\begin{array}{c}\text { My partner(s)/ roommate } \\
\text { and me more or less } \\
\text { equally }\end{array}$ & $\begin{array}{c}\text { Mostly } \\
\text { someone else }\end{array}$ & $\begin{array}{c}\text { Only } \\
\text { someone else }\end{array}$ \\
\hline & & & & \\
\hline
\end{tabular}

2. What is your Gender?
Woman
Man

3. In which federal state do you live?

\begin{tabular}{|l|l|}
\hline Baden- & \\
Württemberg & \\
\hline Bayern \\
\hline Bremen \\
\hline Hamburg \\
\hline Niedersachsen & \\
\hline Schleswig-Holstein & \\
\hline Hessen & \\
\hline Nordrhein- & \\
Westfalen & \\
\hline Rheinland-Pfalz & \\
\hline Saarland & \\
\hline Berlin & \\
\hline Brandenburg & \\
Mecklenburg- & \\
Vorpommern & \\
\hline Sachsen & \\
\hline Sachsen-Anhalt & \\
\hline Thüringen & \\
\hline
\end{tabular}

4. When have you born? 19 
5. Could you say what is approximately your total household net incomes monthly in $€$ ? (Please check one box)

\begin{tabular}{|l|l|l|l|l|l|l|l|}
\hline$<900 €$ & $900-$ & $1.500-$ & $2.000-$ & $3.200-$ & $4.500-$ & & No \\
& $1.499 €$ & $1.999 €$ & $3.199 €$ & $4.499 €$ & $5.499 €$ & $>5.500$ & answer \\
\hline
\end{tabular}

6. What is the highest level of formal education you have completed? (Please check one box)

\begin{tabular}{|c|c|c|c|c|c|c|}
\hline $\begin{array}{c}\text { Basic school } \\
\text { leaving } \\
\text { certificate }\end{array}$ & $\begin{array}{c}\text { Advanced } \\
\text { Vocational } \\
\text { Certificate }\end{array}$ & A-level & $\begin{array}{c}\text { University } \\
\text { degree }\end{array}$ & PhD & $\begin{array}{c}\text { Still at } \\
\text { school }\end{array}$ & $\begin{array}{c}\text { No qualification } \\
\text { (as yet) }\end{array}$ \\
\hline
\end{tabular}

7. How often do you buy organic food products per week in the following stores?

\begin{tabular}{|l|l|l|l|l|l|l|}
\hline & $\begin{array}{l}\text { Several } \\
\text { times per } \\
\text { week }\end{array}$ & $\begin{array}{l}\text { About once } \\
\text { per week }\end{array}$ & $\begin{array}{l}\text { About twice } \\
\text { per week }\end{array}$ & $\begin{array}{l}\text { About once } \\
\text { per month }\end{array}$ & $\begin{array}{l}\text { Less than } \\
\text { once per } \\
\text { month }\end{array}$ & $\begin{array}{l}\text { Almost } \\
\text { never }\end{array}$ \\
\hline $\begin{array}{l}\text { Hypermarket (Real or } \\
\text { Kaufland) }\end{array}$ & & & & \\
\hline $\begin{array}{l}\text { Supermarket (Edeka, } \\
\text { Rewe) }\end{array}$ & & & & \\
\hline $\begin{array}{l}\text { Discounter (Aldi, Lidl) } \\
\text { Week market }\end{array}$ & & & & & \\
\hline $\begin{array}{l}\text { Fruit shop } \\
\text { Organic supermarket }\end{array}$ & & & & & \\
\hline $\begin{array}{l}\text { Organic shop/ Health } \\
\text { shop }\end{array}$ & & & & & \\
Direct marketing & & & & & \\
\hline
\end{tabular}


8. Now there is a list of issues about the purchasing of food. Please check a box that you better agree with them.

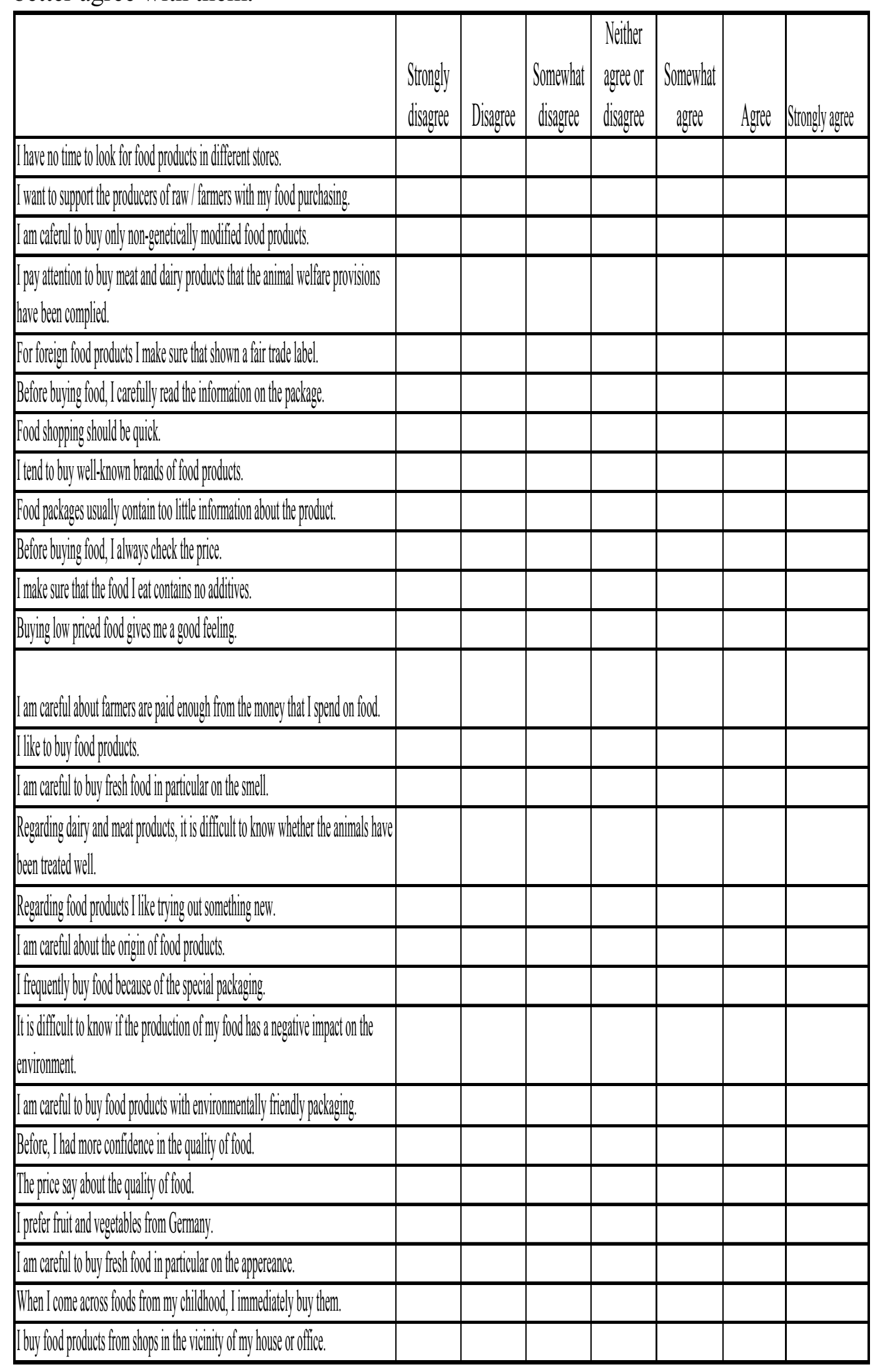


9. Now a few questions about your cooking behaviour please check a box that you better agree with.

\begin{tabular}{|c|c|c|c|c|c|c|}
\hline $\begin{array}{l}\text { I think that meals should be fast to prepare. } \\
\text { I like to cook. } \\
\text { Ilike cookbooks. } \\
\text { I like to try new recipes. } \\
\text { I think that meals should be easy to prepare. }\end{array}$ & Strongly disagree & Disagree & $\begin{array}{l}\text { Somewhat } \\
\text { disagree }\end{array}$ & $\begin{array}{c}\text { Neither agree or } \\
\text { disagree }\end{array}$ & $\begin{array}{c}\text { Somewhat } \\
\text { agree }\end{array}$ & Agree Strongly agree \\
\hline
\end{tabular}

24. Many people talk about organic products, but the meaning is often different. How would you describe organic products? (Please give three aspects).
A:
B:
C:

25. Which of the organic label (certification) shown below do you? Please check all that you know.

1
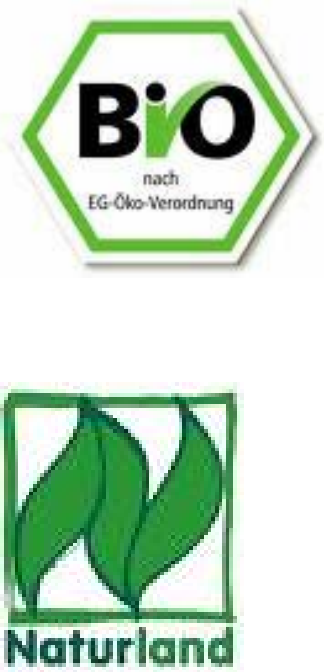

2

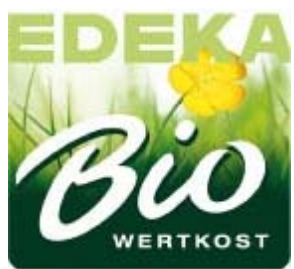

5

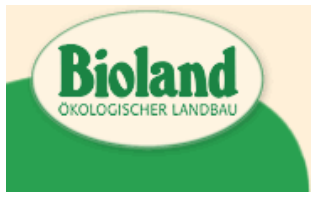

3

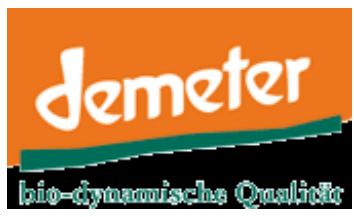

4

\section{BIOBIO}

26. Have you ever purchased organic food? (Products with someone of the label showed above?

Yes

No 
12. How much time do you have purchasing organic products?

\begin{tabular}{|c|l|l|l|l|l|l|}
\hline $\begin{array}{c}\text { Less than } \\
\text { one year }\end{array}$ & $1-2$ Years & 2-3 Years & 3-4 Years & 4-5 Years & 5-6 Years & $\begin{array}{c}\text { More than 6 } \\
\text { Years }\end{array}$ \\
\hline & & & & & & \\
\hline
\end{tabular}

13. How often do you buy the following nutritional groups in organic quality?

\begin{tabular}{|l|l|l|l|l|l|l|}
\hline & Always & $\begin{array}{c}\text { Almost } \\
\text { always }\end{array}$ & Frequently & Ocasionally & Rarely & Never \\
\hline $\begin{array}{l}\text { Eggs } \\
\text { Meat and meat } \\
\text { products }\end{array}$ & & & & & \\
\hline Bread and pastries & & & & & \\
\hline Fruits & & & & & \\
\hline Cereals an muesli & & & & & \\
\hline Vegetables & & & & & \\
\hline $\begin{array}{l}\text { Milk and Dairy } \\
\text { products }\end{array}$ & & & & \\
\hline
\end{tabular}

Thank you very much for your previous answers, for our research on consumer behaviour, it is important to know what of the followings values and principal in life are important to you.

14. There are below different principals and values that might play a role in life. Please score them according to the importance that these have in your life.

\begin{tabular}{|c|c|c|c|c|c|c|c|}
\hline & $\begin{array}{l}\text { Extremely } \\
\text { important }\end{array}$ & \begin{tabular}{|l}
$\begin{array}{l}\text { Very } \\
\text { important }\end{array}$ \\
\end{tabular} & \begin{tabular}{|l} 
Moderately \\
important
\end{tabular} & Neutral & \begin{tabular}{|l|l}
$\begin{array}{l}\text { Low } \\
\text { importance }\end{array}$ \\
\end{tabular} & \begin{tabular}{|l|l} 
Not at all \\
important
\end{tabular} & \begin{tabular}{|l|}
$\begin{array}{l}\text { Contrary to } \\
\text { my viewpoint }\end{array}$ \\
\end{tabular} \\
\hline \multicolumn{8}{|c|}{ Feeling of security and belonging } \\
\hline \multicolumn{8}{|c|}{ Close relationships with other people } \\
\hline \multicolumn{8}{|c|}{ Secure environment } \\
\hline \multicolumn{8}{|l|}{ Enjoying life } \\
\hline \multicolumn{8}{|c|}{ Enjoying the world and life } \\
\hline \multicolumn{8}{|c|}{ To be recognised and respected } \\
\hline \multicolumn{8}{|c|}{ To perform well and achieve something } \\
\hline \multicolumn{8}{|c|}{ Self-fulfillment and self-development } \\
\hline \multicolumn{8}{|c|}{ An exciting, varied life } \\
\hline \multicolumn{8}{|l|}{ Social justice } \\
\hline \multicolumn{8}{|c|}{ Respect for fellow humans } \\
\hline \multicolumn{8}{|l|}{ Maintain traditions } \\
\hline Earn a lot of money & & & & & & & \\
\hline
\end{tabular}


10. Please check two values that you consider are the main important in your life

\begin{tabular}{|l|l|l|}
\hline & Important & Not important \\
\hline Respect for fellow humans & & \\
\hline A job which fulfils me & & \\
\hline Self-fulfillment and self-development & & \\
\hline An exciting, varied life & & \\
\hline To perform well and achieve something & & \\
Maintain traditions & & \\
\hline Social justice & & \\
\hline Readiness to help people in need & & \\
\hline Feeling of security and belonging & & \\
\hline Secure environment & & \\
\hline Environmental protection & & \\
\hline Enjoying the world and life & & \\
Earn a lot of money & & \\
\hline Close relationships with other people & & \\
\hline To be recognised and respected & & \\
\hline
\end{tabular}

11. Where did you grow up?

\begin{tabular}{|l|l|l|l|l|l|}
\hline & In a bigger & In a small city & In a bigger city & $\begin{array}{l}\text { In a } \\
\text { metropoli }\end{array}$ & $\begin{array}{l}\text { In a } \\
\text { metropoli } \\
\text { more than }\end{array}$ \\
$\begin{array}{l}\text { In a small village } \\
\text { (less than } 500\end{array}$ & village $(501$ & $(5,001-$ & $(20,001-$ & $(100,001-$ & more \\
habitants) & habitants $)$ & 20,000 & 100,000 & 500,000 & 500.000 \\
& habitants $)$ & habitants $)$ & habitants $)$ & habitants \\
\hline & & & & & \\
\hline
\end{tabular}

12. Where do you live now?

\begin{tabular}{|c|c|c|c|c|c|}
\hline $\begin{array}{l}\text { In a small village } \\
\text { (less than } 500 \\
\text { habitants) }\end{array}$ & $\begin{array}{l}\text { In a bigger } \\
\text { village ( } 501- \\
5,000 \\
\text { habitants) }\end{array}$ & $\begin{array}{l}\text { In a small city } \\
(5,001-20,000 \\
\text { habitants })\end{array}$ & $\begin{array}{l}\text { In a bigger city } \\
(20,001- \\
100,000 \\
\text { habitants })\end{array}$ & $\begin{array}{l}\text { In a metropoli } \\
(100,001- \\
500,000 \\
\text { habitants })\end{array}$ & $\begin{array}{l}\text { In a metropoli } \\
\text { more than } \\
500.000 \\
\text { habitants }\end{array}$ \\
\hline & & & & & \\
\hline
\end{tabular}


13. How many people are living at home?

14. Do you live with? I live ....

\begin{tabular}{|c|c|c|c|c|c|c|c|c|}
\hline Alone & $\begin{array}{c}\text { Alone, the } \\
\text { children } \\
\text { moved }\end{array}$ & $\begin{array}{l}\text { With couple } \\
\text { and children }\end{array}$ & $\begin{array}{c}\text { With couple } \\
\text { and without } \\
\text { children }\end{array}$ & $\begin{array}{c}\text { With couple, } \\
\text { the children } \\
\text { moved }\end{array}$ & Single parent & $\begin{array}{l}\text { In a sharing } \\
\text { appartment }\end{array}$ & With parents & With children \\
\hline & & & & & & & & \\
\hline
\end{tabular}

15. How many children are living at home?

16. Could you say us what is approximately the total household net incomes monthly in $€$ ? (Please check one box)

\begin{tabular}{|l|l|l|l|l|l|l|l|}
\hline$<900 €$ & $900-$ & $1.500-$ & $2.000-$ & $3.200-$ & $4.500-$ & & No \\
& $1.499 €$ & $1.999 €$ & $3.199 €$ & $4.499 €$ & $5.499 €$ & $>5.500$ & answer \\
\hline
\end{tabular}

17. Could you say us what is approximately your disposal incomes monthly in $€$ ? (Please check one box)

\begin{tabular}{|l|l|l|l|l|l|l|l|}
\hline$<900 €$ & $900-$ & $1.500-$ & $2.000-$ & $3.200-$ & $4.500-$ & & No \\
& $1.499 €$ & $1.999 €$ & $3.199 €$ & $4.499 €$ & $5.499 €$ & $>5.500$ & answer \\
\hline & & & & & & & \\
\hline
\end{tabular}

18. Can you estimate how much do you spend per week in the purchasing of food? 